\title{
Vadose Zone Modeling Workshop Proceedings, March 29-30, 1993
}

\author{
R. Khaleel
}

Date Published

August 1993

Prepared for the U.S. Department of Energy Office of Environmental Restoration and Waste Management

(28) Westinothouse $\begin{aligned} & \text { P.O. Box } 1970 \\ & \text { Hantord Compamy }\end{aligned}$ 


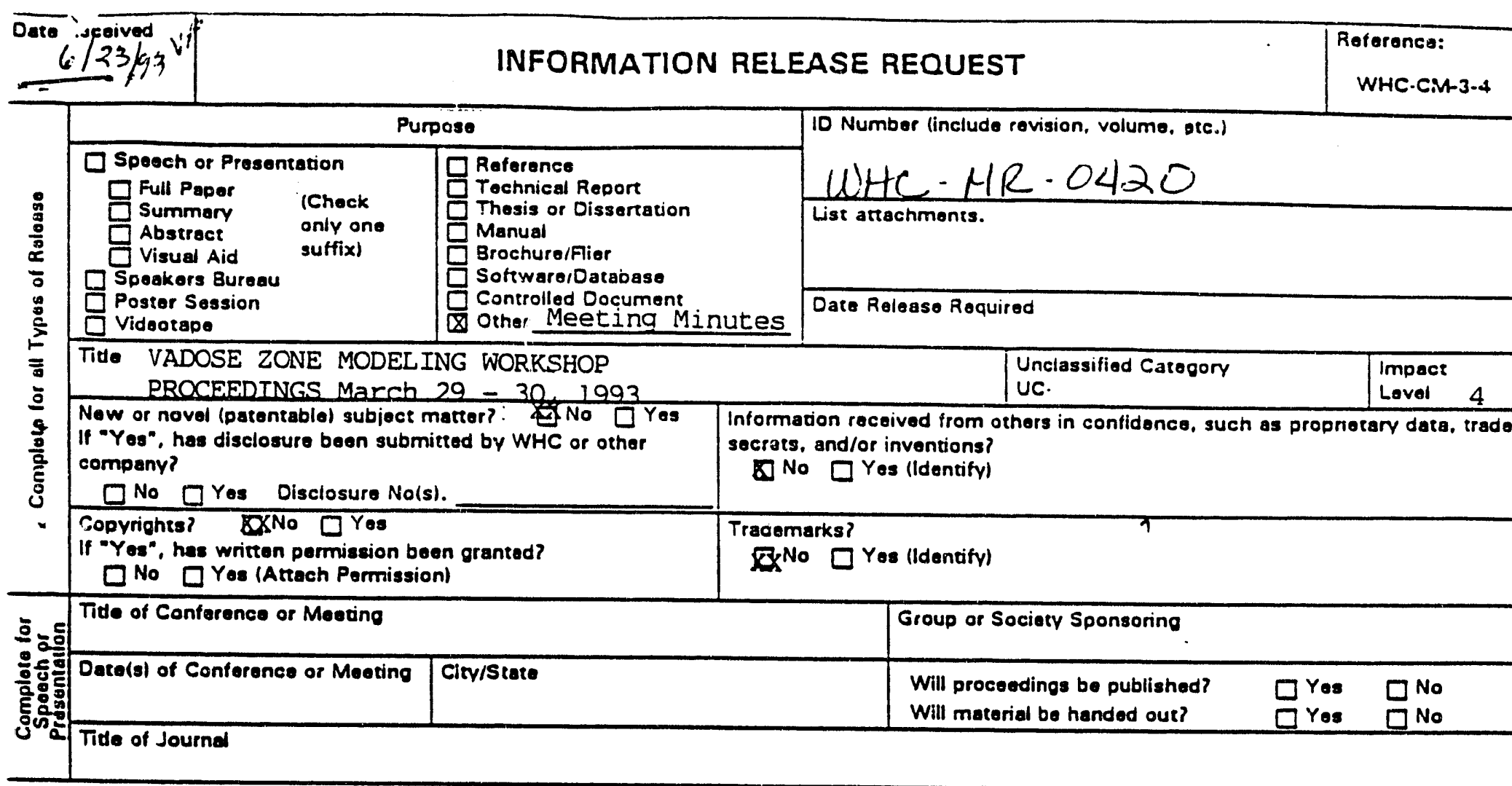

\section{CHECXLIST FOR SIGNATORIES}

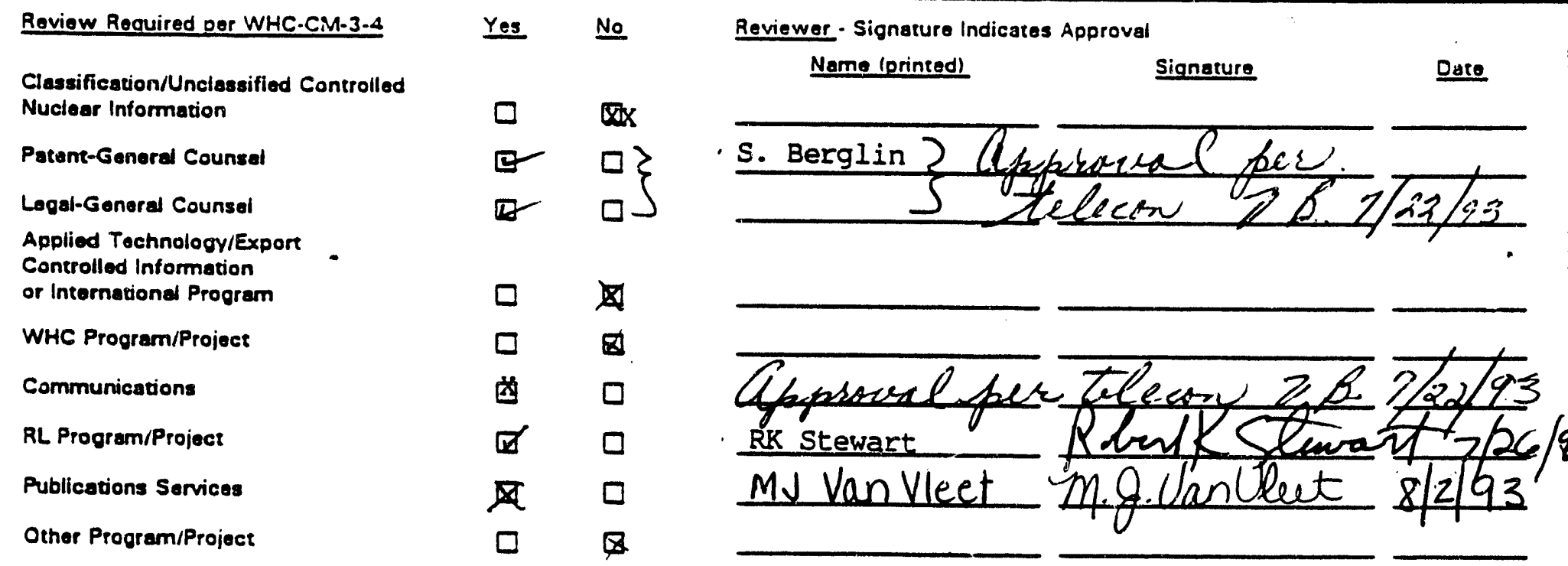

Information conforms to all applicable requirements. The above information is cercified to be correct.

Reforences Available to Intended
Audience

Author/Requestor (Printed/Signatura)

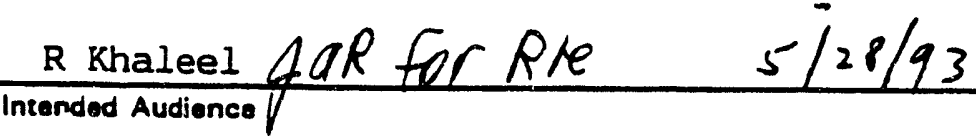

$\square$ Intarnal $\square$ Sponsor Xexternal

Responsible Manager (Printed/Signature)

Dato
INFORMATION RELEASE ADMINISTRATION APPROVAL STAMP

Stamp is required before release. Release is contingent upon resolution of mandatory comments.

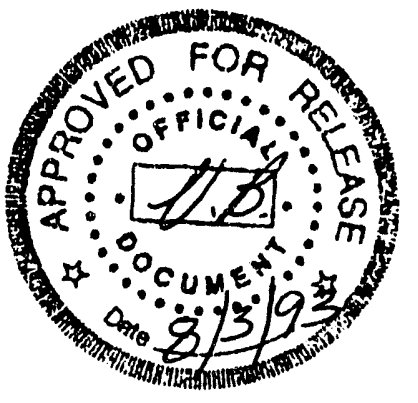

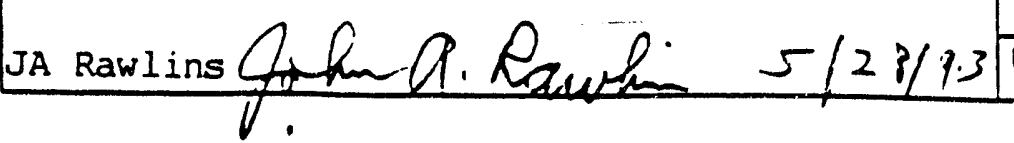
Date Disepproved 
WHC-MR-0420

Document Title: Vadose Zone Modeling Workshop Proceedings, March 29-30, 1993

Prepared by:

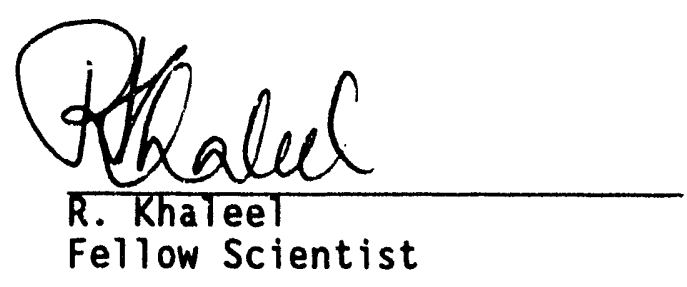

Approved by:
Gl Q. Ravelin

. A. Rawlings, Manager

Environmental Risk \& Performance Assessment
$7 / 20 / 1993$

Date

$\frac{7 / 28 / 93}{\text { Date }}$ 


\section{VADOSE ZONE MODELING WORKSHOP}

\section{BACKGROUND}

At the Hanford Site, the record of decision for remediation of CERCLA sites is largely based on results of the baseline risk and performance assessment of the remedial action alternatives. These assessments require the ability to predict the fate and transport of contaminants along appropriate exposure pathways which, in case of the Hanford Site, includes the migration of contaminants through the vadose zone to the water table. Listed below are some of the requirements, as prescribed by the regulators, relative to CERCLA risk and performance assessment at Hanford.

- "Assumptions and approximations used in the models or input data must be clearly documented by a thorough audit trail and the predictive uncertainties associated with these approximations should be quantified. This will aid in the understanding of model results and will provide a framework in which the predictive models can be improved as more and better data are collected and a better understanding of the site evolves."

"Data input to predictive models shall be of a quality acceptable to the regulators and of sufficient quantity to statistically describe spatial and temporal variability. This requirement will assure that uncertainty due to variable input can be quantified and, as more and better data are collected, gradually reduced."

The information required in support of site characterization and risk assessment tasks for the RI/FS work at various operable units includes, among others, a characterization of soil hydraulic properties and development of vadose zone flow and transport models. Due to the broad scope and complexity of these tasks and their application to most, if not all, operable units, they fall outside the scope of any individual operable unit RI/FS work plan and are better addressed in a well-organized, comprehensive, and coordinated site-wide effort. The tasks identified in the TPA milestone M-29-02 document, "Groundwater Model Development Plan in Support of Risk Assessment, "DOE/RL-9162, December, 1991 are in support of the requirements prescribed by the regulators.

A workshop was organized by the Environmental Risk and Performance Assessment Group, Westinghouse Hanford Company on March 29-30, 1993 at the Richland Best Western Tower Inn. During the workshop, an assessment was made of the need for and scope of various tasks being conducted or planned as part of the Hanford Site waste isolation performance assessment/risk assessrient activities. Three external, nationally-recognized experts served as part of a review panel for the workshop: a. Professor Lynn Gelhar of MIT, b. Professor Peter Wierenga of University of Arizona, and C. Dr. Rien van Genuchten of U.S. Sal inity Laboratory, Riverside, Cal ifornia. The technical experts provided their perspectives on the current state-of-the-art in vadose zone flow and transport modeling. In addition, the technical experts provided an outside independent assessment of the work being performed or planned in support of various activities identified in TPA Milestone M-29-02. 
This document includes the following:

o Recommendations from the three peer reviewers.

$0 \quad$ Areas of expertise of the three peer reviewers.

$0 \quad$ Workshop agenda.

- Copies of viewgraphs (where availabie) from presenters at the workshop.

- Workshop minutes.

o List of workshop attendees. 
WHC-MR-Oิaิ20

\section{FiECOMMENDATIONS FROM THE THREE PEER REVIEWERS}


WHC-MR-0420 
United States

Department of Agriculture
Agricultural

Research

Service
Pacific West Area

U.S. Salinity Laboratory 4500 Glenwood Drive Riverside, CA 92501

Tel. 909-369-4847

Fax. 909-369-4818

April 13, 1993

Dr. Raz Khaleel

Westinghouse Hanford Co.

P. O. Box 1970

Mail Stop H4-14

Richland, WA 99352

Dear Raz:

Enclosed is my report about the Vadose Zone Modeling Workshop. I trust that my comments are interpreted in a constructive manner and eventually will help to improve interactions and productivity at the site.

Please let me know how else, at any point in the future, I can help your program at WHC. Again, I enjoyed the discussions in Richland and hope that something constructive will come out of the meeting.

Sincerely yours,

Rién van Genuchten

Research Leader

Enclosures 
Comments by $M$. Th. van Genuchten regarding the

\title{
VADOSE ZONE MODELING WORKSHOP
}

\author{
ENVIRONMENTAL RISK AND ASSESSMENT GROUP \\ WESTINGHOUSE HANFORD COMPANY \\ RICHLAND, WA
}

March 29-30, 1993

\begin{abstract}
My comments below are based on information provided in TPA Milestone M-29-02 document "Groundwater Model Development Plan in Support of Risk Assessment, "DOE/RL-91-62 (December 1991), and on information given by investigators during the above two-day workshop (March 29-30, 1993). This material was insufficient for me to get a clear view of the scope and depth of all activities. Hence, several of my comments are of a very preliminary and somewhat superficial nature. Nevertheless, I hope that they will benefit ongoing and planned activities at the Hanford Site. Several comments directly or. indirectly address organizational and program integration issues, while others focus on more specific technical issues.
\end{abstract}

1. From the presentations at the workshop it is clear that some excellent basic and applied research is being carried out, especially in the site characterization and mocieling areas. Creative work is clearly needed to conceptually understand, measure and model water flow and chemical transport at Hanford in view of the deep and highly heterogeneous unsaturated zone. Expertise to carry out the necessary work is certainly available among scientists and engineers at Westinghouse Hanford Company and its collaborators including Pacific Northwest Laboratories.

While the expertise is available, there appears to be a lack of coordination and integration of site-wide activities. Improved coordination appenrs needed both "horizontally" across institutional and disciplinary barriers among the researchers sitewide (Comment No. 2), and "vertically" in a program integration sense involving researchers, managers and especially regulators (No. 3).

2. Informal technical meetings or internal seminars, including workshops such as the present Vadose Modeling Workshop, are important mechanisms for interaction and feedback to, and dialogue among, scientists and engineers at what I would like to call the "grass-roots" level. Such opportunities for information exchange at the grass-roots level should be provided on a regular (e.g., monthly) basis, and possibly with input and scrutiny from outside researchers. The purpose of such meetings is not to satisfy regulatory milestones, but to provide feedback to, and interaction among, scientists and engineers. They provide a mechanisms to identify and address shortcomings in current research plans and procedures related to specific topics, e.g.; recent developments in modeling, coordination of computer codes, review of instrumental methods, clarify data needs for modeling purposes, and provide informal reviews of progress. It is imperative that the ciscussions be relatively open to, and at times with active participation from 
outside researchers. I believe that such informal grass-roots interaction will increase research productivity and effectiveness, and will also increase morale among the scientific staff.

3. Comment 2 pertains to interaction among scientists. In addition, I believe that there is a major need for improved site-wide program integration. Since multiple groups, institutions, and interests are involved (WHC, PNL, USGS, on-site and off-site contractors, scientists, managers, regulators), effective interaction and communication between these different parties is critical. In part because of institutional barriers, there appears to be a lack of coordination among scientists involved in field data collection activities, laboratory analyses of field samples, and modeling efforts. Lack of coordination seems to be exacerbated by a lack of communication between researchers and regulators. Researchers should be given a high degree freedom to interact with peers across institutional and disciplinary barriers. Examples which could benefit from improved coordination are area-wide characterization of the unsaturated hydraulic properties (see also comment No. 4), the modeling efforts (No. 5), and improved communication between modelers and experimentalists. It seems that at present no specific individual or group is responsible for this type of integration and communication. Hence, I strongly recommend that some type of area-wide coordination or oversight committee be put into place. It is imperative that researchers, managers and especially regulators representing different groups participate in such an oversight committee.

It is crucial for the site-wide oversight/coordination committee to realize that the ultimate goal of activities at the Hanford site is the long-term safe management and/or cleanup of radioactive and other wastes, and that these goals may go beyond satisfying certain CERCLA RI/FS procedures and TPA Milestones. The committee should ensure that data collection and modeling activities are commensurate with each other. The ultimate goal of a data collection program is not to satisfy a long list of data appearing in a pre-determined list for the RI/FS process, but for use by modelers to make risk assessments, predict future events, or investigate the appropriateness of alternative remediation strategies. In other words, data collection, sample analysis and use by modelers is an ongoing dynamic process which requires continued and intense interaction.

The committee can help immensely by facilitating communication among scientists sitewide as advocated under comment No. 2, and especially between scientists and regulators. While "data input to predictive models shall be of a quality acceptable to the regulators...", it is imperative that they also be acceptable to the scientific community at large. I am afraid that if the broader approach is not taken now, it is not unlikely that eventually the entire CERCLA RI/FS process and the usefulness of TPA milestones will be questioned. Acceptance by the scientific community at large requires scrutiny and feedback from the scientific community. This also means the participation of Hanford scientists in professional meetings and publication of research results in the 
open literature. Payoff of such an open perspective are easily documented.

I am stressing these points in part also because of my impression that much work is being done to physically get soil samples from the field, but that a comprehensive analysis of the samples and their use in RI/FS modeling is not commensurate with the expensive field sampling program (notable regarding the deep drilling effort).

4. This comment relates to hydraulic properties measurements. Hydraulic property estimation is an area of work where area-wide coordination is a especially important. A wealth of high-quality data is currently being collected, but by different groups, for different purposes, and for different waste sites. I propose that a site-wide hydraulic or soils database management system be established. This database should be continually updated and regularly distributed among the various parties and researchers involved (including modelers, field people, laboratory personnel). The database should contain all measured hydraulic and related data such as the measured particle-size distributions and other information affecting the hydraulic properties.

The database eventually may yield enough information to correlate the hydranlic properties with soil textural information at the Hanford site. While fractal approaches appear useful, other approximate indirect methods also exist which correlate the hydraulic properties with soil texture and related properties such as organic matter content, bulk density, and soil mineralogy (Rawls et al., 1992; Baumer, 1992, Carsel, 1992). Again, a site-wide database is a prerogative for such an effort.

Area-wide coordination of hydraulic measurements also will permit comparisons of different methods for measuring the hydraulic properties of soils, e.g., predicted results based on measured retention data (Khaleel), and measured results obtained using Tempe-cell type samples (Relyea), and the centrifuge method (Conca).

Some other comments related to the hydraulic properties:

a. Hydraulic properties currently are measured mostly on small cores. I believe that it is important that eventually a field experiment be carried out to measure the unsaturated soil hydraulic properties in situ. The standard approach would be to implement a instantaneous profile type (or gravity drainage) experiment, although other methods are also availabie. Several promising scaling procedures (Shouse et al., 1992; Vogel et al., 1991) are now available for analyzing the hydraulic properties of layered or otherwise heterogeneous soil profiles. Although the in-situ experiments are generally limited to measurements in the upper few meters of soil (they could be easily extended to deeper layers at Hanford), they should give more realistic field estimates of the hydraulic properties, and provide a comparison with the core measurements in the laboratory. The field experiment could be implemented using neutron probes, tensiometers were applicable (may not work well in gravel), TDR, and other instrumentation. 
b. There should be a site-wide delineation/inventory of soil textural heterogeneities and their hydraulic characterization using a combination of direct and indirect measurements. Some of the small core hydraulic data by Conca show extensive vertical differences in hydraulic conductivity. This information could be extrapolated laterally using information of soil textural stratification and, with some additional theoretical work, provide a means for estimating the anisotropy in the macroscopic scale hydraulic conductivity. The bottom line here is to synthesize/extract as much information as possible from available field samples, especially the very expensive samples coming from the deep boreholes. Again, this effort requires some creative and publishable developmental research.

c. The accuracy of the predicted hydraulic conductivity functions obtained with the van Genuchten-Mualem equations should be validated at low water contents. The predicted conductivity equations should be matched with a measured value (or measured values) at relatively low water contents (data of the type collected by Conca provide appear excellent for this purpose). Matching the equations at saturation is not realistic because (1) the saturated hydraulic conductivity, $K_{s}$, may be a poor indicator of unsaturated hydraulic conductivity at low water contents because of the dominant effects of soil structure on $K_{s}$ and (2) the need for extrapolating to conductivities close to the average recharge rates at the Hanford Site. Some of these issues are discussed by Luckner et al. (1989).

d. An accurate description of the hydraulic properties at low water contents is important. A two-phase (air-water) flow description may be needed for the prevailing water contents at Hanford. If the single-phase Richards' equation is used, problems surrounding the residual water content $\left(\theta_{r}\right)$ must be addressed. I know that some work in this area has been done by Gee and colleagues at PNL, or is being done, but this was not discussed at the workshop.

e. Anisotropy in the unsaturated hydraulic conductivity may not be an important issue for steady-state vertical flow simulations. However, anisotropy is important for single high-intensity point infiltration events when the soil profile controls the infiltration rate (e.g., spills and leaks such as those at the 241-T-106 tank site).

f. Similarly, very precise estimates of the hydraulic conductivity may not be needed for long-term flow simulations close to steady state. The most important issue is then having accurate estimates for the recharge rate $\left(q_{0}\right)$ and precise estimates of the effective water contents throughout the vadose zone.

5. Area-wide coordination is also needed in the modeling area. It may be worthwhile to implement a centralized modeling group which continually improves and updates adopted computer codes, and which provides support to smaller units involved in the application of models to specific waste sites. It is my feeling that the use of publicdomain codes should be preferred (e.g., the PORFLO set of codes), so that there is in- 
house expertise and less reliance on outside contractors. This will also facilitate a more immediate and expeditious adaptation of the models to the various waste sites. Again, this approach presumes that institutional barriers can be eliminated or kept to a minimum.

6. This comment addresses model validation and calibration. First of all, the possibility of true model validation is increasingly being questioned in the scientific literature. I believe that TPA Milestone M-29-02 document (DOE/RL-91-62) provides a realistic assessment of what can be done in the groundwater and vadose zone modeling area, except that the term "validation" should not be interpreted in the most strictest sense $n$ f culy being able to validate a particular model. The usefulness of the term "model validation" has justifiable been questioned (e.g., Konikow and Bredehoeft, 1992), although not by all (Maleszewski and Zuber, 1992; Beven, 1989). At a minimum, there should be a clear distinction (Narasimhan, 1987) between model verification (accuracy of the numerical methods used for solving certain differential or other equations), and model validation (accuracy ard applicability of conceptual processes embedded in the adopted differential equations).

With all its shortcoming, it appears that the Richards' equation is still the model of choice for simulating variably-saturated flow, although variants are possible (e.g., for two-phase flow simulations involving water and air at very low water contents, or stochastic approaches). Some of the processes which likely will not be included accurately in the near future are, among other things, macropore flow, unstable flow at soil textural interfaces, small-scale heterogeneities; chemical and temperature effects on hydraulic properties (notable for liquids leaked from the tanks, two-phase air-water flow at low waier contents), and root growth and root water uptake as a function of water stress. These complexities show that one must remain modest about model predictions, and that a high level of uncertainty will remain. It also means that model validation is a fairly site-specific exercise and, unless demonstrated otherwise, should hold only for similar initial and boundary conditions (e.g., similar recharge rates) as used for the calibration exercise.

The best use of models, then, is to consider them to be pragmatic tools in research and management. Ultimately, models are the most effective tools for geiting a better understanding of the unsaturated zone at the Hanford site. Any prediction should be treated with some suspicion, yet as being the best avaiiable estimates, subject to continuous adjustment. No model should ever be declared "valid". This is because no model, however sophisticated, will ever represent reality since a model, by definition, is an approximation of reality.

For the above reasons, model calibration at the Hanford site should be a continuous, never-ending effort. An excellent example of model calibration involves the work by Gee and colleagues at PNL on estimation recharge (see also Comment No. 9). This type of model calibration and model use (not validation) could provide a good example 
for other activities (e.g., modeling and model calibration at the tank farm).

8. The tailing (elution) part of the nitrate distributions below the 241-T-106 tank leak site may provide unique data for model calibration.

9. Current work by Gee and colleagues at PNL on recharge measurements (and related model calibration) is critical and should continue with a high level of priority. Real payoffs of this work is (was) only possible after many years or decades of persistent work. This research is now showing significant results. Perhaps different tracers could be added (e.g., $\mathrm{Cl}$ or $\mathrm{Br}$ at intervals of several years) to provide additional information many years from now. I disagree with Gee's disappointment that suddenly recharge was observed, but not "predicted" with the previously calibrated Richards' equation. Inis discrepancy does not validate or invalidate Richards equation (we know that Richards' equation has its problems but, again, it may be the best model available at present). I advocate a more pragmatic approach. The issue here is not the "validity " of the Richards' equation but how best to use this equation for analyzing the data, and then to extrapolate in time (or space), i.e., its use as a long-term management tool. Model calibration should continue in this case also because the initial calibration did not include time periods during which recharge was high. [Was there some preferential flow in the lysimeters? What about along the walls? Or could it be that the lysimeters were not completely at equilibrium until this relatively wet winter?].

10. Actual water contents should be used when simulating transport in the surface sandy gravel systems near tank site 241-T-106. While water contents and hydraulic properties may be measured without the gravel and stones, they must be corrected before their use in the actual simulations, Otherwise they will underestimate the actuai pore-water velocities $\left(v=q_{0} / \theta\right)$ in the simulations of contaminant transport below the leaking tank site.

11. A do-nothing baseline attitude may be unacceptable for certain situations. This is especially true for the high infiltration situation at the 241-T-106 tank leak site. The surface silty sandy gravel backfill layer induces $5-15 \mathrm{~cm}$ recharge per year, while the sloping (?) tank surface may further increase point recharge. This situation irreversibly promotes infiltration and increases the rate of transport of radioactive and chemical wastes into and through the vadose zone. This issue is a high-priority one and should be addresses without delay. Common sense says that some action is needed here.

12. Modeling radionuclide transport using the $K_{d}$ approach may not be accurate. For example, Cs transport may be controlled by major ion chemistry. Complexing with chelating agents/solvents such as EDTA may lead to significantly lower $K_{d}^{\prime}$ s, and hence increased transport rates. Also, retardation factors $(R)$ are functions of the water content and should not be taken constant over the whole profile or versus time. 


\section{References:}

Baumer, O. W. 1992. Predicting unsaturated hydraulic parameters. In: M. Th. van Genuchten, F. J. Leij and L. J. Lund, Indirect Methods for Estimating the Hydraulic Properties of Unsaturated Soils, pp. 341-354, University of California, Riverside.

Carsel, R. 1992. Development of agricultural soils database for estimating soil water retention characteristics. In: M. Th. van Genuchten, F. J. Leij and L. J. Lund, Indirect Methods for Estimating the Hydraulic Properties of Unsaturated Soils, pp. 369-378, University of California, Riverside.

Beven, K 1989. Changing ideas in hydrology - The case of physically-based models. J. Hydrol. 105:157-172.

Konikow, L., and J. D. Bredehoeft. 1992. Ground-water models cannot be validated. Adv. in Water Resour., 15: 75-83.

Luckner, L., M. Th. van Genuchten, and D. R. Nielsen. 1989. A consistent set of parametric models for the two-phase flow of immiscible fluids in the subsurface. Water Resour. Res. 25:2187-2193.

Maloszewski, P., and A. Zuber. 1992. On the calibration and validation of mathematical models for the interpretation of tracer experiments in groundwater. Adv. Water Resour., 15:47-62.

Narasimhan, T. N. 1987. Some thoughts on mode! verificution. In: D. D. Evans and T. J. Nicholson (eds.), Flow and Transport Through Unsaturated Fractured Rock, pp. 185187, Geophys. Monogr. 42, Am. Geophys. Union, Washington, DC.

Rawls, W. J., L. R. Ahuja, and D. L. brakensiek. 1992. Estimating soil hydraulic properties from soils data. In: M. Th. van Genuchten, F. J. Leij and L. J. Lund, Indirect Methods for Estimating the Hydraulic Properties of Unsaturated Soils, pp. 329-340, University of California, Riverside.

Shouse, P. J., J. B. Sisson, T. R. Ellsworth, and J. A. Jobes. 1992. Estimating in situ unsaturated hydraulic properties of vertically heterogeneous soils. Soil Sci. Soc. Am. J. 56:1773-1679.

Vogel, T., M. Cislerova, and J. W. Hopmans. 1991. Porous media with linearly variable hydraulic properties. Water Resour. Res., 27(10):2735-2741. 
LYNN W. GELHAR

HYDROLOGIC CONSULTANT

41 NASHOBA ROAD

CONCORD, MASSACHUSETTS 01742

TELEPHONE (617) 369.0427

\section{MEMO}

TO: Raz Khaleel, WHC, H4-14 FROM: Lynn Gelhar RE: Vadose Zone Modeling Wdesfoghop, 29-30 March

Enclosed are copies of the transparencies I used during my talks at the workshop. In the following I have outlined a few quick points reflecting my initial impressions about the material presented at workshop. Please recognize that these comments are based on the limited written information. I may alter some of these points after having reviewed more explicit data and results.

1) Judging from the 200-BP-1 modeling effort, the parameters for the vadose zone modeling are not being derived in any systematic way from the field data on unsaturated hydraulic properties which are being collected at that site. The selection of parameters for the presumed homogeneous zones seems to be largely arbitrary and subjective, and consequently the model does not seem very defensible to me.

2) The elaborate model being used in the 200-8P-1 simulations seems to be far too complex and basically is incompatible with the crude input parameters which are being used. It would be more reasonable to begin with a series of simplified screening models of increasing complexity which are used systematically to identify the dominate transport process for this site.

3) It doesn't seem that the site characterization/data collection effort at the 200-BP-1 site is being designed at all to meet the needs of the modeling work. It seems to me that there should be much closer coordination between the field sampling activities and the modeling zi乇ะr:s.

$\because$ a zrachasz: framework to design tiela sampling programs, Evaluate 


\section{R. Khaleel}

4 April 1993

page 2

model parameters from small-scale field data, evaluate predictive capabilities of models, and assess model uncertainty would seems to be a much more defensible framework than the largely ad hoc and unrelated approaches which are currently being used to address these issues. The stochastic approach continues to be controversial, but I don't see any serious alternatives being put forth to address these issues in a unified
fashion.

5) The program to measure unsaturated hydraulic conductivity is producing important new data that are essential if you are to be able to predict multidimensional transient flow and transport. The data clearly show that the standard van Genuchten/Mualem method (based on saturated conductivity and moisture retention data) fails dismally as a predictor of unsaturated hydraulic conductivity. This is in line with our experience and strongly suggests that unsaturated hydraulic conductivity must be measured directly.

6) The 200-BP-1 site seems to be marginal as a case study for model evaluation (validation); the currently available site characterization data are not likely to be adequate for this purpose.

$\therefore$ The Injection Test Site experiment has a much better prospect of being useful for model evaluation; this effort should be continued and expanded. Some additional drilling to assess current moisture (neutron logging) and solute (cores) distributions would allow evaluation of independently developed model predictions.

3) Reliable predictions of recharge are central to most of the vadose zone inodeling efforts. Continued field experimentation at the Hanford site on this problem is very important.

9) Formal and informal communication, coordination and ccoperation among those working on the vadose zone at Hanford should be enhanced. The adose zone seminar suggested by Pete Wierenga would certainly be worthwnile. 
WHC-MR-0420

Dr. John A. Rawlins, Manager

Environmental Risk and

Performance Assessment

Westinghouse Hanford Company

P.O. Box 1970

Richland, WA 99352

Dear Dr. Rawlins:

Please find enclosed my outside, independent assessment of the activities of the Environmental Risk and Performance Assessment Group of Westinghouse Hanford Company as presented at the Workshop on March 29 and 30, 1993.

I don't believe that I ever got the Tri-party agreement milestone M-29-02 document and so I cannot comment on this document.

I did get the "Groundwater Model and Development Plan in Support of Risk Assessment" published December, 1991. This document is well written and complete and shows a good insight in models, model development, and validation issues.

If you have further questions on my report of the Workshop, please contact me at my office in Tucson, (602) 621-7228).

Sincerely yours,

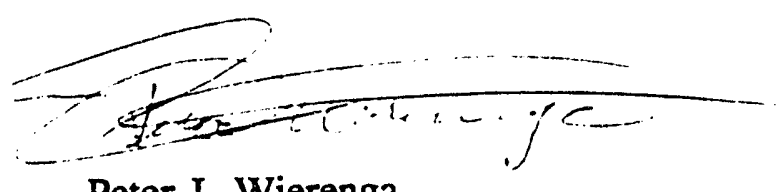

Peter J. Wierenga

Professor and Head of Department

PJW:jer

Enclosure 


\section{Comments by Peter J. Wierenga, University of Arizona Third Party Review \\ Environmental Risk and Performance Assessment Group Westinghouse Hanford Company}

March 29 and 30, 1993

The purpose of the Workshop was to review the activities of the Environmental Risk and Performance Assessment Group of Westinghouse Hanford Company. Many of the activities conducted by this group were represented in a clear and adequate way. However, this reviewer would have greatly benefited from an overview at the beginning of the workshop of the waste disposal problems at the Hanford site, before the presentations of individual programs. A map showing the major problem areas would have been most helpful. Without such an overview, it becomes diffirult for outside reviewers to place a particular site within the context of the waste disposal problems at the Hanford site.

It would have also been most useful to have a high official of DOE give a presentation on what DOE plans to do, over the next several years, about improving the waste situation at the Hanford site. Without such an overview, it appeared that the present studies are done somewhat in isolation and that the group is working in a vacuum. This comment is similar to the comment made by Dr. Weeks during the third-party technical review conducted in 1989. In the 1989 report, Dr. Weeks commented that a well-thought out staged data collection program in support of site-wide system understanding seems to be lacking. From the two-day workshop in March, 1993, it is not clear that such a well-thought out data collection program is now in place, although considerable data on vadose zone hydraulic properties are now being generated.

A second comment, which was also made by Dr. Weeks in 1989, is that people responsible for data collection in the field or in the laboratory are not closely working with people responsible for model development and testing. In programs such as these, it is absolutely imperative that modelers and experimentalists work closely together. The experimentalists need to know which data are required for the various models and the modelers need to know which data realistically can be expected from the experimentalists.

The lack of interaction between modelers and experimentalists seems to go beyond just these two groups. This reviewer perceived a lack of interaction between scientists operating at the Hanford site. Collaboration between various scientists at Westinghouse and at PNL also needs to be encouraged. This can be accomplished by seminars, workshops, and funding of cooperative projects. For example, a seminar series on vadose zone hydrology could be considered.

More specifically, the following:

1. Modeling should be site specific. It might also be useful to employ simplified models including analytical solutions, as first approximations to understanding the phenomena observed at a particular site. Many analytical and numerical models already exist, which 
observed at a particular site. Many analytical and numerical models already exist, which could be used with good success without much modification. Lynn Collard and John Smoot showed good use of existing models.

2. In applying models to existing data sets, one should not only look at the retarded species such as cesium or strontium, but also at nitrates. Apparently large amounts of nitrates were disposed of in the cribs. The tail ends of the nitrate breakthrough curves may reveal a lot about the displacement of nitrate containing solutions by nitrate-free solution after the cribs were no longer used. This would give insight into total amount of water percolating through the cribs. The same should be done for tritium.

3. Rather than drilling expensive holes at a limited number of sites, I would prefer to have small diameter holes drilled for neutron access tubes, to measure the distribution of water below cribs or leaking tanks. The depth distribution of water under a tank or crib, is of importance for modelling solute transport to the groundwater. During installation of neutron access tubes samples could be collected and analyzed for tritium, nitrate, cesium, strontium and other elements.

4. The group seems to have made good progress in characterizing the unsaturated hydrologic properties of the soils at a number of sites. The data from these characterizations will be useful in vadose zone predictions of flow and transport. It is recommended, however, to consider using faster methods for determining the unsaturated hydraulic conductivity as a function of water content or tension. In particular, the upward infiltration method and the centrifuge method should be looked at in a systematic manner. Although neither of these methods have been around very long, they look promising and will greatly improve the speed with which the data can be obtained.

5. Dr. Glendon Gee has done an outstanding job in collecting data on recharge at the Hanford site. This work should be supported and continued for many years. Recharge is the driving force for contaminant movement to the groundwater table and a clear understanding of recharge under the various soil and vegetation conditions at the Hanford site is of great importance.

6. Scenaios should be developed to cover the cribs and tank sites to reduce recharge at these sites. This work should be started as soon as possible to reduce further pollution of groundwater at the Hanford site. Models such as UNSATH which take into account evaporation and evapotranspiration could be used for refining the various scenarios.

7. It would be useful to test all models used at the Hanford site with data from the Las Cruces Trench Site. Several scientists are presently testing their model with data from the Las Cruces Trench Site. Testing of modeis used at the Hanford site on this data set would give there models further credibility. 
8. For model testing I prefer experiments on non-contaminated sites. Such an experiment should be done on a site where full control over the boundary and initial conditions is available and where the amount of water and solute added to the site can be precisely controlled. Such a site would also require adequate characterization of the physical and chemical properties before such a model testing experiment could be initiated. Testing a model at an existing site such as at a leaking crib site or a leaking tank site, is basically curve fitting, unless predictions are made and data are collected over time (e.g. 5-10 year intervals). 
WHC-MR-0420

\section{AREAS OF EXPERTISE OF THE THREE PEER REVIEWERS}


WHC-MR-0420

This page left intentionally blank. 


\title{
VADOSE ZONE MODELING WORKSHOP
}

Panel members were carefully selected to provide a broad group of nationally and internationally recognized experts with individual and collective experience in stochastic hydrology, vadose zone hydrology, and soil physics. Further, members were selected to assure independence and, therefore, none were selected from Westinghouse, prime contractors at other U.S. Department of Energy Sites, or the U.S. Department of Energy.

Suggestions for panel members were provided by Westinghouse Hanford Company professionals and other nationally-known experts in the disciplines of interest. The technical areas of interest, the selected individuals, and their exclusive unique capabilities are as follows.

1. Lynn W. Gelhar

\author{
Raiph M. Parsons Laboratory \\ Department of Civil \& Environmental \\ Engineering \\ Massachusetts Institute of Technology \\ Cambridge, MA 02139
}

Dr. Gelhar is a nationally and internationally recognized expert in stochastic groundwater hydrology. He is currently a professor at the department of Civil and Environmental Engineering, Massachusetts Institute of Technology. Dr. Gelhar has been awarded numerous research grants in the area of stochastic applications in groundwater hydrology by U.S. Environmental Protection Agency, National Science Foundation, Nuclear Regulatory Commission, United States Geological Survey, American Petroleum Institute, National Institute for Environmental Health, and Tennessee Valley Authority. He has received several highly-prestigious awards and honors from technical and professional societies. Dr. Gelhar has published extensively in peer-reviewed journals such as Water Resources Research, American Society of Civil Engineers, and Ground water. His unique expertise in stochastic applications was of special interest to the vadose zone modeling workshop in addressing issues such as uncertainties in estimates of groundwater travel times and radionuclide release rates.

2. Peter J. Wierenga

Department of Soil and Water Science University of Arizona Tucson, AZ 85721

Dr. Wierenga is a nationally and internationally recognized expert in vadose zone hydrology. He is currently a professor and Head of the Department of So iT and Water Science, University of Arizona. Dr. Wierenga has been awarded numerous research grants by federal agencies such as U.S. Environmental Protection Agency, U.S. Department of Interior, National Science Foundation, and Nuclear Regulatory Commission. He has received several highly-prestigious awards and honors from technical and professional societies. He has conducted experiments at a variety of scales including laboratory columns and field scales. Dr. Wierenga was the Principal Investigator of the Las Cruces Trench 
Site experiments. These experiments were designed to test a variety of models for predicting flow and transport in the vadose zone. Under his guidance, a large set of field data was collected and this data is now being used to test a variety of models. Several groups in the country are participating in this model validation effort including several national laboratories. Dr. Wierenga has published extensively in peer-reviewed journals such as Water Resources Research, Soil Science Society of America, and Soil Science. His unique expertise in vadose zone hydrology was of special interest to the workshop in addressing issues such as calibration and validation of vadose zone flow and transport models.

\title{
3. Martinus Th. van Genuchten
}

\author{
U. S. Salinity Laboratory \\ 4500 Glenwood Drive \\ Riverside, CA 92501
}

Dr. van Genuchten is currently research leader at the U. S. Salinity Laboratory and an adjunct professor in soil physics at the University of California, Riverside. His expertise is in the areas of soil physics and hydrology, particulariy as pertaining to the unsaturated (vadose) zone. Or. van Genuchten is internationally known for his development and application of a variety of computer models and related software for predicting water flow and chemical transport in one- and multi-dimensional variably-saturated soil profiles. This work has focused on flow and transport in homogeneous soils, as well as on transport in structured media involving macroporous soils or unsaturated fractured rock. Dr. van Genuchten also developed several popularly used parameter estimation ("inverse") models for estimating the hydraulic and solute transport properties from more easily measured laboratory or field data. Additionally, he proposed a set of quasi-empirical functions to describe the hydraulic properties of unsaturated soils; the "van Genuchten" functions are being widely used in research and management to predict water flow into and through the vadose zone. Dr. van Genuchten has published extensively in peer-reviewed journals such as Water Resources Research, Soil Science Society of America, and Soil Science. He was elected Fellow in the American Geophysical Union, the Soil Science Society of American, and the American Society of Agronomy. His expertise was of special interest to the workshop since the "van Genuchten" functions are extensively used in vadose zone modeling work at Hanford. 
WHC-MR-0420

\section{WORKSHOP AGENDA}


WHC-MR-0420

This page left intentionally blank. 


\section{VADOSE ZONE MODELING WORKSHOP AGENDA}

Environmental Risk and Performance Assessment Group

Westinghouse Hanford Company

Richland. WA

March 29, 1993

Location: Richland Tower Inn

8:00 Opening remarks

- Welcome - John Rawlins, WHC

- Introduction of Panel members - John Rawlins, WHC (10 min.)

- Workshop Agenda - John Rawlins, WHC (15 min.)

- Statement of workshop goals and objectives - Raz Khaleel, WHC

8:40 Performance/Risk Assessment Overview

$(15 \mathrm{~min}$.

- Types of wastes and waste sites - Raz Khaleel, WHC $(20 \mathrm{~min}$.

- Performance/risk assessment at a CERCLA site at Hanford Len Collard, WHC (30 min.)

\section{9:30 BREAK (15 min.)}

9:45 Vadose zone modeling issues

- What are the issues? - Raz Khaleel, WHC (30 min.)

10:15 Code Calibration/Validation Issue

o :Vhat constitutes a validation experiment? - Lynn Gelhar, MIT $(45 \min$.

- Discussion - Rien van Genuchten, U.S. Salinity Lab., Riverside

11:30 LUNCH BREAK

1:00 Code Calibration/Validation Issue (Cont'd.)

- Las Cruces Trench Experiment - Peter Wierenga, Univ. of Arizona (40 $\mathrm{min}$.

- Injection test site at Hanford - Allen Lu and Raz Khaleel, WHC

- 241-T-106 Tank Leak - John Smoot, PNL (20 min.)

(30 $\mathrm{min}$.

2:30 BREAK (15 min.)

2:45 Code Calibration/Validation Issue (cont'd.)

- A calibration/validation proposal - Charley Kincaid, PNL (30 min.)

- Open Discussion on Calibration and Validation Issue 


\section{VADOSE ZONE MODELING WORKSHOP AGENDA}

March 30, 1993

Location: Richland Tower Inn

8:00 Modeling Data Requirements

- Laboratory measurement of hydrologic properties - John Relyea, WHC (30 min.)

- Unsaturated hydraulic cond. measurement - Jim Conca, WSU/TC (30 min.)

- Use of van Genuchten-Mualem relationships - Raz Khaleel, WHC

- Use of Arya-Paris model to generate moisture retention data (30 min.)

\section{0:00 BREAK (15 min.)}

10:15 Modeling Data Requirements(Cont'd.)

- Dispersivity estimates (Lynn Gelhar, MIT - $45 \mathrm{~min}$.)

- Open Discussions ( $1 \mathrm{hr}$. )

\section{2:00 LUNCH BREAK}

\section{1:00 Modeling Data Requirements(Cont'd.)}

$0 \quad$ Evaluating uncertainty and spatial variability (Lynn Gelhar, MIT)

(45 min.)

\section{1:45 Open Discussions}

- Wrap-up, Feedback, Summary

- Other modeling issues: Use of Kd's, Recharge estimates, moisturedependent anisotropy.

3:00 BREAK (15 min.)

3:15 Executive Session (ER and ERPA personnel, DOE-RL, and regulators).
- Summary
$0 \quad$ Objectives 2 and 3 
WHC-MR-0420

COPIES OF VIEWGRAPHS 
WHC-MR-0420

This page left intentionally blank. 


\section{TYPES OF WASTES AND WASTE SITES}

RAZ KHALEEL WESTINGHOUSE HANFORD COMPANY

LIQUID WASTES DISCHARGED AND SOLID WASTES BURIED AT HANFORD: $1944-1989$

- 1.7 trillion liters (444 billion gallons) of water and liquid wastes discharged to the soil Irom all sources.

- 625,000 cubic meters $(820,000$ cubic yards) of solid wastes buried in three generai locations over a 429-acre area.

- Liquid wastes discharged through the use of cribs, trenches, ponds, and ditches, as well as through leaks and spills.

LIQUID WASTES DISCHARGED AND SOLID WASTES BURIED AT HANFORD: 1944-1989

- Contanninated siles contain 5.6 million curies of radioactivily. Much higher quantilies of radionuctides have actually been disctiarged. but those with a short half-life are not represented in the 5.6 million curies.

- Radioactive liquid contaminants include cesium, strontium. tritium, jodine, technetium. uraniurn. and plutonium. Some of the chemical contaminants are nitrates. sodium, phosphate. suifate. ammonia. fluoride. and carbon telracilioride. 


\section{VADOSE ZONE MODELING WORKSHOP}

\section{OBJECTIVES}

\section{WHY FOCUS ON MODELING ISSUES?}

- Predictive reliablility is essential because real-time testing to confirm the waste disposal design and to confirm the long-lerm predictions is impossible.

- Regulatory requizenents do indeed provide a major locus for performance assessment However, it is important to achieve an appropriate level of understanding of the lechnical basis in predicting isolation potential of a waste sile.

- In any predictive ellort, in the interest of available resources and time, there must be a cornpromise between the additional data needed and the loss of predictive reliability when the additional data are not available.

\section{VADOSE ZONE MODELING WORKSHOP OBJECTIVE 1}

Develop a conselisus position by alf "3lakellolders (lechillologisis.

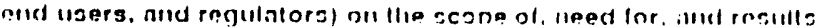
ol tasks being conducted or plamed as part of llie nctivilies identllled under TPA milestone M-29.02 document, DOE.RL.91.62. In partlcular. lhe lollowing questions heed to be answered:

- Are llie dala collected to dale sulficient lo provide a technically defelisible performancentisk assessment modeling in support of a CEFCLA Record of Decision $(R O D)$ ? (Yes or $110 ?)$

- Il the answer is "no: ivhat is : : a appropriate level of sile characterization data ara sace calibration: validation work llecessarvi s stify techmcally delensible performance tish assessinem activilles? 
VADOSE ZONE MODELING WORISHOP OBJECTIVE 2

Obtain a cominiment from all stakeholders to support (according to each attendee's individual responsibilities) the results, findings, and/or recommendations of the workshop.

- Will the Environmental Restoration Program (and other funding sources) commit to continued funding in support of resolution of the vadose zone modeling issues?

VADOSE ZONE MODELING WORKSHOP OBJECTIVE 3

Identify action items that each stakehoider must periorm to ensure that the results, findings, and/or recommendations of the workshop are realized.

- How do we get froin where we are to where we need to be?

- Who needs to do what by when to assure that the results of the workshop are executed as agreed upon and as planned?

\section{VADOSE ZONE MODELING ISSUES}

RAZ KHALEEL WESTINGHOUSE HANFORD COMPANY 


\section{VADOSE ZONE MODELING ISSUE: CODE CALIBRATION/VALIDATION}

- Flow and transport codes that are currently being used in performarice assessment evaluation of a waste site are not calibrated and validated to have sulficient confidance in their ablility to predict contaminant migration.

- Limited data for code calibration/validation are available at Hanford but have not been fully utilized.

\section{VADOSE ZONE MODELING ISSUE: EVALUATE APPLICABILITY OF MODELS TO ESTIMATE UNSATURATED K'S}

- Current appraach uses a single measurement of saturated hydraulic conductivity, combined with laboratory-ineasured moisture relention dala, 10 predict the unsaturated $K$ 's over the entire range of moisture content.

- Approach untested for unsaturated soils will conductivities varying roulinely over several orders ol inagnilude.

\section{VADOSE ZONE MODELING ISSUE: DISPERSIVITY ESTIMATES}

- Lilerature data for saturated media suggest linat dispersivilies lend lo increase wilh llie scale of experillent. Also, lab measurements on dispersivily are inuch amaller than those based on lield tracer experiments.

- Pepresenting inacrodispersion over large dislance and lime scales in helerogeneous unsalurated media by "conslant" values of dispersivily appears to be questlonable.

- Under ungaturated condltions. Il la reasonable to expect liat dispersivitles vill increase as the scale of the experiment increases as observed in case of the saluraled flow.

- Few llela-scale dispersivilies have been reporled for llie unsaturated media. Possible use of one ol Hauford's "unplanued Iracer lest" lo esllinale dispersivilies? 


\section{VADOSE ZONE MODELING ISSUE: UNCERTAINTY AND SPATIAL VARIABILITY}

- Uncertainty refers to lack of knowledge about the variation in input data and in the accuracy of inodels used. Sources of uncertainty include ineasurement error, lack of understanding of physical processes of the system, and extrapolation into the distant future.

- Spatial variability relers to variability of sediment properties as characterized by the statistical properties of the spatial distribution such as the mean, variance, and spatial correlation lengths.

\section{VADOSE ZONE MODELING ISSUE: EVALUATE APPLICABILITY OF PSD DATA IN ESTIMATING MOISTURE RETEIJTION}

- Presence of a sigrilicant correlation oetween llie particie size distribulion (PSD) and moisture retention can potentlally lead to a large cost reduction in slle characterization activilies alıned at obtanilg moisturn retentlon data lor various operable unils.

- Demonsirale the success (ol lallure) of using existing models le.g. Arya.Paris lecliniquel of translaling lihe partlcle size distribution data 10 moisture retenllion curves. Bulk densily as well as the narticte-riza. distribution are line only data needed 12: applicalion of the Arya.Paris model.

\section{VADOSE ZONE MODELING ISSUE:$$
\mathrm{K}_{\mathrm{d}} / \mathrm{R}_{\mathrm{d}} \text { APPROACH }
$$

- Lack of delailed knowledge about site-specific geochemical conditions, and the difficulty of incorporating detailed geochemical reations 11 transport calculations, result in use of $K_{d}, R_{d}$ approacil.

- Using conslant values of $K_{d}$ in transport caiculations for bolh salurated and unsaturaled media.

- Using $K_{d}$ s measured under laboratory concilions that are vastly dilferent from those likeiy under field conditions. 


\section{WHC-MR-0420}

\section{VADOSE ZONE MODELING ISSUE: EVALUATE MOISTURE-DEPENDENT ANISOTROPY}

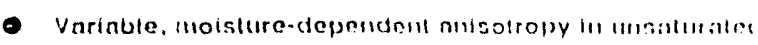
soils is an ellectlve. large-scale (Inacroscopic) llow properly resulling from media lextural helerogenellies at a smaller scale.

- For unsalurated inedla, lateral K to vertlcal $K$ rallo is entanced with a decrease in moisiure conterit of the solls: l.e.. the anisotropy increases with a decrease in soluration.

- It It is demonstrated that the moist jre-dependent anisotropy is a signilicant procesj for Hanturd solls. appropriate credit can be assignerd to account for such a phenoinenon in compuling groundwaler travel limes and radionuclide flux rales. 


\title{
MOISTURE-DEPENDENT ANISOTROPY FOR HANFORD SOILS: INJECTION TEST SITE
}

\author{
RAZ KHALEEL \\ WESTINGHOUSE HANFORD COMPANY
}

MACROSCOPIC ANISOTROPY BASED ON STOCHASTIC THEORY

Yeh et al., (1985)

$$
\frac{K_{12}}{K_{22}}=\exp \left[\frac{\sigma_{z}^{2}-\sigma_{p}^{2} H^{2}}{I-\lambda A \cos \gamma}\right]
$$

\footnotetext{
$\sigma:^{2} \quad=$ variance of $\ln \mathrm{k}$, randon tield,

$\sigma_{A}^{2} \quad=$ variance of the slope of the in $K$ vs. "l relationship

$H=$ mean value of $\mu$,

$i=$ correlation length for both $\mathrm{lnk}$, and $\beta$ random field in the direction perpendicular to stratification.

A $=$ mean slope of $\ln K$ vs. $\psi^{\prime}$, and $\because \quad=\quad \begin{aligned} & \text { angle between the soil layer stratification and the } \\ & \text { horizontal. }\end{aligned}$
} 
WHC-MR-0420

RHC-3i-ン́

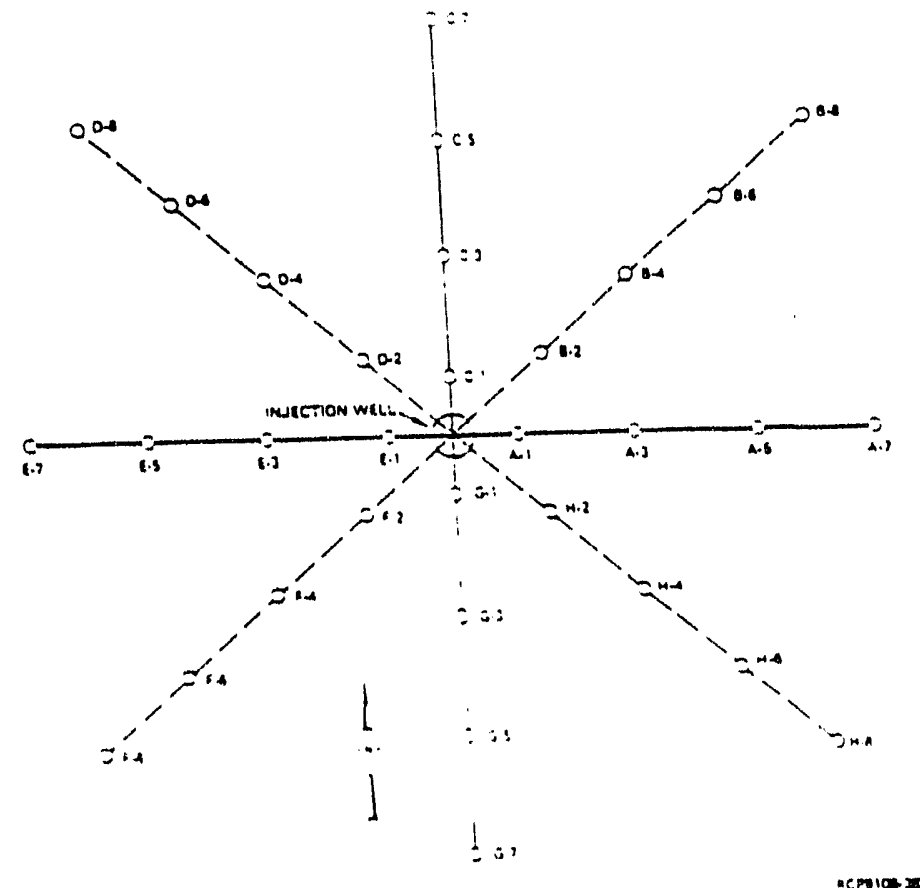

FICURE 6. Weii inmoering seneme.

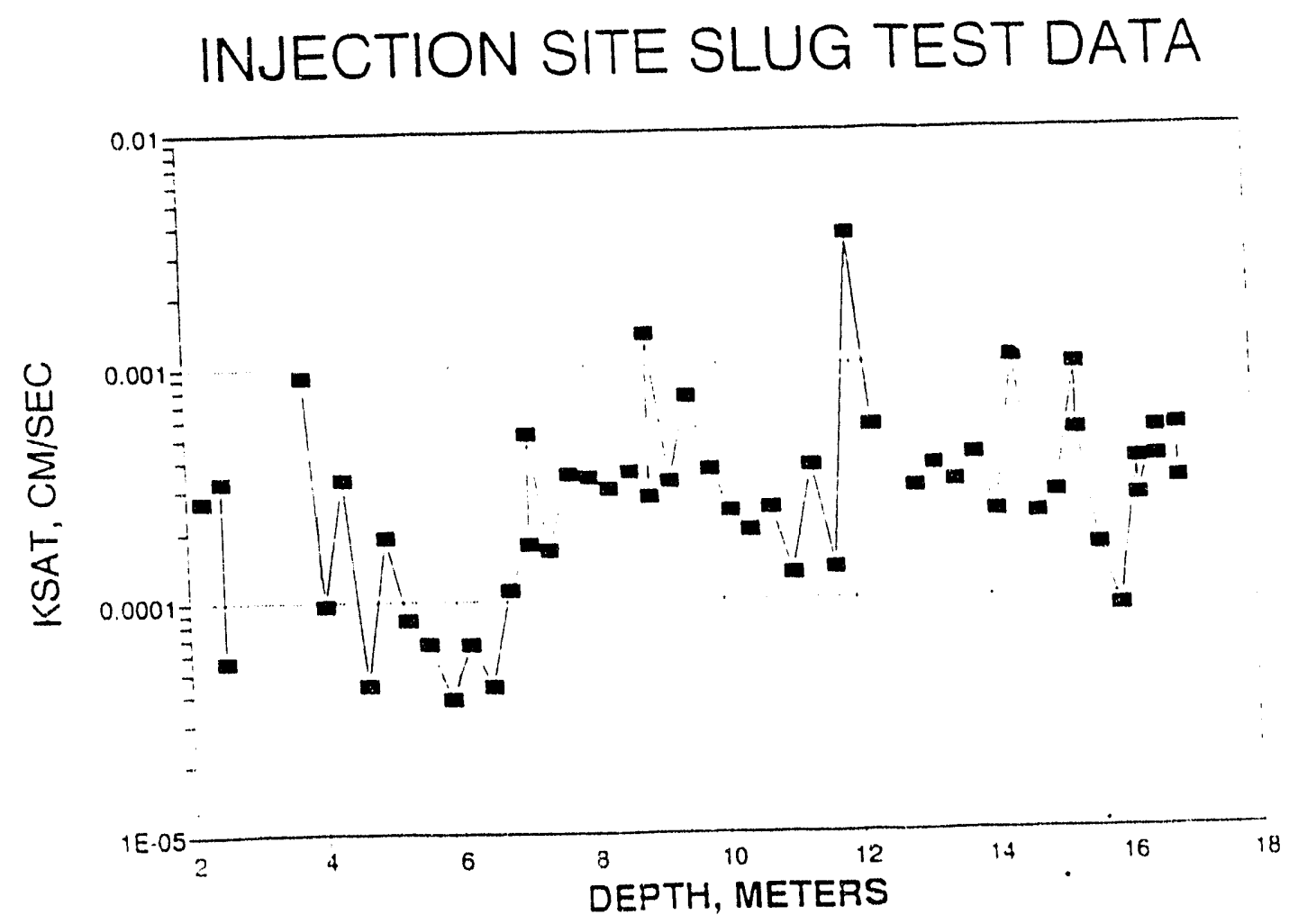




\section{INJECTION SITE SLUG TEST DATA: \\ Ksat Histogram Results}

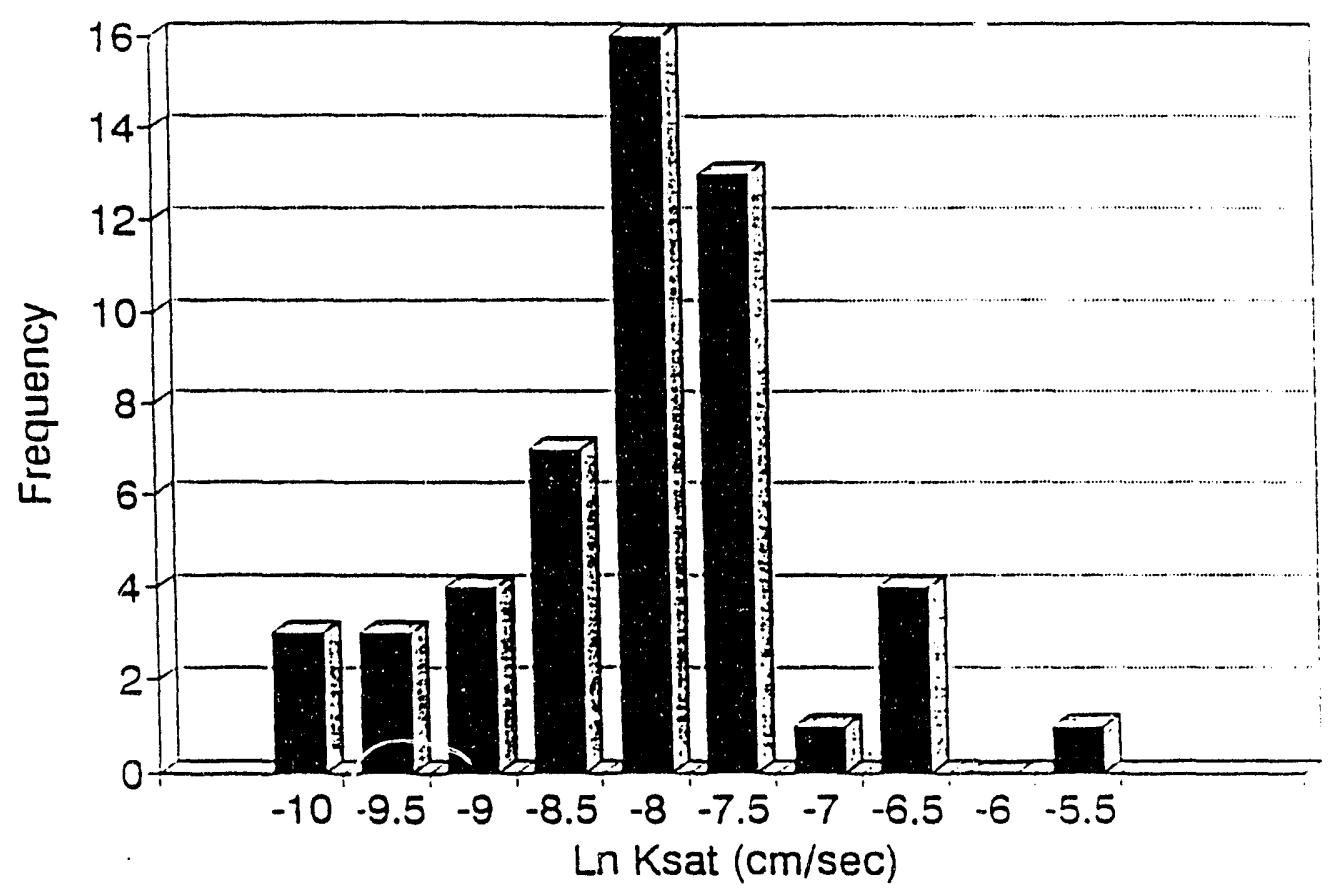

INJECTION SITE PERMEAMETER DATA. Ksat Histogram Results $(N=19)$

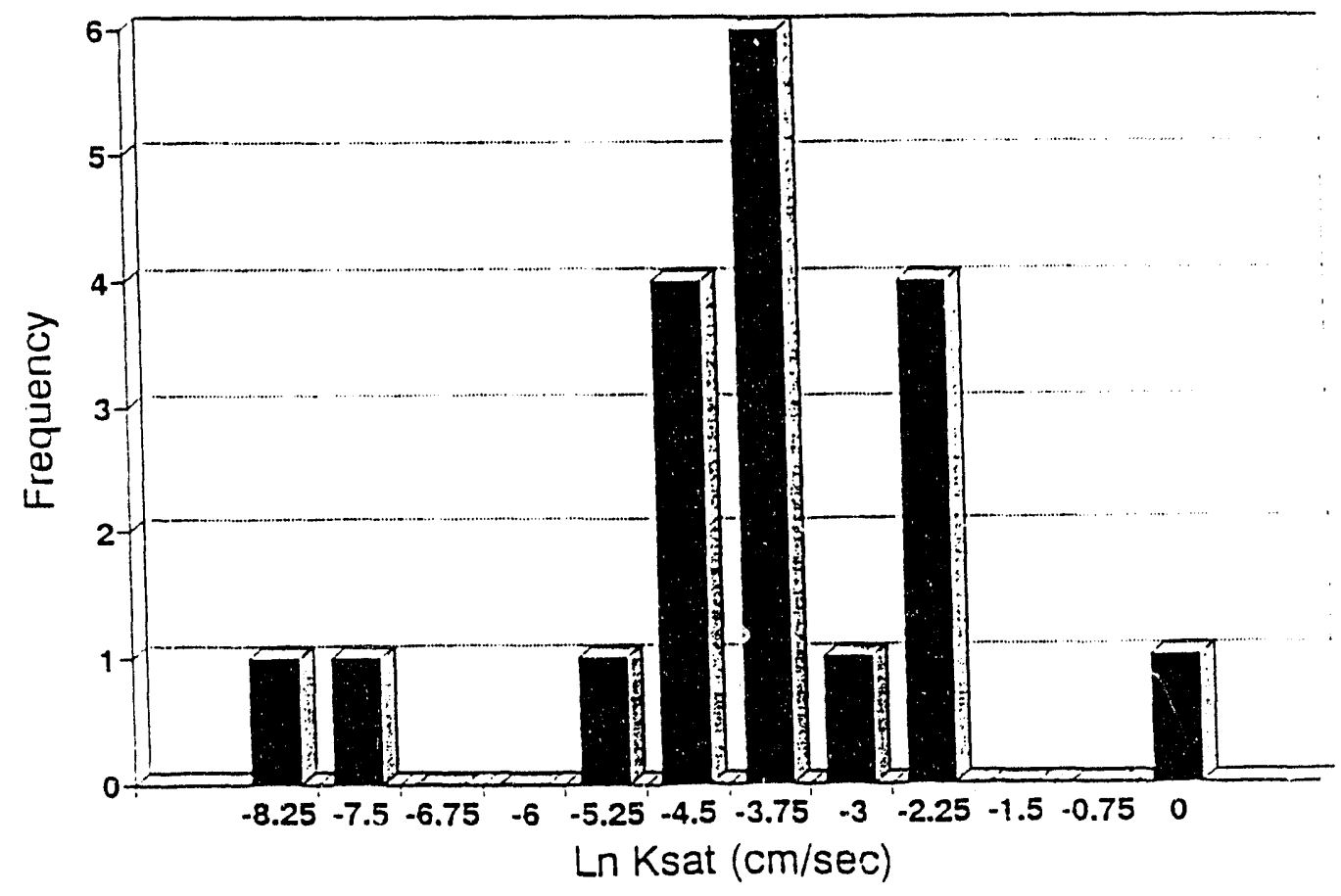


Injection Site Farmeameter Data:

$\mathrm{K}_{\text {sat }}$ Probability Plot

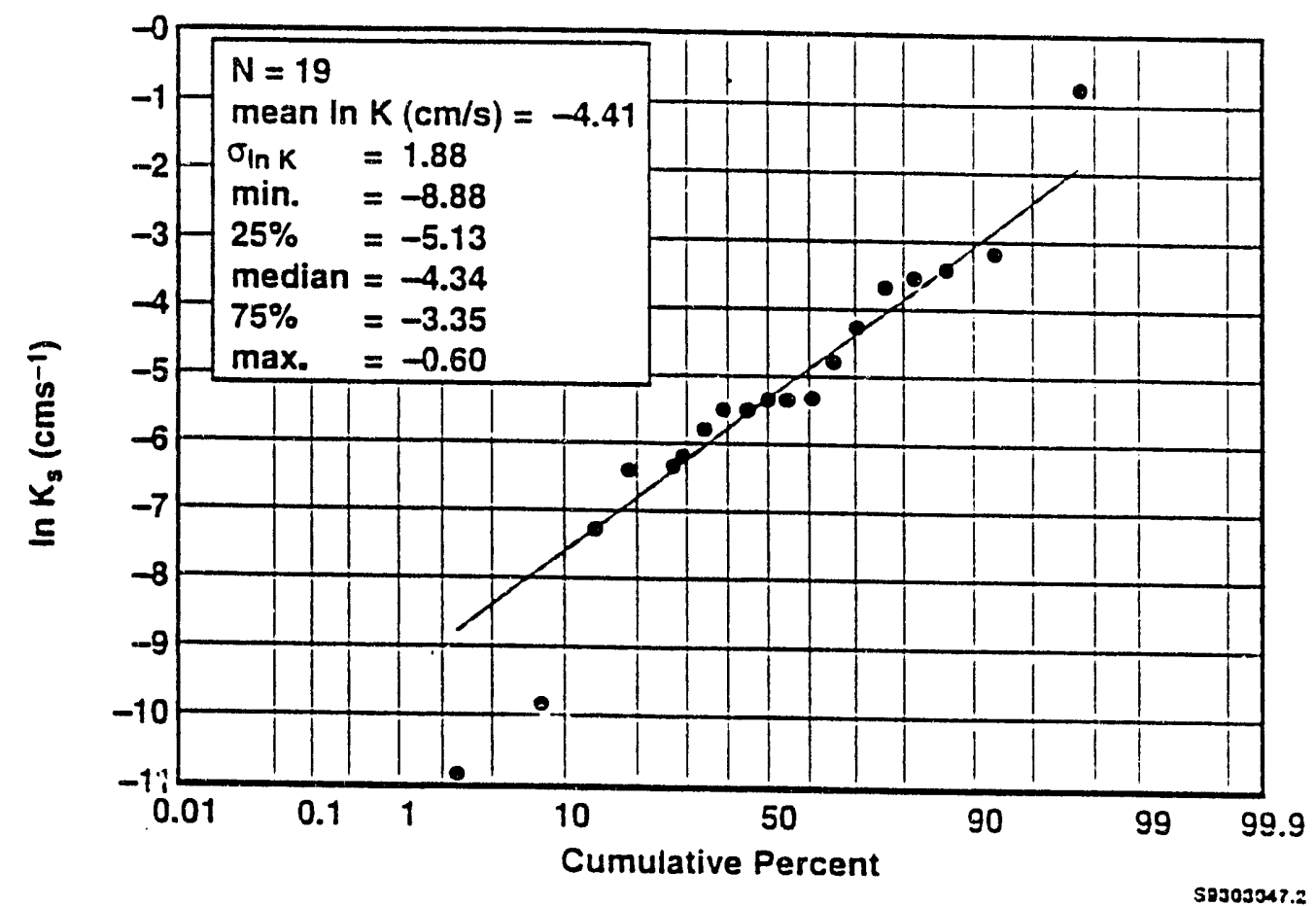

Injection Site Slug Test Data: $\mathrm{K}_{\text {sat }}$ Probability Plot

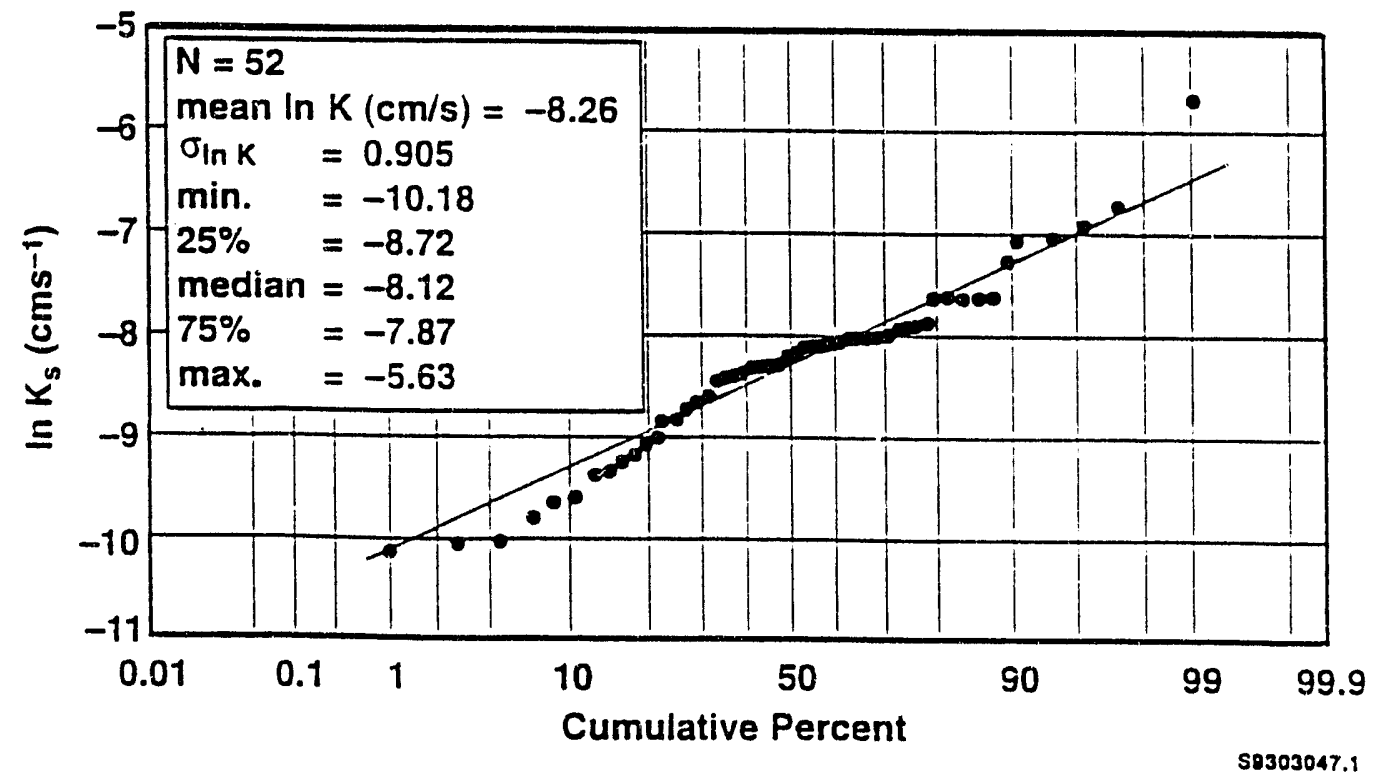


WHC-MR-0420

INJECTION SITE SLUG TEST DATA

Experimental and Fitted Variograms

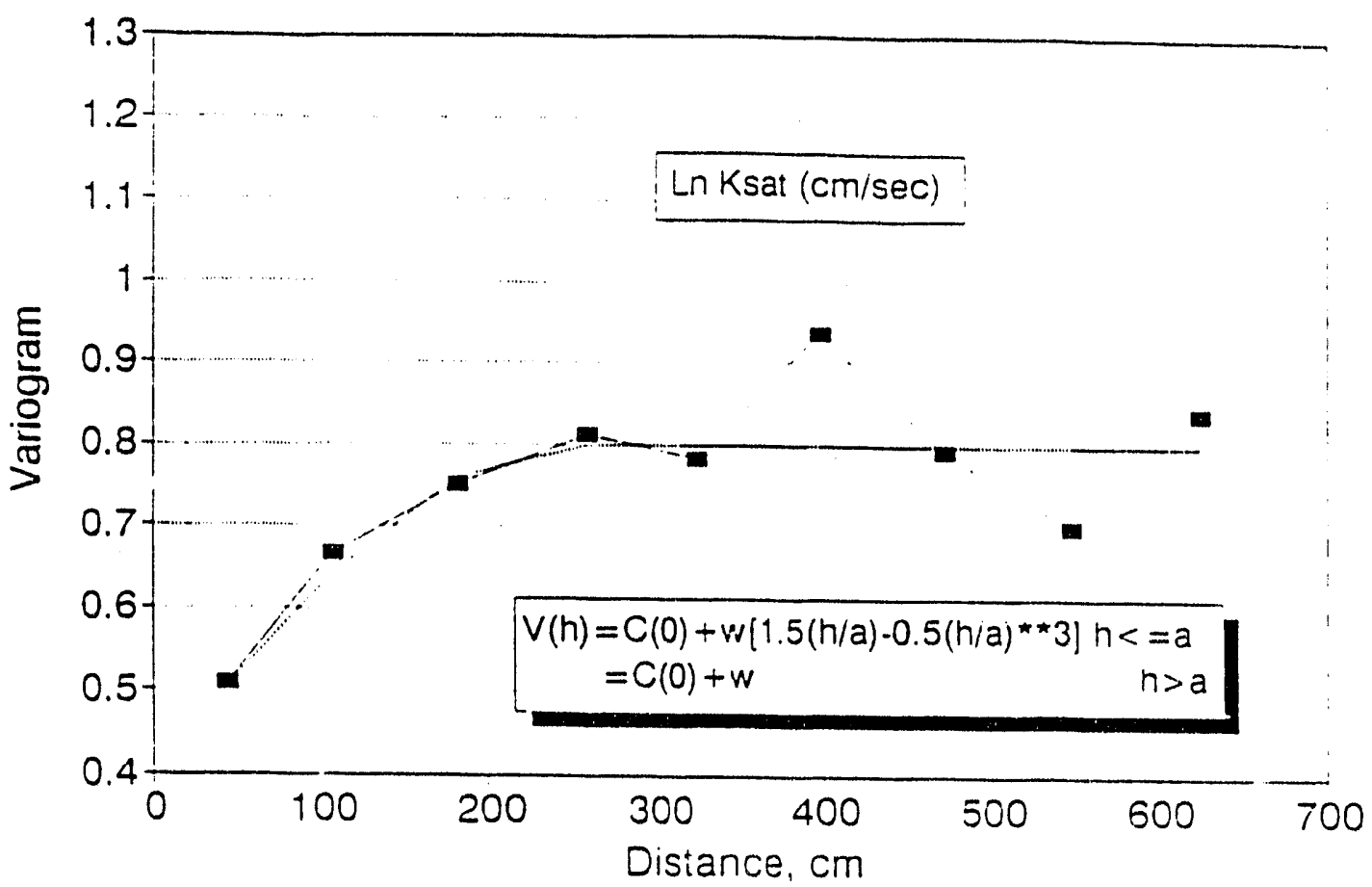

INJECTION SITE DATA

17 samples analyzed for

$\theta$ vg. $\psi$

$K(\theta)$ re. $\theta$

Simultaneous fit for v6-m parameters: $\theta_{r}$. $\theta_{s}, k, l, k_{s}$, and $n\left(n=1-y_{n}\right)$

Filed un cat. $K(\theta)$ data transformed using:

$k(\theta)=k_{1} e^{-\beta \psi}$

Obtained slope of

en kr va. $\varphi$ plot

Mean sind Variance

Jor $\beta$ of 17 samples

39 

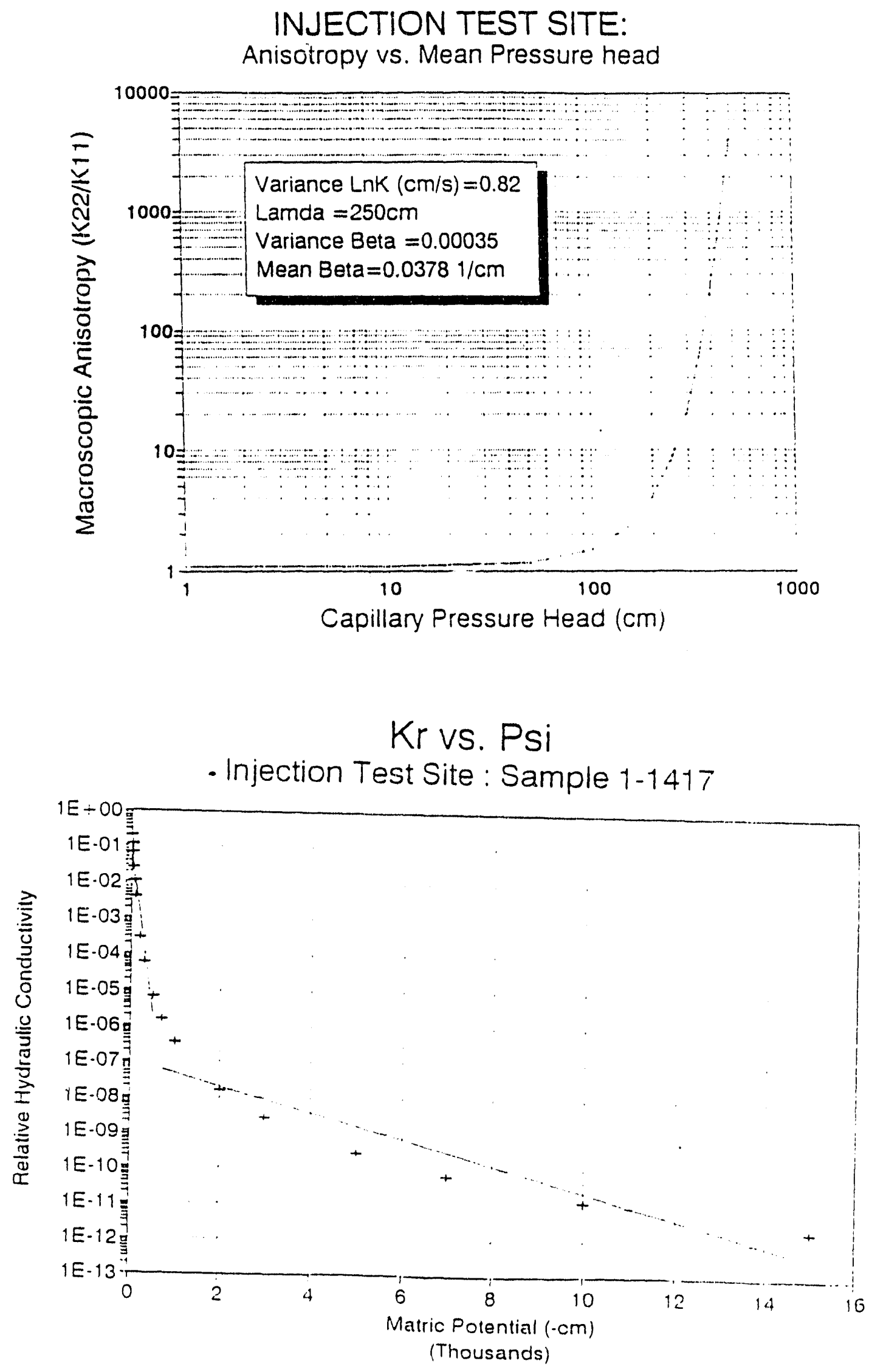
Performance/Risk Assessment at a CERCLA Site at Hanford

\author{
Len Collard \\ Westinghouse Hanford Company
}


WHC-MR-0420

This page left intentionally blank: 


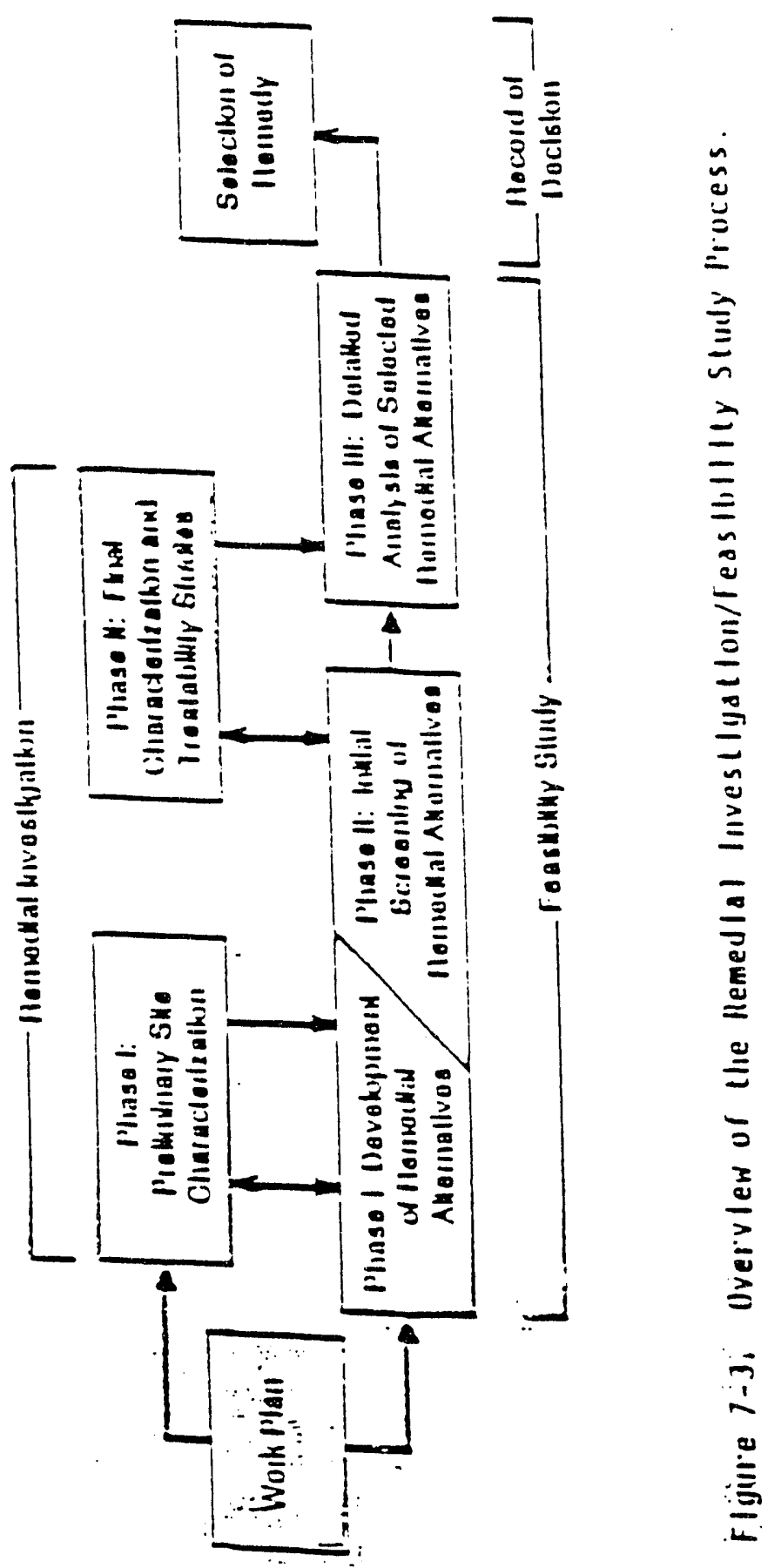




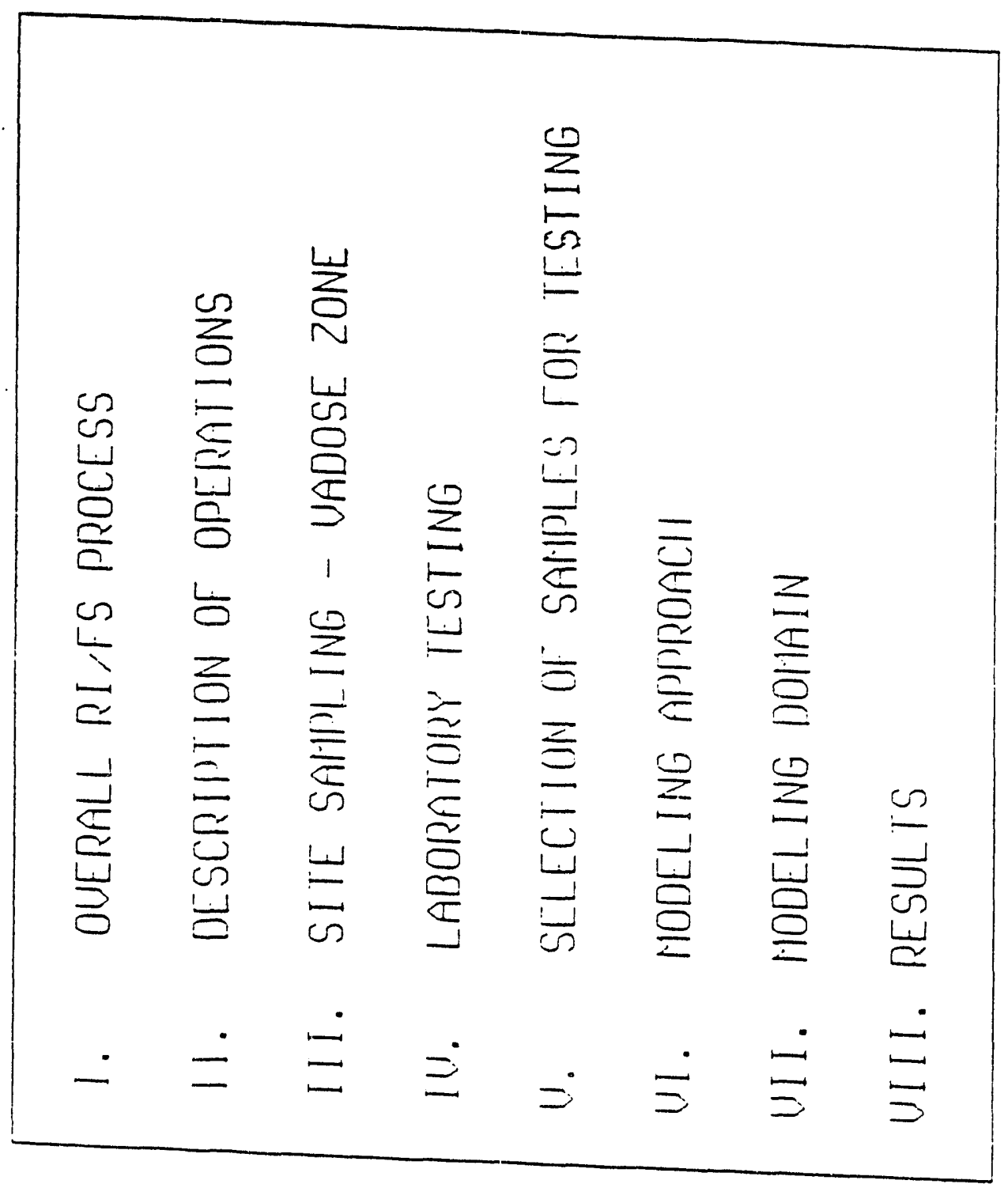




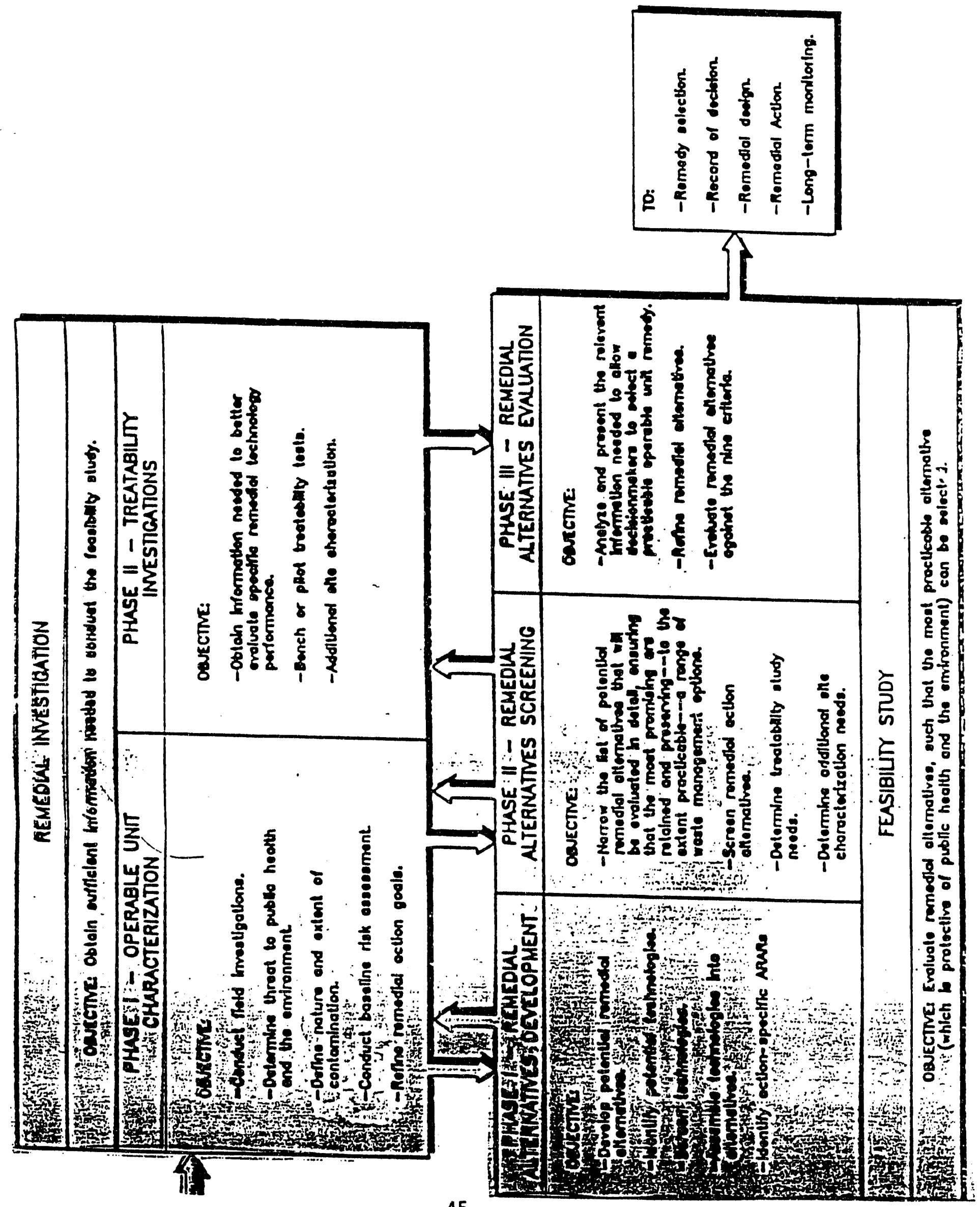




\section{WHC-MR-0420}

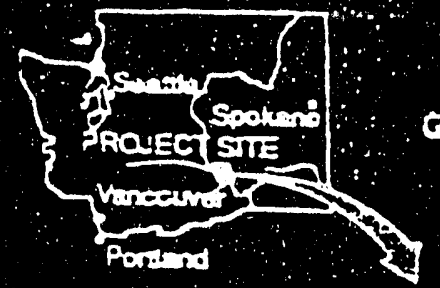

Priest Rapids Dam<smiles>c1ccccc1</smiles><smiles>CCCCCCC(C)C</smiles><smiles>C1CCCCCC1</smiles><smiles>C1CC[Te]CC1</smiles><smiles>[TeH][TeH]</smiles>

Han Site Boundary<smiles>CCCCCCI(C)I</smiles>

Rantesnake Hilts

EENTON Co.

Gist co.

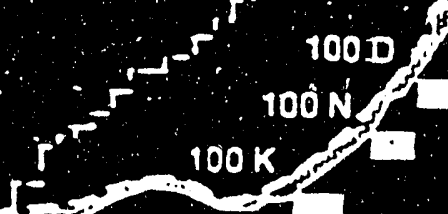

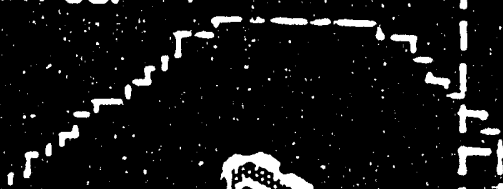

Dans ca<smiles>[3H]CC[3H]</smiles>

$1008 / C$
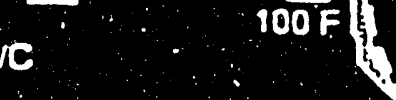

12 


\section{Acres}

243 - 849: iow-ievel waste from tributyly phosphate process in 221-U. Sludge from 24l-8Y tanks transferred to $U$ Plant, dissolved in nitric acid, and extracted with TBP. Acid solution was made alkaline. Wastes were treated with potassium ferrocyanide as a cesium scavenger. Supernatant was decanted to B43 - B49. 11/54 - 12/55

E50 - B57 : In tank heating of 241-BY tanks created evaporates which were condensed and discharges to B5O and B57. 3/65 - 1/74 


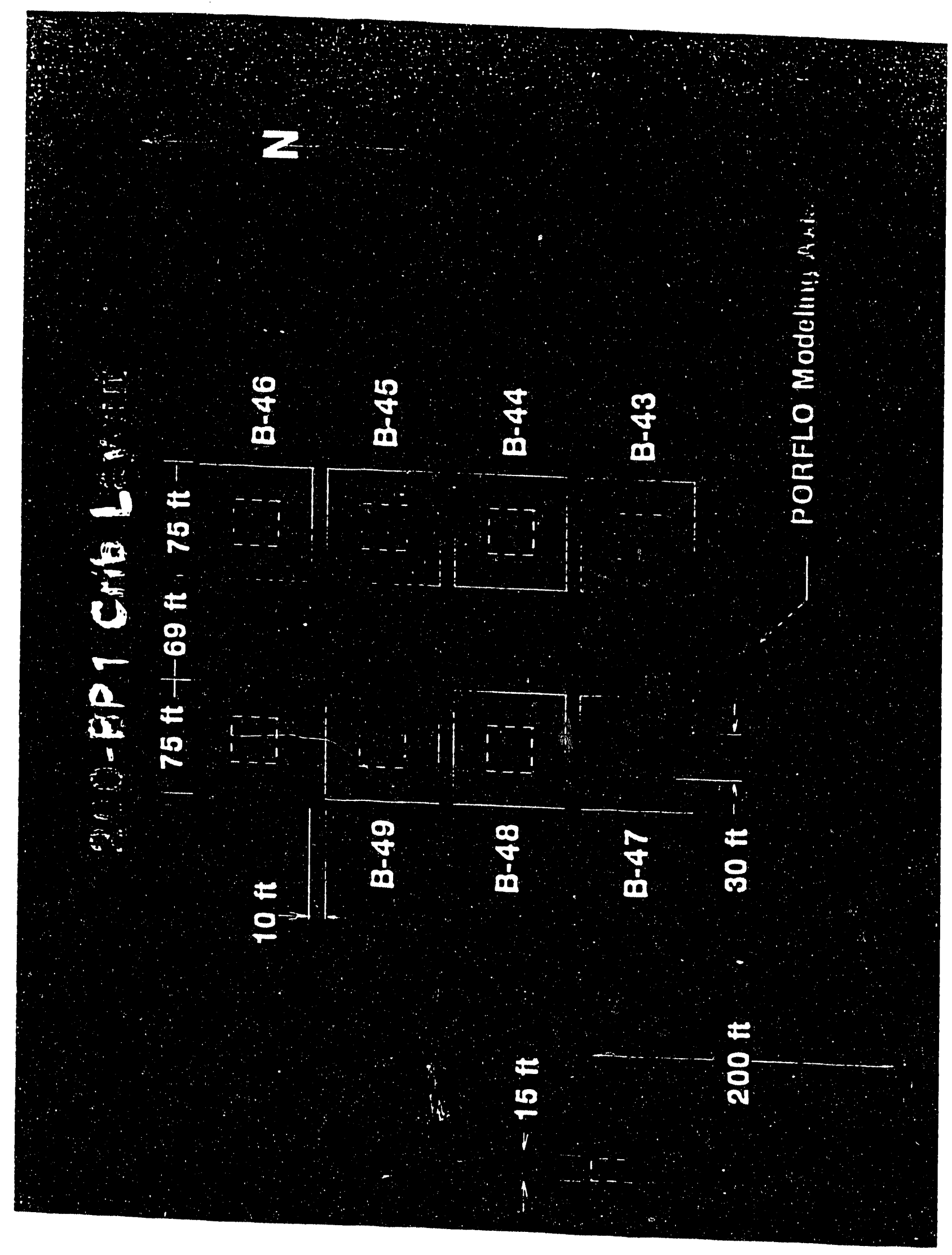




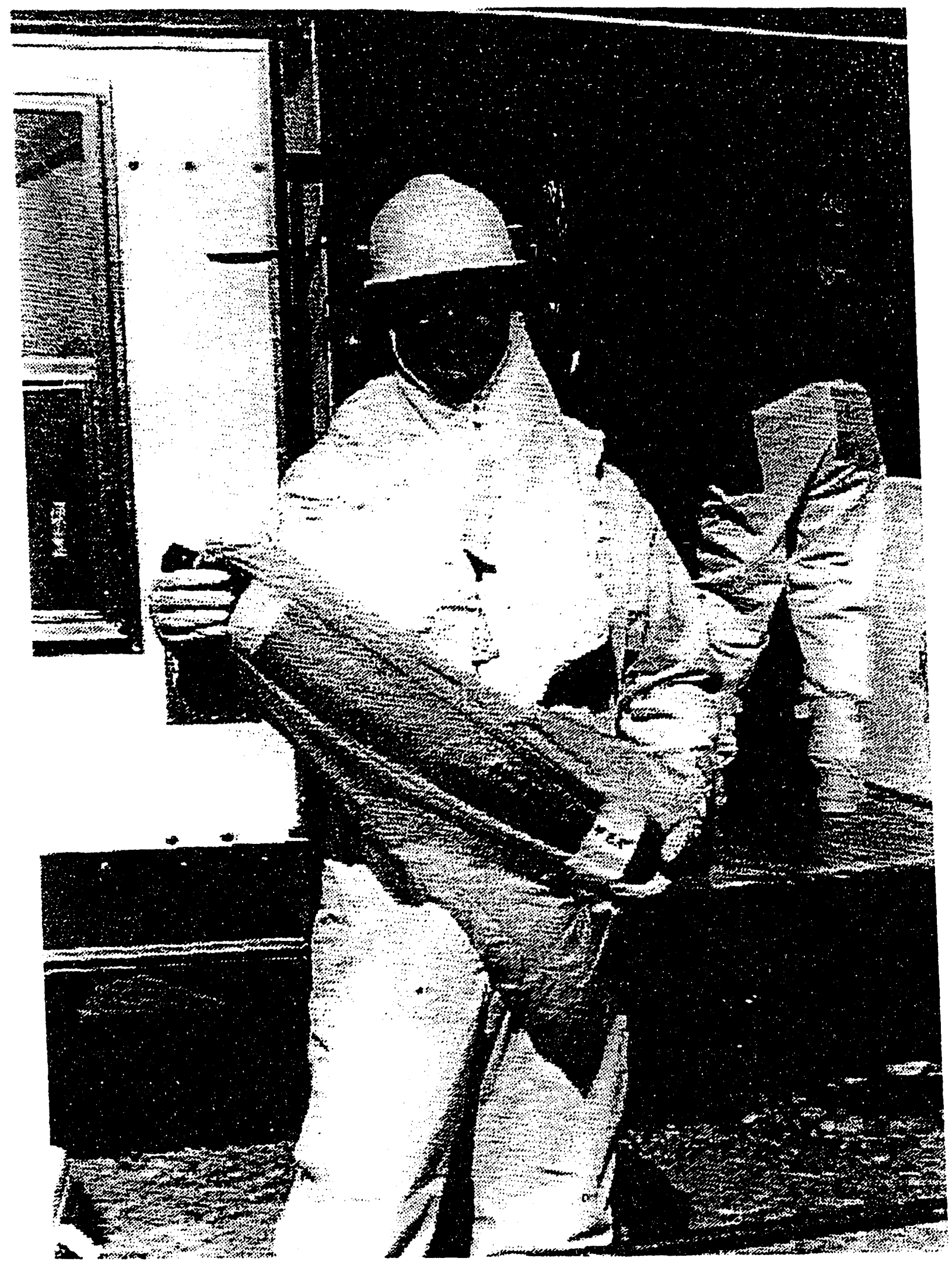


WHC-MR-0420

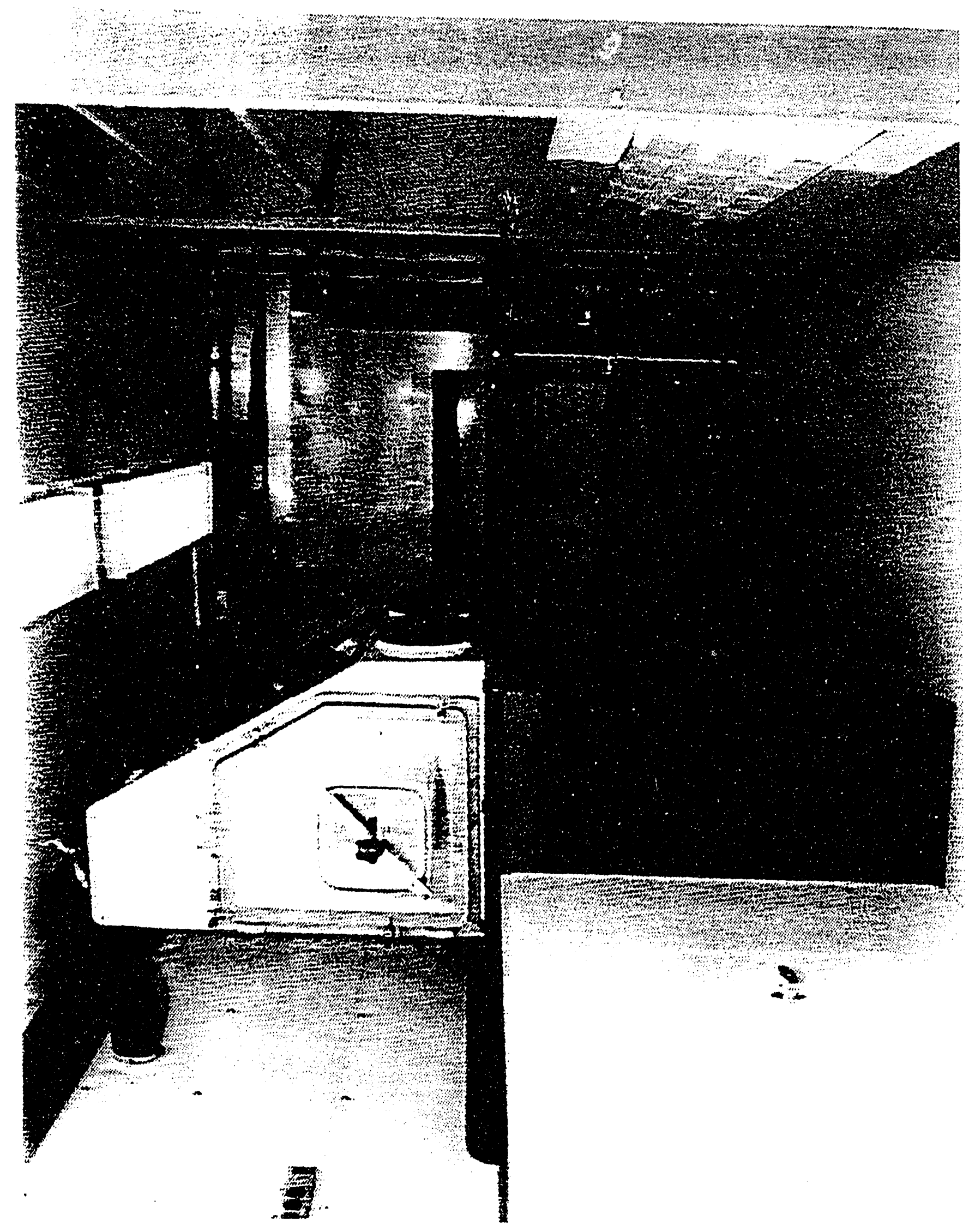


WHC-MR-0420

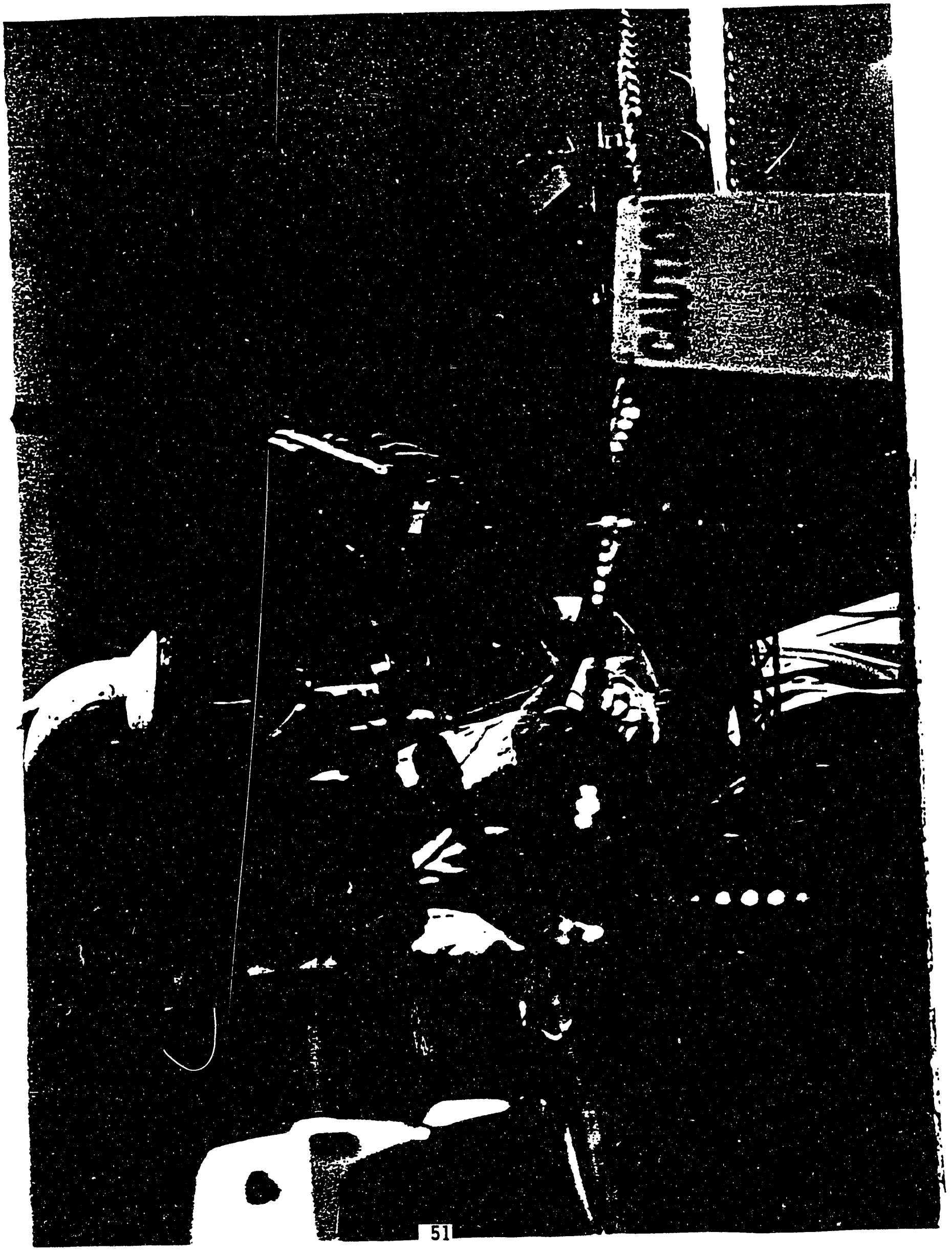




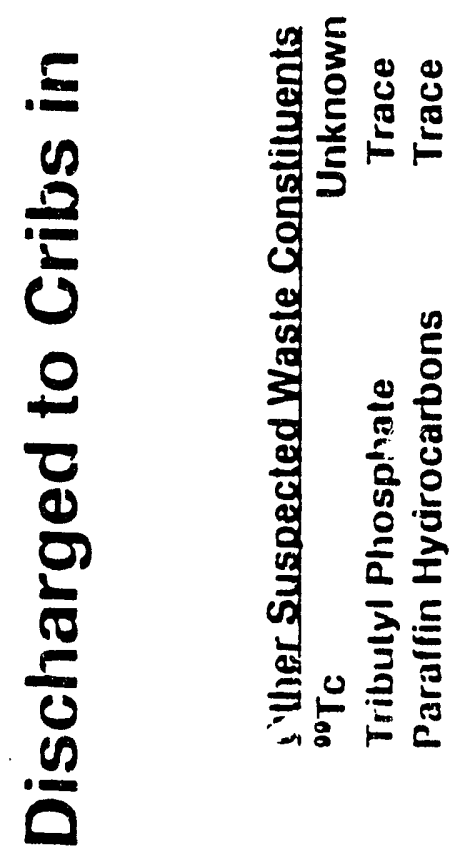

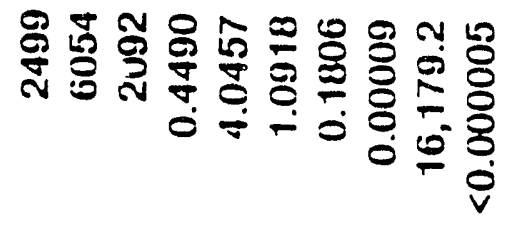
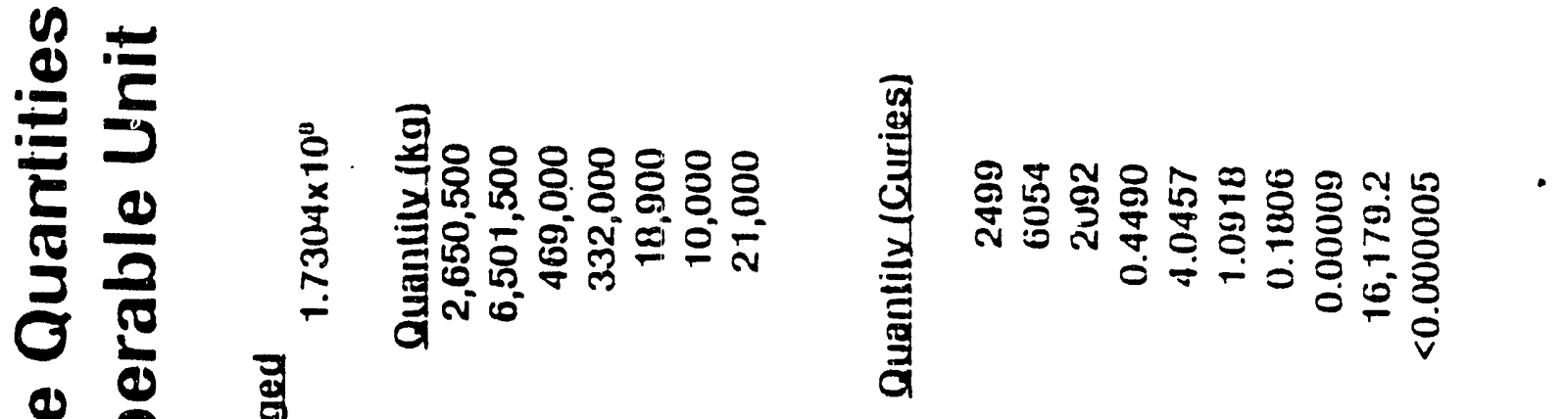


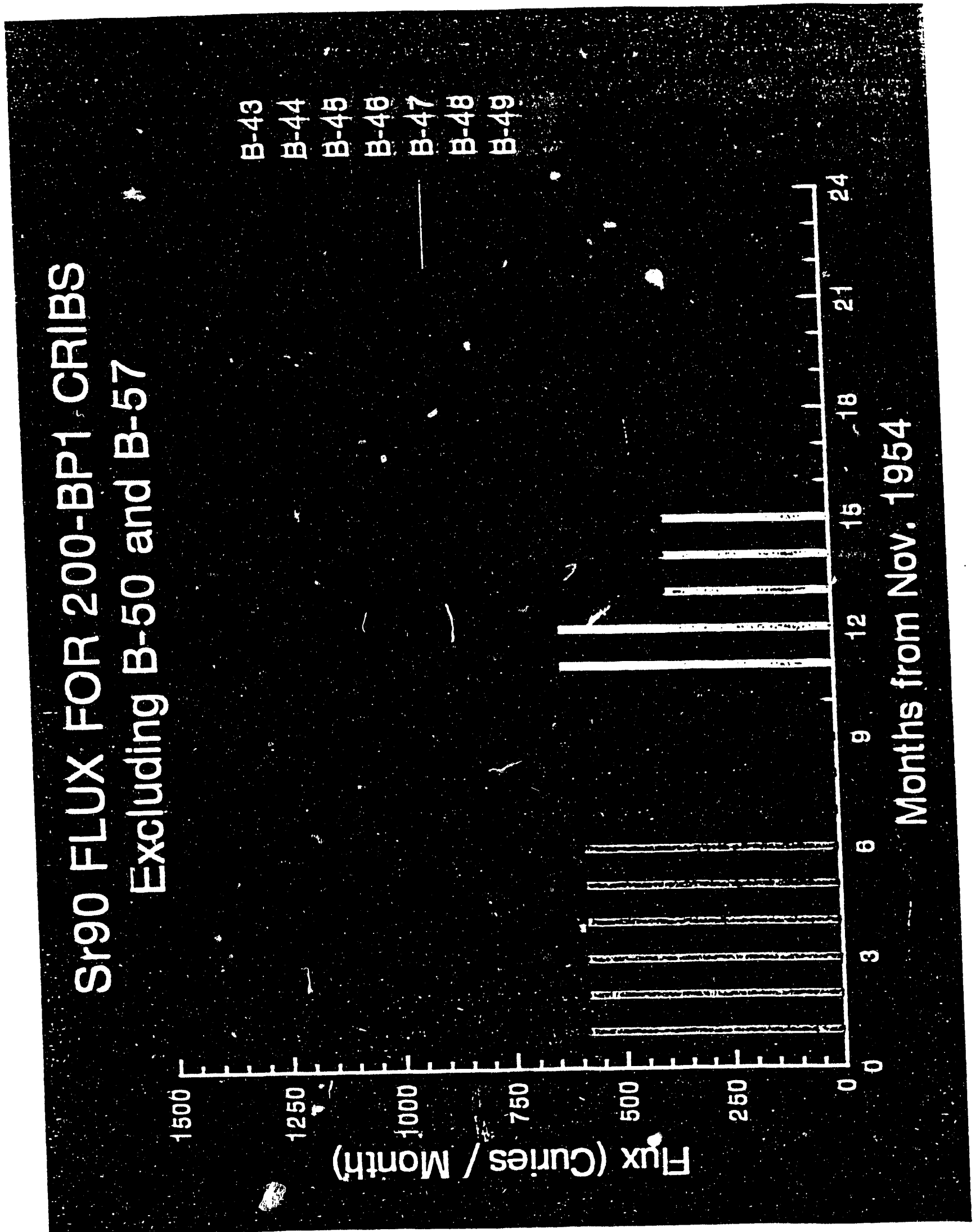




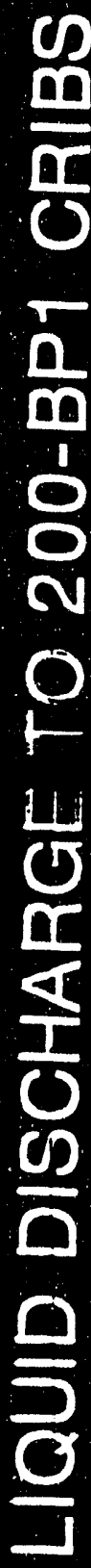

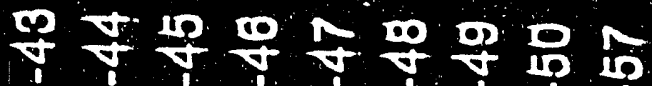

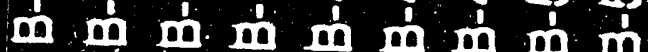

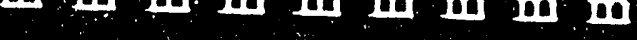
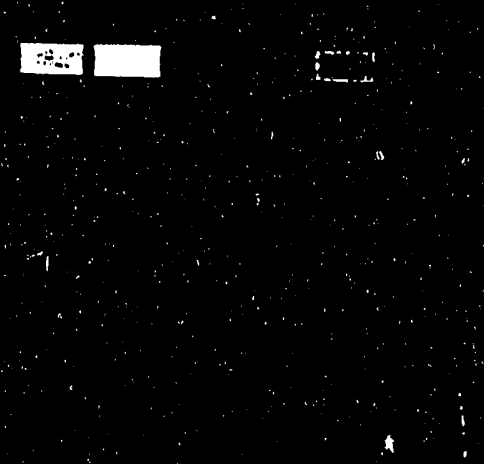


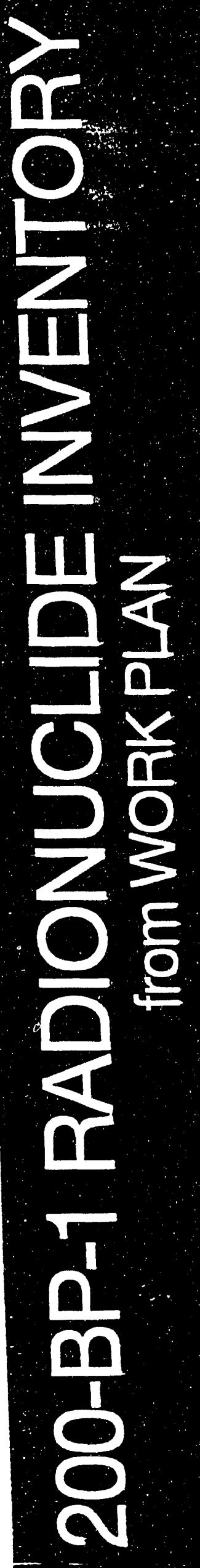

WHC-MR-0420

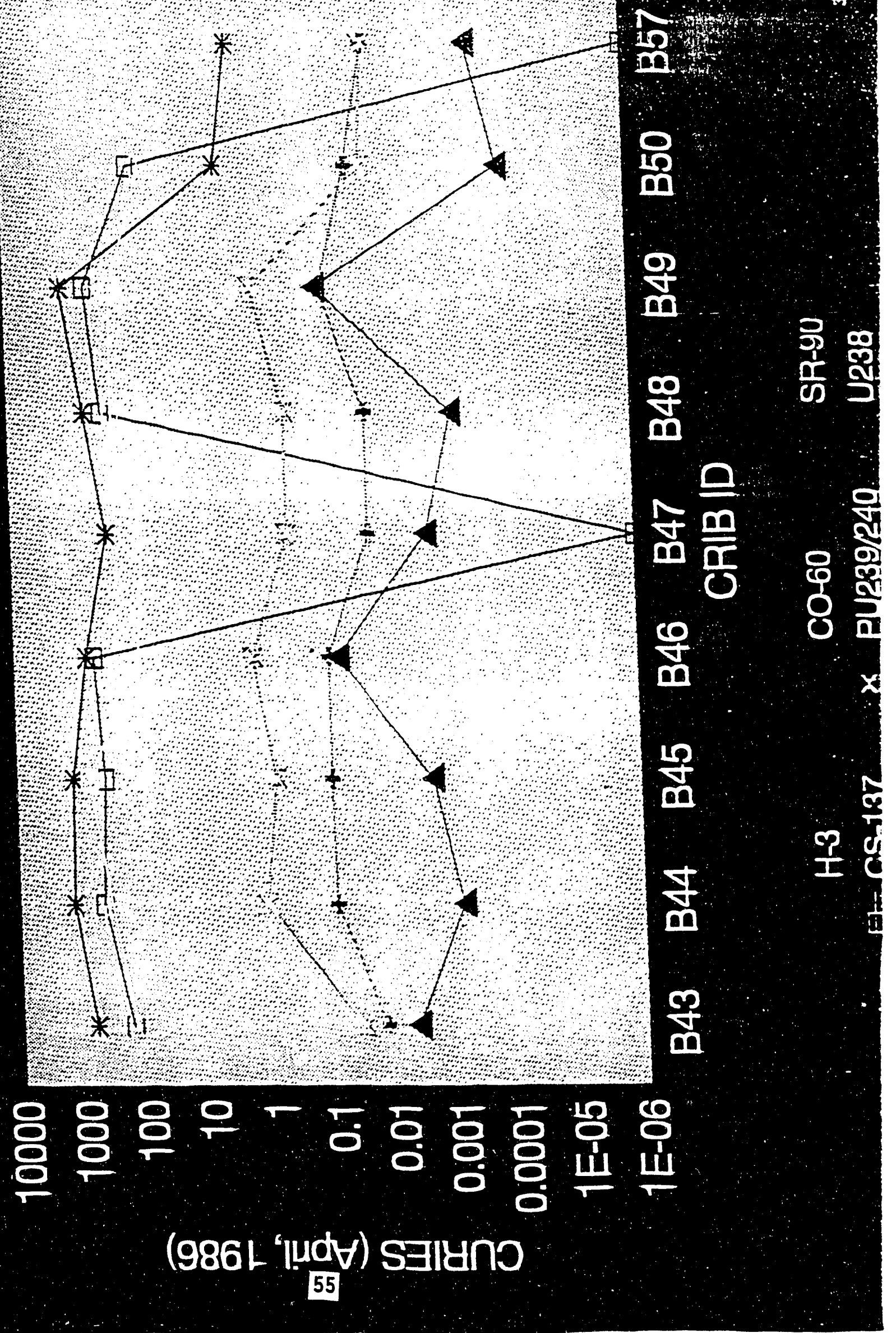


WHC-MR-0420

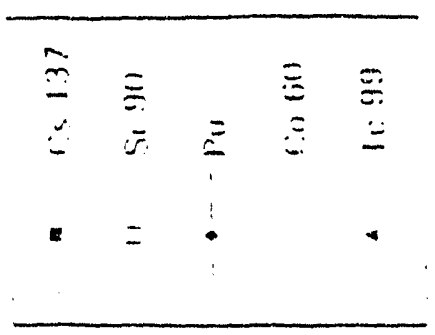

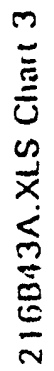

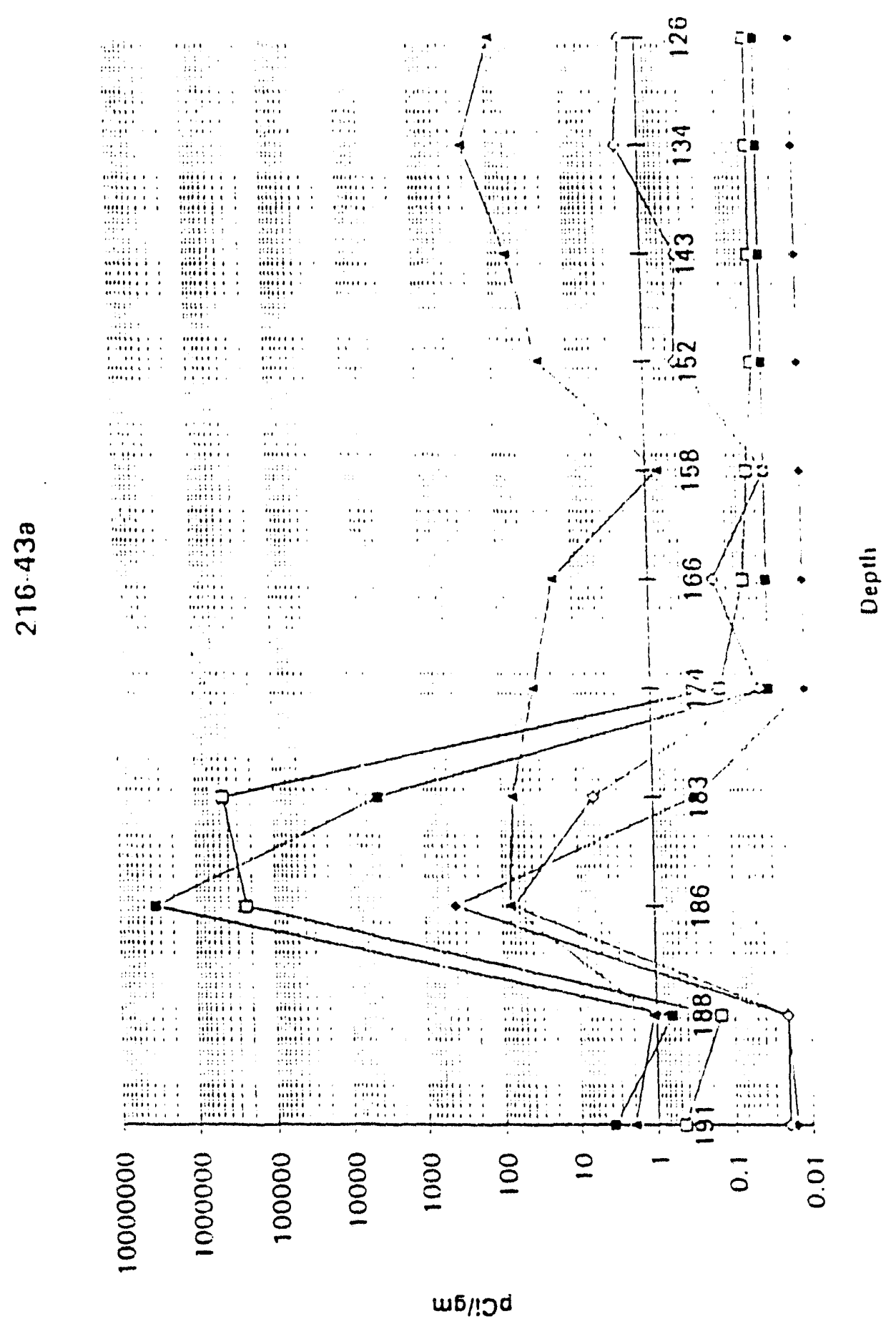




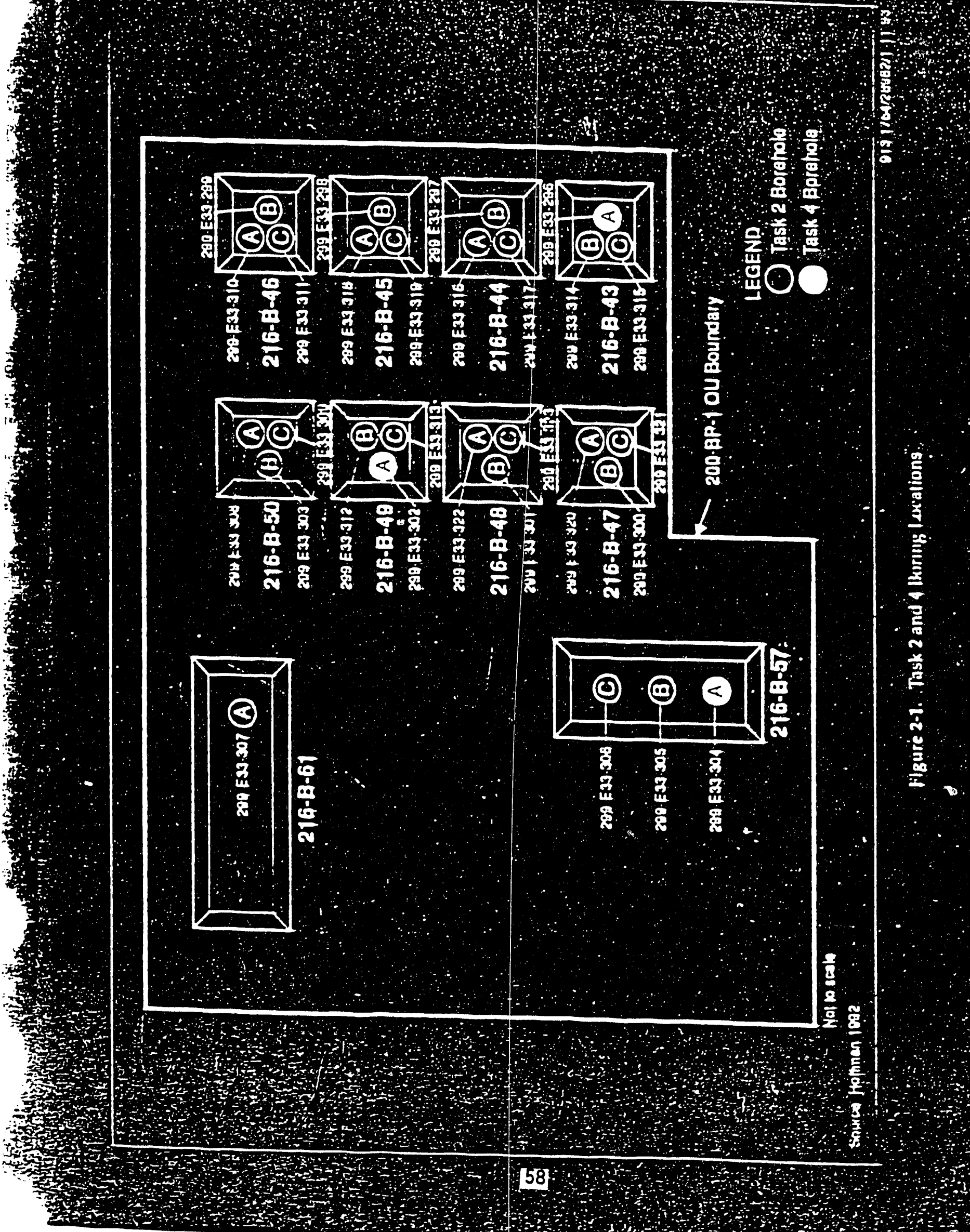




\section{0-BP-1 Vadosezone Crib Borehole

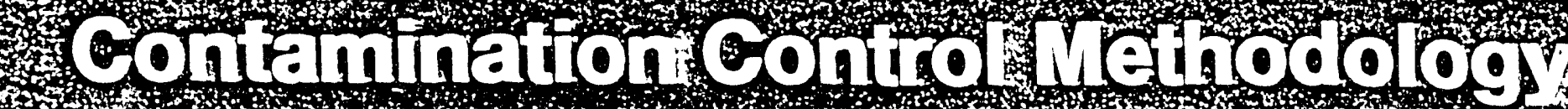

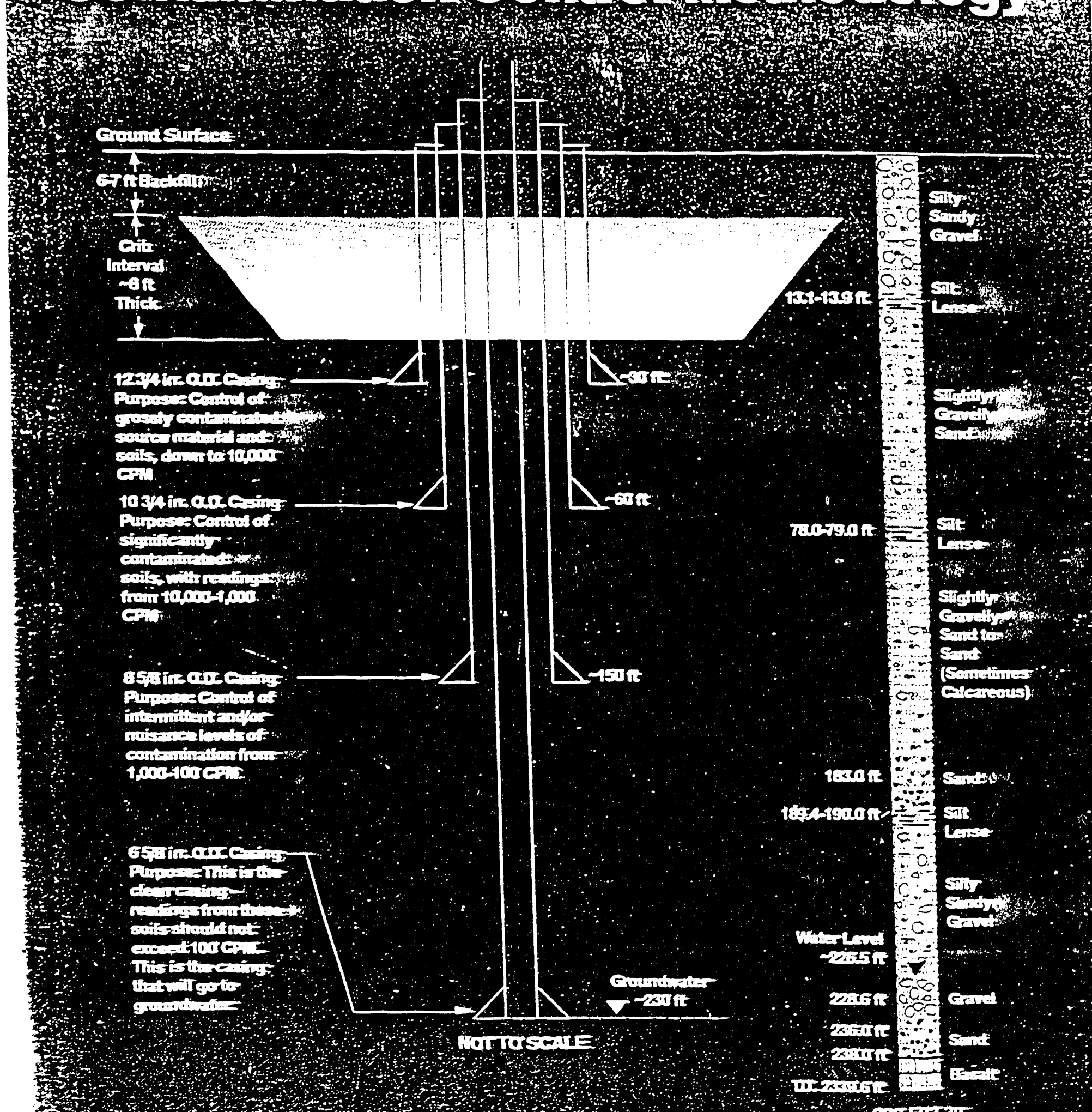




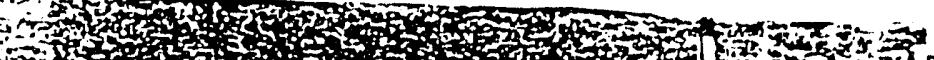

WHC-MR-0420

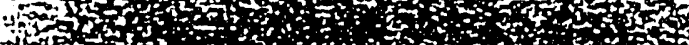

itis

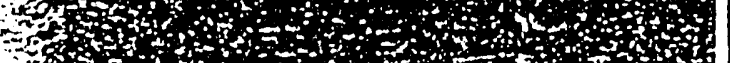

13)

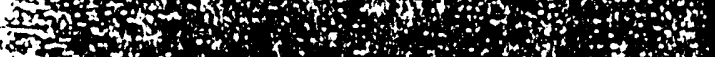

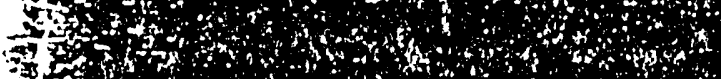

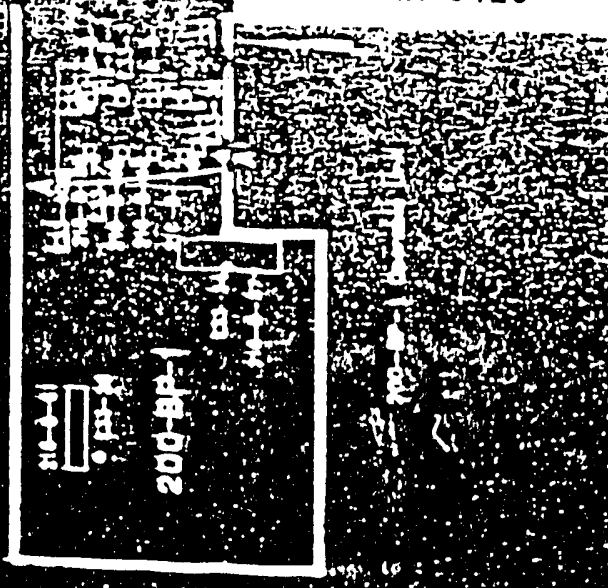

(3)

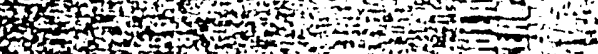

$2=$

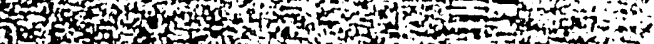

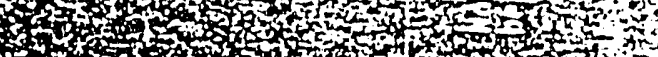

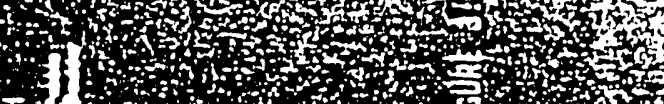

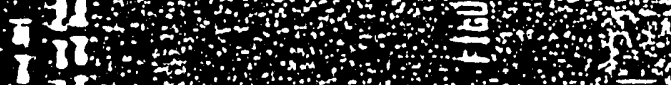
III

38

tif

3it

ins

ind

.
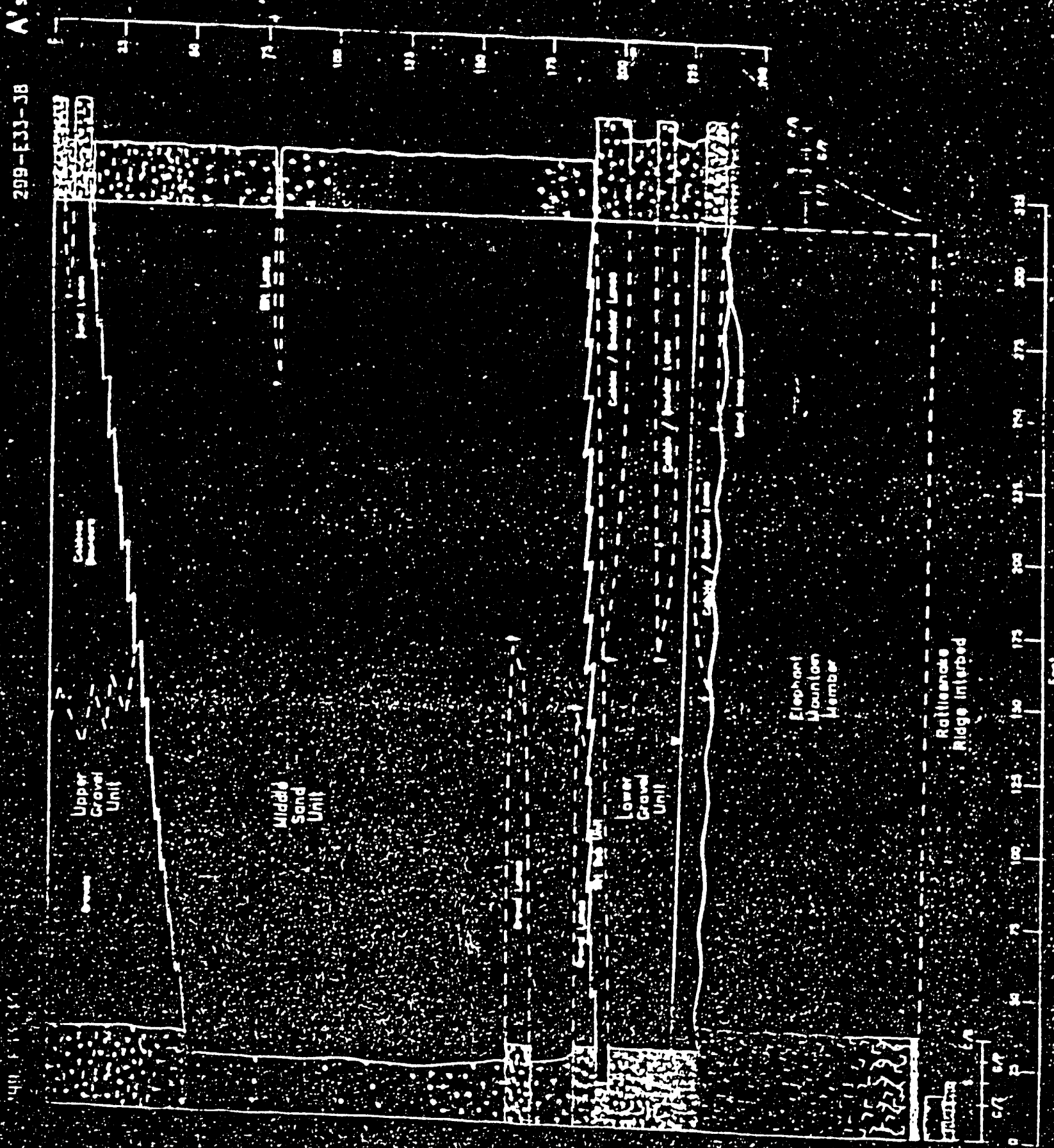

60 


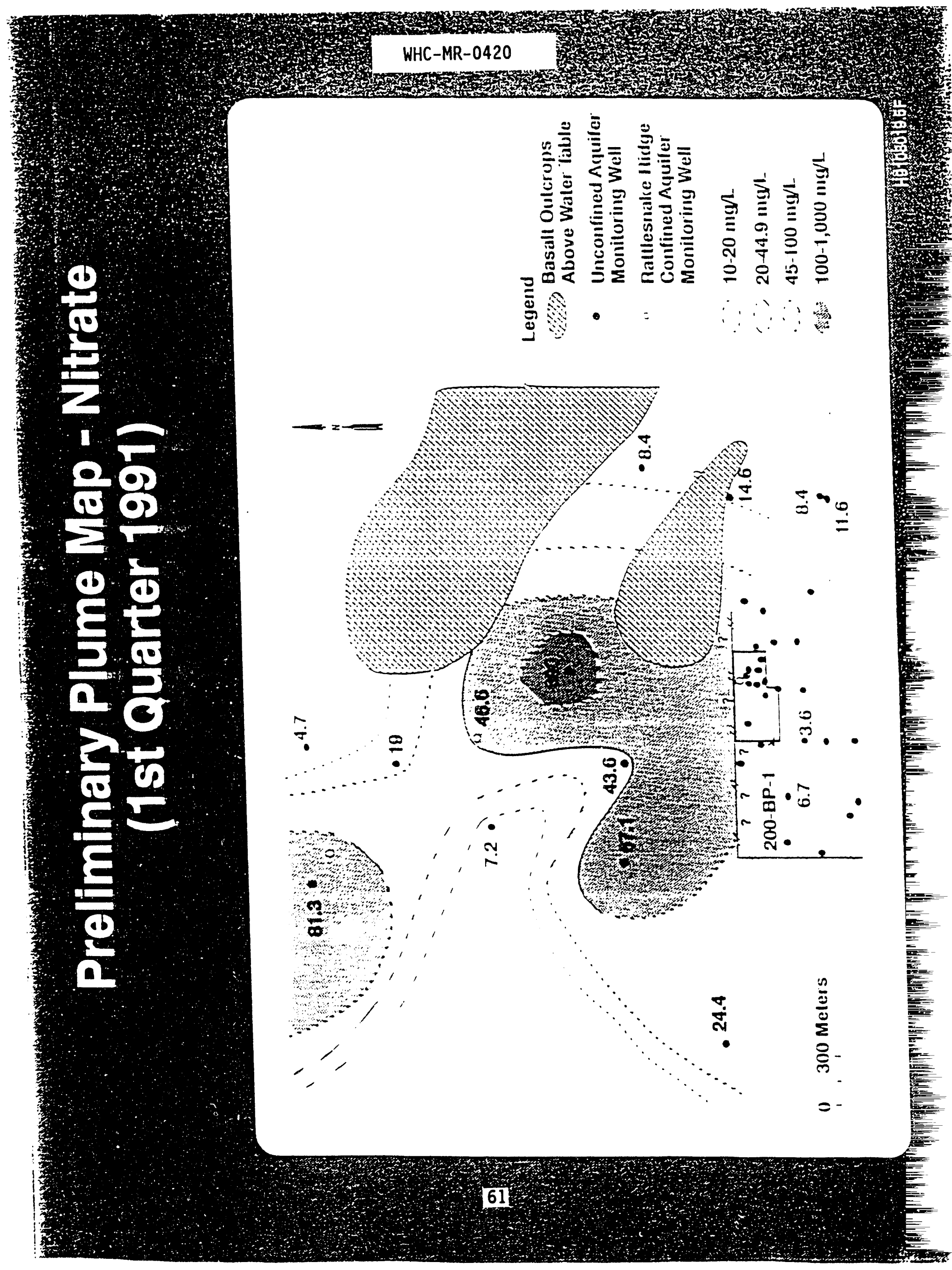




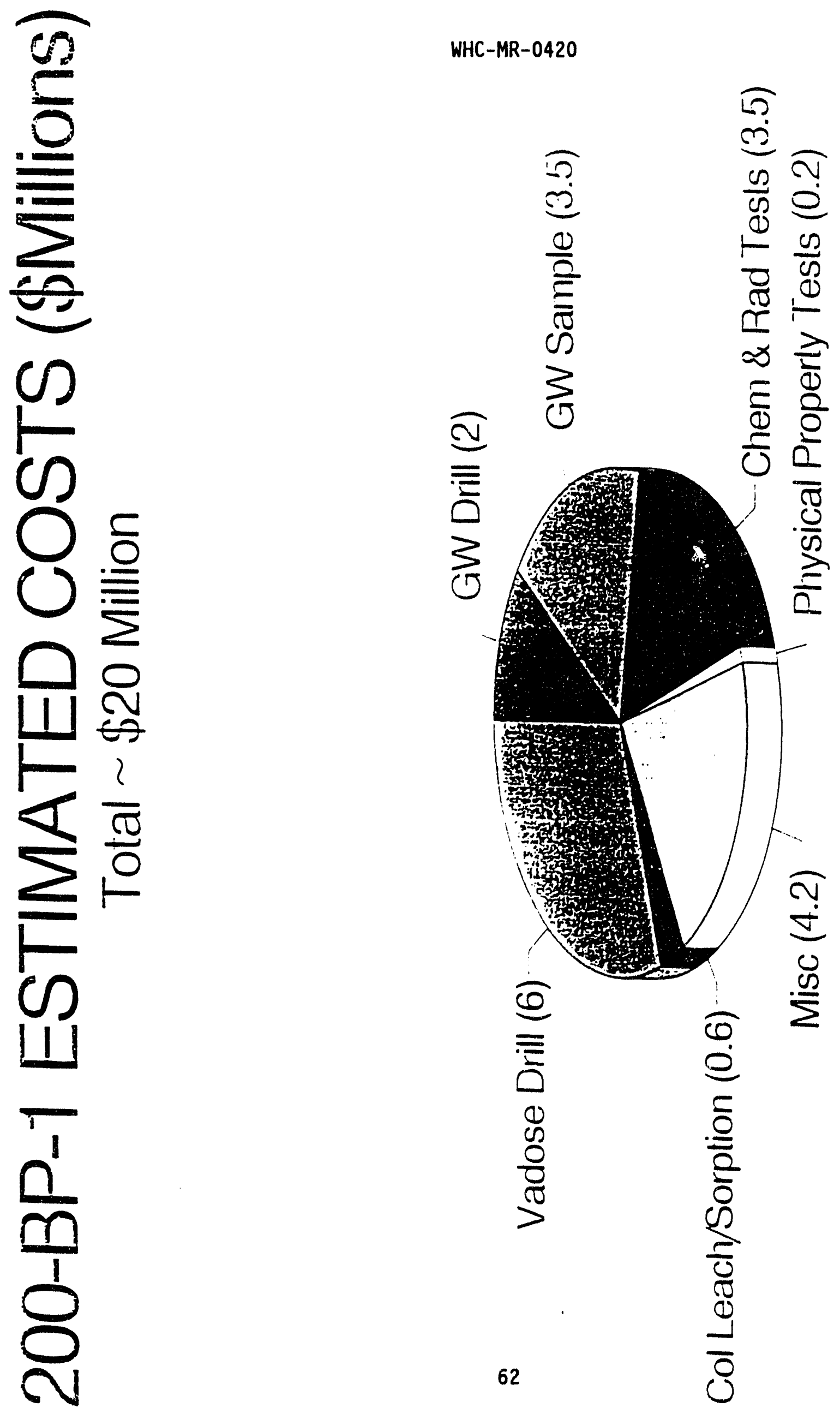


RLS Spectral Gamma-Ray Borehole Survey Project: 200-EF-1 Eorehole: $29 \subseteq-5 j J-296$ (E-4JA) Ancl. DGie: Aug 20, 92

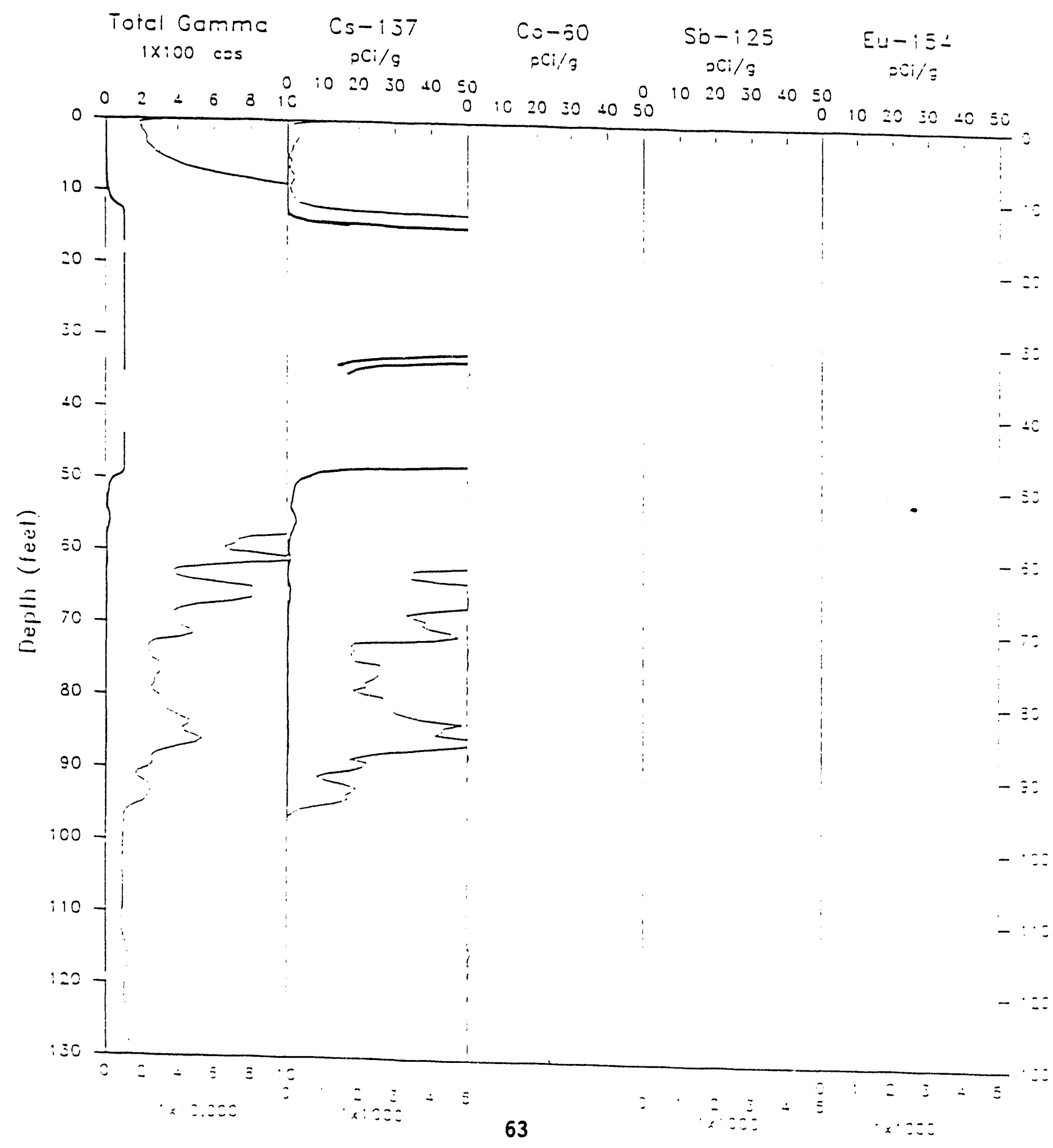


WHC-MR-0420

RADIONUCLIDE LOGGING SYSTEM

¿. IN SITU CHARACTERIZATION PROBE - 5 INCH DIAMETER PIPE

iI. APPROXIMATELY $\$ 10,000$ EACH

III. CESILI! 1:37, COBALT 60, AND OTHERS

IY. QUICK TURIIAROUNO

У. STILL OEVELOPING TECHHOLOGY 
RLS Spectral Gamma-Ray Borehole Survey

Project: 200-8P-1

Log Date: Jan 27, 92 Borehole: 299-Ej3-296 (B-4JA) Ancl. Date: Aug 20, 92

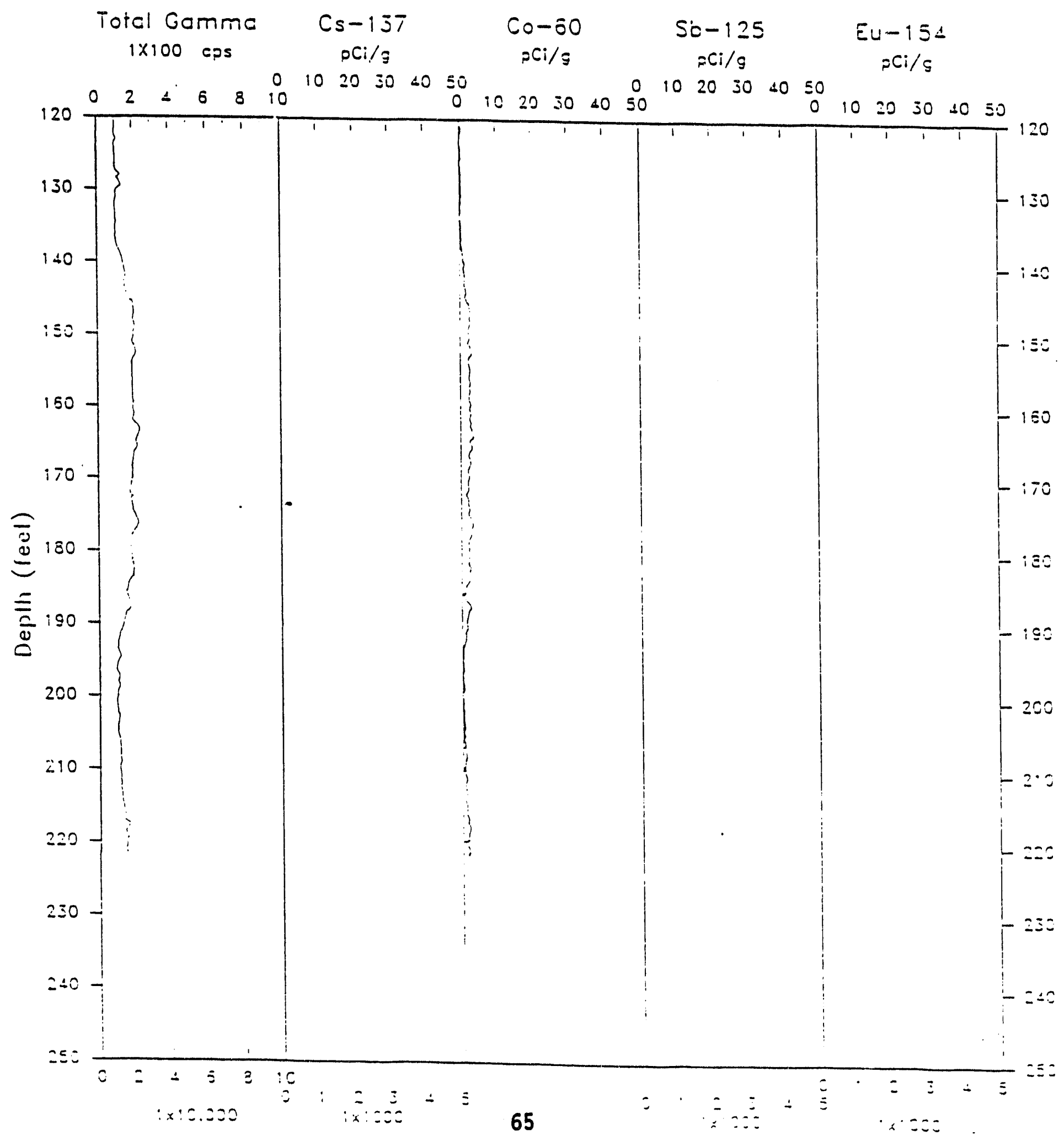




\section{RLS Spectral Gamma-Ray Borehole Survey}

Froject: 200-BP-1

Borehole: 299-EjJ-j02 (E-49A) Anal. Date: Jul 20, 92

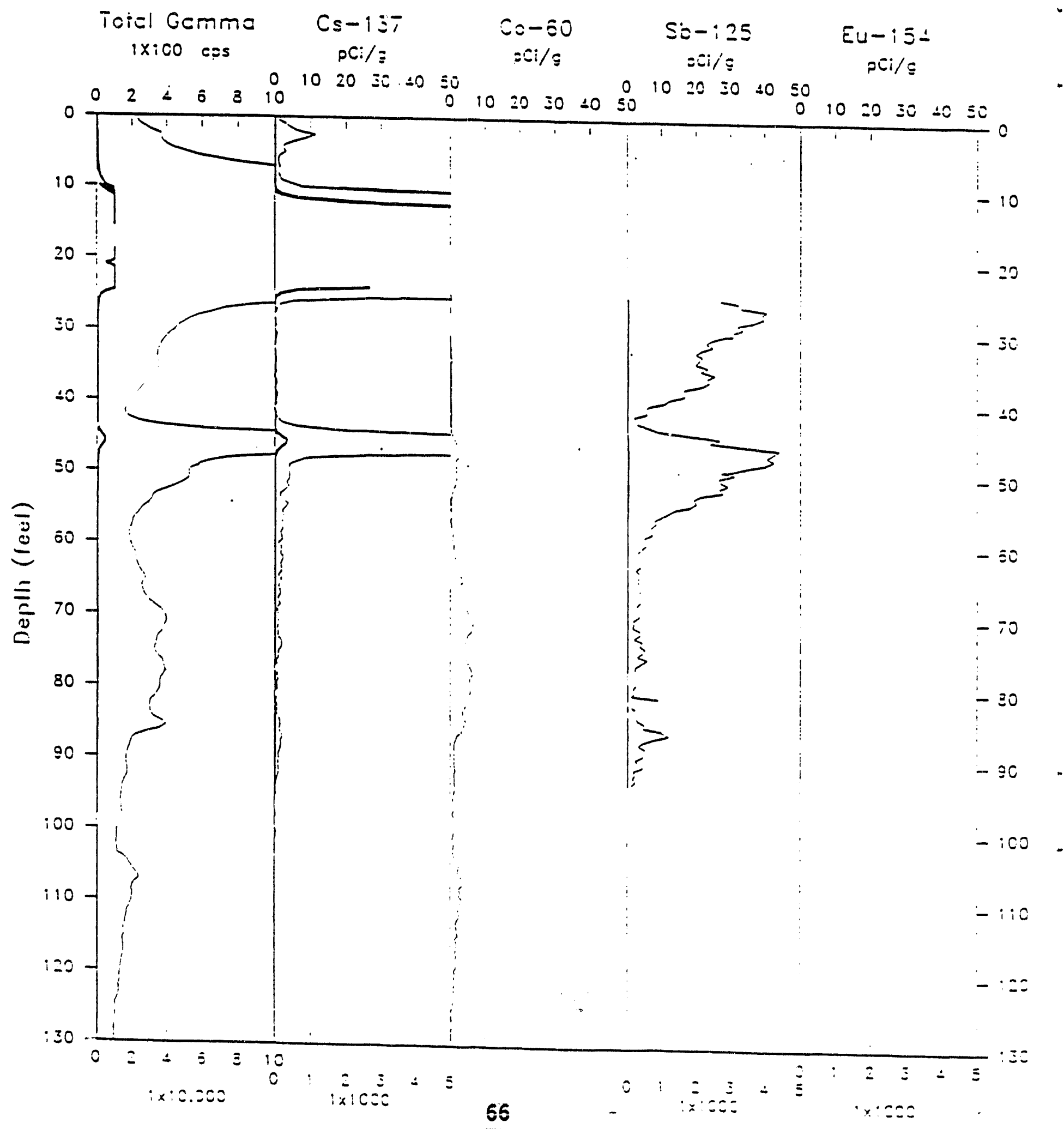


RLS Spectral Gamma-Ray Borehole Survey

Projest: $200-B P-1$

Log Daie: Sepi 5, 92

Borehole: 299-Ej3-302 (E-49A) Anal. Daie: Jul 20. 92

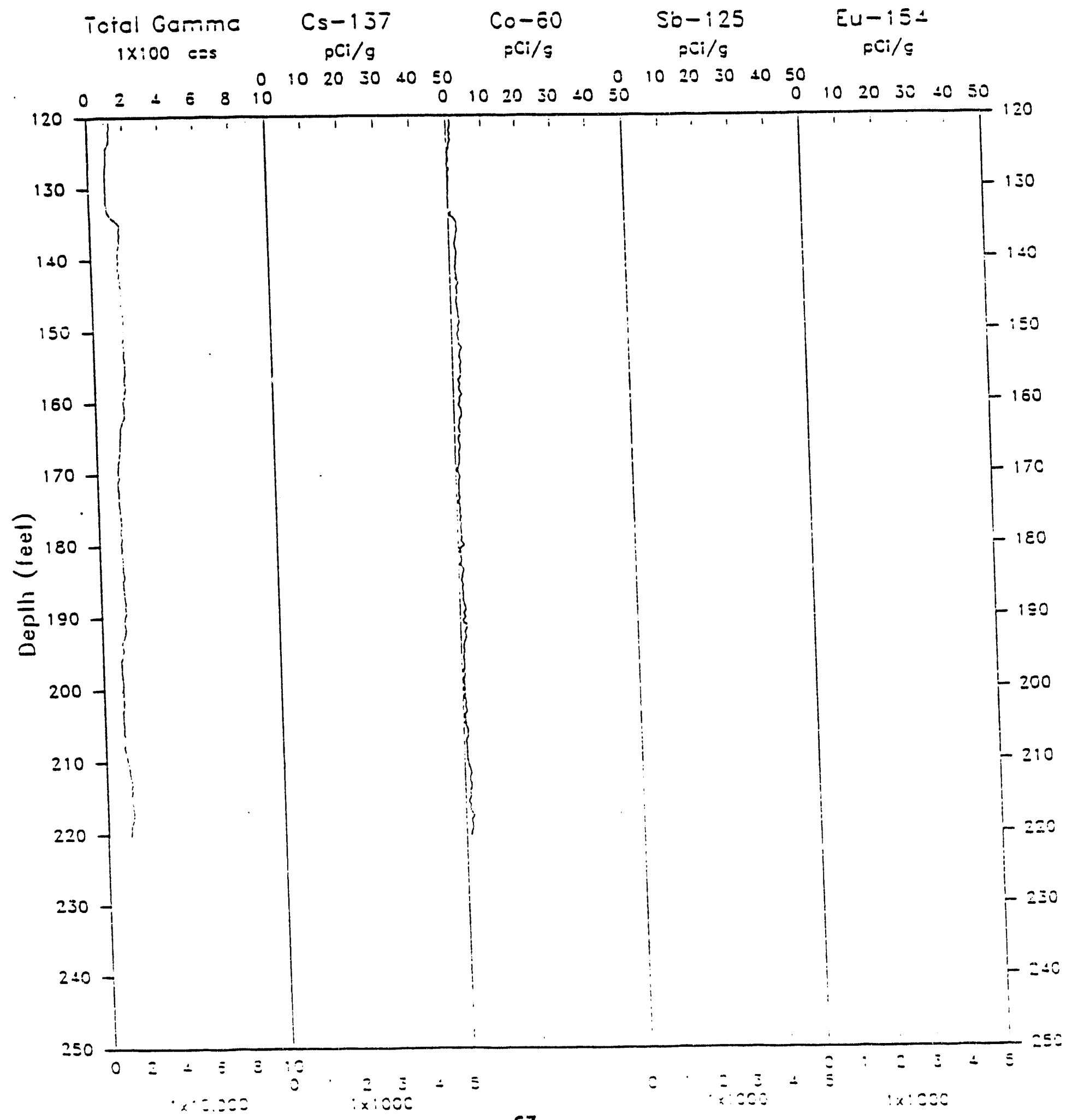




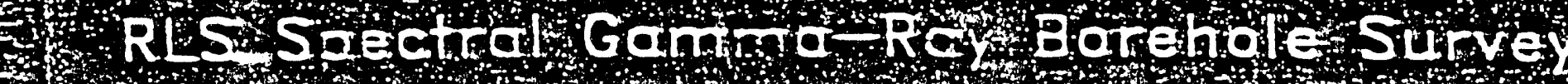

m.5.6.

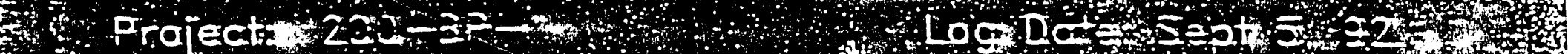

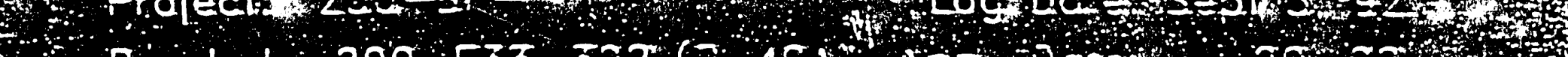

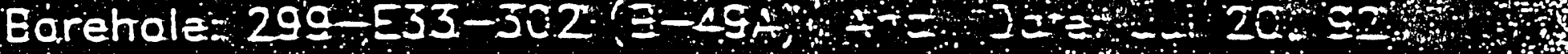

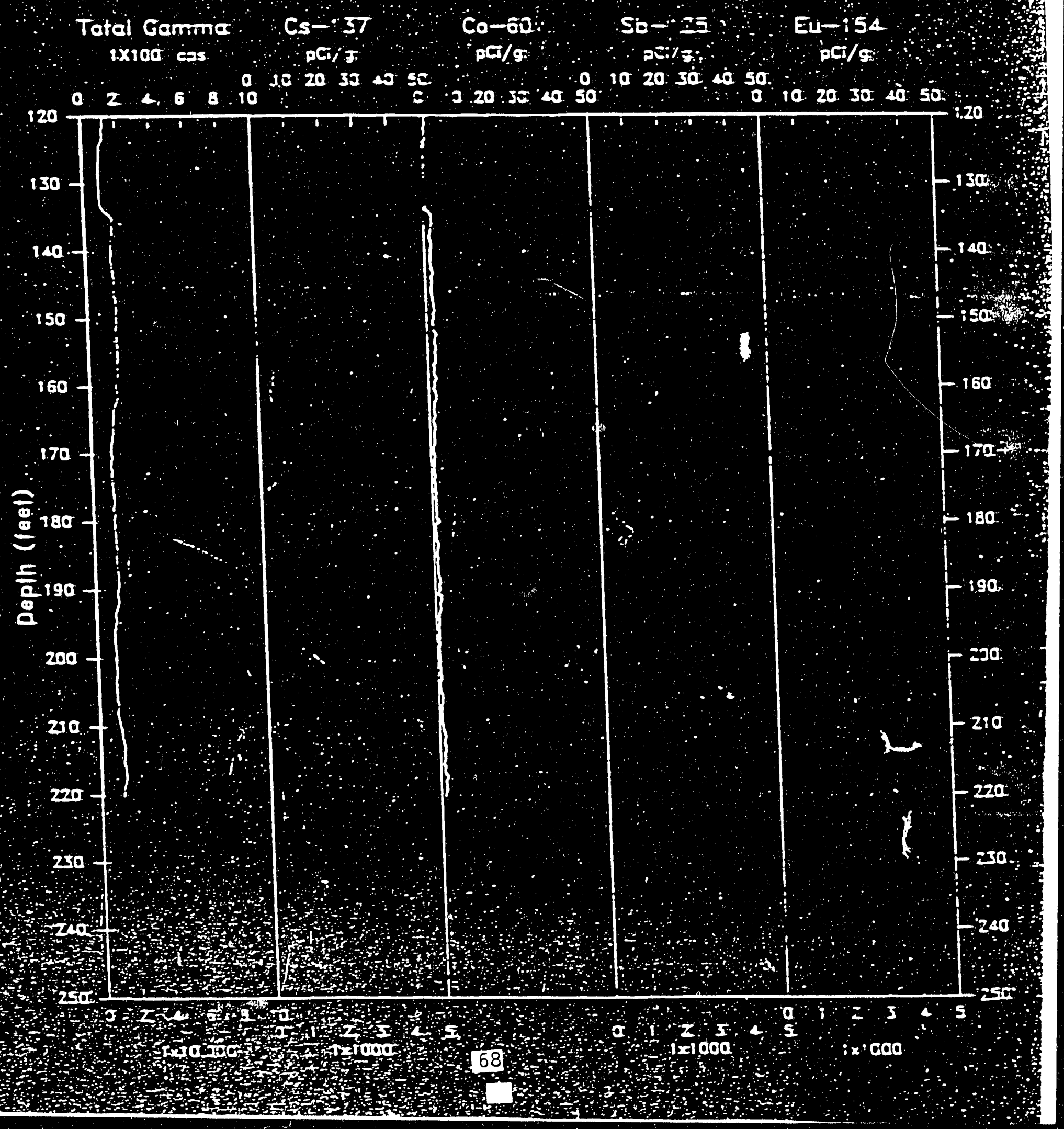




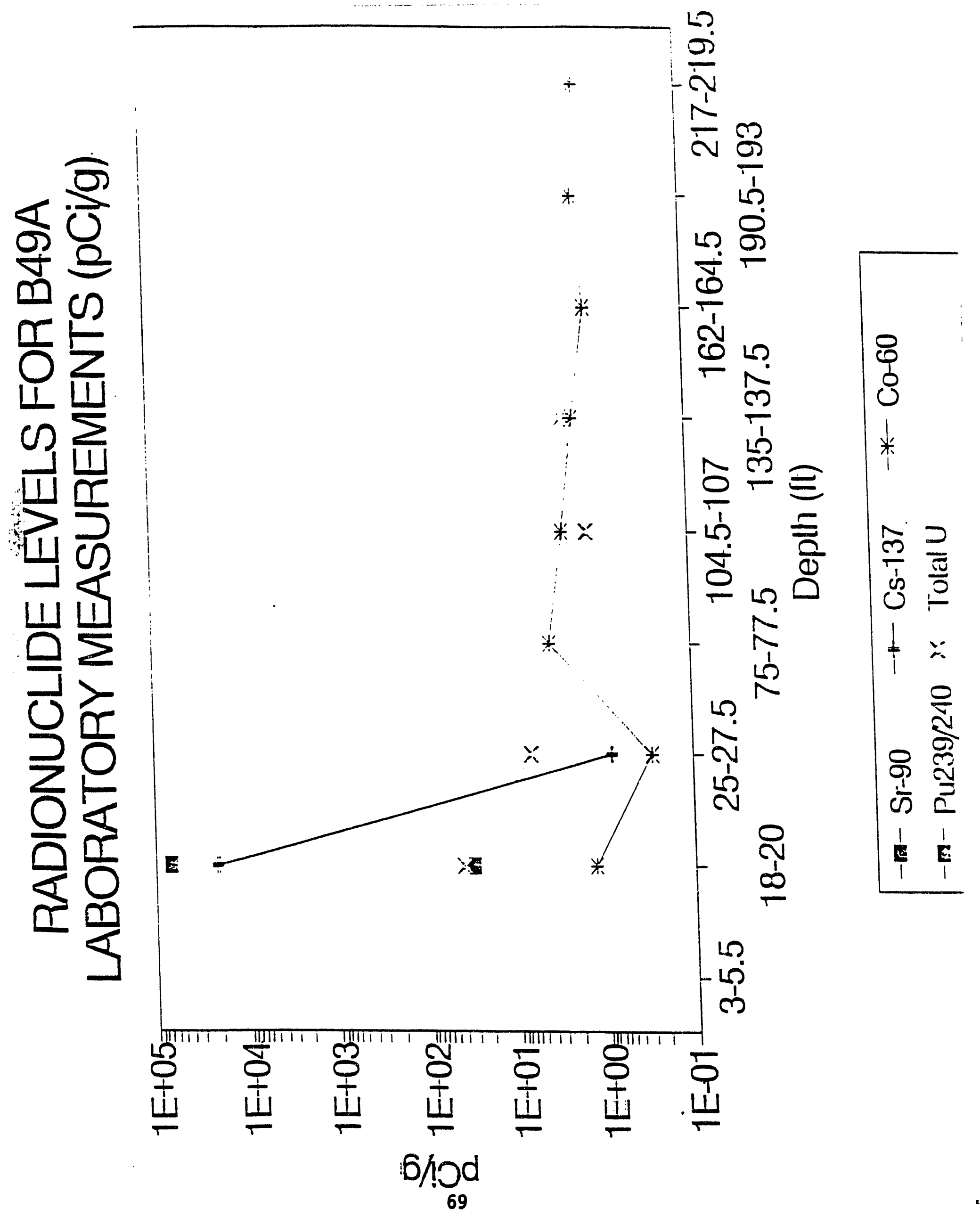




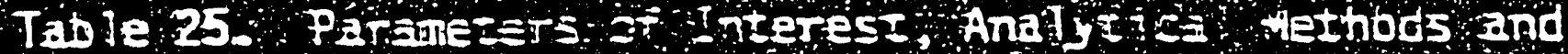

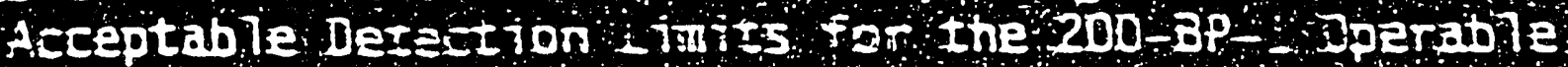
Unit Remedial Investigat tons:

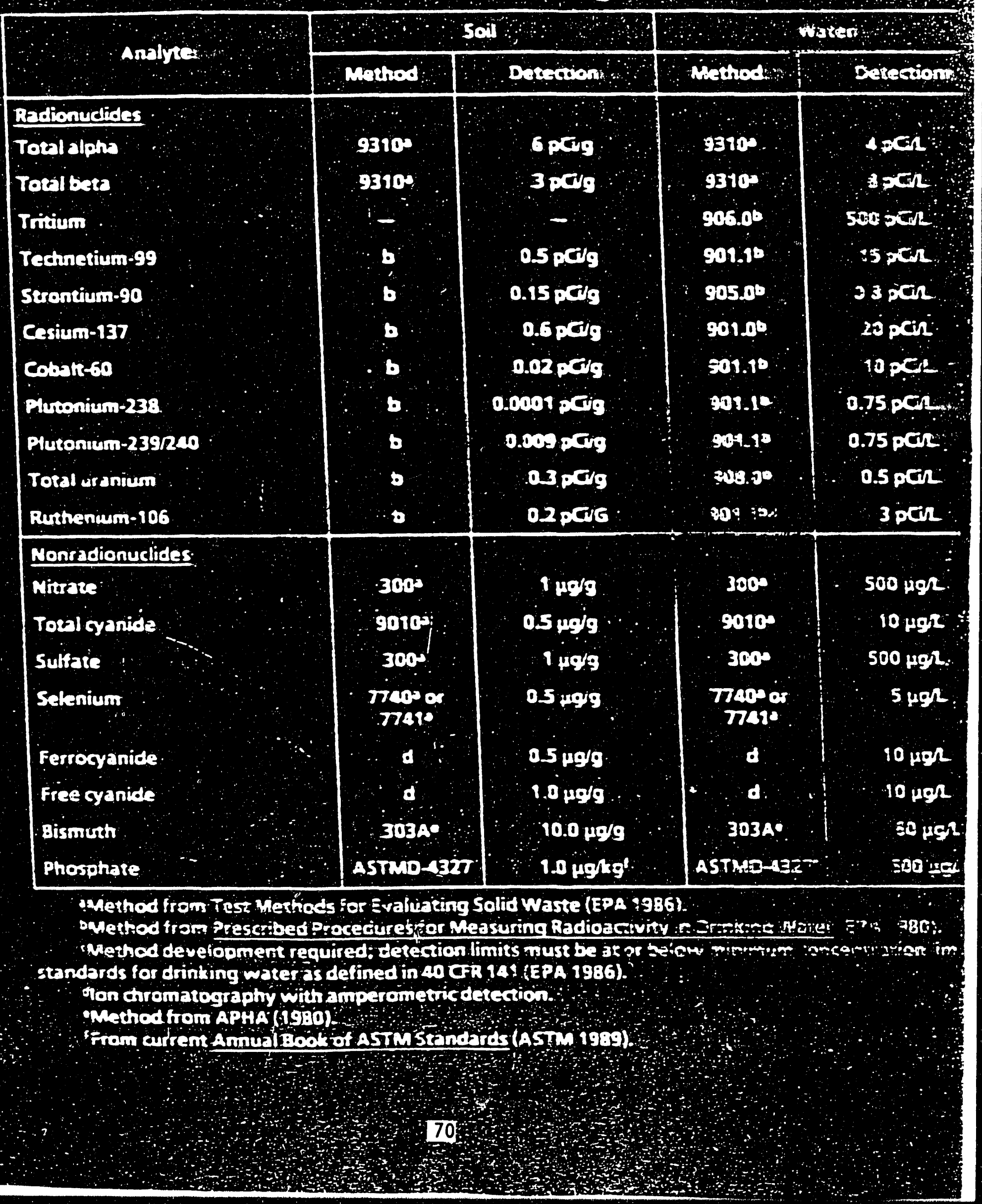


WrIL-I'In-U+LU
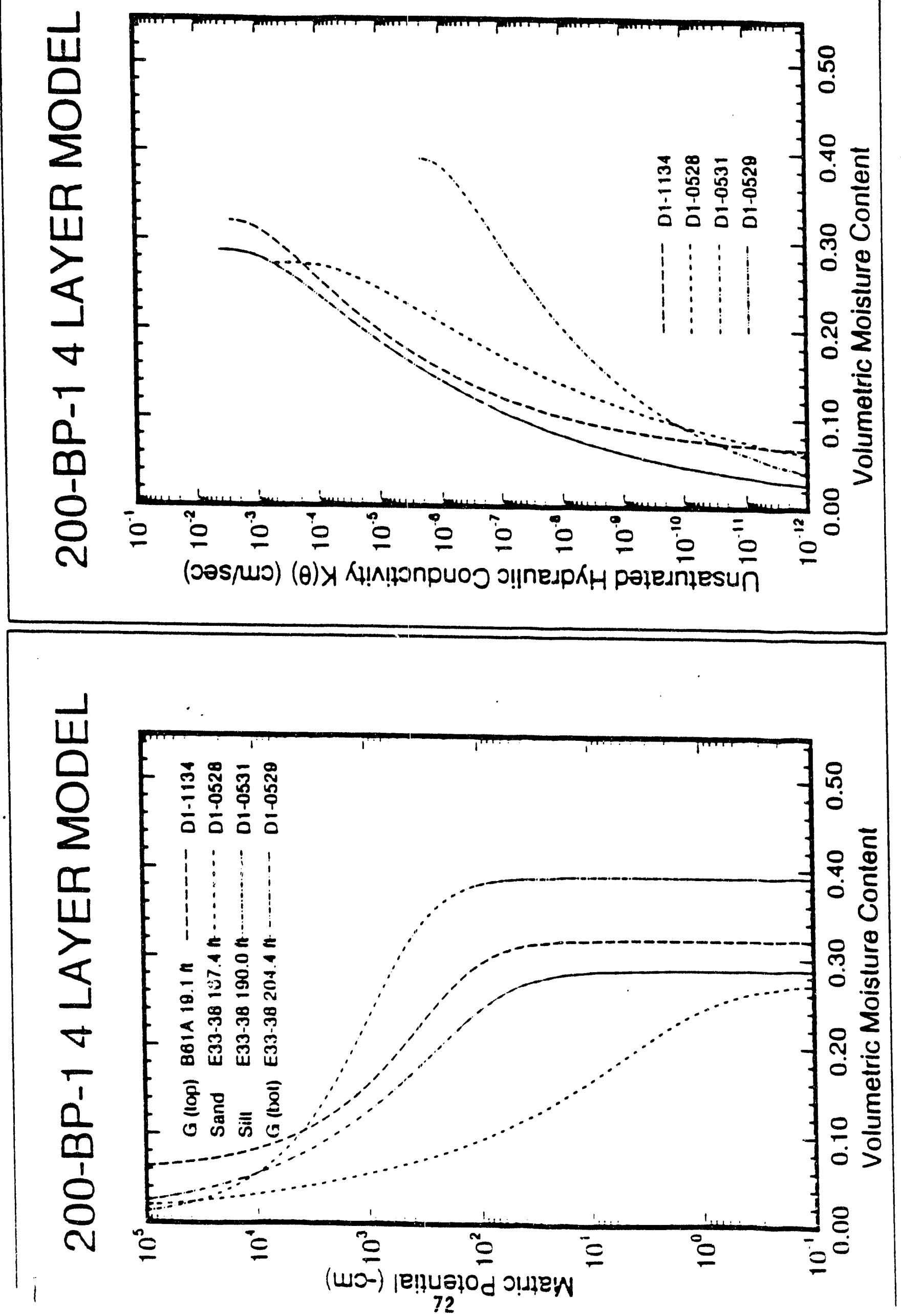


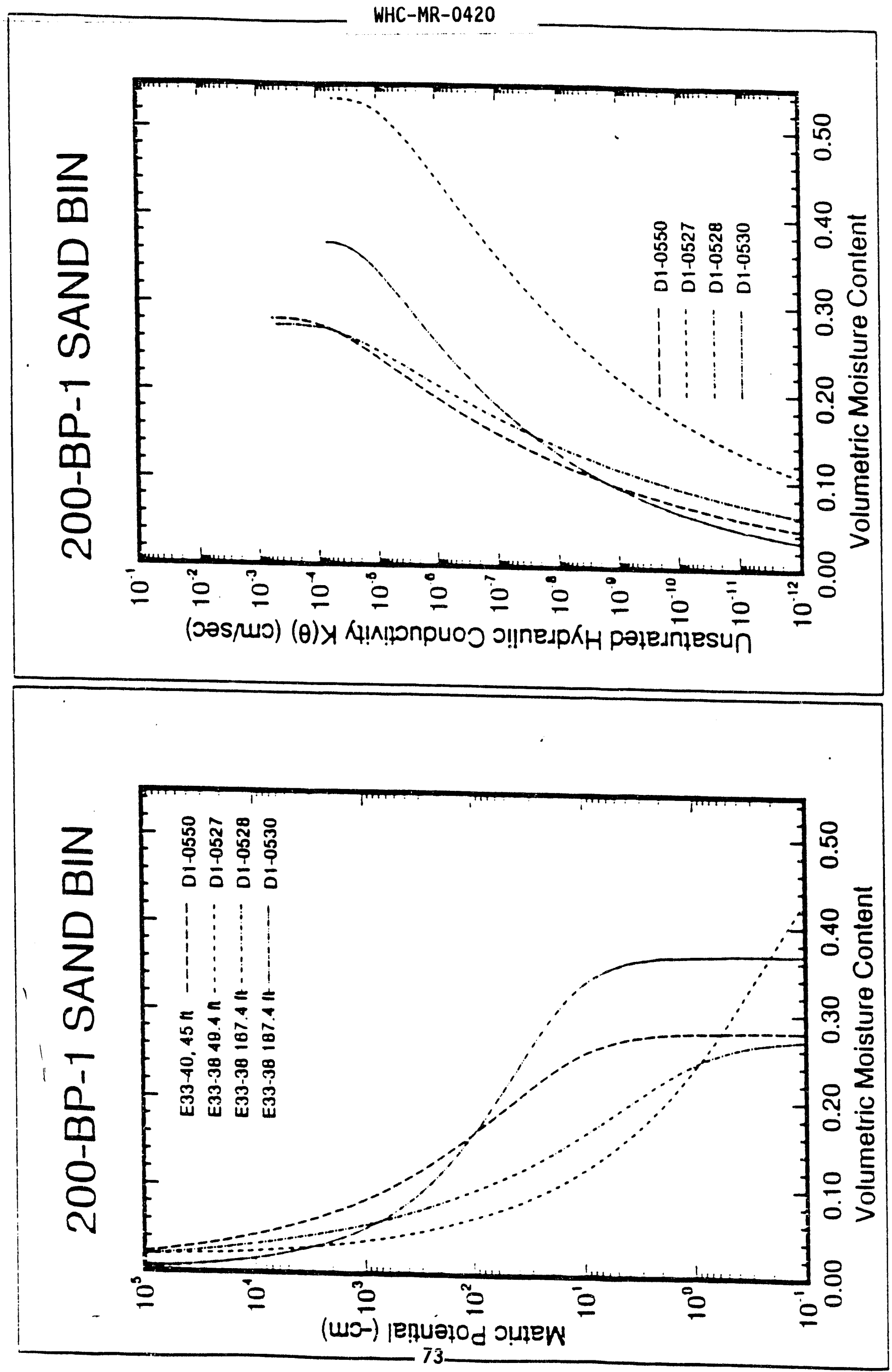


WHC-MR-0420

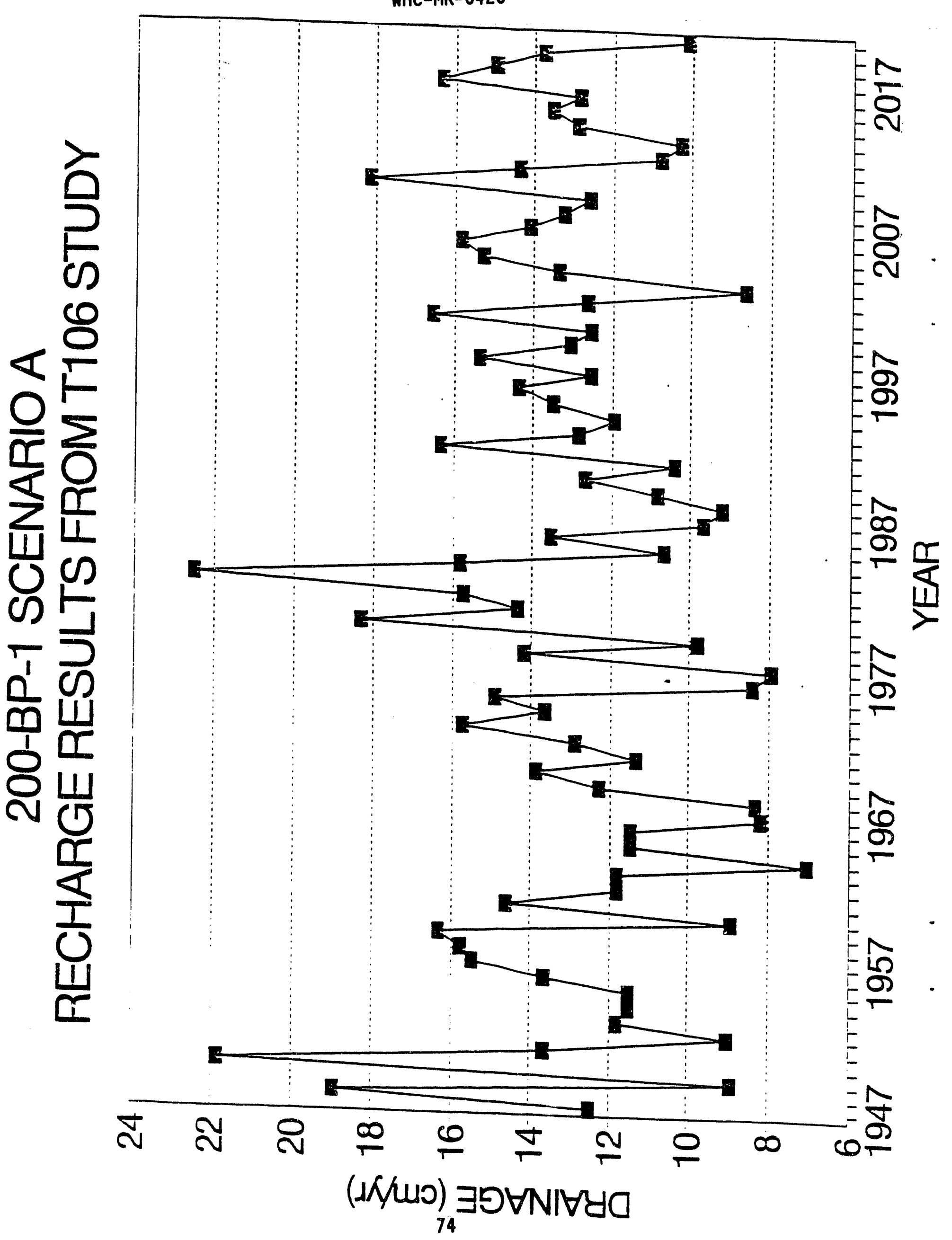




\section{PHYSICAL PROPERTY TESTS}

BULK DENSITY / POROSITY

SPECIFIC GRAVITY FOR PARTICLES

SATURATED HYDRAULIC CONDUCTIVITY

MOISTURE RETENTION (no unsaturated hydraulic conductivity tests were run)

CALCIUM CARBONATE CONTENT

IN SITU MOISTURE CONTENT

PARTICLE SIZE DISTRIBUTION

COLUMN LEACH

SORPTION

OTHER PHYSICAL PROPERTY DATA (from literature)

\section{DISPERSIVITIES}

MOLEEULAR DIFFUSION

SPEZ:ZIC STORATIVIT!

RADIOACTIVE DECAI RATE

UNTESTED DISTRISUTION COEZFICIEITS 
TABLE 1. Sumary of $K_{d}$ Data Spectfic to Hanford.

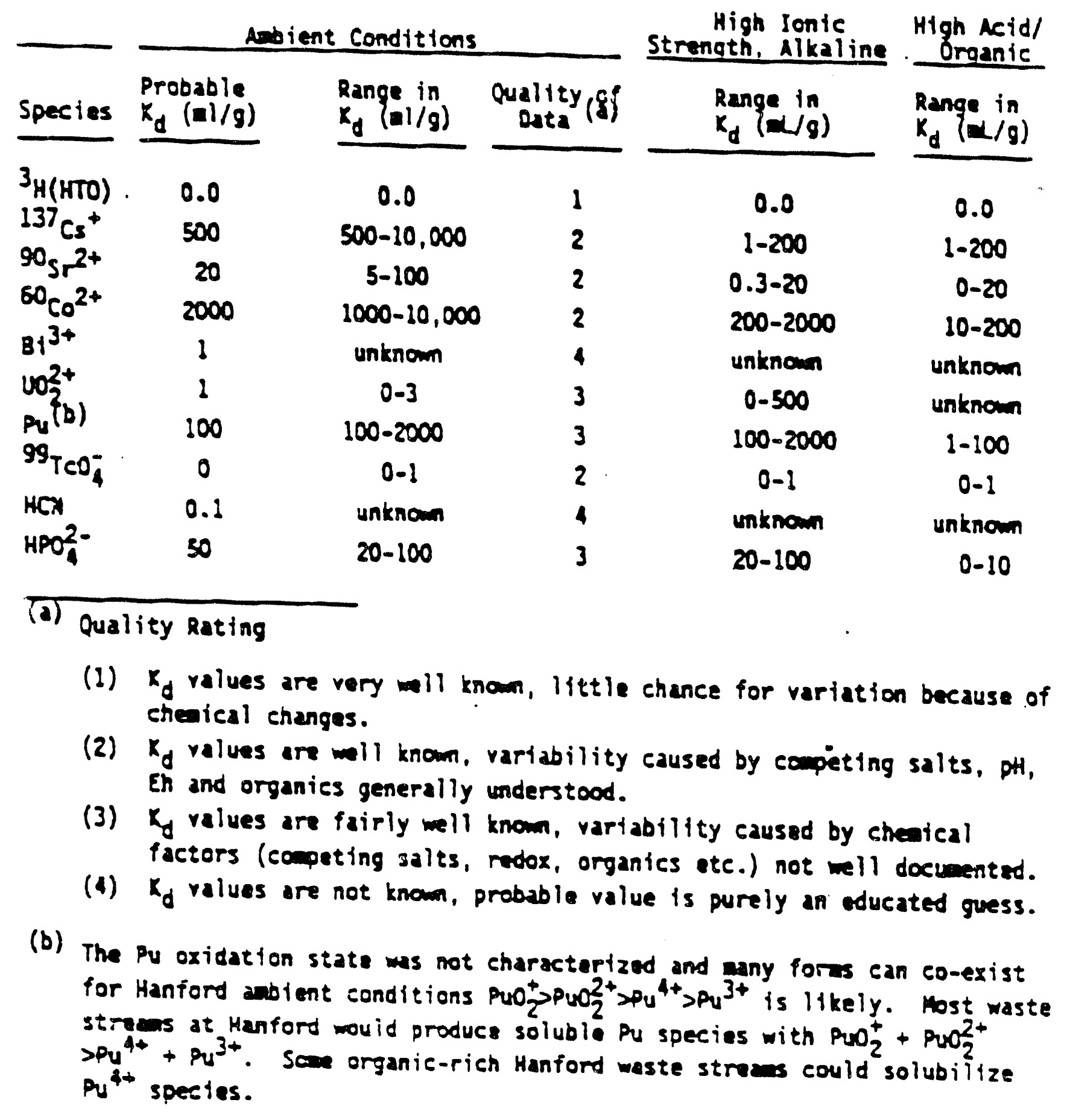


WHC-MR-0420

SELECTION OF SAMPLES FOR TESTING

I. Provide Data for Major Layers to be Modeled

i!. Problems and Difficulties

A. Individual Sample Integrity and Representativeness

B. Use of Multiple Liners

C. Lack of Data in Hot Zone

D. Possible Overdriving of Sampler (bulk densities $>2 \mathrm{~g} / \mathrm{cc}$ )

E. True Behavior of Gravels

$F$. Sensitivity of Laboratory Technique

1) Moisture Retention Sample $\equiv$ Size

2) Equipment Available

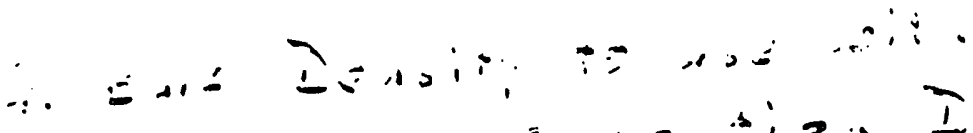

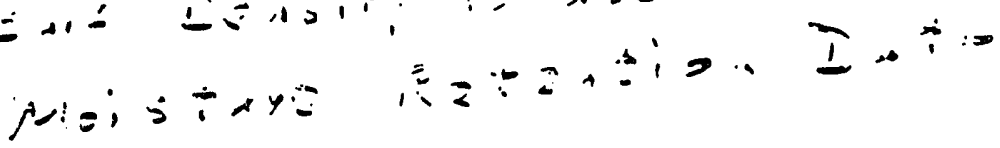

77 
MODELING APPROACH

I. Conceptual Model - Composite Soils, Single Crib

II. Computer Program Selection

III. Contaminants of Concern
A. Inventory
B. Screening Process

IV. Scenarios - Conservative, but Not Unrealistic
A. Start with Reported Inventor:
6. is:. kd from range
c. Vercical Movemeni to Grouncidaiser
i. Isotropic
D. Lateral Migration
!. Anisotropic
2. "Uorld" Model for !nterac:ions
E. PEcinarge Rates.

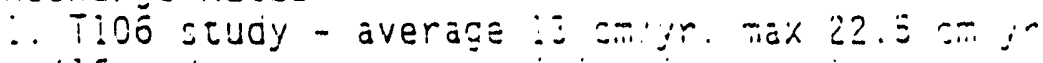

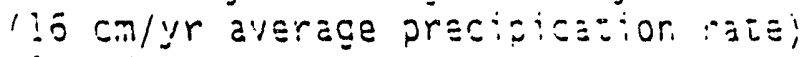
2. $1 \mathrm{~cm} / \mathrm{yr}$
¿. $10 \mathrm{~cm} / \mathrm{yr}$ (preliminary gioia barrier: 


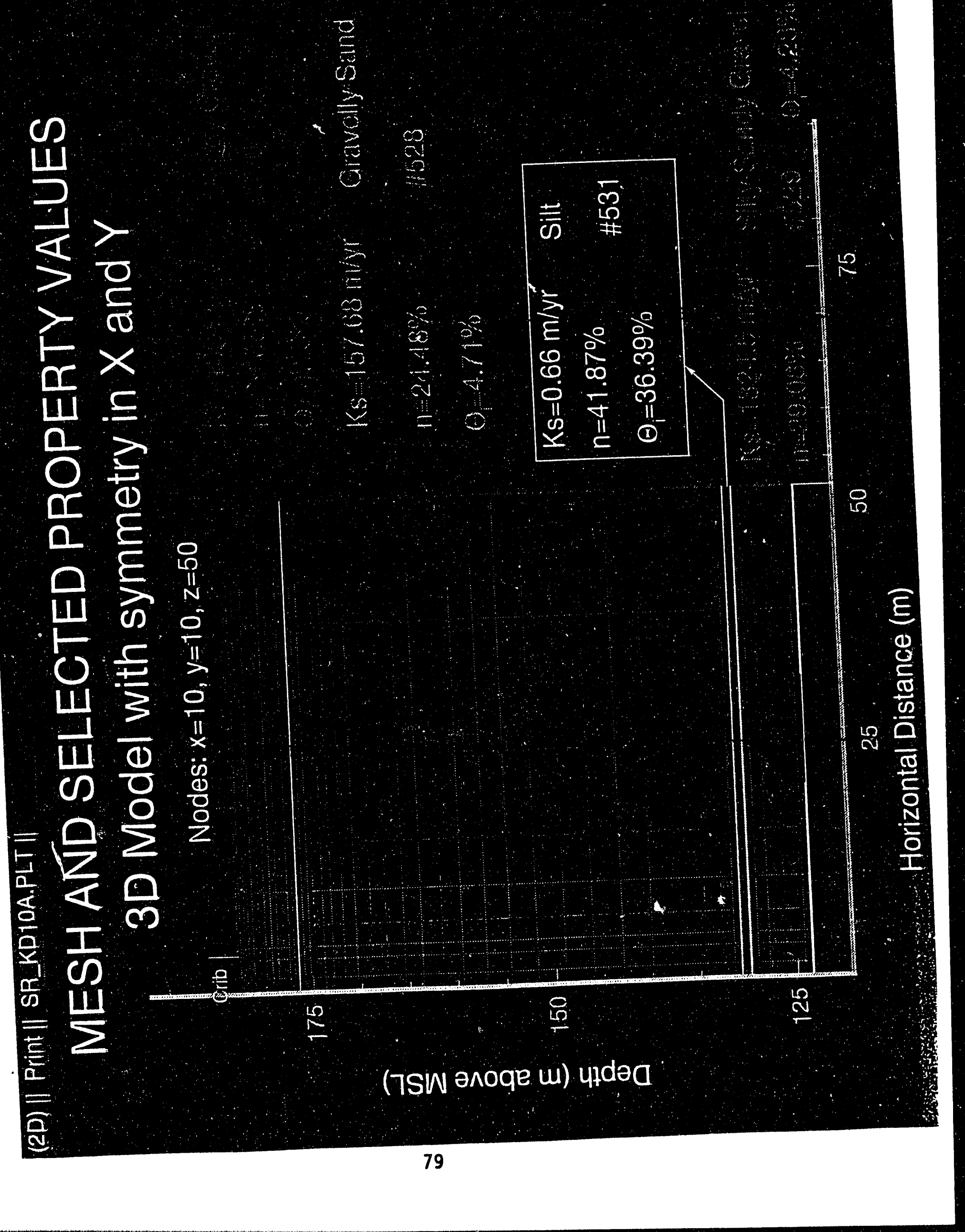




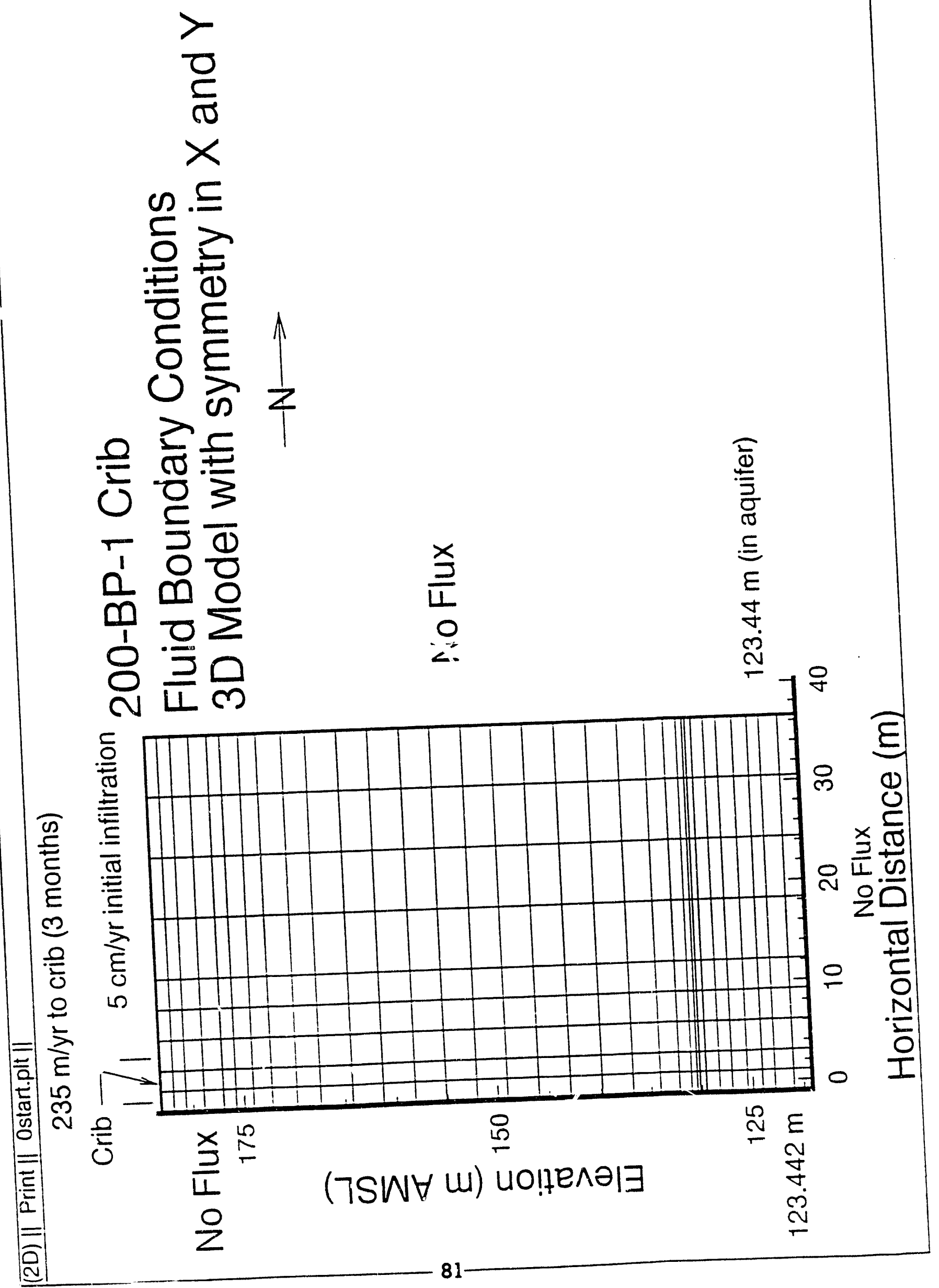



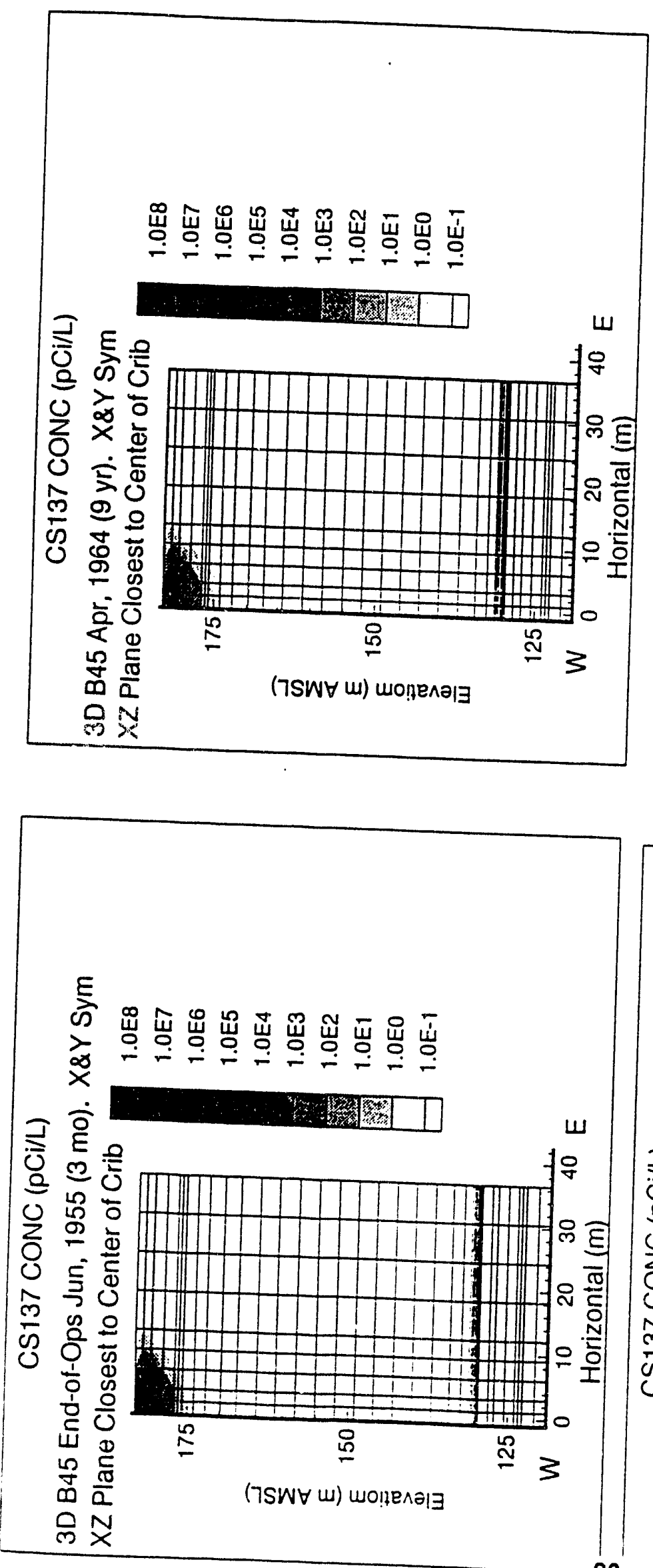

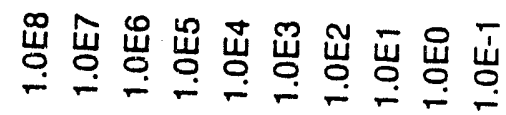
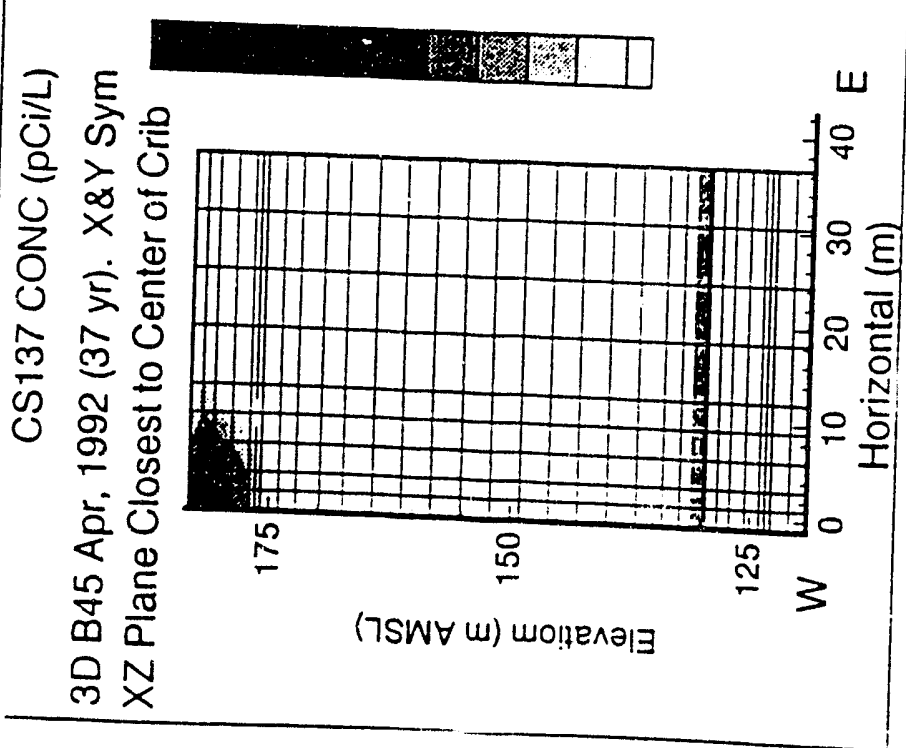
N

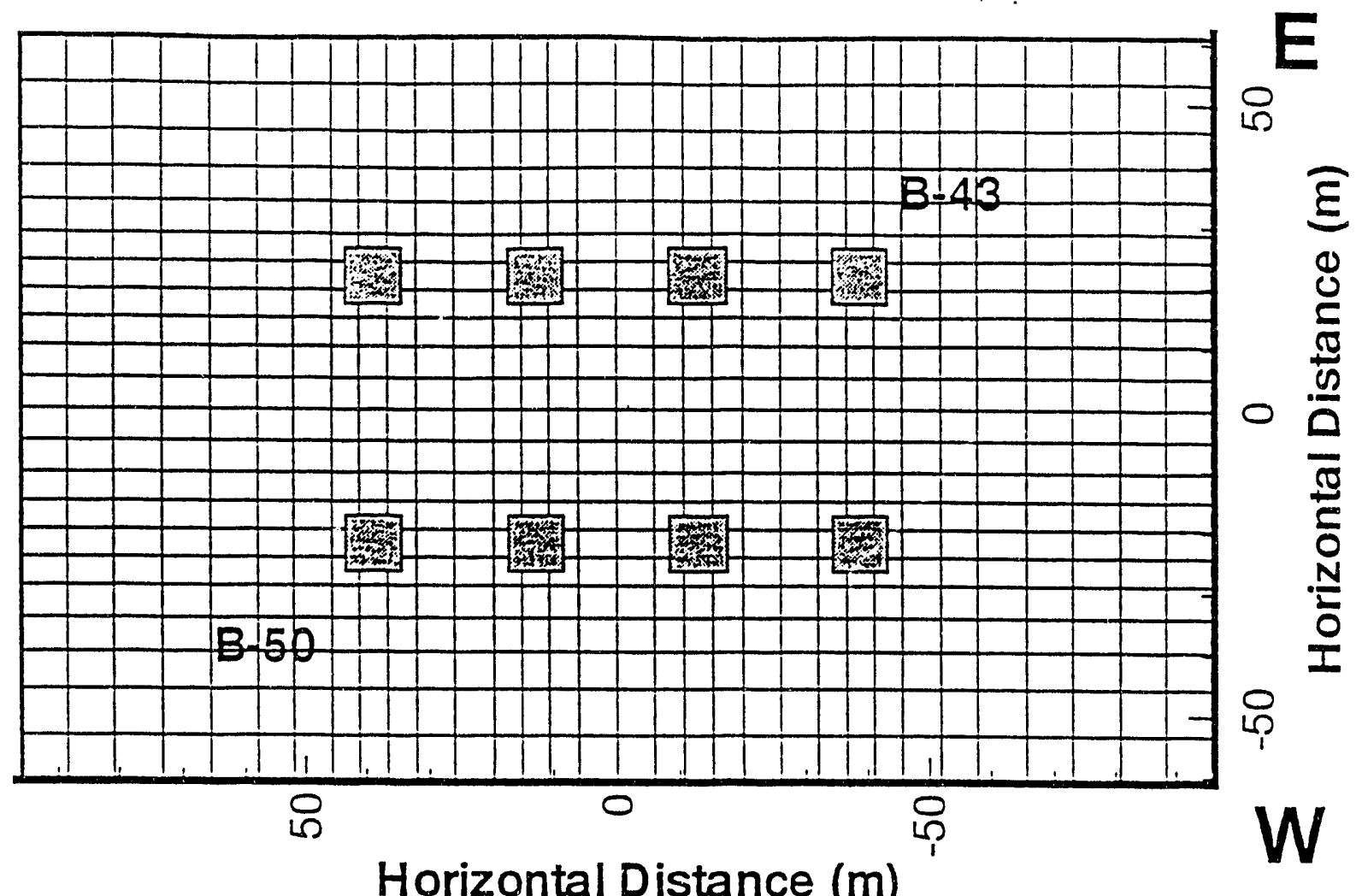

N

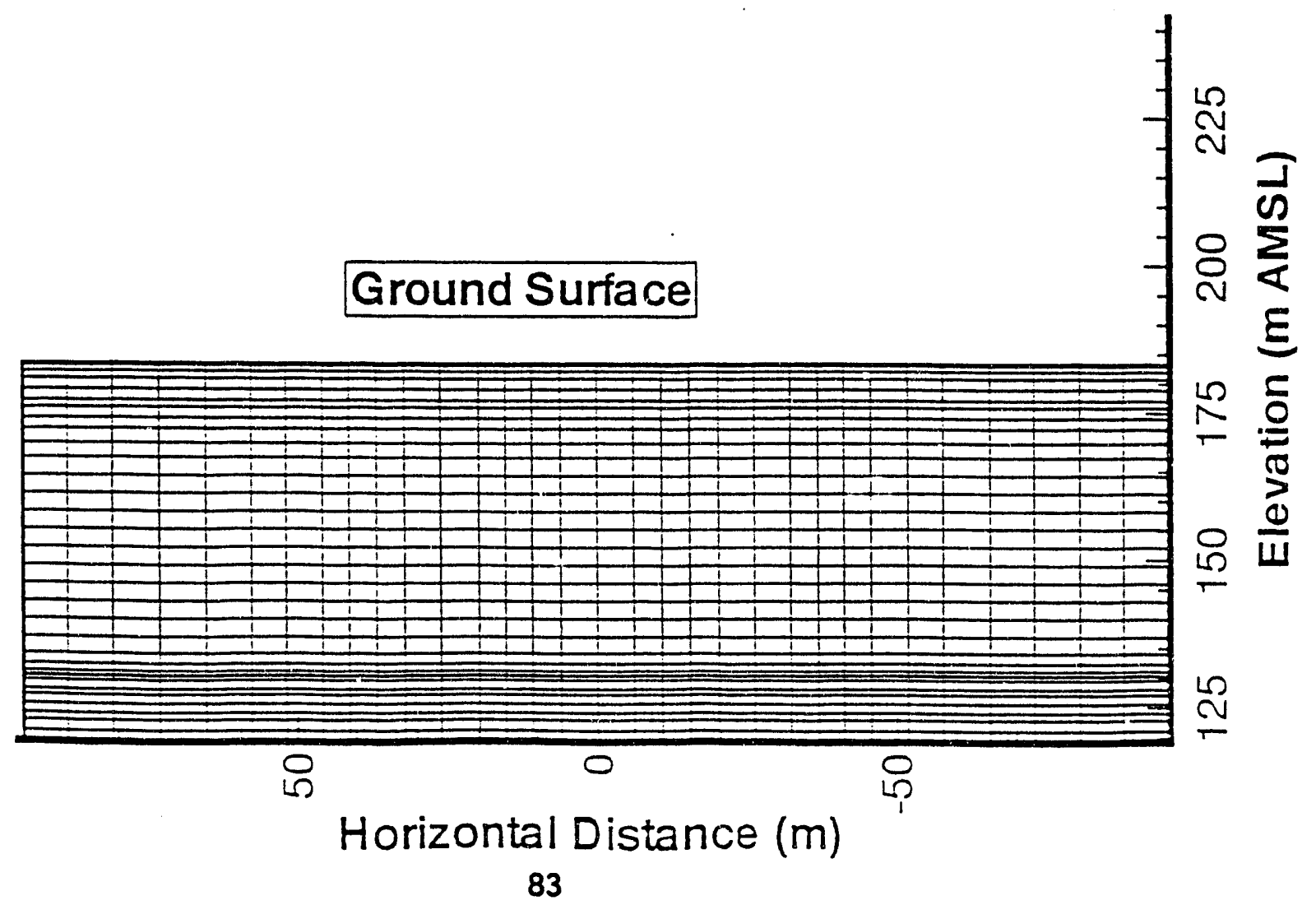




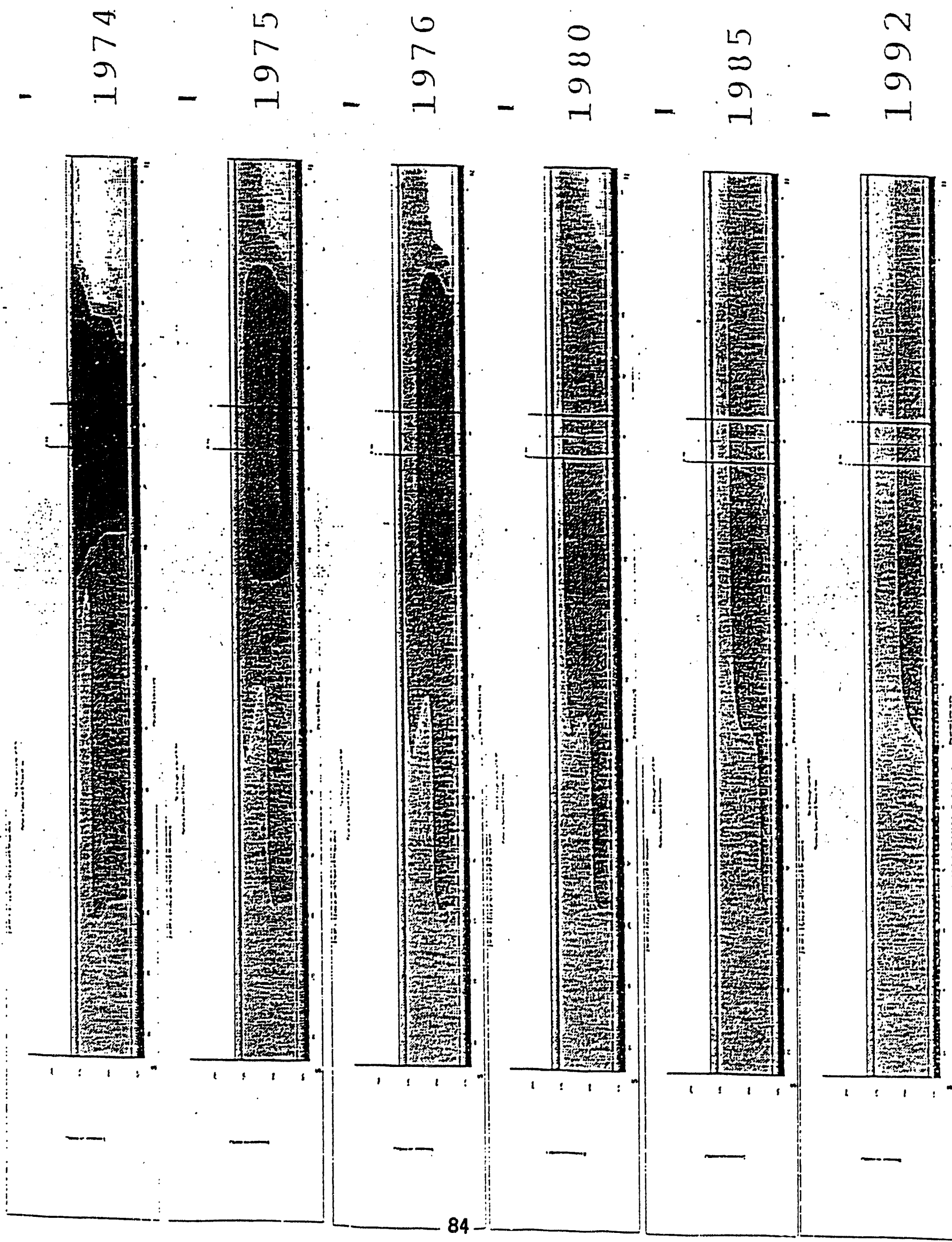


WHC-MR-0420

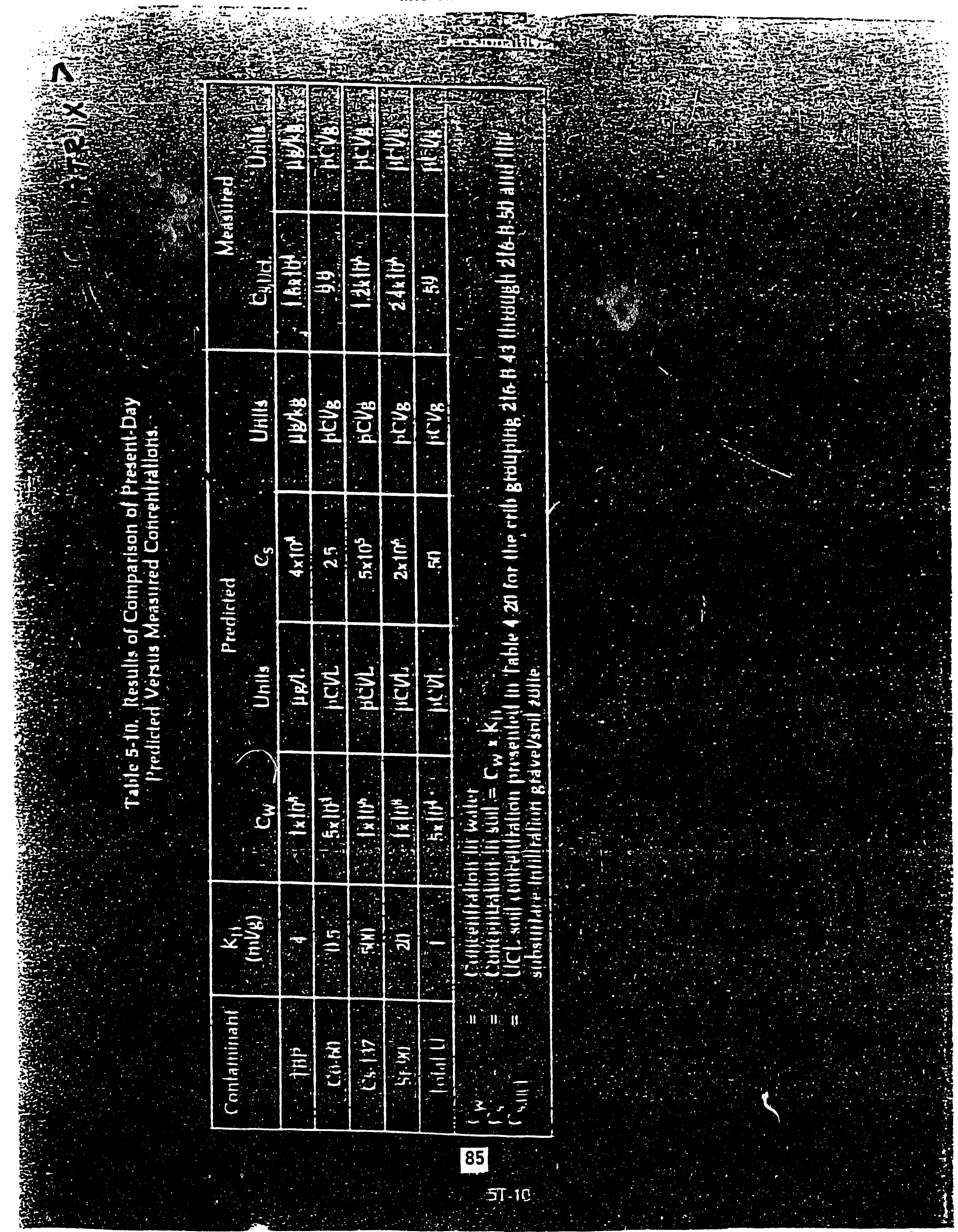


\%

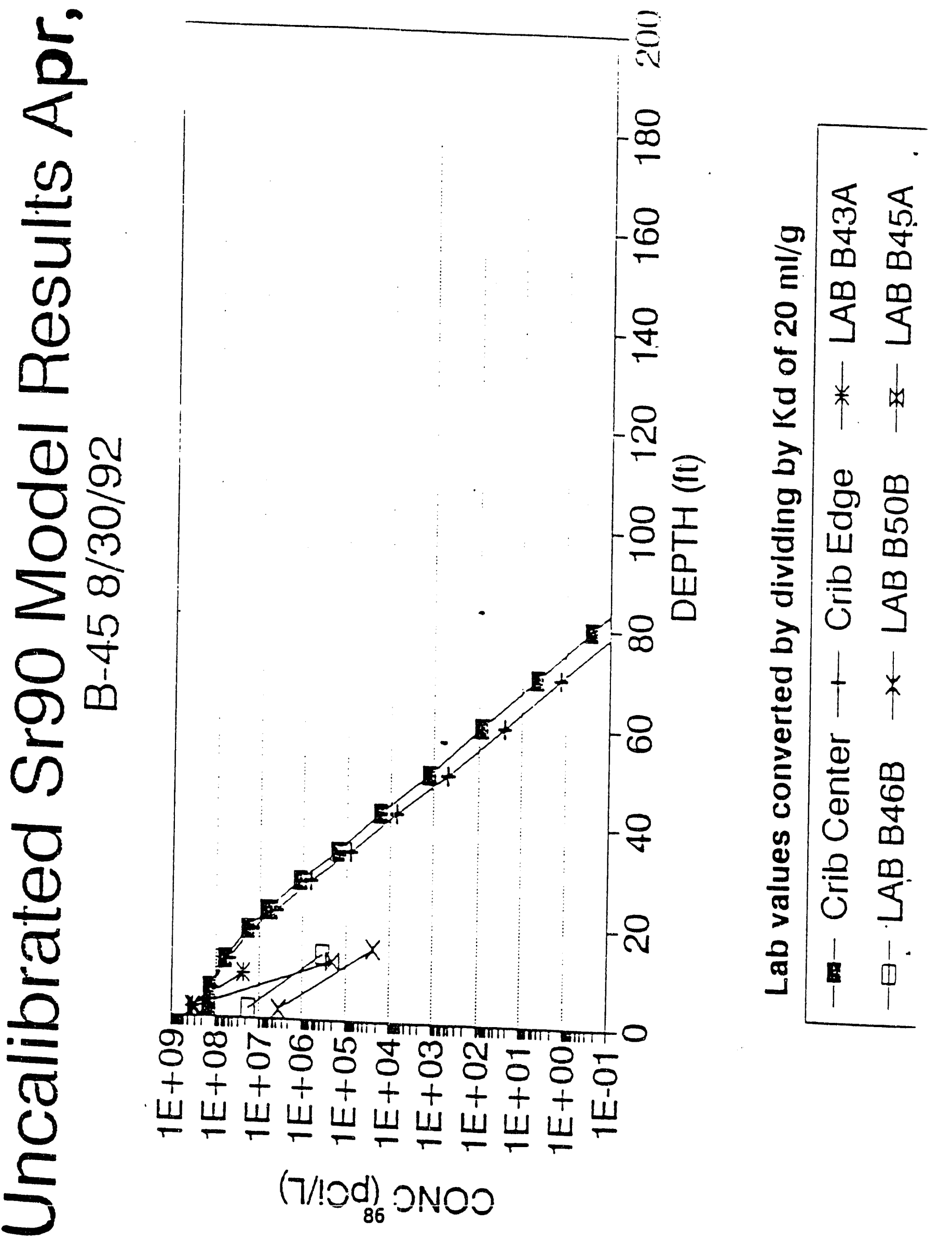




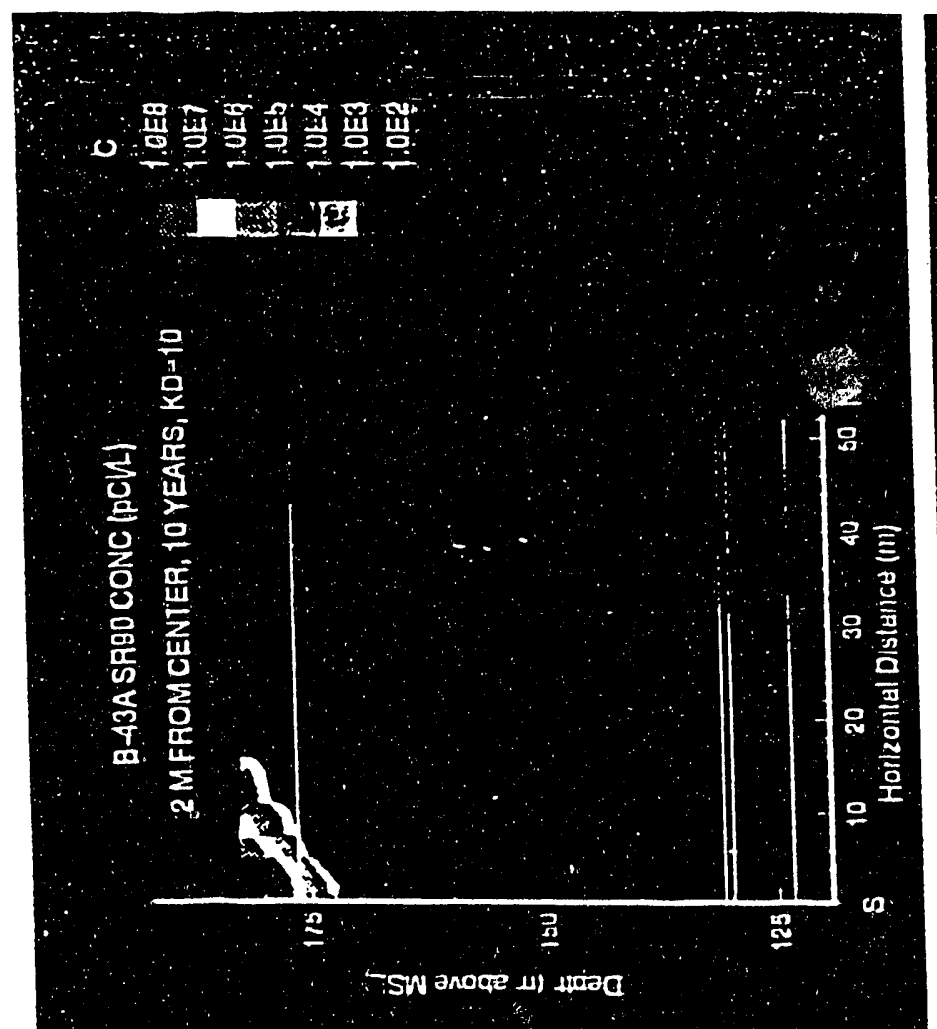

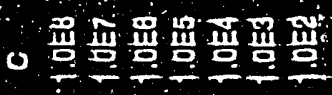

(1)

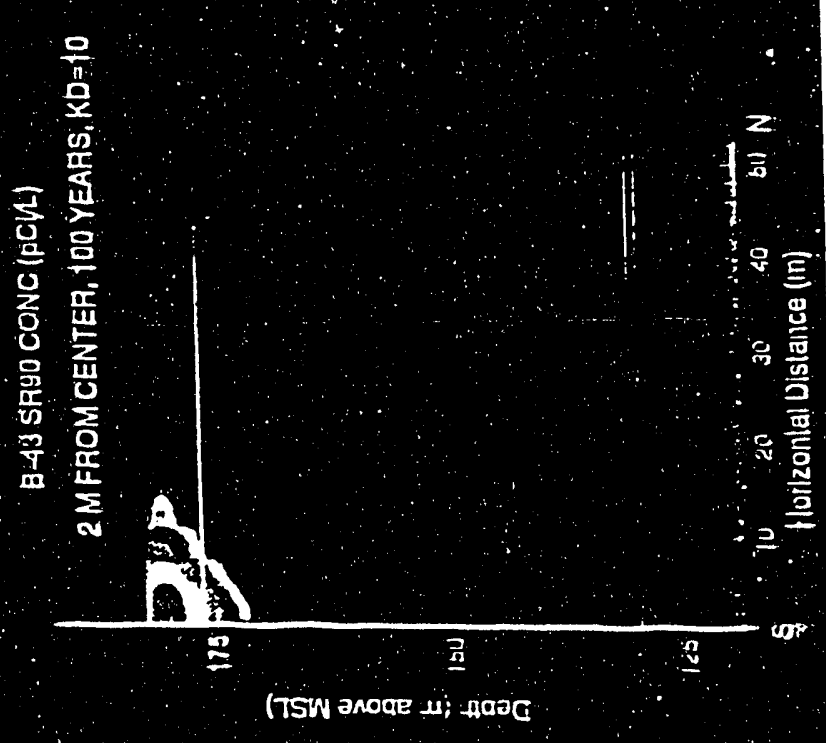

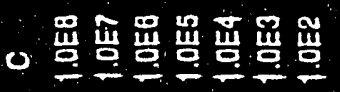

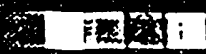

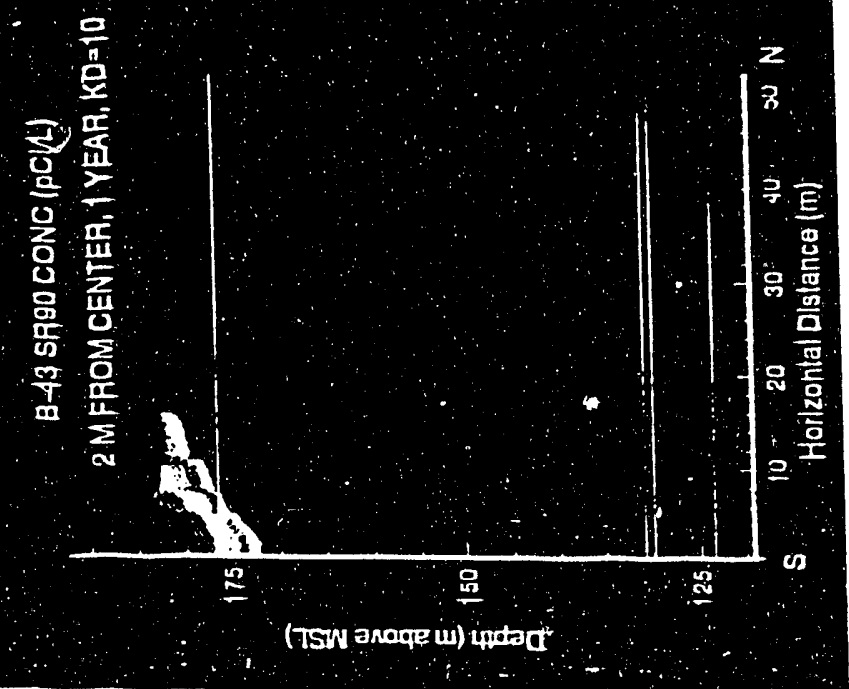

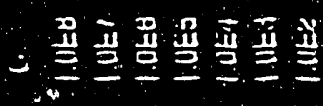

结通

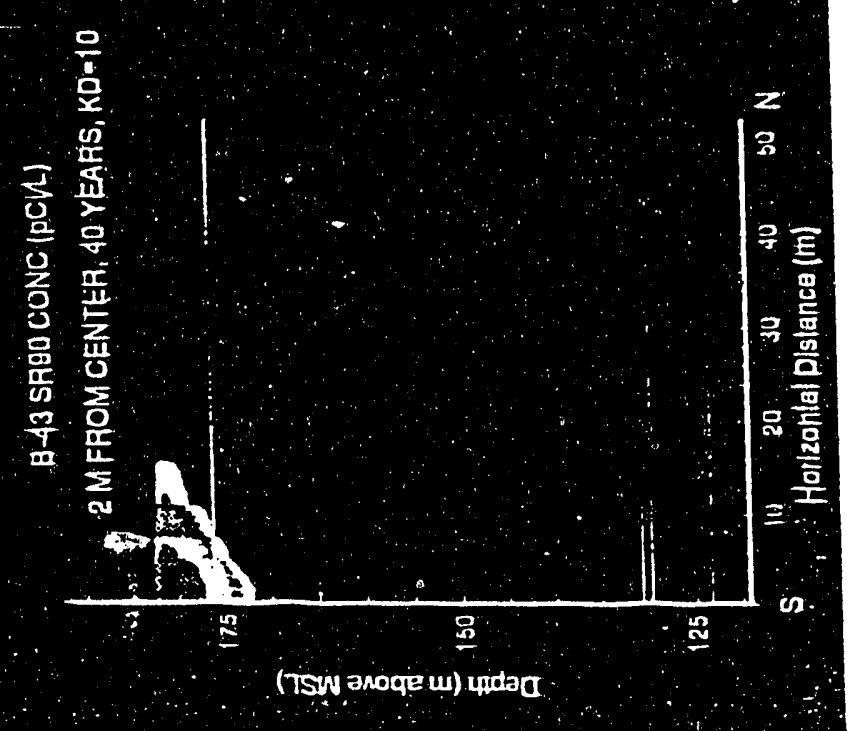



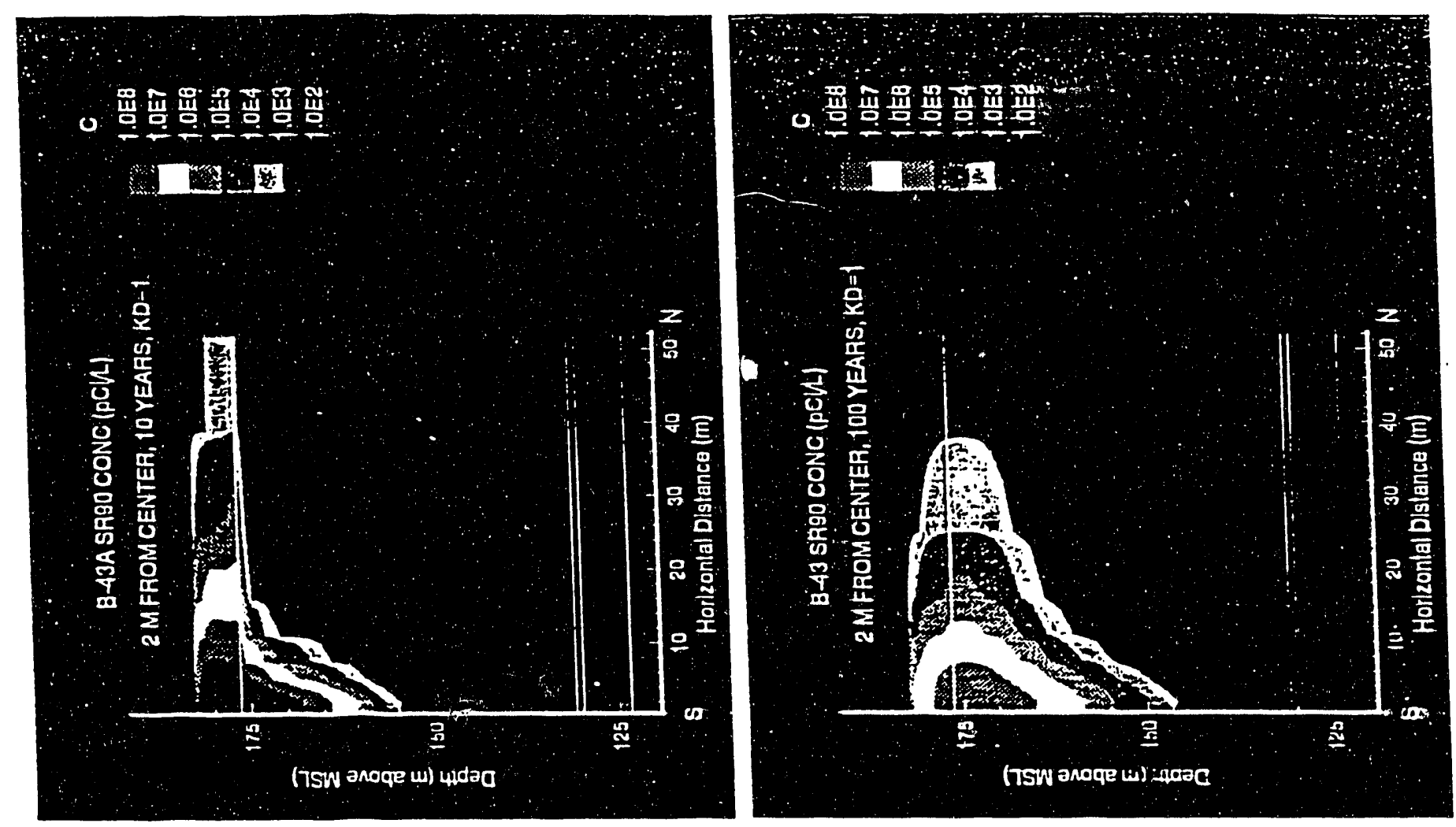

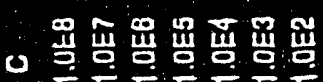

\section{$\therefore$ 级目}

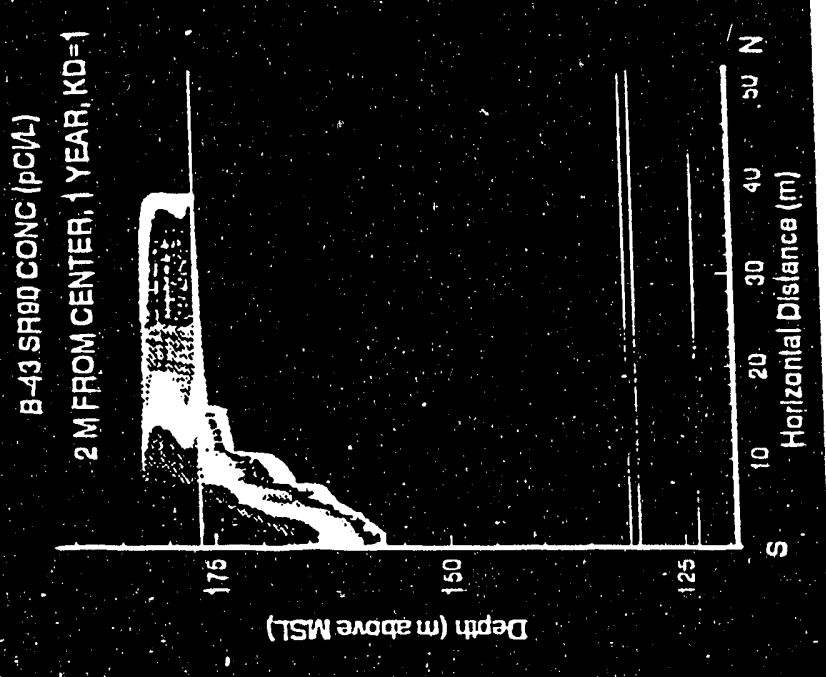

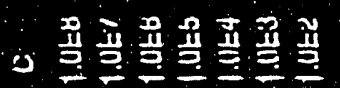
1

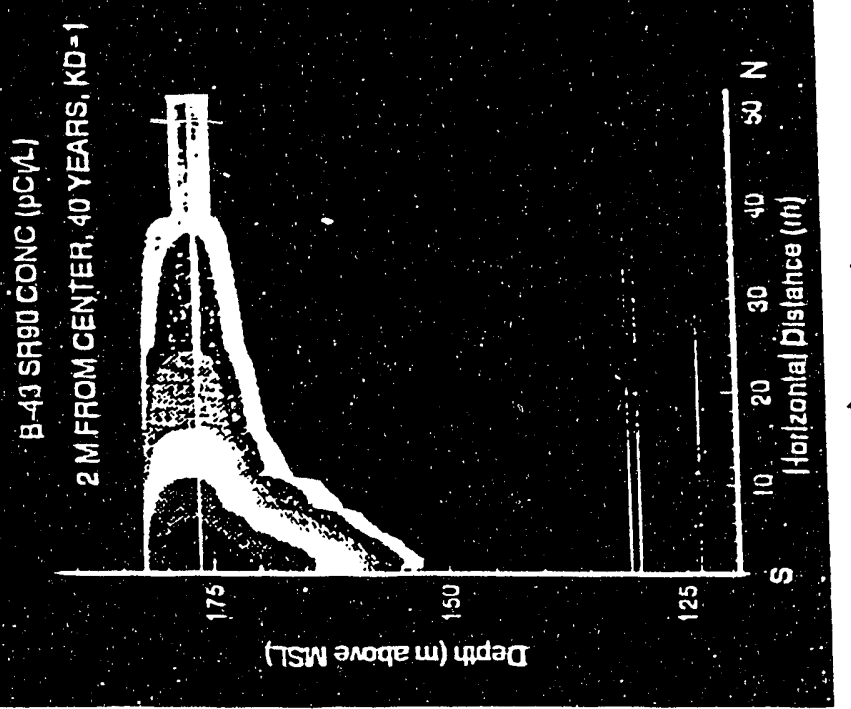


WHC-MR-0420

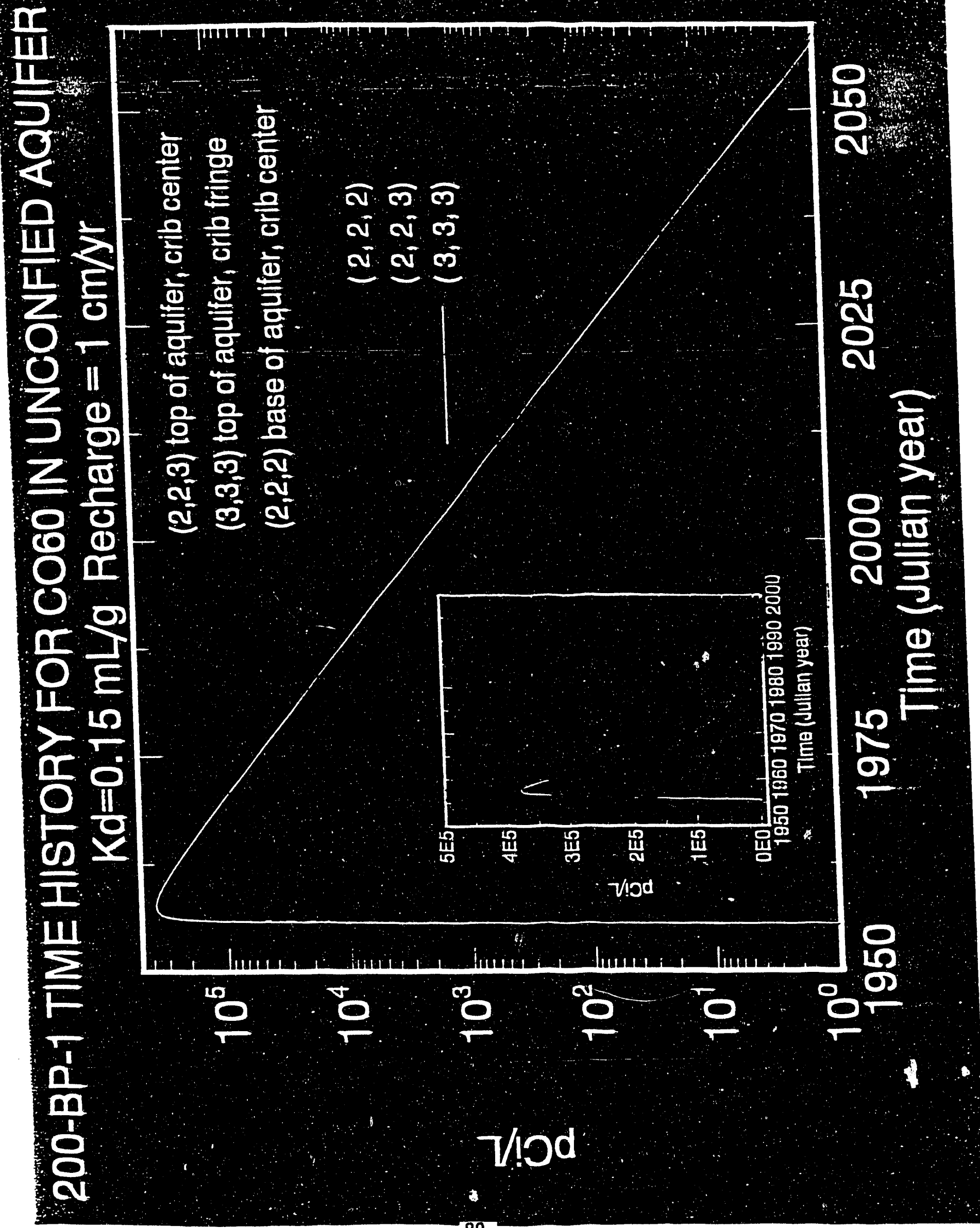


응 응응 응은 옹 - 응

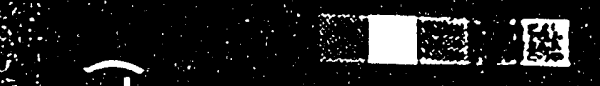

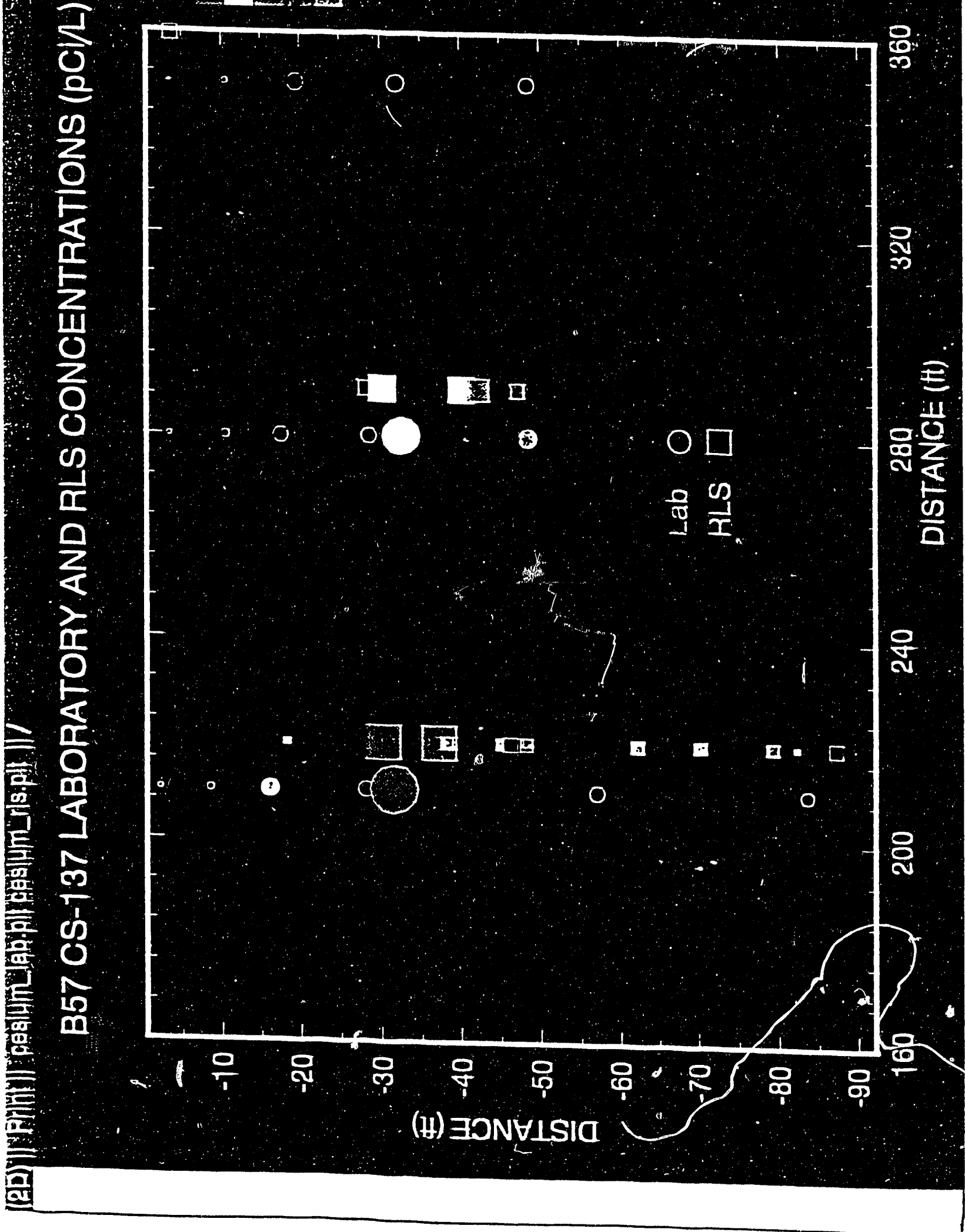



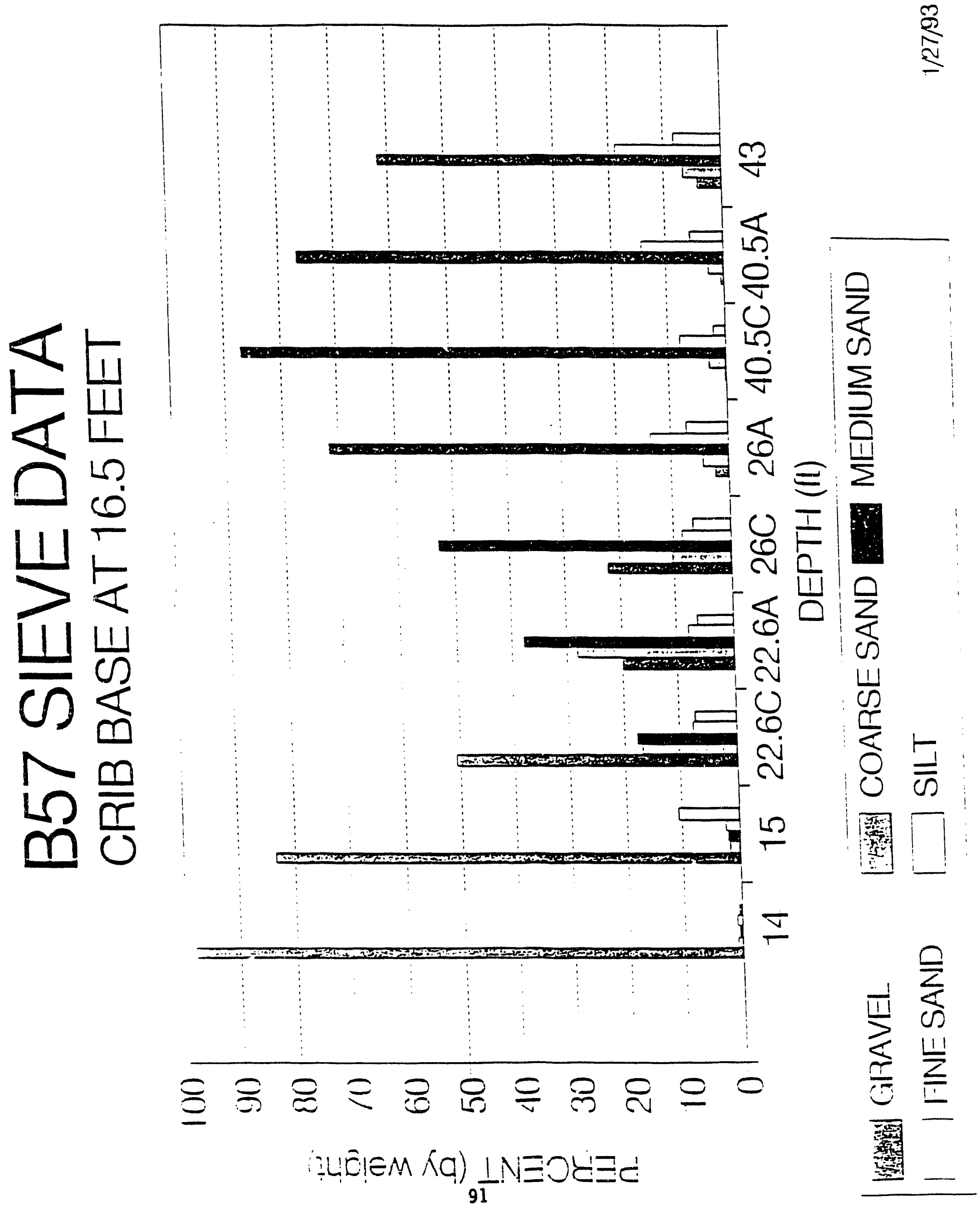

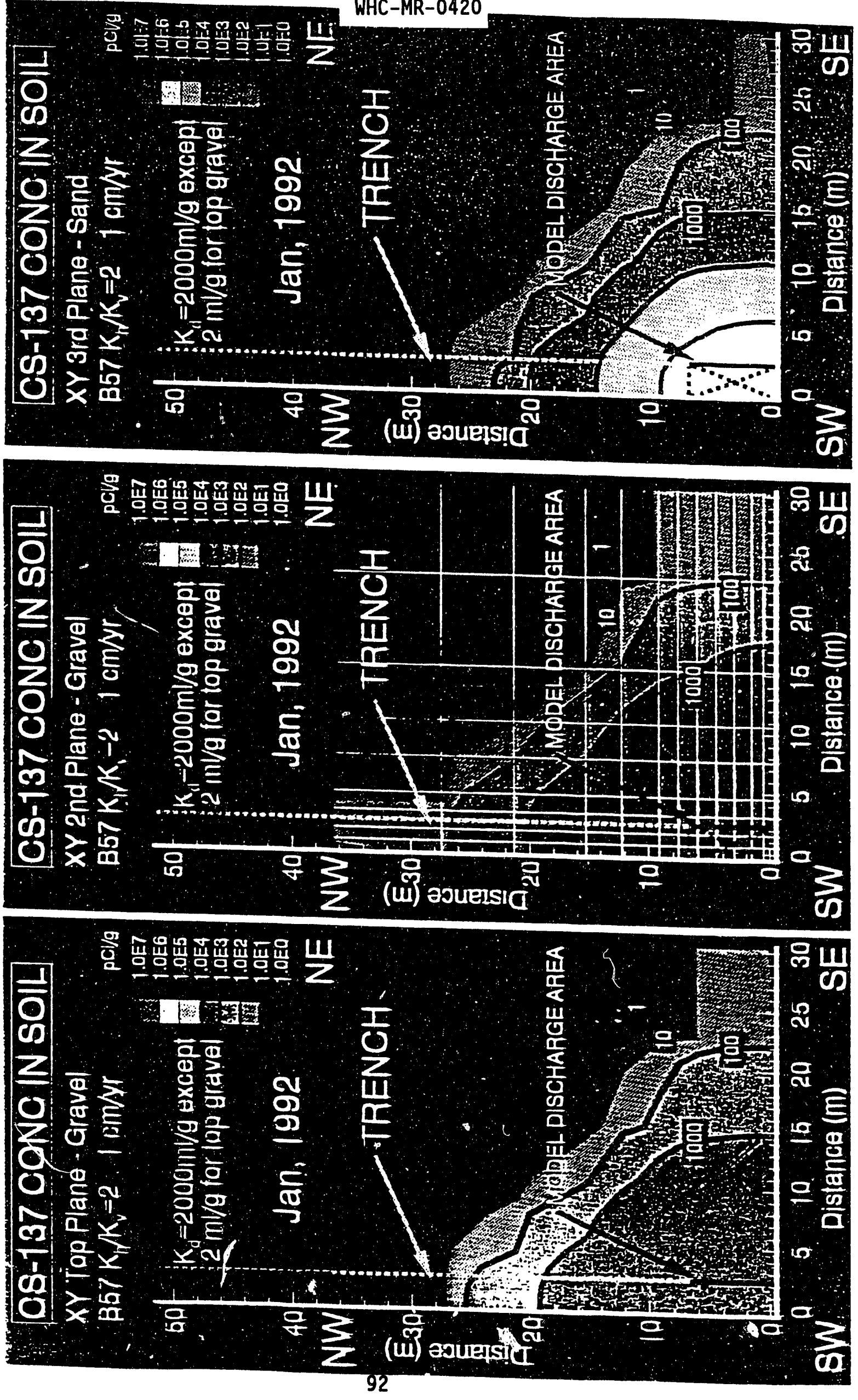


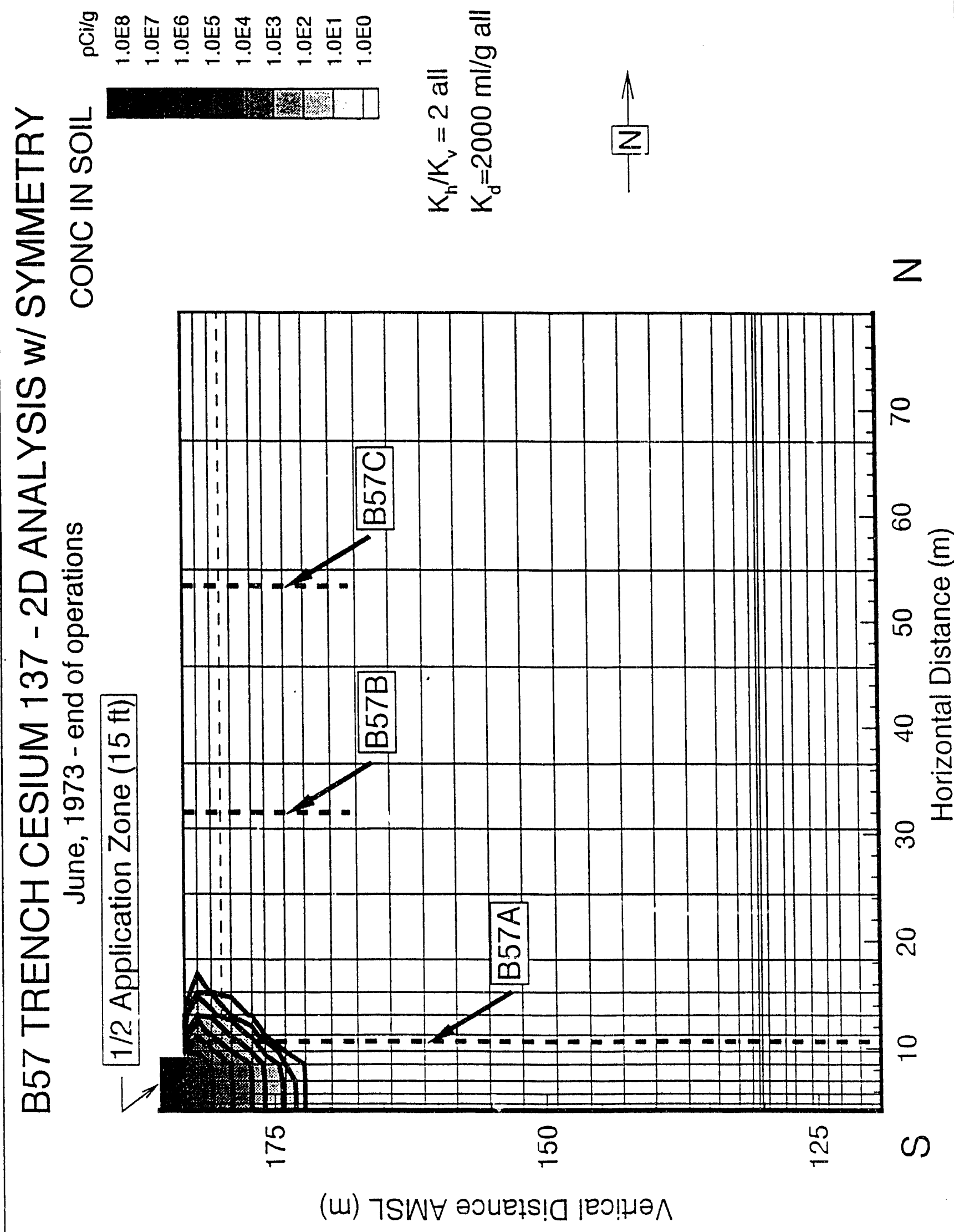




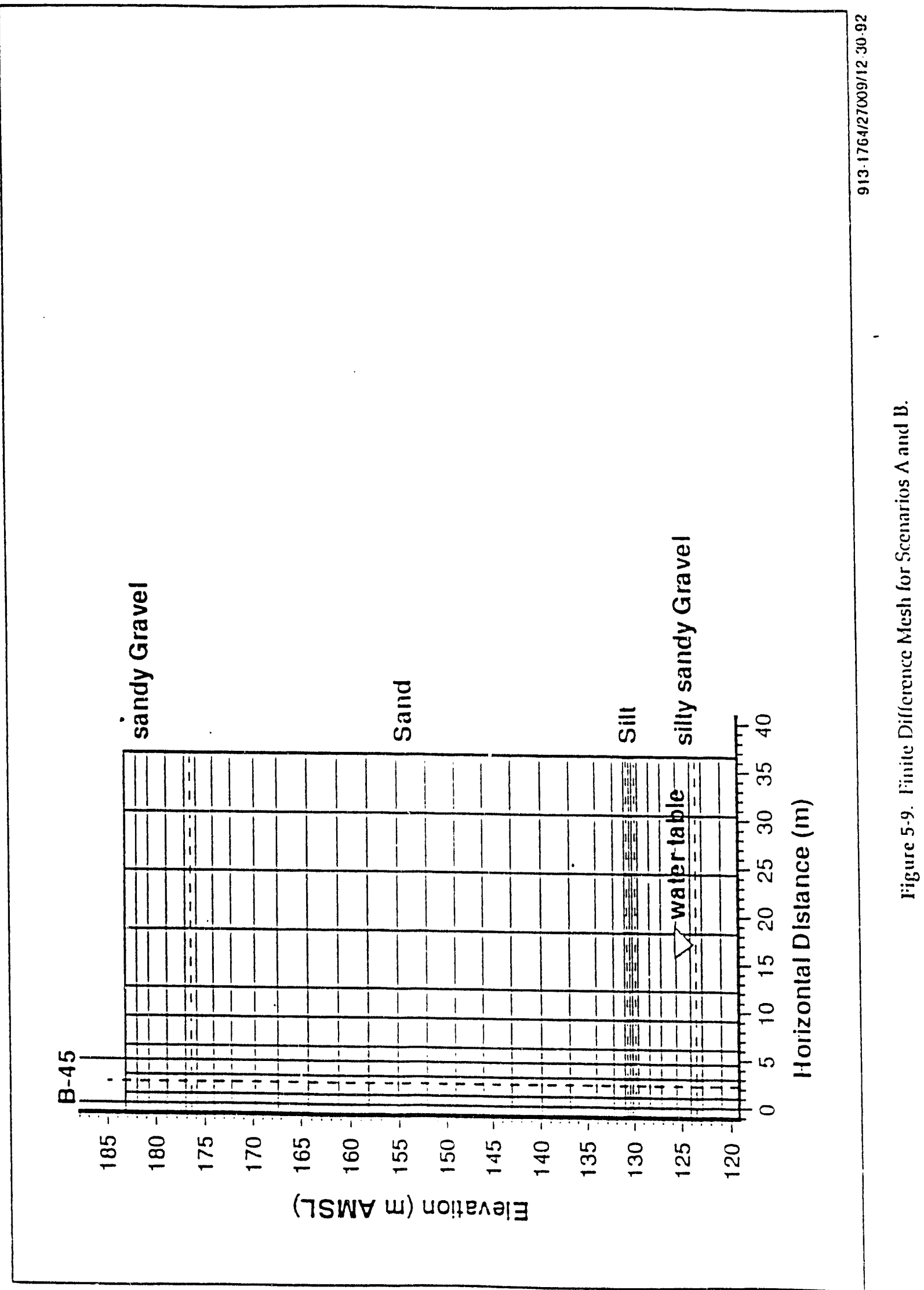


$3.75^{\prime \prime}$ I.D. Split Spoom WHC-MR-0420

Physical Samples - Every $10^{\prime}$ OR LITHOLOGT :HANGE

PAGE 30 O:

$= \pm=$

$0-$

$\cong$

-

10

60

20

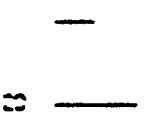

$-$

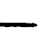

100

$\infty$
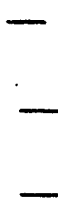

100

160

-

$-180$

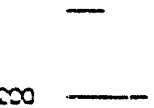

$\$ \infty$

-

$=2$

$=2=-$
SAMPUNG SCHEME FOR DE:= BOREHOLES - CRIES 216-B-43 AND 49

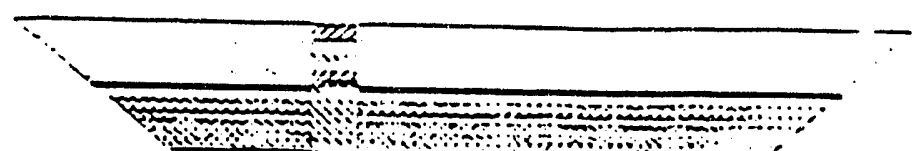

- CHE:ACAL ANO COL! IN LEACA SAivif!

"RA CHESACAL SAMPLES ILY

PHYSICAL ANO ARC: TE SARPLES
METE THAT COLLN LAOH! HPLE

MUL CHLY BE ETTUCT: : . LE

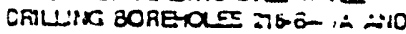

:26-a.5i: 


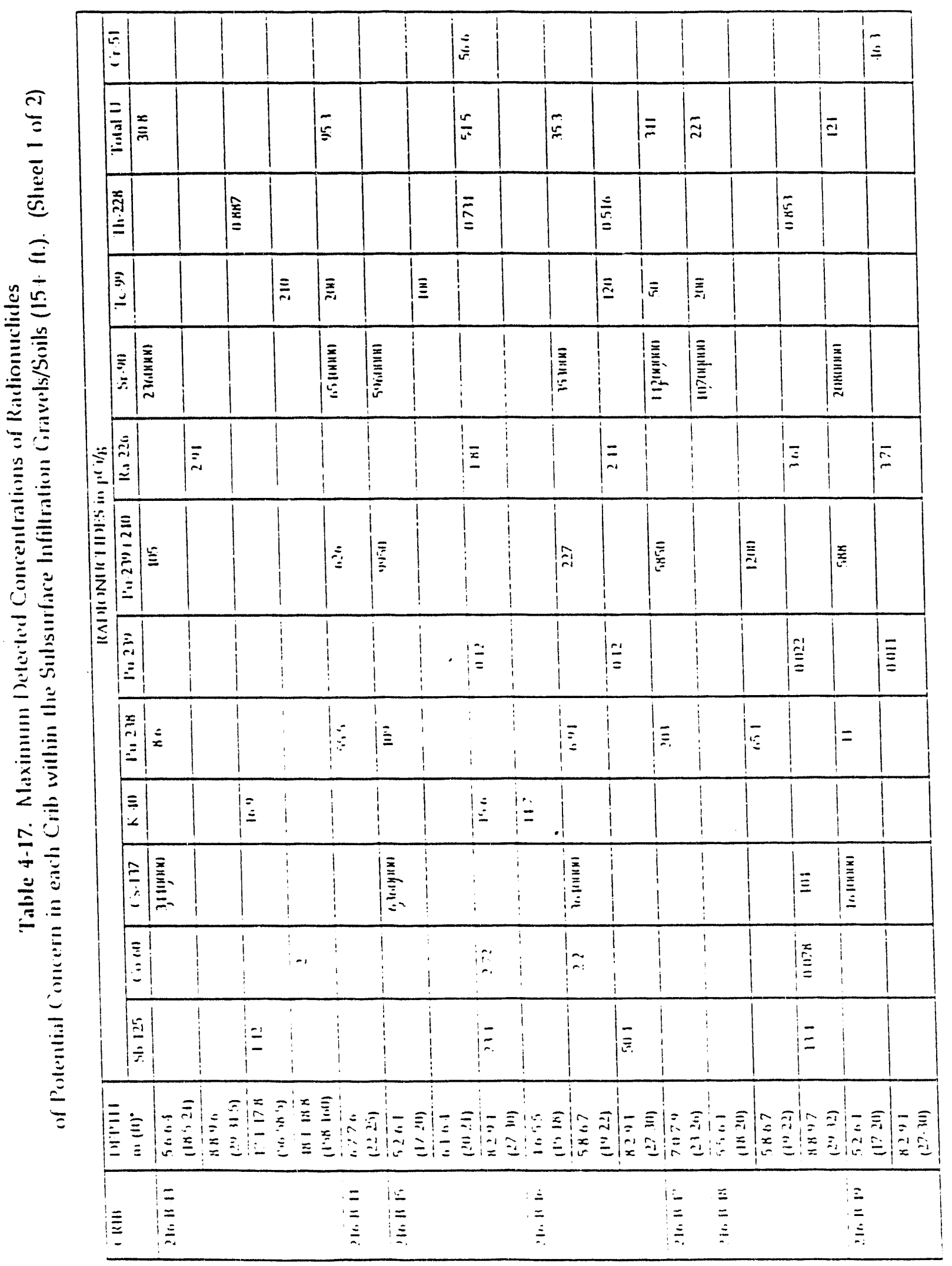


WHC-MR-0420 :

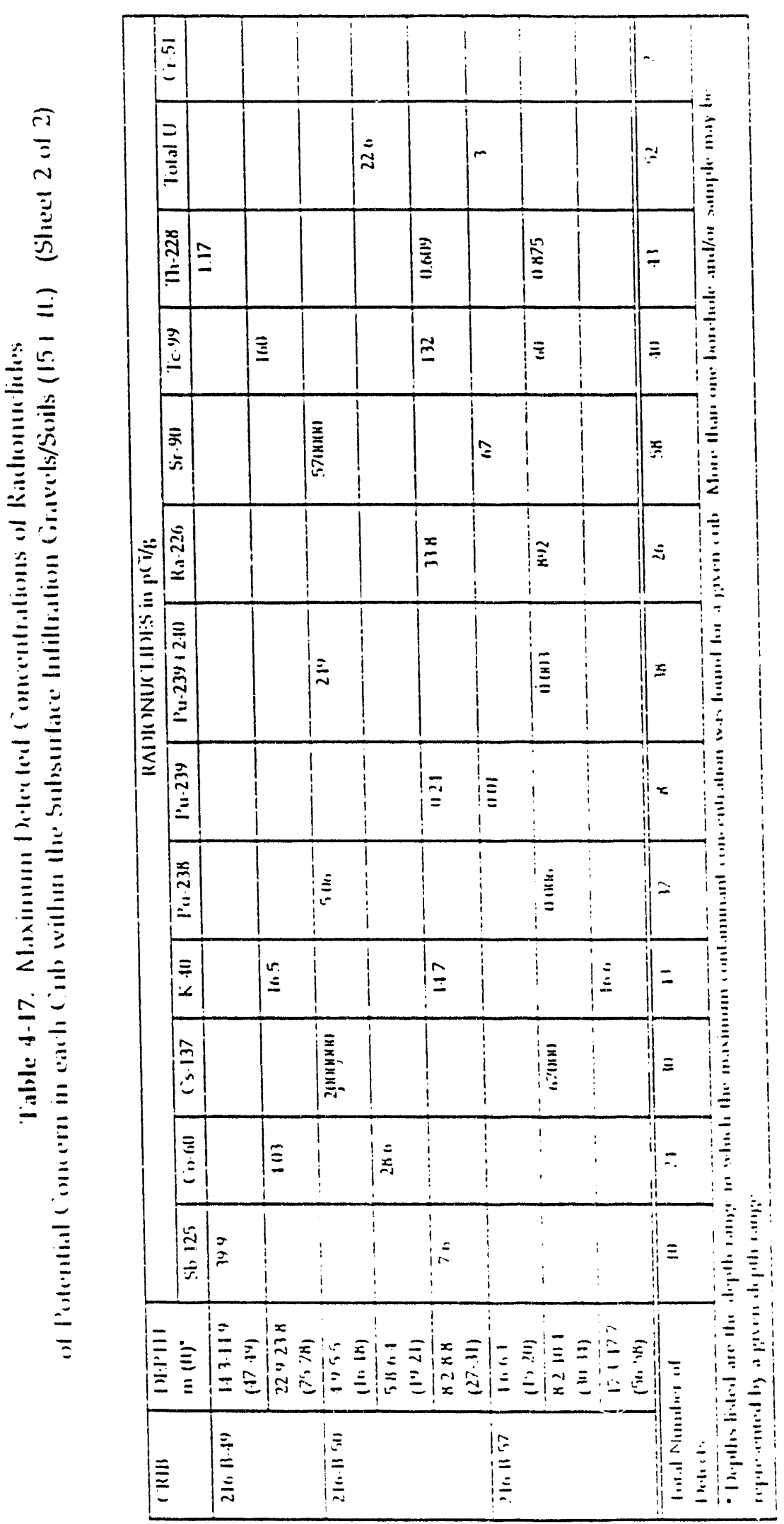


WHC-MR-0420

This page left intentionally blank. 


\section{CALIBRATION/VALIDATION OF VAMI3D MODEL USING INJECTION TEST DATA AT HANFORD}

Allen Lu and Raz Khaleel

- Injection Test Site (RHO-ST-46; Sisson and Lu. 1984)

- Graphical Presentation of the Field Data

- Modeling Scenarios

- Model Esaluation Criteria

- Samples of Modiel Results and Comparisons

\section{FIELD EXPERIMENT}

o The Iniecion Tesi Site is in :he 200 East Area

D About :000 gai of enater were jeiivered for acal ji ten consecutive weekly injecrions.

- A $20 \cdot \mathrm{mL}$ solution of the radiological iracers ${ }^{95} \mathrm{Sr}$ anci ${ }^{134} \mathrm{Cs}$ were mixed and injected.

- Field data was obtanned by lowering sensors io ille desired aedths in the ouservation wells.

- Soii samoles taken from the irso monitoring wails during ariiling were saved. In silu conducivitues ifere determined in welis adjacent 0 the test stte

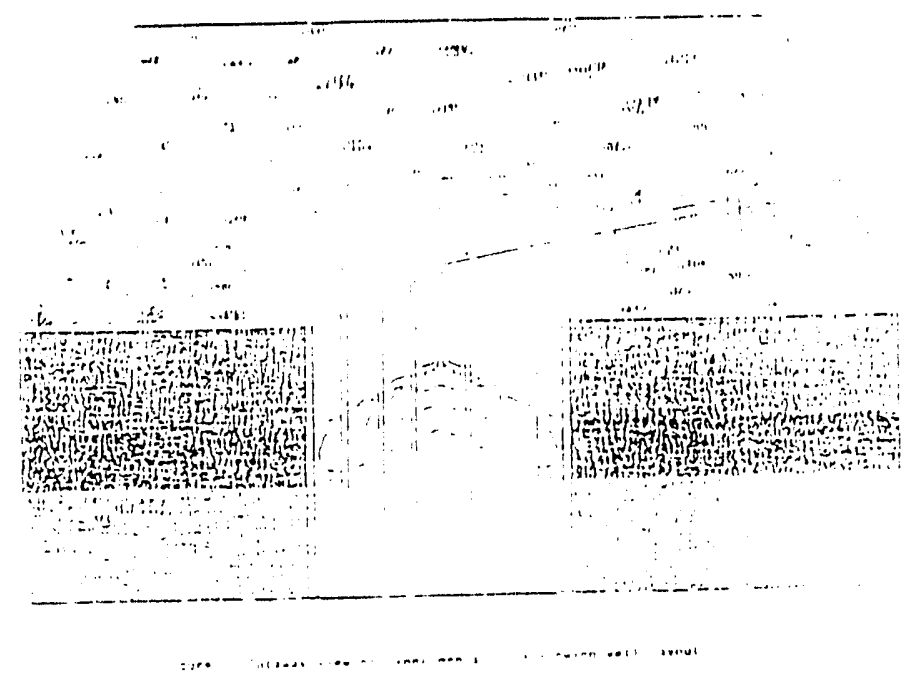




\section{WHC-MR-0420}

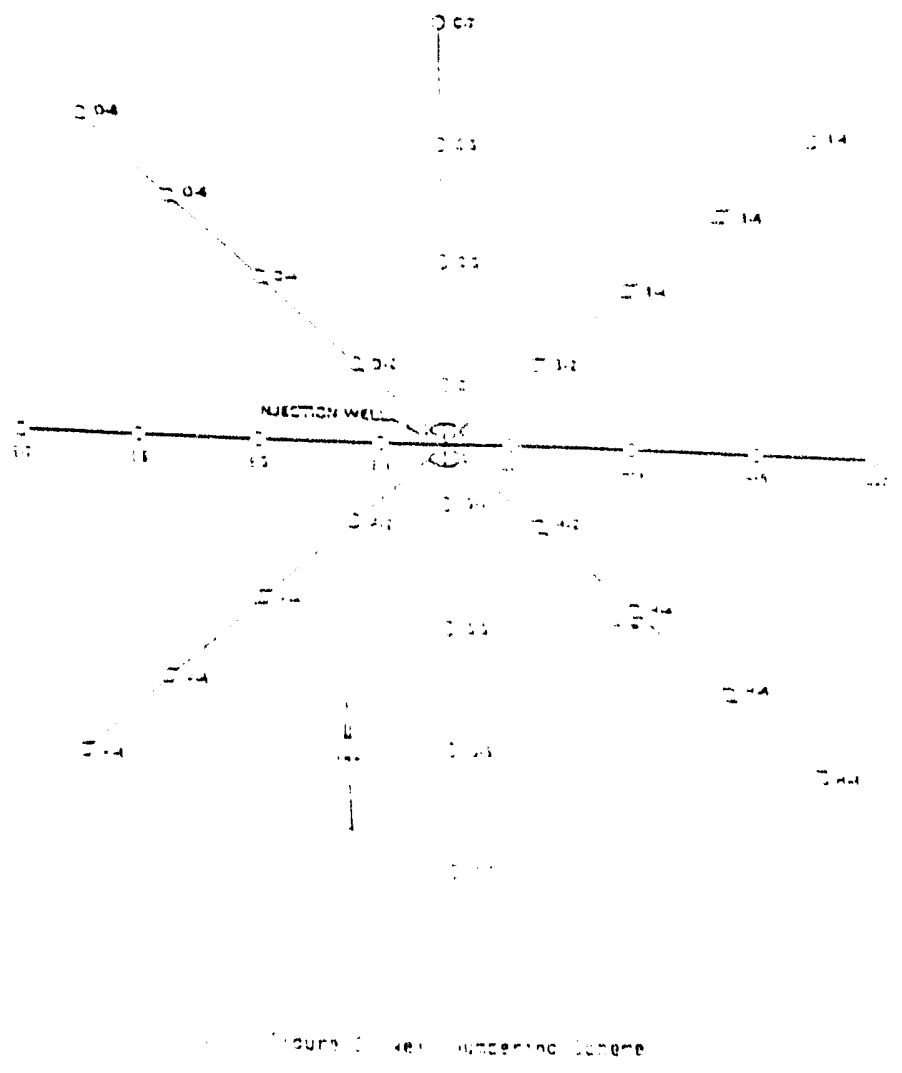

Cumuiatlve volume of Inlecied Tracer Solution

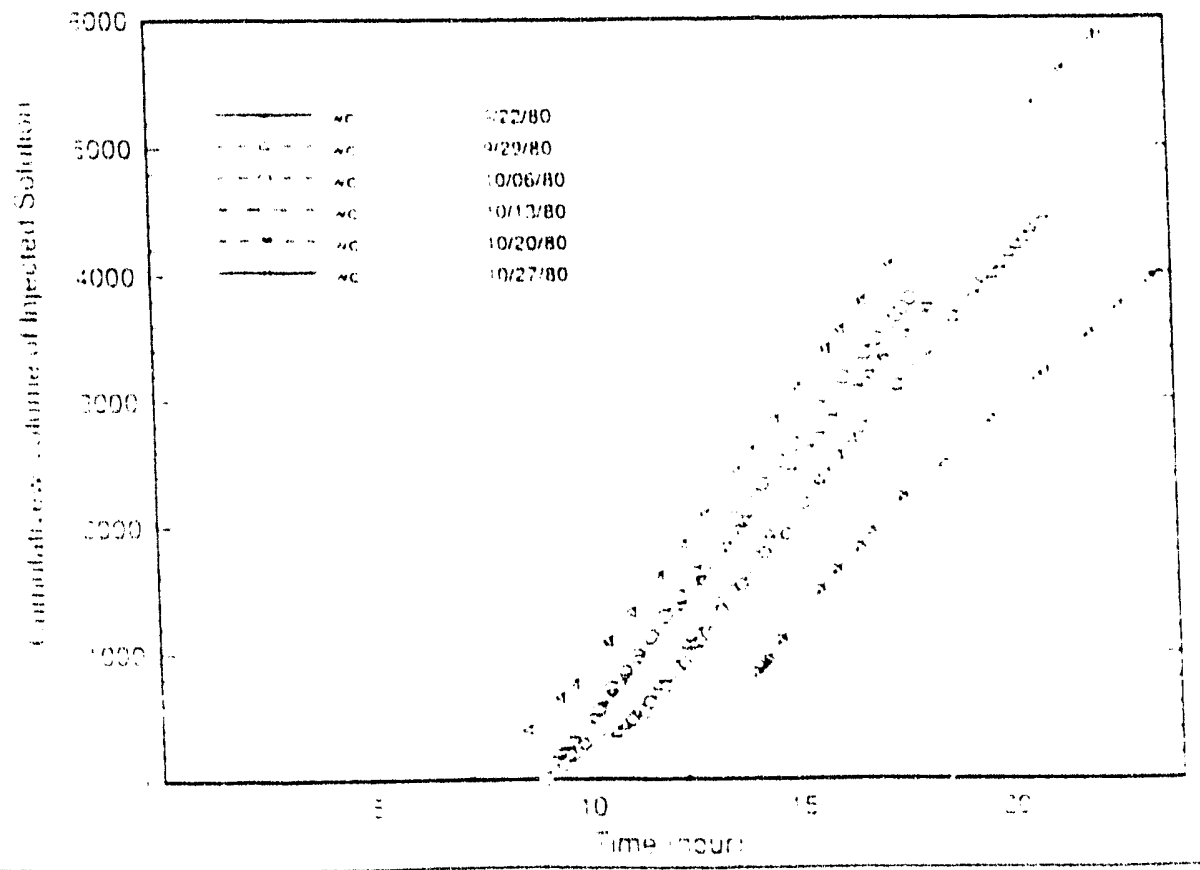



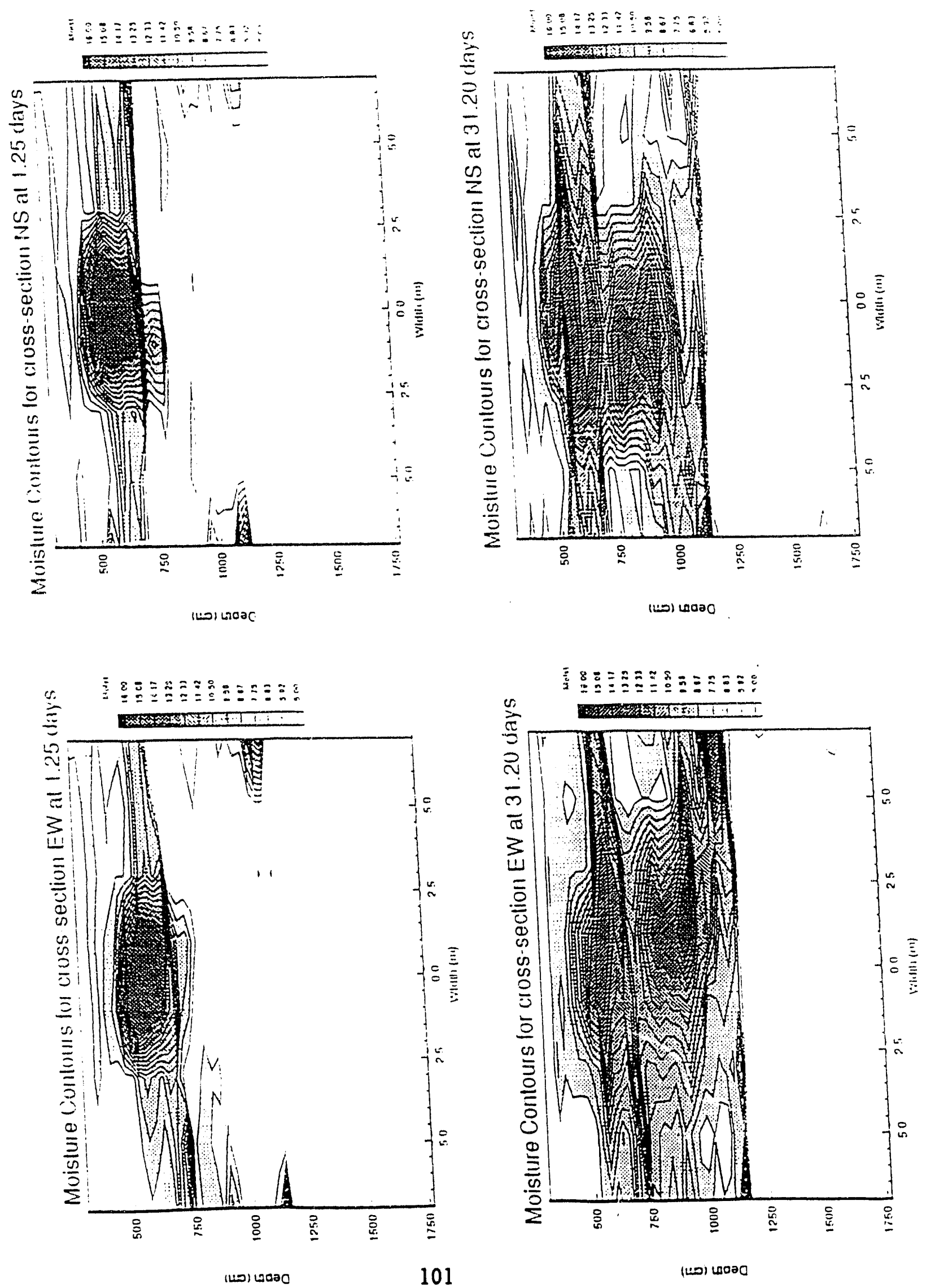

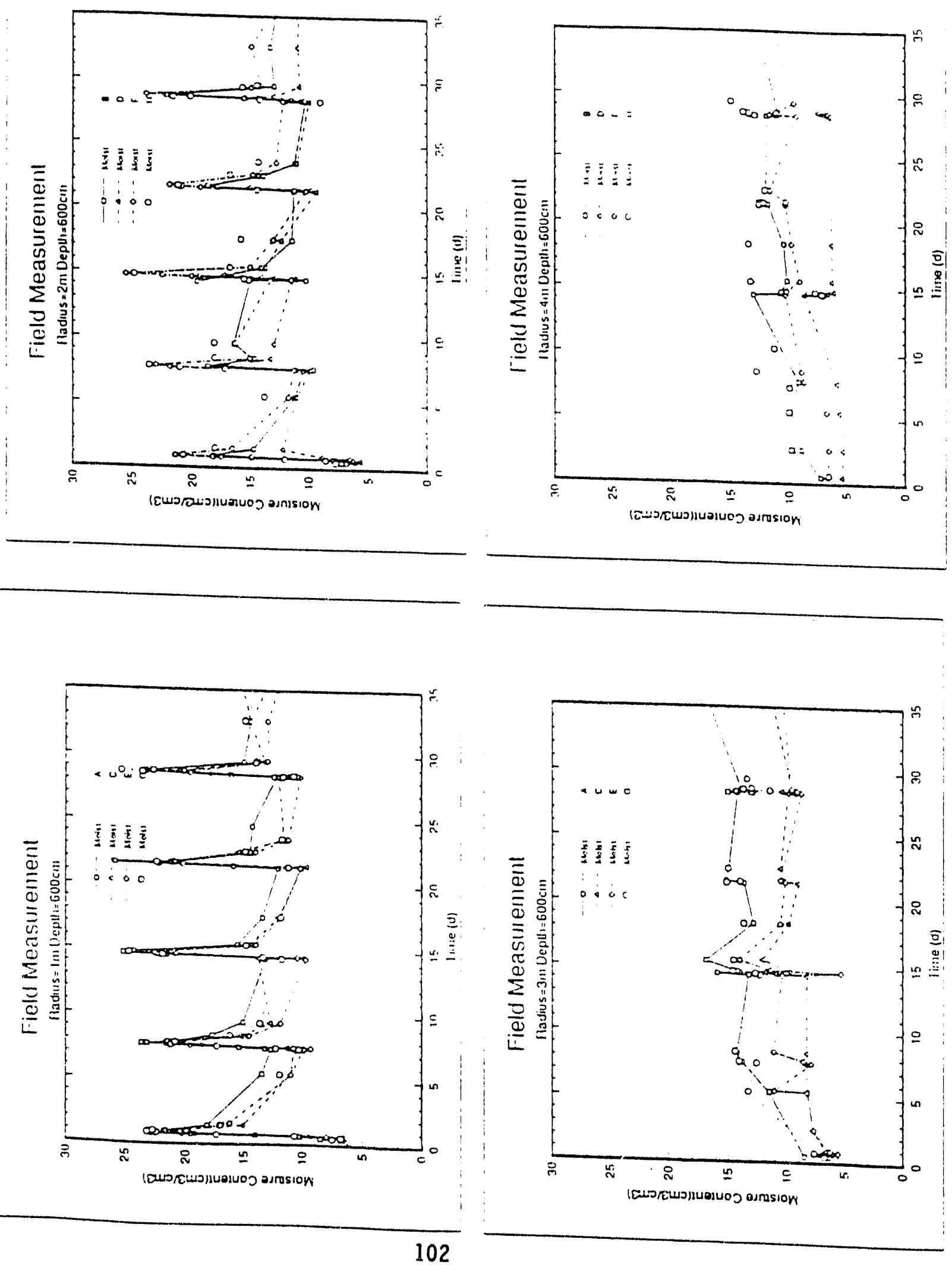

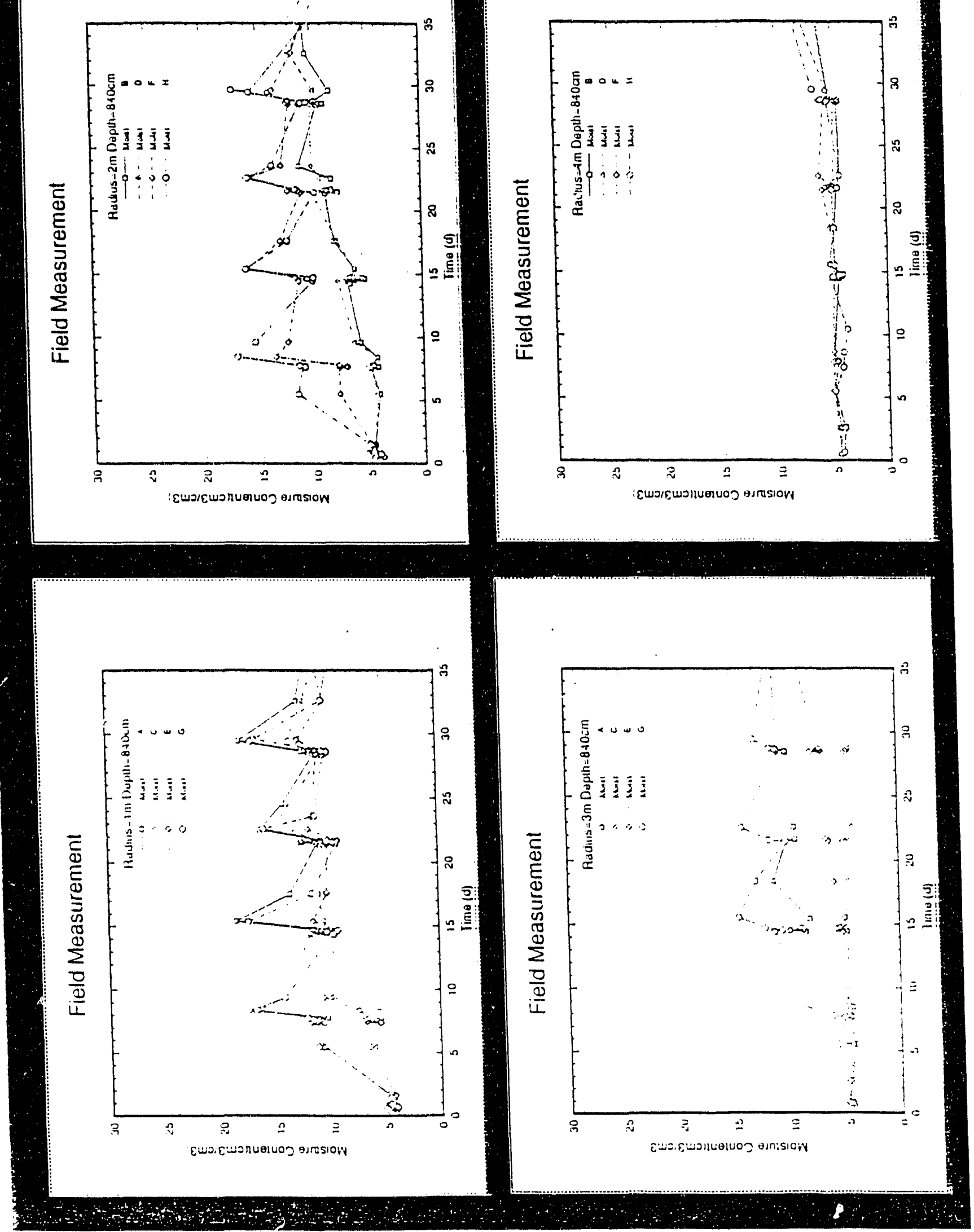
Six different model simulation scenarios were evaluated:

1) Uniform isotropic soil with soil properties given by averages obtained from soil core data from test site. it

2) Uniform but anisotropic soil with saturation dependent anisotropy incorporated in the model.

3) Non-uniform (10 layersl, but isorropic soil.

4) As Scenario 3, except that the effect of hysteresis is accounted for in the model simulation.

5) Nont-uniform, but anisotropic soil with saturation dependent anisotropy incorporated in the model.

6) As Scenario 5, but only saturated hydraulic conductivities were allowed in he anisotronic.

.

MODEL EVALUATION CRITERIA

- A visual comparison of field data and simuiated results in $(A)$ contour plots: and $(B)$ historical piots.

- A spatial moments (Kool and $N(1,1991$ ) of water content vill be compused and :ompared.

- The zero-order moinemt is used to ineasure total mass change in the chosen simulation domain.

- The first-order moments is used to indicate the change in location of radial and vertical center of mass of the water plume, respectively.

0 The second-order moments is used to measure the water spreading in the honzontal and vertical tirecions, respectively 


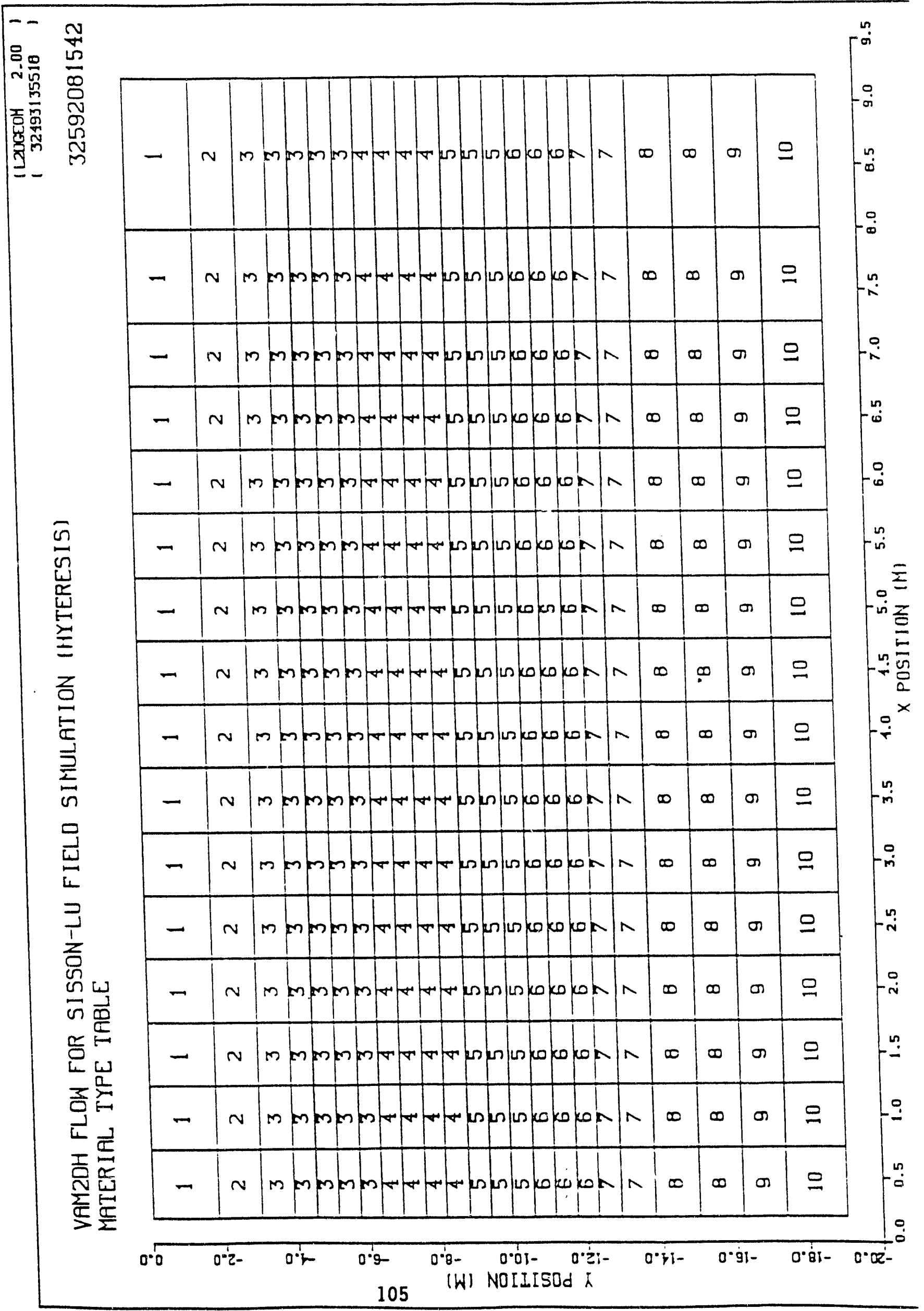



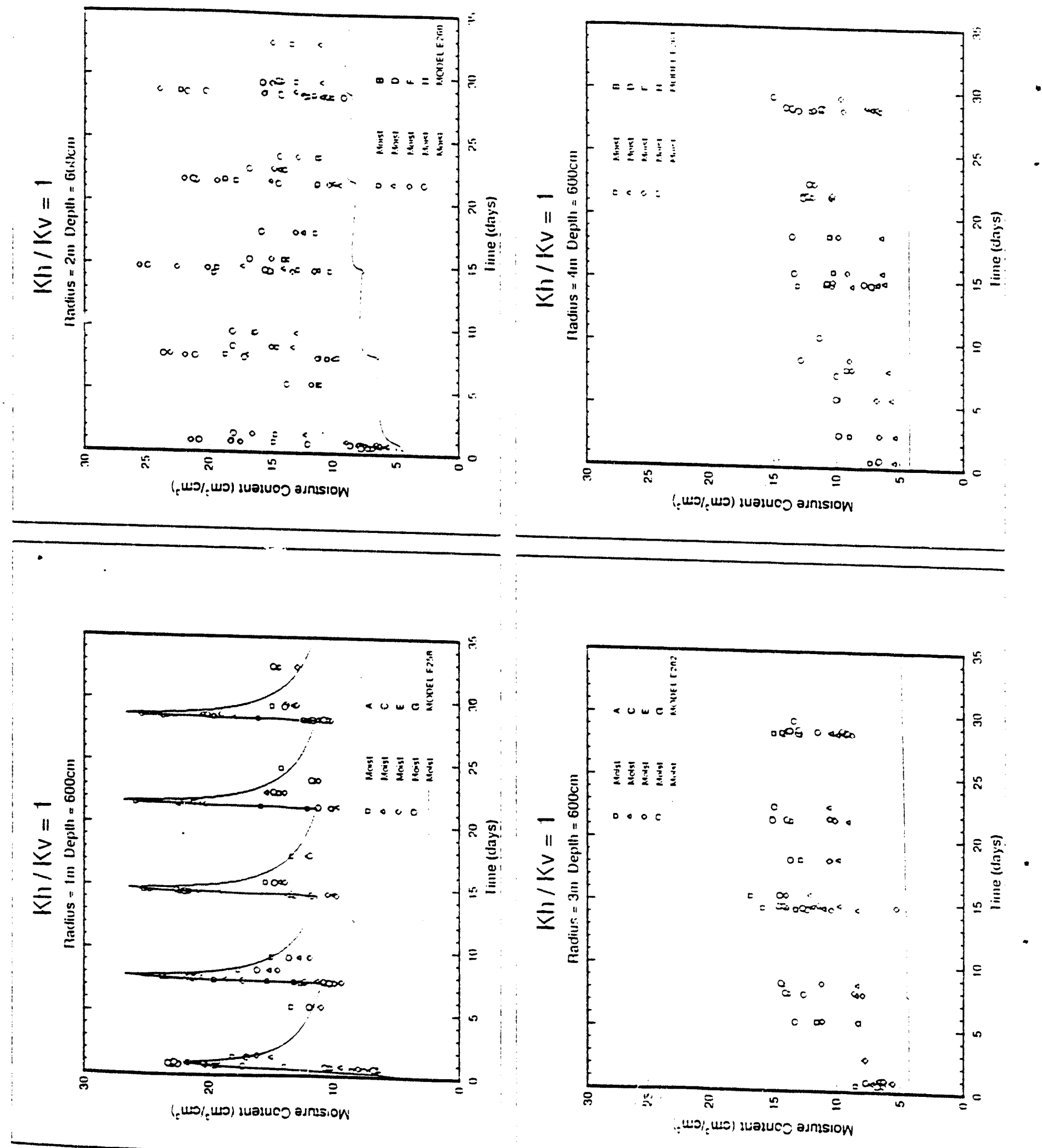


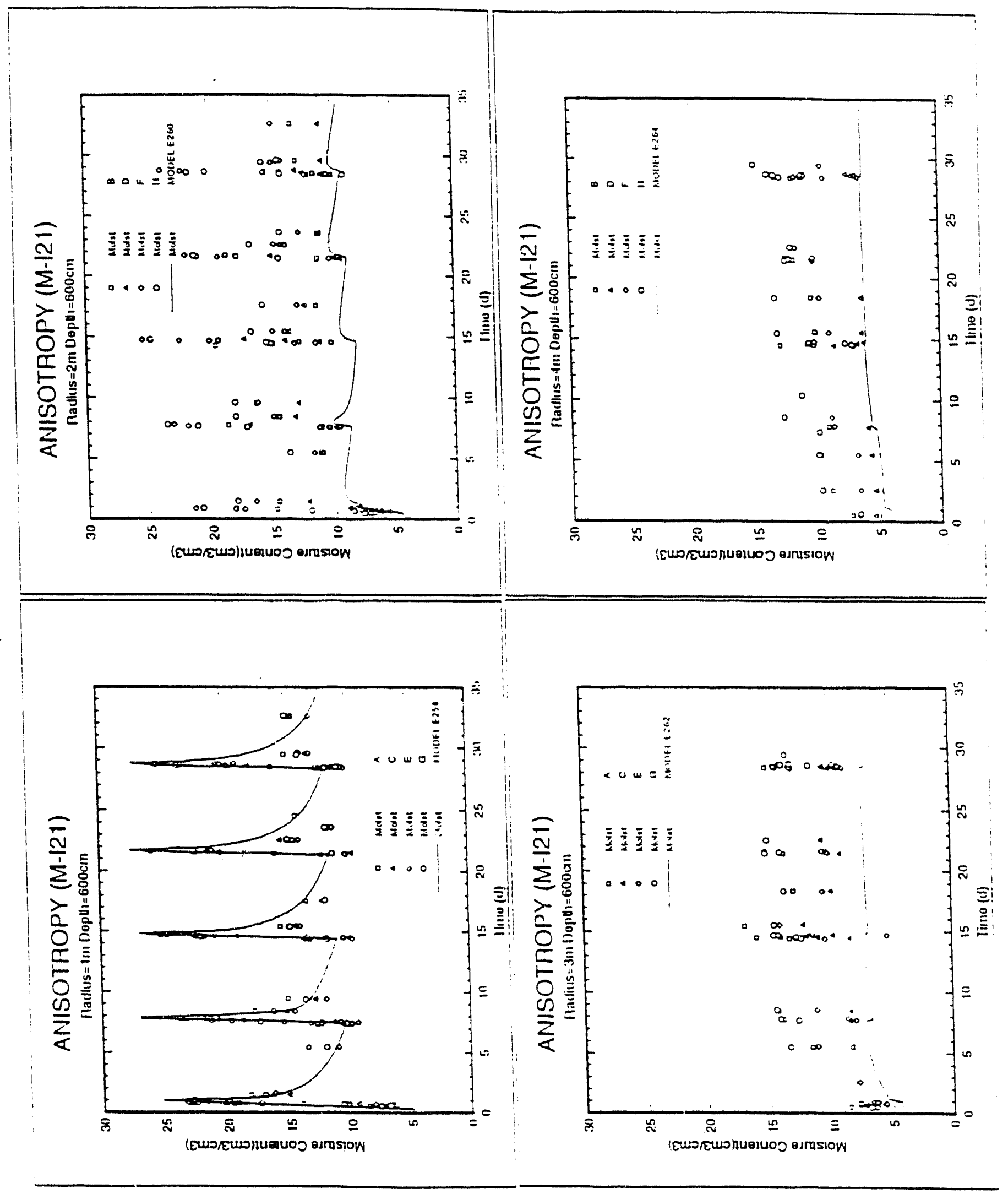



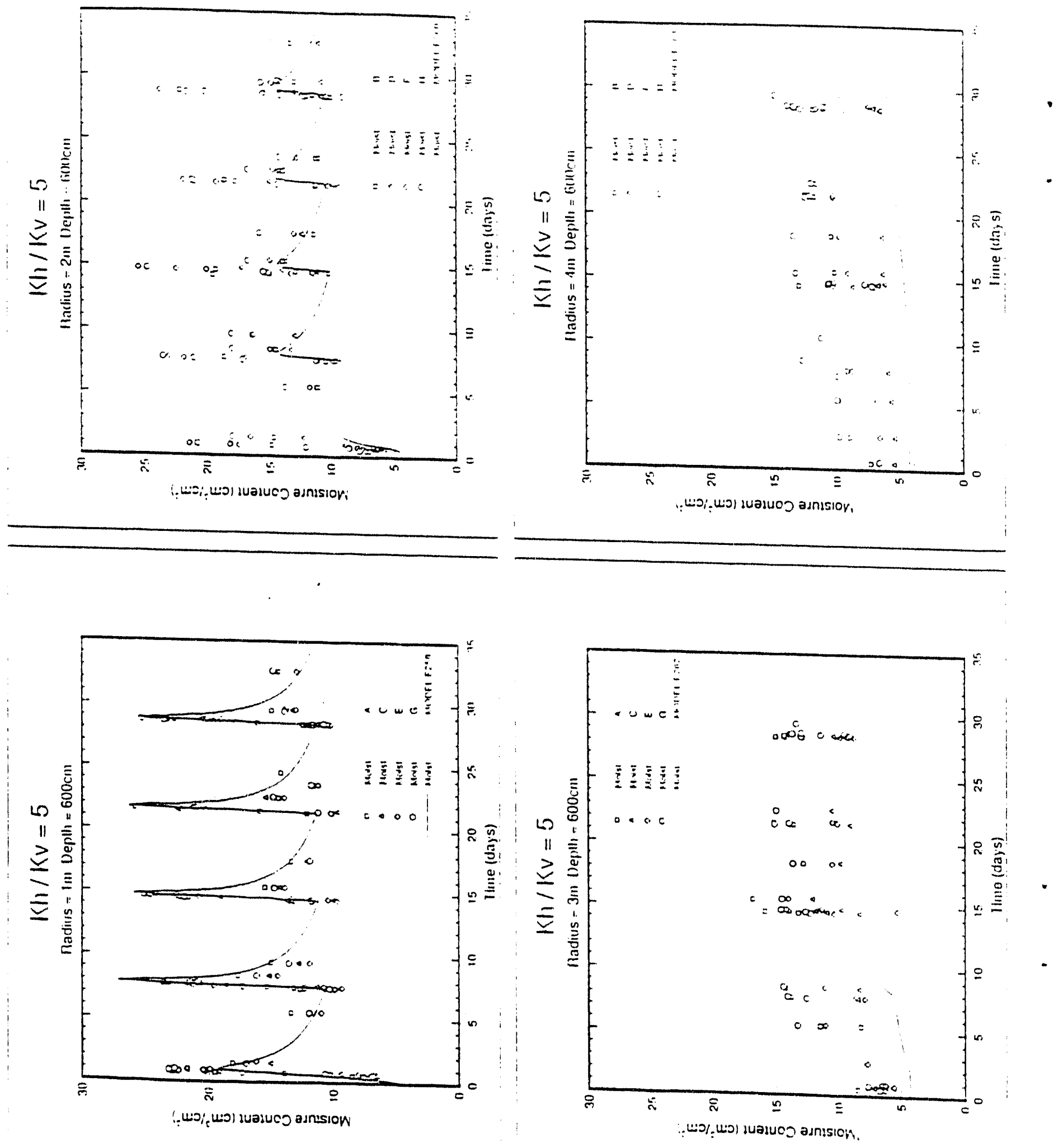

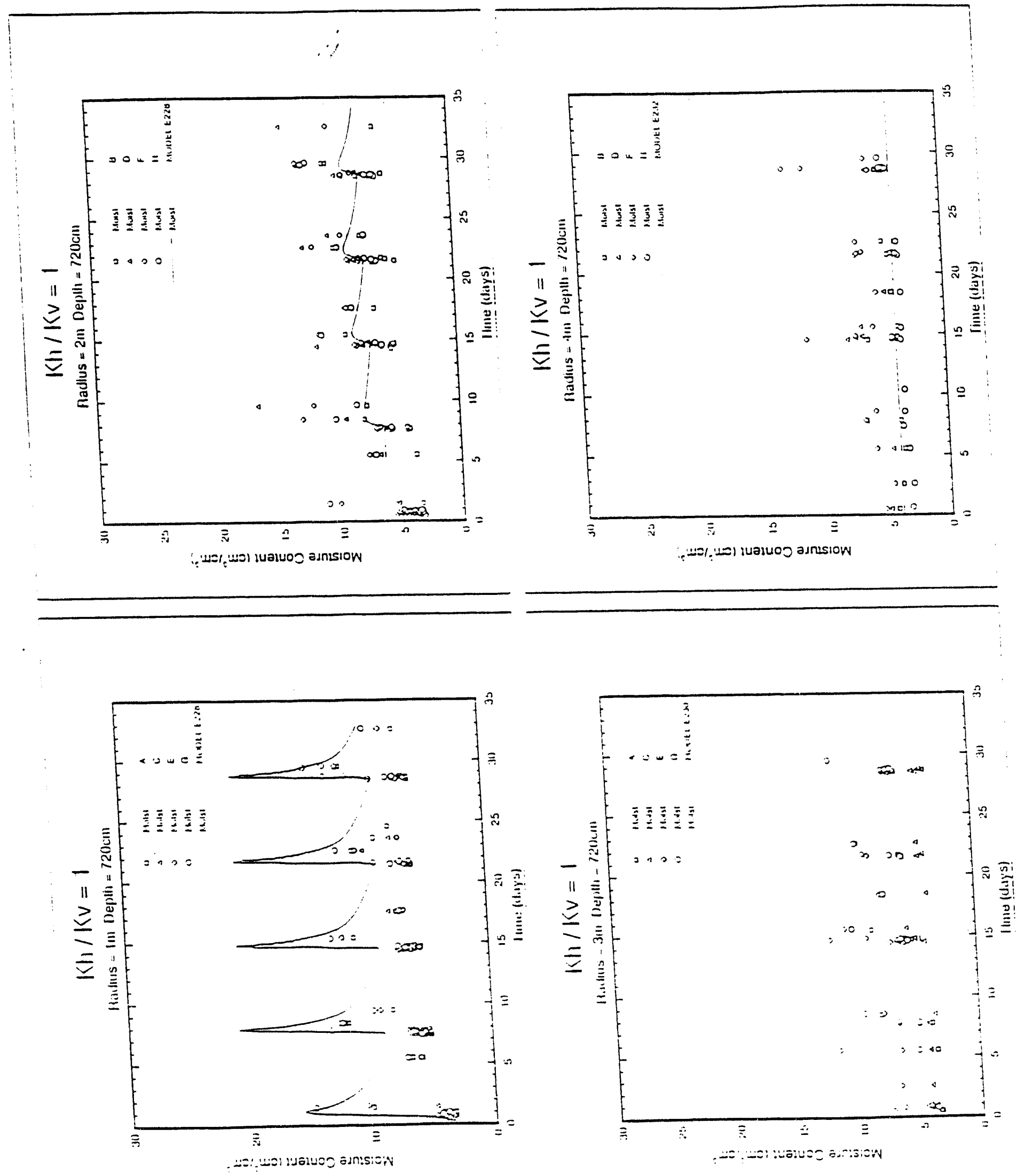


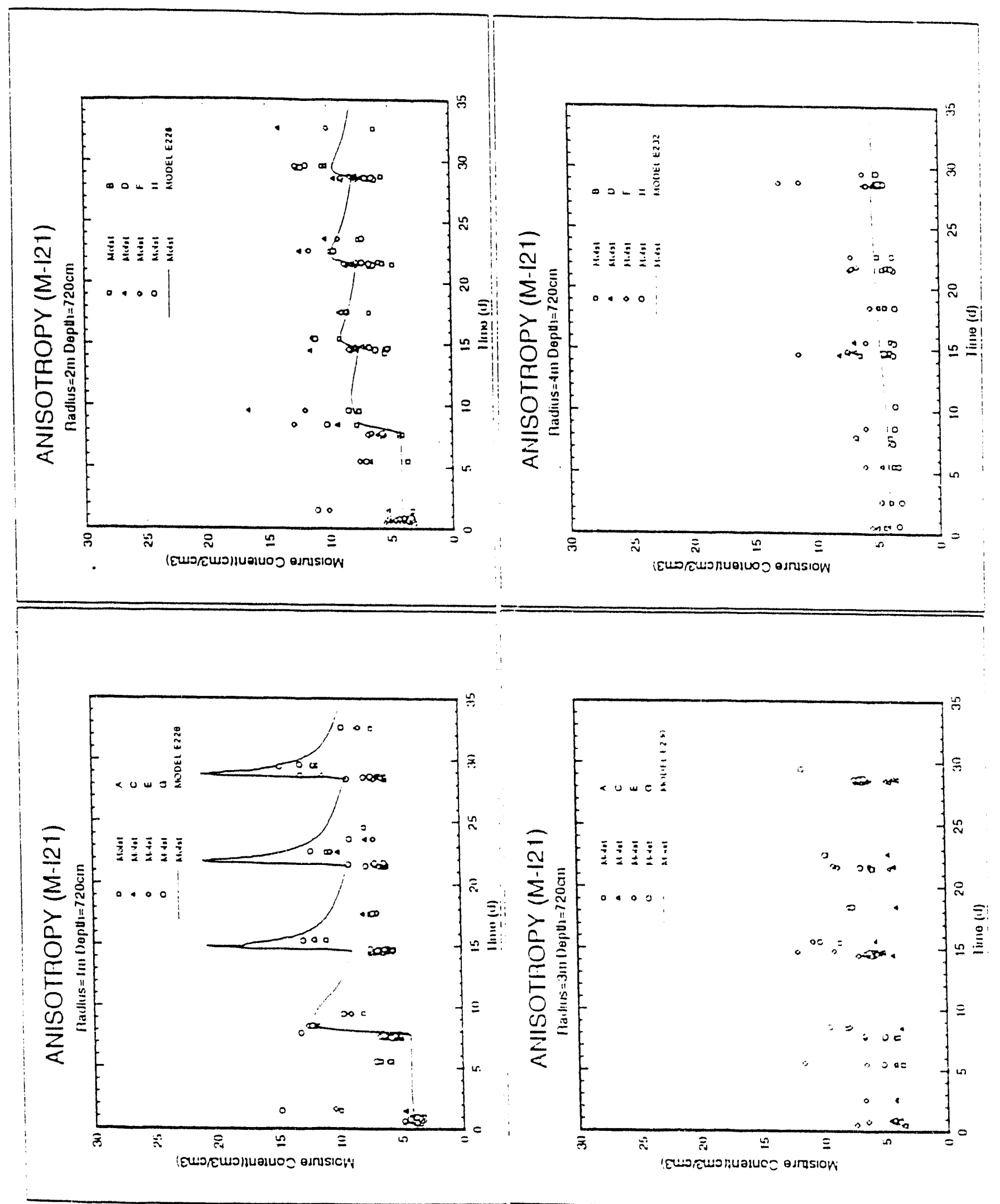



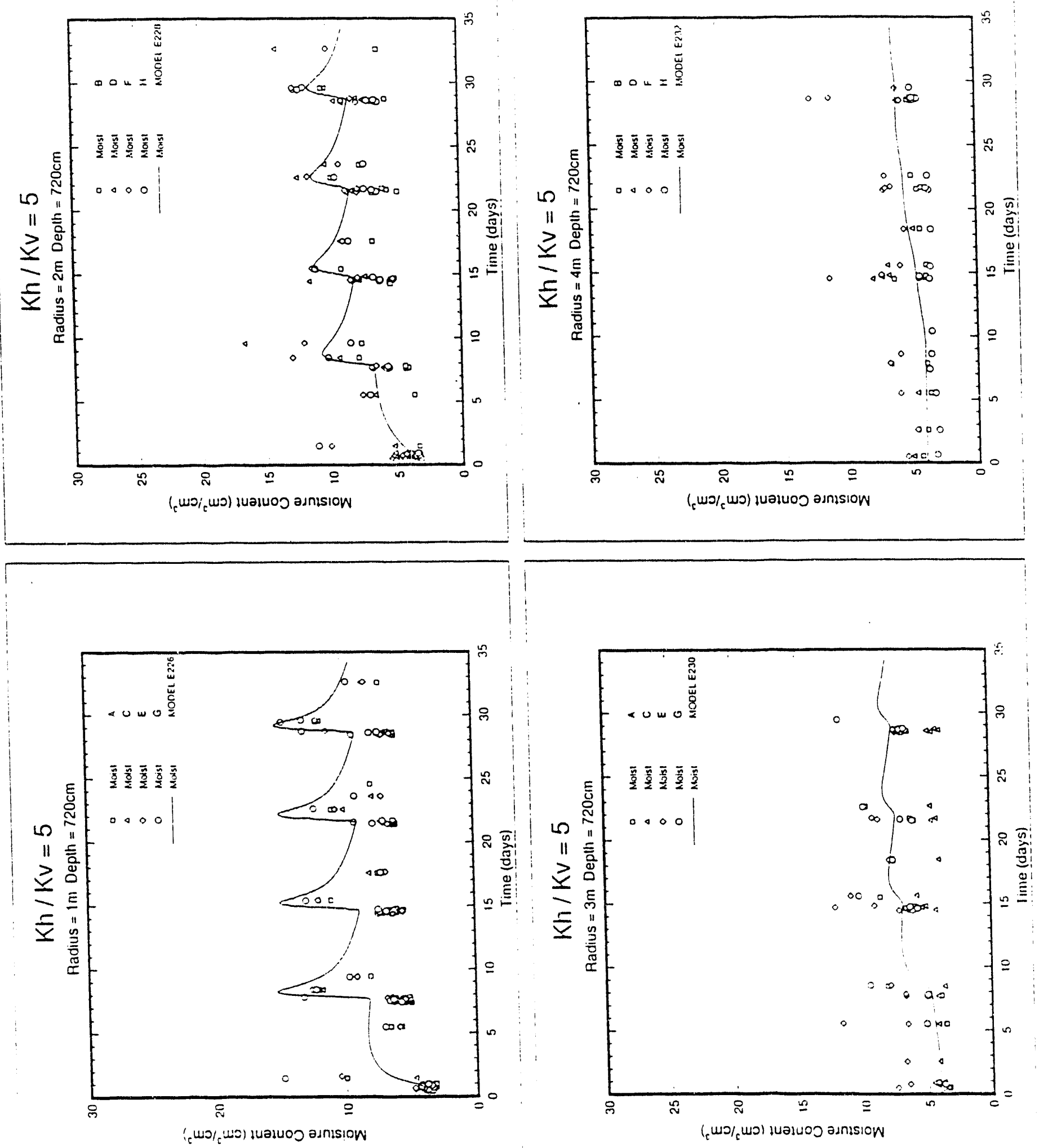
WHC-MR-0420

This page left intentionally blank. 
THAEE-DIMENSIONAL SIMULATIONS OF CONTAMINANT PLUMES IN THE SANOS AND GRAVELS UNDERLYING THE HANFORD SITE 'NASHINGTON

Jonn L Smoot

Pacifie Northwest Laboratory

P. O. Box 999, M.S. KG-T

Rienland. 'NA 99352

ABSTRACT

Aoproximately $450 \mathrm{~m}^{3}$ at liousd centaining :adoactive ane enemical wastes leaked trom the $269+i .106$ single-snetl ank nio the raoose sone at the U.S. Jeoanment of Energy's Hantore Site in soutncentral 'Nasnington in :973. Sinqie-sneil ianks ar Hantoro are juried in generally coarsu-grained seaiments that overile Estumoia River Jasalts in the Paseo Basin. The exient of '37C.j. 'A4.ja, and 10044 Eontaminant Jumes in :he vacose isne were esumated in : 973 and :978 by gamma soectromeiry in monitong wells. 'Molsture retention ano tadonuc:ice iransoon prodernies were estimated from measurements of soll

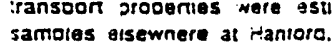

Three-aimensional, : ransient PORFLO.J simulauons of siume miqtatuon

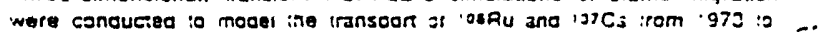
1990. The mecel oumain measures $50 \times 38 \times 51 \mathrm{~m}$ in me $\times$. 1 . ano: zirections. escectively. il inls seale. zach scil aver winin tre zomain is assumed io ae at sonstant incxness. Ho flow is alloweo

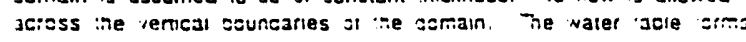
the ower souncary. anc gressure is assumeo $=$ remain ixeo at the itmosoneric alue. i fniorm niltration s acsiled 'o he joder

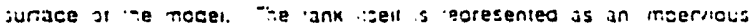

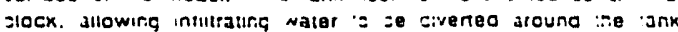

Simulations :or ecfiu ane is7r,j jooroximateo :ne 372 measureo

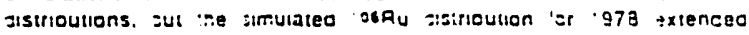

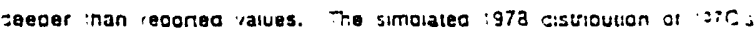

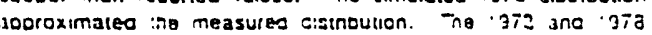

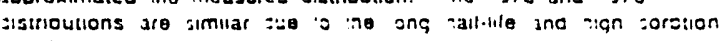
serficient at art.

INTRODUCTION

-rorotogic :tow and :anscort cemouter esces NIII So used to covalc:

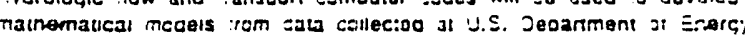
DOEj ites :o qualuate ho ertects of grocoseo nasto cissosal

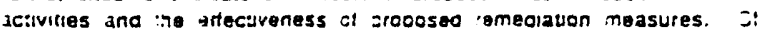

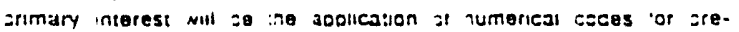

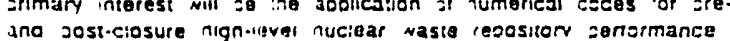

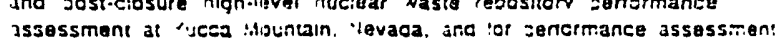
$\|$ zoveral cecaces of zimense wasies roused at several CCE :lies

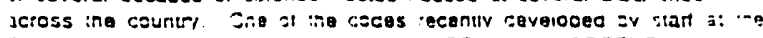

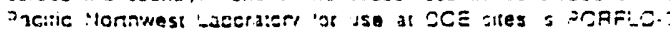

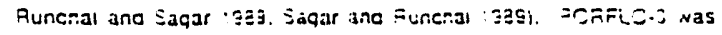

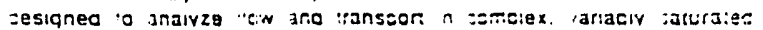

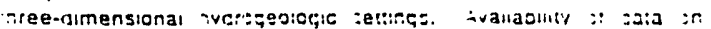

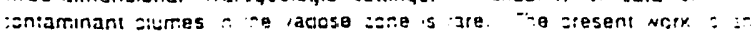

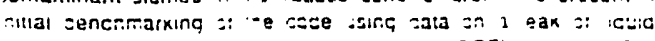

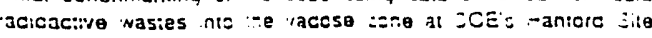

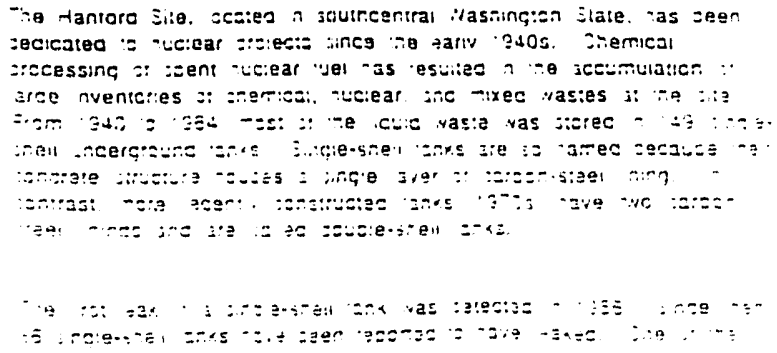


biggest laaks wes dotucted in June of 1973 in the 241-T-108 singlesnell tank locatod in the T Tank Farm in the 200 Wesr Aroa. Based on the monitonng of liquid levels inside the lank. it was esumased that adoroximatety $435 \mathrm{~ms}$ (115.000 gallons) of liduld containing

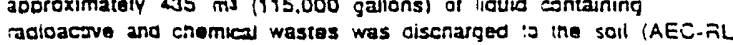
1973: ARHCO 1973: Gillerto 19731. Observation noles wero anlled around the leak site to dalineate the contaminant stume.

Concsnyravons of ragionuctide sDecres wero esumated tram iopal gamma onergy in the ooservatoon holes in 1973 (ARHCO 1975) and :97a (Rourson ot 2l. 1979). The colectre of the oresent study is io use inis field data with the PORFL 0.3 code 10 calibrate a cemelex numerical modol of ine $T=106$ tank site.

STTE HYOROGEOLOGY AND MODEL INPUT

Singte-snoll tanks at Hantord are suried in Generally esarsemraineo seaiments that overtie Columbia Piver oasalts in ne Paseo alasin. The sedirnentes are dividse into ine Miocene-Pliocene Aingold Formation and jvertinng Pleistocone Hantoro formation. secaratea sy a gisinct aver anown as ine Plio.Ploistocene unit. The Pingdid Jormauon is gonerally cearsemgranned bur with occasional inin lavers of ine sano or sile Nith small amounts of ctay. Callcrie is odserved in satn the casal Fingold Formation and Plio.pleistocene unis. The Hantero cormauon contains a very coarse-grained thood facies called the Pasco Gravel ano a linerjraneo tacies known as the Touctiot beas.

Laxage trom ine $i+: 06$ lank aodears 10 nave =een centained within in j1.m vaoese sene: hetetore. The modeling anaivsis wil 'ocus on

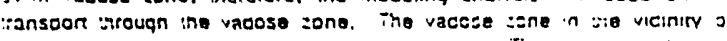
ho $a n k=a n=0$ civiceo into live mator avers. he ingie-shesl anks

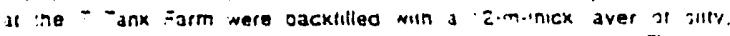

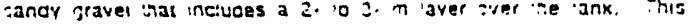

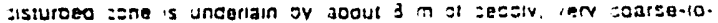

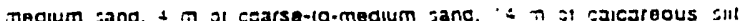
and tine sana. and $22 m$ of sanoy gravel. The 'surs ayer of alcareous jilt ano tine sand may contain a weil-oeveroses sallcte roction al

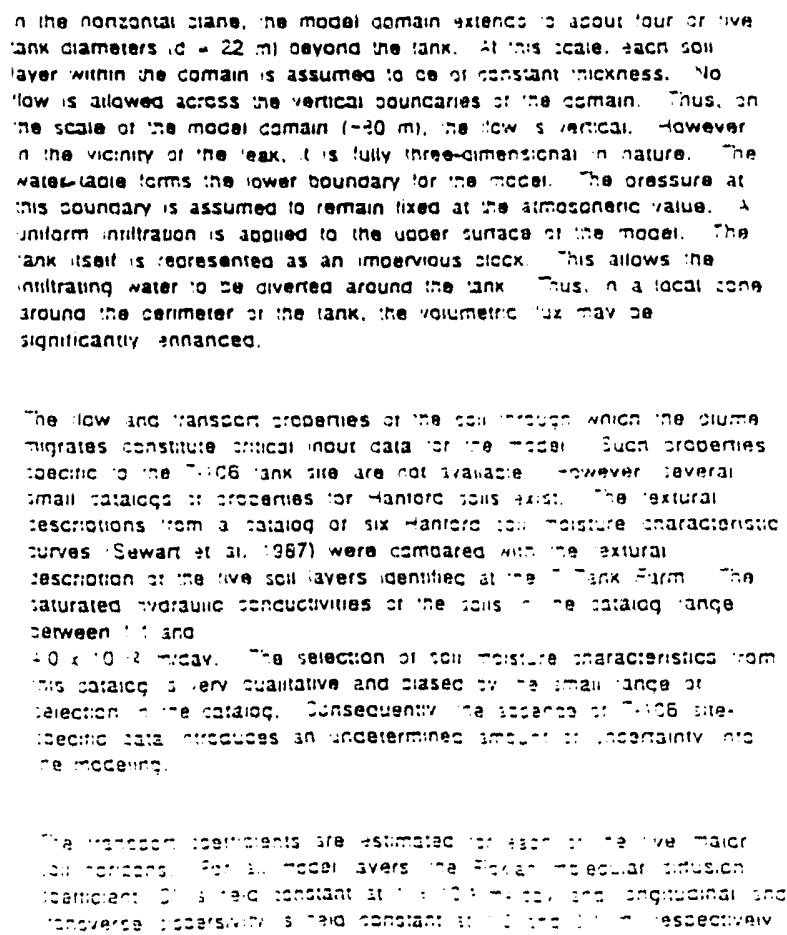


For those modal simulations incorooraung intarcauon, a sistrbution coerticient (Ka) of $S \times 10.7 \mathrm{mN} / \mathrm{g}(0.5 \mathrm{~mL} / \mathrm{g})$ was used for $100 \mathrm{Pu}$ and : $\times 10 \rightarrow \mathrm{ms} / \mathrm{g}$ (100.0 mUa) lor 13rCs.

At of near the base of the southeast side of the i.106 tank. sooroximateiy $\$ 35 \mathrm{~mJ}^{\mathrm{j}}$ of sudernate containing ranous radionuclides leaked into unis soil column ounng an approximataiy jo-day jerioa in ise late sonng of 1973 (Routson ot 31. :979). The most modile consutuent in the tank was 100Ru: by the summer it 1973 it nao migrated abous

$15.20 \mathrm{~m}$ laterally and about $20 \mathrm{~m}$ verocally bolow the soutnoest jase of the tanX (AAHCO 1973). For purcoses of modeling, colease was assumeo to occur at an unitom rate over re leak auration. The tank was assumed to be an imoermeade zone within the How teld: ro contaminants wore introducad into ine soll column along a sontion of the soutneasi oase of tha lank wnere data indicales ine :eak onginateo.

\section{SIMULATIONS}

Model callbratuon stared with a inal simulation neoroorating all of ine collected cala. The inttal simulated loshu slume adoeared :0 jo vigger than the actsal olume. Whan the genene saturated goll vertical nyaraullic conducjuilles in Jll tive model lavers were reouced oy a

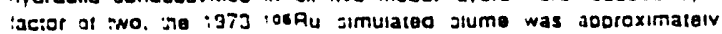
similar to no measurea stume. A smaller salurateo nyoraulic

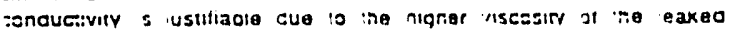

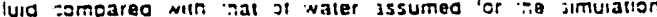

Jsing the meditied nyoraule sonouctivily, io 36 fiu ziume Tigration was inan simulated trom 1973 to :989. =ollowing 57 :easonaote

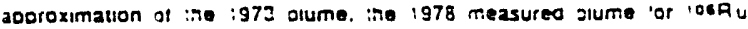
sould not se matcred. The measured slume ' $c$ r 37 a nolcates ilte

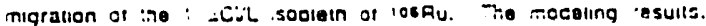
however naics:e hat he alume gxoancs jignilleanuy $n \cdot 978$. The nowever. naca:e hat the alume gxoancs signillcanuy n

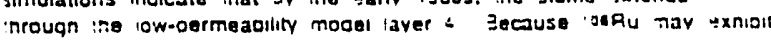

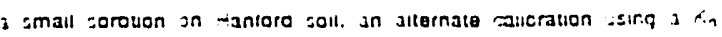

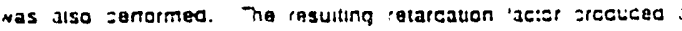

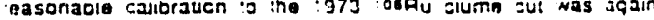
nsulfic:ent a 2000 ine calculated osfu zluma jiztionary $: 973$ snc

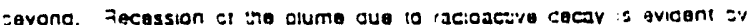
ne hariv $380 \mathrm{~s}$ tor soln cases out s most sronourcao in ro $\mathrm{k}$, imuiation.

Join simulations, rowever. nacated a signilicant woening it tho slurce or :978. Jis tesult appears io oo ine censequence of mign

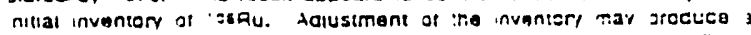
matening 973 slume: Towever. :iss adusiment was not : Teo. There nav 3150 se otner axclanations. such as use it - on-site-scectic :all zrocentes in the mocel and errors in measured sata. =irtier nvestication it hase inout zarameters nay so varrantes.

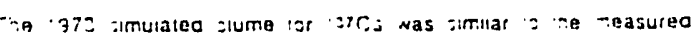

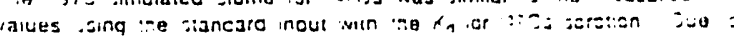

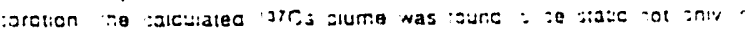

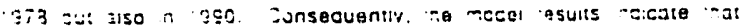

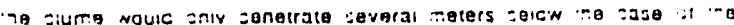

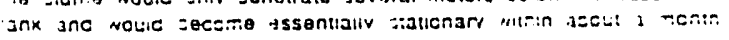
ifter $\rightarrow a$ ank was juncea out, sicsoing the eak.

IUMMARY

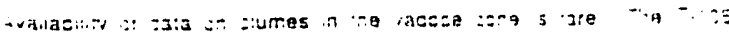

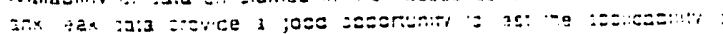

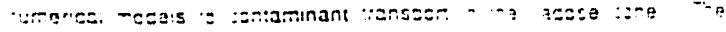

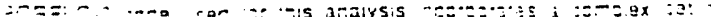

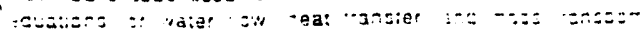

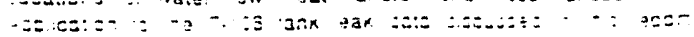

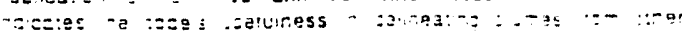
:-iะes 
Desoite the discreoancy botween the calculated and measured 1978 100Ru ptume. here is surficiont ovidence that the numerical moceat can succosstully simulate tre vansient jenavior of a infer-ainensional jlume in the vacose zone. The modeling lesults incicate that Obfu wil jlume in the vadose 20 ne. The modeling lesults incicate that corfu neeced to reconcte differences becween the simulatuons and ine moesureo data

in summary:

- In the absence of soll procemes at the i-106 tank site. Jenenc soll grobermes were inour to the mocel.

- Minor adiustminis of the jeneric soll grooerties in tho nooei

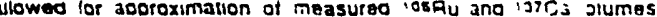
with ing model.

- For long-term zerrormance ussessment. i would ze greteraole :0 odtan measured soll jrocernes from ine $-\cdots 06$ inn sile.

- Peeliminary calibration tesults neicate nat 'or ine irst so Ja;s. Eonvection is ine cominant snenomena and the stume ingration is sominateo oy the larçe leax yolume catnes than the tocnarge cate.

- At later simulaton umes, eouct:on in the iodpu slume or adiozcive cecay is very avicent.

ACKNOWLEDGE:AENT

This work was suczereo av no U.S. Jezarteent a: Erepryt neer

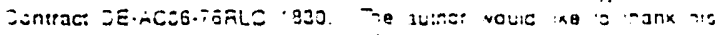

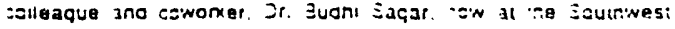

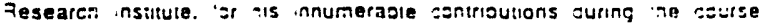
at the stuar.

TEREREYCES

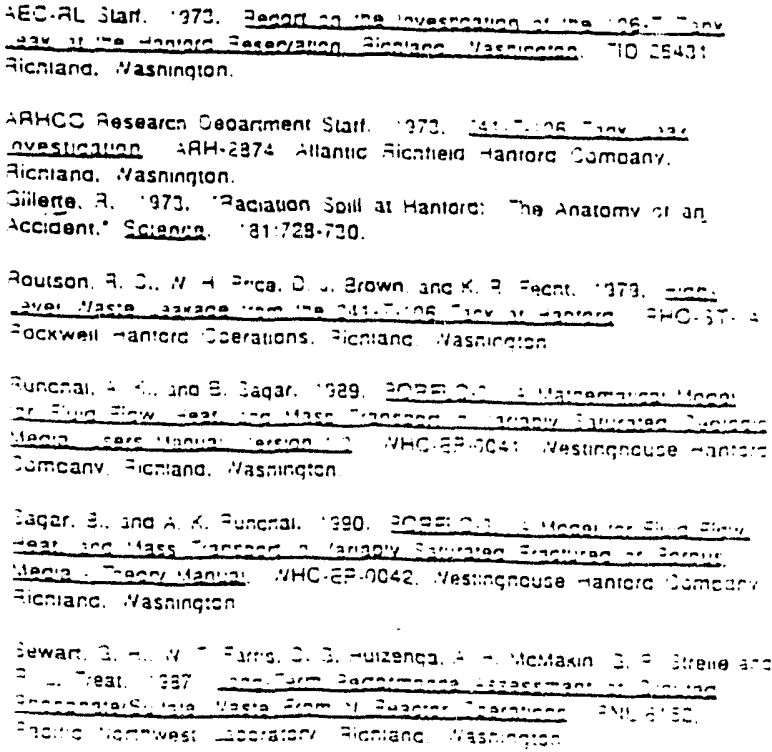




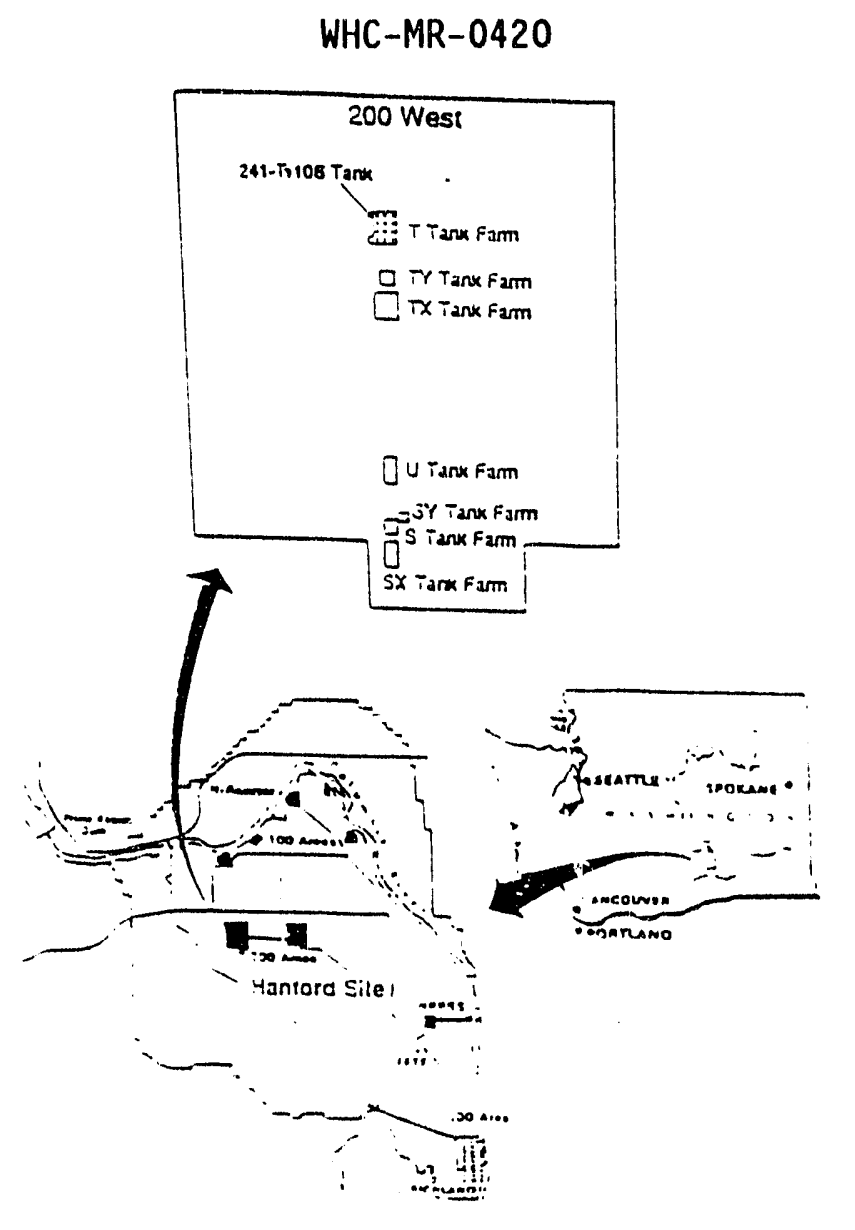

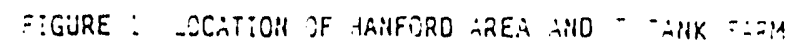

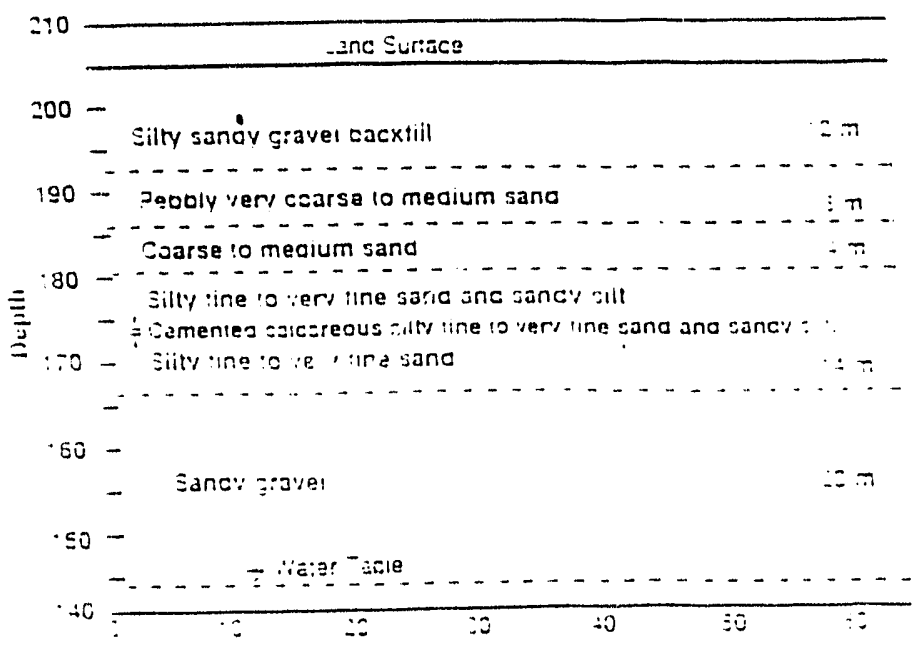

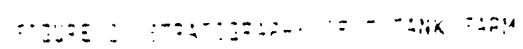



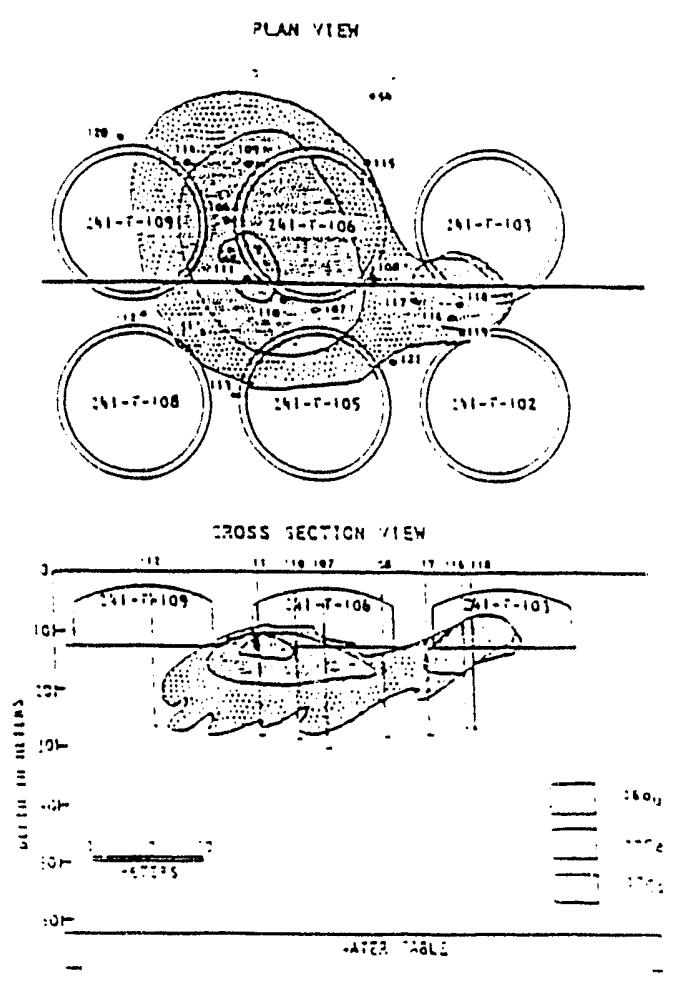

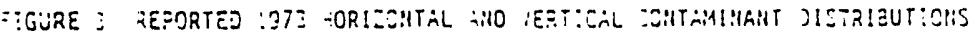

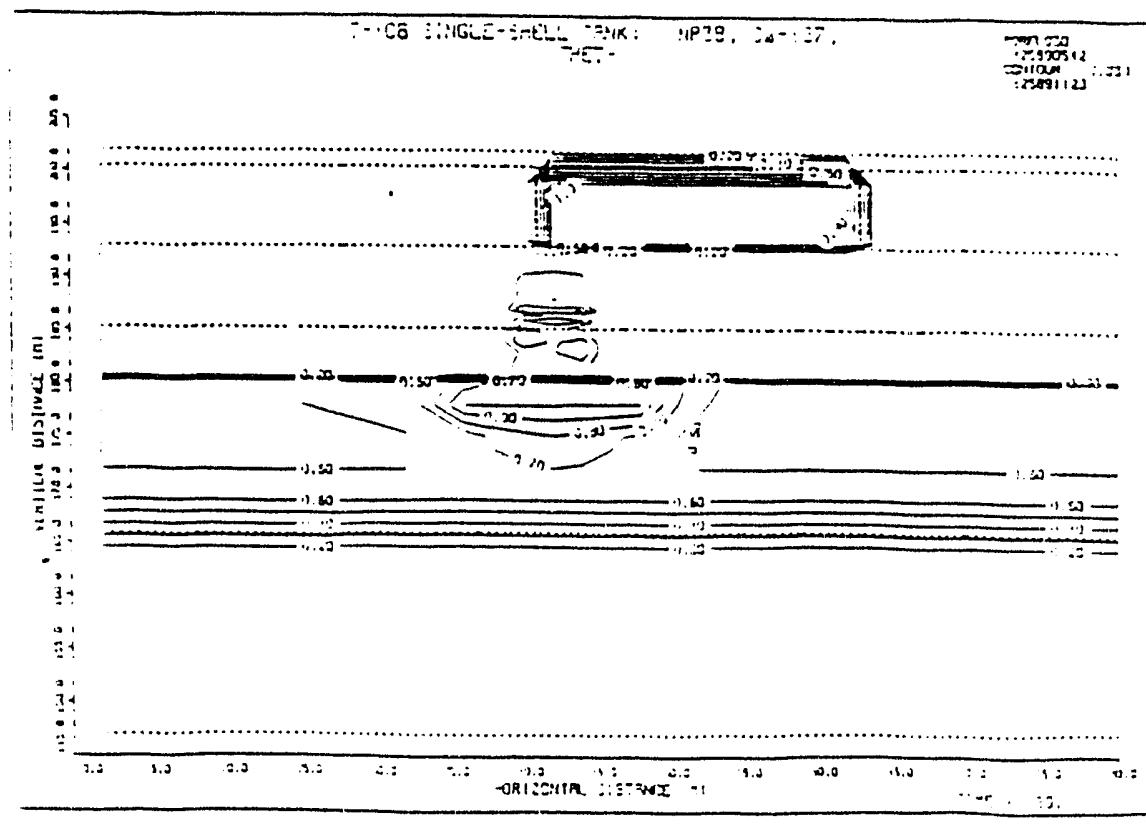

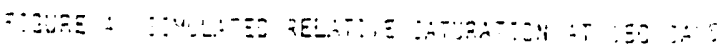


WHC-MR-0420

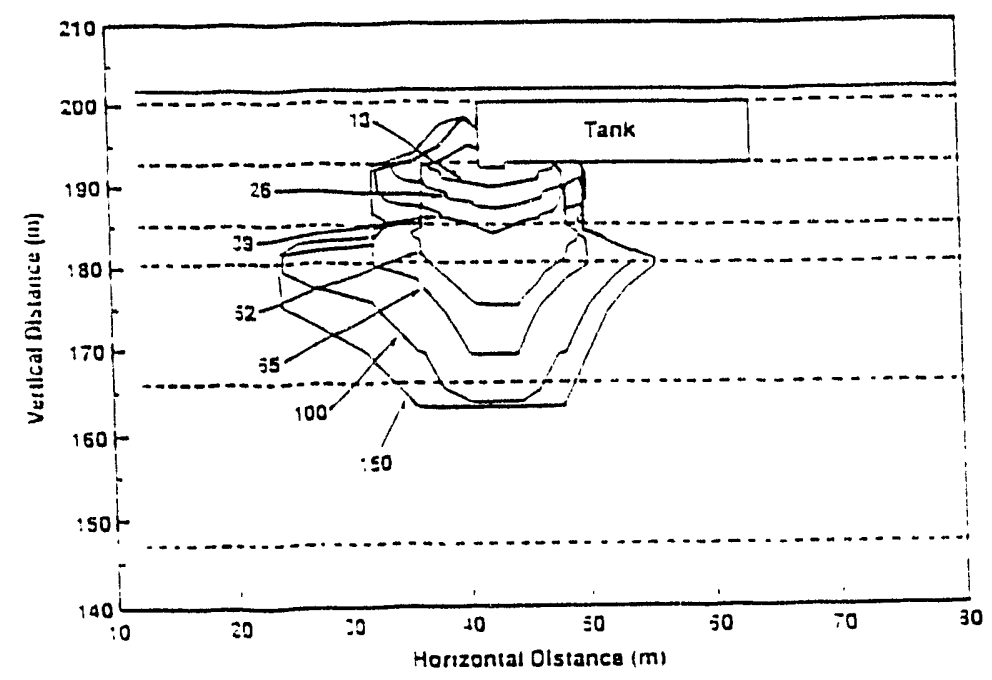

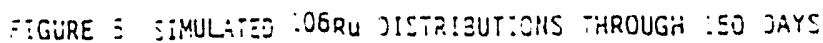

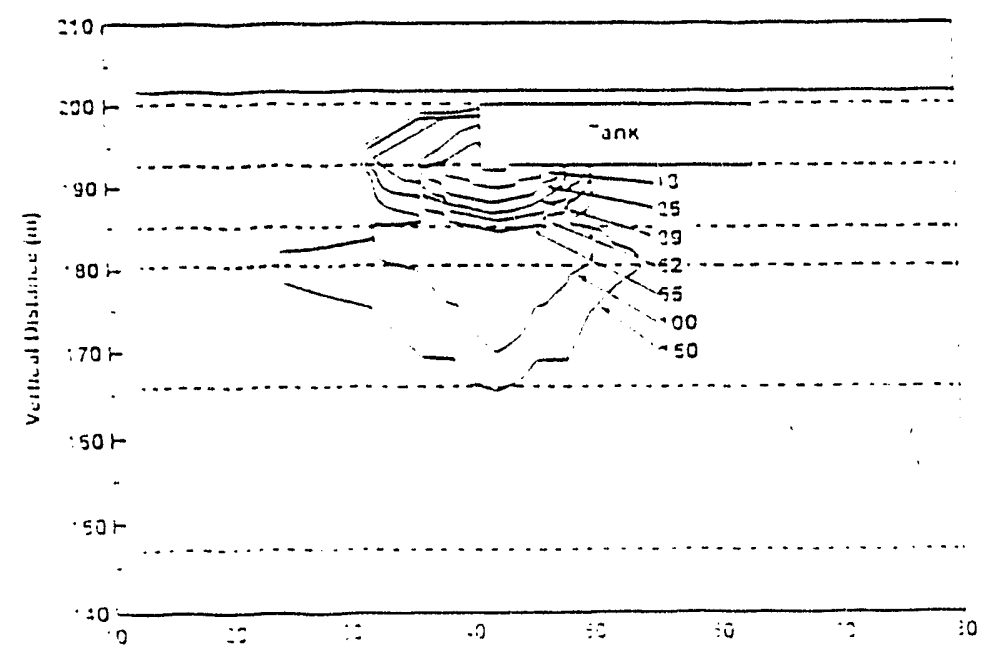

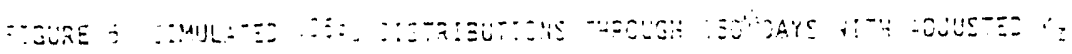




\section{WHC-MR-0420}

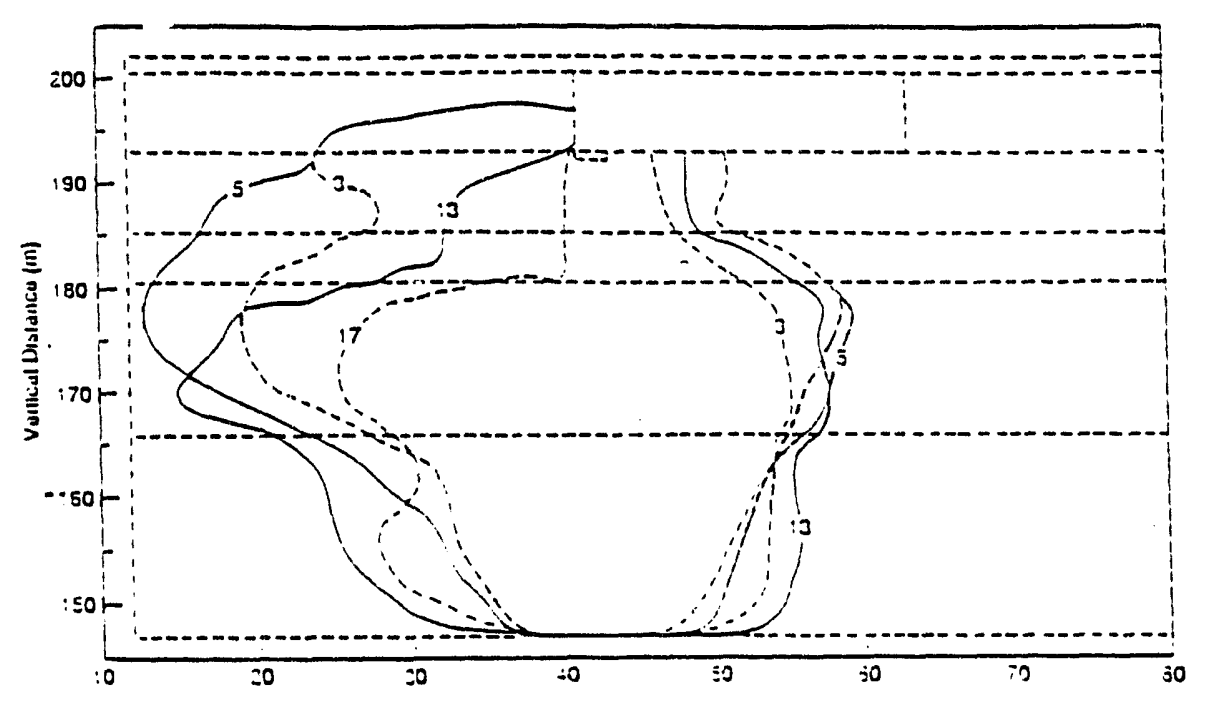

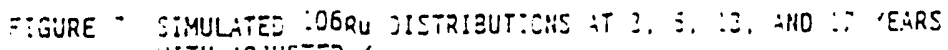
ITiA

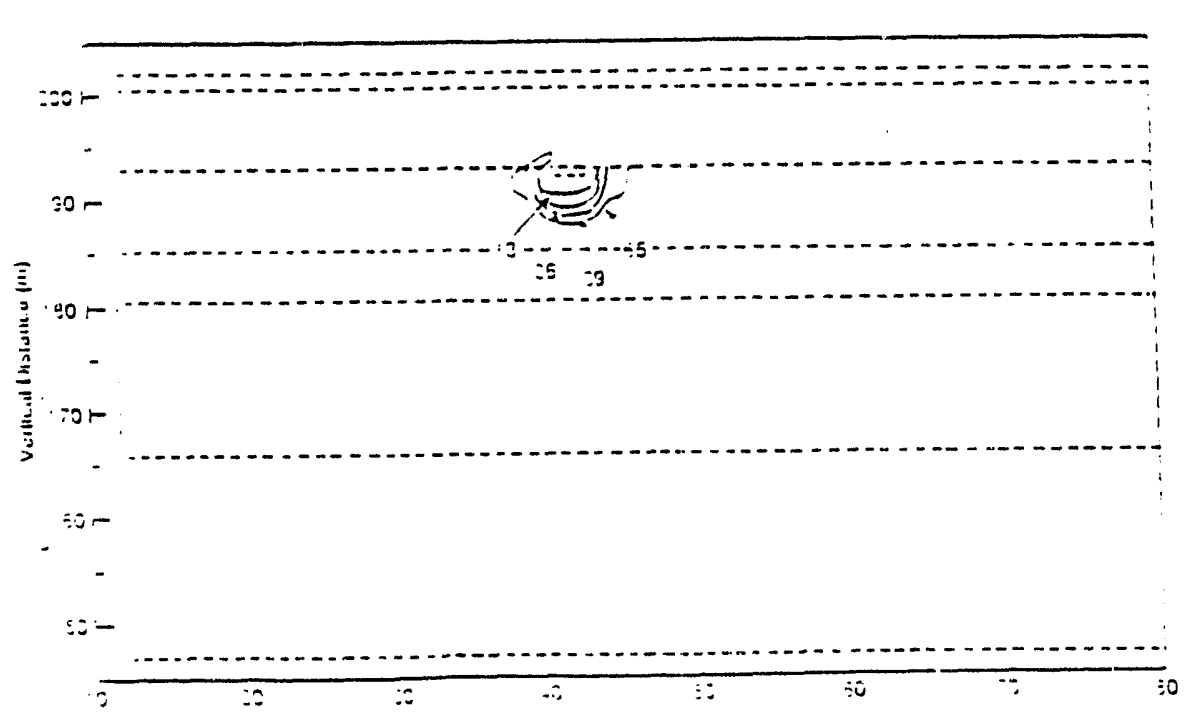

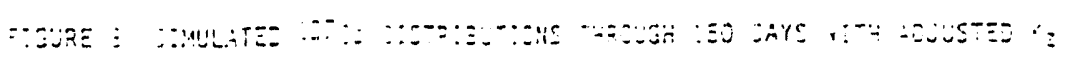




\section{CALIBRATION/VALIDATION}

A PROPOSAL

Charley Kinczıa

P3c:iic Nortrives: 'accratory

Marci 29.

PURPOSE

- TC EETAEL!SH DCE. BEA. MASTINGTCN-CCE JIALCEUE SN SCDES. :ACDE:S, IALIDATICN

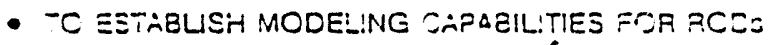

Femecial Investigations

Feasicitity Sludies

Zameciaiion Decisions

- =emeciaucn Design Hcriz:irg झ̈aluatuon

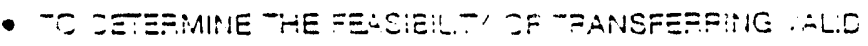
MCEE:_

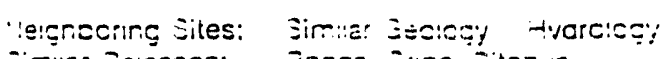

:-rilar Deteases: =arza. Z::Ls. Zitcrus 


\section{WHC-MR-0420}

\section{BACKGROUND}

- mCDeung capabilities for what ??

- STATUS AND ELPECTATIONS

- Current - Unvalidated Mocets - Low Reliance in RCDs

- Future - Validated Mccels - Medium to Hign Reliance

- POTENTTAL COMPLESITI

- No-Action Alternative - the Baseline

- sentaminant release

- cotentia: of interierence among reteases

- jeonverologi

- Alternate Remediations

- snysics. chemisiry, miciodiology or ireatment

D NHY?

- Ciecicte Inour :o aisk iszessment

- Succori 'or 'ess scziiy emeclaticris

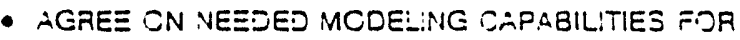

2 Peccess incceis

Leuic Water anc Conser/at!ve Solute

Giher Fules anc =rases

aeac:ive Gontaminants

- Tirre Frame ano Scalla Scale

Lenc.:erm ano Frr.Find Aquier! Forecasis Encri:erm anc ilear.Fie!c Vacosel Peectci:cns

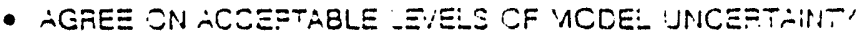
CONFICENICE, . ICOURAC' ... IALICIT!

2 sarameters . Fielc : Measurade and Mocelade

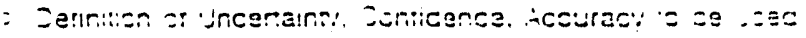

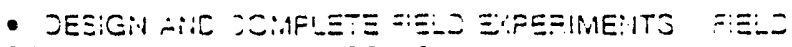

STUCIES OF =OICP DISPCSALI

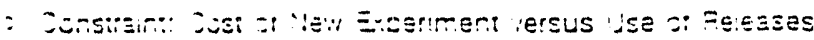

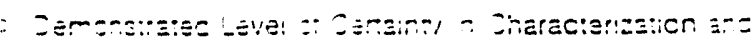

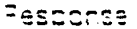

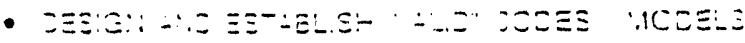

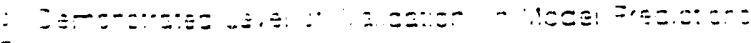

$=\because-15: 5: 2$ 
- DETEAMINE NEEDS OF DOE, EPA, W-DOE. (OTHER STAKE:HOLDEAS)

- Processes

? Time and Soatial Scales

D Measures oi Accuracy, Conridence. Uncerainty

IDENTTFY AND/OR DEJ/E:CP CODEs AND MODE:S FOR REGULATORY APPUCATION

o Expected Documentation

- Soitware Avaliablity, Maıntenance, and Suppor

- IDENTIFY ANO/CR CONDUCT APPRCPRIATE FEE:D

EKPEEIMENTS CR STUDIES FOR REEULATCRY APOICATICN

- DCCUMENT MCDE! PEFFCRMANCE

z Anaivsis s: Ercerimenis

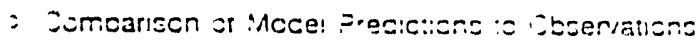

V issessment of vicce! 1/alle:t!

$=$ issessment of Mcce! Transiaraculity

CONSTRAINT: TYPICAL DOCUMENITATION FOR CODES MOCE:S

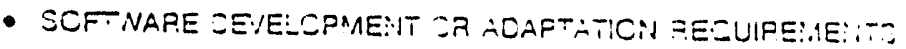

- scf nafe jesigin

- scFinape jsea guicance

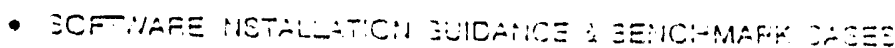

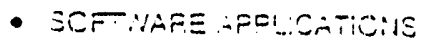


CONSTRAINT: TYPICAL DOCUMENTATION FOR FIELD STUDIES

- Design of fie:D elpeaiments / Studies

- Objective and Plan for the Exceriment / Study

- Measurement Techniques, Acsuracy, and Error ior Characterization and Monitoring

- NEPA DOCUMENTATION

- REPORTS ON ELPE:AIMENTAL RESULTS

- Site Ciaraceerization and Analysis of Proderties and intial Conaitions

- $1^{\text {st }}$ Phase. $2^{\text {nd }}$ Phase. .... Peccrts

- Posi-Mortern Charac:erizanon ano Comolete Analysis

THIS AEVIEN: ADVICE AHD INPUT

- SCOPE OF A SUCOESSFUL "ALLCATICA" EFFOAT

- Fhysics, Chemisir, Mic:ss:ciç̣y

- Long-ierm. as-iletc and/oi sncritzrm, rear-ilelc

- Dimensicnältv

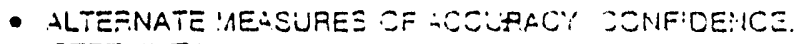
UNCEETIINTI

- Level of "/alloaticn" Acrievec:e

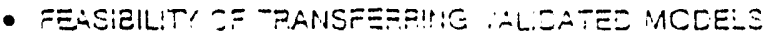

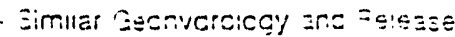


WHC-MR-0420

\title{
What Constitutes a Validation Experiment?
}

\author{
Lyrin Gelhar \\ Massachusetts Institute of Technology
}


WHC-MR-0420

This page left intentionally blank. 
WHC-MR-0420 Cerumetici sad $\neq$ inciàel

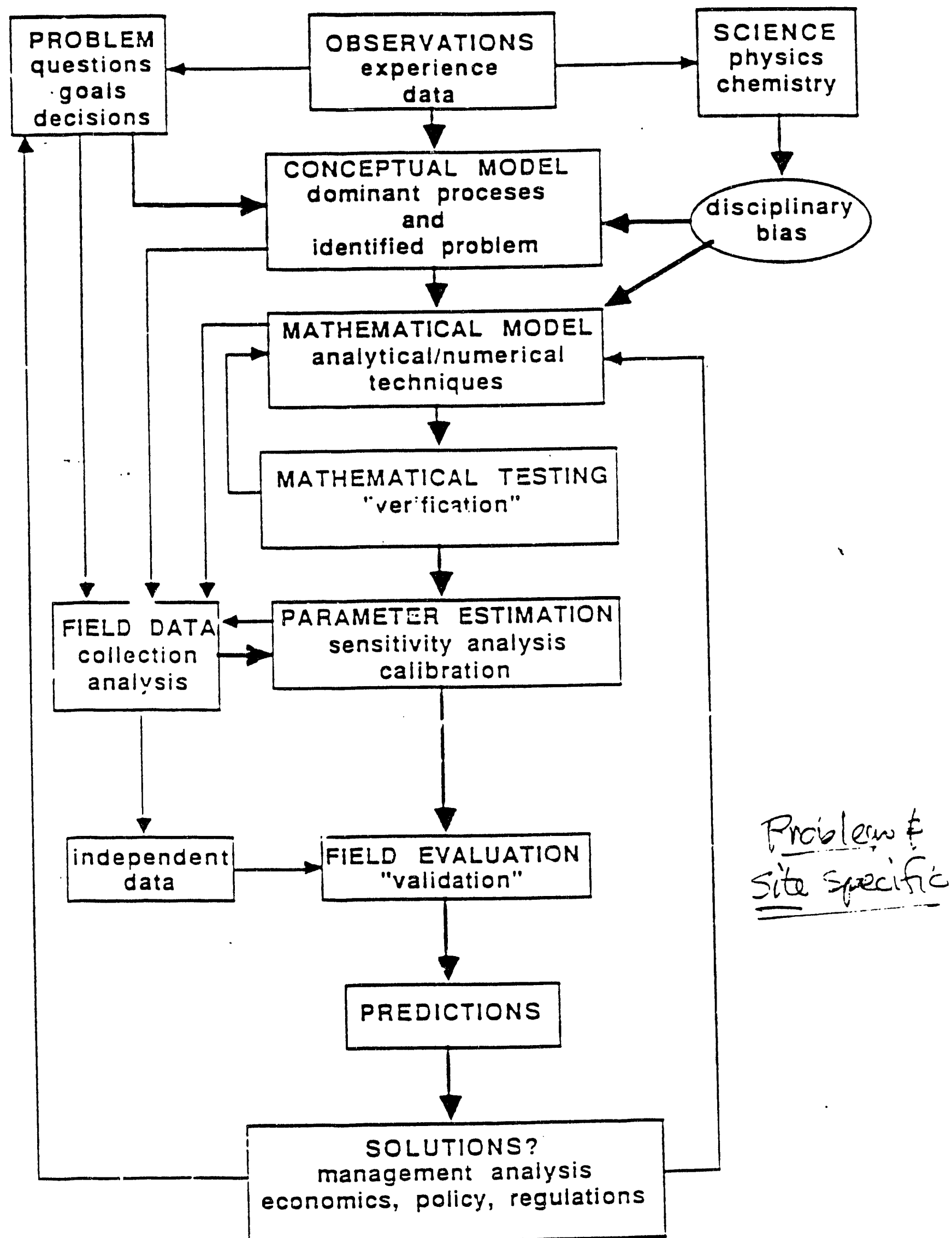




\section{POSSIBLE MODELING APPROACHES}

A. Deterministic Numerical Models

(i) Classical - homogeneous layers or zones

- ignores spatial variability

- no error measure

- mocíd parainetrers not directlej measurable

(ii) Detailed w/"actual" heterogeneity

- measurements impractical 3D!!

- computationally infeasible? $10^{9}$ nodes

\section{B. Stochastic Models}

Intraformational heterogeneity represented by spatial random fields characterized with a small number of statistical parameters

mean $\Rightarrow$ form of large scale p.d.e. + effective properties $C$ a method for determinina model parameters from direct smail-scale measuremt.

variance $\Rightarrow$ model error provides a systematic basis for model validation (and ealibration) 
WHC-MR-0420

STOCHASTIC UNSATURATED FLOW THEORY re Yeh.....WRR (1985) ; Mantoglou... WRR (1987)

Polmann (1990)

$$
K=K_{S} e^{-\alpha \psi}, \quad h K=\ln K_{S}-\alpha \psi
$$

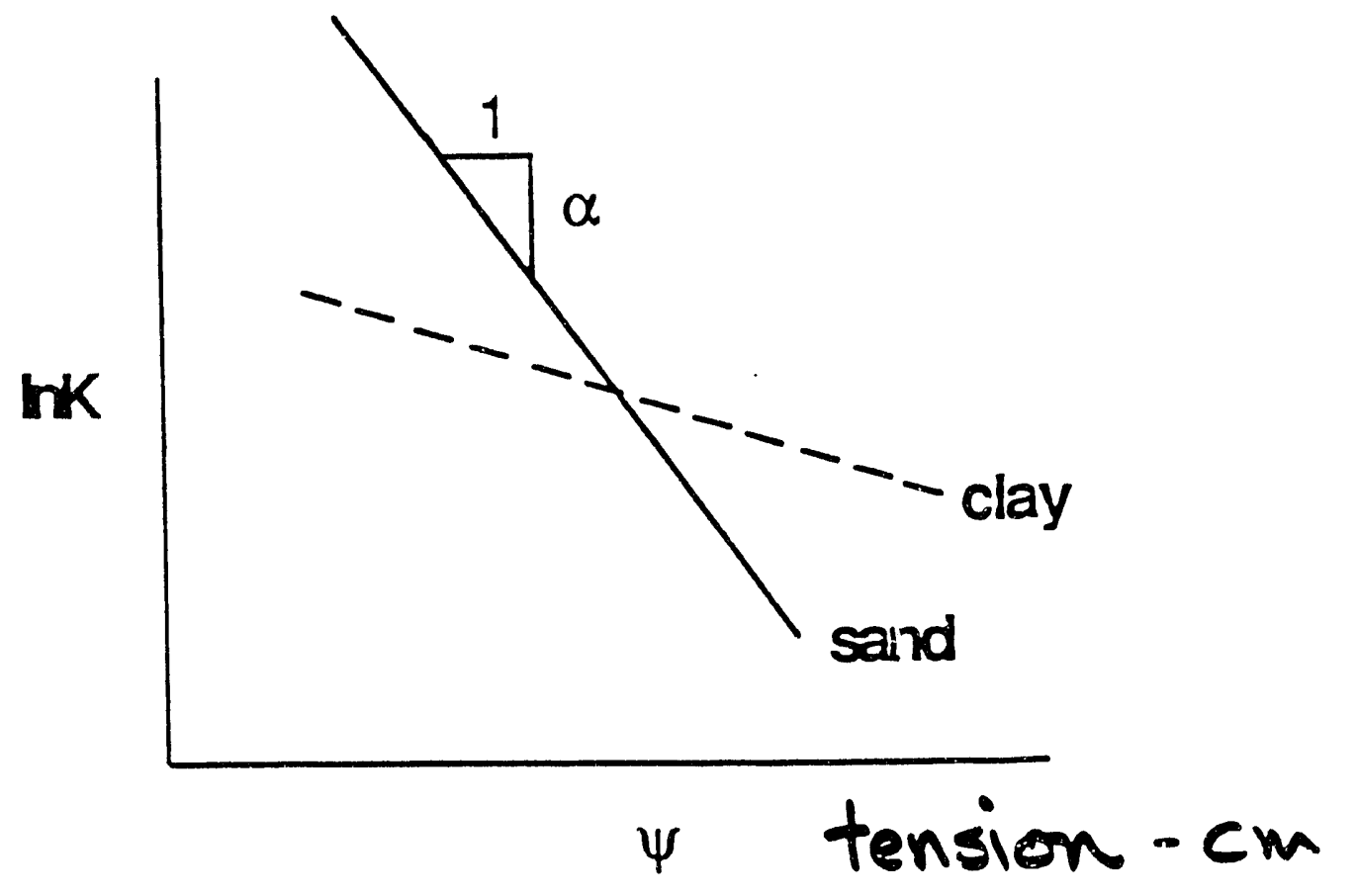

$$
\begin{array}{lll}
\mathrm{WK}_{S}=\operatorname{lnK} \mathrm{K}+\mathrm{f}, & E[\mathrm{f}]=0, & \sigma_{f}^{2}=E\left[f^{2}\right] \\
\alpha=A+a, & E[a]=0, & \sigma_{a}^{2}=E\left[a^{2}\right] \uparrow .
\end{array}
$$

luks $\& \alpha$ raudoun fields w varicines corralation scales $\lambda_{i}$

$\sqrt{2}$ 
WHC-MR-0420

Maddock sandy loam

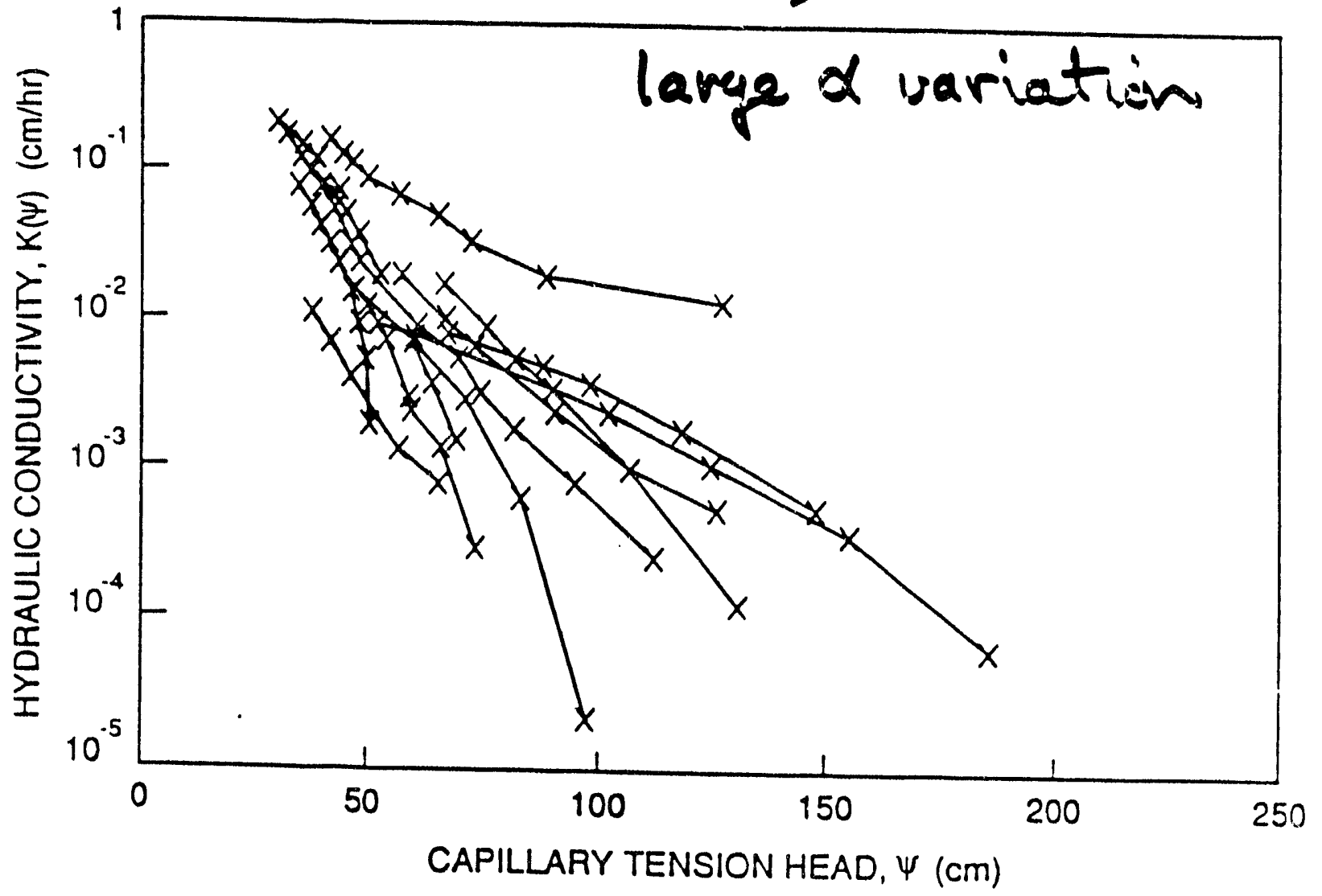

130

3 
N
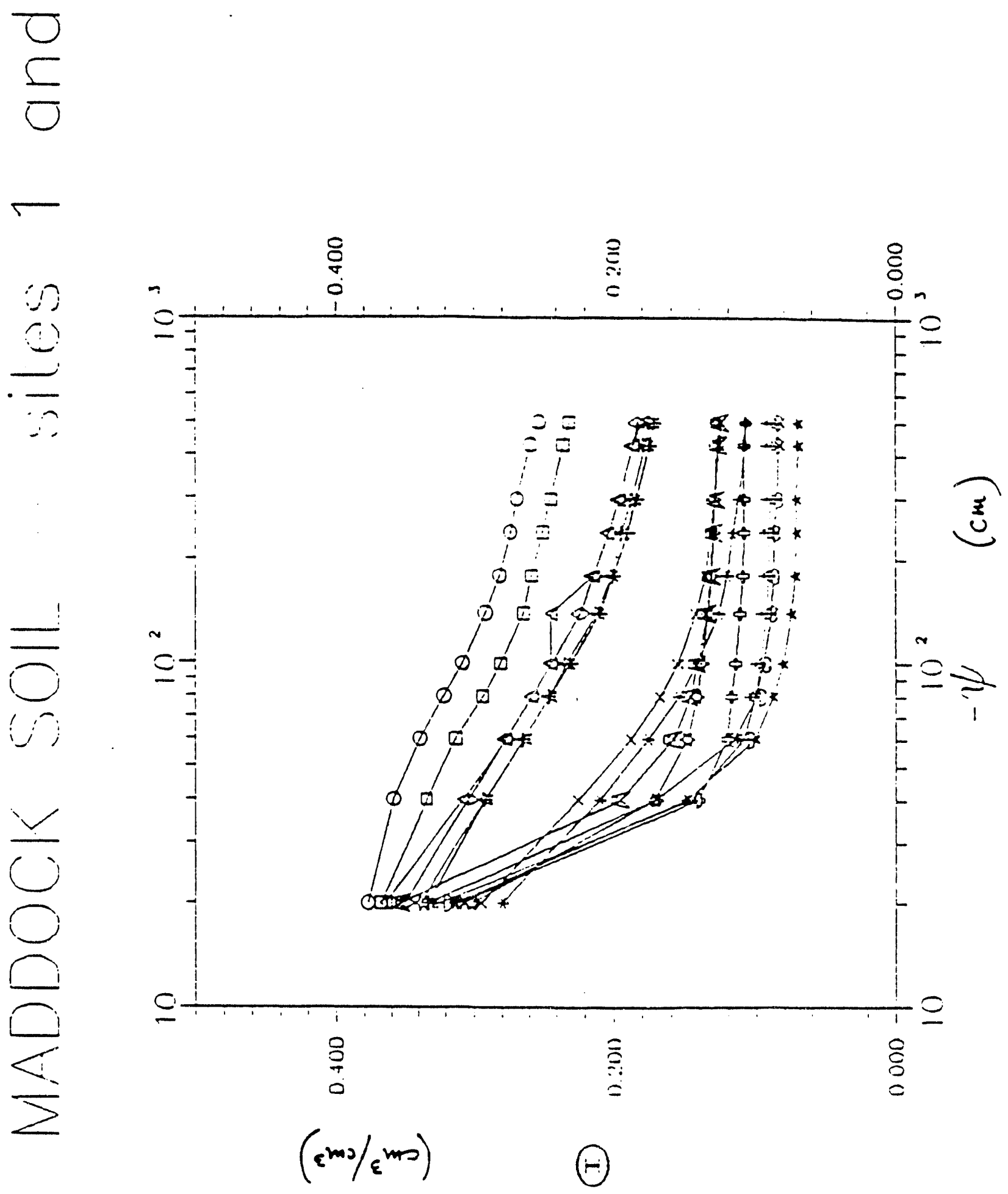


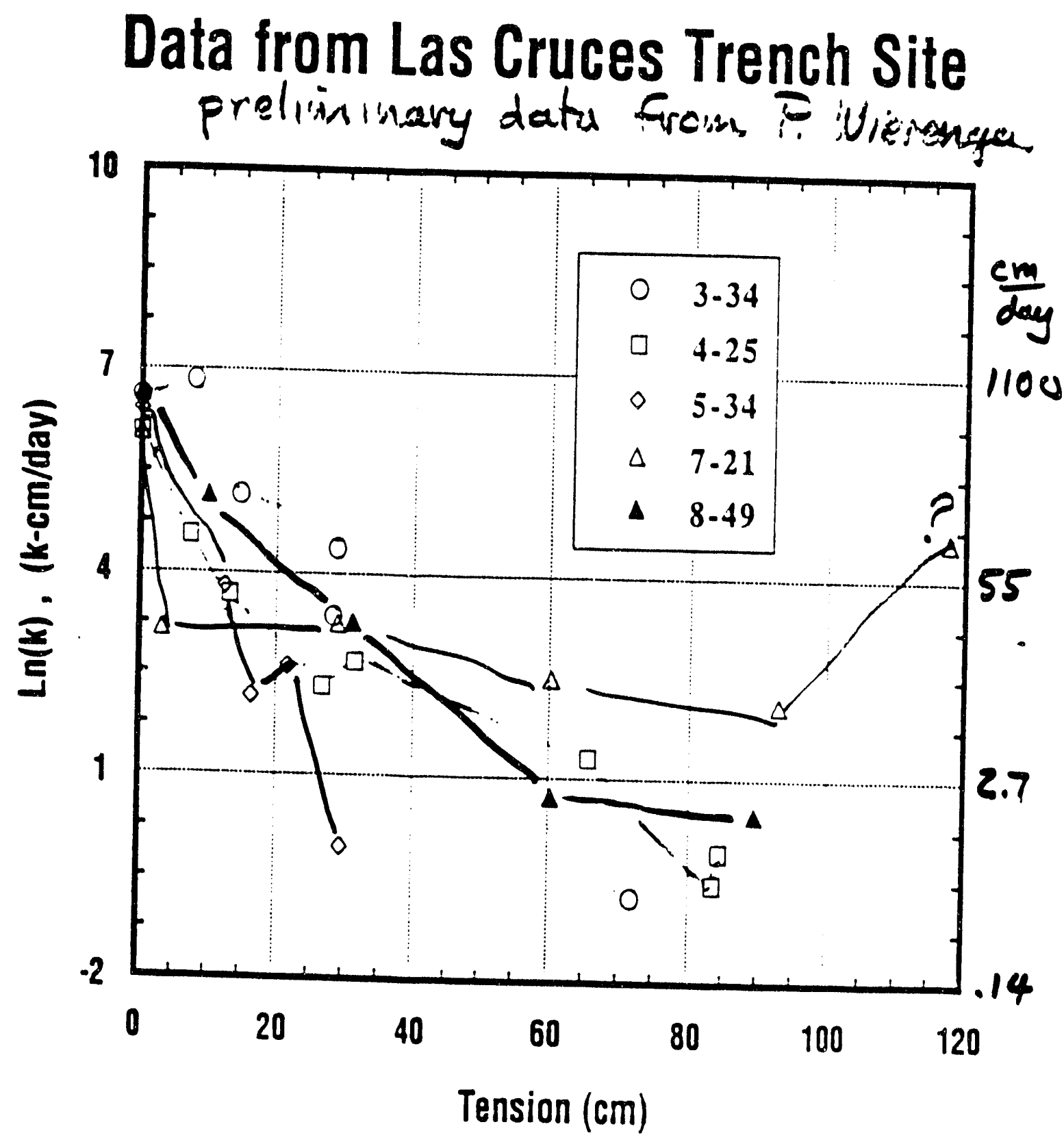




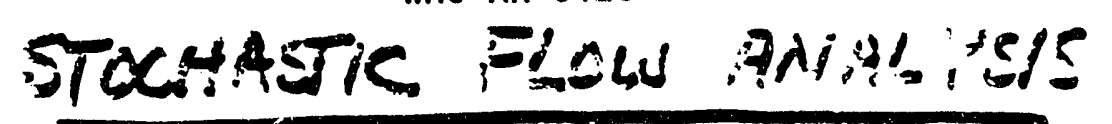

(6)

re Yeh atal, 1985; Maxtogloa .. 1987

conservation afmess

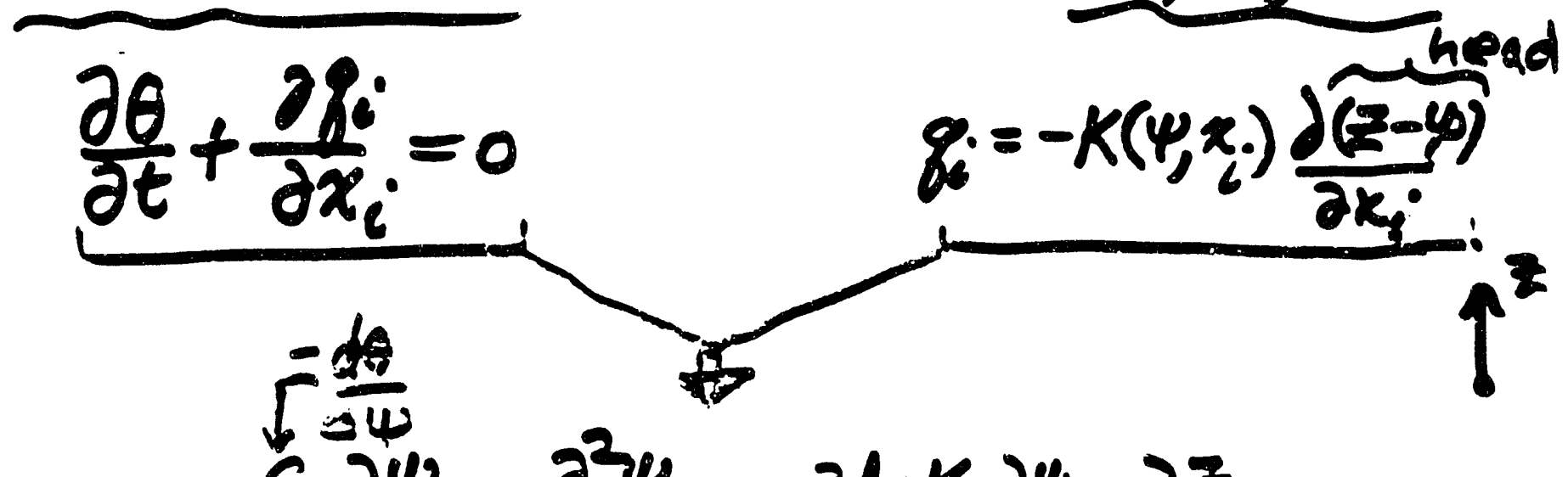

Darey equation

$e^{\frac{C}{C}+x_{i}} \frac{\partial \psi}{\partial t}=\frac{\partial^{2} \psi}{\partial x_{i}^{2}}+\frac{\partial \ln K}{\partial x_{i}}\left(\frac{\partial \psi}{\partial x_{i}}-\frac{\partial z}{\partial x_{i}}\right)$

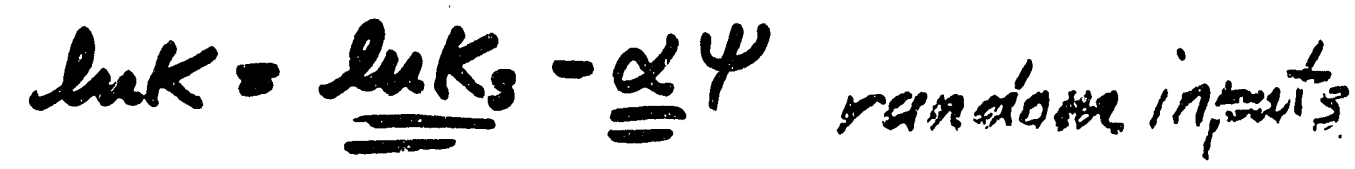

Lot

$$
\begin{array}{rlrl}
\psi & =H+h, & E[h]=0 \\
\ln _{s}=F+f, & F[f]=0 \\
\alpha & =A+a, & E[a]=0 \\
C=\Gamma+\gamma, & E[\gamma]=0
\end{array}
$$

Solve for $h \Rightarrow \sigma_{h}^{2} \equiv E\left[h^{2}\right], \ldots, E[a h]$, etc

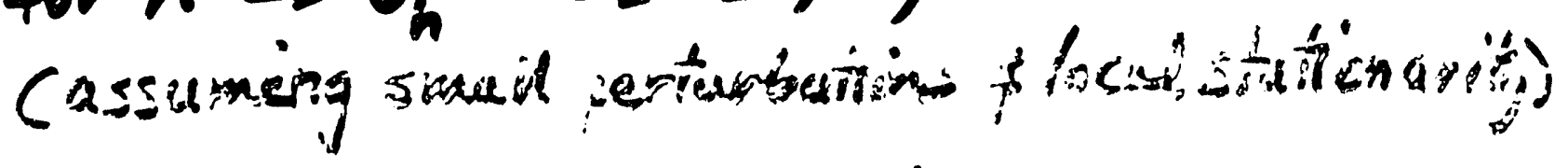

Mean equation: (anisid sras)

$$
\frac{\partial \Theta\left(H, \frac{\partial H}{\partial t} ; \sigma_{f}^{2} \cdots\right)}{\partial t}=\frac{\partial}{\partial x_{i j}}\left[\hat{K}_{i j} \cdot\left(H, \frac{\partial H}{\partial t} \frac{\partial H}{\partial x_{i}} ; \sigma_{f}^{2} \cdots\right) \frac{\partial(E-H)}{\partial x_{i}}\right]
$$

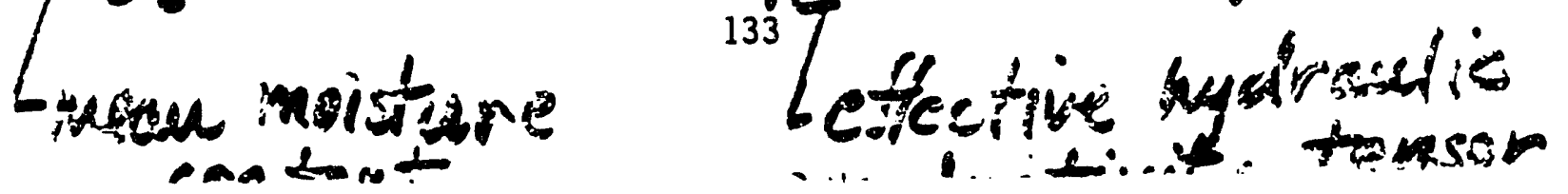


WHE-MK- $\mathbf{v} 420$

TENSION VARIANCE

司

Steady flow / layered soil / unit gradient

$$
\sigma_{h}^{2}=\frac{\left(\sigma_{f}^{2}+\sigma_{a}^{2} H^{2}\right) \lambda_{1}}{A\left(1+A \lambda_{1}\right)}
$$

for $\ln k_{g} \& x$ uncervelutid

note strong dependence on mean tension $\mathrm{H}$

$$
\text { large } H=>\sigma_{h} / H \rightarrow \text { constant }
$$

$$
\begin{aligned}
& A \lambda_{1} \ll 1 \\
& A \lambda_{1} \gg 1
\end{aligned}
$$

$$
\begin{aligned}
& \sigma_{h}^{2} \propto \lambda_{1} / A \\
& \sigma_{h}^{2} \propto A^{-2} \text { independent of } \lambda_{1}
\end{aligned}
$$

If ok o perfectly

$$
\begin{aligned}
& a=\xi_{j}^{\rho}, \quad\left(\sigma_{f}^{3}+\sigma_{a}^{2} H^{2}\right) \rightarrow \sigma_{f}^{2}(1-\xi H)^{2} \\
& \text { on } \rho=\frac{\sigma_{a}}{\sigma_{f}} \\
& H_{134}=1 / 3 \Rightarrow \sigma_{h}^{2}=0 \\
& \text { SSP Yeh(1989) WRR 25 }(10) \text { pZl'q }
\end{aligned}
$$


WHC-MR-0420

EFFECTIVE CONDUCTIVITY

Steady Flow Results:

$$
f i=E[\psi]
$$

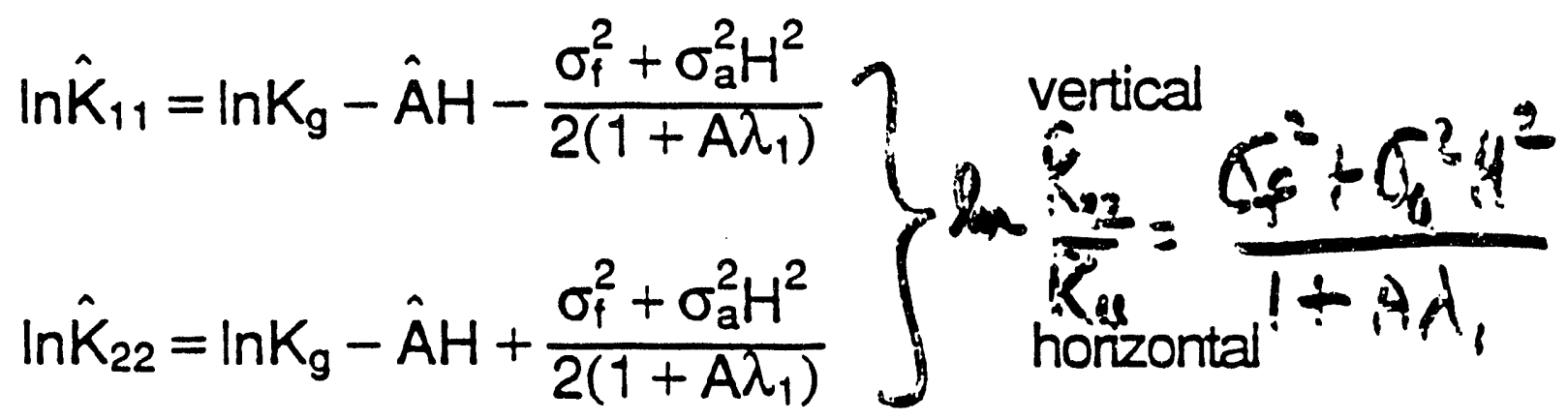

$$
\begin{aligned}
& \hat{A}=A\left(1-\frac{\sigma_{a}^{2} \lambda_{1}}{A\left(1+\lambda_{1} A\right)}\right), \quad \lambda_{1}=\text { correlation scale }
\end{aligned}
$$

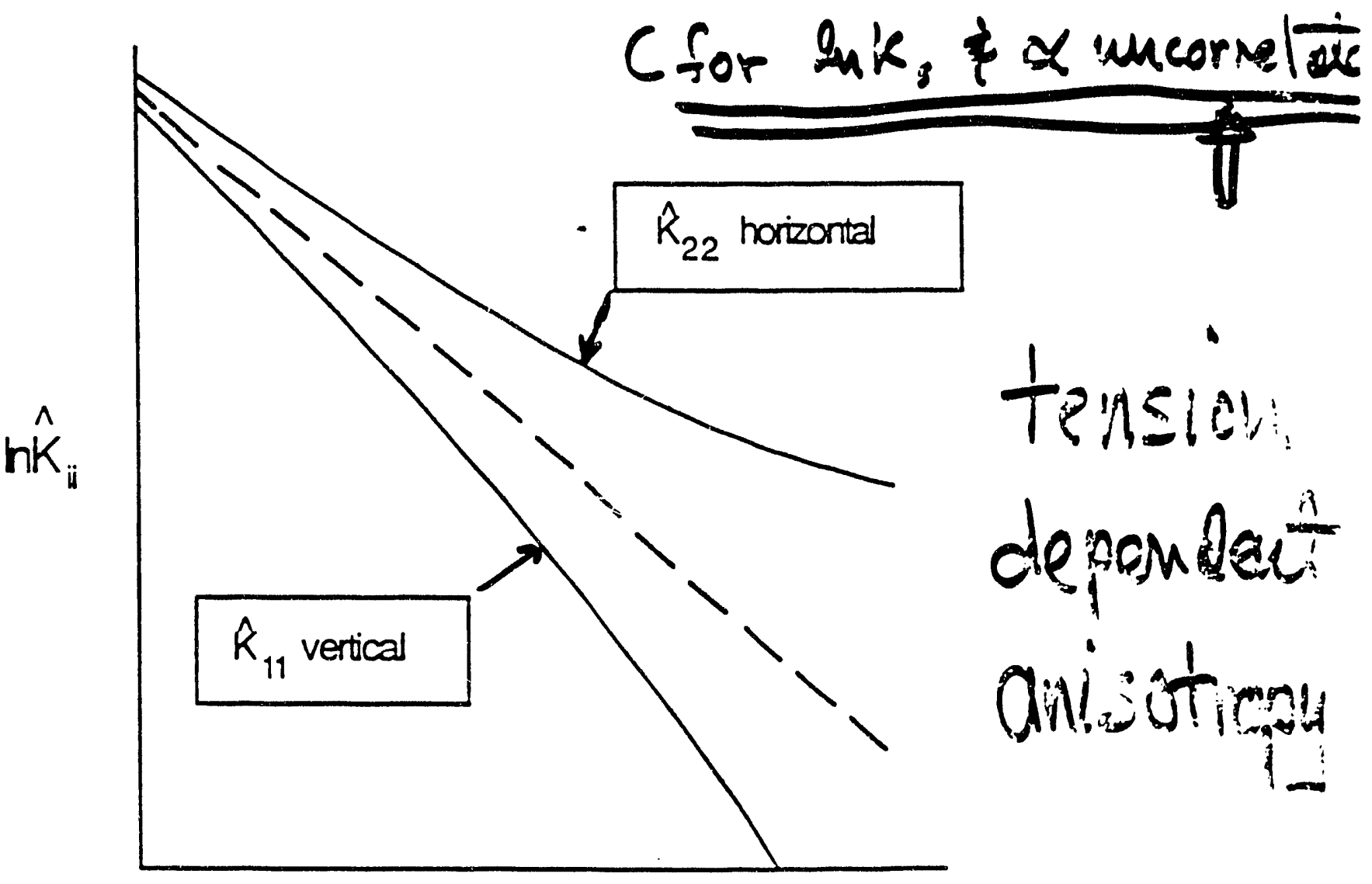

$$
\because \quad \text { Nate: } \sigma_{\psi}^{2}=\frac{\left(\sigma_{f}^{2}+\sigma_{t}^{2} H^{2}\right) \lambda_{1}}{A(i+\Delta \lambda .1} \quad 18,
$$


WHC-MR-0420

UNSATURATEO FLOW

EFFEGTIVE HYDRAULLC CONDUCTIVITY

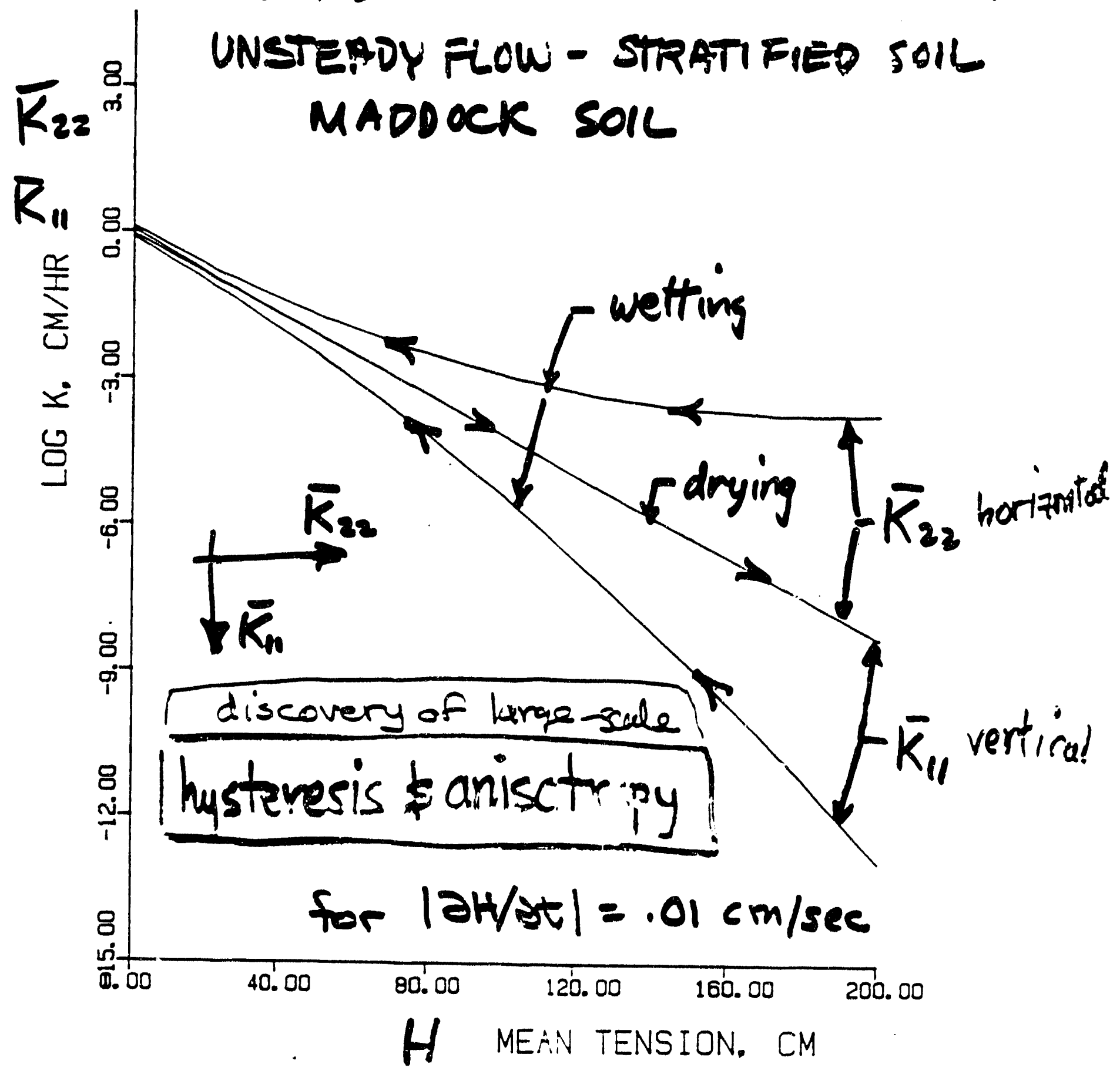

136

$\bar{c}$ 
WHC-MR-0420

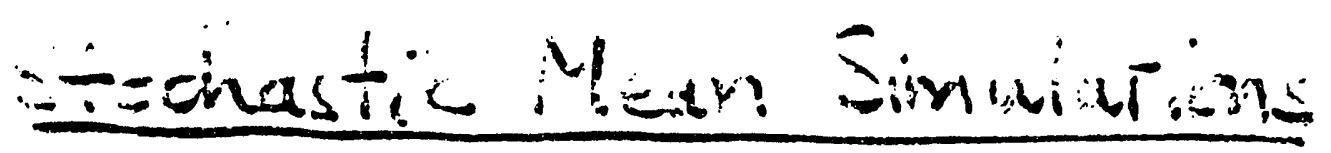

101

re Pd mann at al (1990) were

Lara - scale model w/ effective parameters

$$
\begin{aligned}
& \frac{\partial \theta}{\partial t}=\frac{\partial}{\partial x_{1}}\left(\hat{K}_{11} \frac{\partial\left(H+x_{1}\right)}{\partial x_{1}}\right)+\frac{\partial}{\partial x_{2}}\left(\hat{K}_{22} \frac{\partial H}{\partial x_{2}}\right) \\
& \Theta=\Theta\left(H \frac{\partial H}{\partial t}, \frac{\partial H}{\partial x_{i}} ; \sigma_{i}^{2}, \lambda_{i}, A, \ldots\right) \\
& \left.\hat{K}_{i i}=K_{i i}\left(H, \frac{\partial H}{\partial t}\right) \frac{\partial H}{\partial x_{i}} ; \sigma_{i}^{2} \cdots\right)
\end{aligned}
$$

Numerical approximation:

$\rightarrow$ Galerkin finite element discretization

$\rightarrow$ modified Picard iteration

$\rightarrow$ mass conserving "mixed "formulation $(\theta, H)$

137 
Stochastic Modeling of Large-Scale Flow in Heterogeneous Unsaturated Soils

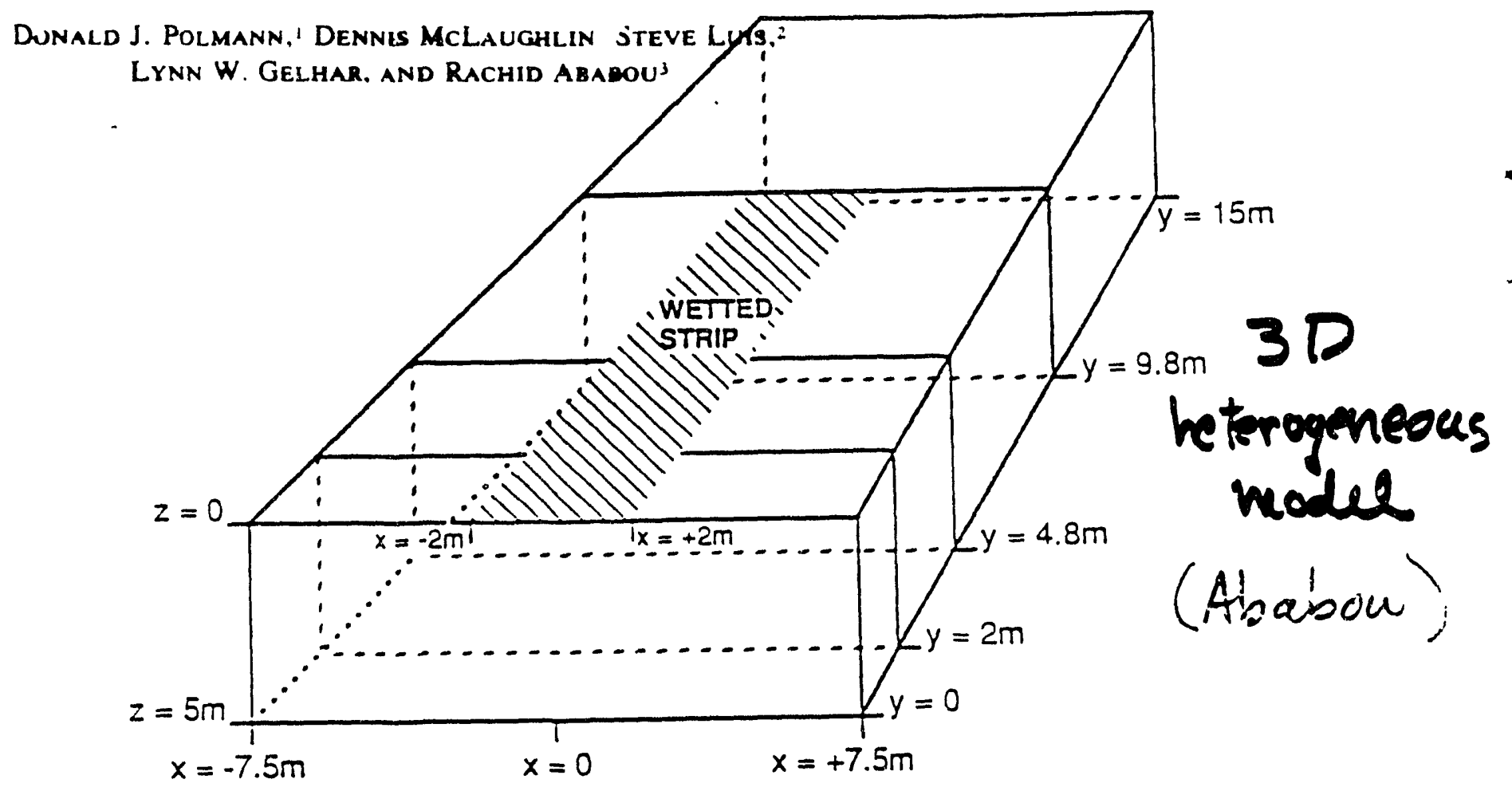

Figure $x$-xa. Schematic representation of the Ababou flow domain.

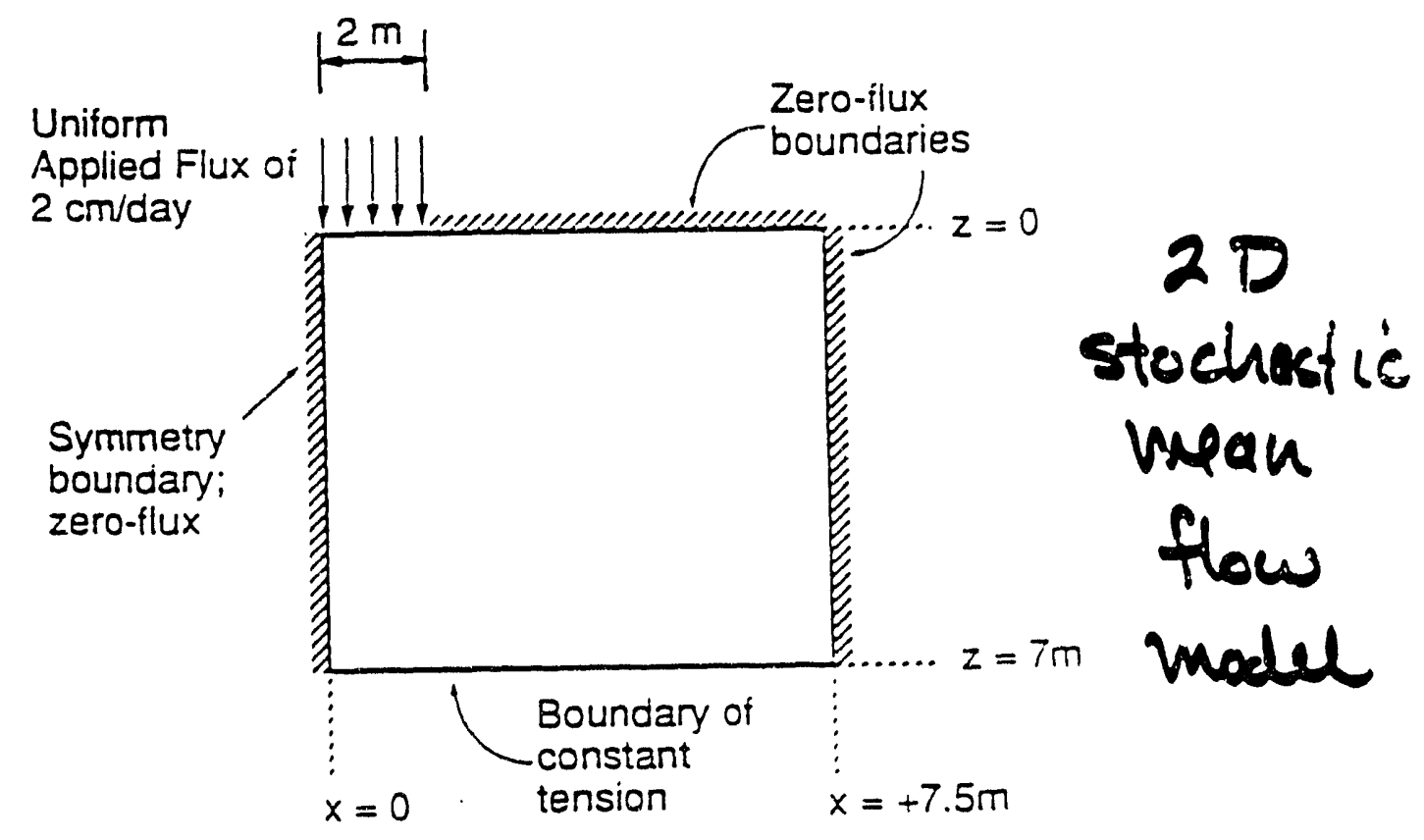

Figure $x-x b$. Schematic representation of the mean model fow domain. 

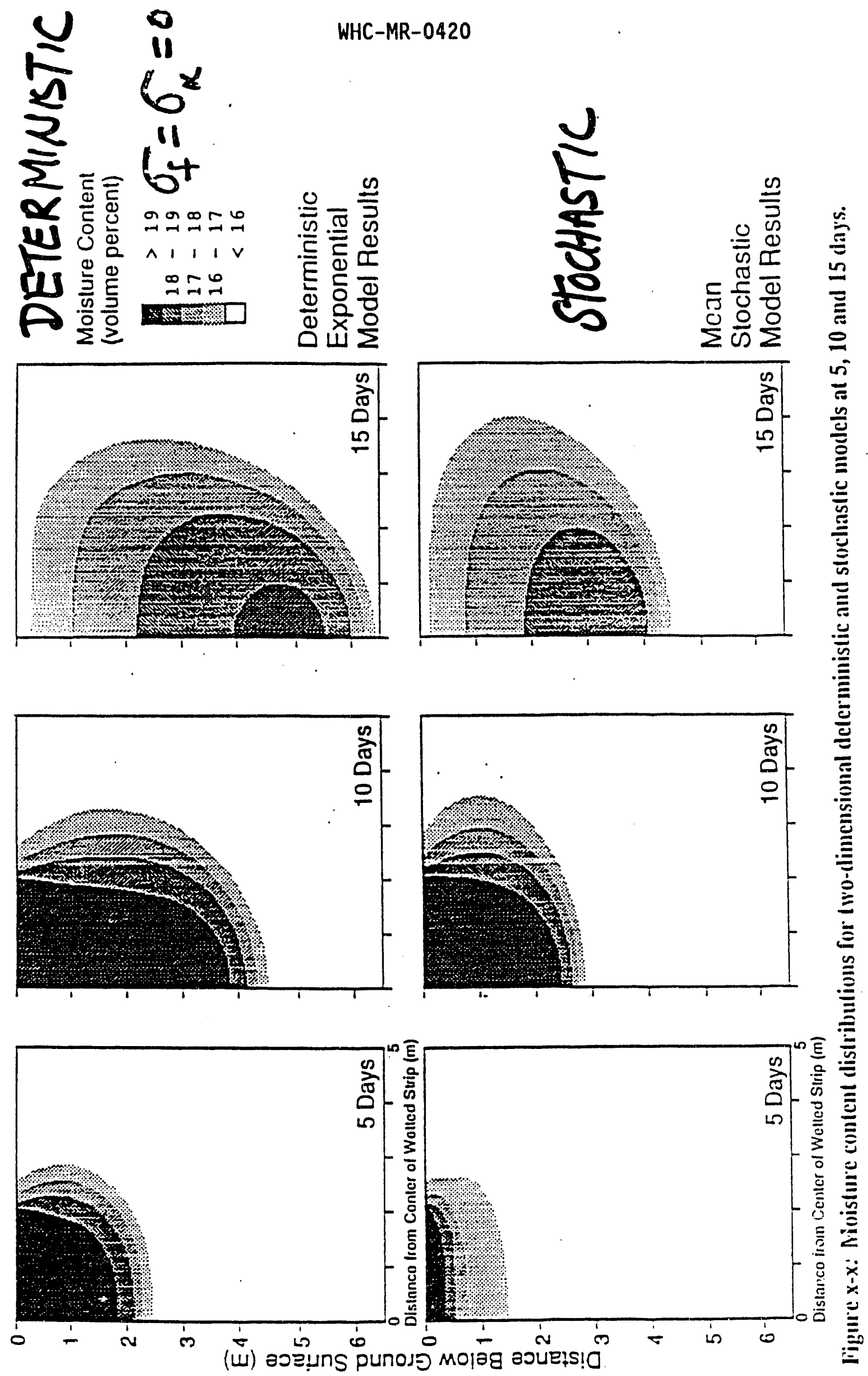
15 days

WHC-MR-04:0

(13)
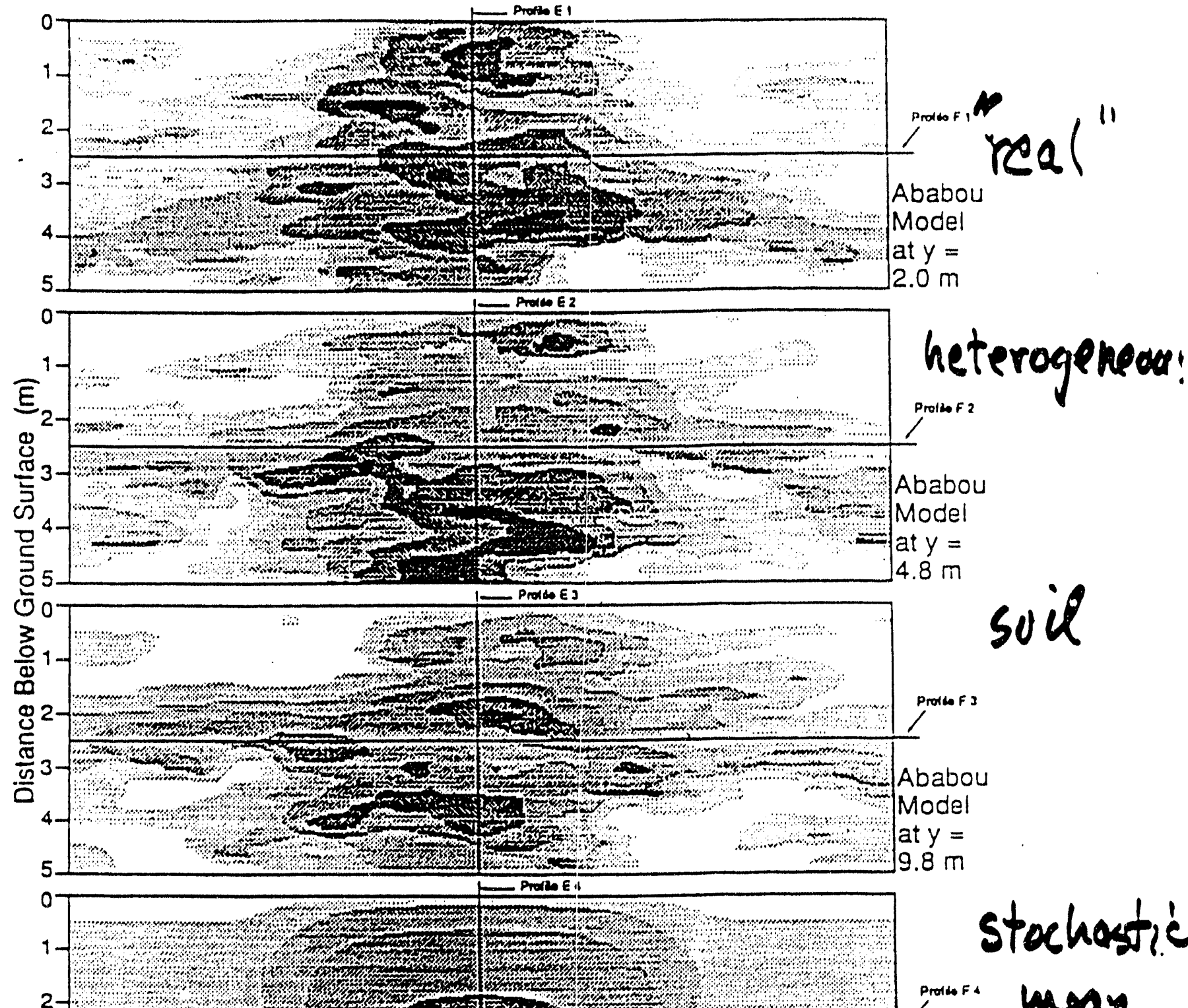

stochastic

3

4

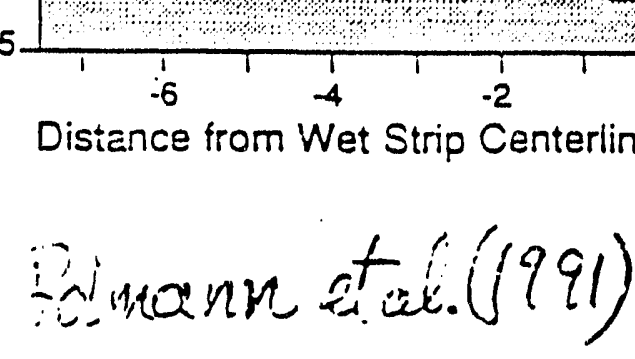

Figure $x-x$. Tension distributions for Ababou and mean stochastic models at 15 day's.

UR. $7 c$

i. 


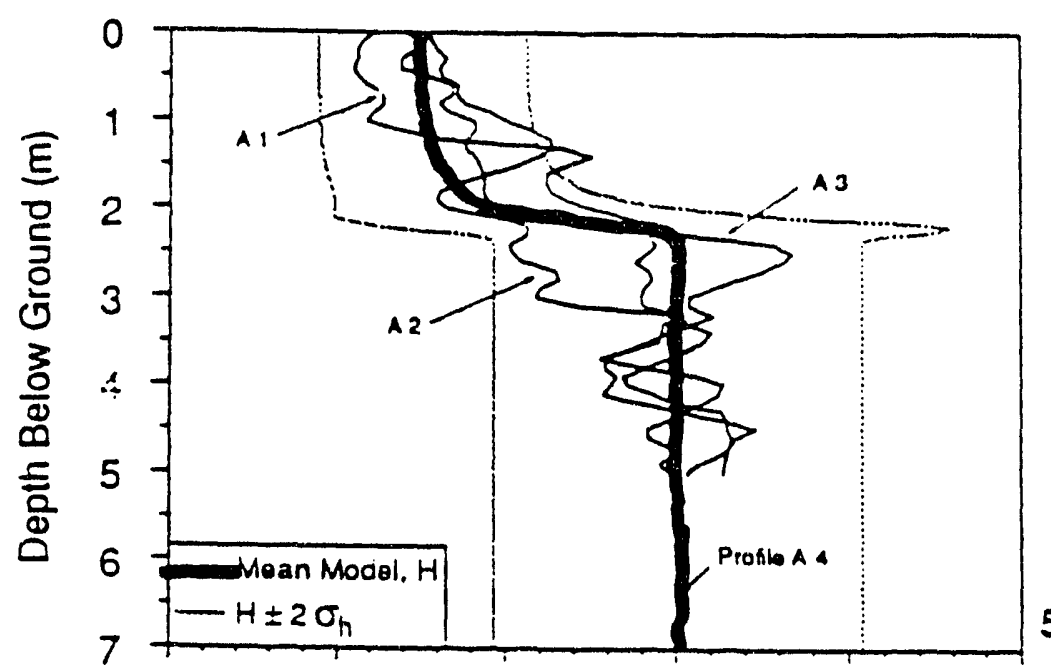

5 Days

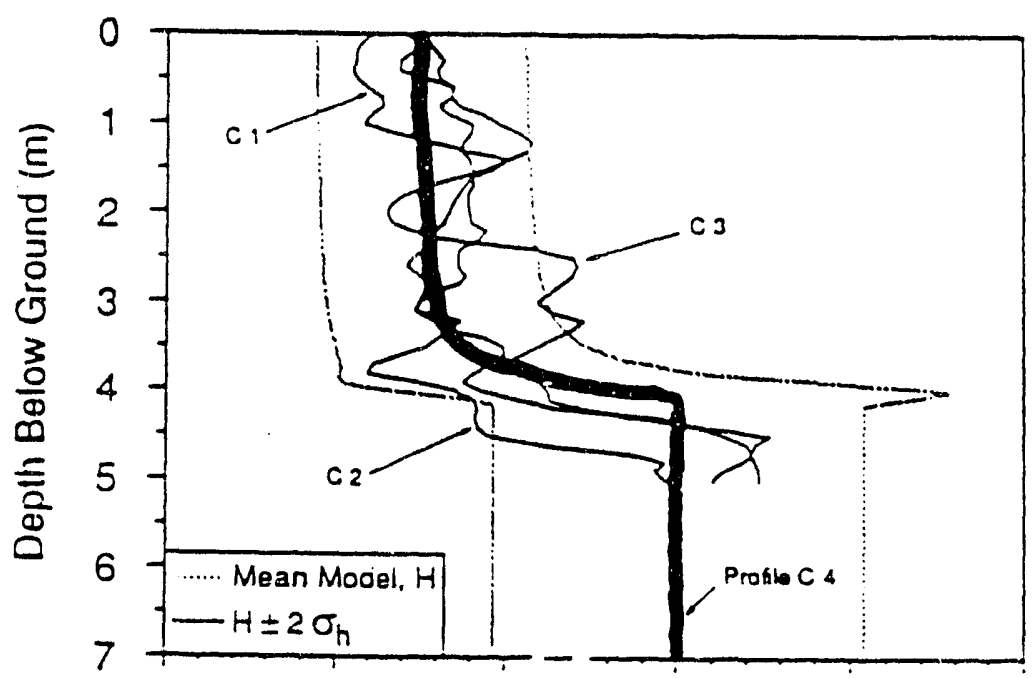

10 Days

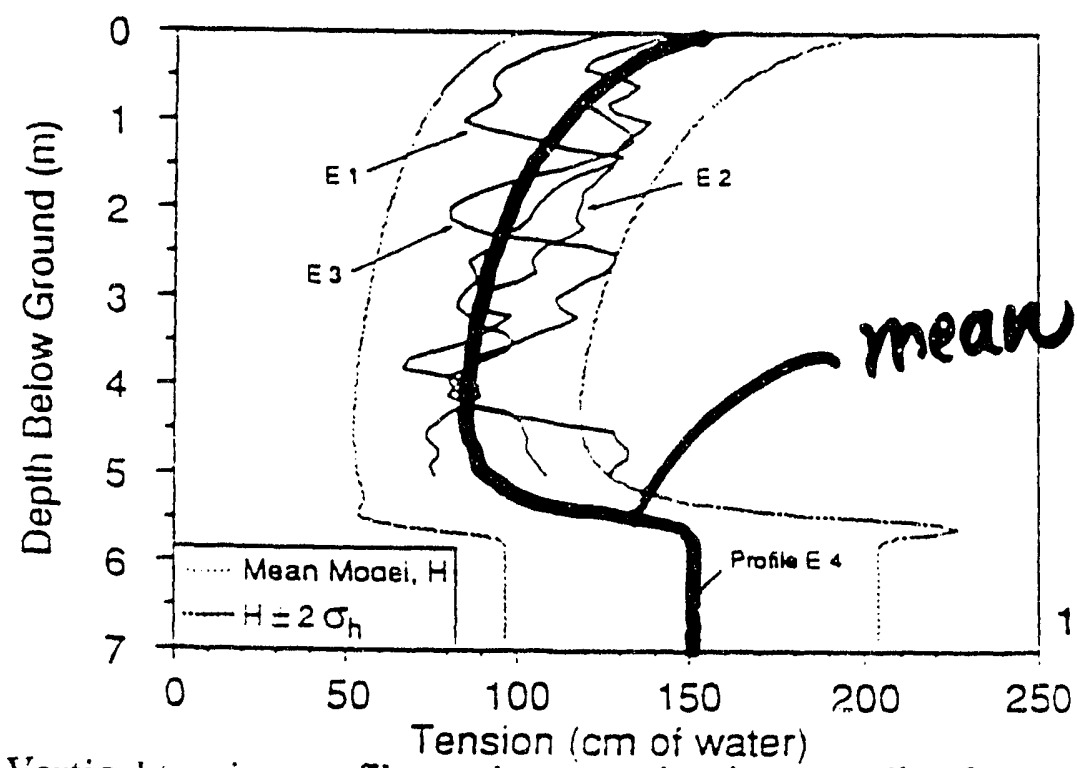

15 Days

Figure $\mathrm{x}-\mathrm{x}$. Vertical tension profiles under wetted strip centerline for $A b a b o u$ and mean model simulations (profile numbers refer to Figure $x-x$ ). soe Luis s Mclaugńlich (19a2), Qdv. WR. 15, i5-32 for

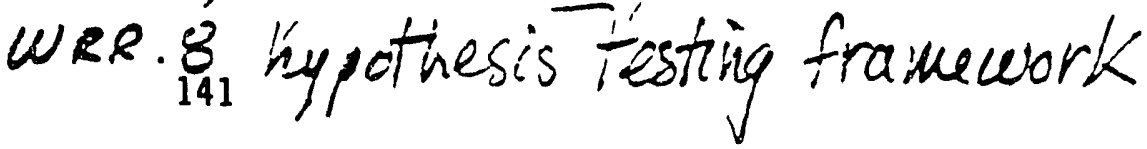




\section{hysteresis effect $\& 0.5 \mathrm{~m}$ deep}

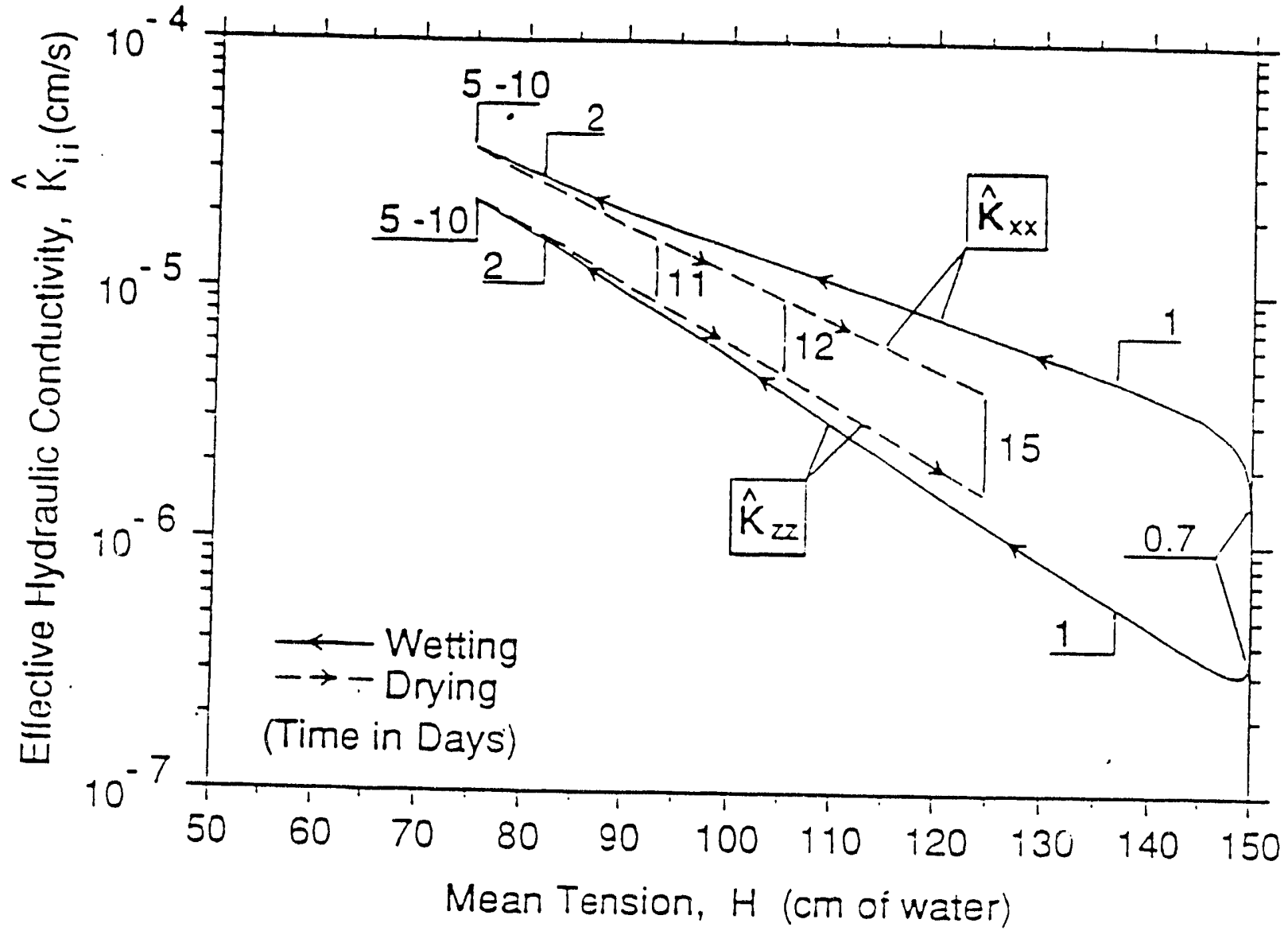

Figure $x \cdot x$. Hysteresis and anisotropy of effective conductivity at $0.5 \mathrm{~m}$ depth below centerline of wetted strip.

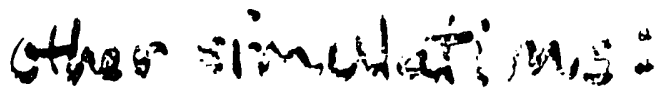

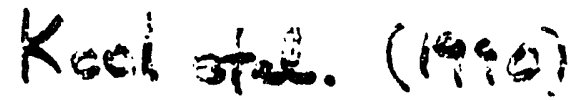

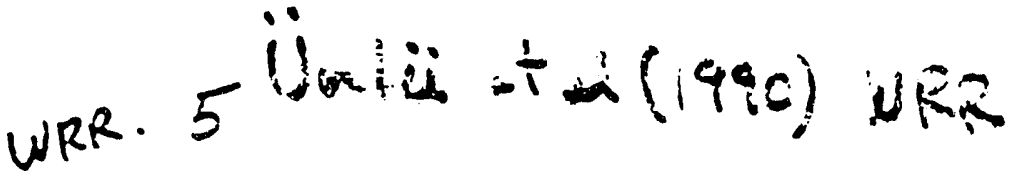


UNSATURATED FLOW EXPERIMENT

re Stephens \$ Herman (1288), WRR 24(5),770-778

NEW MEXICO TECH lab experiment

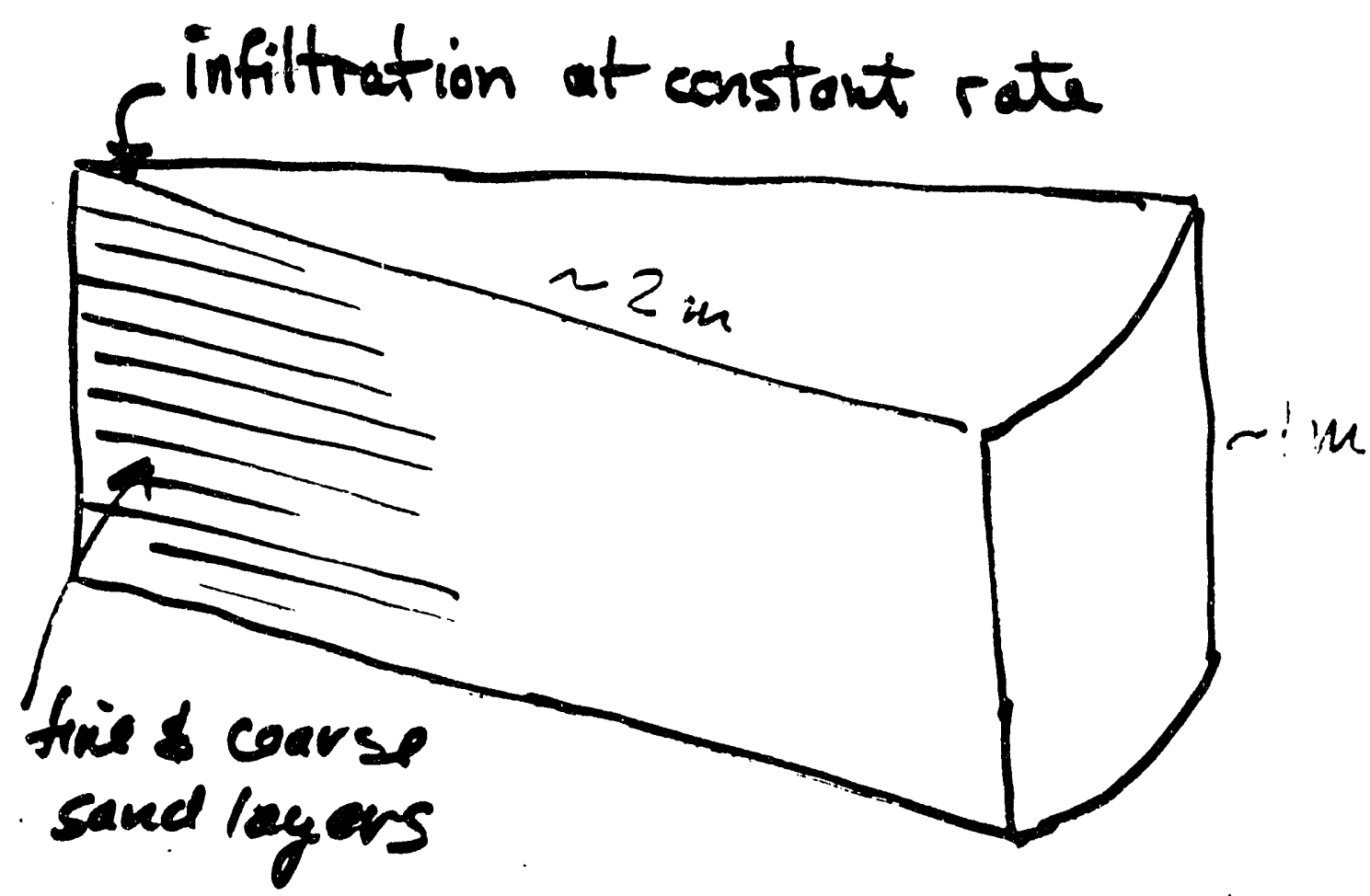

$\left.\begin{array}{ccc}\text { Slide } & \begin{array}{c}\text { Flow } \\ \text { rate } \\ \frac{m e / h r}{1}\end{array} & \frac{\text { tenie-hr }}{24} \\ 2 & 20 & 1 \\ 3 & 100 & 4 \\ 4 & 100 & 12 \\ 5 & 100 & 12\end{array}\right\} \sim$ same volume


WHC-MR-0420

$\sqrt{20}$

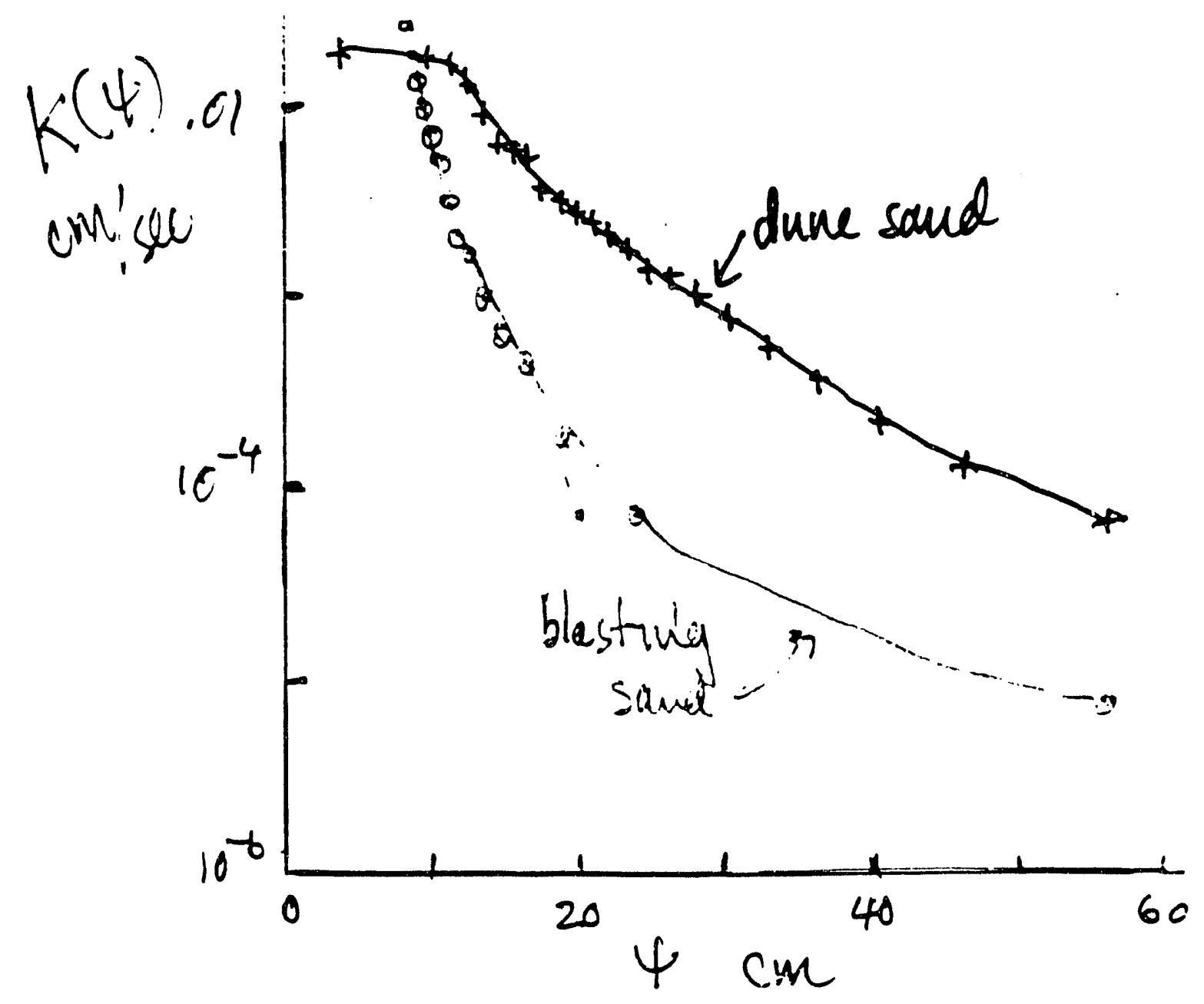

144 
WHC-MR-0420

\title{
LABORATORY MEASUREMENT OF HYDROLOGIC PROPERTIES
}

J.F. Relyea, H.L. Benny and R.T. Mcintosh

\section{GEOTECHNICAL ENGINEERING} LABORATORY

\author{
PARTICLE SIZE DISTRIBUTION \\ Sieves $(1 \overrightarrow{0} \mathrm{~cm}$ to $.0075 \mathrm{~cm})$ \\ Hydrometer $(.0034 \mathrm{~cm}$ to $.0001 \mathrm{~cm})$ \\ Calcium Carbonate (AVG $=0.7 \%$; \\ STDEV $=1.2 \%$
}

Cementation by $\mathrm{CaCO}_{3}$ is found in the fine fraction (less than $0.2 \mathrm{~cm}$ ) of nearly all samples. Particle size data is probably not useful for estimating moisture retention curve without removal of carbonate.

\section{POROSITY \\ Bulk Density}

Measured on oyen dry soil and volume of sample sleeve filled by sample.

Specific Gravity / Grain Density Measured on fine and course fraction. Average llsed for calculation of porosity. 


\section{MOISTURE CONTENT}

Moisture content is measured on sainples as received from the lield. Some are stored in the field for several weeks to months before testing and dry substantially. Others are soaked by melting ice hefore reaching the lab.

\section{SATURATED HYDRAULIC CONDUCTIVITY}

- Saturated hydraulic conductivity data are measured on both intact (as received) and recompacted samples (specified bulk density if required).

- Samples range from 1 " to 4 " diameter. Length is limited by length of all thread-available

- Head is usually supplied by a water column or by air pressure over water for more impermeable samples

\section{SATURATED HYDRAULIC CONDUCTIVITY}

- Tests methods are (1) Falling head, (2) Constant head and (3) Falling head. Rising tail.

- Falling head used for more permeable samples: down to $1 \mathrm{E}-4 \mathrm{~cm} / \mathrm{sec}$.

o Constant head from 1 E.4 down to 1 E.A ran sers.

- Falling head . Rising tail used for least permeable samples: $1 E \cdot 3$ down to $1 E \cdot 10 \mathrm{~cm} / \mathrm{sec}$.

o Ranges overiap an order of magnitude or inore. 


\section{SATURATED HYDRAULIC CONDUCTIVITY}

- Pressure must be controlled to prevent channeling

- Filter paper or screen needed to prevent small particle movement..

- Conductivity measured on empty apparatus to determine head loss of equipment. Needed to correct values for high permeability samples.

o Have flexible wall permeameter capability

\section{MOISTURE RETENTION CURVE}

- Moisture retention curves (soil water content versus matric potential) are measured on the fine fraction (less than $0.2 \mathrm{~cm}$ ).

- Data generated is on an oven dry weight basis and converted to volumetric by inultiplying by the sample bulk density.

- Kettle and CX-2 drying curves are matched to the Tempe drying curve by multiplying the curves by the Tempe Cell bulk density.

\section{MOISTURE RETENTION CURVE}

TEMPE CELL .

- Wetting and drying curves are ineasured between 0 and 1 bar. removing the sample and 13 after cleaning the cell for reuse.

- Moisture content is measured on sample 11 after filling the cell and $(2$ after the run is terminated.

D The moisture content of the final sample is matched to that of the sample at the last measured data point. 


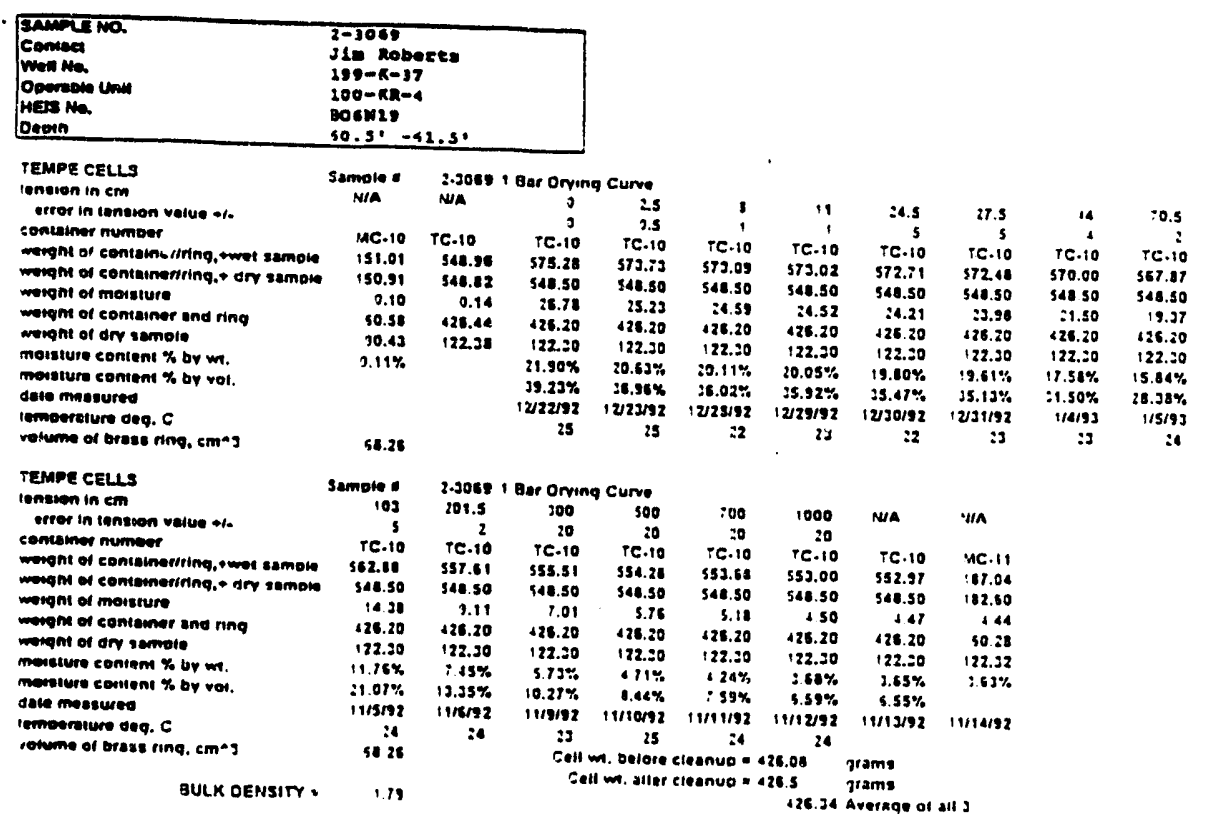

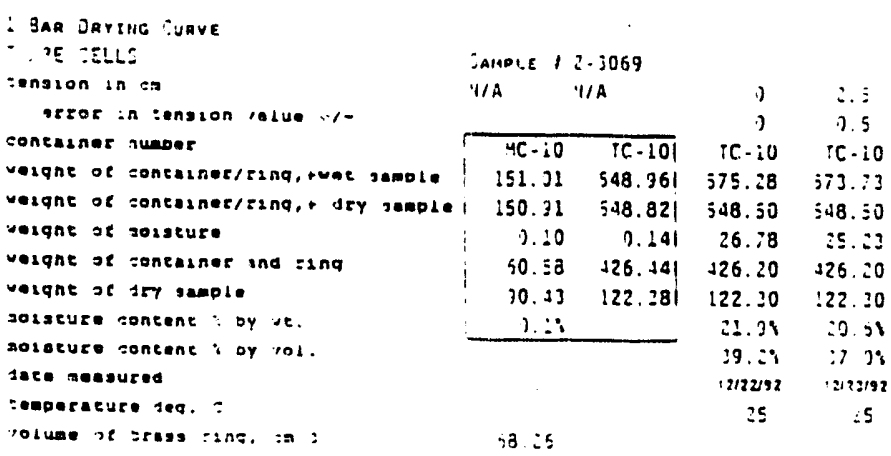

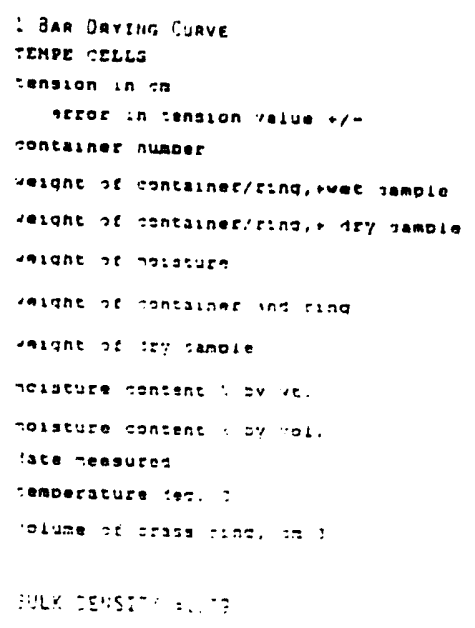

\begin{tabular}{|c|c|c|c|}
\hline \multicolumn{4}{|c|}{$\begin{array}{c}\text { AMPLE : } \\
\text { IOD } \quad 1069 \\
\quad 1000\end{array}$} \\
\hline 20 & 20 & & \\
\hline$: C \cdot: 0$ & $i c-101$ & $i c-i 0$ & ${ }^{\circ}$ “. - i!! \\
\hline$\$ 53.58$ & 533.201 & 352.37 & $: 87 \quad 341$ \\
\hline $5: 8 \quad \vdots 0$ & $\$ 48.501$ & $\$ 48.50$ & $: 32 \quad 201$ \\
\hline$\vdots .3$ & $2 \leq 01$ & 1.17 & ו \\
\hline$: 25.20$ & $: 26.201$ & $125: 9$ & 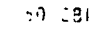 \\
\hline$: 22 .: 2$ & .22 .201 & $: 22 .: 0$ & $:: Z: Z !$ \\
\hline$: \because$ & $\therefore 91$ & ?. & $\therefore 5: 1$ \\
\hline$=1$ & $5.5 \% 1$ & 3. & \\
\hline 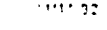 & $4: 12 / 921$ & - $1112: 92$ & $\cdots 18.921$ \\
\hline$\therefore$ & $: 9$ & & \\
\hline CELL - T & $: 25.28$ & $2 \pi .8145$ & \\
\hline$\therefore \ldots$ & $\begin{array}{r}: \div 5 \vdots \\
: \div 5: 2\end{array}$ & $\begin{array}{l}: \pi \wedge 115 \\
\because \cdots+2 \geq n\end{array}$ & \\
\hline
\end{tabular}




\section{PRESSURE KETTLE}

- Data points are measured from 0.5 to 10 bars in kettles.

- Volumetric moisture contents are obtained by multiplying the moisture content (weight \%) by Tempe cell bulk density.

- Data points are rreasured in du $r$ licate.

\section{MOISTURE RETENTION CURVE}

CX-2

- The CX.2 measures the water activity (relative humidityl of a sample to $0.1 \%$. This corresponds to matric potentials from 5 to $>1000$ bars.

- The water activity is the total of matric and osmotic potentials. Salts must be leached from the sample before testing to measure the matric potential.

- Osmotic effects are not significant on well samples. Grout samples; however, are dominated by osmotic effects.

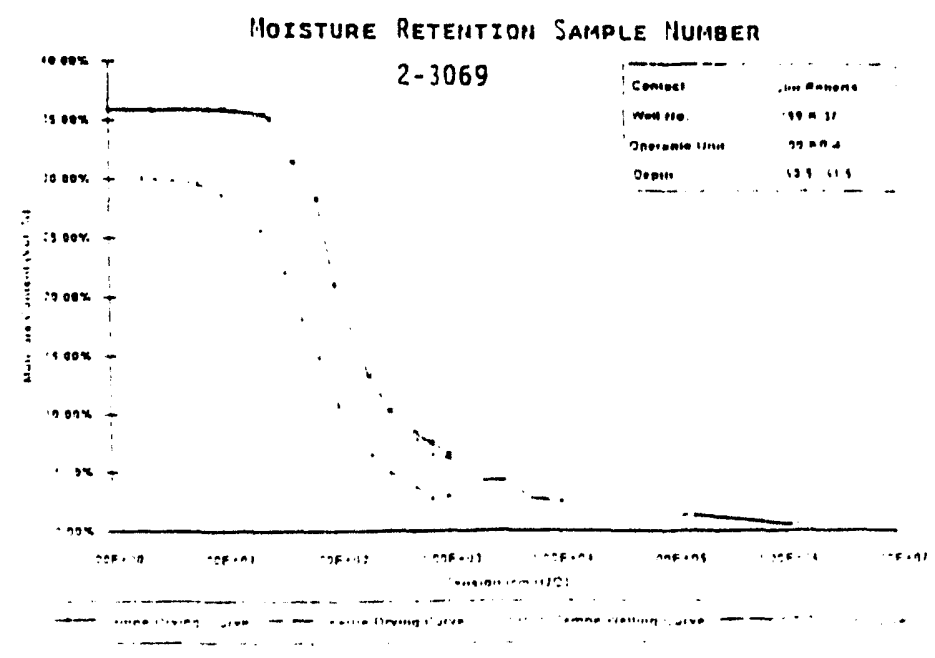




\section{UNSATURATED HYDRAULIC CONDUCTIVITY}

- Unsaturated hydraulic conductivity data are gathered on both intact and recompacted samples (usually $10 \mathrm{~cm}$ diameter by $15 \mathrm{~cm}$ longl.

- Samples are brought to specified matric potential using air pressure lirst. Then vacuum is applied to the top and bottom plates.

\section{UNSATURATED HYDRAULIC CONDUCTIVITY}

- Mariotte llask is used at low matric potential 10 to $-100 \mathrm{~cm})$ to maintain constant head. Large reservoir is used at higher matric potential, falling head not significant.

- Leaks in vacuum/pressure system are the biggest problem. Mariotte flasks at higher tensions are not practical. Any small leak leads to water entering the vacuum system.
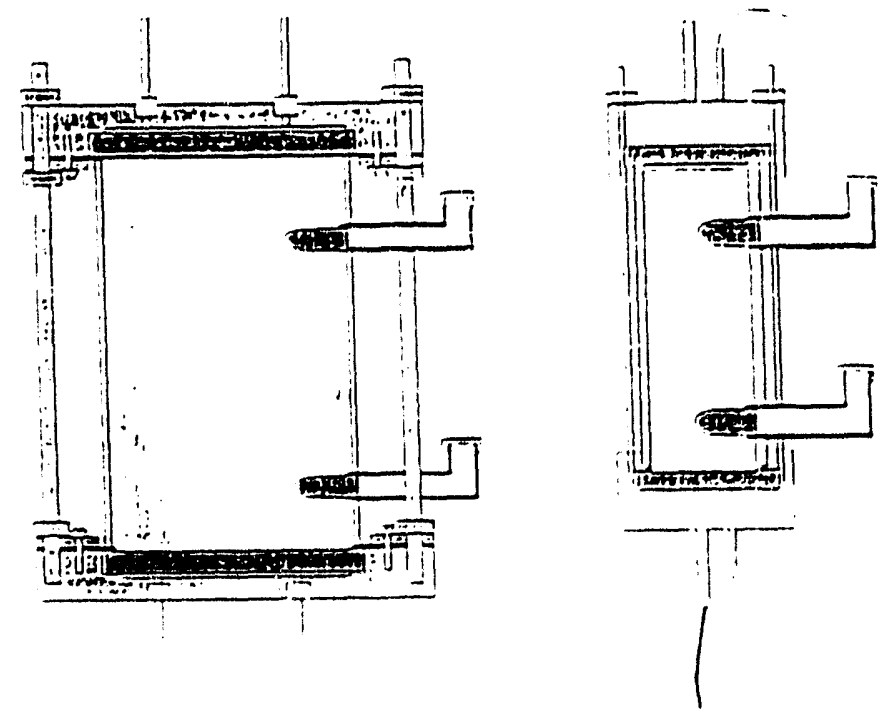
WHC-MR-0420

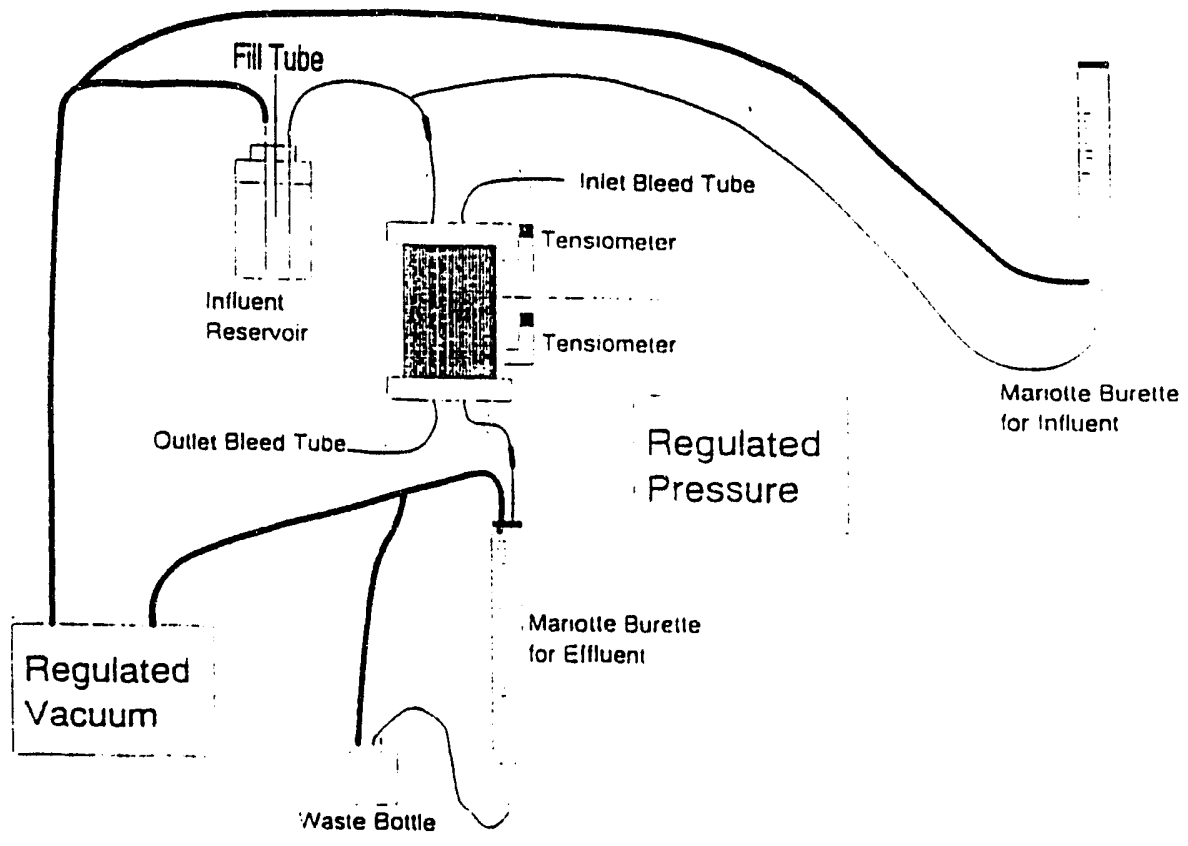

\subsection{6}

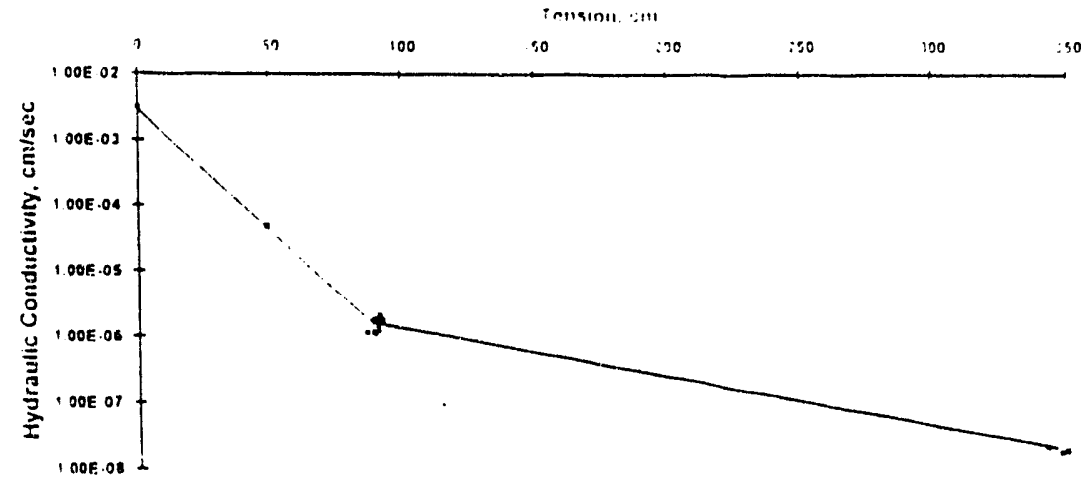

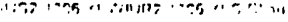

intent.e $2 \cdot 1306$

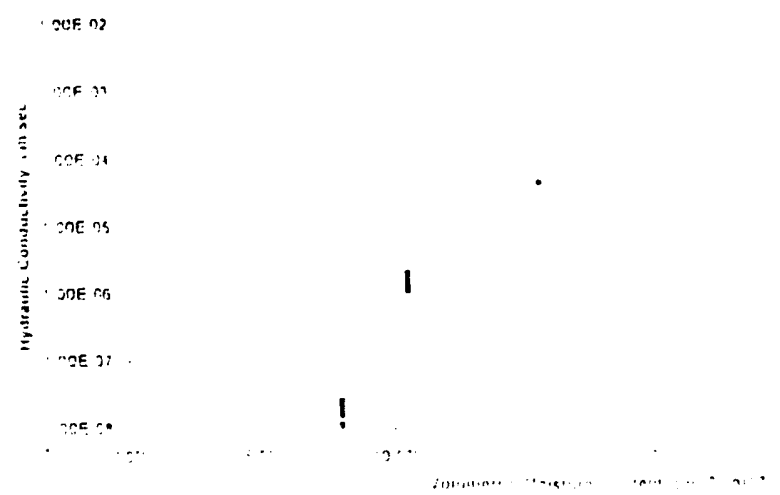


WHC-MR-0420

This page left intentionally blank. 


\section{Centrifuge Technology for Vadose Zone Transport Measurements}

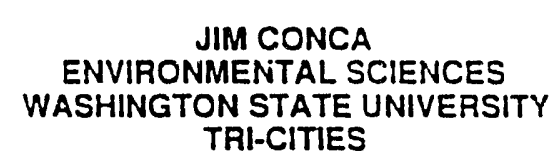

JIM CONCA WASHINGTON STATE UNIVERSITY TRI-CITIES

\author{
JUDITH WRIGHT \\ ENVIRONMENTAL MANAGEMENT \\ OPERATIONS \\ PACIFIC NORTHWEST LABORATORY
}

\section{The Primary Transport Parameters}

$\mathrm{K}$ - Hydraulic Conductivity (water permeability)

$$
q=K \cdot \nabla \psi
$$

water flux $=$ conductivity $\times$ pressure gradient

$D$ - Diffusion Coefficient (static diffusion of single ions)

$$
J=D \cdot \nabla \psi
$$

ion flux $=$ diffusion coefficient $\times$ concentration gradient

$R_{f}$ - Retardation Factor (Chemical sorption and exchanges!

$$
R_{f}=V_{g w} \div V_{c}
$$




\section{Limitations of Traditional Methods}

- Traditional methods require months to years to conduct experiments at the low water contents characteristic of arid sites.

- Estimations derived from data obtained under saturated conditions are difficult to support without sufficient unsaturated data.

- Traditional laboratory methods cannot test remediation strategies under low water contents and field conditions prior to field testing.

\section{UNSATURATED FLOW APPARATUS (UFA)}

- combines ultracentrifugation with precision fluid flow through a rotating seal

- attains hydraulic steady-state in hours instead of weeks to years at low water contents

- acceleration is a whole body force analogous to gravity (no physical changes in sample - no compaction, no density changes, no induced iracturing or grain rearrangements 
Some Advantages of the UFA

- Determines the transport behavior of soils and sediments for aqueous and non-aqueous phase fluids. These data can be used to develop remediation strategies and predict contaminant migration.

- Soil and sediment samples in the UFA ${ }^{\mathrm{TM}}$ reach hydraulic steady-state with any fluid in 1 to 10 hours over a range of saturated to highly unsaturated conditions.

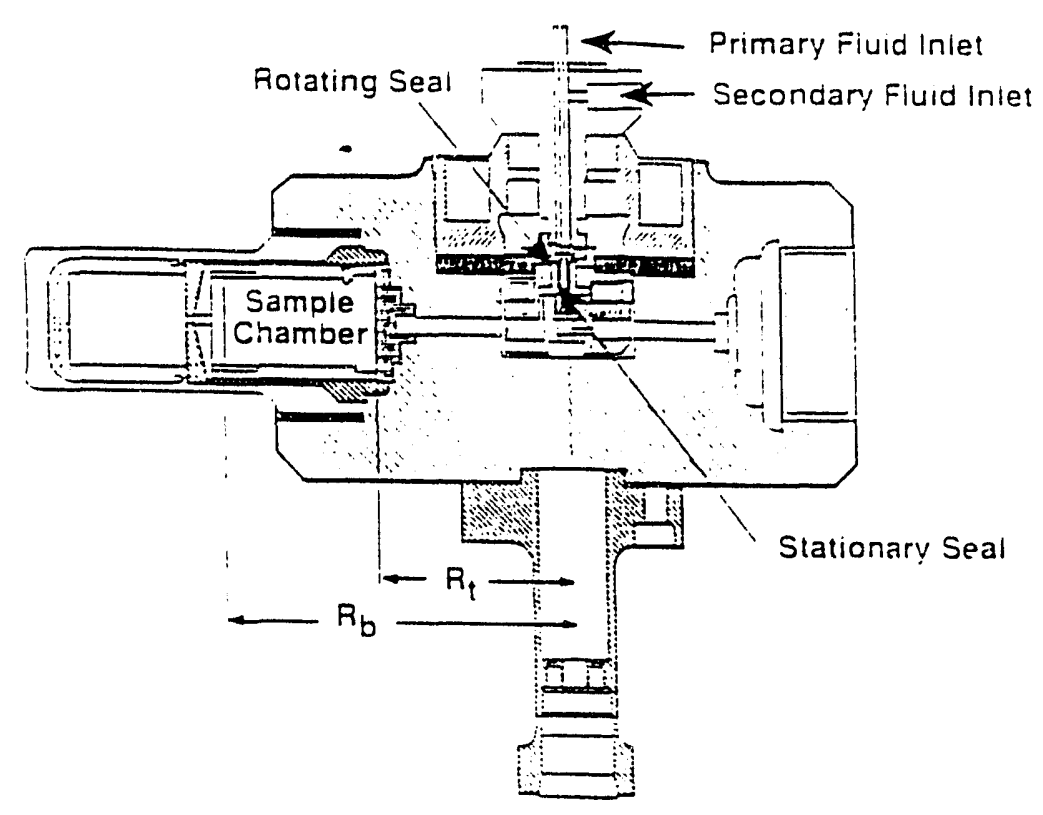

Eiane 1 The CFA Rotor and Seal Assemuis. 


\section{UNSATURATED FLOW APPARATUS (UFA)}

- multiphase/multicomponent capabilities

$.0^{\circ}$ to $150^{\circ} \mathrm{C}$

- flow rates to $0.1 \mathrm{ml} / \mathrm{hour}$

- accelerations up to $20,000 \mathrm{~g}$

After the water content is fixed in the sample and the material is at hydraulic steady-state, transport parameters can be determined from a variety of associated methods.

- Hydraulic conductivity can be determined from the driving force and flux.

- The electrical conductivity can be measured for determination of diffusion coefficients using the Nernst-Einstein equation.

- The effluent can be monitored/collected/analyzed for chemical changes, retardation effects, and determination of partitioning. 
Under a centripetal acceleration in which the water is driven by both the matric potential and the centrifugal force per unit volume, $\rho \omega^{2} r$, Darcy's Law is given by:

$$
q=-K(\psi)\left[d \psi / d r-\rho \omega^{2} r\right]
$$

where:

$$
\begin{aligned}
& q=\text { flux density } \\
& \mathbf{K}=\text { hydraulic conductivity } \\
& \psi=\text { matric potential } \\
& \rho=\text { bulk density } \\
& \omega=\text { rotation speed } \\
& r=\text { radial distance from axis of rotation }
\end{aligned}
$$

At speeds above about $300 \mathrm{rpm}$, provided that a sufficient flux density exists,

$$
\text { d } \psi / d r \ll \rho \omega^{2} r
$$

and Darcy's Law reduces to:

$$
K(\psi)=q / \rho \omega^{2} \mathbf{r}
$$

As an example, a 45-gram sample of a silt accelerated to $2,000 \mathrm{rpm}$ with a flow rate into the silt of $3 \mathrm{ml} / \mathrm{hr}$ achieved hydraulic steady state in two hours at the target volumetric water content of $22.4 \%$ and an unsaturated hydraulic conductivity of $2.5 \times 10^{-7} \mathrm{~cm} / \mathrm{s}$. 
Darcy's Law is a bulk property and does not depend upon the type of driving force

- Positive Pressure Gradient (High Pressure Cells)

- Negative Matric Potential (Weirenga Cell)

- Gravitational Acceleration (Soil Columns)

- Centripetal Acceleration (UFA ${ }^{T M}$ )

- Miagnetic/Electric Potentials (Magstream)
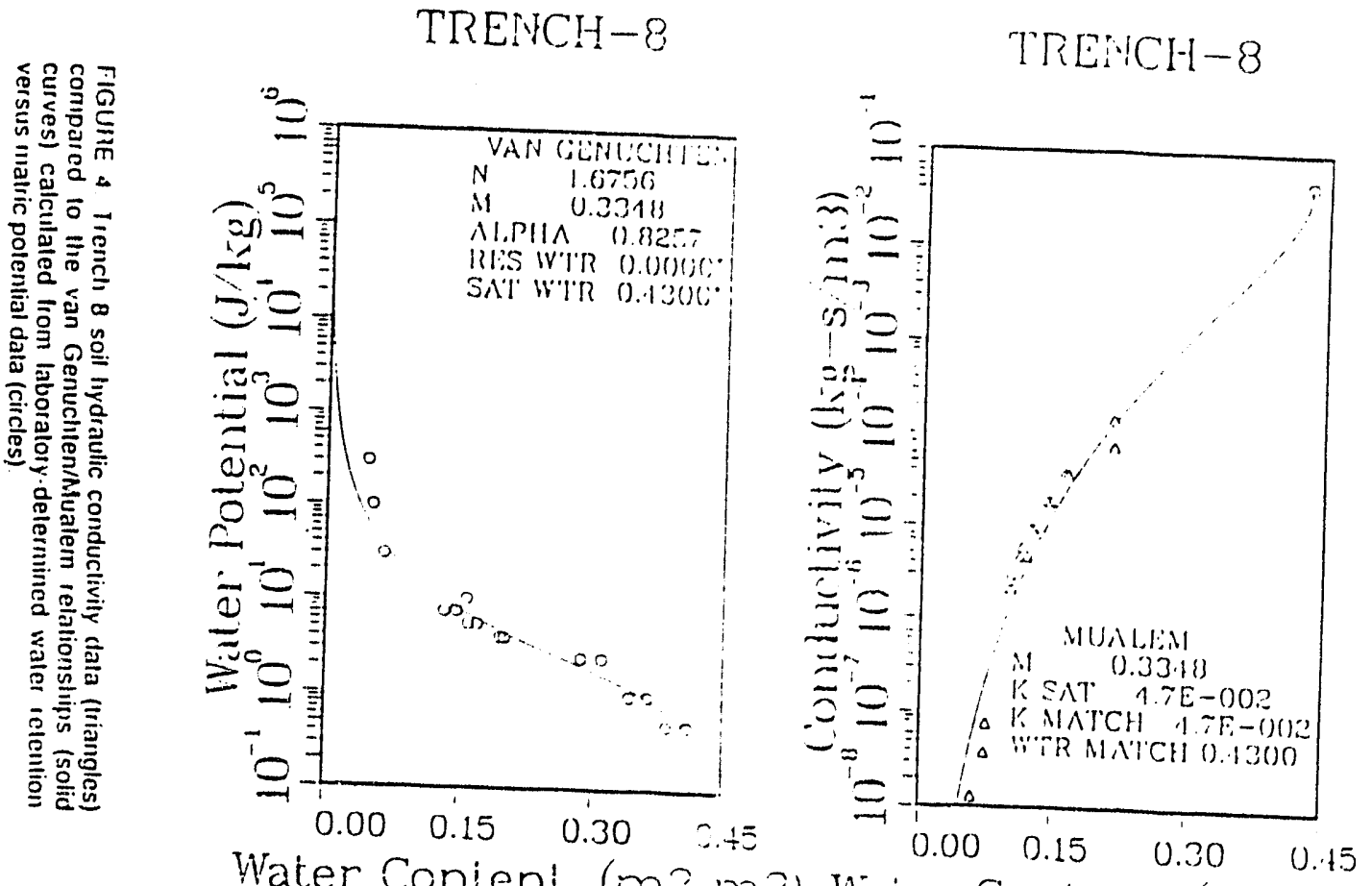
The following figure compares the UFA centrifuge method with three traditional techniques for determining hydraulic conductivity for soil from the Buried waste Test Facility at Hanford. Open squares are direct measurements of hydraulic conductivity using the UFA under unitgradient conditions obtained in a three-day period. Open triangles are direct measurements of hydraulic conductivity using a traditional column flow experiment under unit-gradient conditions, requiring a total experimental time of more than a year. The closed circle is the 13-year field lysimeter data obtained under unit-gradient conditions at water contents in the field, a point which ties the laboratory data to the field conditions. The solid line is an estimation derived by curve-fitting to water retention data using a Mualem relationship.

The agreement of the UFA data with all three methods is excellent and demonstrates the validity and rapidity of the UFA method relative to traditional methods.

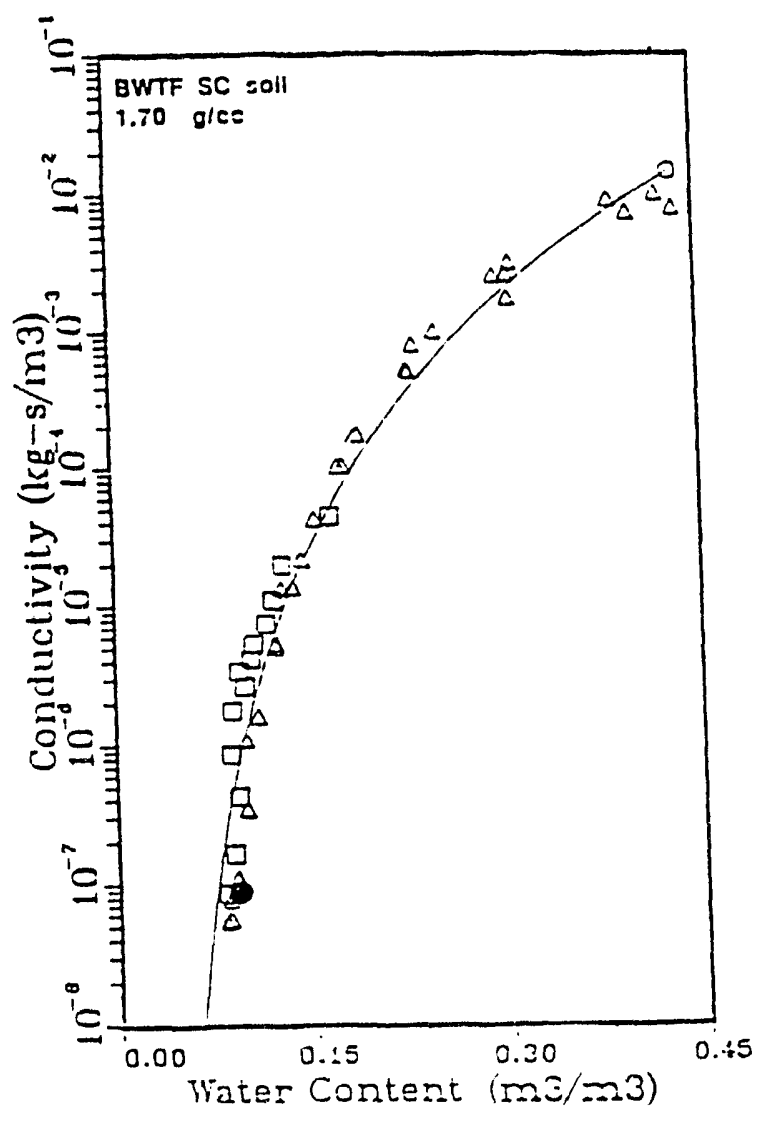

UFA $^{\text {mm }}$ measuremen:s made over 3-days

$\triangle$ Traditional column experiments mace over 1-year

- Average of fleld lysimeter measurcments made over 13-years

- Mualem estimation derlved prom curve-flited water-retention data mage over 5-y ? soys 


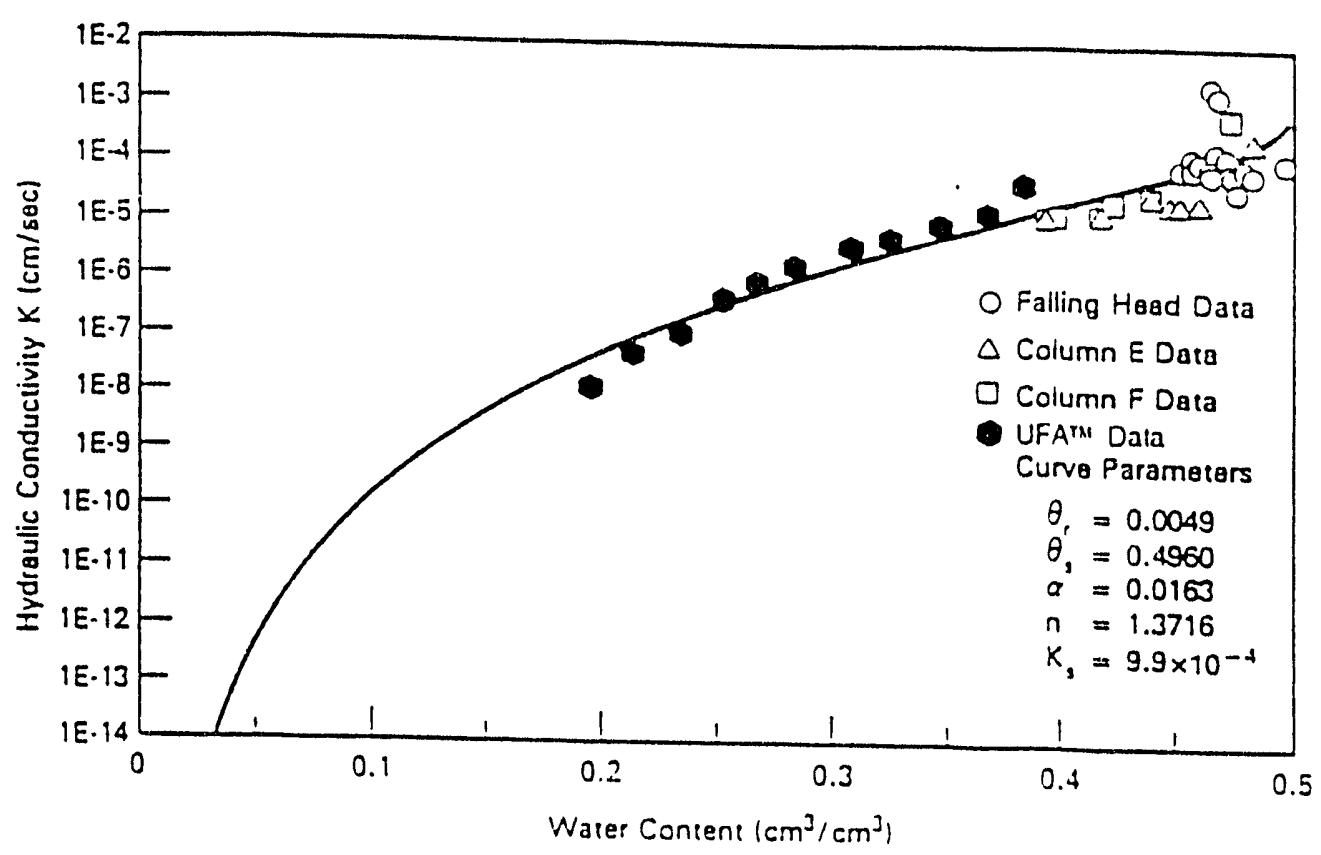

EigURE A.3. Heasures Hydraulic Conductirity Oata and lan Genuciten Function Fit for Flí joll

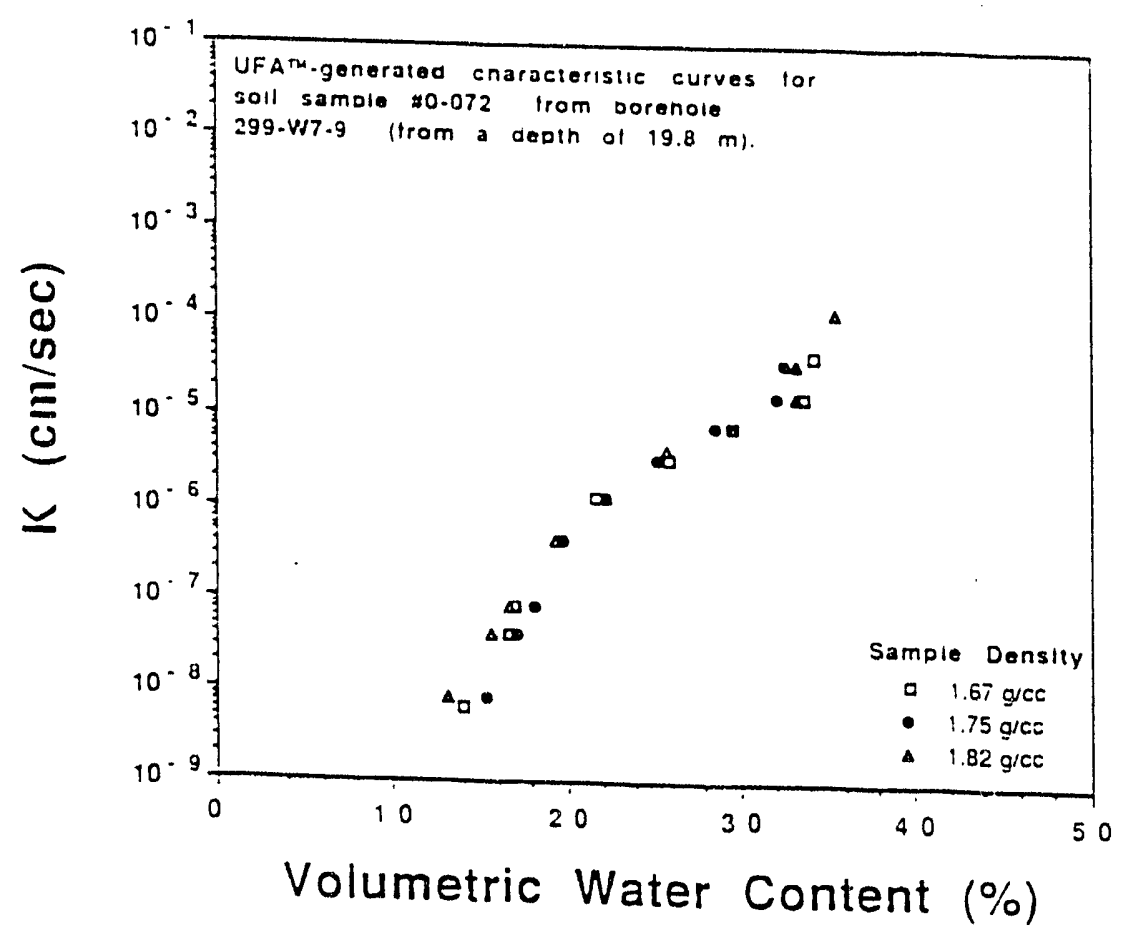


WHC-MR-0420

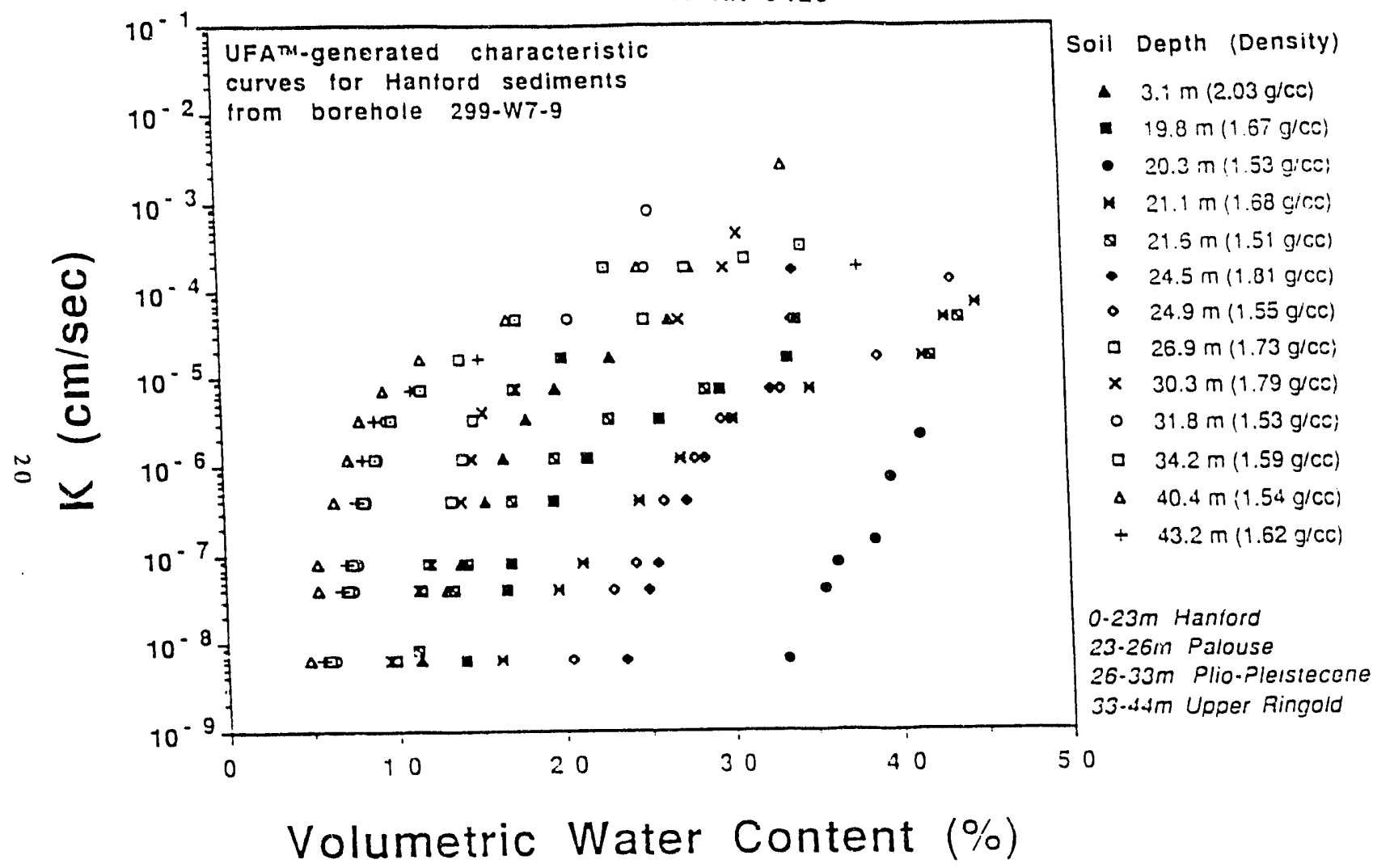

Figure 12

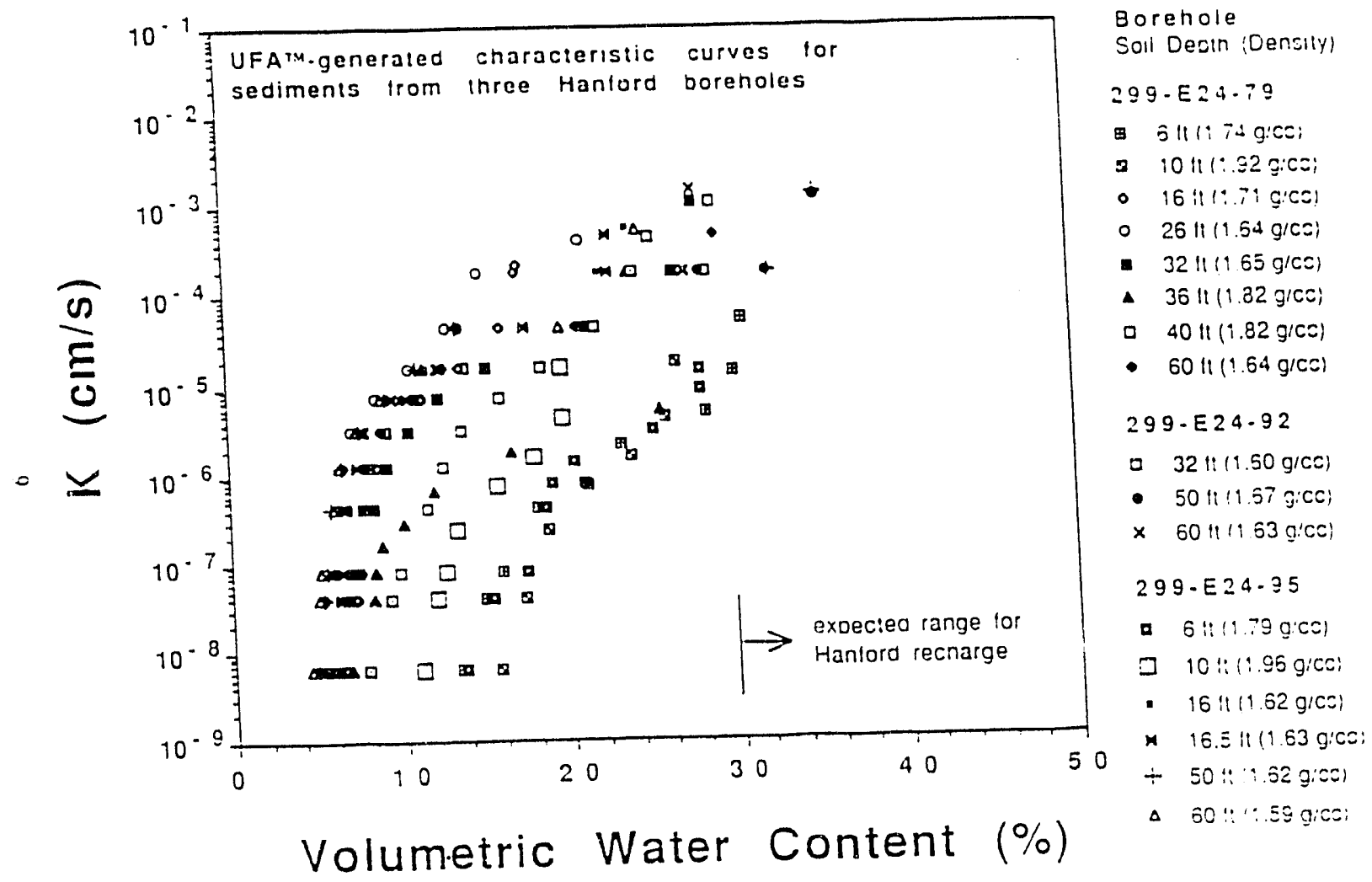

Figure $=$ 


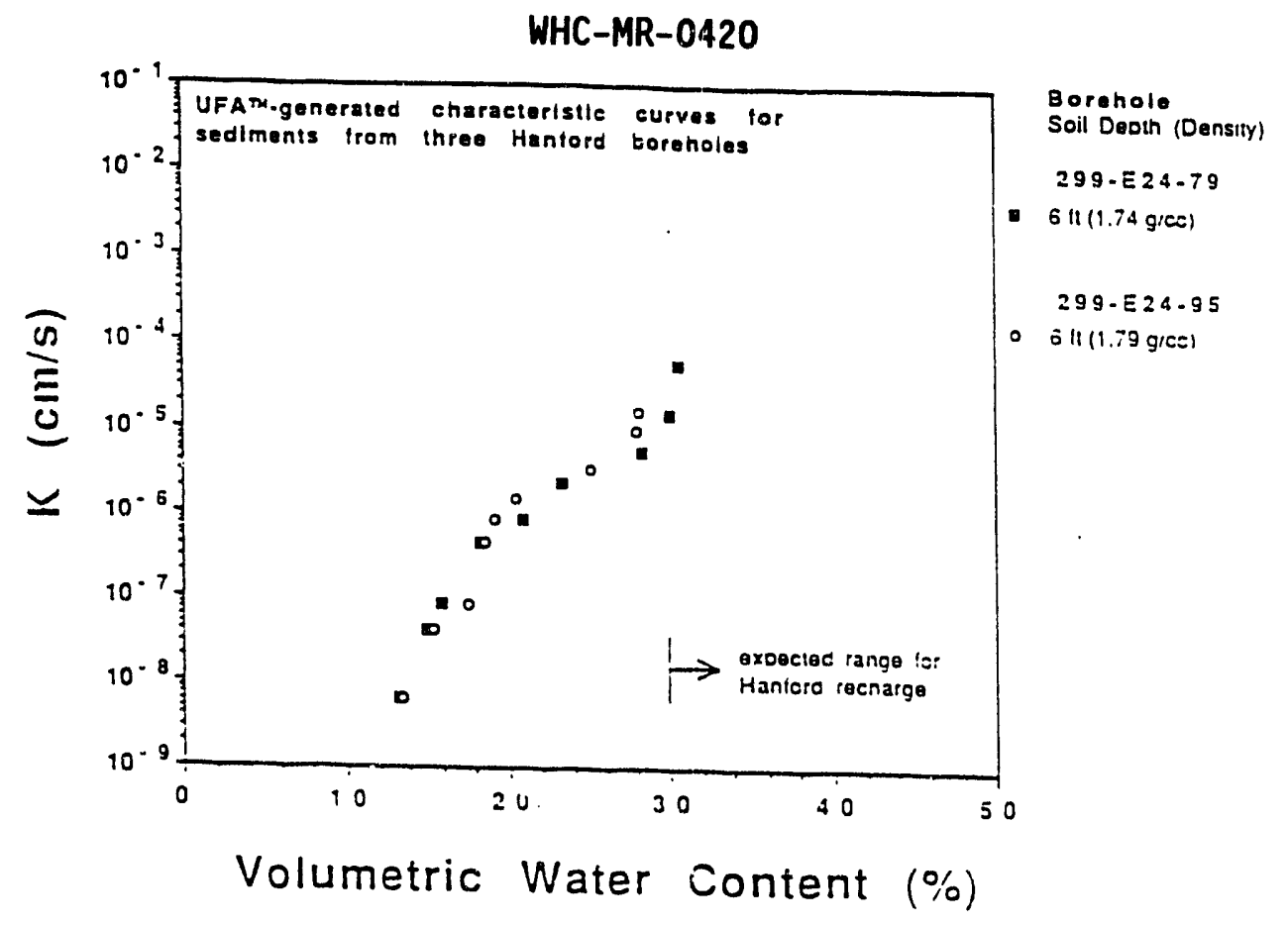

Figure 6

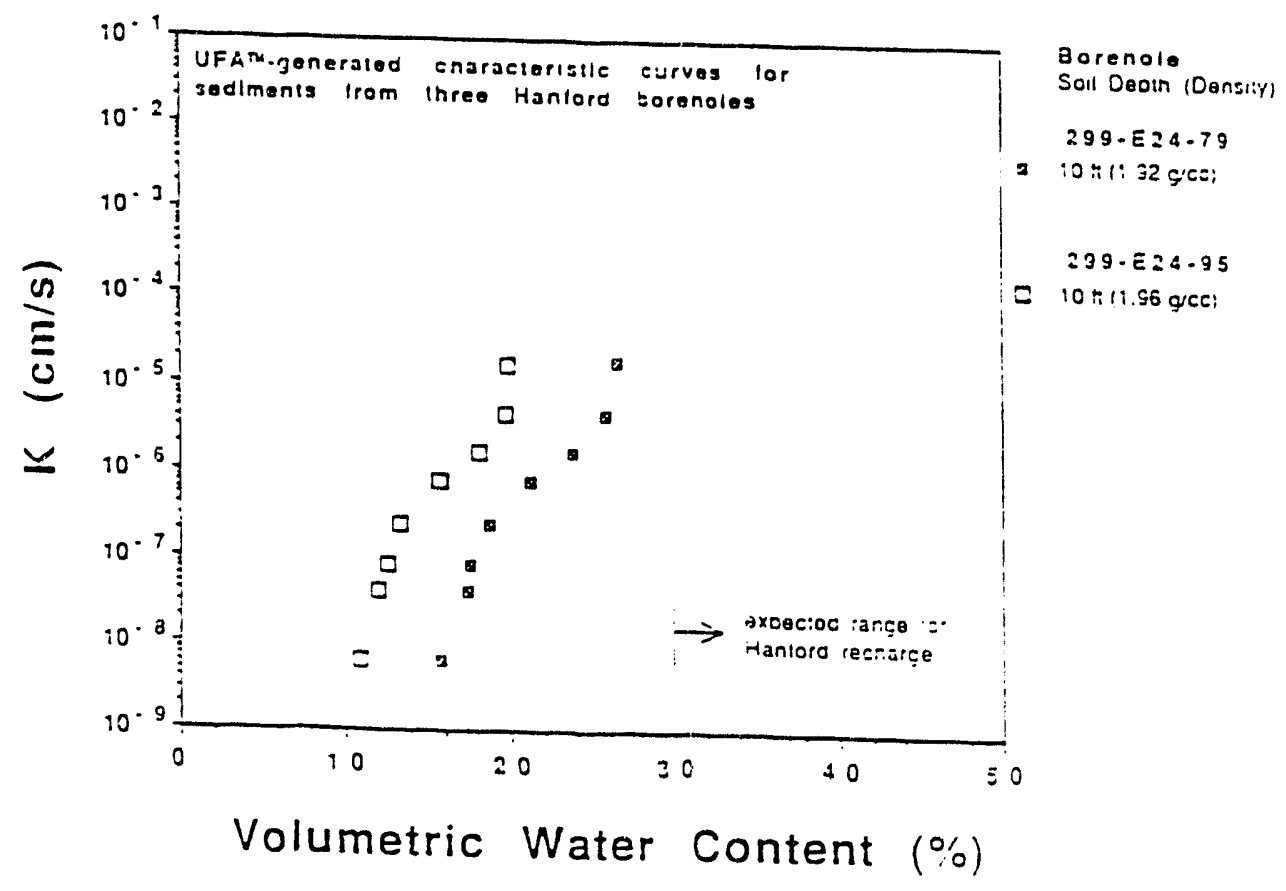

Figure 7 
WHC-MR-0420

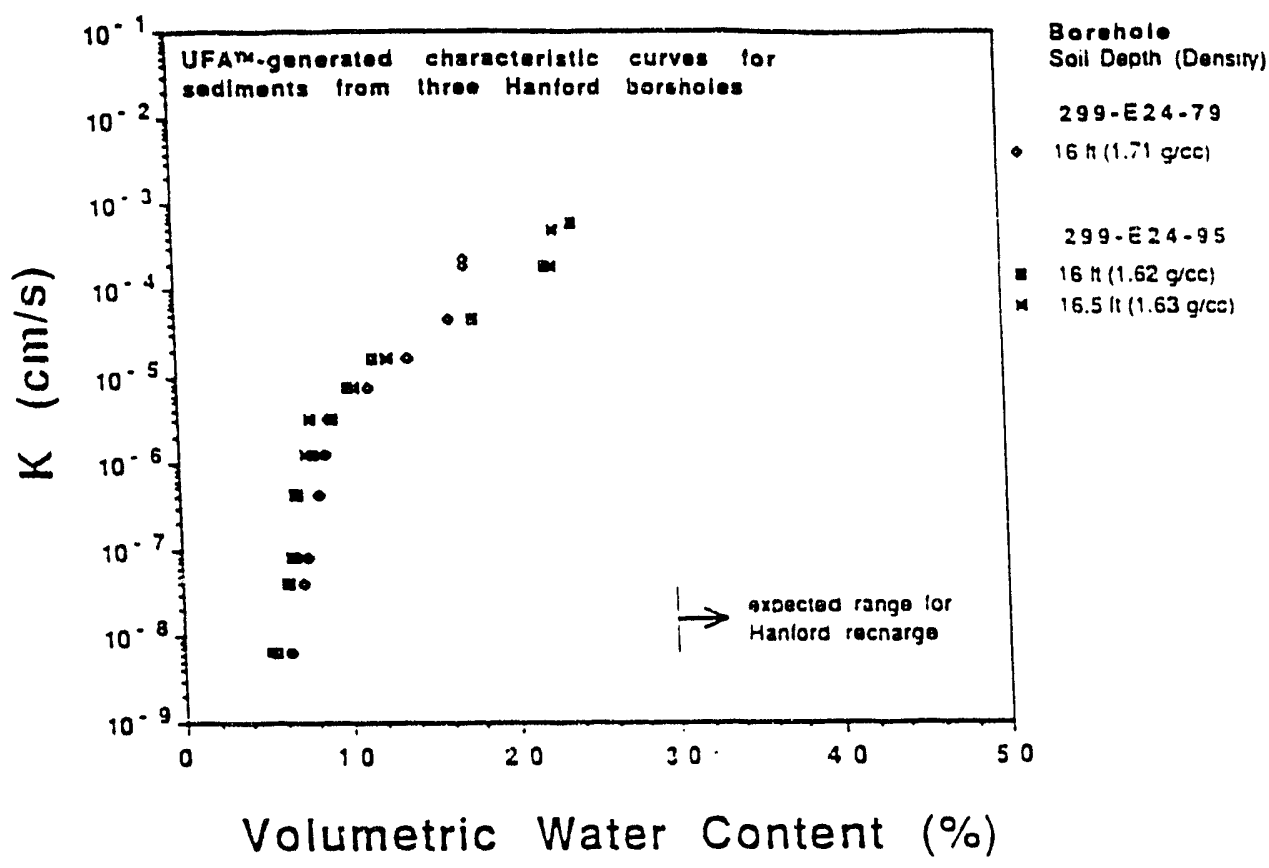

Figure 3

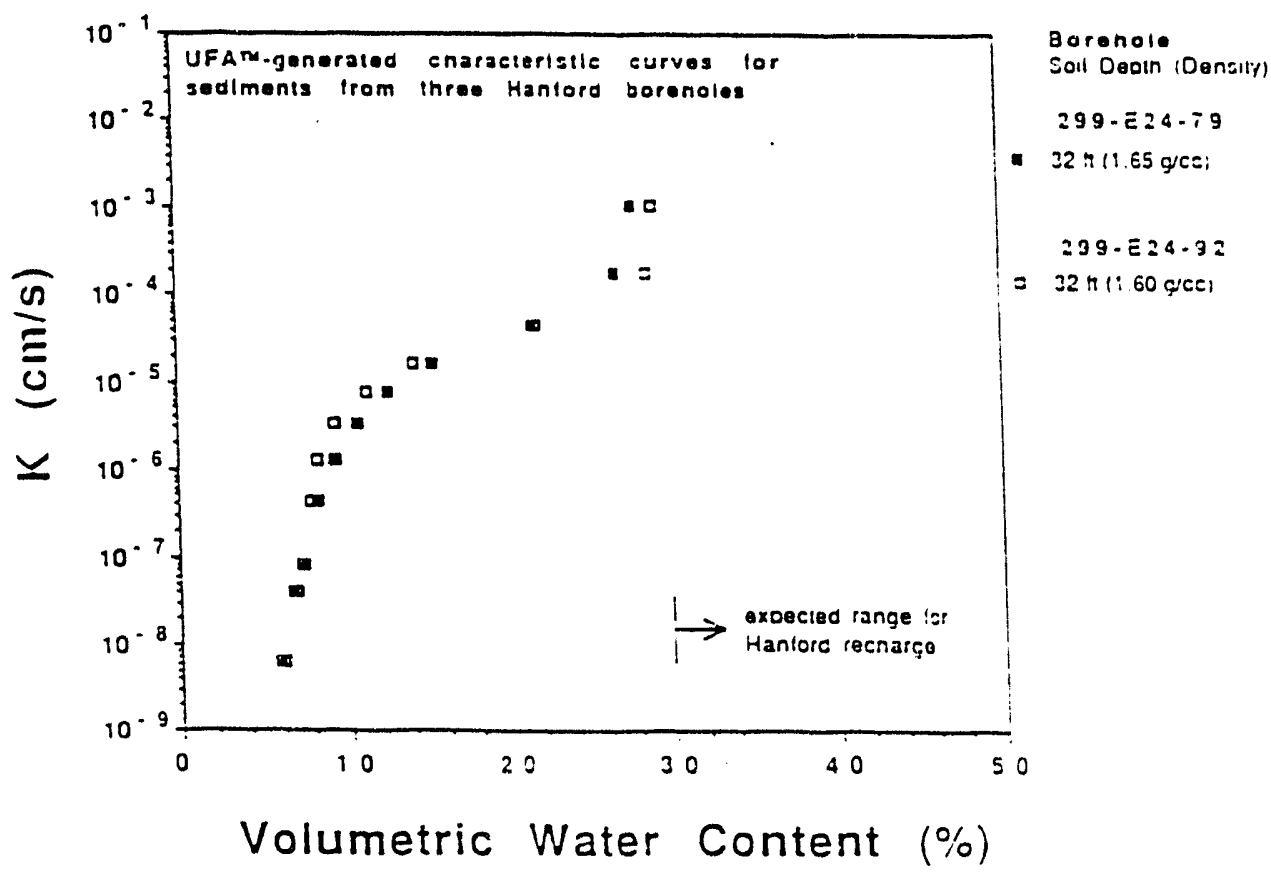

Figure 
WHC-MR-0420

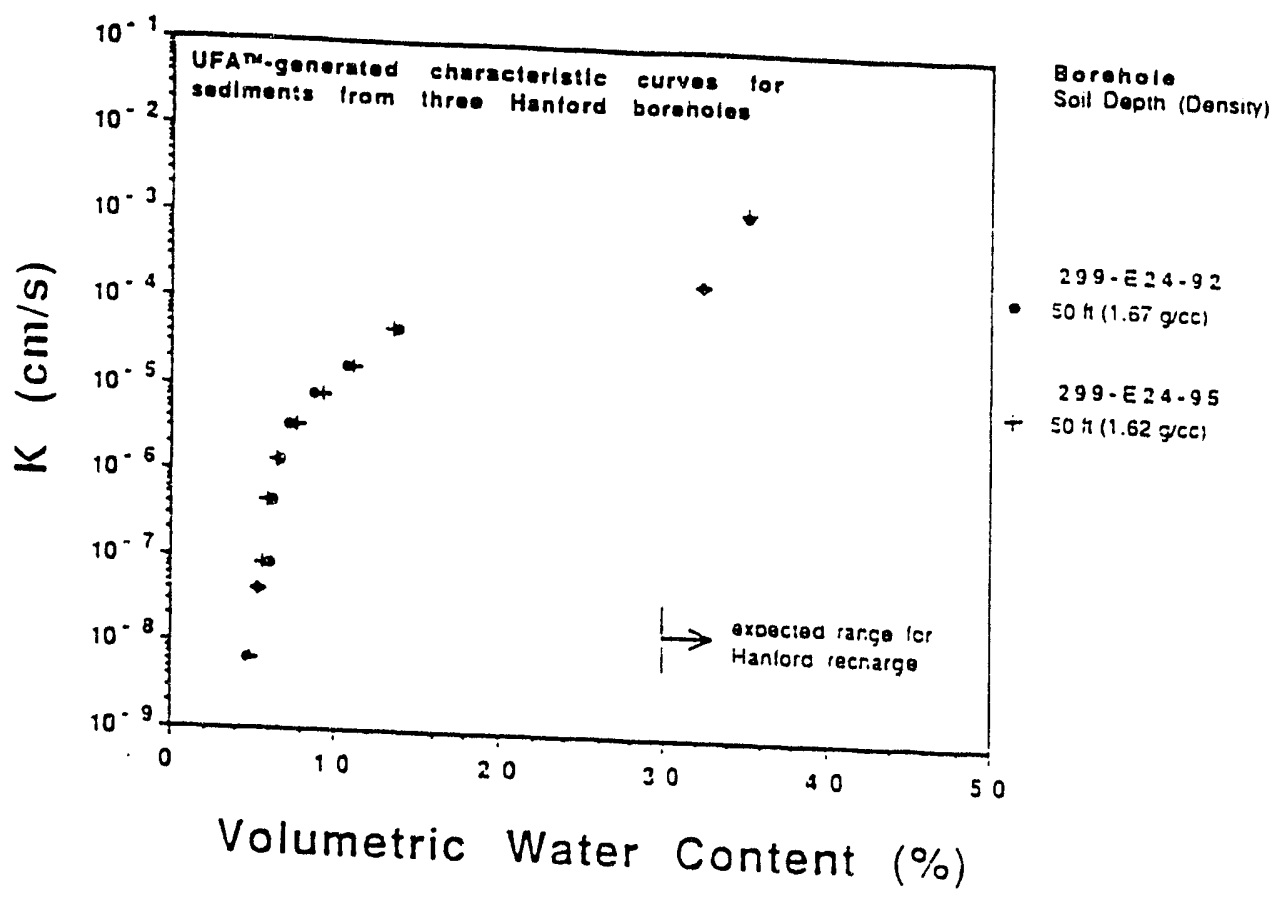

Figure 10

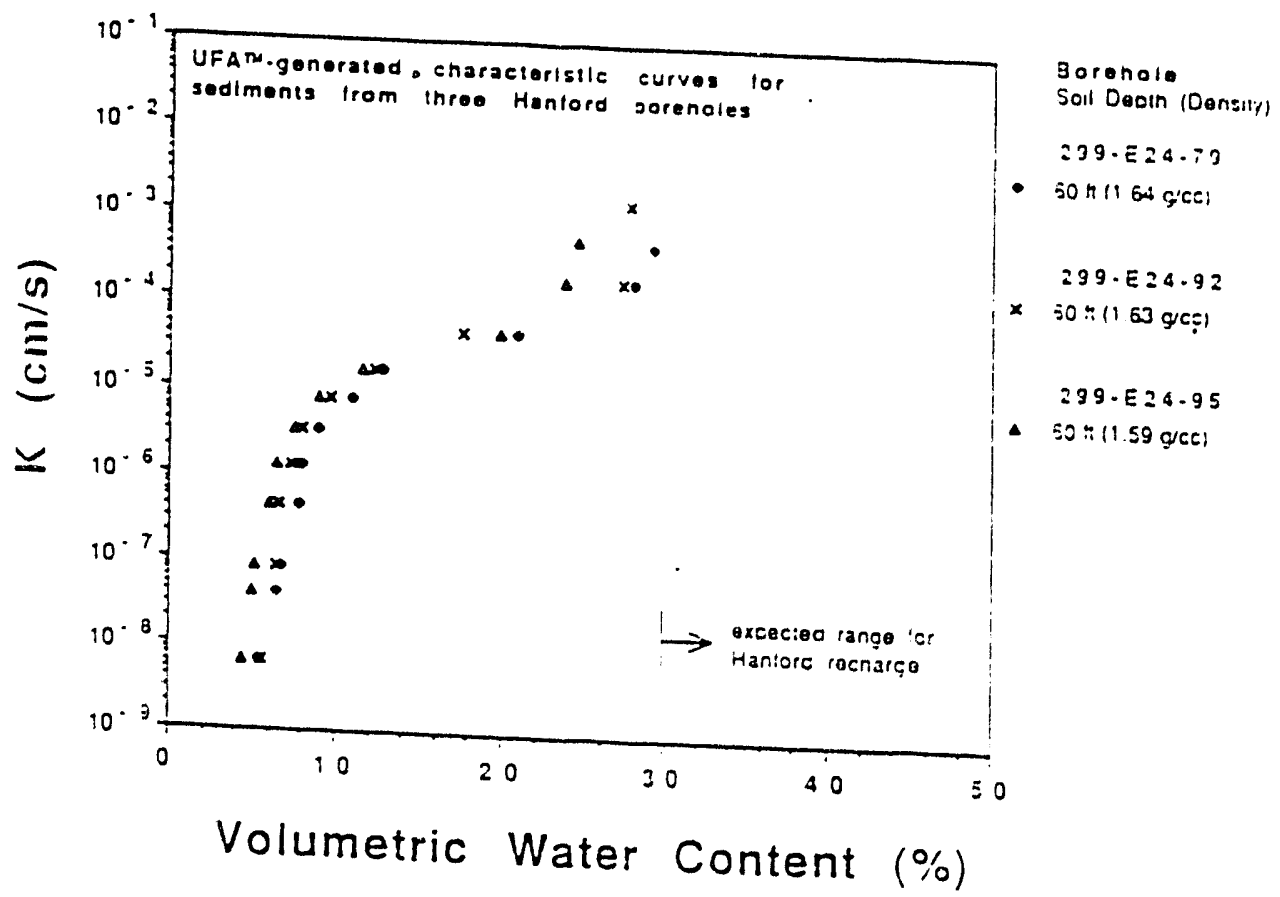

Figure is 

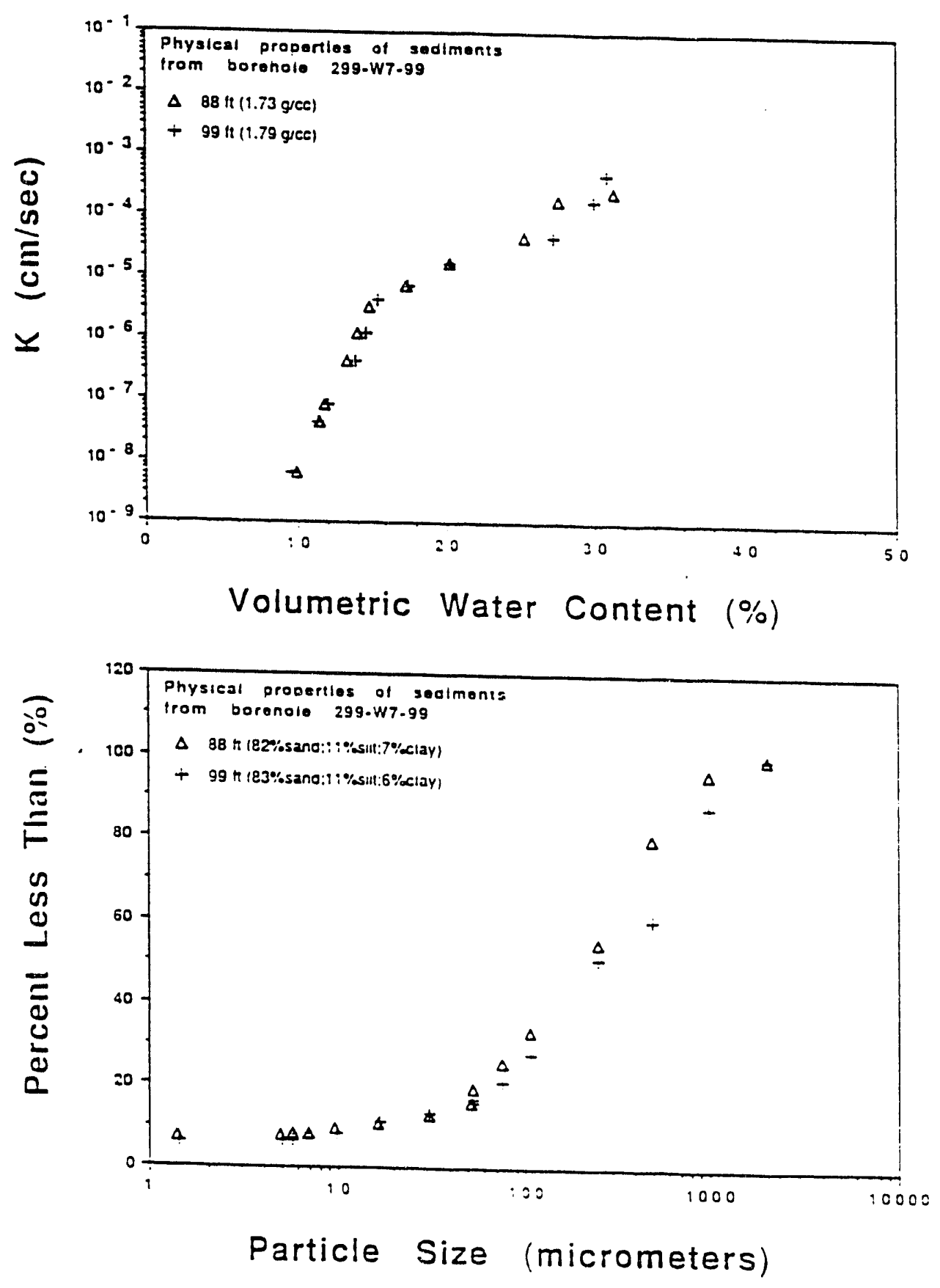

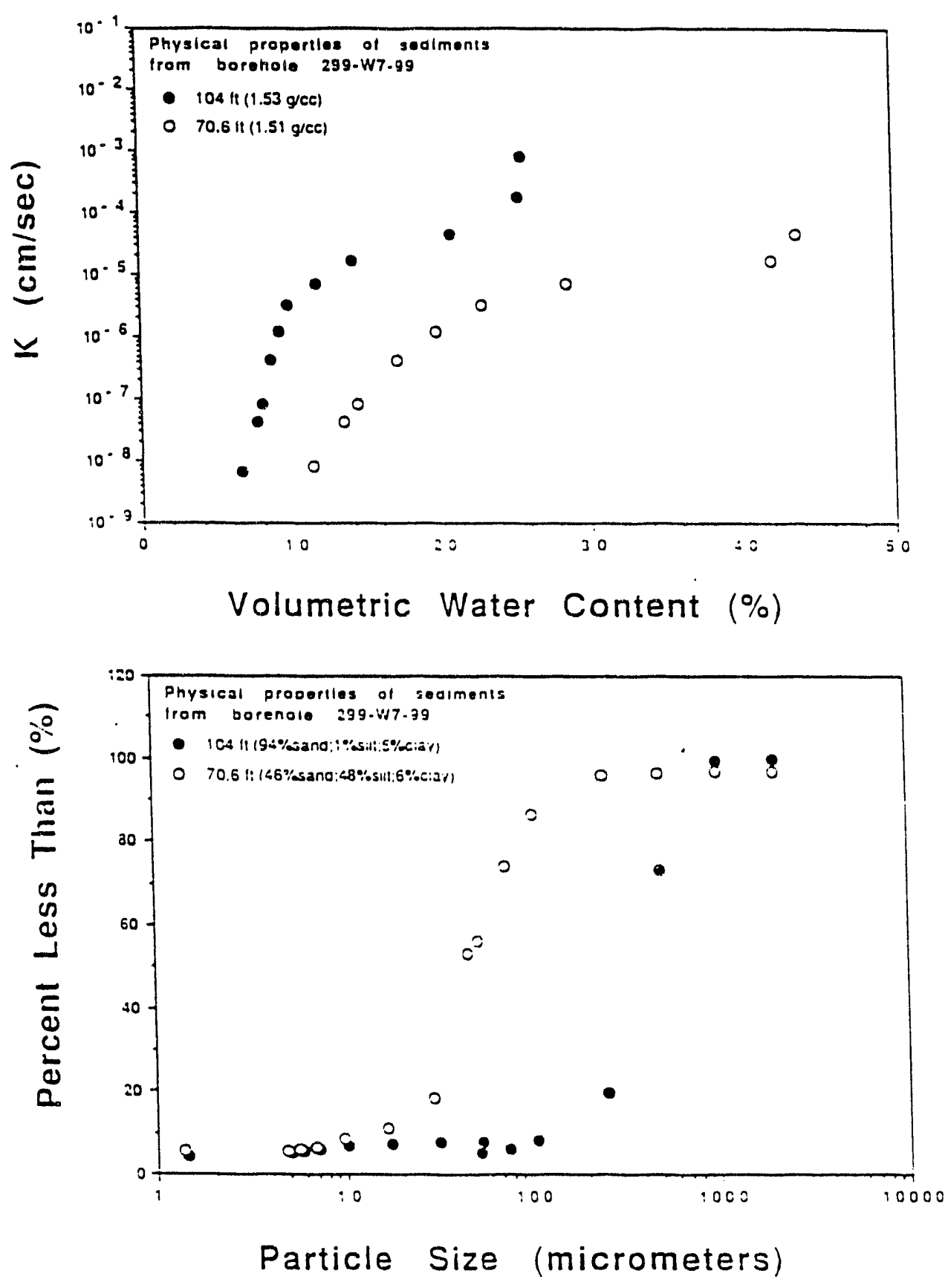
WHC-MR-0420
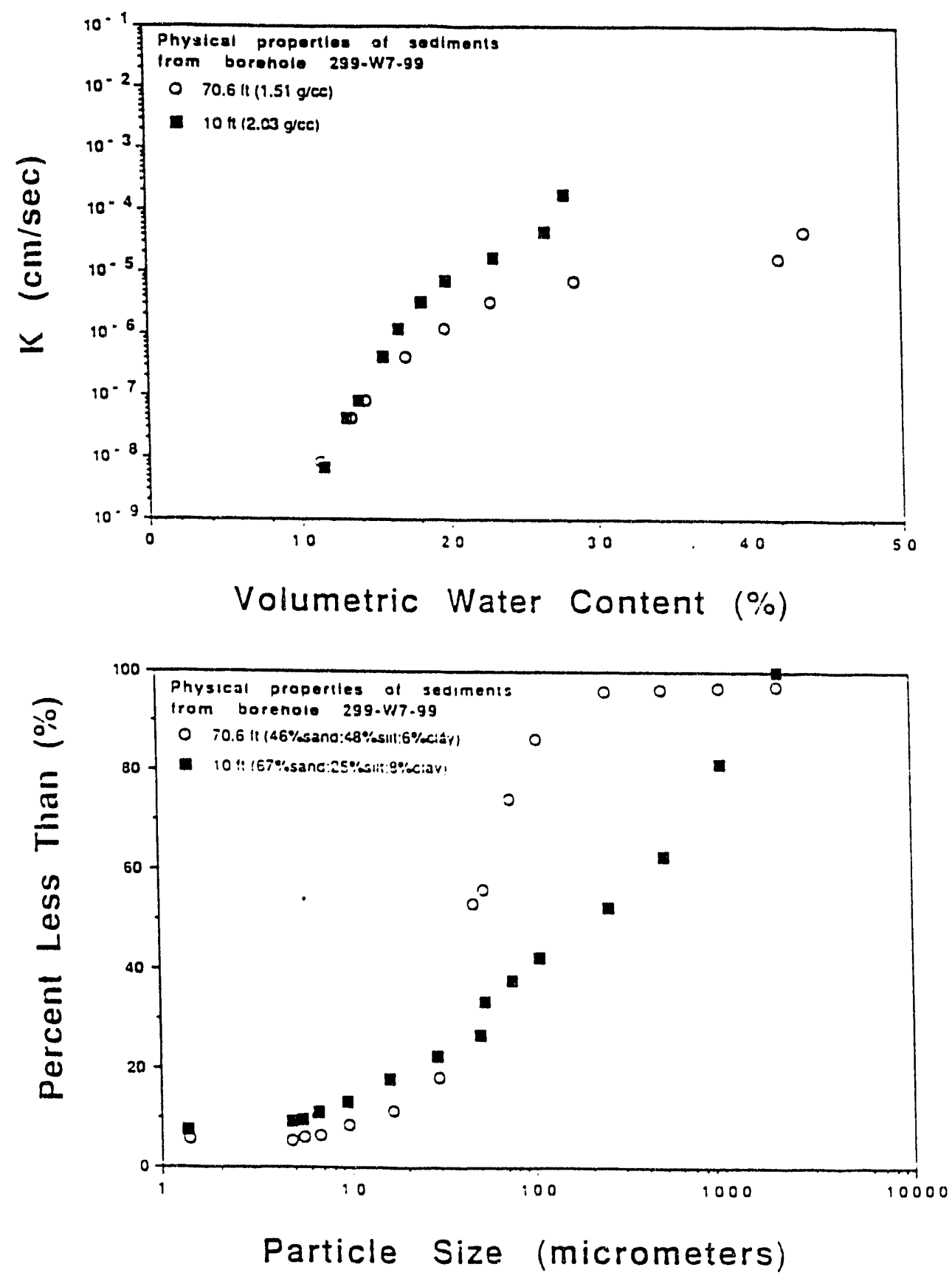
WHC-MR-0420
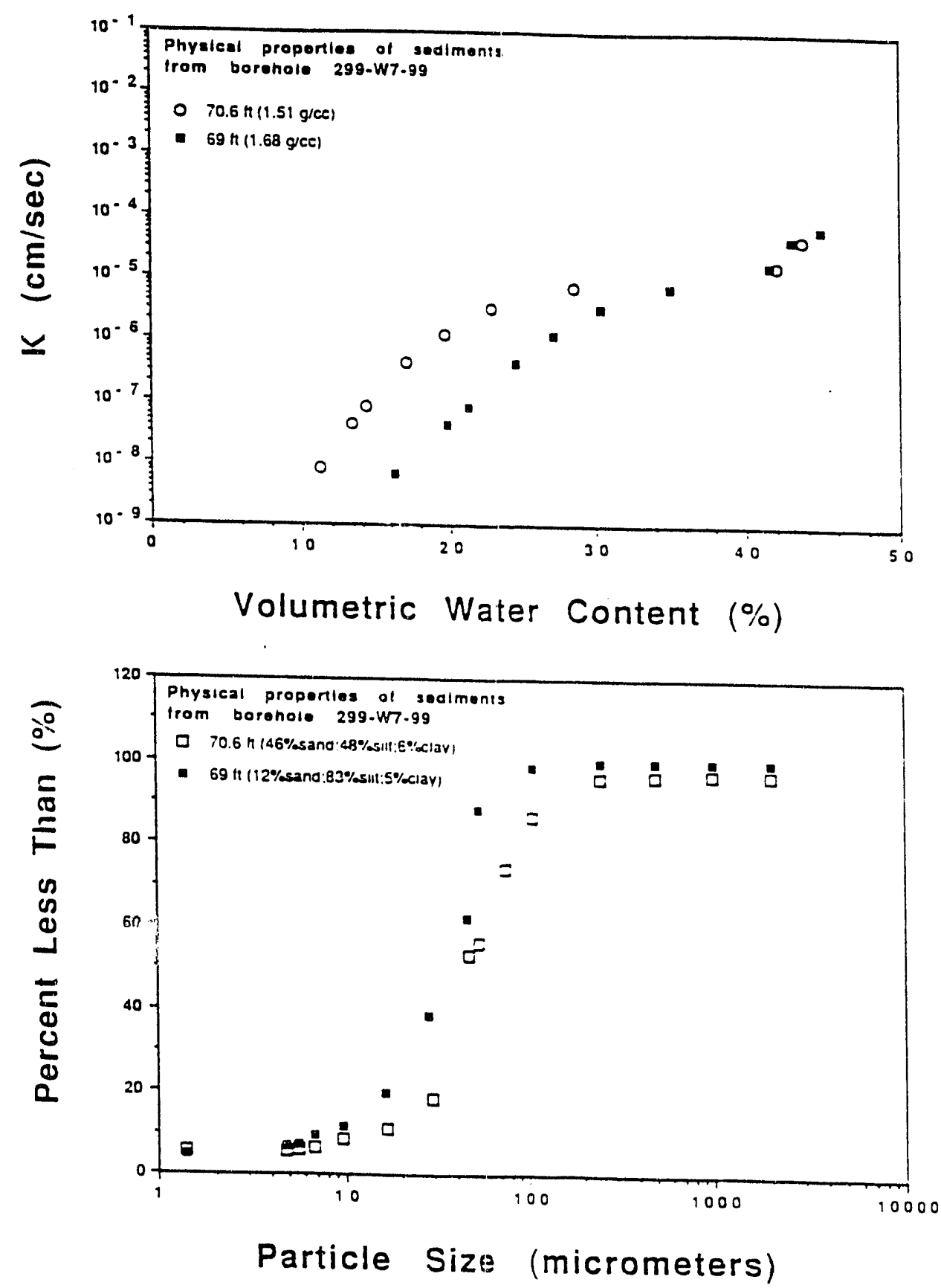


\section{USE OF VAN GENUCHTEN- MUALEM RELATIONSHIPS TO PREDICT UNSATURATED HYDRAULIC CONDUCTIVITY}

RAZ KHALEEL

WESTINGHOUSE HANFORD COMPANY

\section{VAN GENUCHTEN-MUALEM RELATIONSHIPS}

$$
\begin{gathered}
\theta=\theta_{r}+\frac{\left(\theta_{s}-\theta_{r}\right)}{\left[1+(\alpha \psi)^{N}\right]^{M}} \\
\frac{K(\theta)}{K_{s}}=\left[\frac{\theta-\theta_{r}}{\epsilon_{s}-\theta_{r}}\right]^{e}\left\{1-\left[1-\left(\frac{\theta-\theta_{r}}{\theta_{s}-\theta_{r}}\right)^{1 / M}\right]^{M}\right\}^{2}
\end{gathered}
$$

\section{OBJECTIVE}

VALIDATE VAN GENUCHTEN-MUALEM RELATIONSHIPS TO ESTIMATE UNSATURATED HYDRAULIC CONDUCTIVITY FOR HANFORD SOILS, ESPECIALLY AT LOW MOISTURE CONTENTS 


\section{PENALTY FOR USING INCORRECT ESTIMATES FOR UNSATURATED HYDRAULIC CONDUCTIVITY AT LOW MOISTURE CONTENTS}

By not having accurate estimates of hydraulic conductivities at low moisture contents, we will be forced to use conservative estimates and this can potentially lead to overdesigns for various waste disposal sites at Hanford. 
WHC-MR-0420

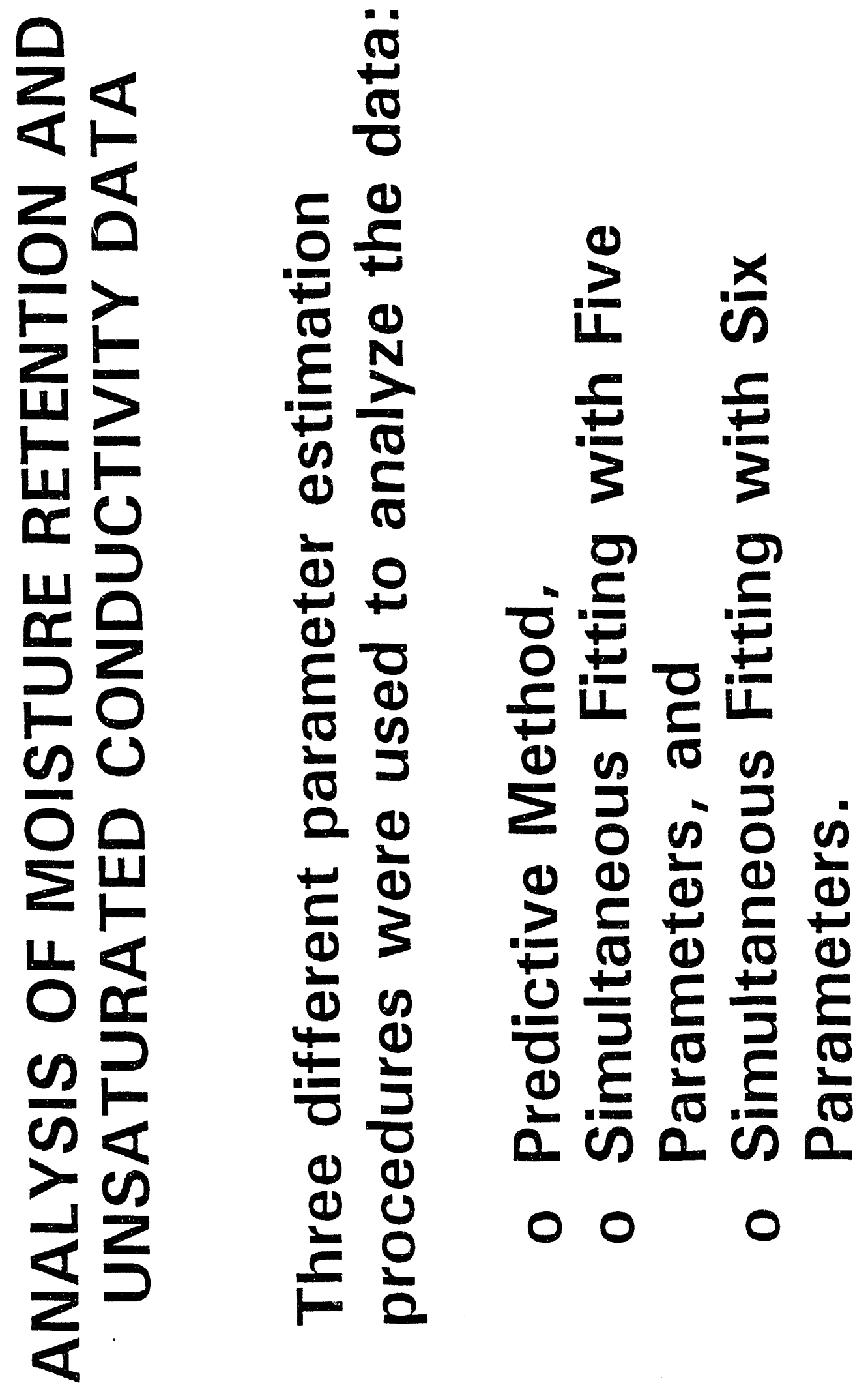




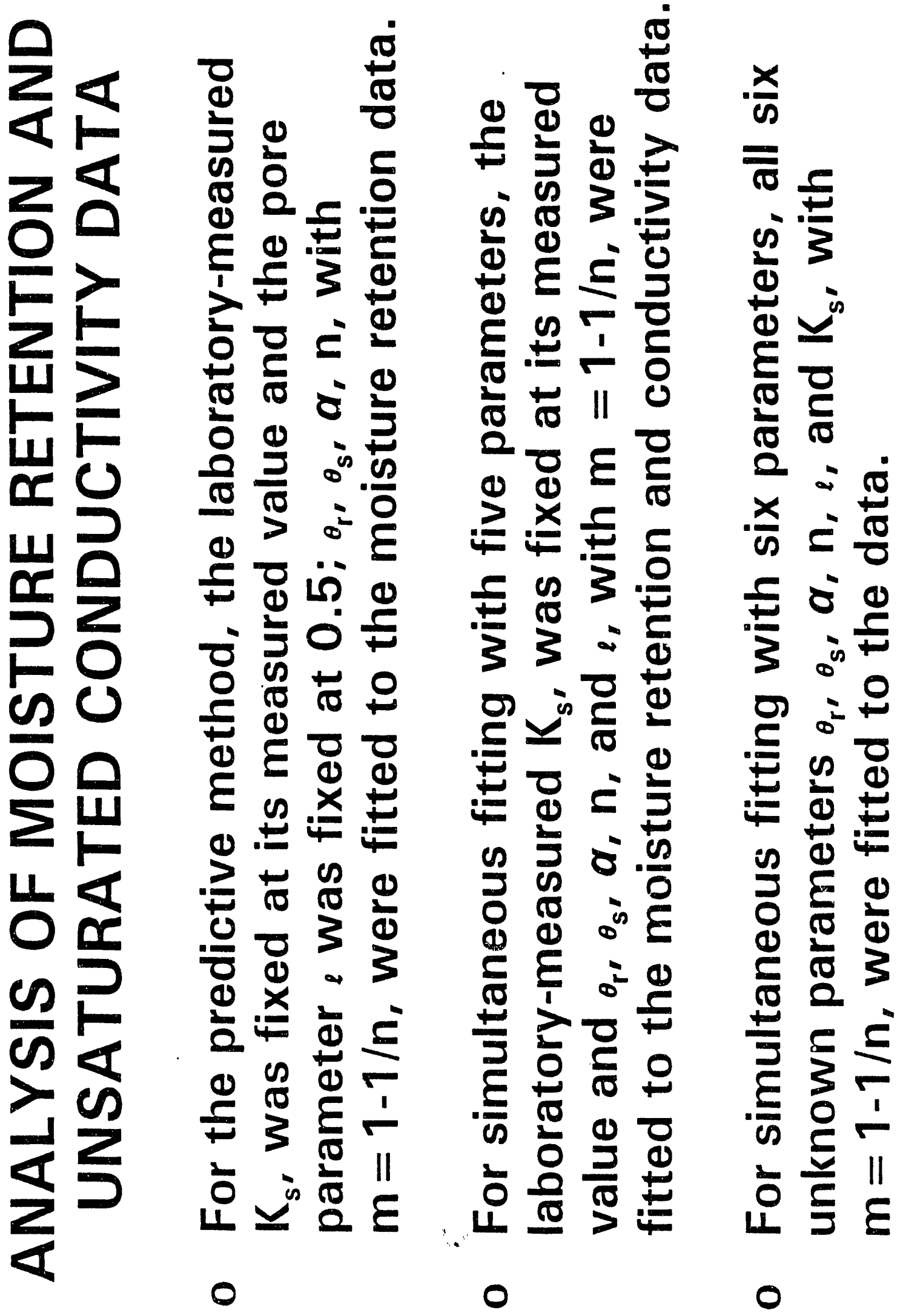



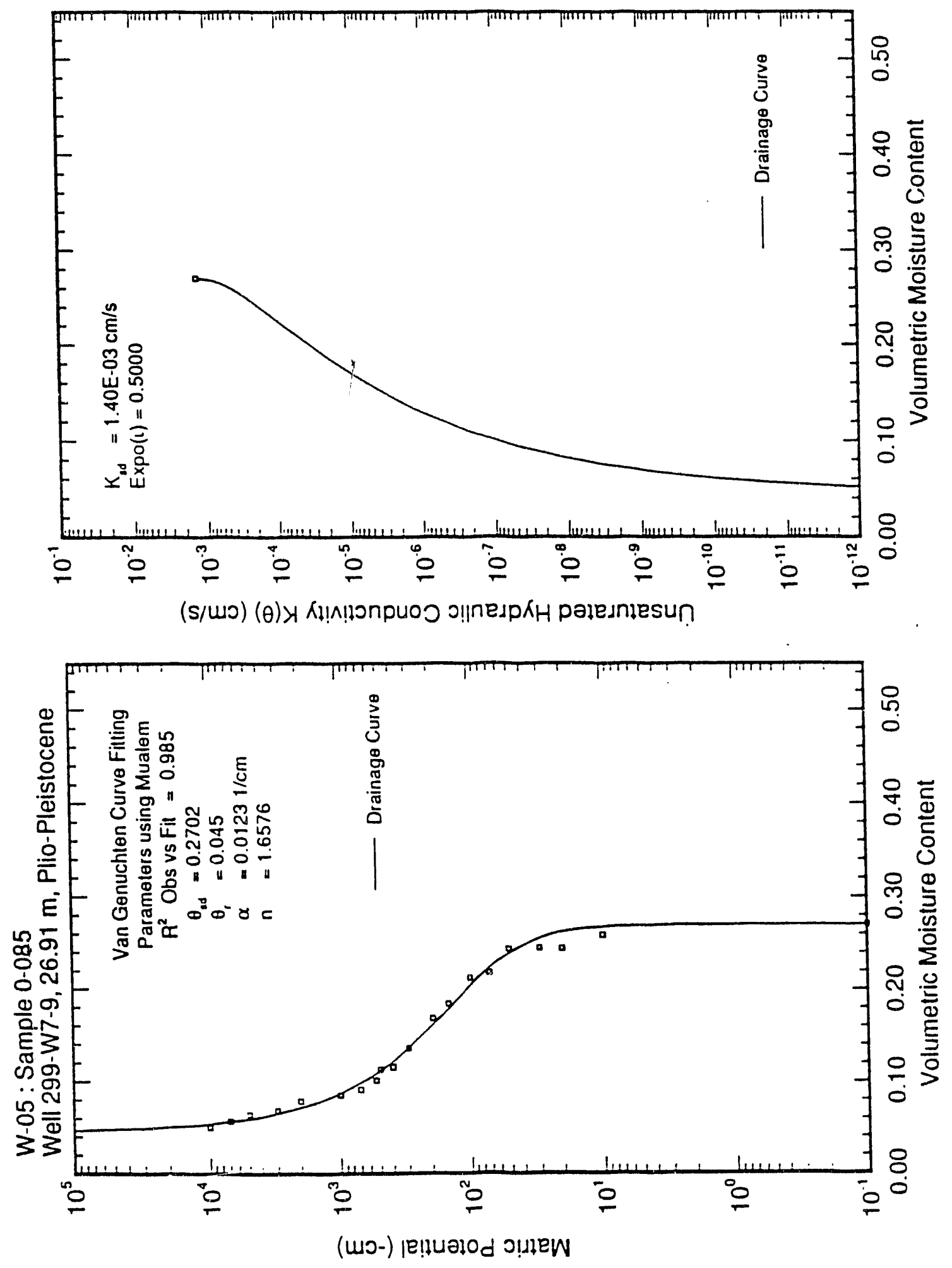


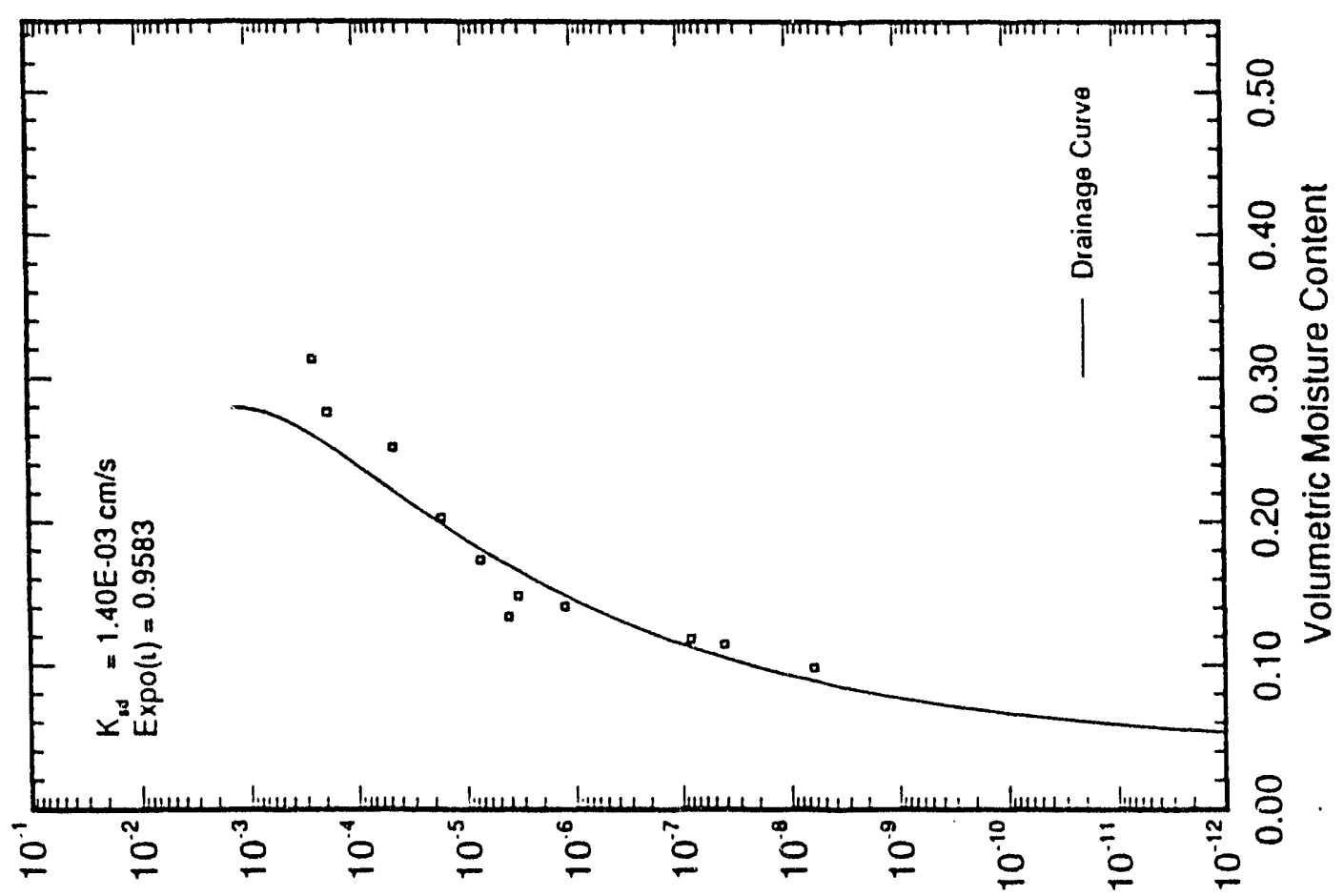

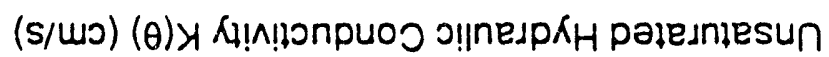

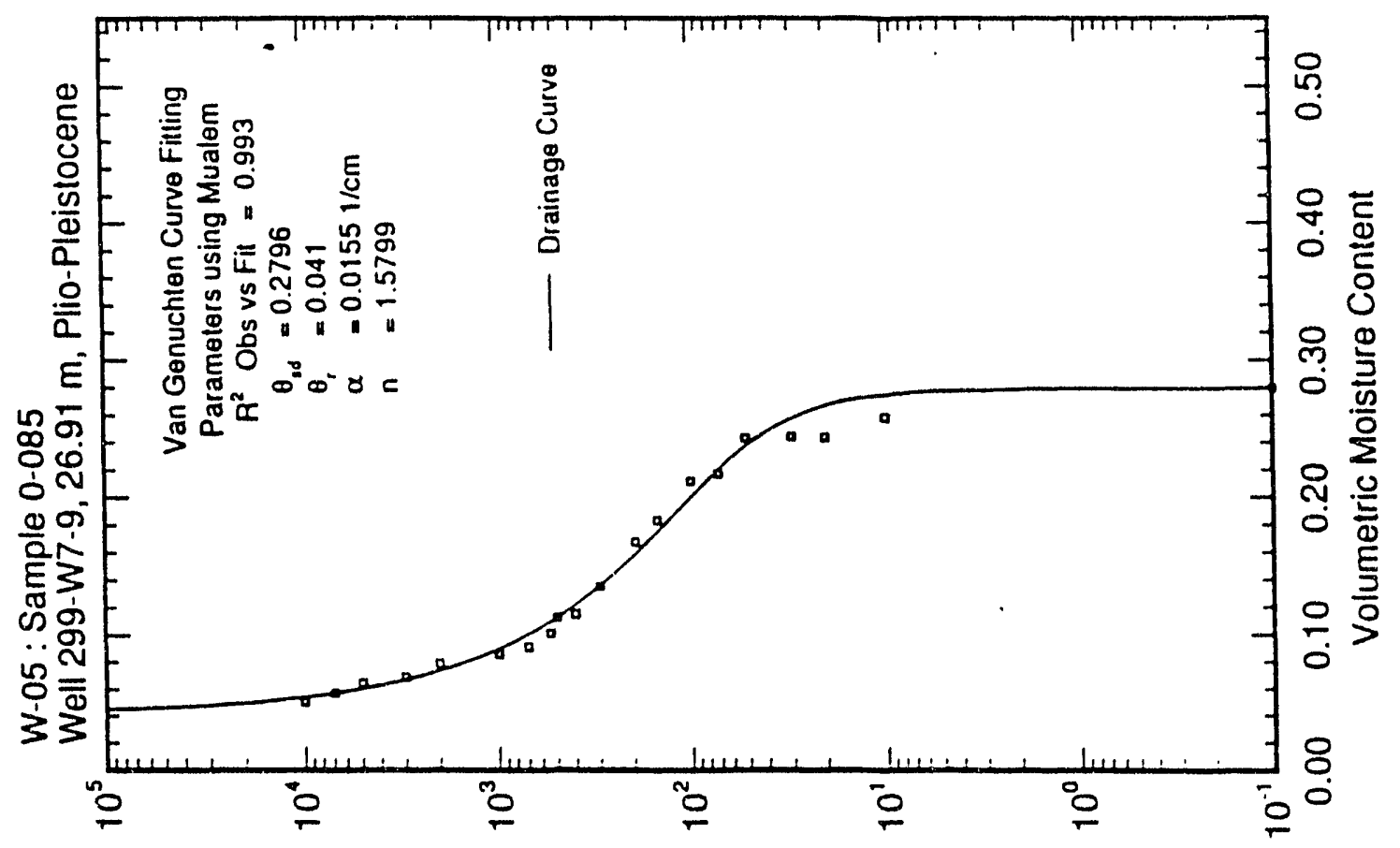

(uว-) ןe!nualod o!new 


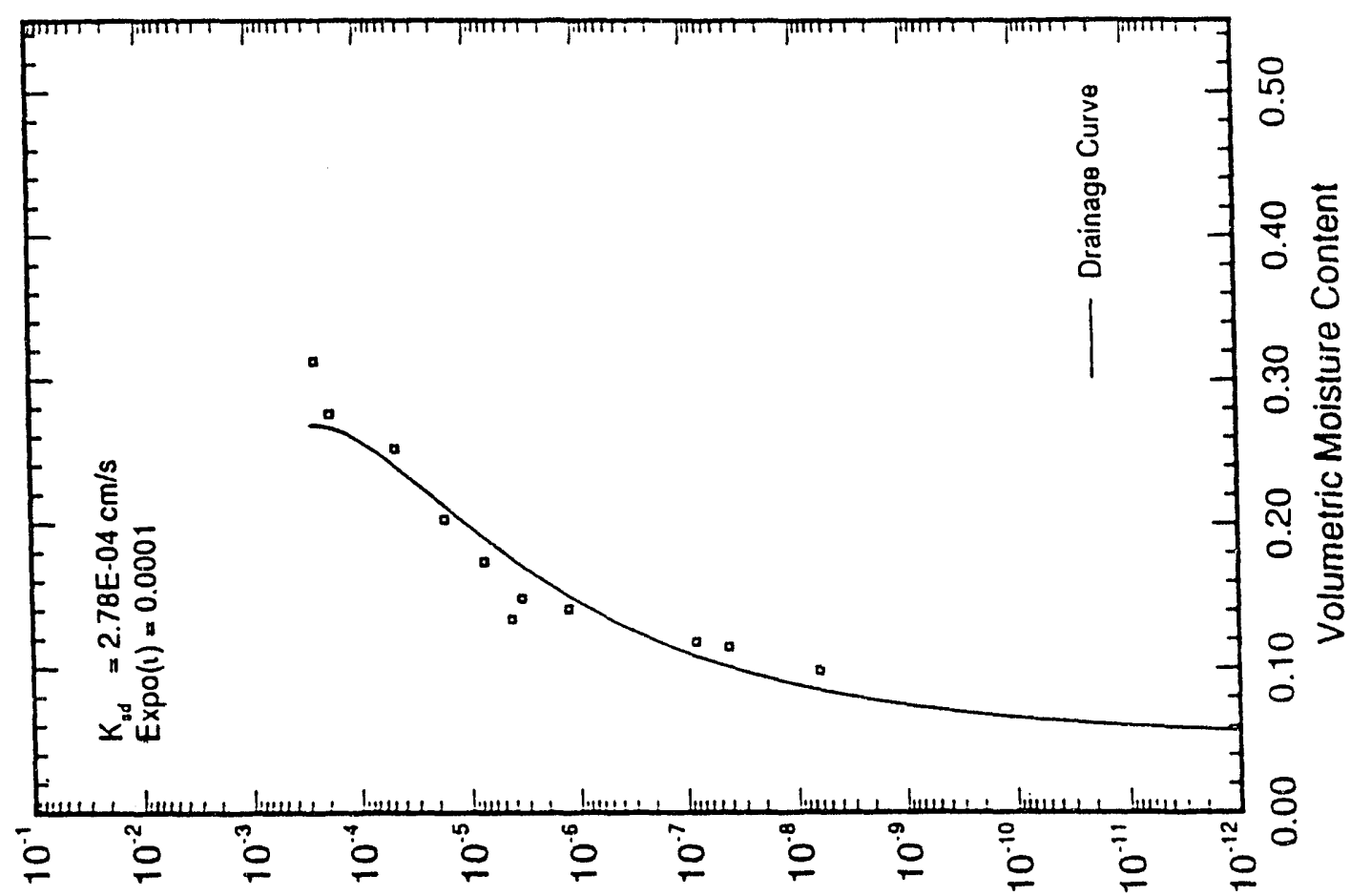

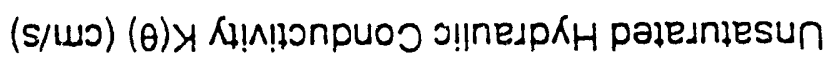

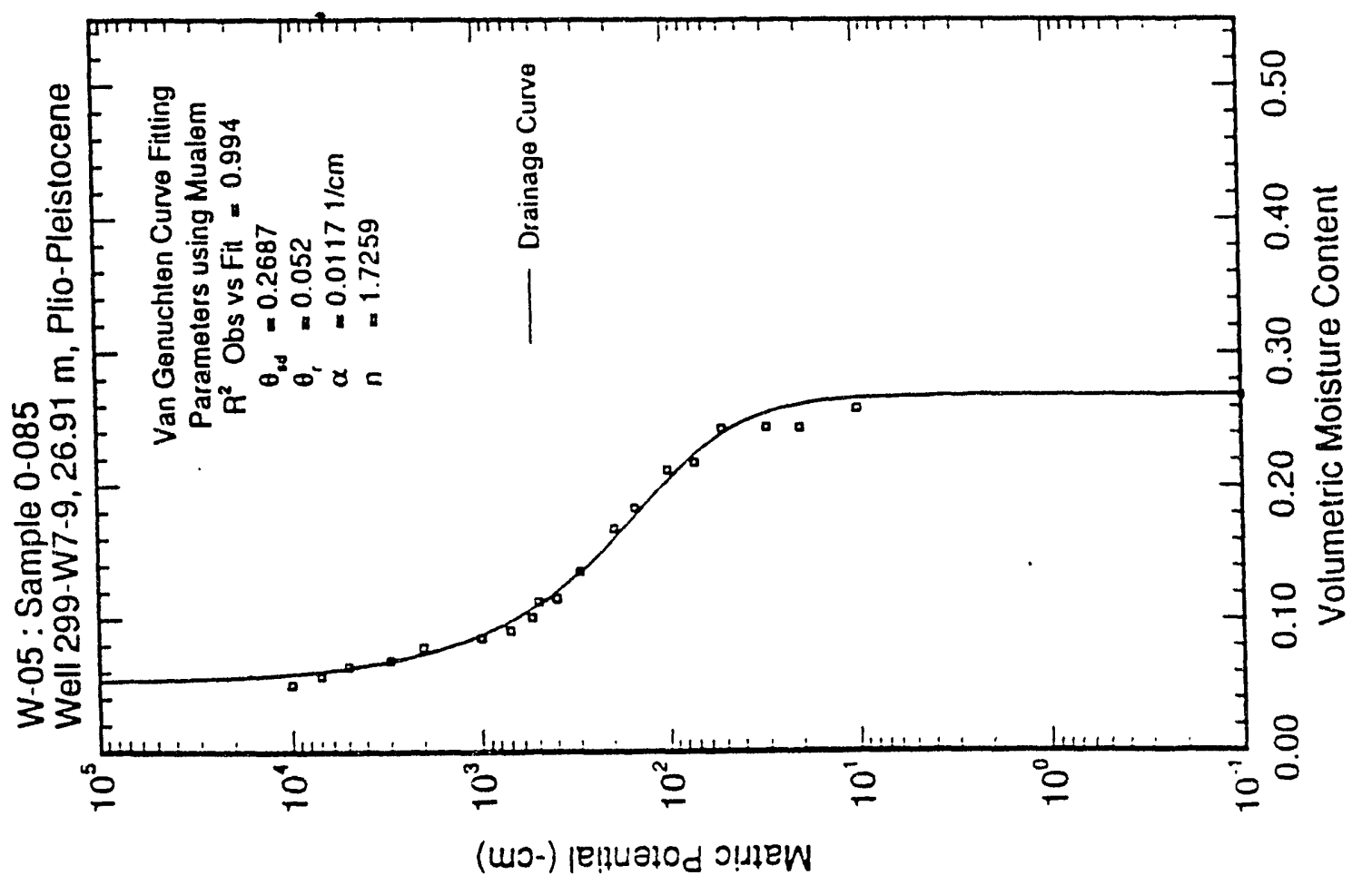




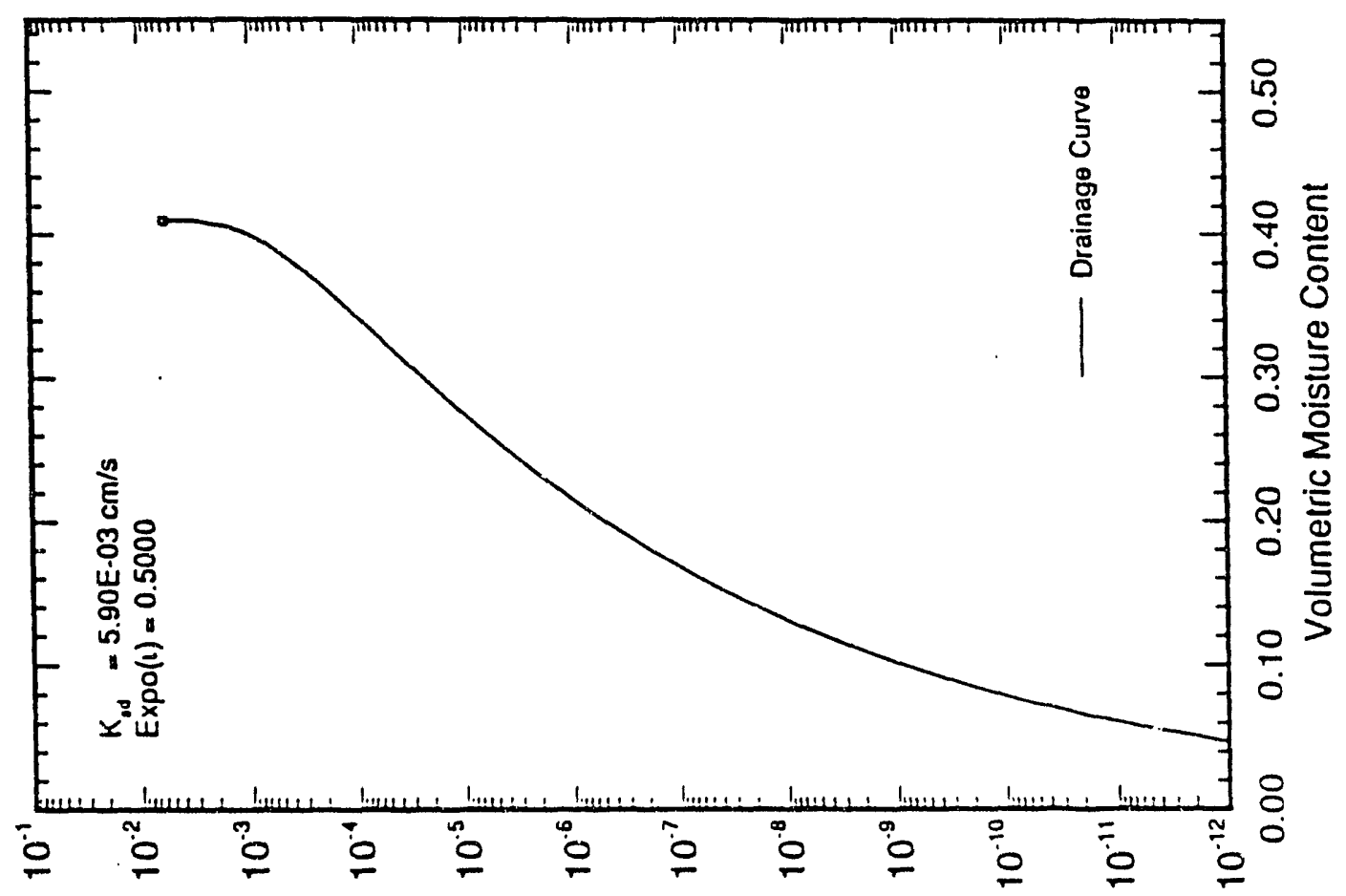

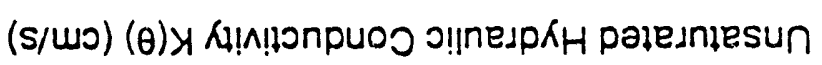

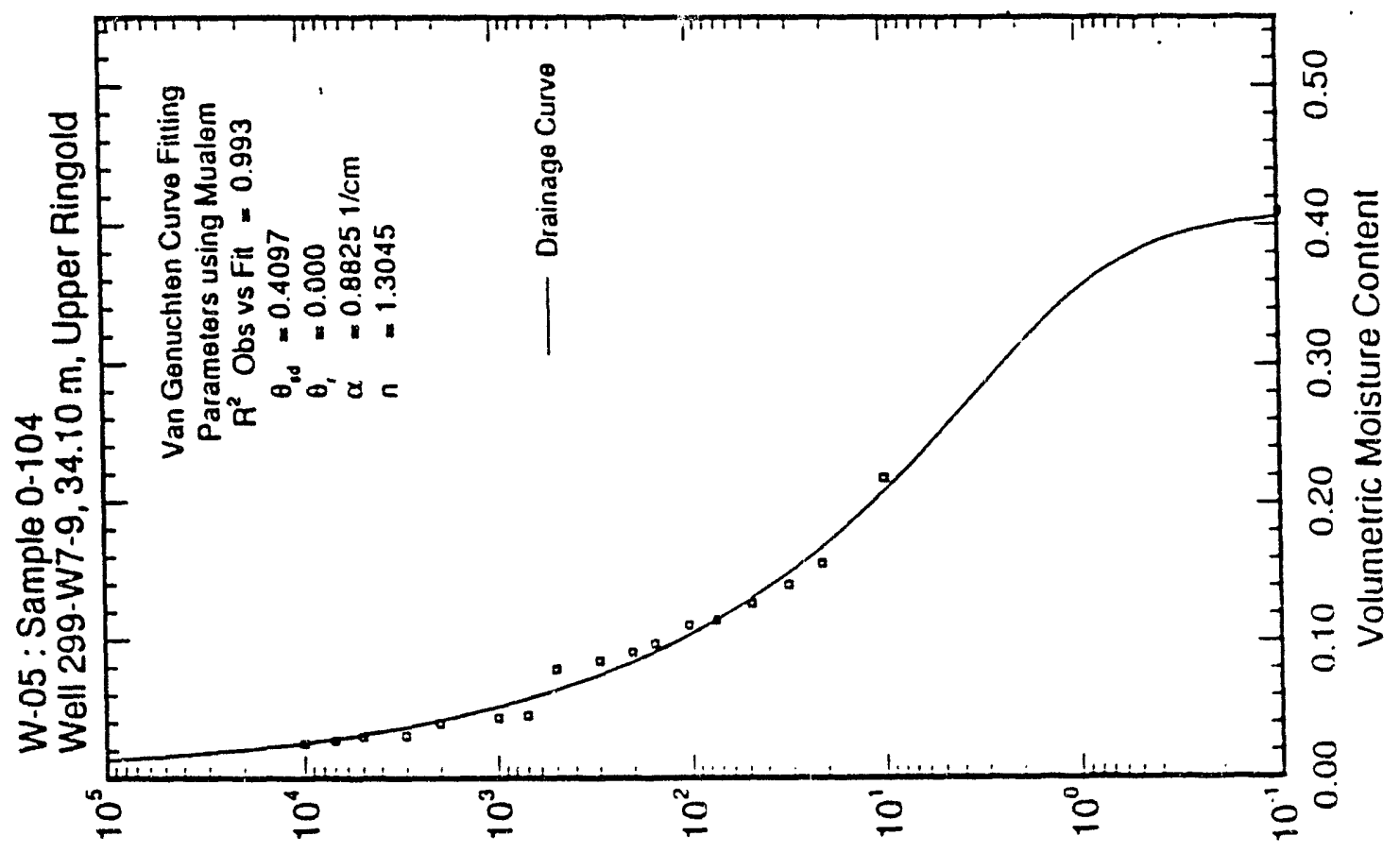

(Wo-) pe!nualod o!new 


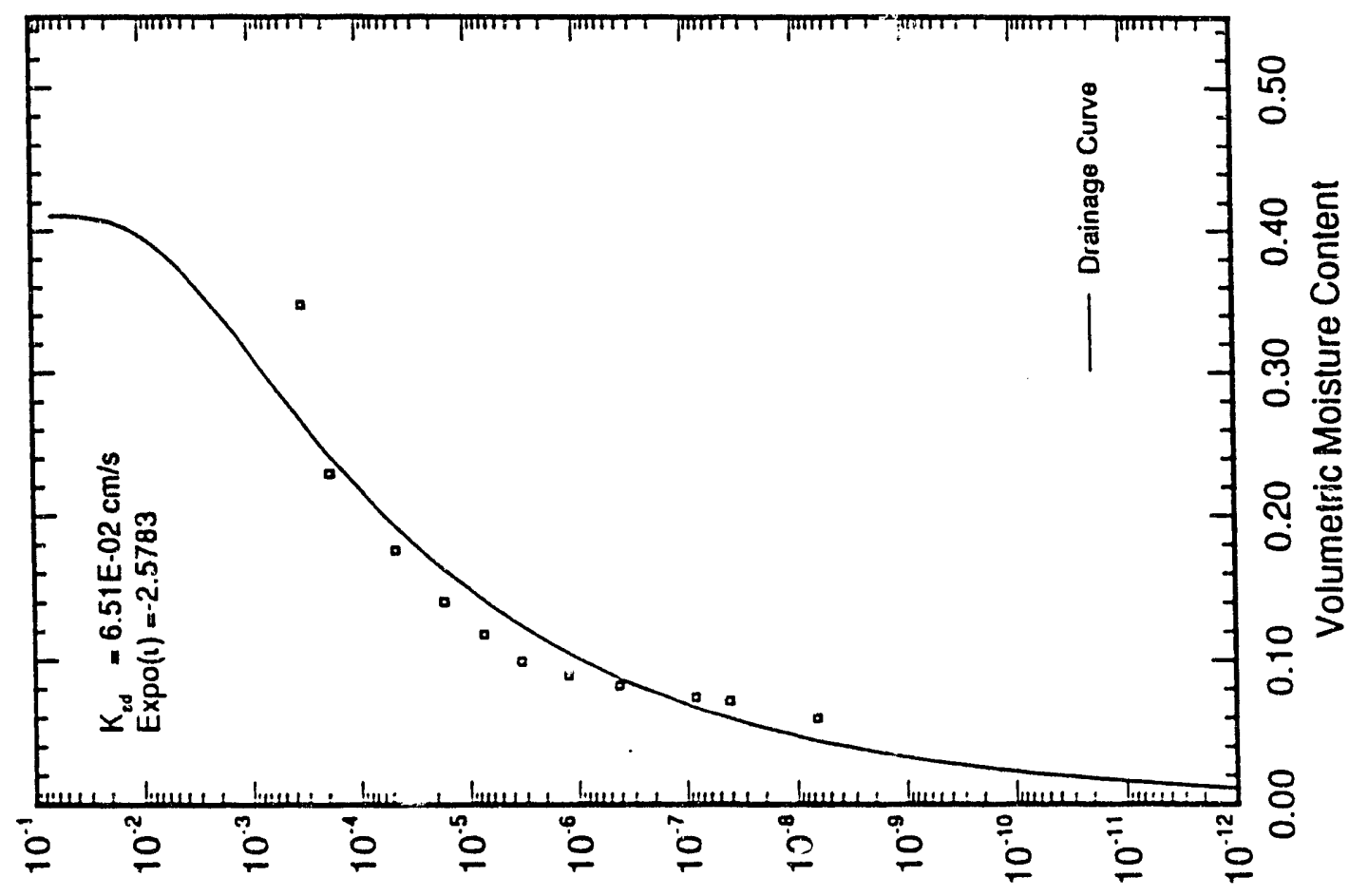

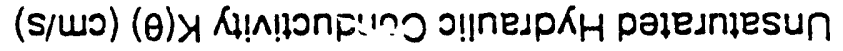

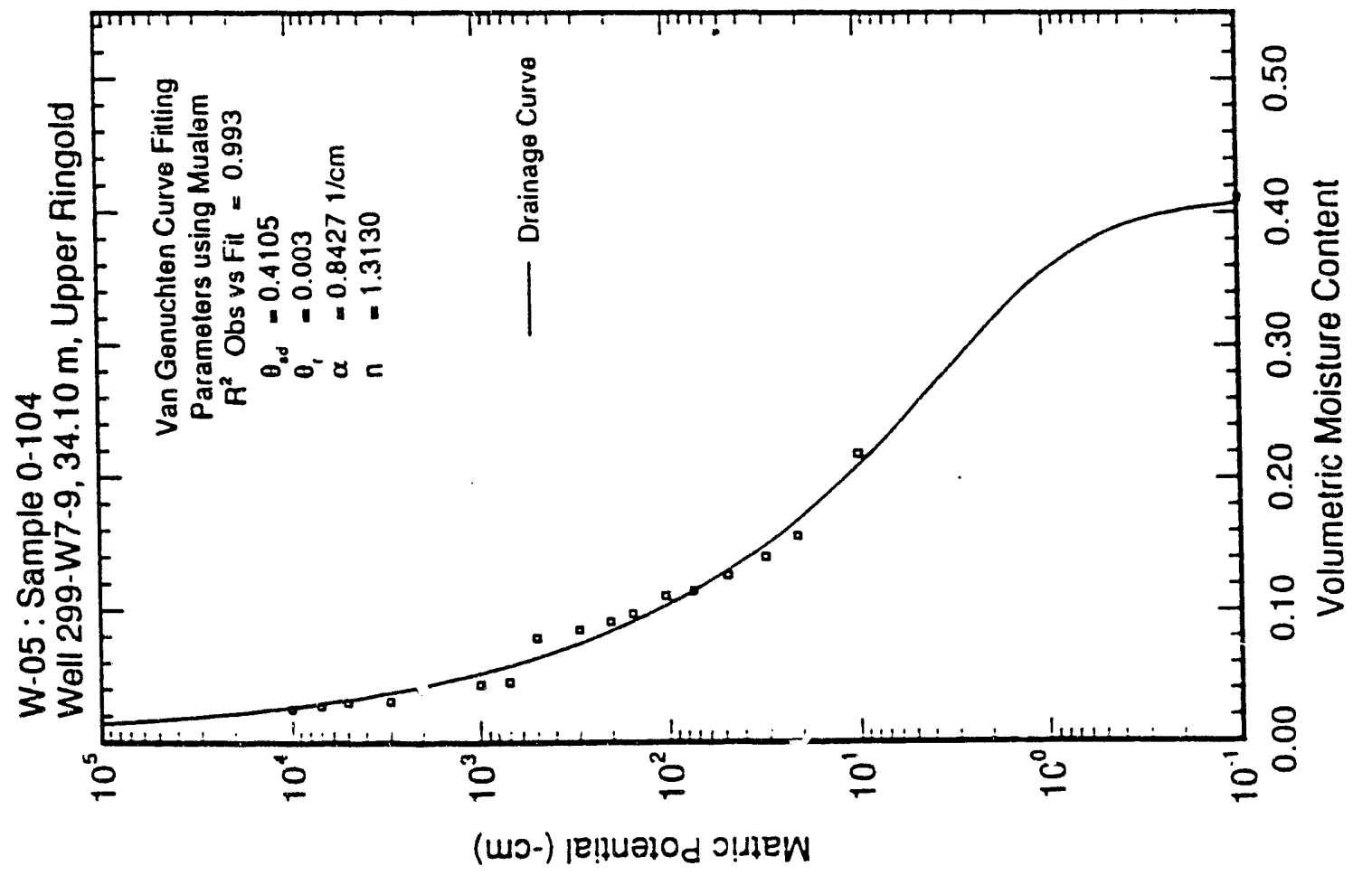




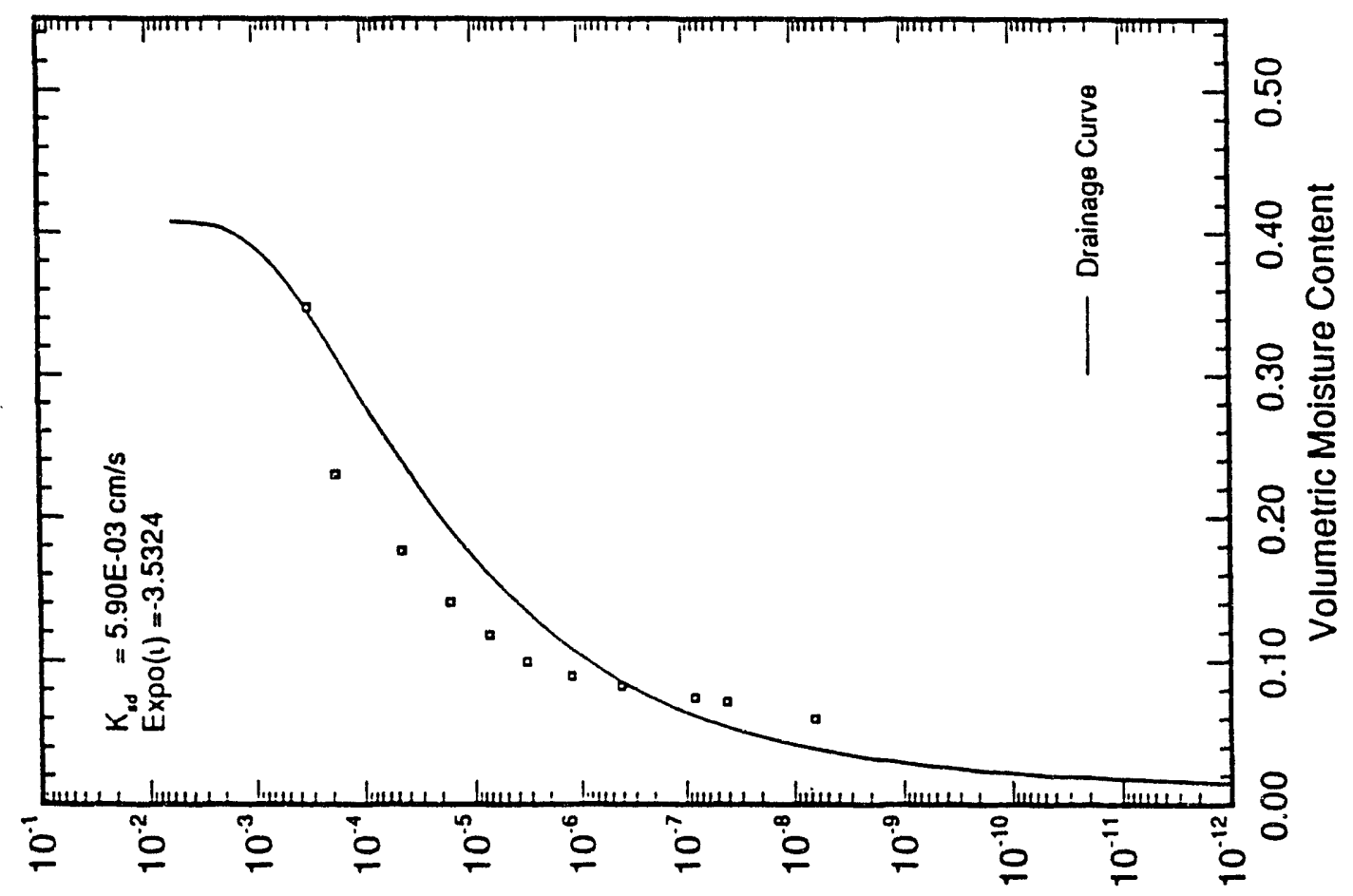

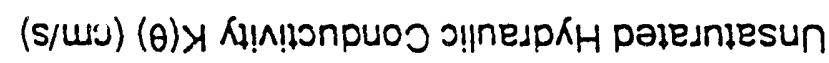

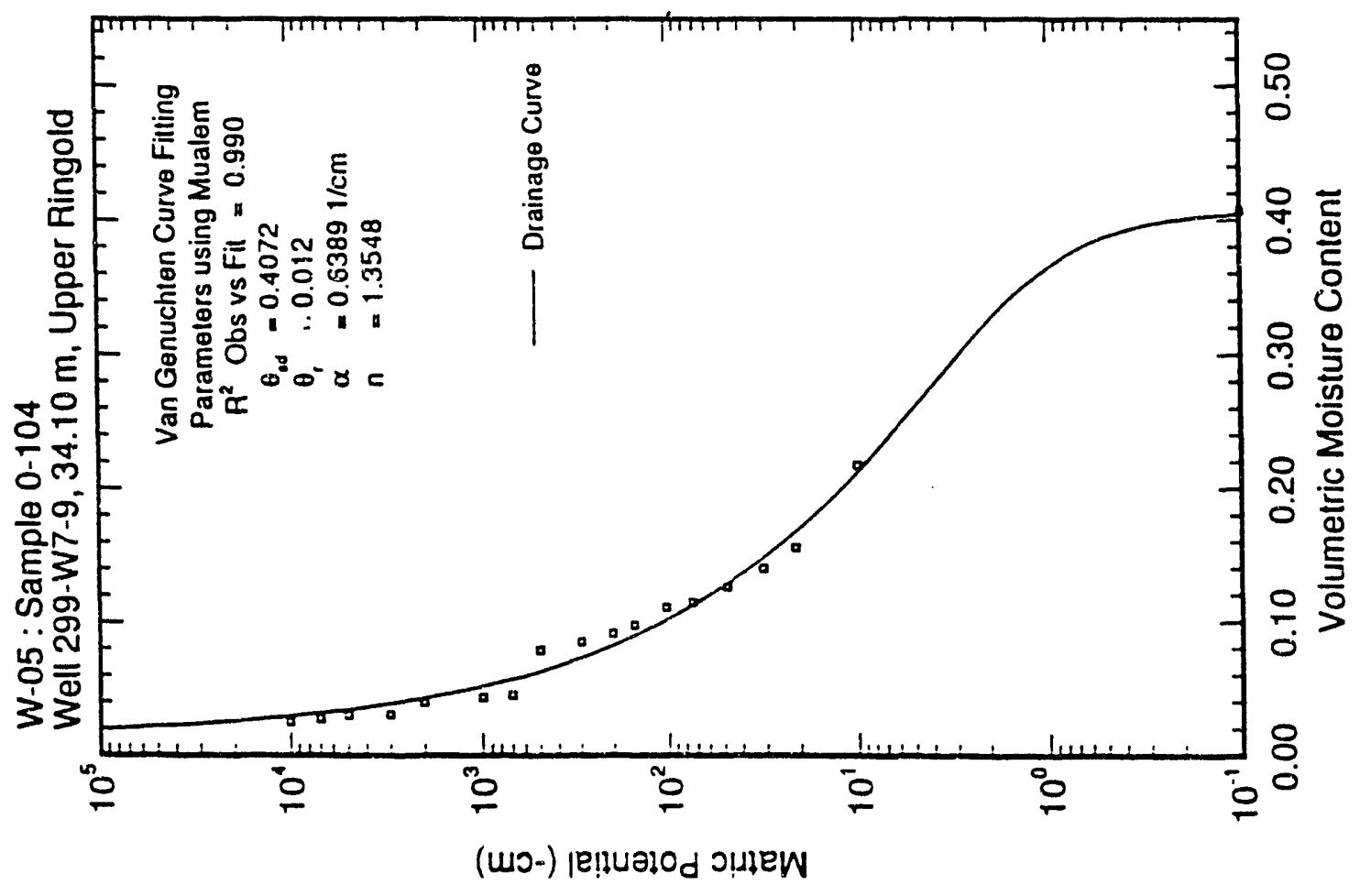




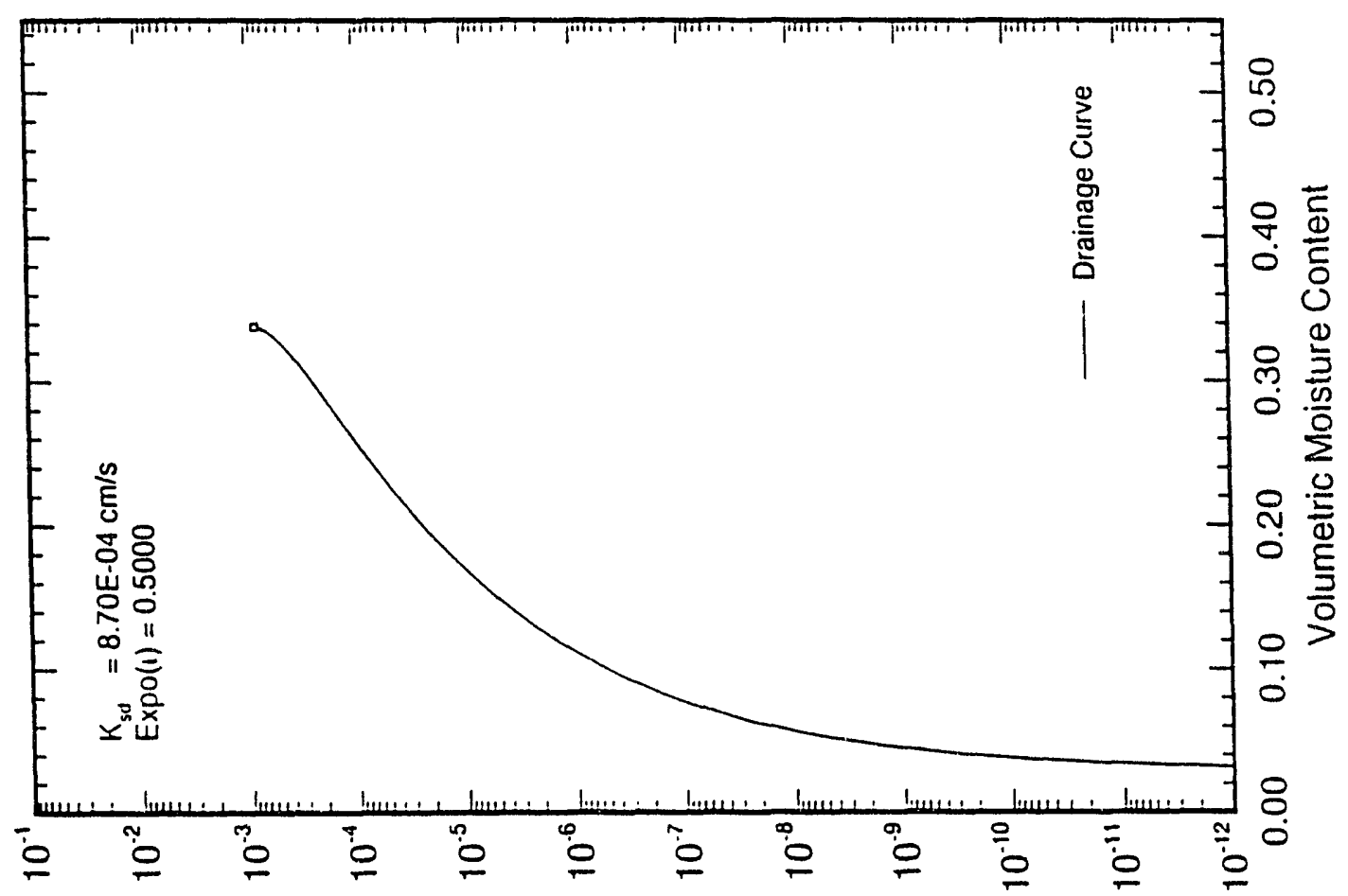

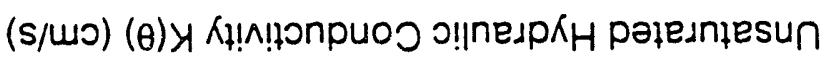

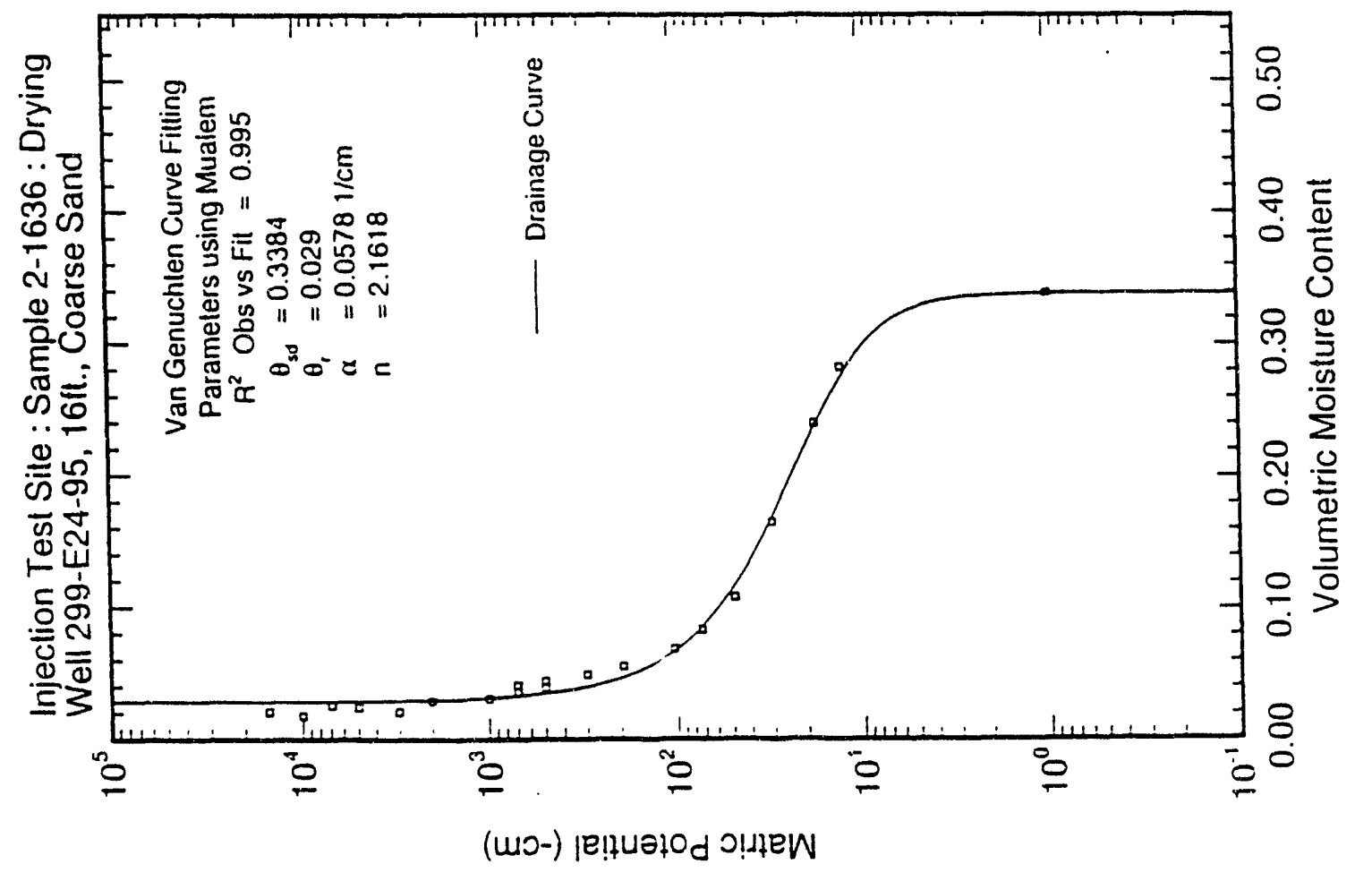



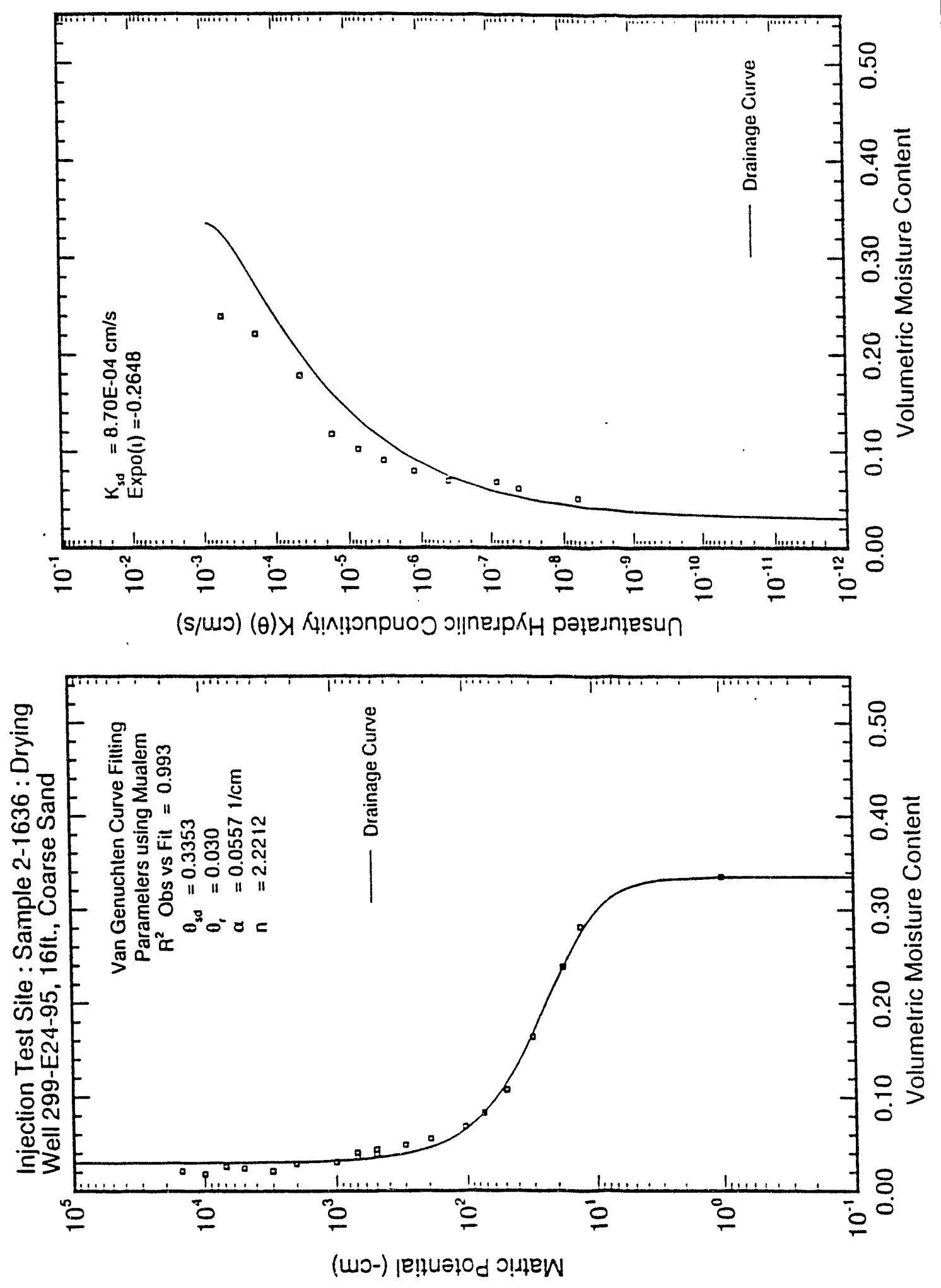


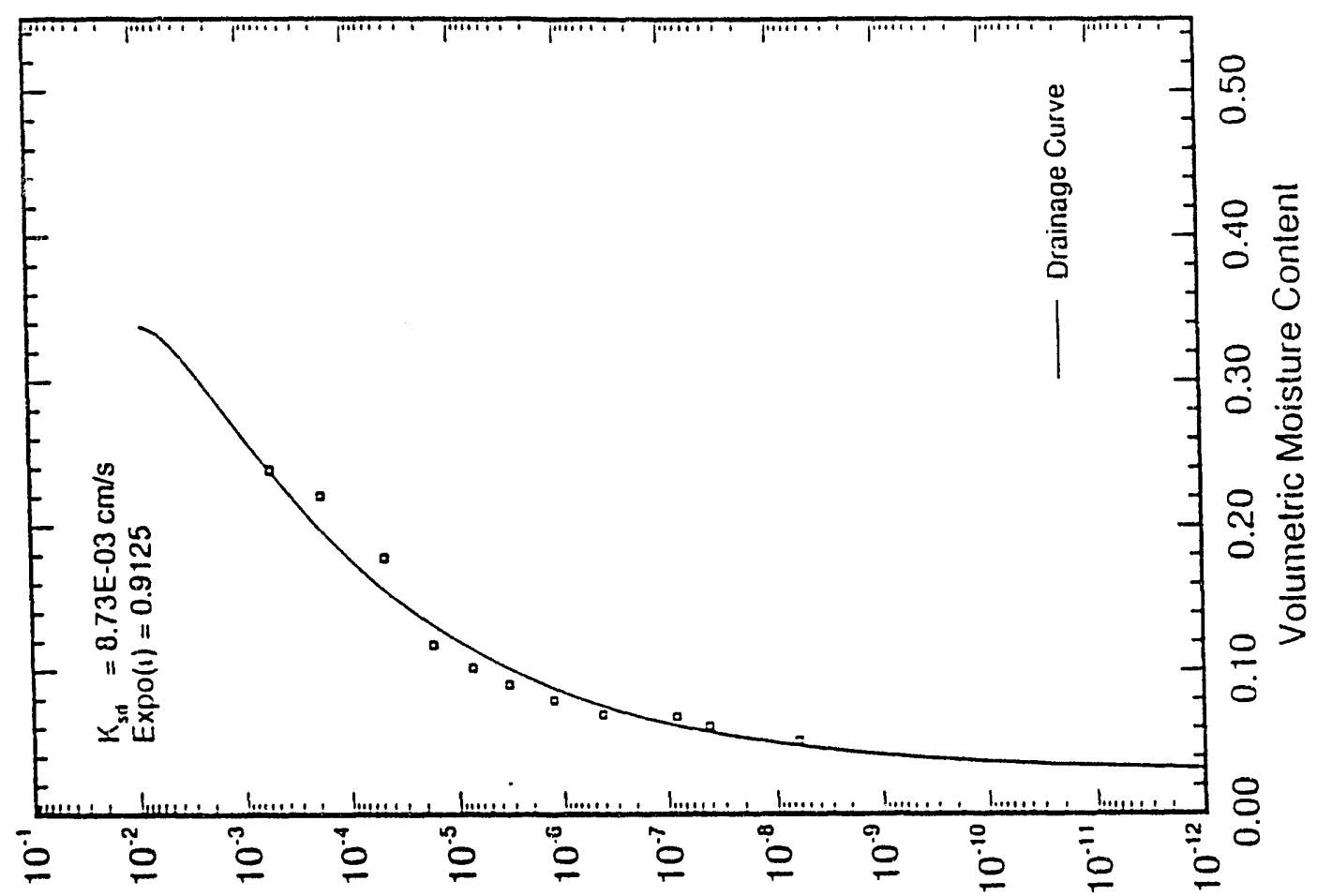

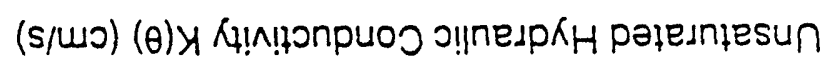

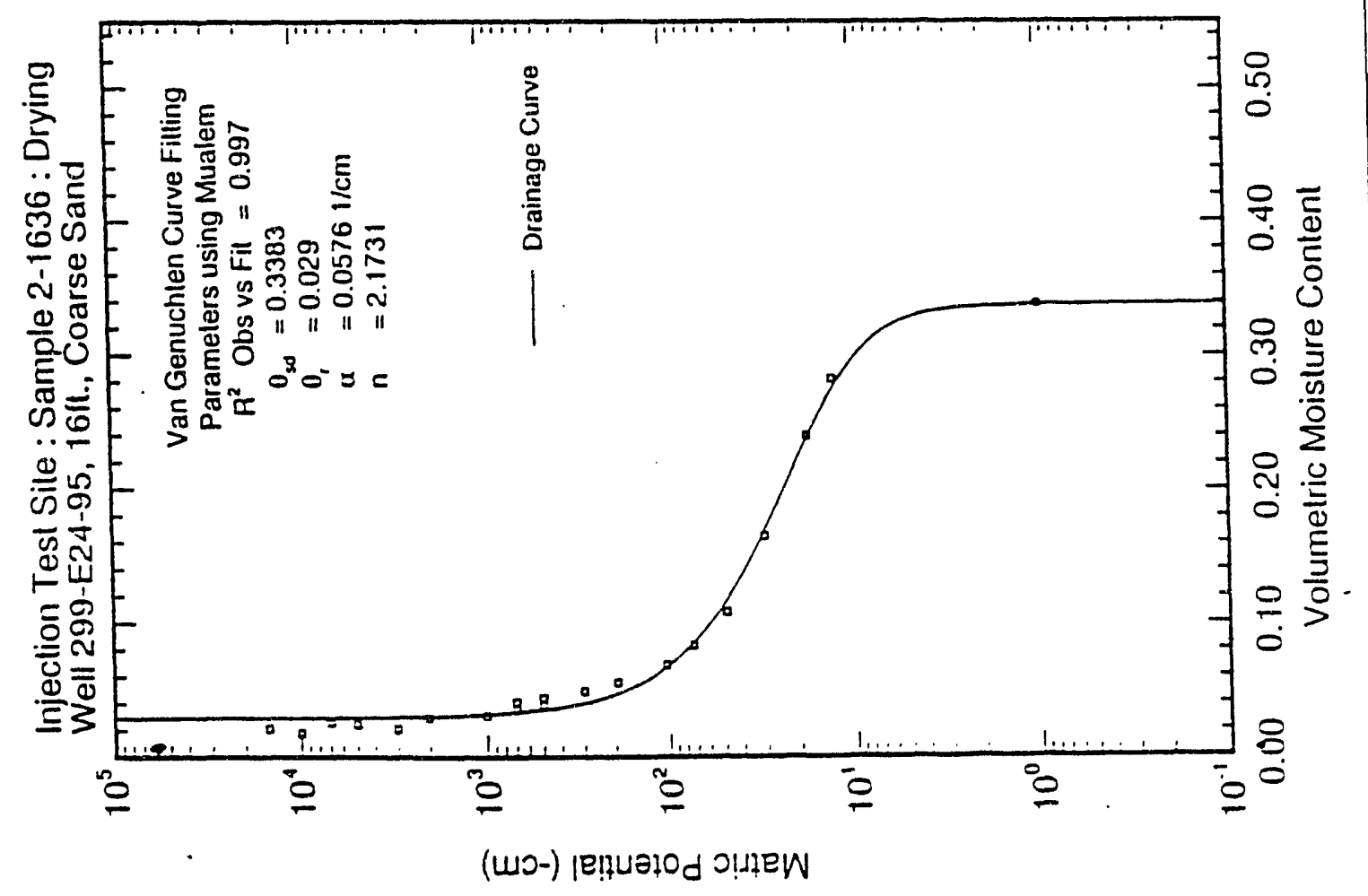



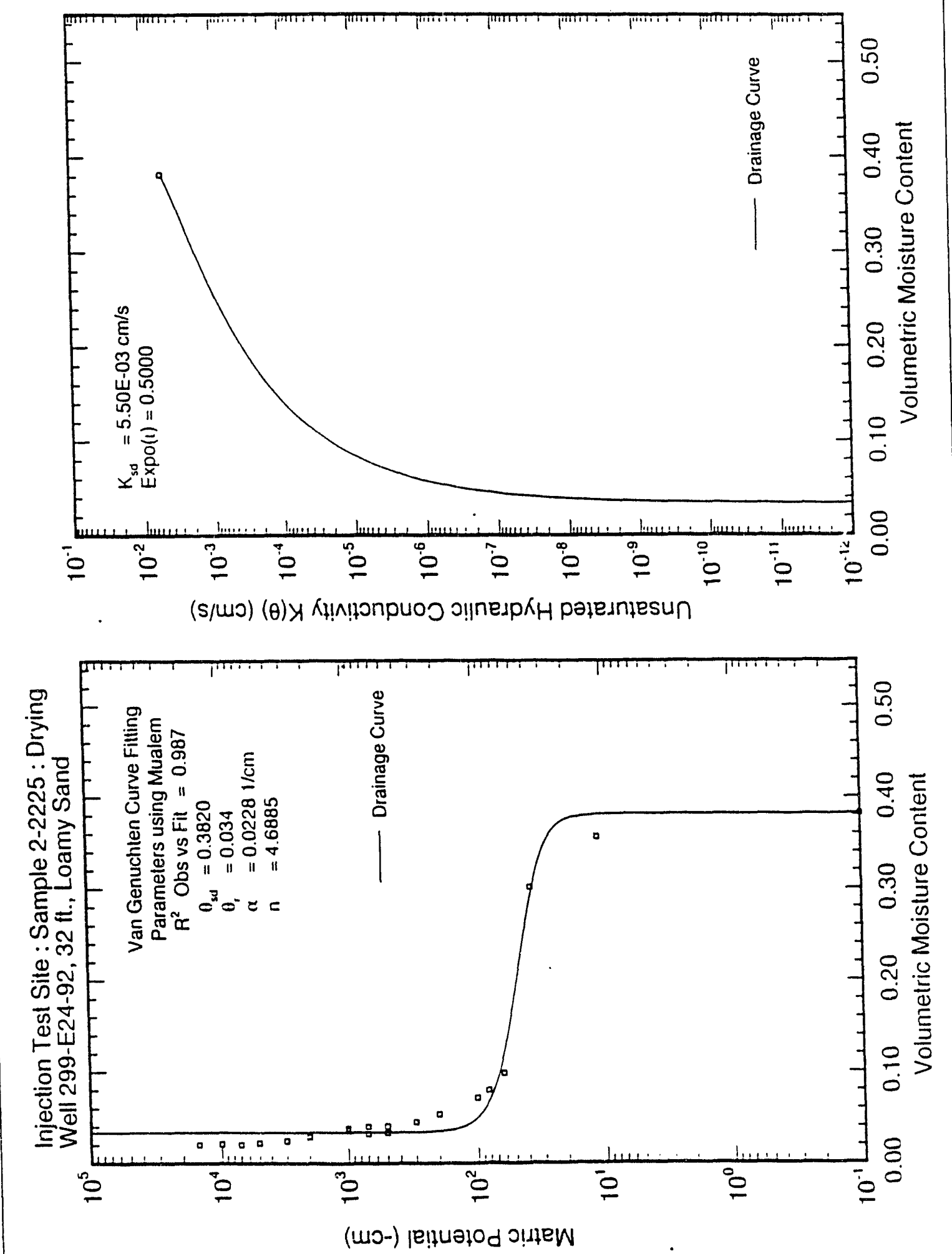


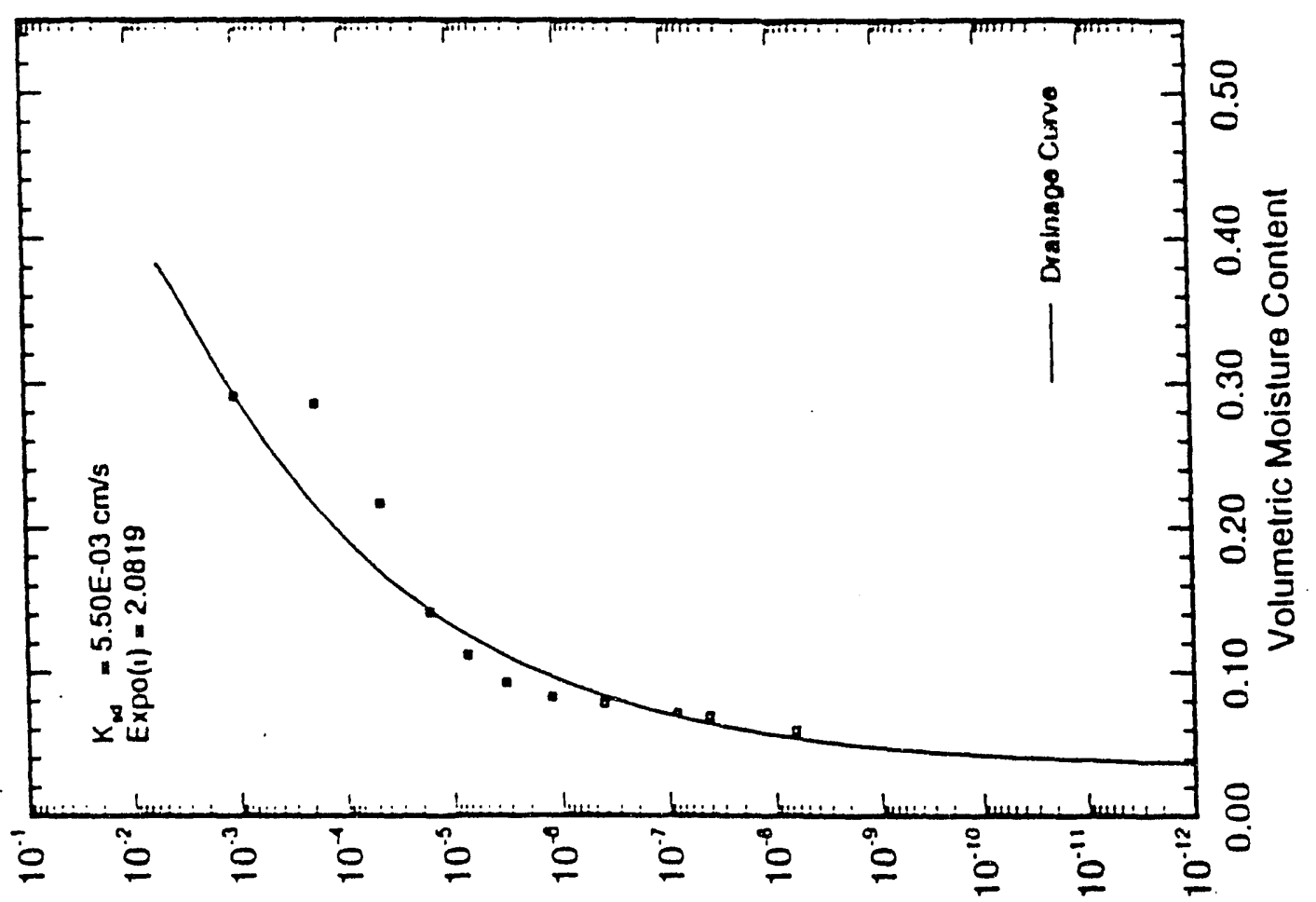

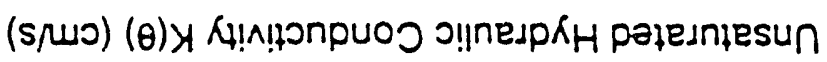

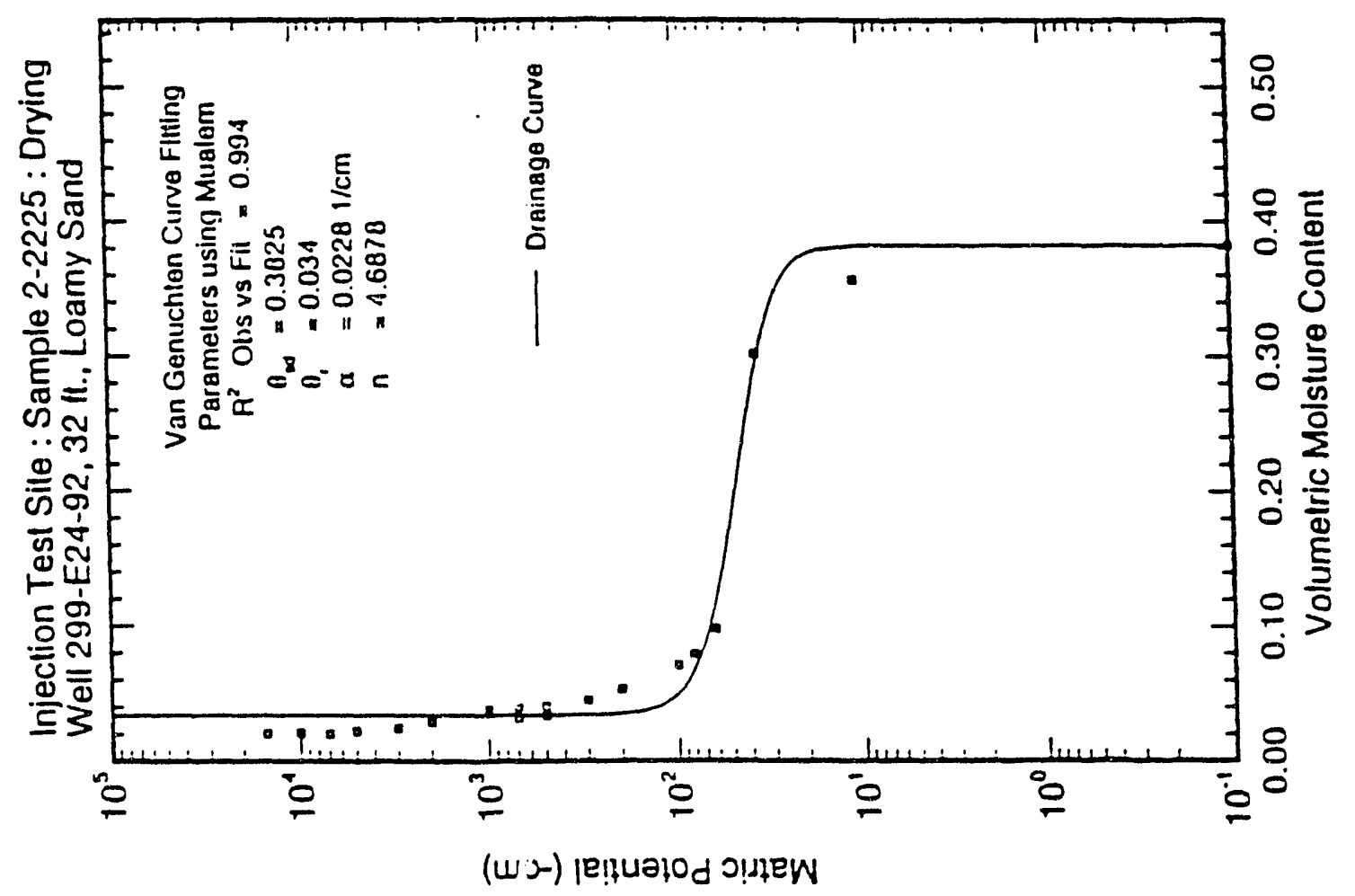




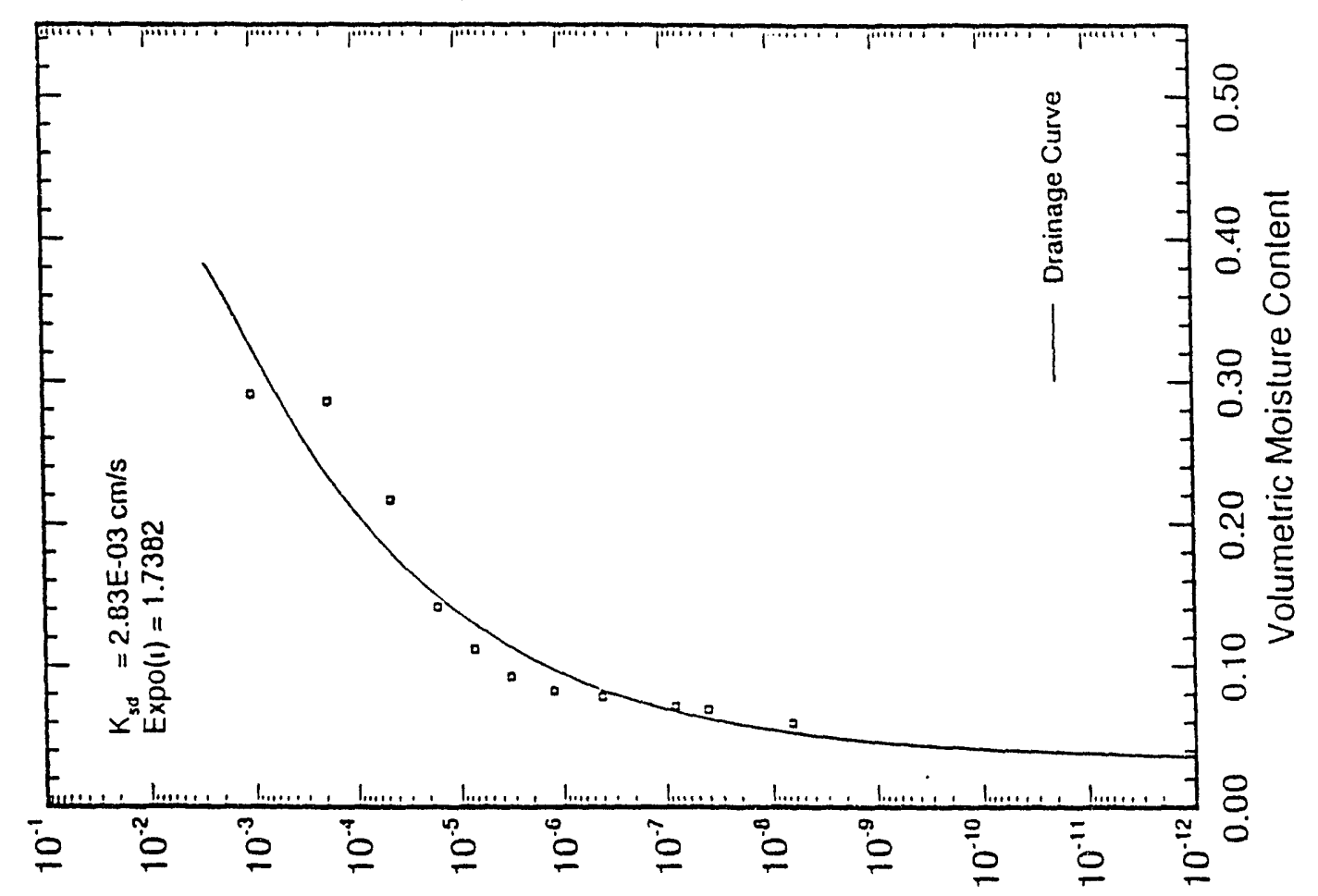

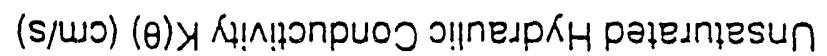

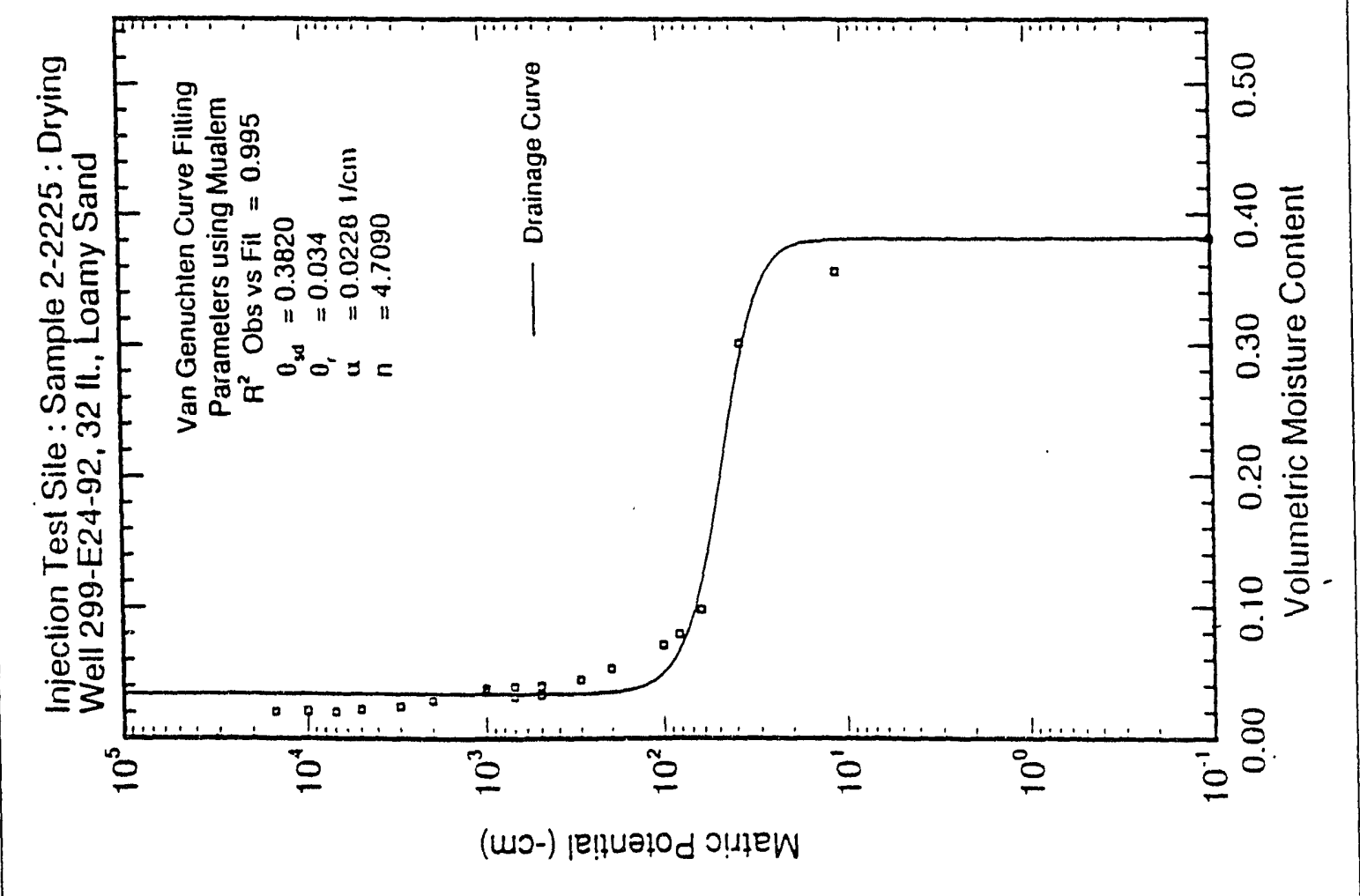




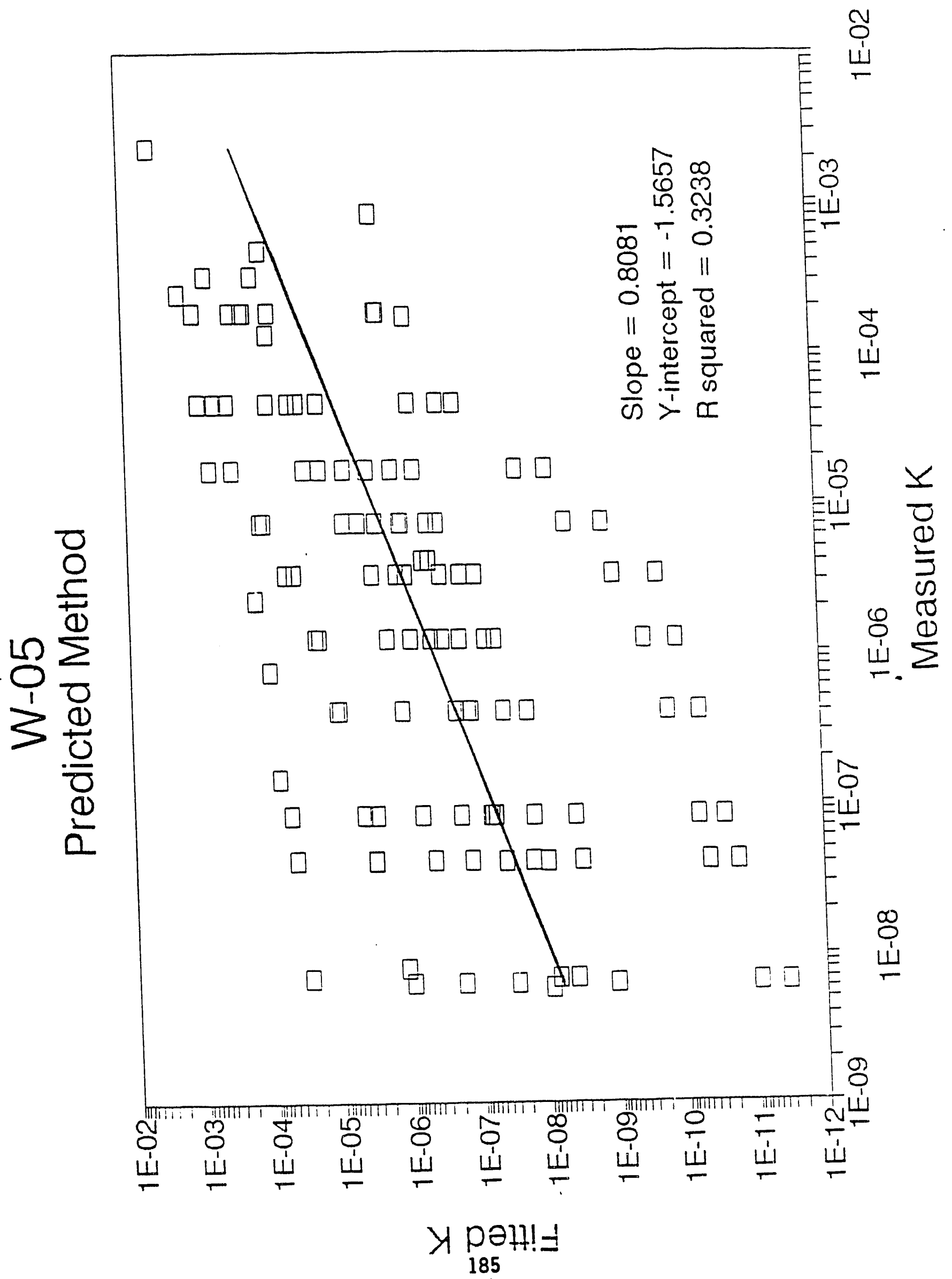




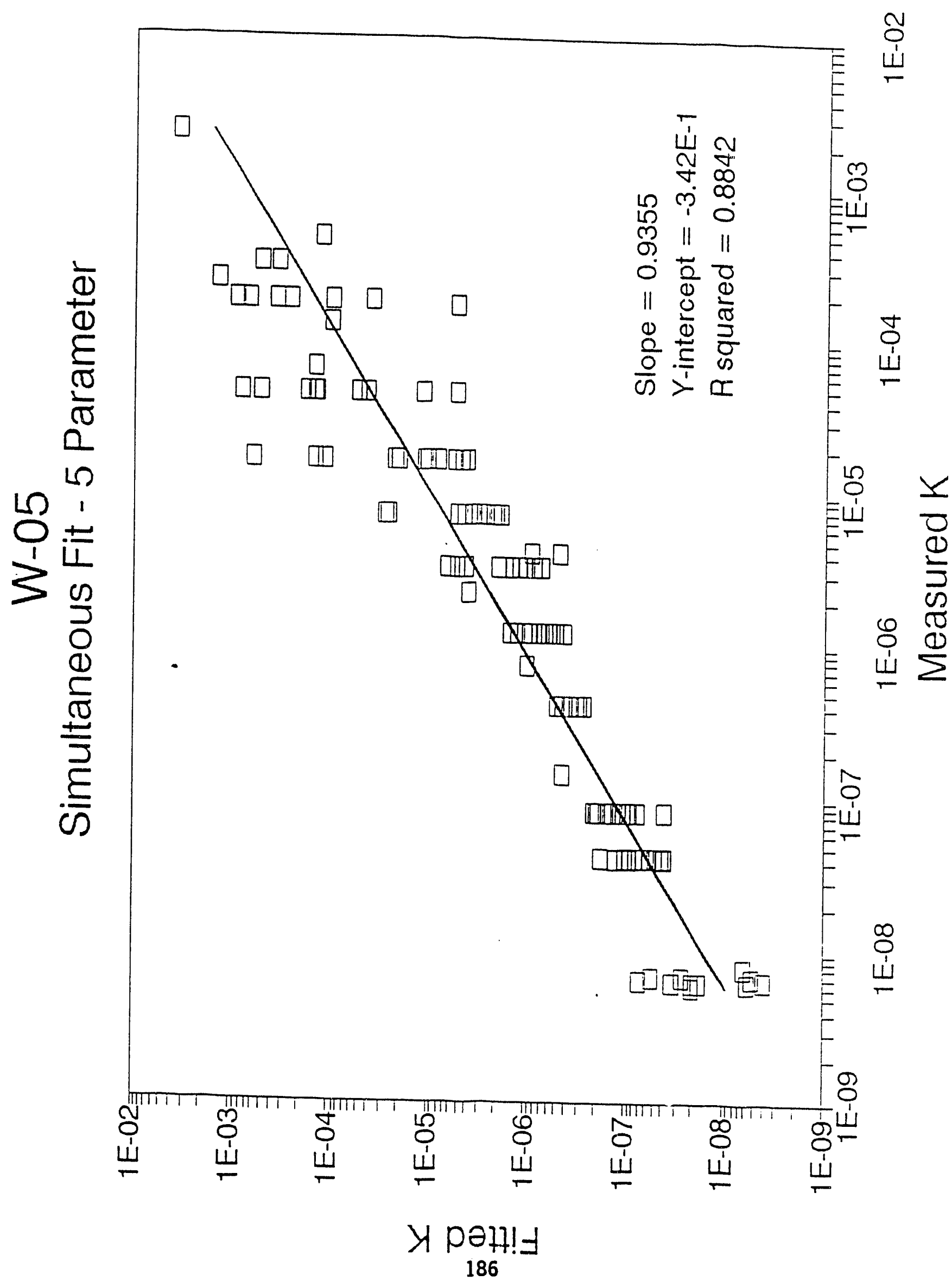




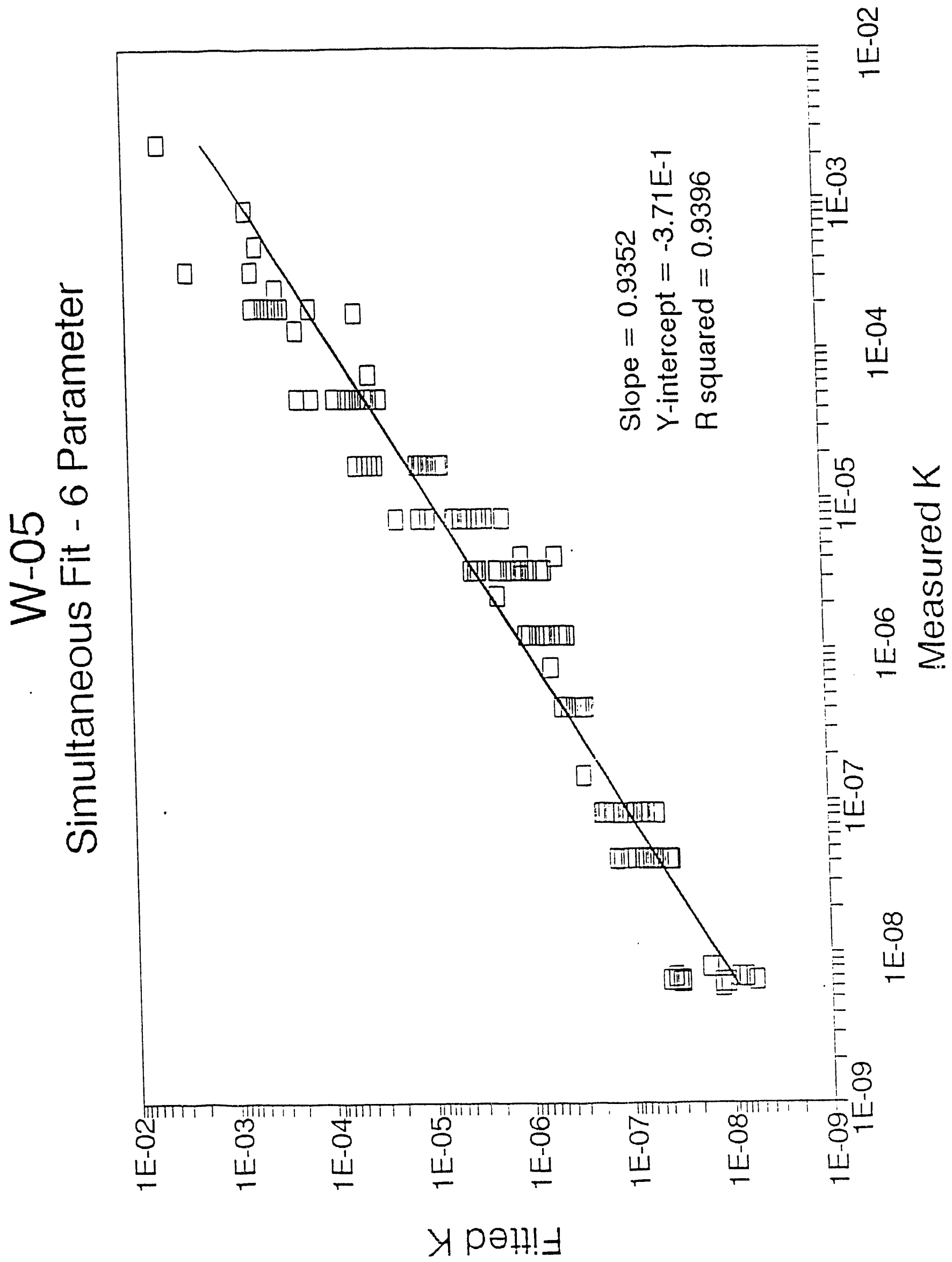




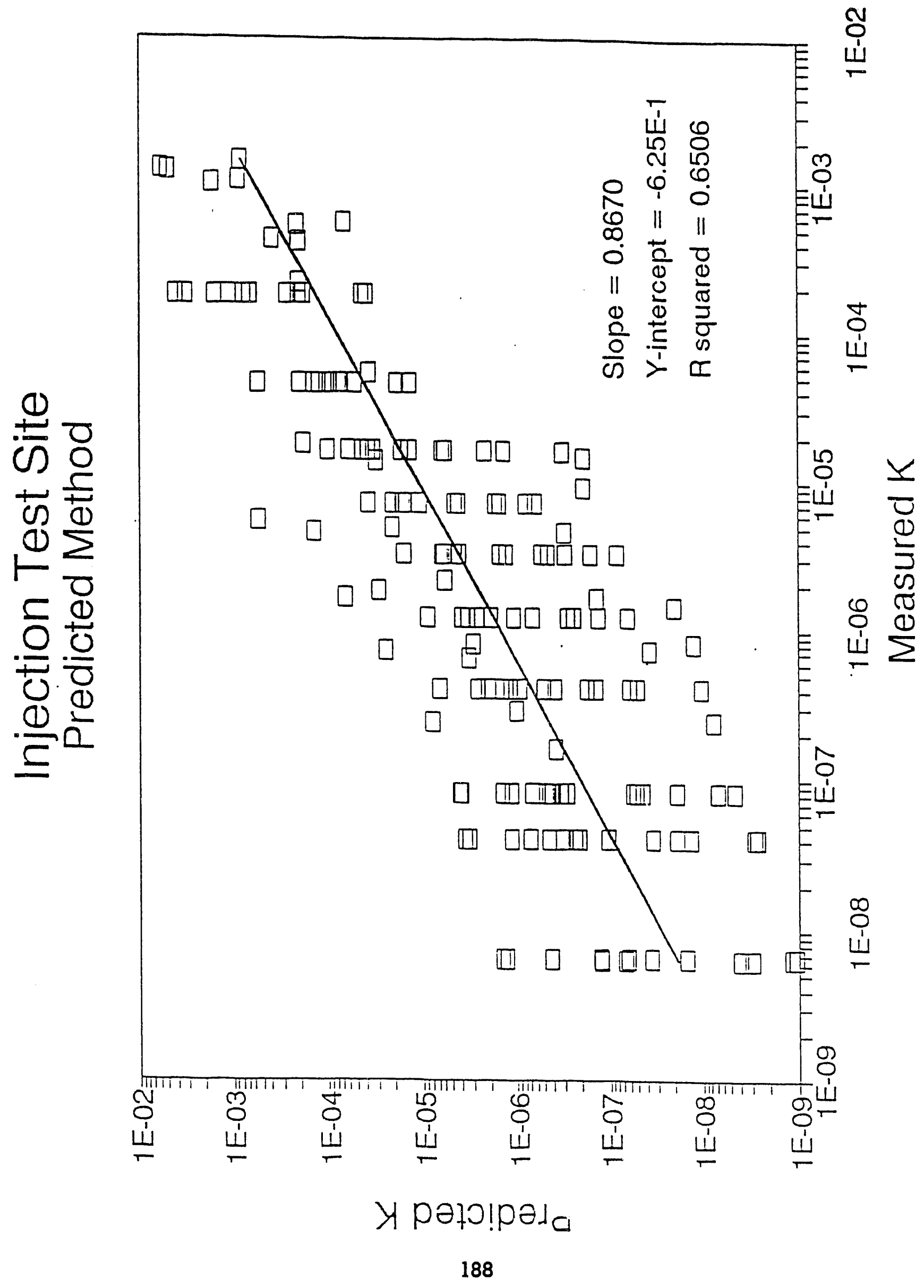


WHC-MR-0420

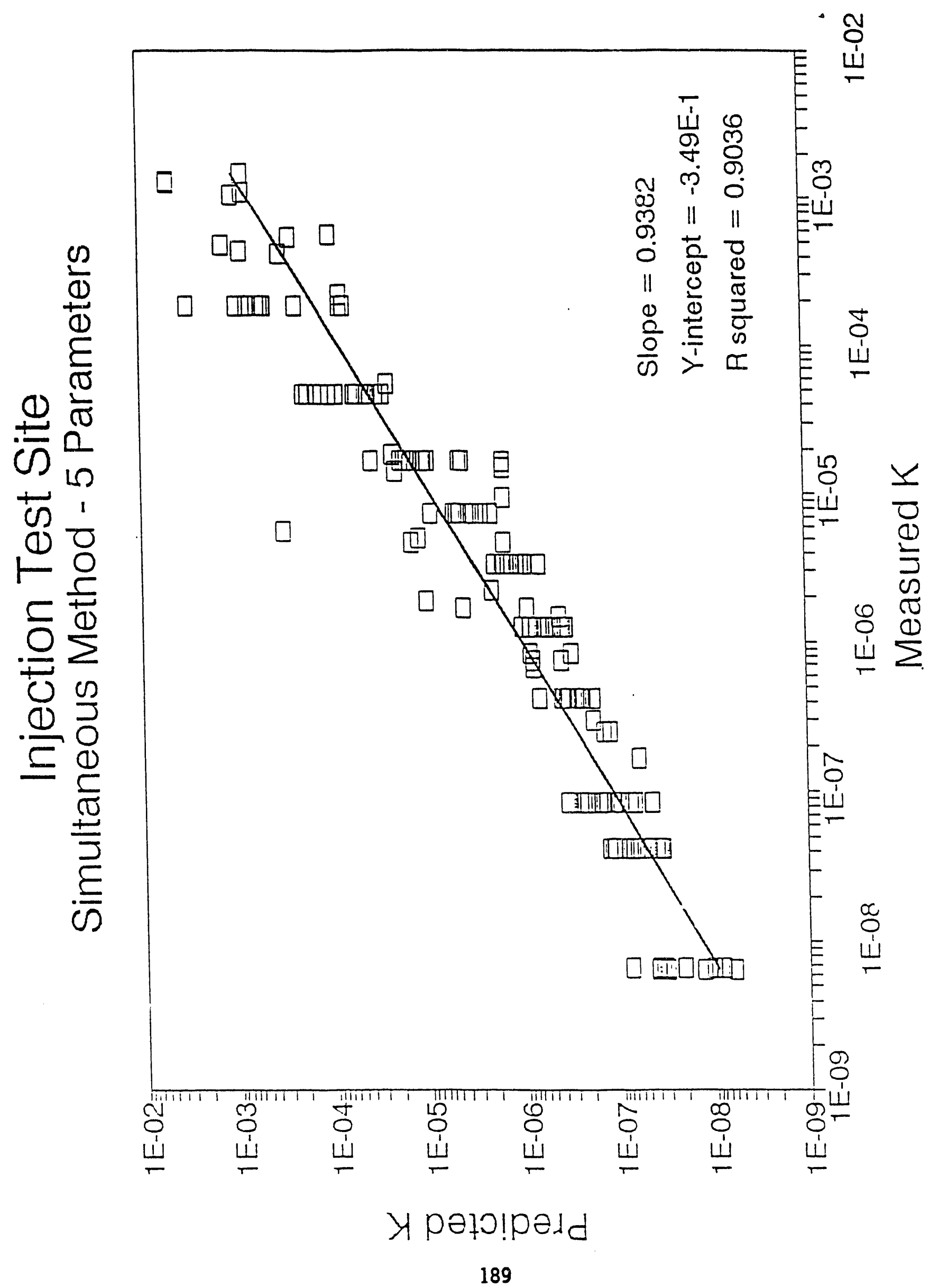


WHC-MR-0420

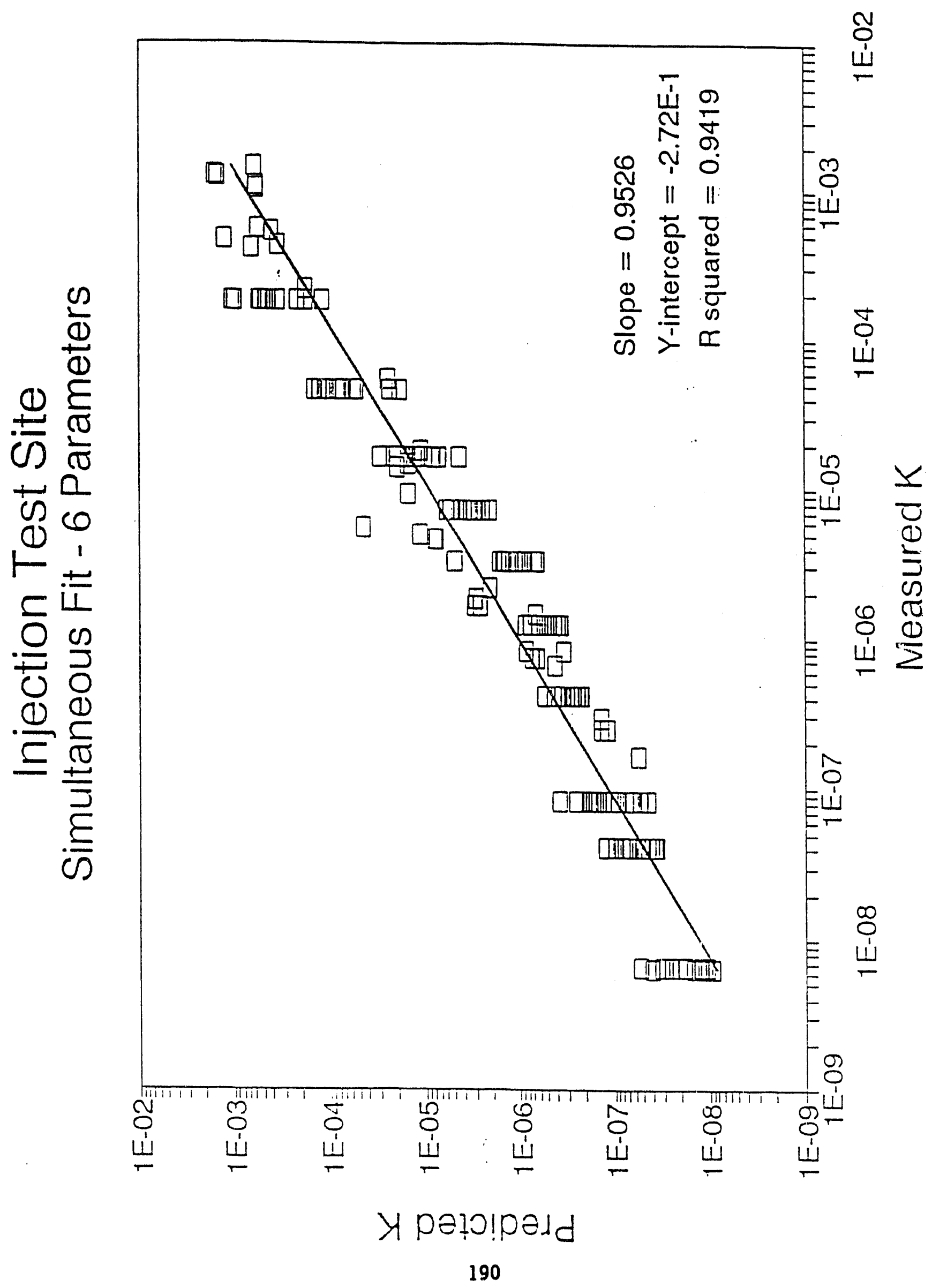




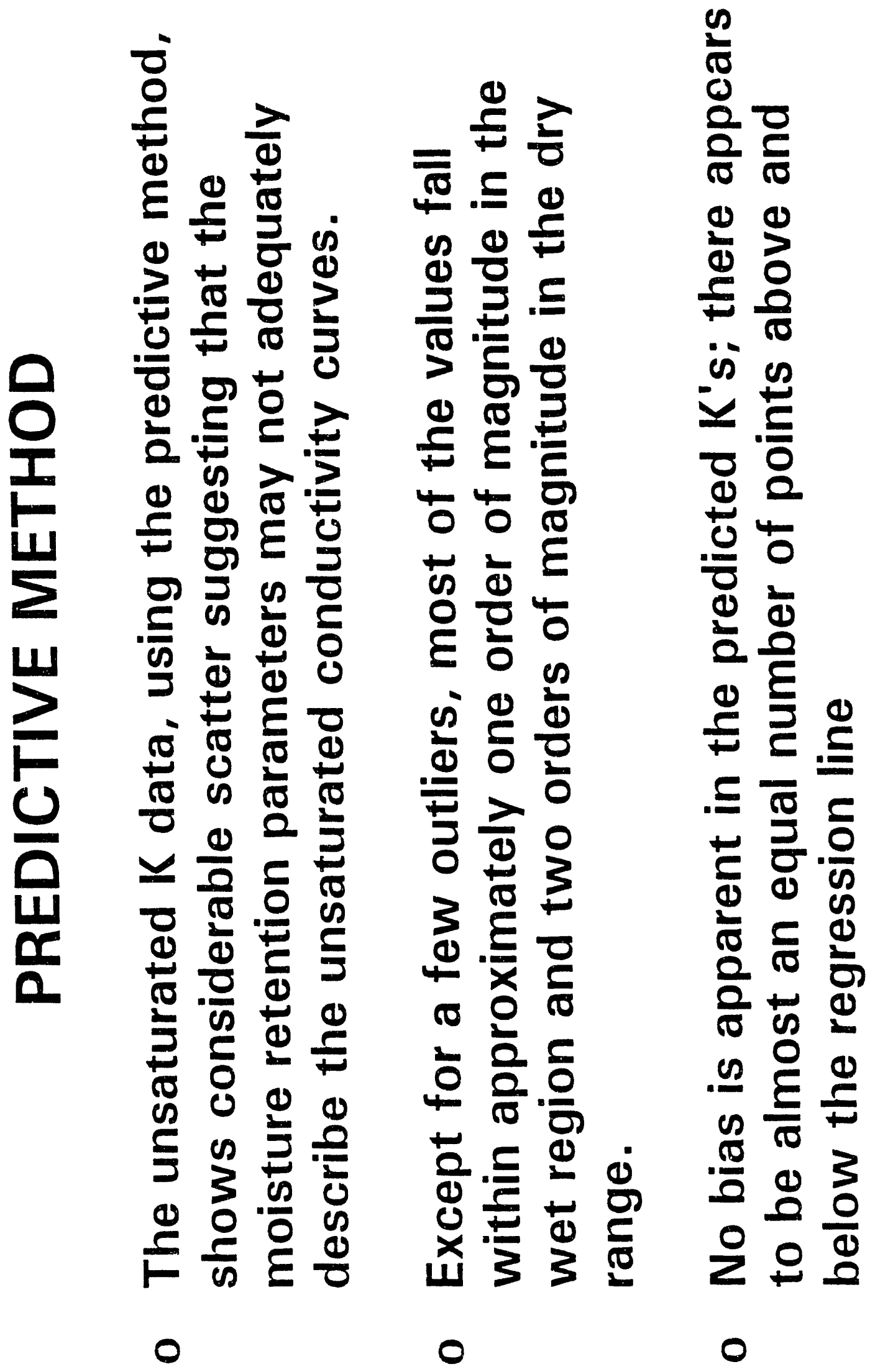




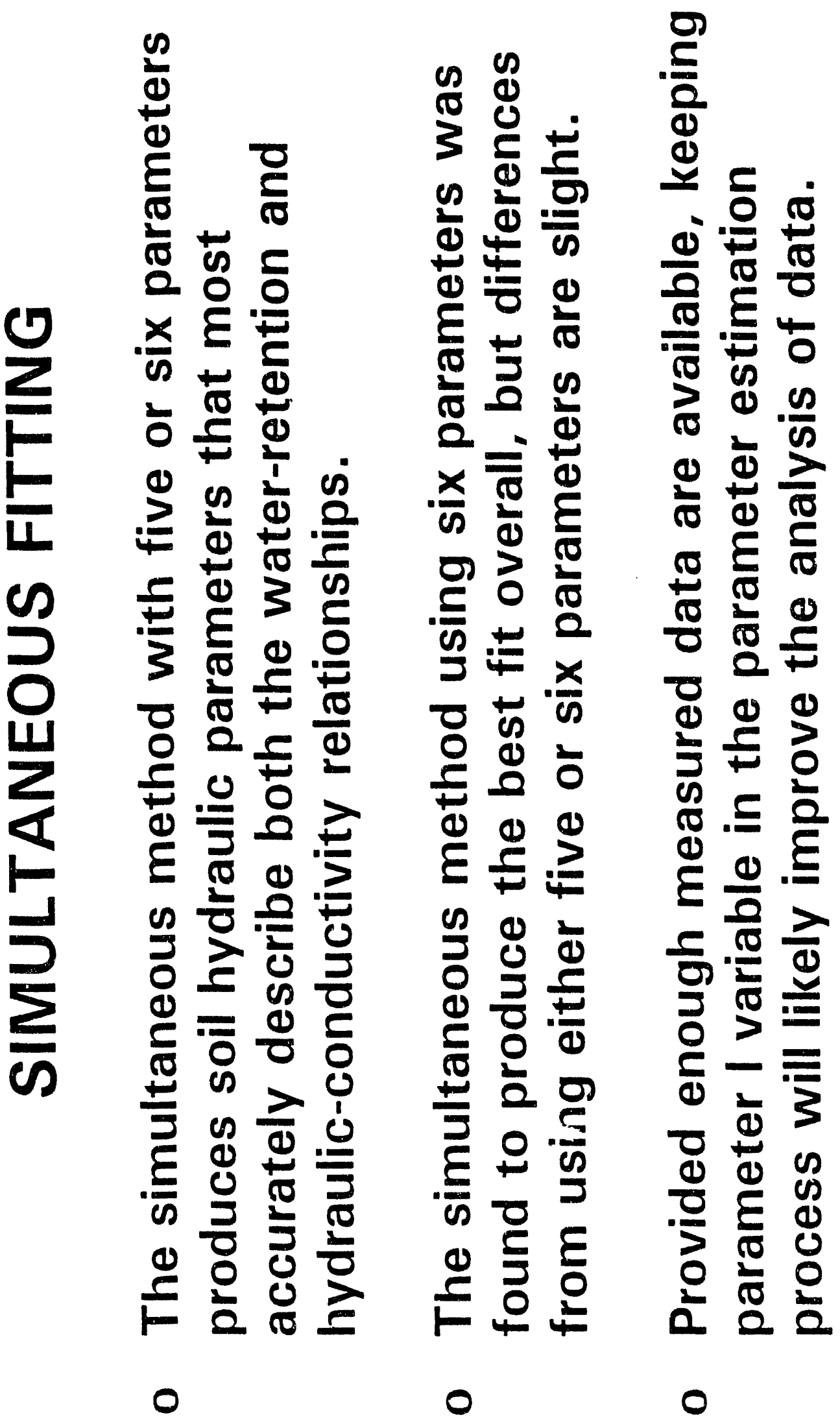




\title{
USE OF ARYA-PARIS MODEL TO ESTIMATE MOISTURE RETENTION DATA
}

\author{
BEN VOLK AND RAZ KHALEEL
}

Why is MRC versus PSD Correlation Necessary?

- Inability to collec: moisture tetention data on every soeracle unit

- Cosi ol Moisture Retention Curve

$\$ 2200$ per sample as compared io $\$ 200$ for Sieve Analysis

- Time 10 Pertorm Moisture Retenion Analysis

27 hours der samole as comoared to 1 hour ior sieve

analysis

- Accuracy of laboratory cerived aata

- Accuracy of in silu field tests

- Reduce number of cnaracierization wells that must be orilled

- Use locally measured soll data lief 200E. 200W, and 300 Areasi to Dredic: contamination movement across the site 


\section{WHC-MR-0420}

Arya and Paris Model (1981)

- An empirical model with constant. a.

- Underlving assumotions:

- Soll particle size related to a corresponding pore diamets:

Model treats the soll as a bundle of captllary :ubes

- Capillary tube volume is a function of

a) particle size

c) empirically determined constant.

- Inout dara needed: Parricie Size Distribution. Bulk Density. ar:z Particle Density.

- Sied 1. Divide the PSD data into M size tractions where ine so:ir mass in the ith Darticle class is equated io ine inass $\partial$. sonerical particies of radius ${ }_{;}$; Volume of the sarticles. $V_{2}$. :

$$
v_{2,} \cdot 4 / 3: N_{1} \times 8 \text {. }
$$

The volume of the volds. $V_{v i}$ can be restesented ov a singre caowlary tupe of radius '

$$
\because \cdots: \cdots
$$

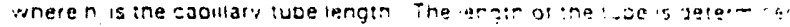

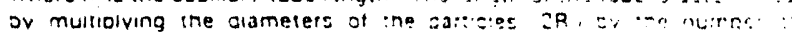
Dartises:

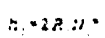

ivhere $a$ is an emoirical constant detween i ine 2 . The g term is isedies to account for the non-sonetical nature at some of the satticles

\section{Arya and Paris Model - Continued}

By combining equations (11), (2), and (2). Arva ano Paris arrives it ine exoression for caollary tude radius. $t:$

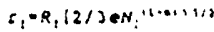

where e is the vold ratio. The rube sadus is inen related to the caolliar. nressure. $\psi_{i}$, via the equation for rise of water in a cjoillary iude:

$$
\rightarrow: 0,000 \theta / 0.3:
$$

vere is is the soll water pressure, is the surtace tension ol water " s

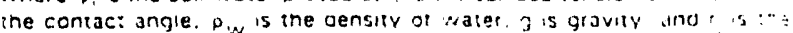
rore ralus.

- Arva-Paris tound that a ranged trom 1.35 to 1.39

\section{Arya and Paris. Determining Alpha}

- Pertorm iterauve analvsis or measureo cjolliary pressures vers . zredic:eo caoillary jessures oy jssurning an dions :arimizing ine root mean seujrec error ifortron Prociram. 
WHC-MR-0420

ARYA ANO PARIS MODEL

Comoutational Procedure

input

Particle Dlam.

Interval cum

Bi $(\mathrm{cm})$

Av. Pagurs

Weight Pore Vol.

$w(9)$ v

7.50E-05

1.75E-04

$3.75 E-04$

7.50E-04

1.25E-03

2.00E-03

3.75E-03

7.50E-03

$1.75 E-02$

3.00E-02

4.25E-02

$7.50 E-02$

0.140

vi

$\frac{v_{v i}}{0.03001}$

$\begin{array}{lll}0.030 & 0.00643 & 0.03644\end{array}$

$\begin{array}{llll}0.074 & 0.01586 & 0.05230\end{array}$

$\begin{array}{lll}0.076 & 0.01629 & 0.06859\end{array}$

$\begin{array}{lll}0.115 & 0.02465 & 0.09325\end{array}$

0.120

$0.02572 \quad 0.11897$

$0.03430 \quad 0.15327$

0.021440 .17470

$0.01393 \quad 0.18863$

$0.01501 \quad 0.20364$

$\begin{array}{lll}0.070 & 0.01501 & 0.20364 \\ 0.020 & 0.00429 & 0.20793 \\ 0.015 & 0.00322 & 0.21114\end{array}$

$\begin{array}{lll}0.015 & 0.00322 & 0.21114 \\ 0.015 & 0.00322 & 0.21436\end{array}$

$\frac{0.015}{1.000}$

$0.5917 \mathrm{~cm} \cdot 3$ 31 gram sample)

Bulk Volume=

Bulk Densilym

Surlace Tension

Contact Angleo

Water Density

Gravilye

Alpha consiani:

Pant. Density-

$1.69 \mathrm{~g}^{\mathrm{cm}} \cdot 3$

72 ornesser

0 degrees

1 grm $\cdots \cdot$

$10 \mathrm{~cm} \cdot 3$

1.38 unilless

$2.65 \mathrm{~g} / \mathrm{cm}^{\cdots} \cdot 3$

B. W. voik

$7-29-92$

Vol. Water Average VWC Number of Mean Pore Soll water

(Theradarvi) Part, ni

$\frac{n+11-a j p h a)}{-}$

agus.II $P S$

6.32E-07 $2.32 \mathrm{E}+05$

2.01E-06 7.29E+04

6.63E-06 2.22E,04

$1.82 E-05 \quad 8.07 E+03$

$4.03 E-05$ 3.65E +03

7.97E-05 1.84E+03

2.34E-04 6.28E+02

$7.54 E-04 \quad 1.95 E+02$

$2.81 E-03 \quad 5.23 E .01$

8.31E-03 1.77E.01

$1.52 E-02 \quad 9.69 E+00$

3.70E- $02 \quad 3.97 E+00$

\section{ARYA-PARIS MODEL CE702}

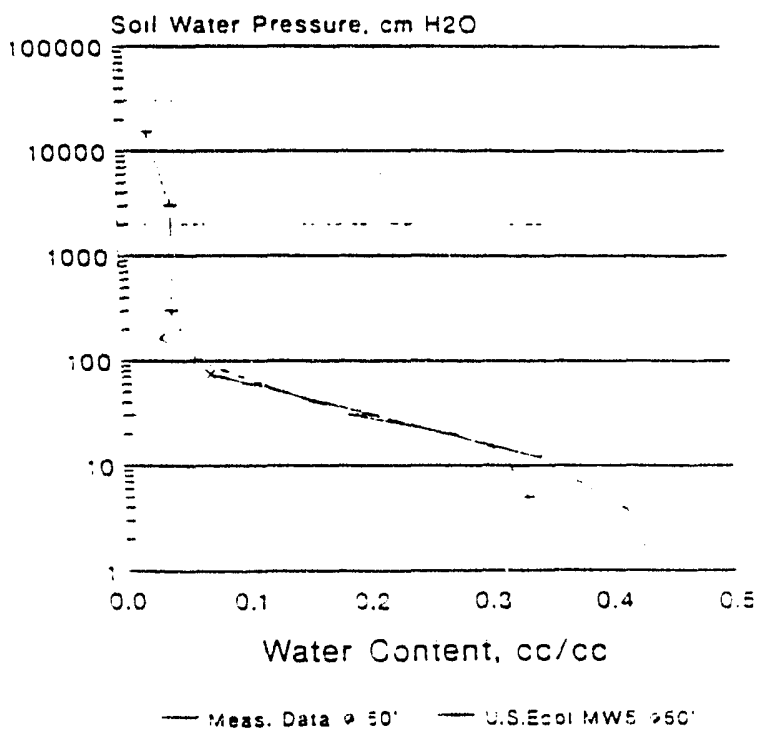

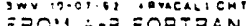

MATYACML=.

$\therefore \angle P H A 2$ 


\begin{tabular}{|c|c|c|c|c|c|c|c|}
\hline & & & Dooun & & & & W. Volk \\
\hline$\frac{\text { Sild }}{\text { FLYF }}$ & Sampie & $\leq$ & Exen & Esmation $T_{y}$ & Solil Tres & 0 & \\
\hline ? & $002-10$ & 1 & $<20$ & Hentord-Udoer Sand & Sill Loam & 2.63 & $\frac{A \log A}{1.17}$ \\
\hline - & $\begin{array}{l}002-16 \\
004-04\end{array}$ & 2 & $<20$ & Hensord-UDDer Sand & Sill Loam & 2.60 & 1.19 \\
\hline - & $004-10$ & 3 & $<20$ & Hentord-Uppor Serd & Sill Loam & 2.52 & 1.20 \\
\hline - & $005-03$ & 5 & $<20$ & Hantora-Udoer Sand & Silt Loam & 251 & 1.19 \\
\hline - & $007-04$ & $\begin{array}{l}5 \\
6\end{array}$ & $<20$ & Henlord-Uoder Sand & Sult Loam & 2.60 & 1.22 \\
\hline - & $008-15$ & $\frac{6}{7}$ & $<20$ & Hantord-Upper Sand & Sil Loam & 251 & 1.20 \\
\hline . & $009-01$ & $?$ & $<20$ & Hunlord-Upoer Sand & Sill Loam & 2.61 & 1.18 \\
\hline$:$ & $009-02$ & 9 & $<20$ & Hanlord-Uoper Sand & Sull Loam & 2.61 & 1.16 \\
\hline : & & 9 & $<20$ & Hanioro-Upper Sand & Sill Loam & 2.61 & 1.18 \\
\hline : & $009-03$ & 10 & $<20$ & Hantord UDDer Sand & Sllt Loam & 2.55 & 1.26 \\
\hline i & $010-04$ & 11 & $<20$ & Hantord-Udoper Sand & Silt Loam & 2.56 & 1.23 \\
\hline : & $011-08$ & 12 & $<20$ & Hantord-Udoder Sand & Sill Loam & 2.51 & 1.20 \\
\hline$\dot{0}$ & $011-08$ & 13 & $<20$ & Henlerd-Udoen Sand & Sill Loam & 2.57 & 1.21 \\
\hline - & $012-14$ & 14 & $<20$ & Hanioco-Upder Sand & Sul Loam & 2.64 & 1.16 \\
\hline - & $013-08$ & 15 & $<20$ & Hesloro-Uooer Sand & Sill Loum & 261 & 1.17 \\
\hline$\dot{1}$ & $014-04$ & 18 & $<20$ & Hentoro-Upoer Sand & Sill Loam & 2.81 & 1.18 \\
\hline U. S. Ecology MW-5 & so & 17 & so & Hantora-Upper Sand & Sand & 2.20 & 1.25 \\
\hline & 70 & 18 & 70 & Hentord- Uoder Sand & Sano & 2.49 & $\therefore .25$ \\
\hline$\dot{0}$ & 90 & 19 & 90 & Hantord-UDoder Sand & Sand & 2.10 & 1.26 \\
\hline : & a 130 & 20 & 120 & Handord-Udoem Sand & Sind & 2.17 & 1.23 \\
\hline : & 170 & 21 & 170 & Mentowa-Udow Sand & Sand & 2.19 & 1.24 \\
\hline : & - 190 & 22 & 190 & Heniora-Lower Sand & Sand & 2.05 & 1.31 \\
\hline - & P 210 & 23 & 210 & Hensora-lower Sund & Sand & 2.04 & 1.21 \\
\hline - & Q 230 & 24 & 230 & Henioro-Lower Sind & Sand & 201 & 121 \\
\hline$\cdot$ & 270 & 25 & 270 & Hanjord-Lower Sund & Loamy Sano & 240 & 1.39 \\
\hline U. S. Ecologr MW- & (1) 14 & 26 & 14 & Heniora-Udoer Sana & Sand & 1.99 & 1.23 \\
\hline 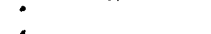 & (A) 145 & 27 & 145 & Maniora-Uoper Sana & Sanoy Loan & 272 & 1.08 \\
\hline$\cdot$ & Q 185 & 28 & las & Haniord-Midodo Sand & Sand & 2.01 & 173 \\
\hline U. S. Ecology MW-10 & (1) 45 & 23 & 45 & Meniora-Uooser Sand & Sand & 2.54 & 1.35 \\
\hline & 86 & 30 & 86 & Handoro-UDDer Sund & Sand & 2.32 & 128 \\
\hline - & ๑105 & 31 & 103 & Henloro-Uopor Sand & Sand & 2.44 & 1.41 \\
\hline - & (ه 125 & 32 & 125 & Henioro-Uoper Sand & Sand & 2.40 & 1.35 \\
\hline - & ค 185 & 33 & 165 & Haniono-UDDer Sand & Sand & 228 & 1.50 \\
\hline - & (7) 195 & ses & 195 & Hensora-ubdolo Sand & Sand & 2.26 & 1.82 \\
\hline - & 205 & 35 & 203 & Handoro-Lower Sand & Sand & 2.27 & 1.60 \\
\hline : & 245 & 36 & 245 & Henverd-Lower Sand & Sand & 236 & 1.28 \\
\hline i & 265 & 37 & 285 & Henioud-Lower Sand & Senc & 233 & 1.27 \\
\hline - & 2as & 39 & 283 & Hendord-lower Sand & Sand & 2.36 & 1.22 \\
\hline 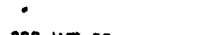 & Q 305 & פב & 205 & Meniend-Lower Seno & Seno & 225 & 1.27 \\
\hline $299-W 7-99$ & $w-069$ & 60 & 10 & Herioro-Uooen Sand & Senoy loam & 2.53 & 1.30 \\
\hline$\dot{1}$ & $w-072$ & 41 & 65 & Haniowa-Uoper Sand & Sanor Loum & 280 & 1.19 \\
\hline - & W-070 & 42 & 69 & Hanjorchlloover Sand & Sill Loam & 2.42 & 1.06 \\
\hline : & $w-080$ & 43 & 71 & Henterd-UDDer Send & Sanco Loam & 251 & 1.13 \\
\hline - & W.082 & $\mu$ & 82 & Manioro-Uoper Sand & Sandr Loam & $2: 8$ & 1.55 \\
\hline - & w- 085 & 45 & 88 & Heniord-Uoder Sand & Loamy Sano & 2.55 & 1.47 \\
\hline - & W-099 & 46 & 99 & Hanlord-Updor Sand & Loamy Sand & 2.54 & 1.56 \\
\hline : & $W-101$ & 47 & 104 & Henloro-Uoder Sand & Send & $2 \leqslant 2$ & 1.21 \\
\hline$\cdot$ & $w-104$ & 48 & 118 & Haniora-Uoper Sana & Sand & 240 & 119 \\
\hline . & $W-107$ & 49 & 132 & Hanioro-Uoper Sand & Sand & 2.45 & 1.15 \\
\hline${ }^{\circ}$ & $w-113$ & $\leq 0$ & 142 & $\begin{array}{l}\text { Hantoro-Uopar Sand } \\
\text { Arutumaue Mean a }\end{array}$ & Sand & $\frac{2.23}{2.43}$ & $\frac{1.10}{1.27}$ \\
\hline & & & & Stancawa Deviation - & & 0.20 & 0.15 \\
\hline Ealubratuen Perameiers & Along & Soer & & & Along & & \\
\hline Send Preduction - & 1.33 & 0.16 & 227 & Loamy Sand Prod. - & 1.47 & $\frac{20.7}{0.07}$ & $2 \leq \frac{0}{2}$ \\
\hline Numbar of Samoles - & 25 & & 25 & Number ol Samoins & ง & & J \\
\hline $\begin{array}{l}\text { Sill Loam Pred. - } \\
\text { Numoer or Samoies }\end{array}$ & 1.18 & 0.03 & 259 & Sandy Loum Prod. - & 1725 & 0.17 & 2.61 \\
\hline rolsamnos & 17 & & 17 & Hurnoer of Sampies & 5 & & \\
\hline
\end{tabular}

\section{ARYA PARIS FITTING PARAMETERS: SO SAMPLES}

\begin{tabular}{lrl} 
Calibration Paramerers & Alpha & SDev. \\
Sand Prediction $=$ & 1.33 & 0.16 \\
\hline \hline Number of Samples = & 25 \\
Silt Loam Pred. & 1.18 \\
\hline Number of Samples = & 17
\end{tabular}

\begin{tabular}{|c|c|c|c|c|}
\hline D & & Alpna & SDov & $D$ \\
\hline 2.27 & Loamy Sand Pred. $=$ & 1.47 & 0.07 & 2.52 \\
\hline 2.59 & $\begin{array}{l}\text { Number of Samples } \\
\text { Sanov Loam Pred. }\end{array}$ & 3 & 17 & \\
\hline & Numb & & 0.17 & 2.51 \\
\hline
\end{tabular}




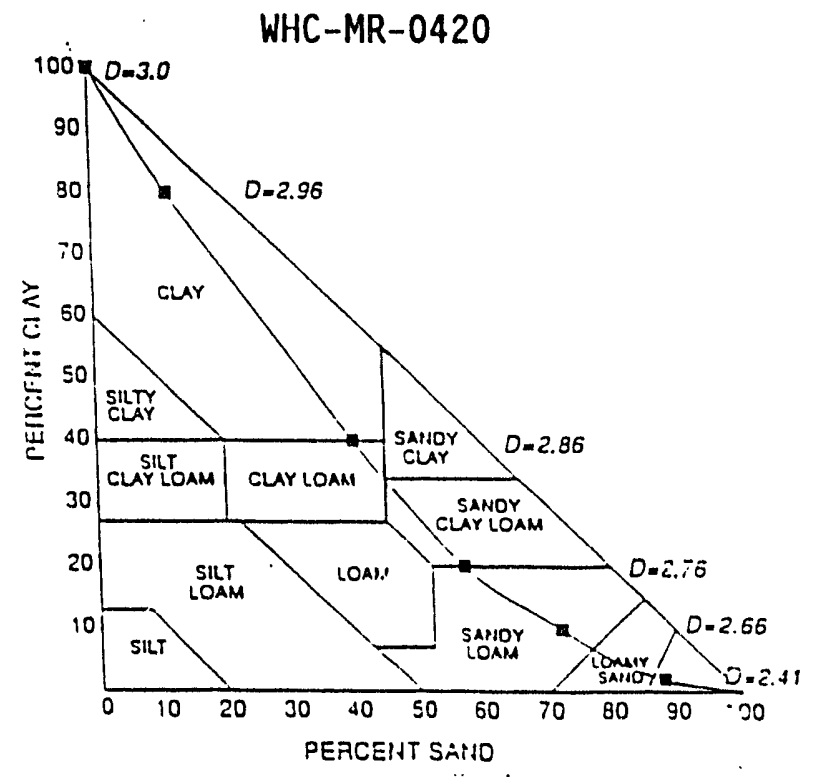

From Tyler and Wheateraft, 1992

\section{Alpha Vs D}

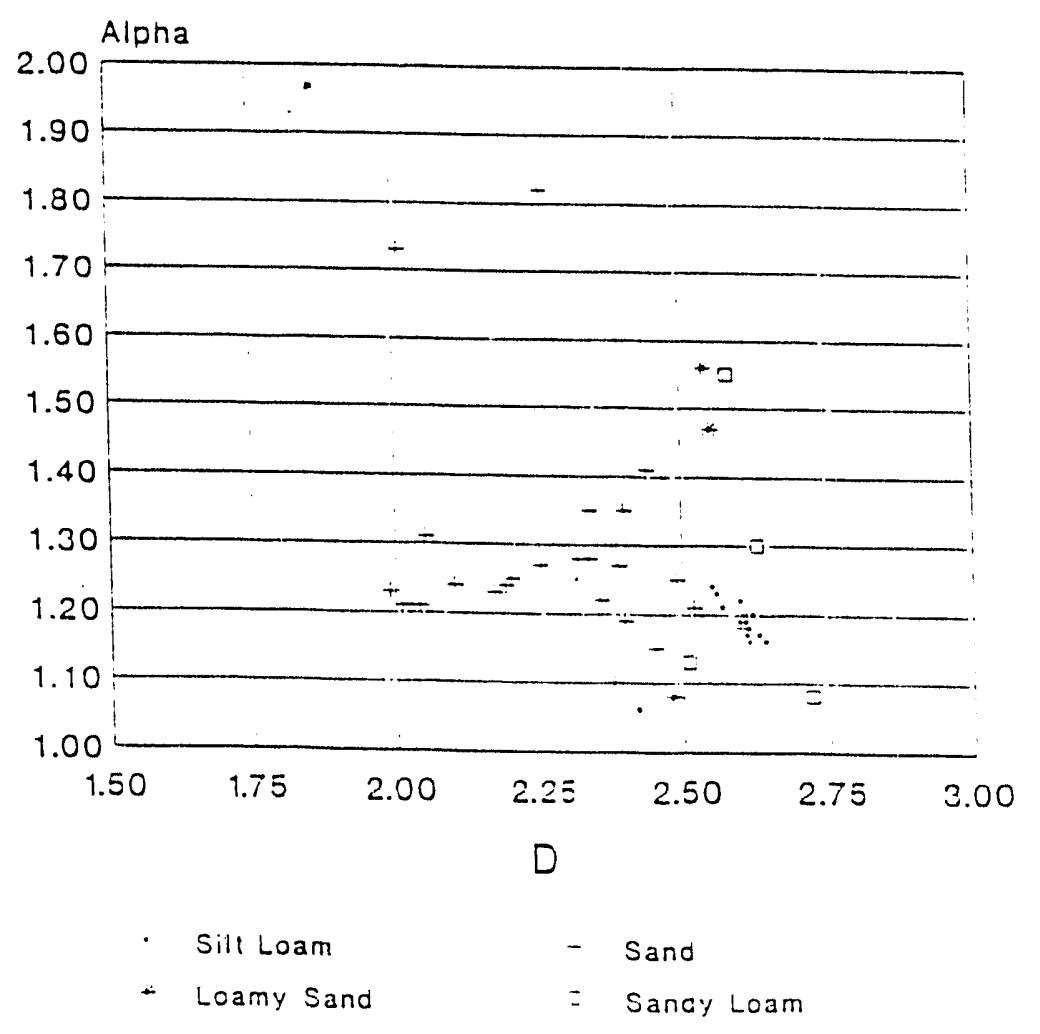

g. W. Volk 03-24-93 

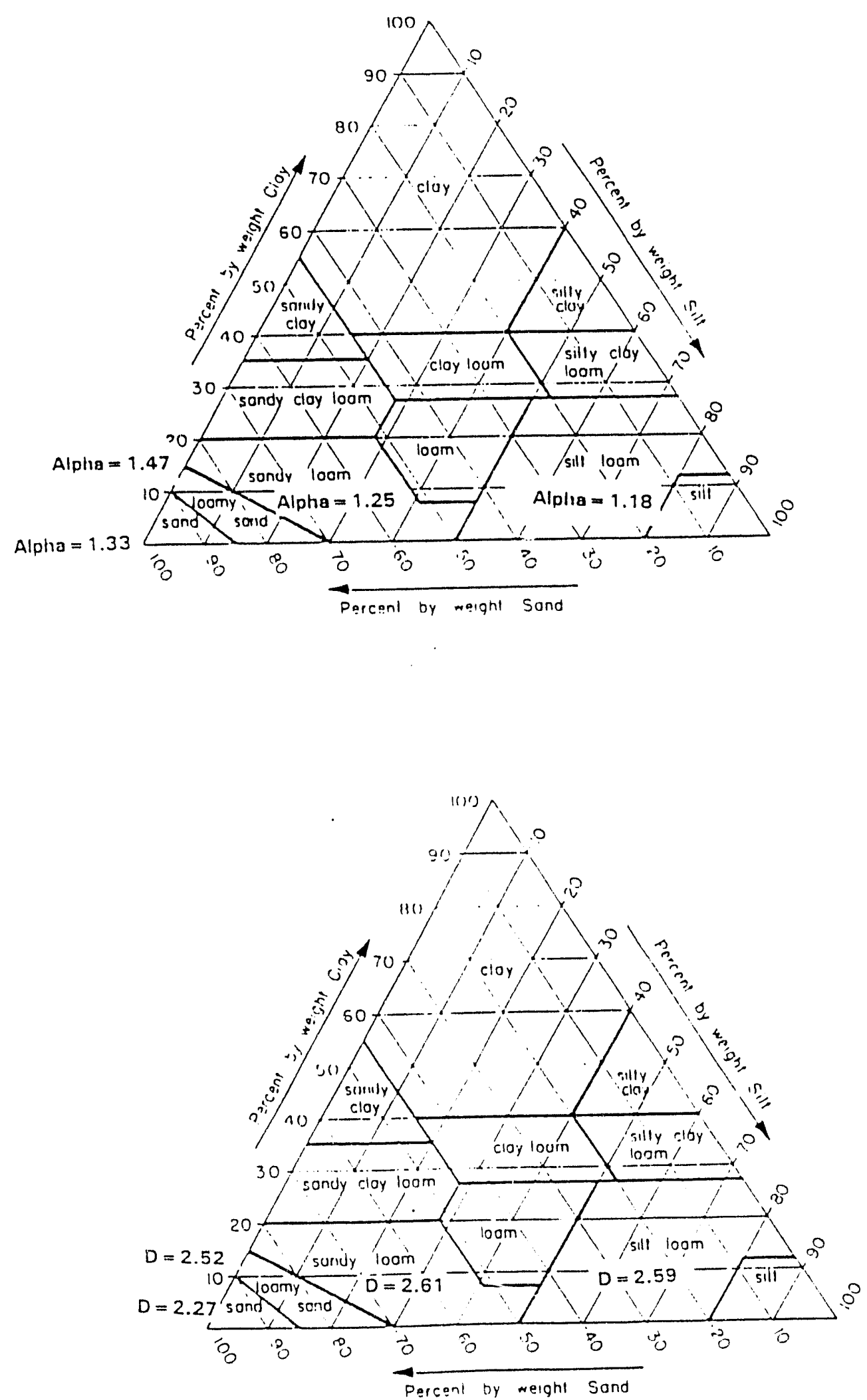


\section{ARYA-PARIS MODEL \\ Inj. Test Site, Sample 2-1636}

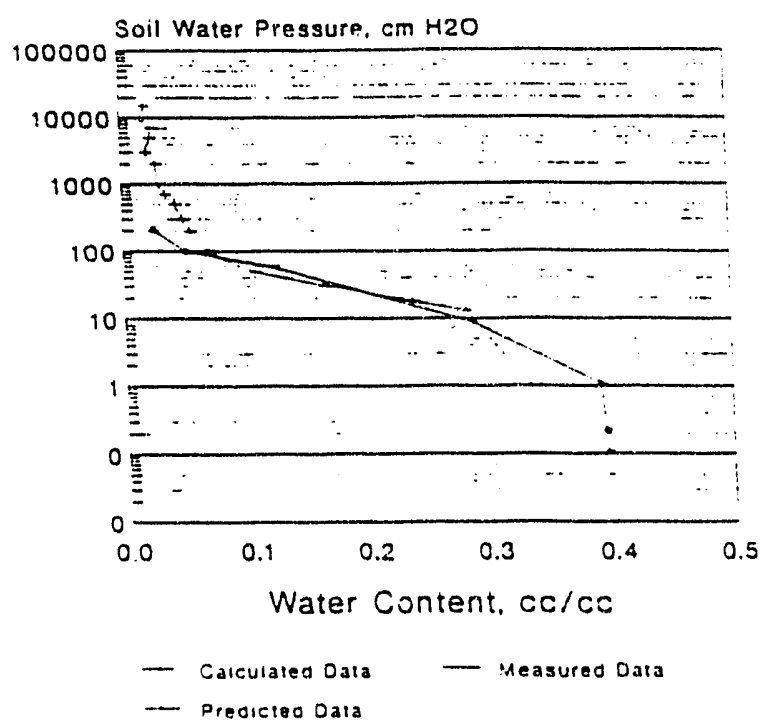

BWV 03-14-93. SAND

From Aotilior. SANO 133 P 0

Calc Alona - i.34, Rsa - 980

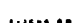

ARYA-PARIS MODEL

inj. Test Site, Sample 2-1639

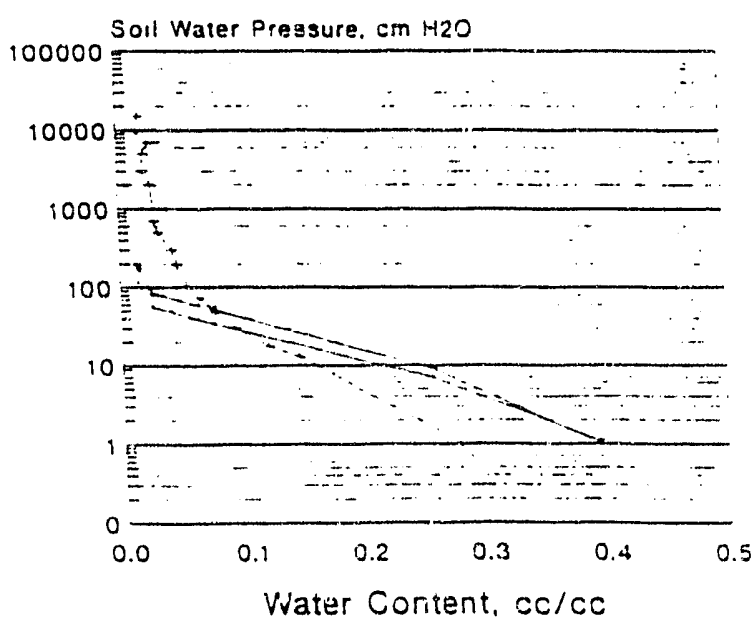

- Calculated Oala - Measured Data
- Predicted Cala

BWV 03-14-93. SAND

Eom Aollt.for. Alona - 133. Asa - $\$ 68$

Salc Alona - 1.23. Rag - 96: 


\section{ARYA-PARIS MODEL}

Inj Test Site, Sample 1-1417

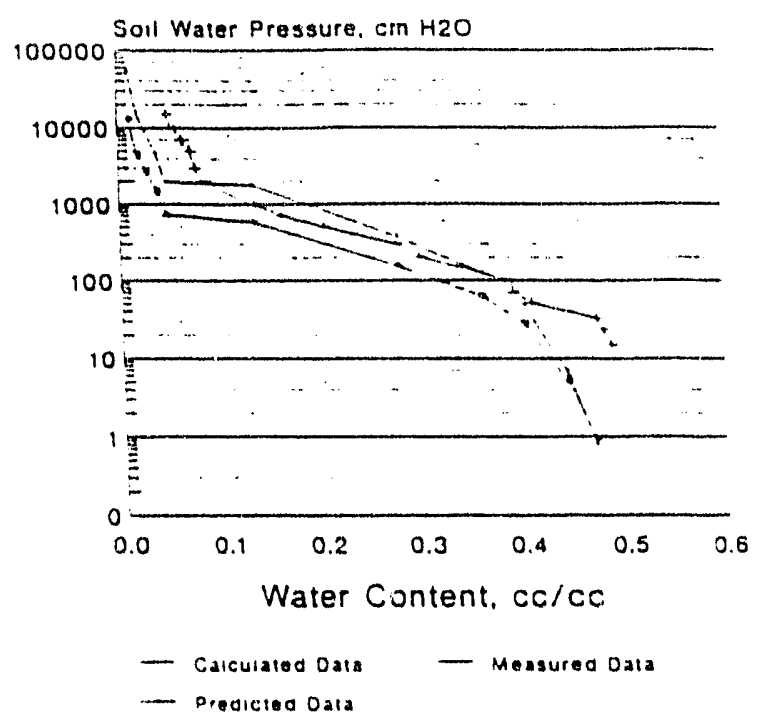

QWV 03.11.93. SANOY LOAM

From Aotlt.tor. Alona - 1.25. HeO - 378

Caic Alone - 1,48. Asa - $98 \mathrm{~J}$

ARYA-PARIS MODEL

Inj Test Site,Sample 2-2230

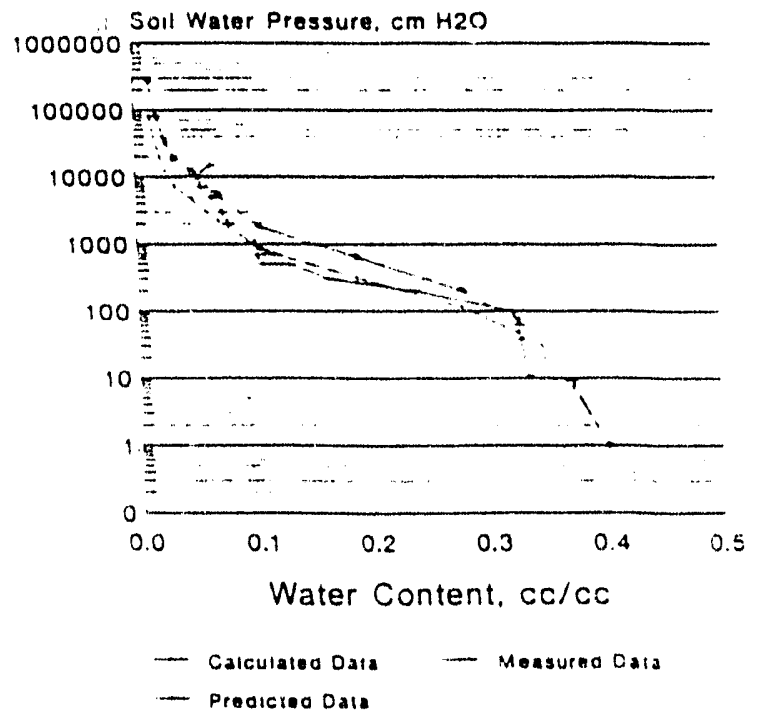

BWV 0J-11-93. LOAMY SAND

From Aollt: or Alona. I, A7. Aso. 947

Salc alena. is Aso. 97 


\section{ARYA-PARIS MODEL 200-BP-1, Sample 1-0531}

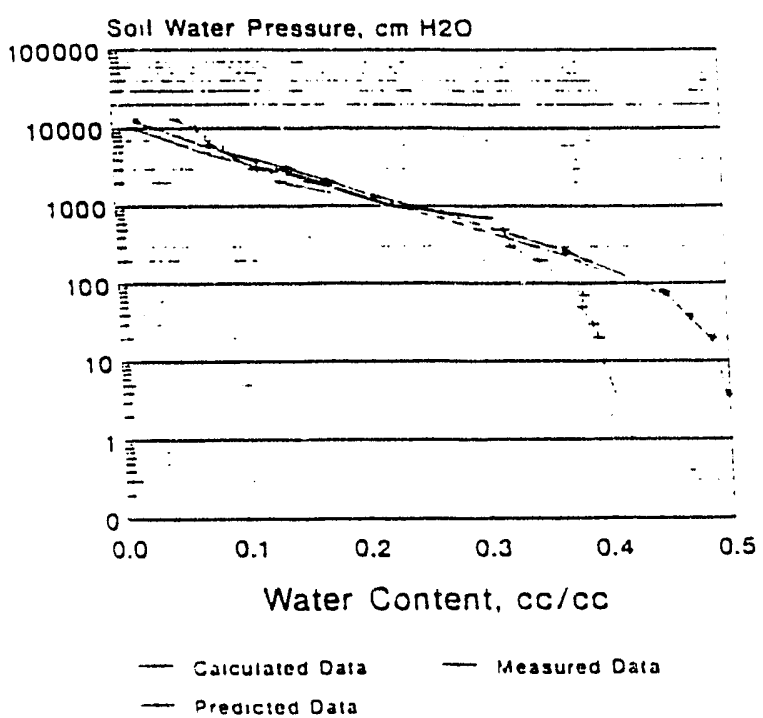

BW' 32-11.93. SILT LOAM

From Aotutior Alona • 18, R90 - 986

ARYA-PARIS MODEL 200-BP-1, Sample 1-0531

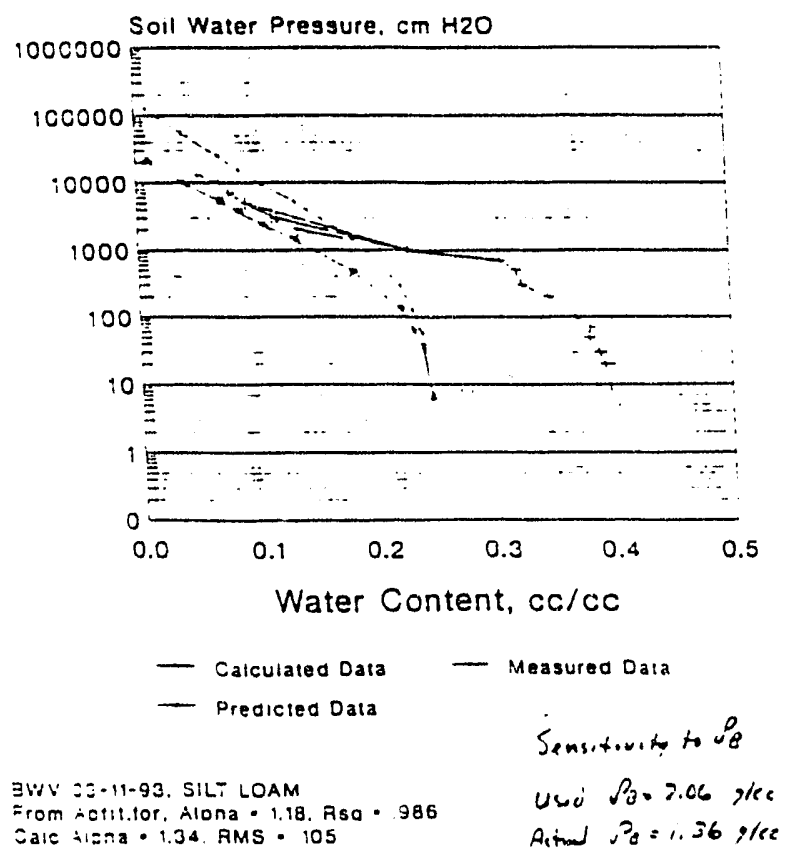




\section{ARYA-PARIS MODEL}

AP Tank Farm, Sample 241-AP-2

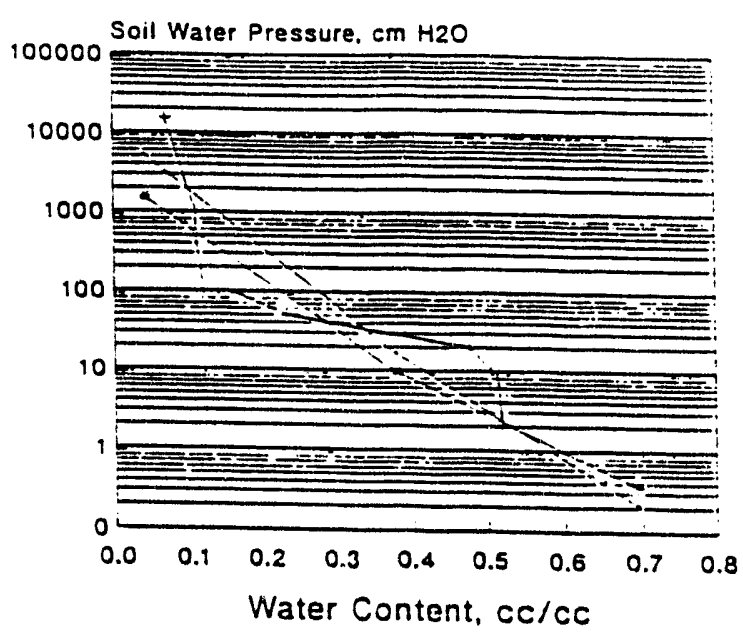

- Calculaled Daia

- Measurnd Data

- Preoicted oala

BWV 33-19-93. 5A110

ARYA-PARIS MODEL

AP Tank Farm, Sample 241-AP-3

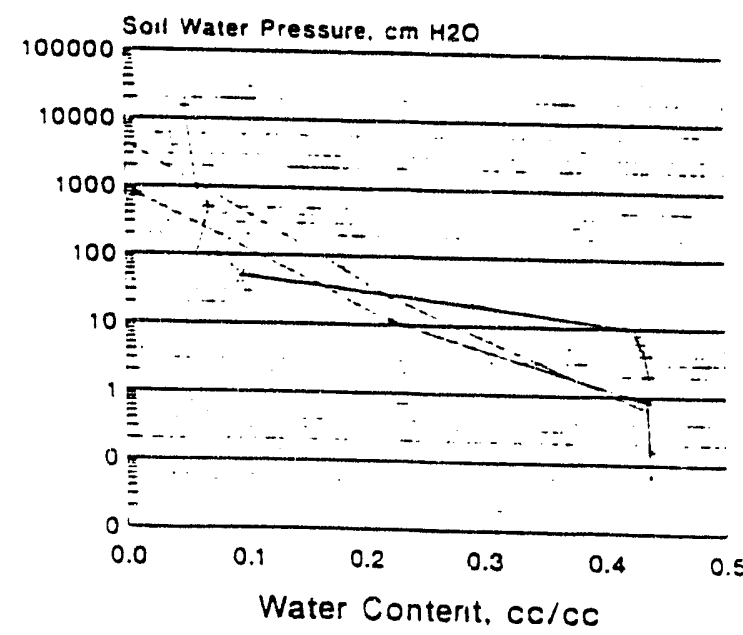

- Calculated Cata - Measured Data 


\section{ARYA-PARIS MODEL \\ AP Tank Farm, Sample 241-AP-4}

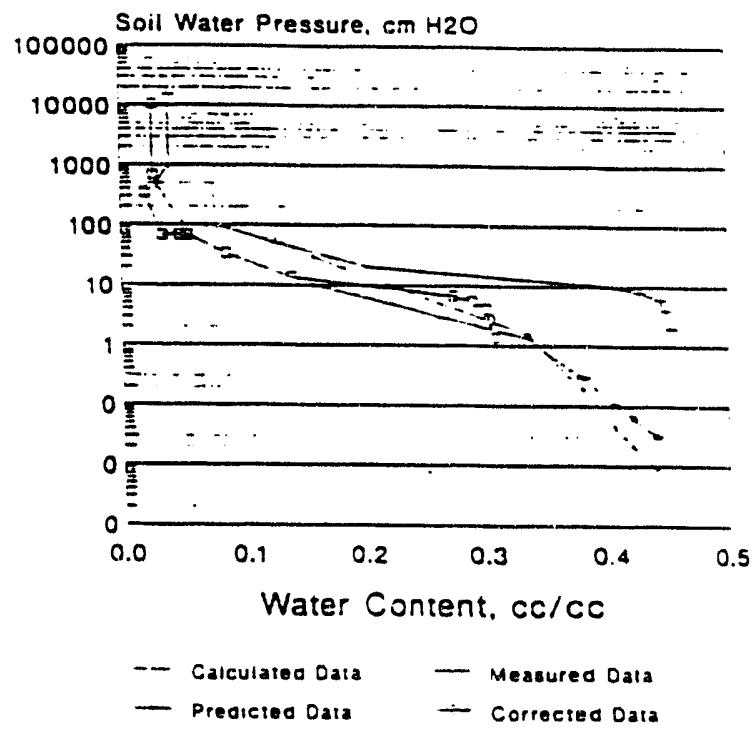

BWV 03-19-93. SAND

From Aofit.for. Alone - 1.33. Aso - 816

Cale Alona $\cdot 1.68$. Red -958

ARYA-PARIS MODEL New Jersey Soil, Sample S58NJ-12

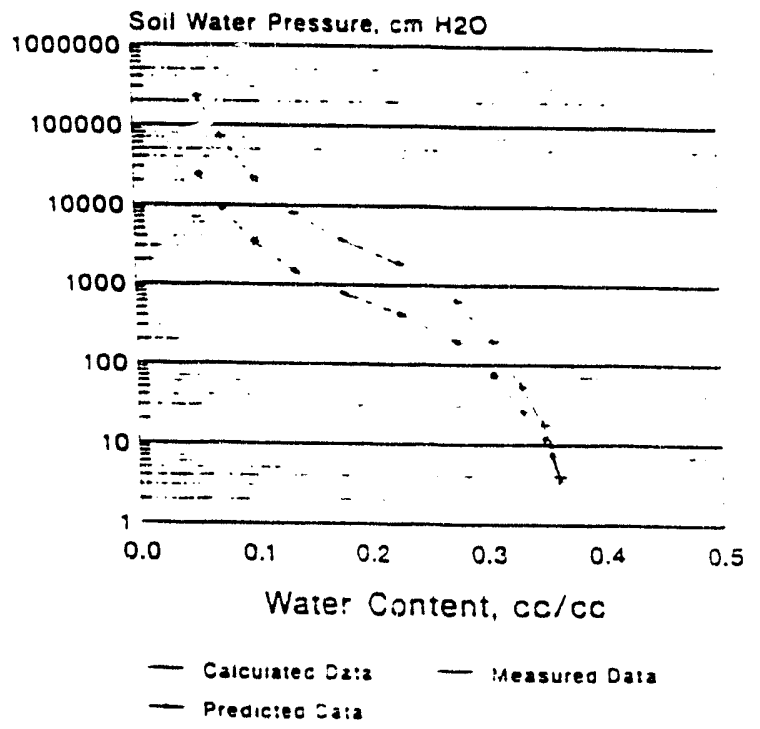

3WV 03-21-93. SILT LOAM

rom Aolit.for, Alona - 1.18. Fse - 32z

Salc Alone $\cdot 1.38$. A $80 \cdot 996$ 


\section{ARYA-PARIS MODEL} New Jersey Soil, Sample S58NJ-12 Sensitivity to Bulk Density

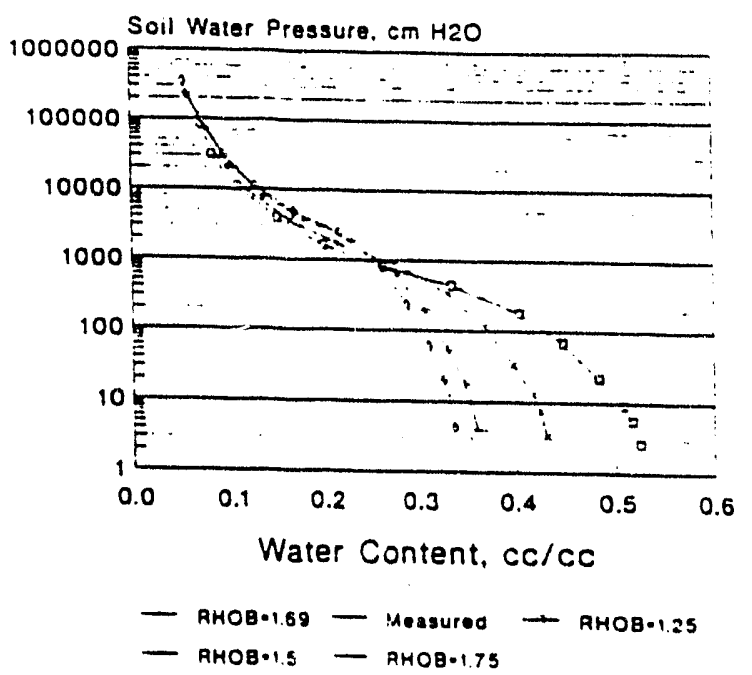

\section{ARYA-PARIS MODEL ISSUES}

- Sensitivity to bulk density

- Sensitivity to Alona

- Reduce by using Arya-Dierolf improved model

- Full sievo analysis

- Dry sieve and hydrometer

- Correct moisture retention curves for $22 \mathrm{~mm}$ size

- Hysteregis

- Model can be based on drying or wetting curve

- Emperical modal

- No physical significance of aloha parameter

- Physical significance with alona.

- Spatial variability

- Geology may be cifficult :o predict

- Calcium carbonate cemeniung 


\section{ARYA-PARIS MODEL CONCLUSIONS}

- Accurate correlation is obtainable provided: - Number of samples in database is increased

- Significant cost reduction:

- less cost per sample

- less wells drilled

- Signiflcant time savings:

- Expedite Hantord Sile characterization and remediation 
WHC-MR-0420

This page left intentionally blank. 
WHC-MR-0420

\title{
Dispersivity Estimates
}

\author{
Lynn Gelhar \\ Massachusetts Institute of Technology
}


WHC-MR-0420

This page left intentionally blank. 
DISPERSIVElY?

- a measure of contaminant spreading and dilution?

- un unpredictable fudge factor?

- depends ox scale?

- determined by small-scall heterogeneity?

209 


\section{STOCHASTIC METHODOLOGY}

$\Rightarrow \operatorname{lnK}$ variability represented by 3D covariance/spectra

re Gelhar \& Axness (1983), WRR 19(1)

Gelhar (1984) NATO ASI \# E82

Gelhar (1987) NATO ASI \# E128

Geikr (1993) Stochatie Subsurfoce Hydrology, P4. with spatial correlation scales $\lambda_{i}, i=1,2,3$

$\Rightarrow$ flow equations with random parameters

$\rightarrow$ stochastic p.d.e.

$\Rightarrow$ perturbation solution using spectral technique assuming local statistical homogeneitv

$\Rightarrow$ generic analytical results

- large scale mean behavior (effective properties)

$\mathrm{K}_{\mathrm{ij}}$ effective conductivity

$\rightarrow A_{i j}$ macrodispersivity

- variance of dependent variables

$$
\begin{aligned}
& \sigma_{h}^{2}, \sigma_{c}^{2} \\
& \text { head conc. } \\
& \text { * cevoriance }
\end{aligned}
$$



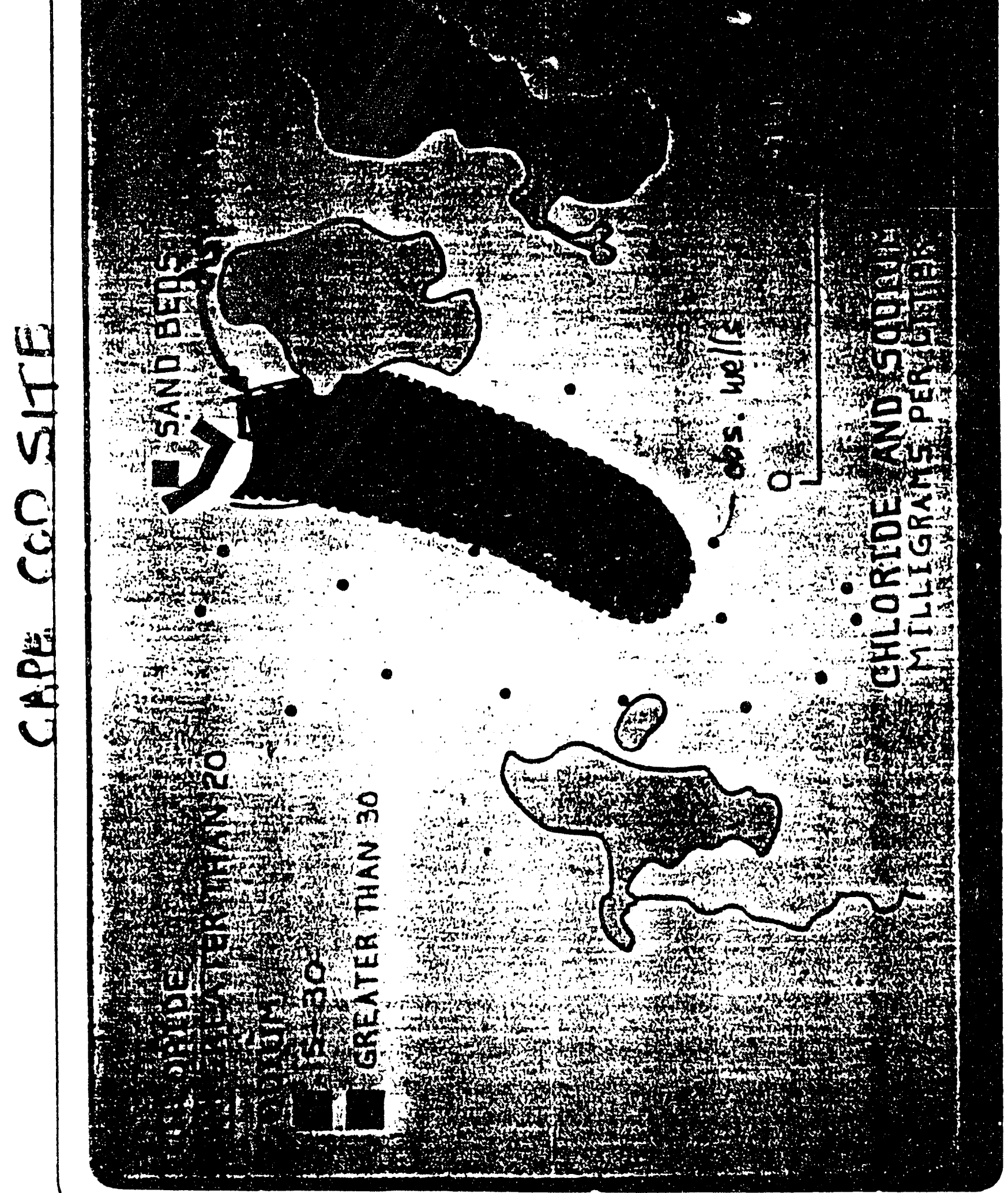


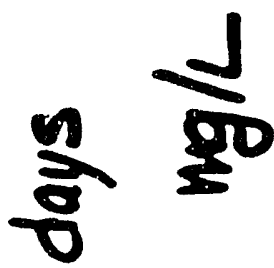

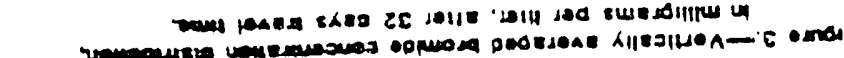

in $\frac{8}{\wedge}$
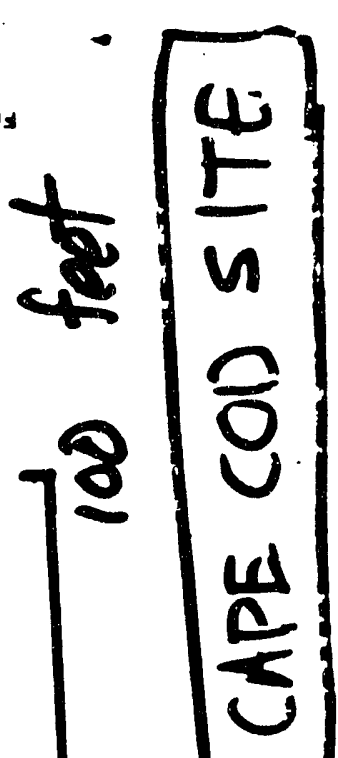

(1)
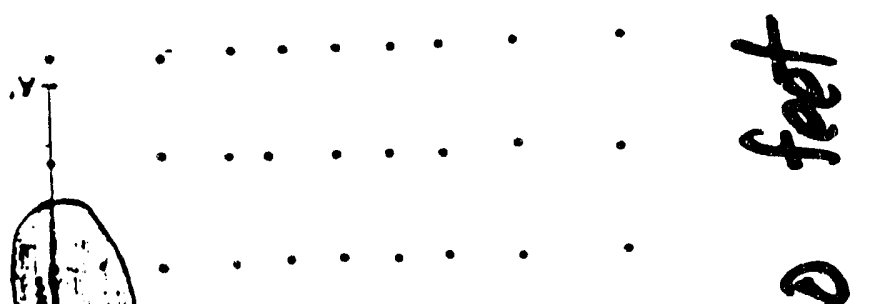

0
8
1
0

a
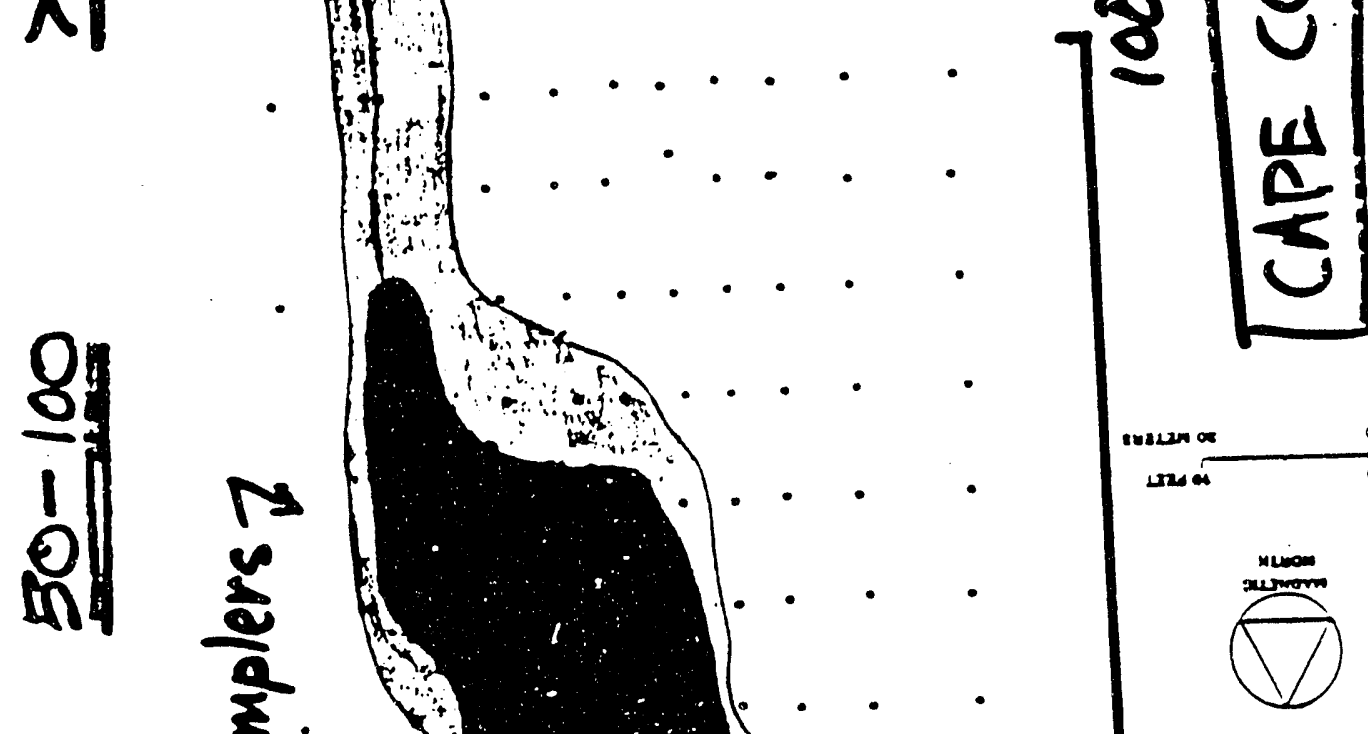

n i

n

z

$\frac{5}{2}$

8

11

(1) 0
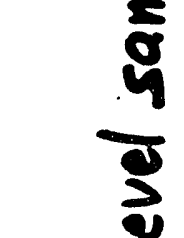

$n$
0

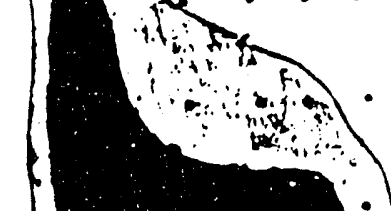




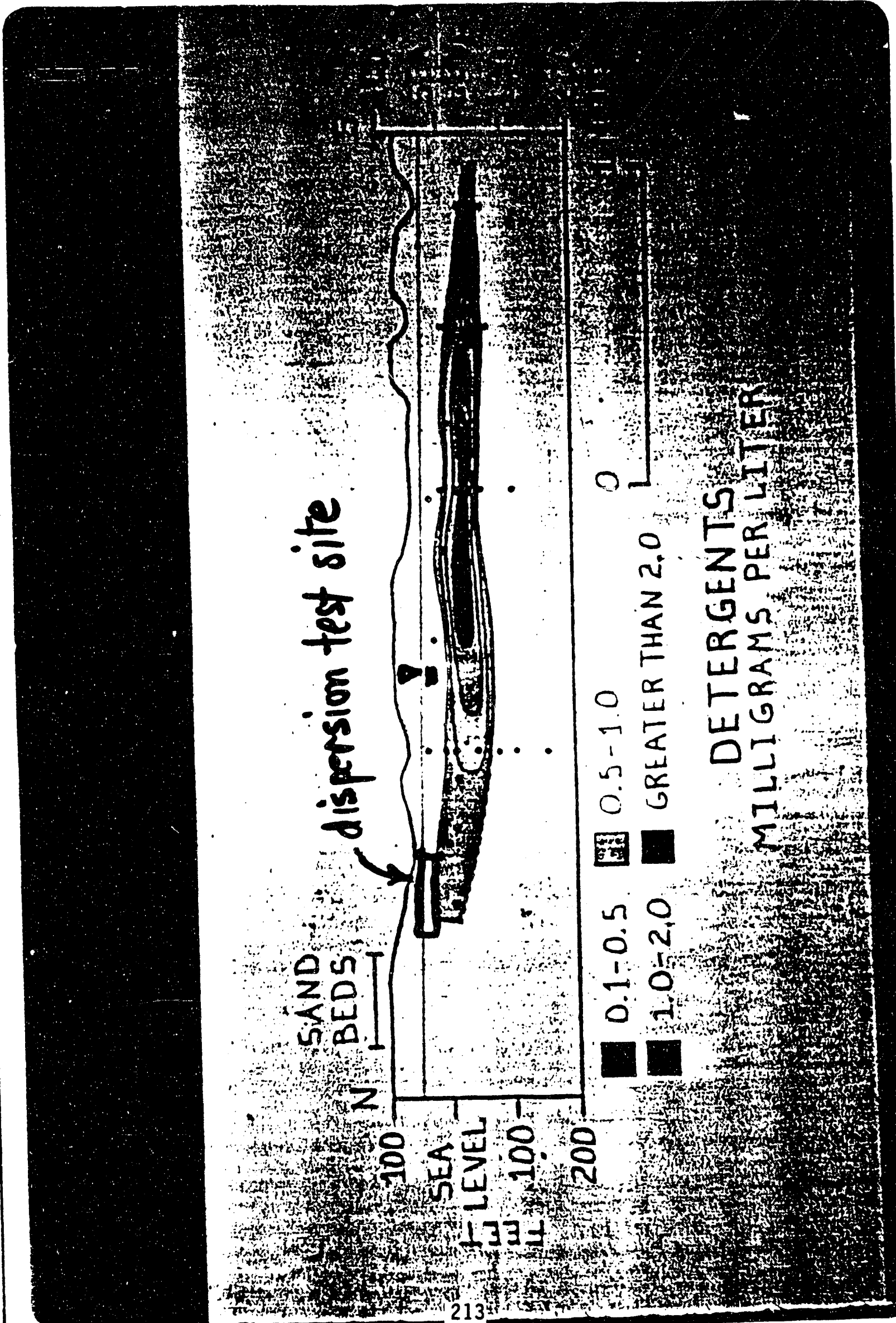


WHC-MR-0420

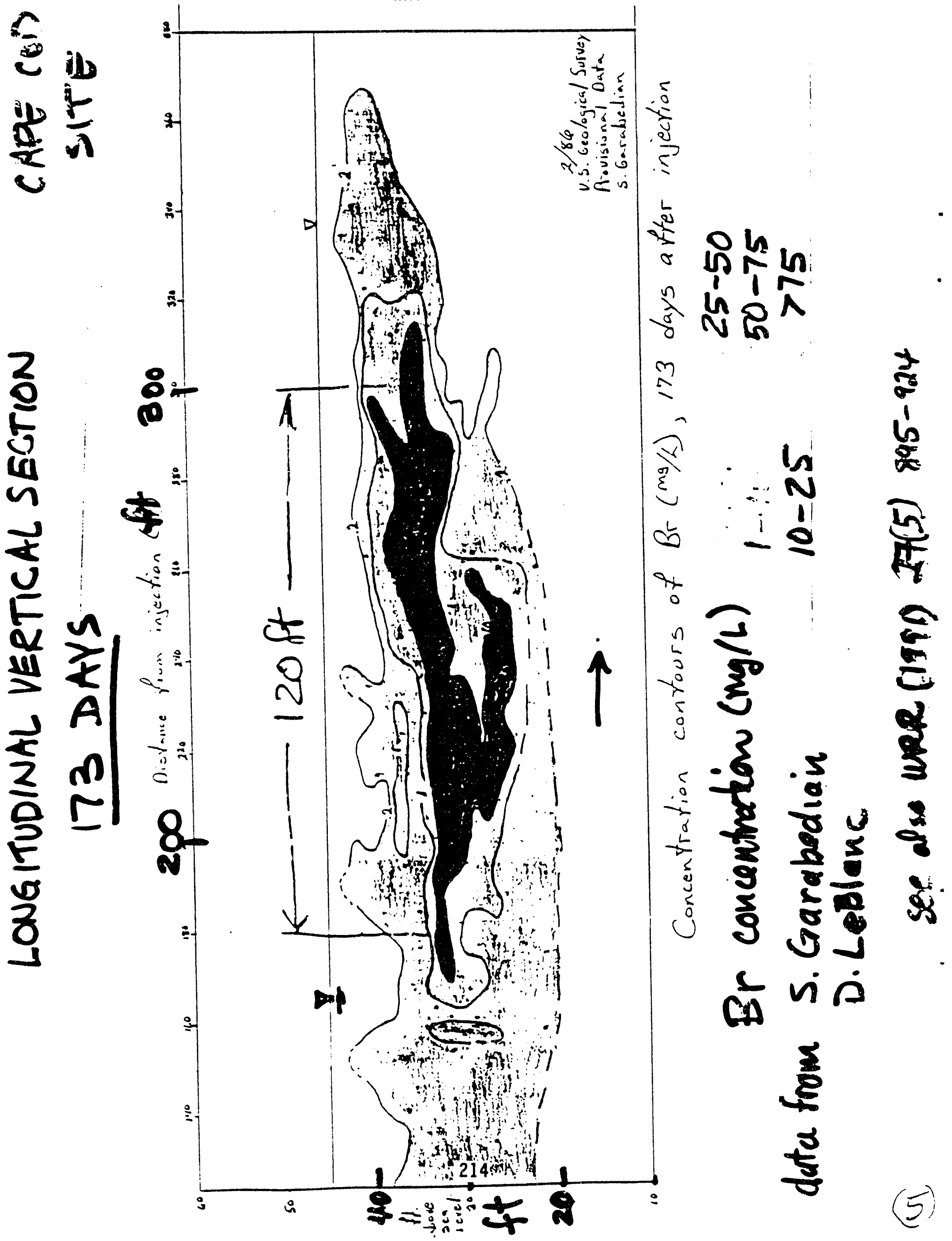




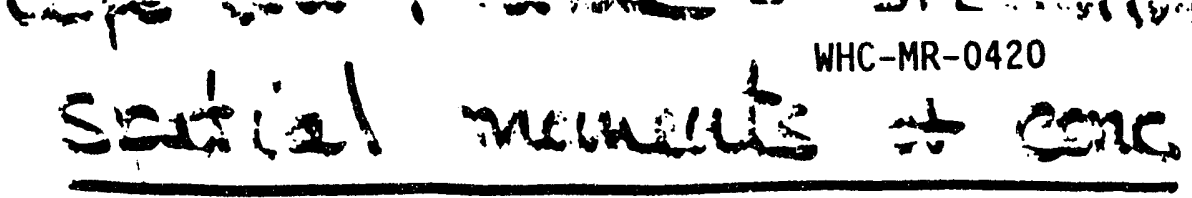

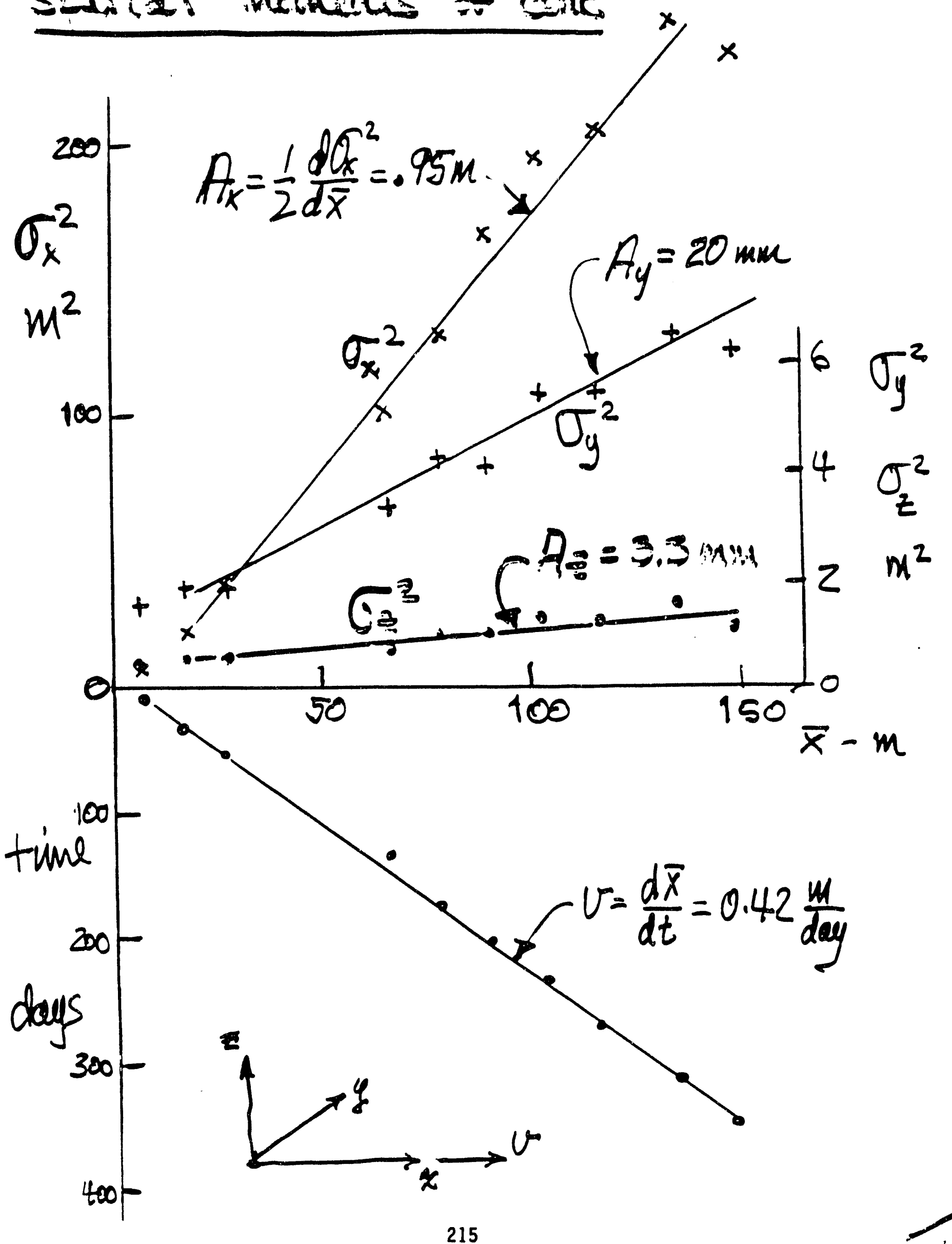


I

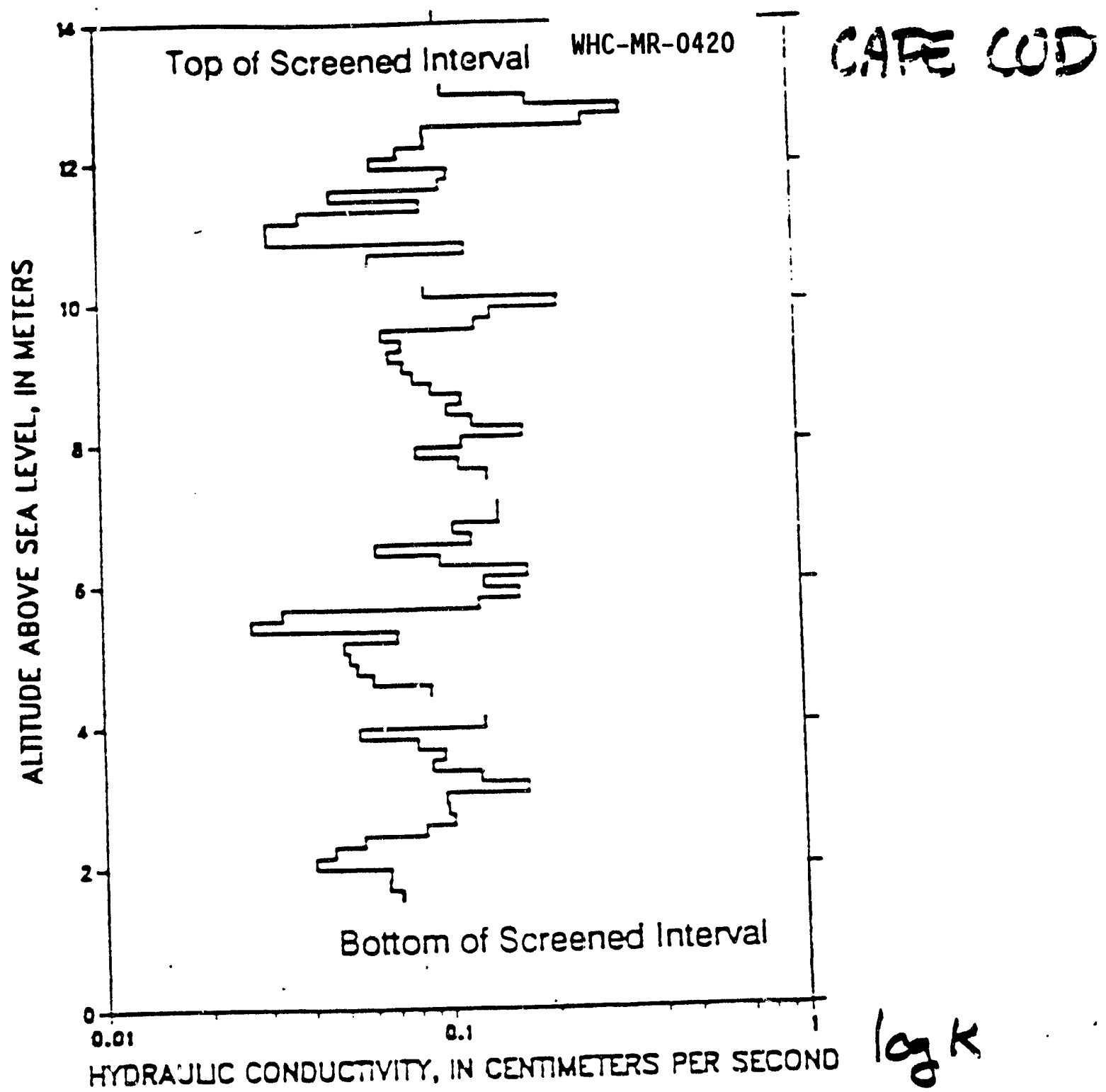

From borehols flowmeter logging

2 inch dia fally-screened well!s

$d=t a$ by $K$. Hess, USGS $f=\operatorname{lnk}$

216 


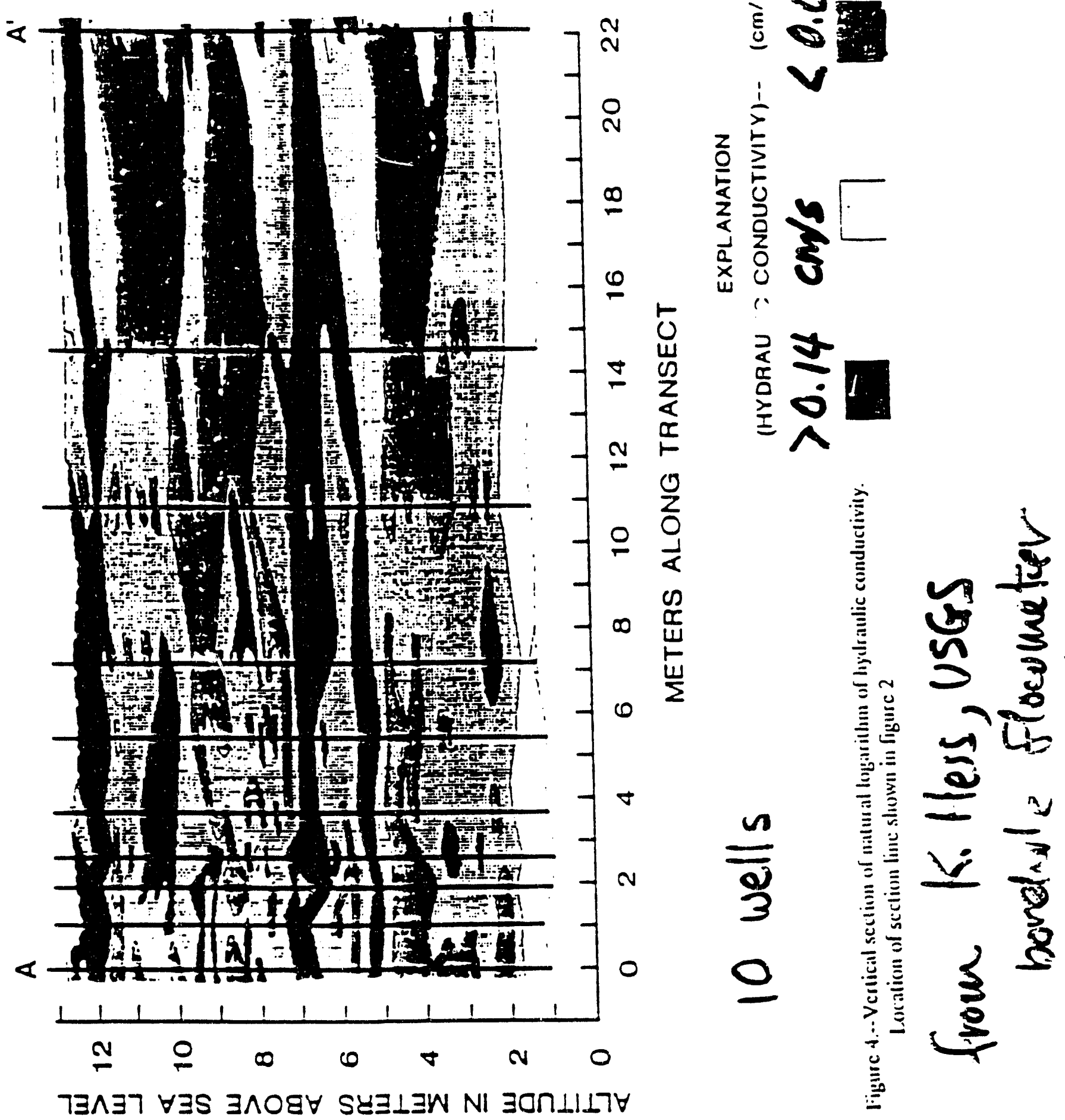


is:

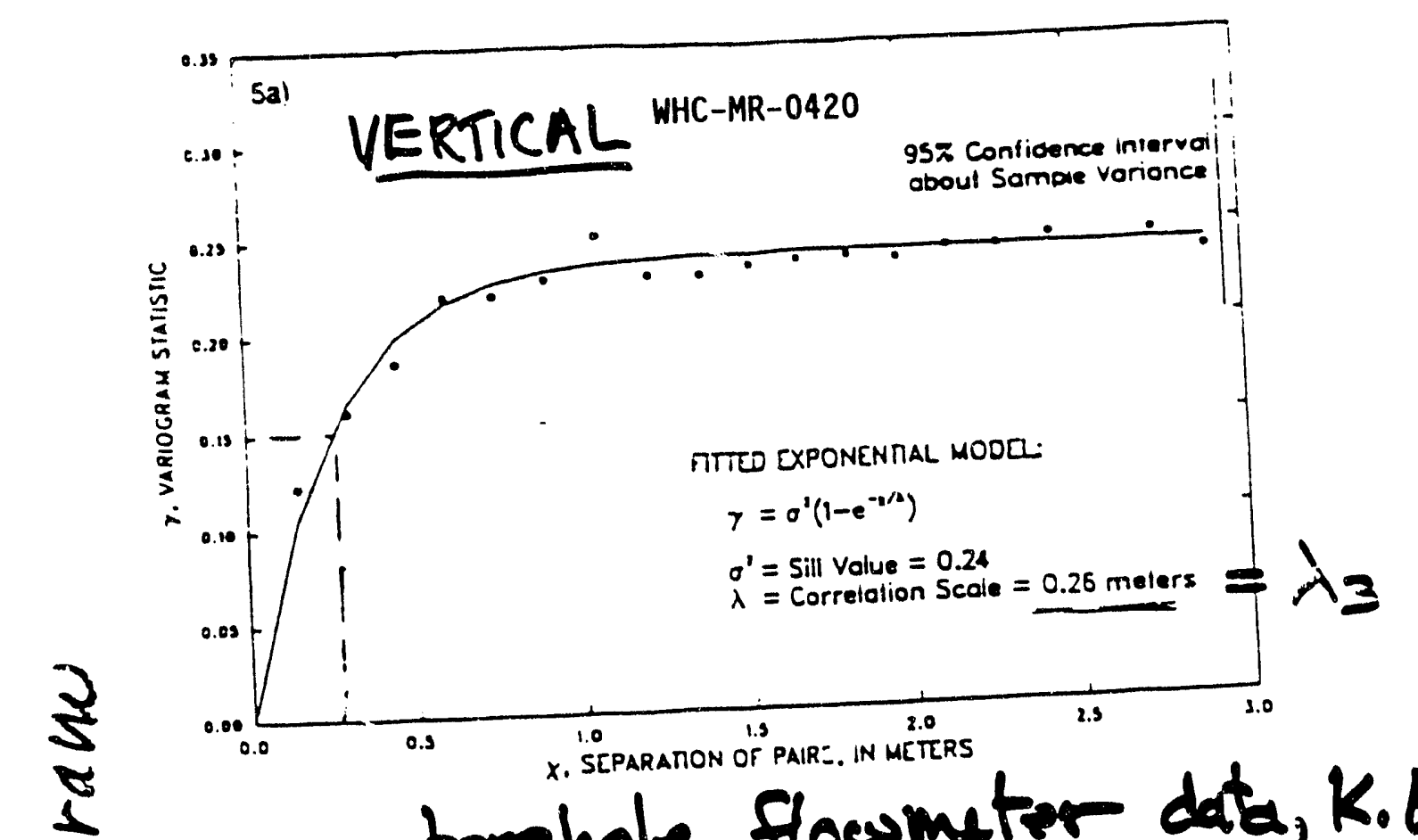

$a$ borehole flowmeter data, K. Hess (rage Uses

STOCHASTIC THEORY :

$A_{11}=\frac{\sigma_{f}^{2} \lambda_{1}}{\gamma^{2}}$ longitudinal dispersivity

$$
=0.98 \mathrm{~m}
$$

$$
\gamma\left(\tilde{o}_{f}^{2}, \lambda_{1}\right)=1.12
$$


HETEROGENEOUS SOKPTION

(10)

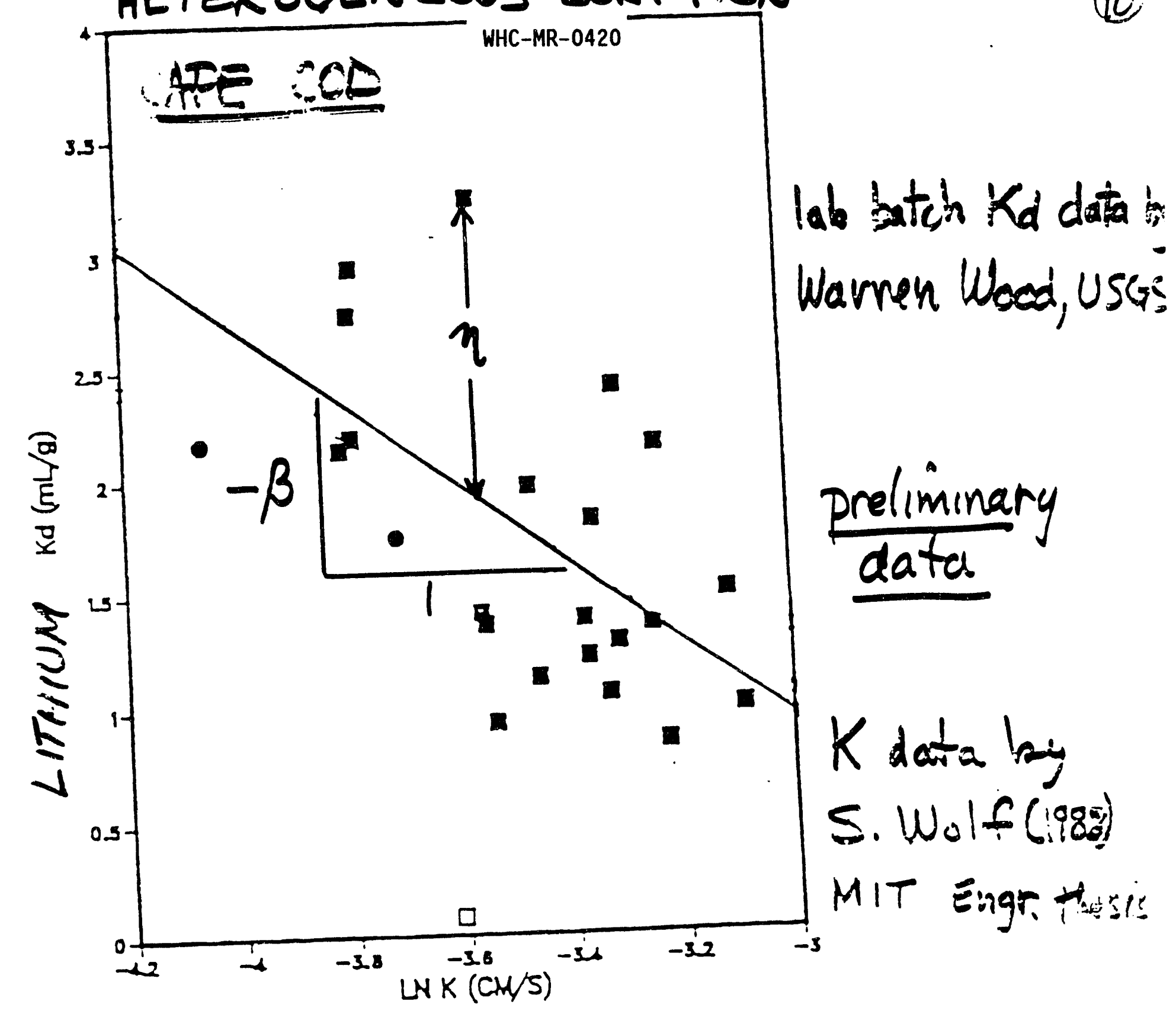

STOCHASTIC THEORY (MIT Parsons Lab Rpt 315, Garabedian...

effective $K_{d}=E\left[K_{d}\right] \Rightarrow R_{\text {eff }}=1+\frac{\rho_{b} \bar{K}_{d}}{n_{2}} \cong 9$

lang. dispersivity

nox-rgtarsind dispersivity

$$
A=\frac{\sigma_{+}^{2} \lambda_{1}}{\gamma^{2}}\left(1-\frac{\gamma \beta_{0} \beta}{n \bar{R}}\right)^{2}+\frac{\rho_{b}^{2} \sigma_{y}^{2} \lambda_{y}}{n^{2} \bar{R}^{2}} \cong 7 A_{0}^{\varpi}
$$

rarrobated. uneouroloturi 
III

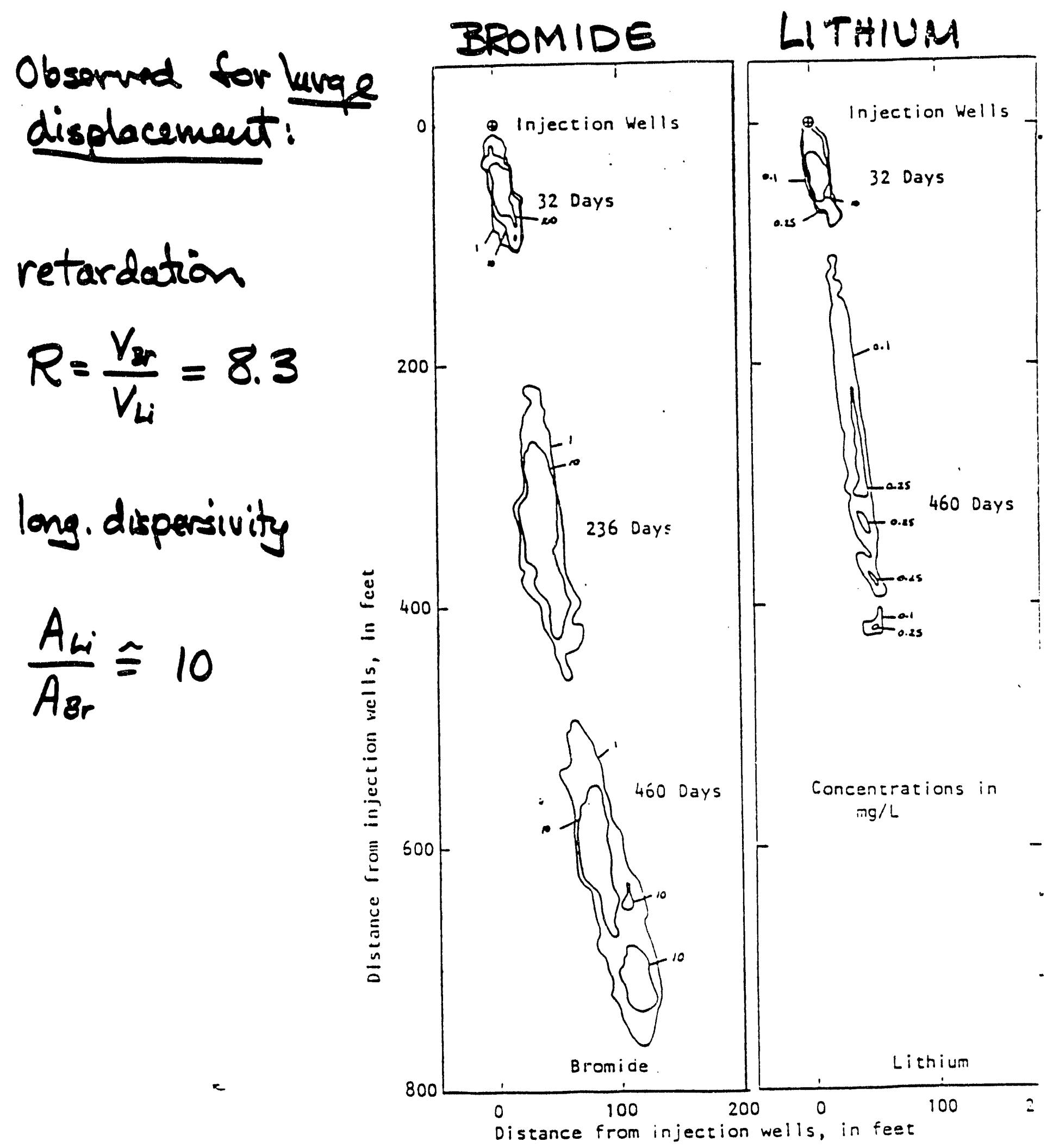

Figure 55. Contours of maximum lithium concentration in $\mathrm{mg} / \mathrm{L}$ in

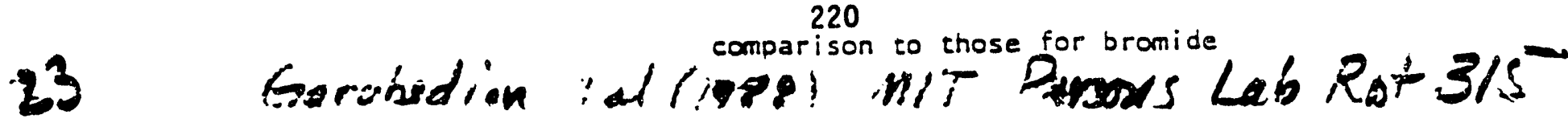




\section{from}

Gelhar, L.N., C. Welty and K.R. Rehfeldt, "A Criticai Review of Data on Eield

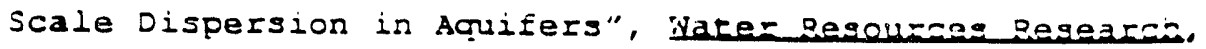

is.

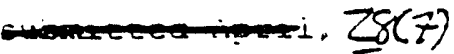

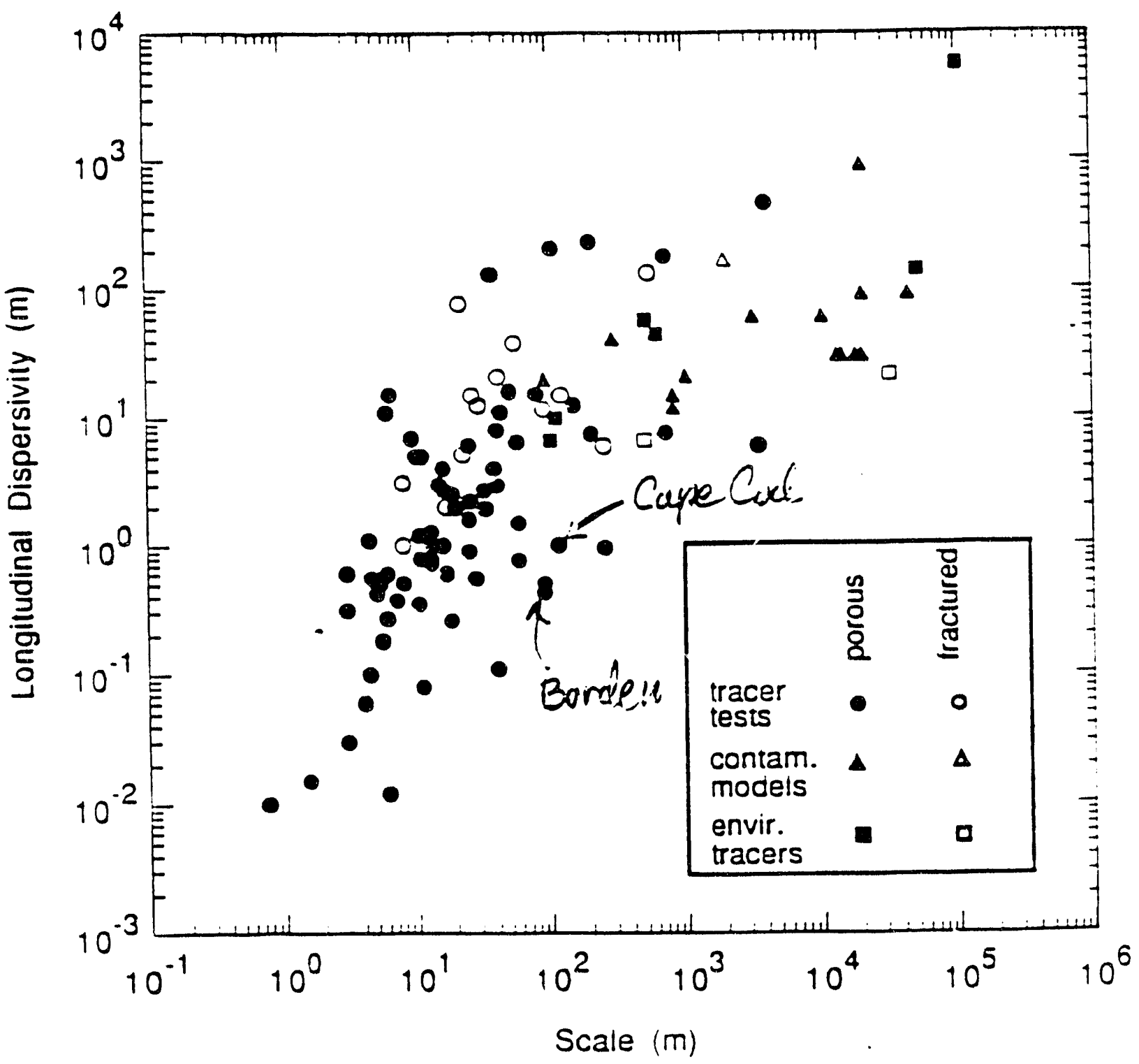

see dlso

Gelhar, L.\#., A. Mantoglou, C. Welty and K.R. Rehfeldt, "A Review Uf E Eeld Scale Physical Soluce Tzansport Processes In Saturated And Unsaturated Poroug Media," Einai Rpt. RP2485-5, Elec. Power Res. Inst., Palo Alto, CA, EPRI EA-4190, Augris: 2985 . 


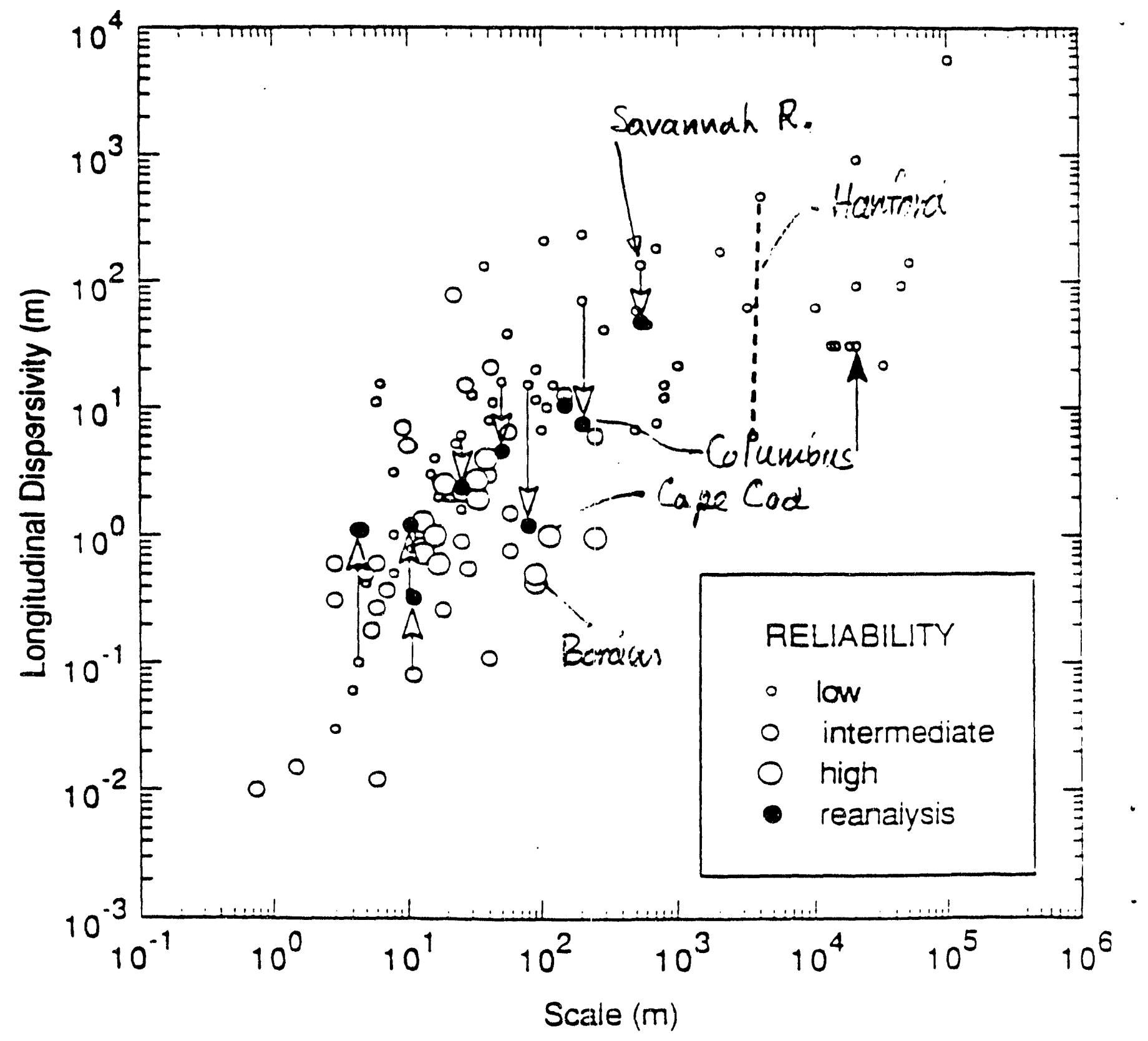




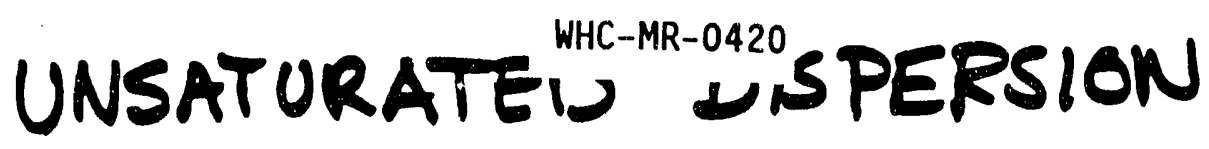

(14)

30 STochastic Heterezengitu

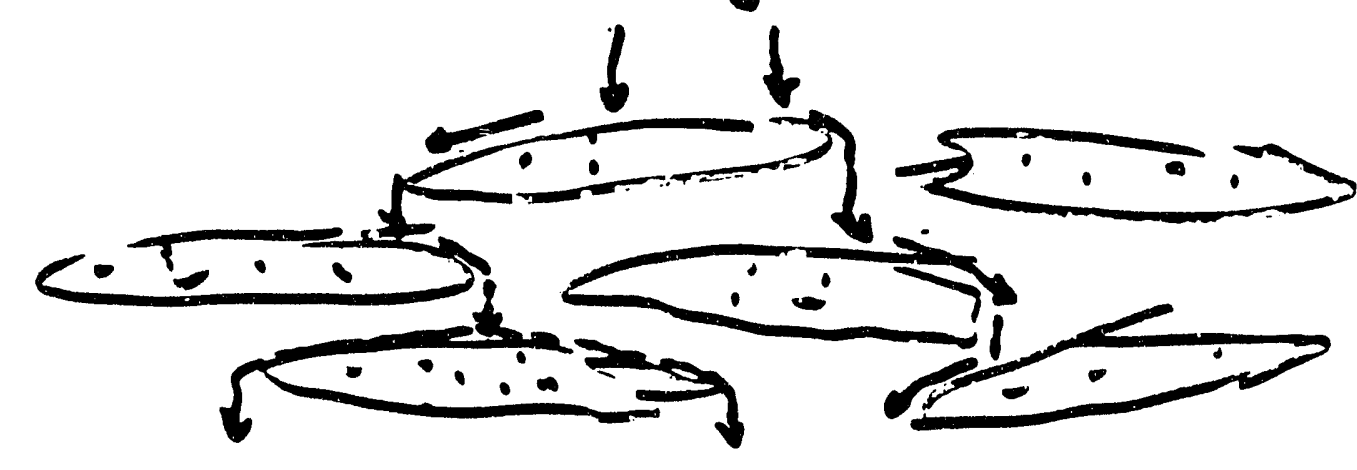

- IP statistically anisotropic random fields represent spatial structure of hydraulic properties

- perturbation solutions to evaluate effective (large-seale) parameters showing mucro-anisotrapy, -hysteresis, -dwapersivity

Composts:

- seemingly realistic 30 heterogeneity

- variance also predicted

- unfamiliar \& untested

- soil statistical parameters difficult to.

- potentially predictive d testable obtain

- works for saturation flow 


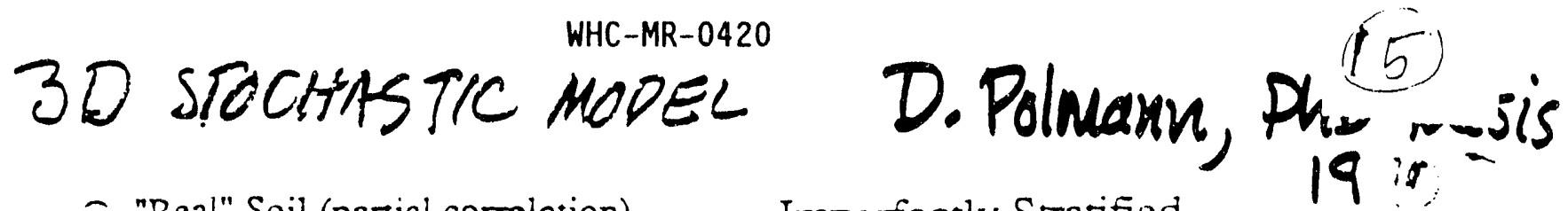

$$
\text { = "Real" Soil (partial correlation) } \begin{gathered}
\text { Imperfectly Stratified } \\
\lambda_{1}=\text { lamb }=25 \mathrm{~cm} \mathrm{\&} \mathrm{lamb}=100 \mathrm{~cm}
\end{gathered}=\lambda_{2}
$$

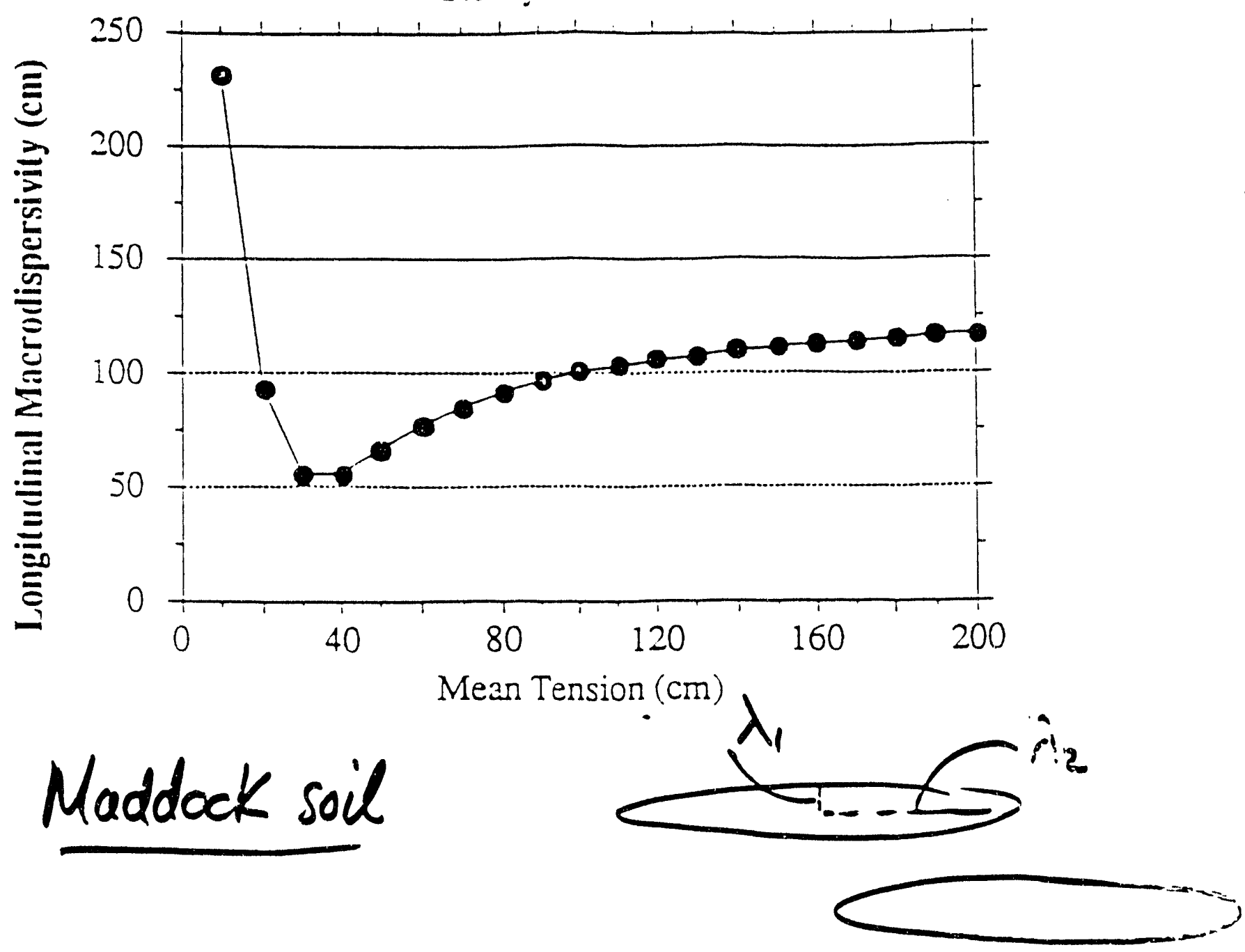

224 


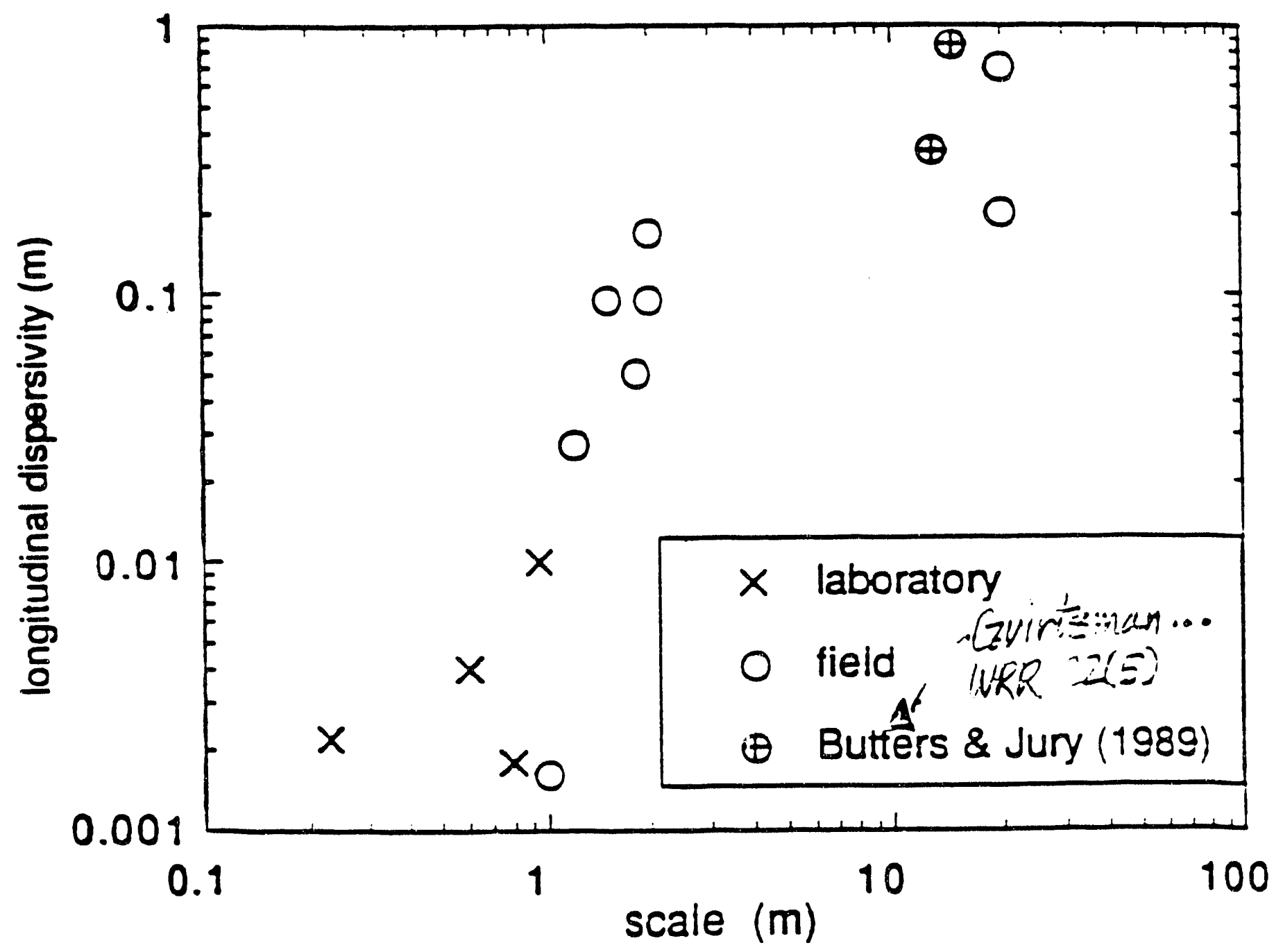

Figure 5.28. Longimuinal dispersivities for unsamurated soils as related to displacement scale (based on Gelhar et al., 1985, Table 34). 

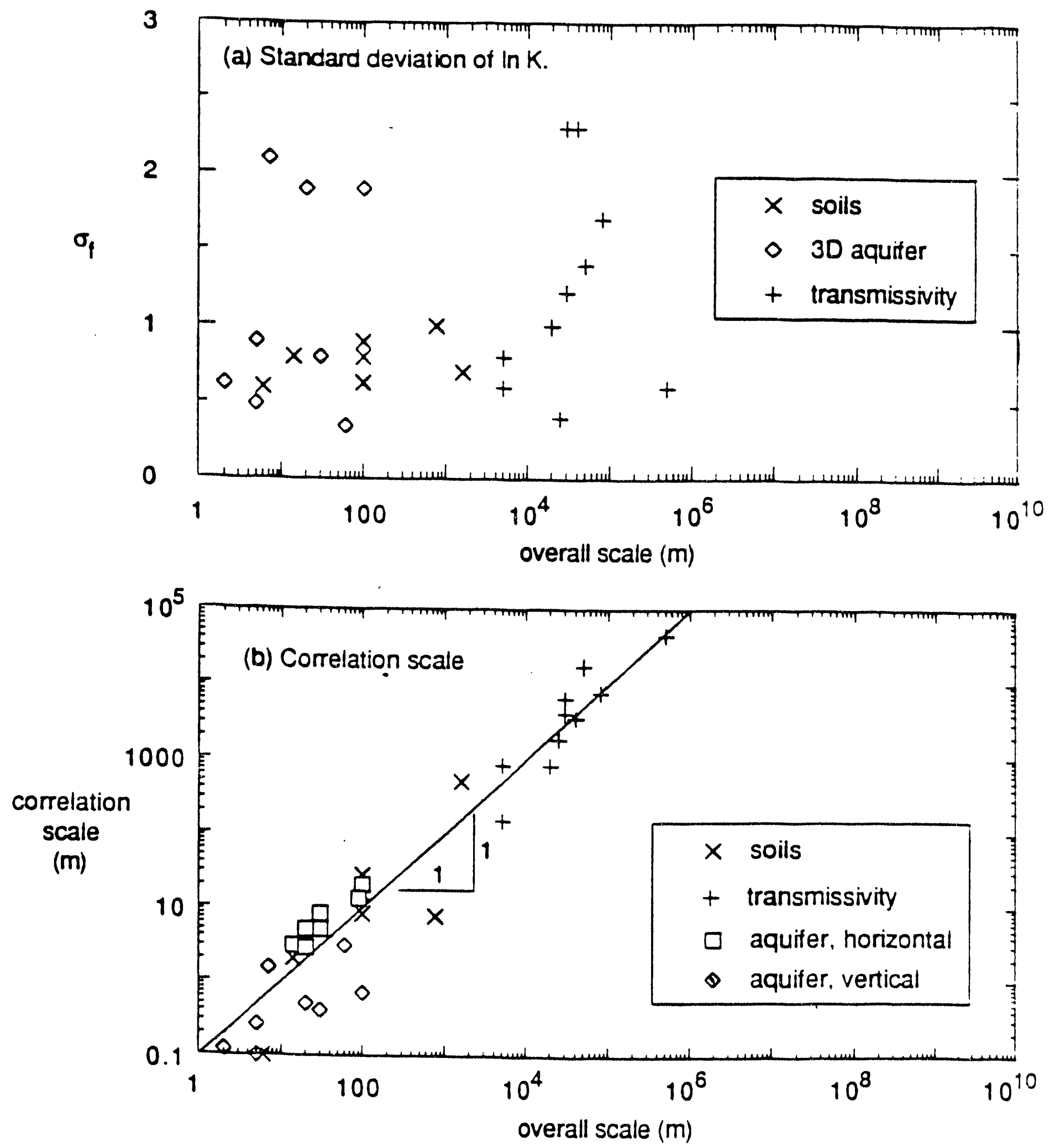

Figure 6.5. Standard deviation and correlation scales of hydraulic conductivity or transmissivity versus overall scale. 


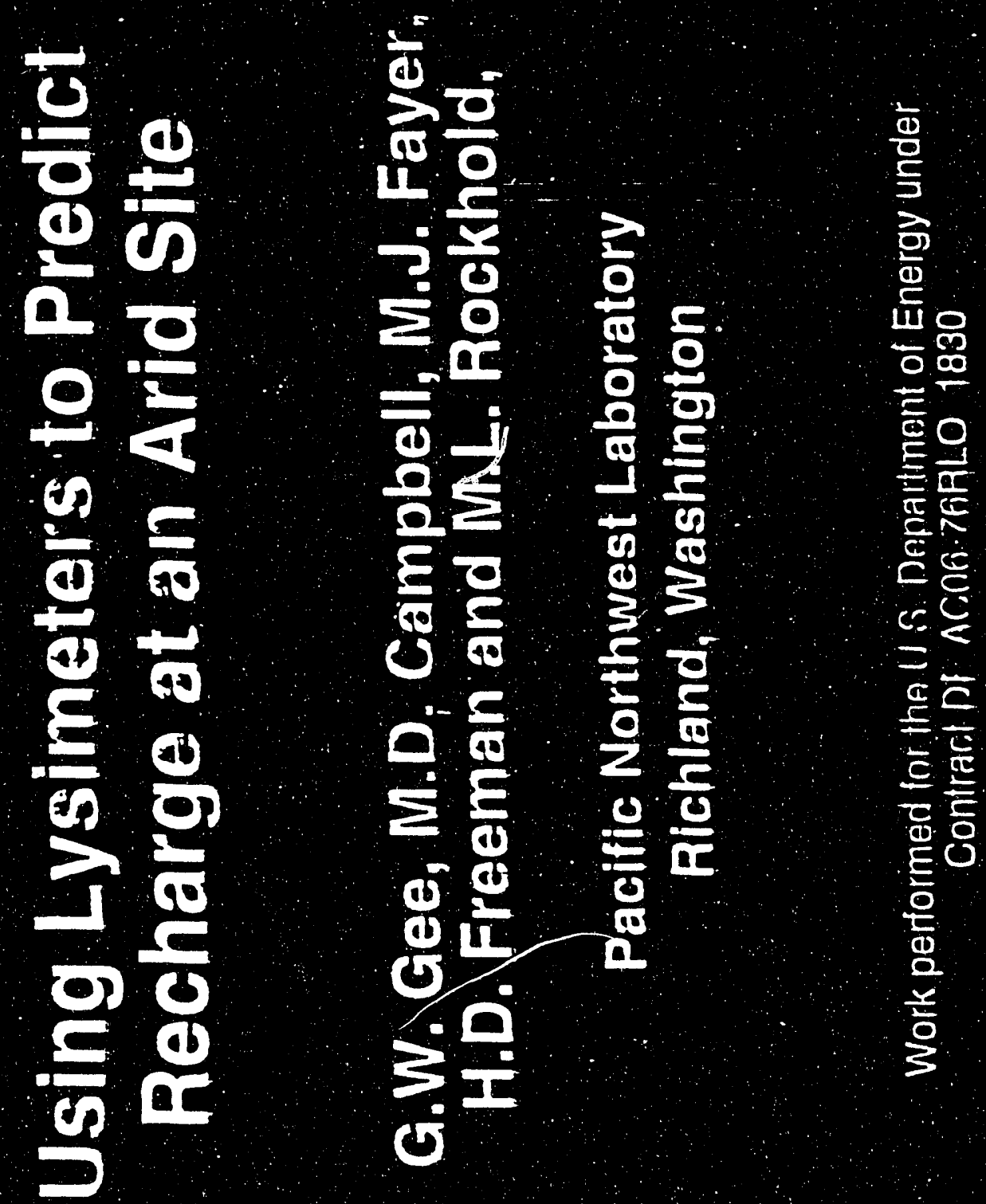


(10 m

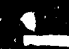

a

$+$

(v)

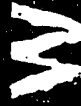

(1)

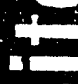

de

8

을

4

c

(10)

T로 $\frac{5}{c}$
$\frac{5}{5}$
0
$\frac{2}{2}$
तi

은

$\pm$

$\frac{2}{0}$

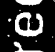

a

o

$\stackrel{2}{\sigma}$

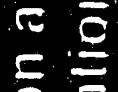

으를

$\bar{\pi}$

응

둔

$\frac{2}{\pi}$

$>F$

u
용

5
$\Xi$
0
0

(1)

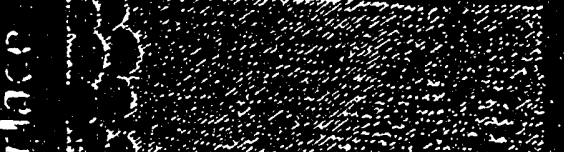

$y$

1.m.

1

,

1.1.

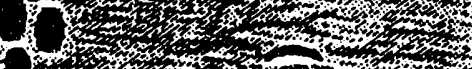

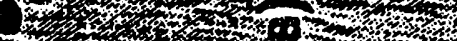

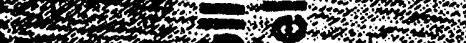

.

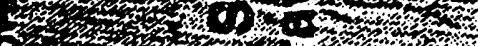

.

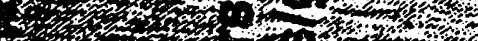

Ds,

$=\stackrel{\bar{E}}{\stackrel{E}{E}}$

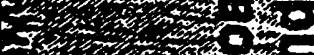

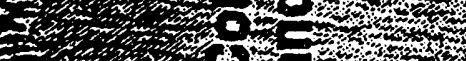

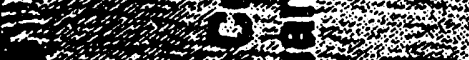

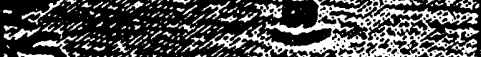

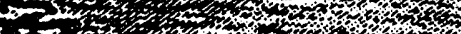

2013

$-12$

10

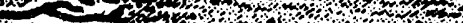

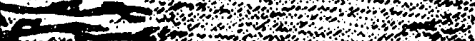

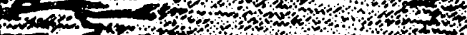

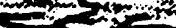

$=2$

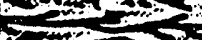

$=\frac{5}{5}$

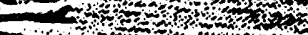

$230-12$

ons

$10=-13-2$

und

1 $57>0$

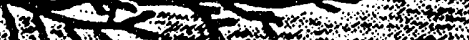

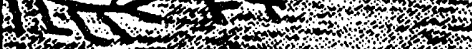

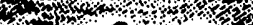

42022

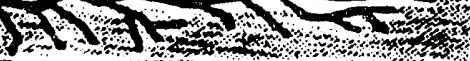

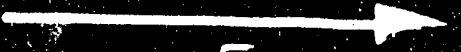

$=\frac{E}{\sigma}$

in o $\varnothing$

1

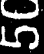

of

d)

as

0

15

$\infty \frac{\sigma}{0}$

a

⿷匚

ธ。

a

อ)

E

$0 \quad \frac{\pi}{a}$

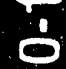

$\frac{D}{E}$




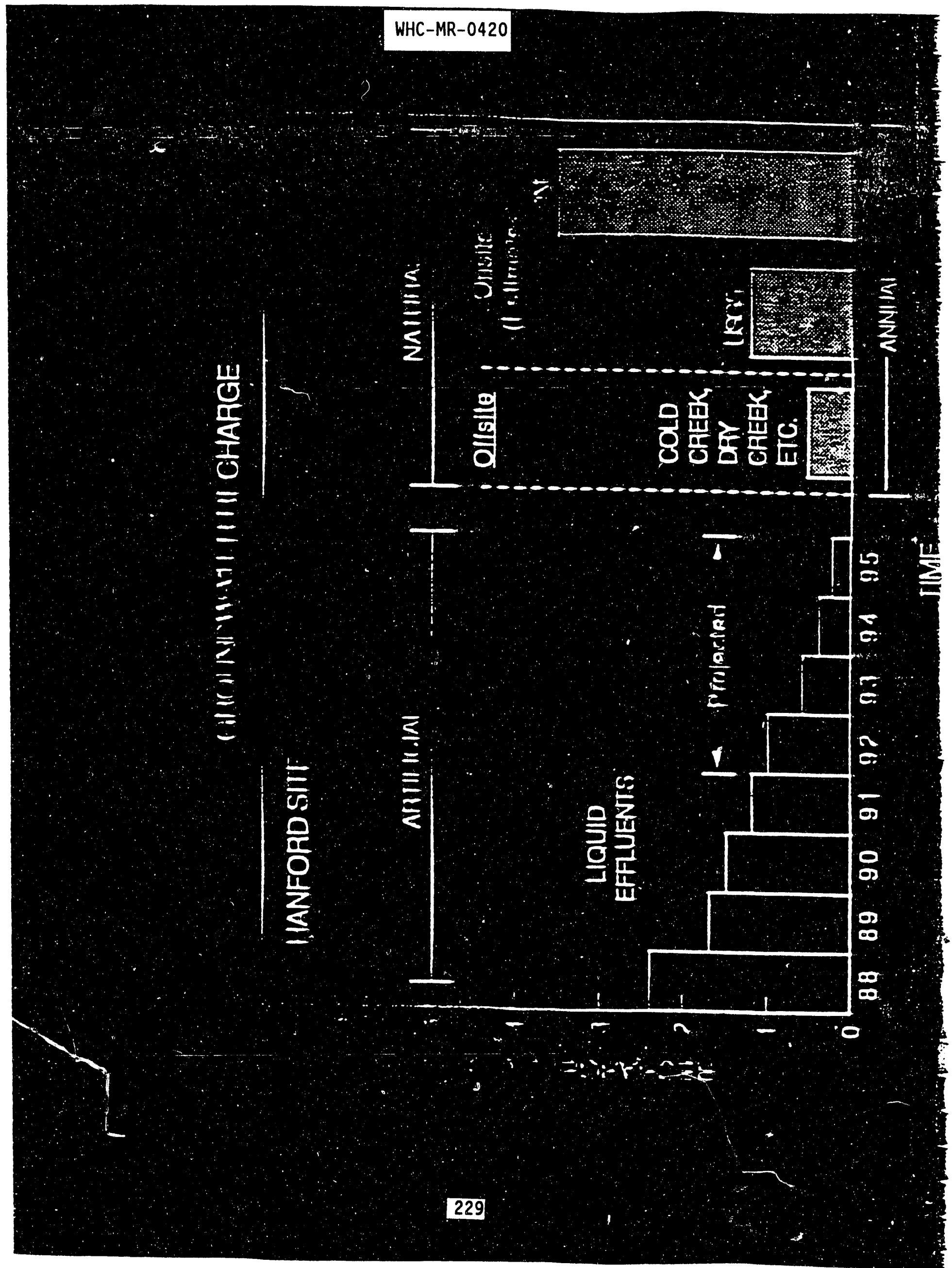


WHC-MR-0420

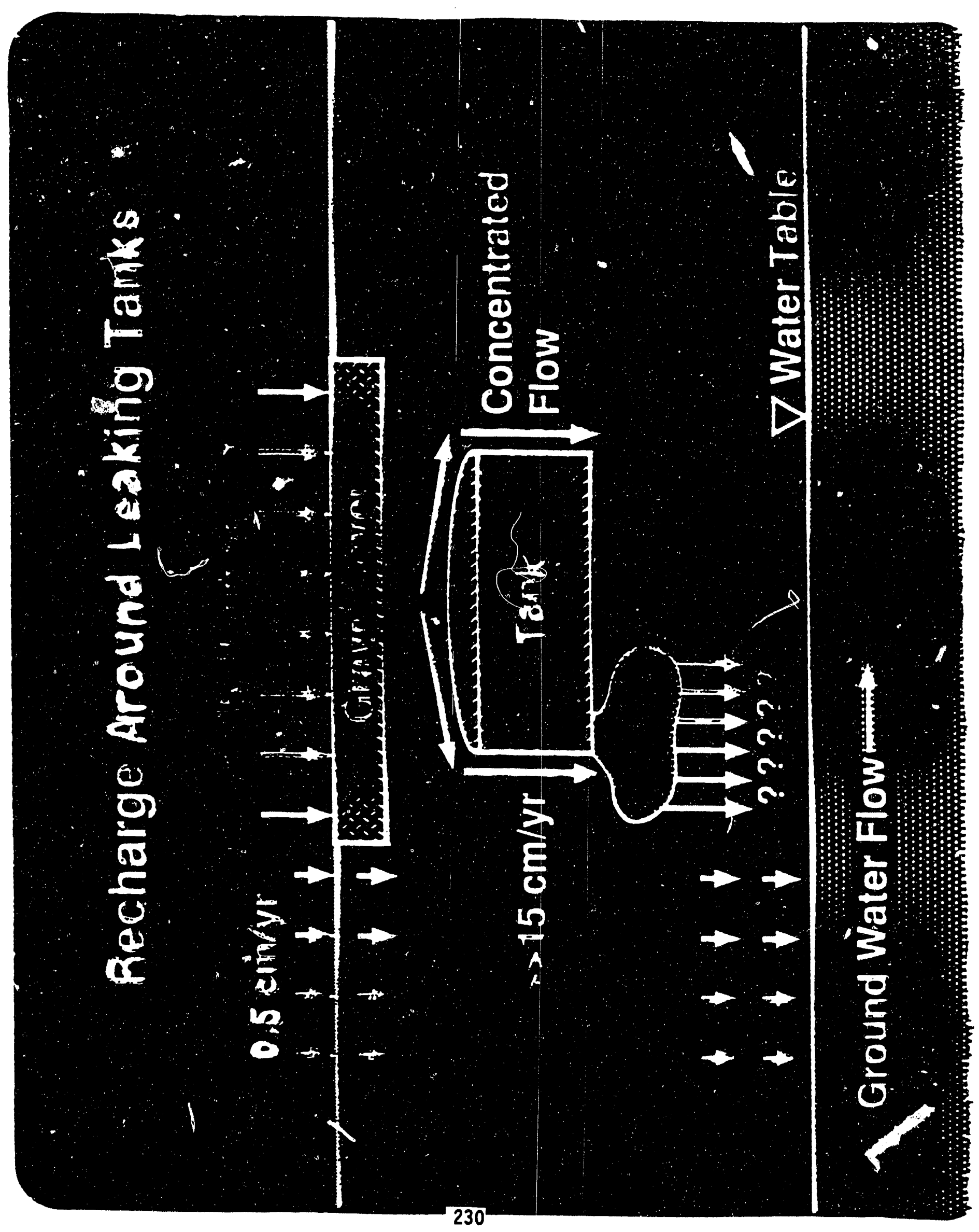




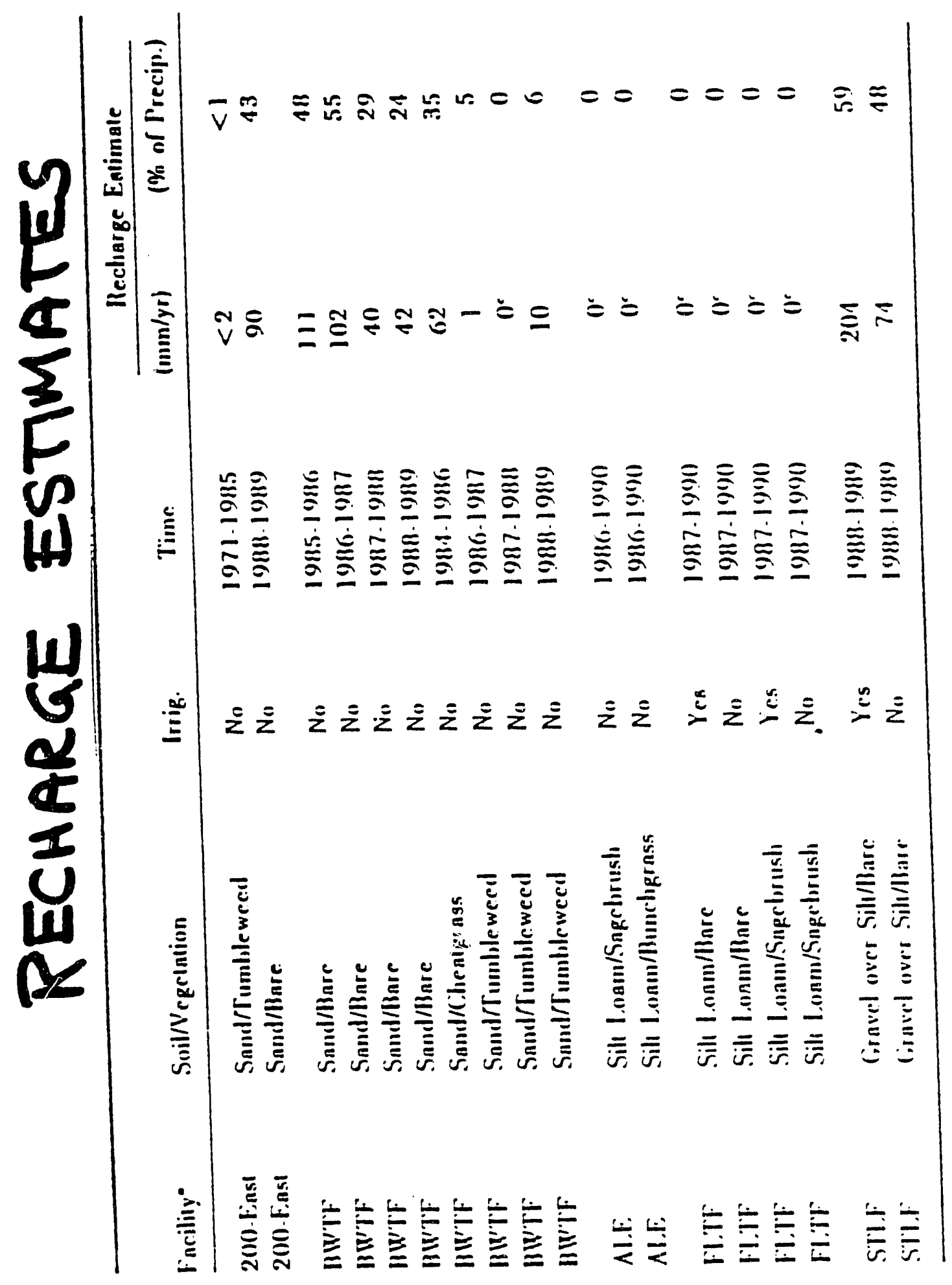




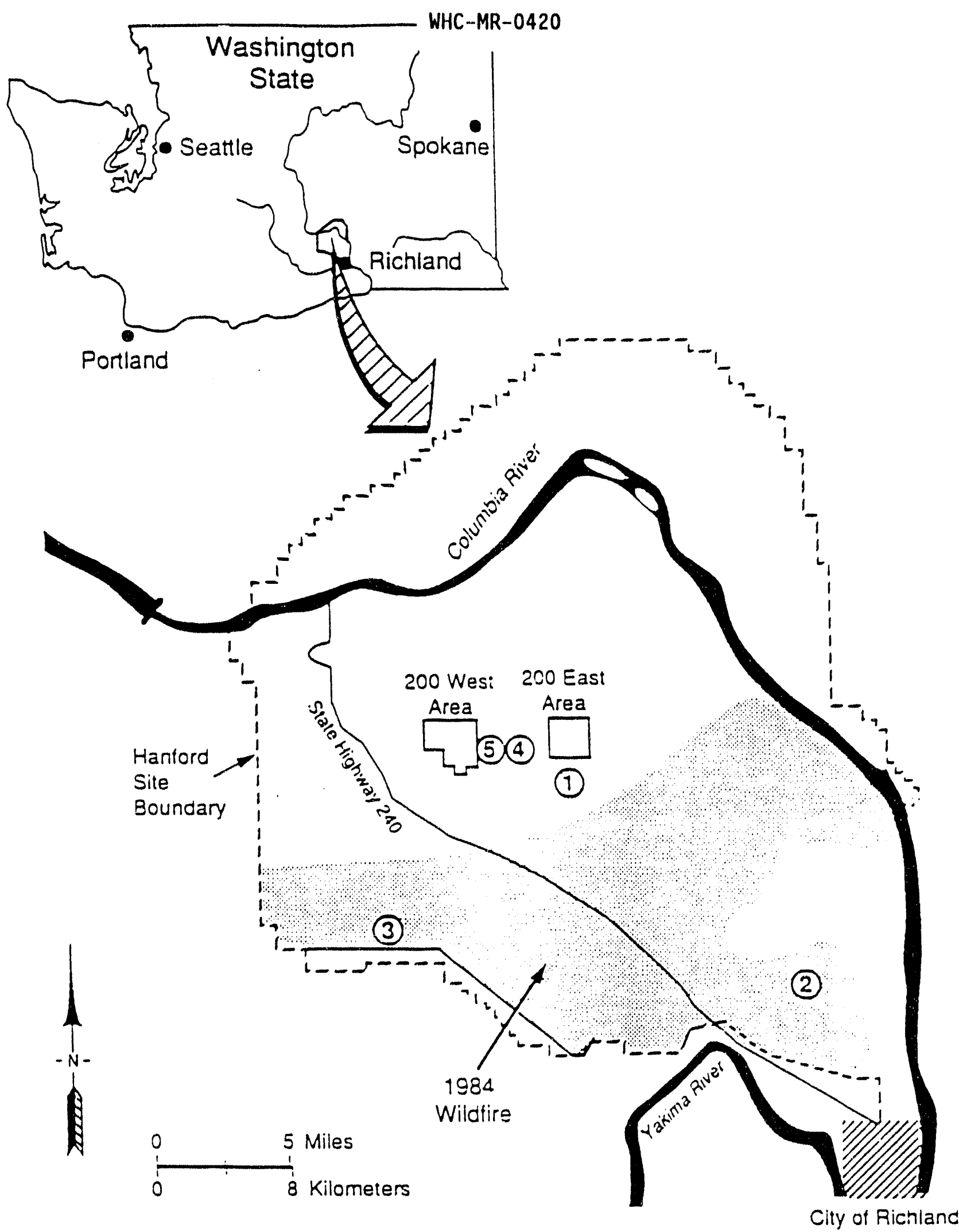

Figure 1. Haniord Site map. Vumbered carcies represent lysimeter :ocacons. Shaded area represents extent of .tugust 1984 widdire. 


\section{HANFARD SITE LYSIMETER FACILITIES}

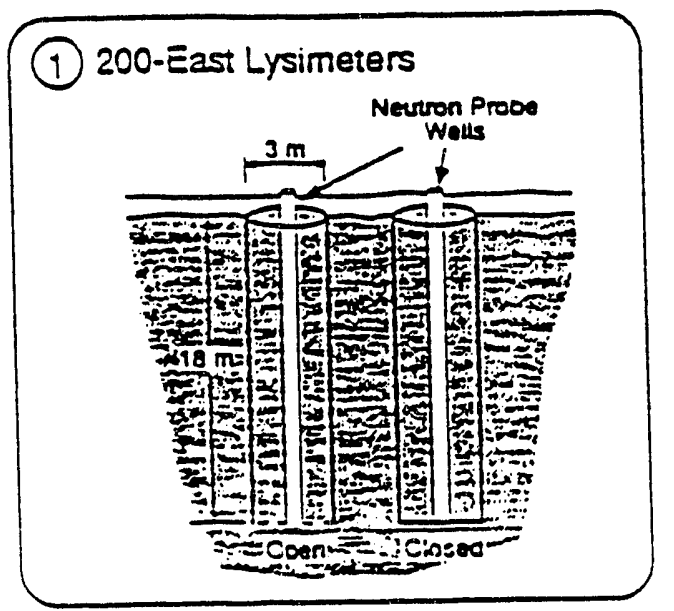

(2) Suried Waste Test Facility
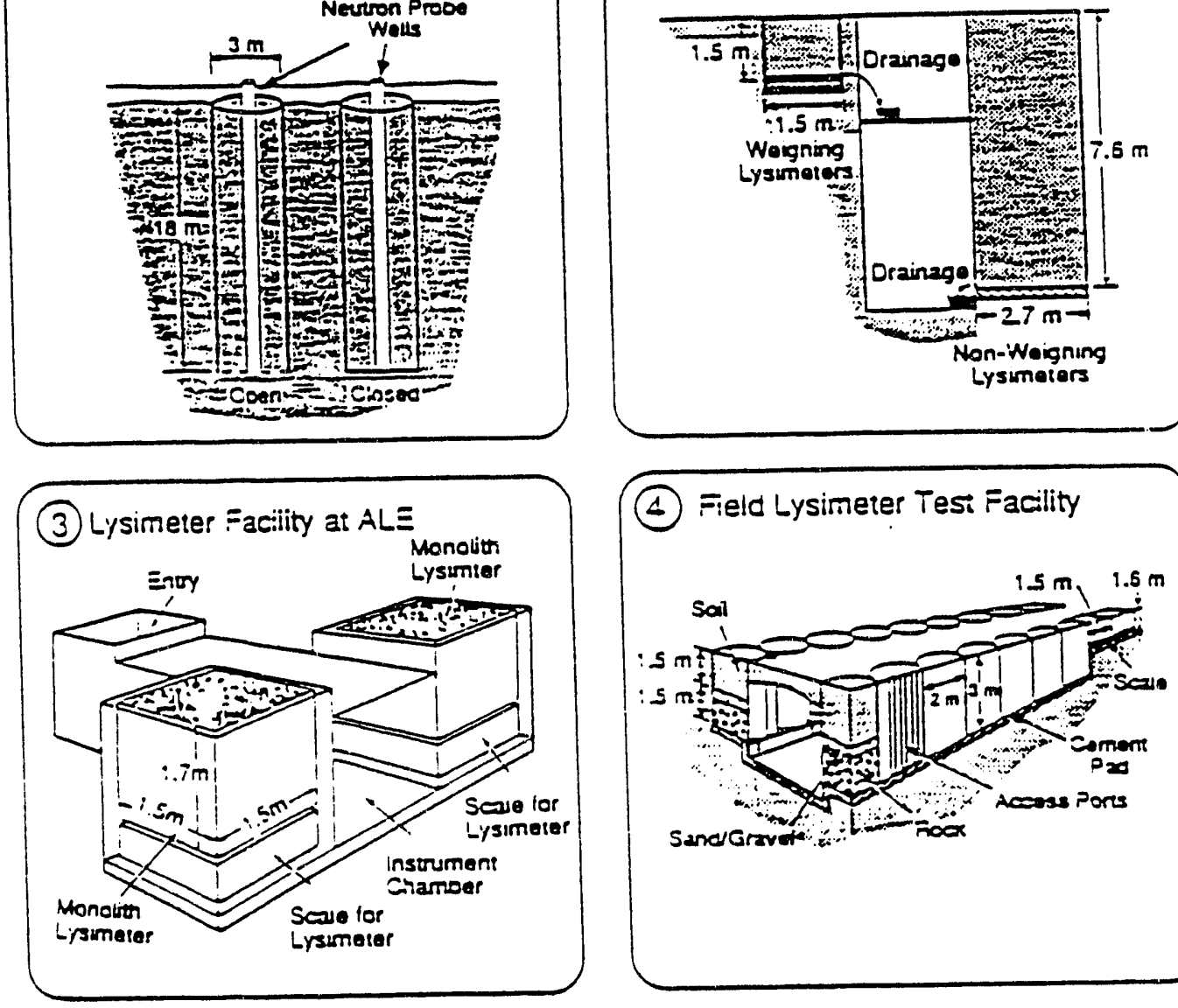

(4) Field Lysimeter Tesi Facility

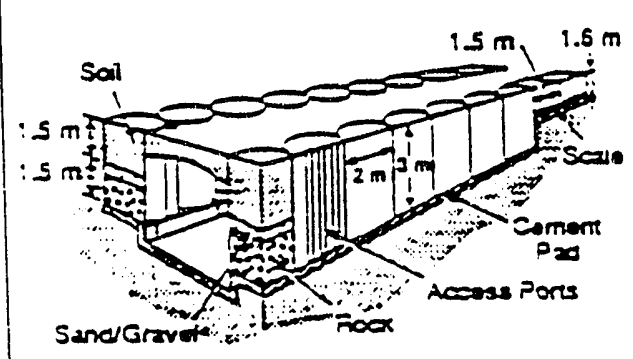

() Small Tube Lysimeter Faciilty

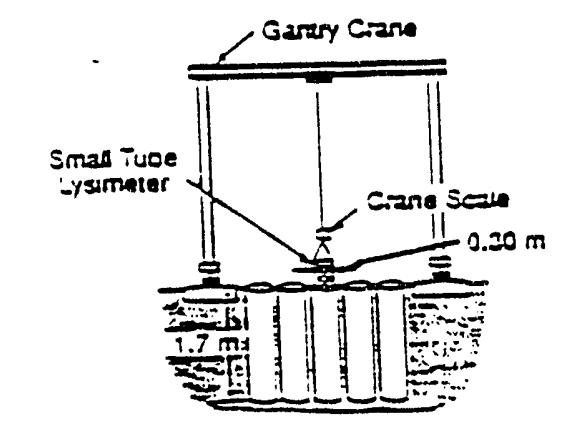




\section{MODEL VALIDATTON?}
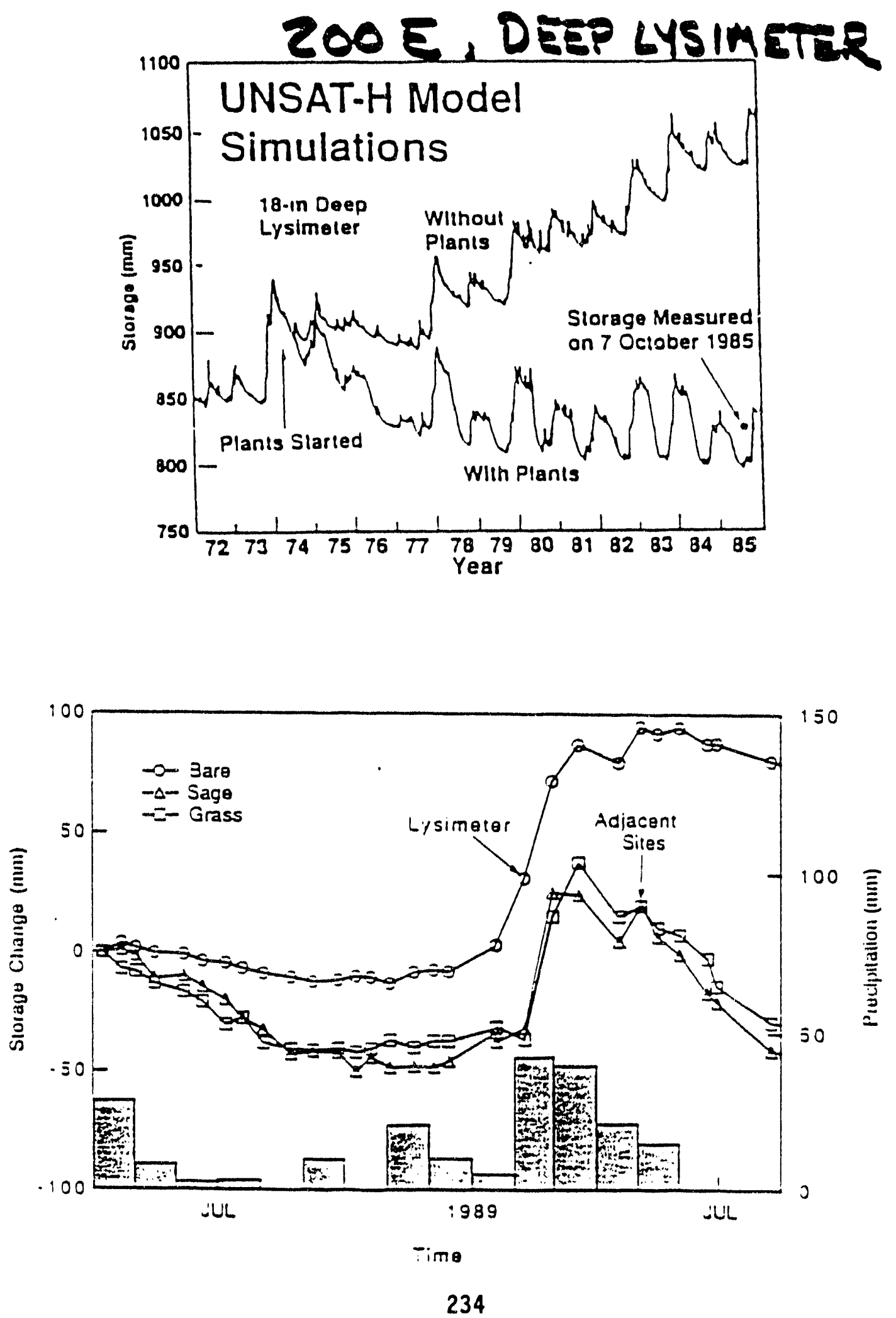
WHC-MR-0420

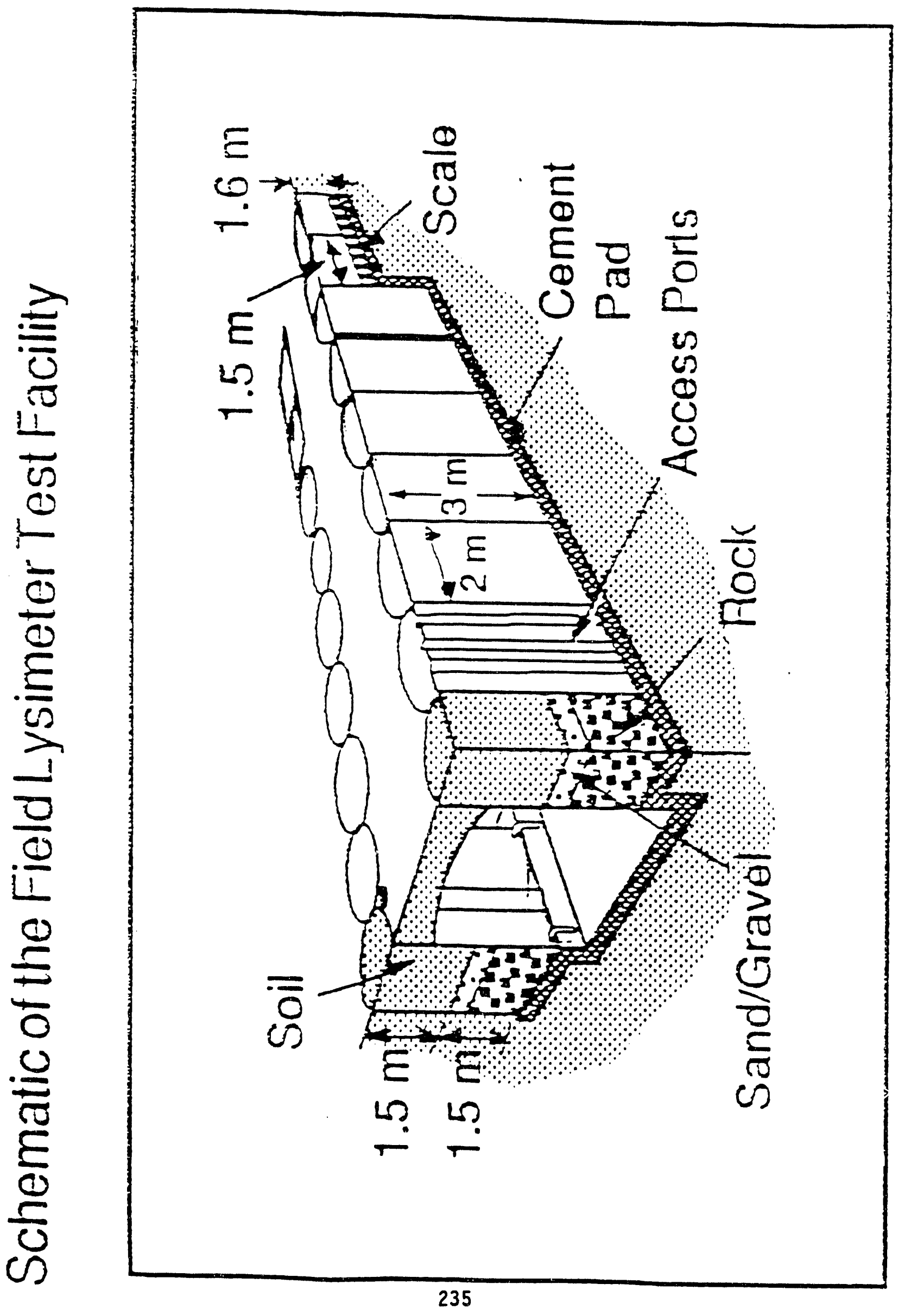




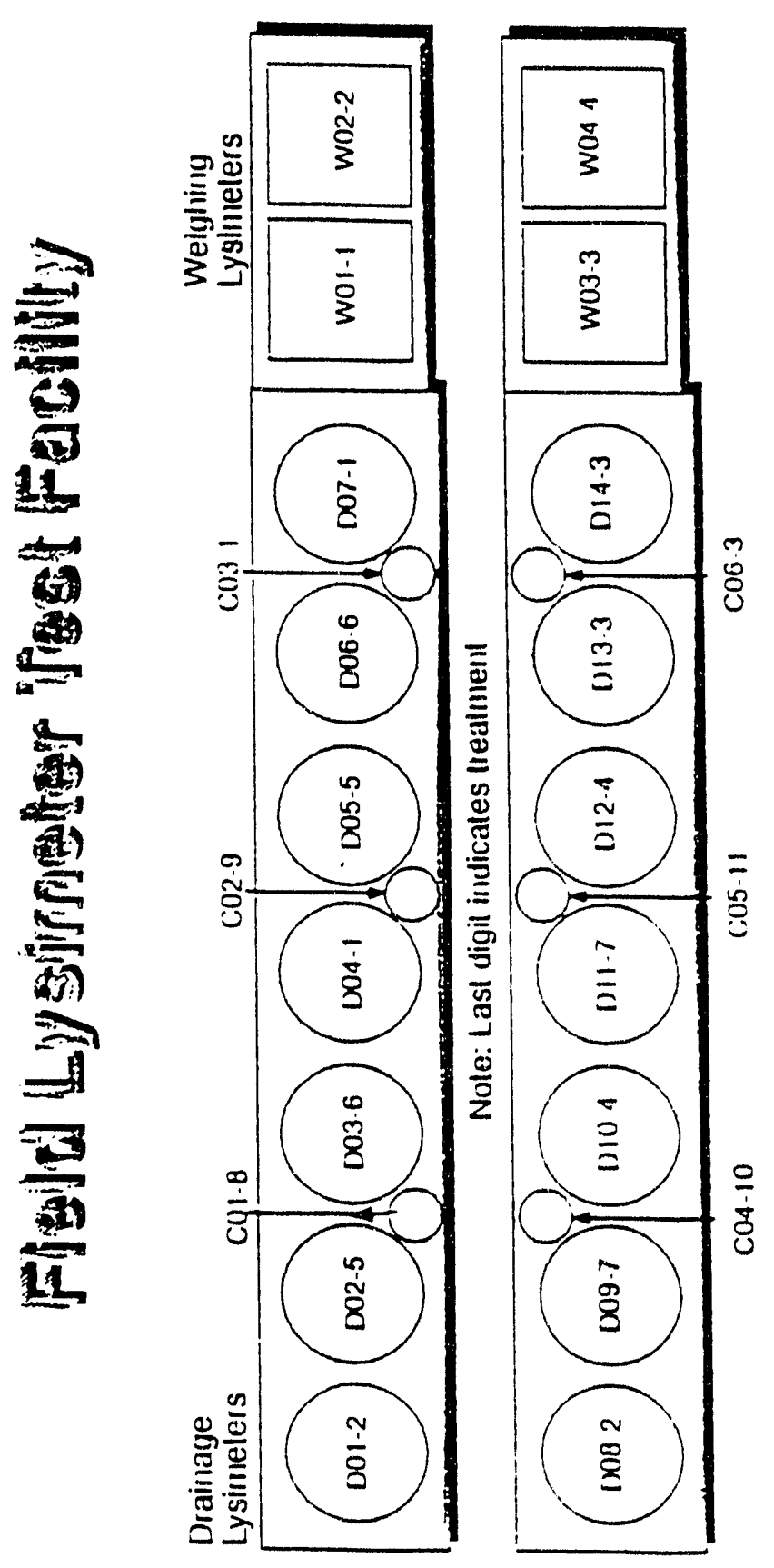

1ि कृ

-

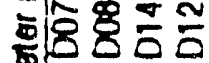

퉁ำ

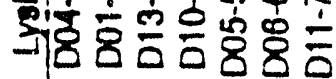

-

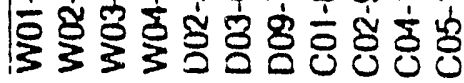

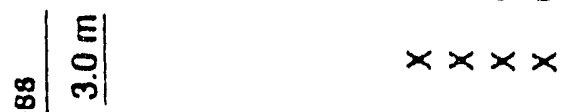

क ल

竞

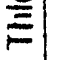

$\mid$\begin{tabular}{l|l}
$\mid$ \\
0
\end{tabular}

क| 总

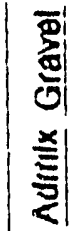
के

\begin{tabular}{|l} 
일 \\
$\frac{5}{5}$ \\
$\frac{5}{2}$
\end{tabular}

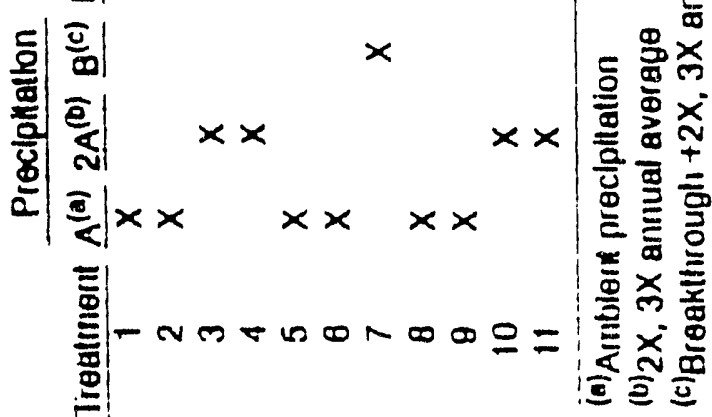


(wu) Ldd

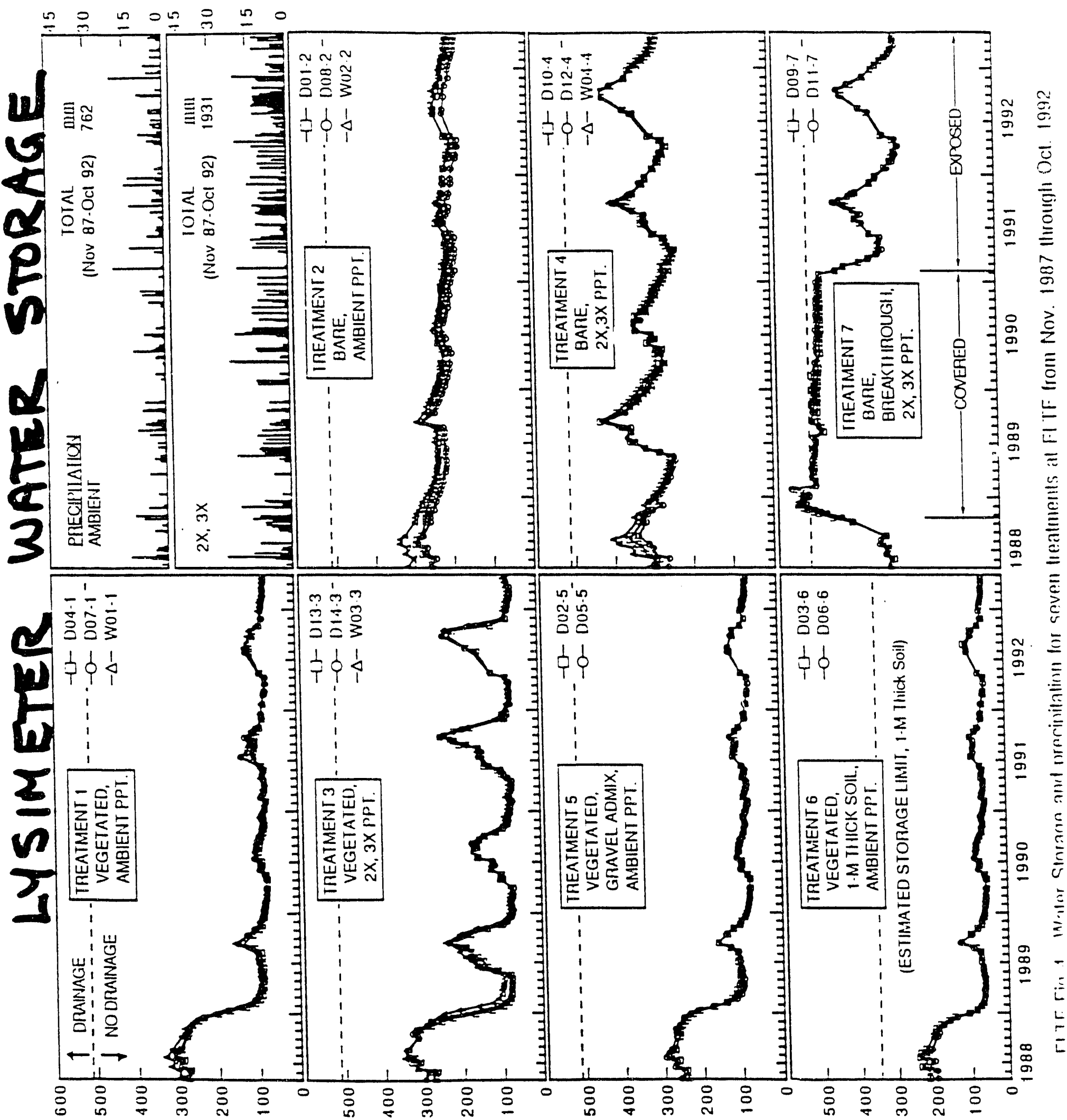

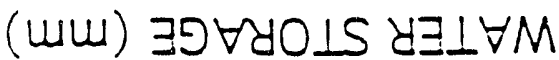


WHC-MR-0420
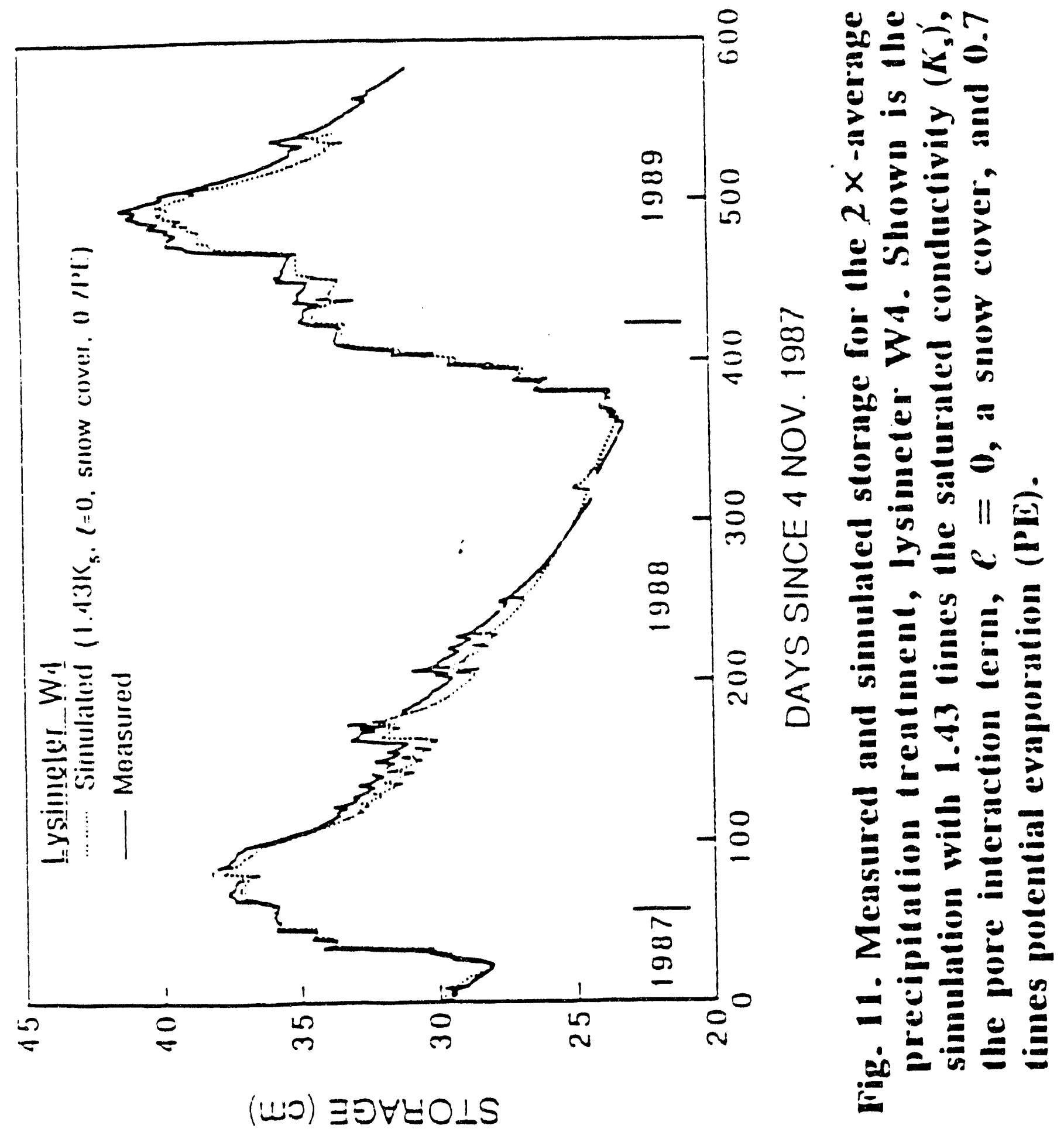


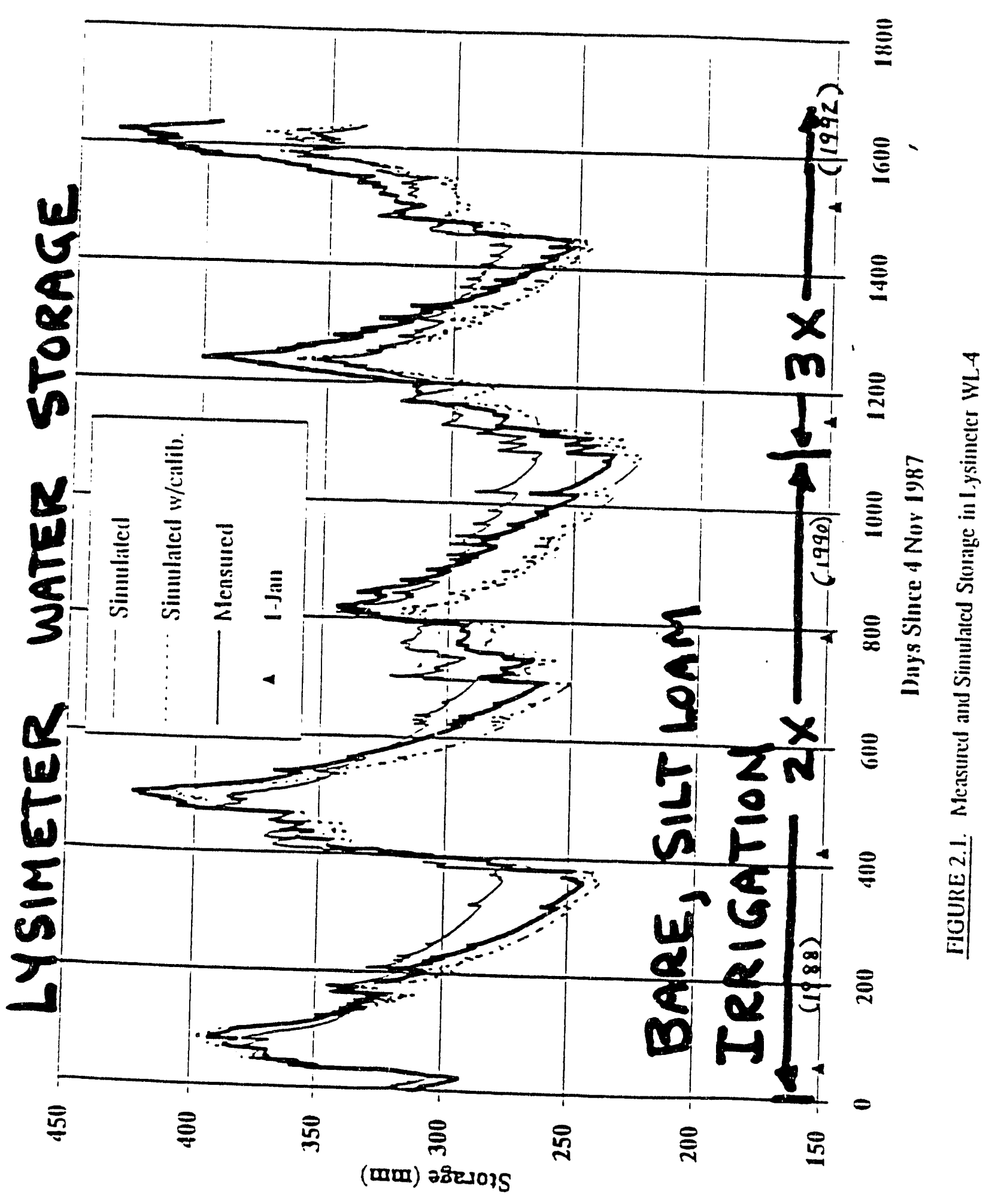


WHC-MR-0420

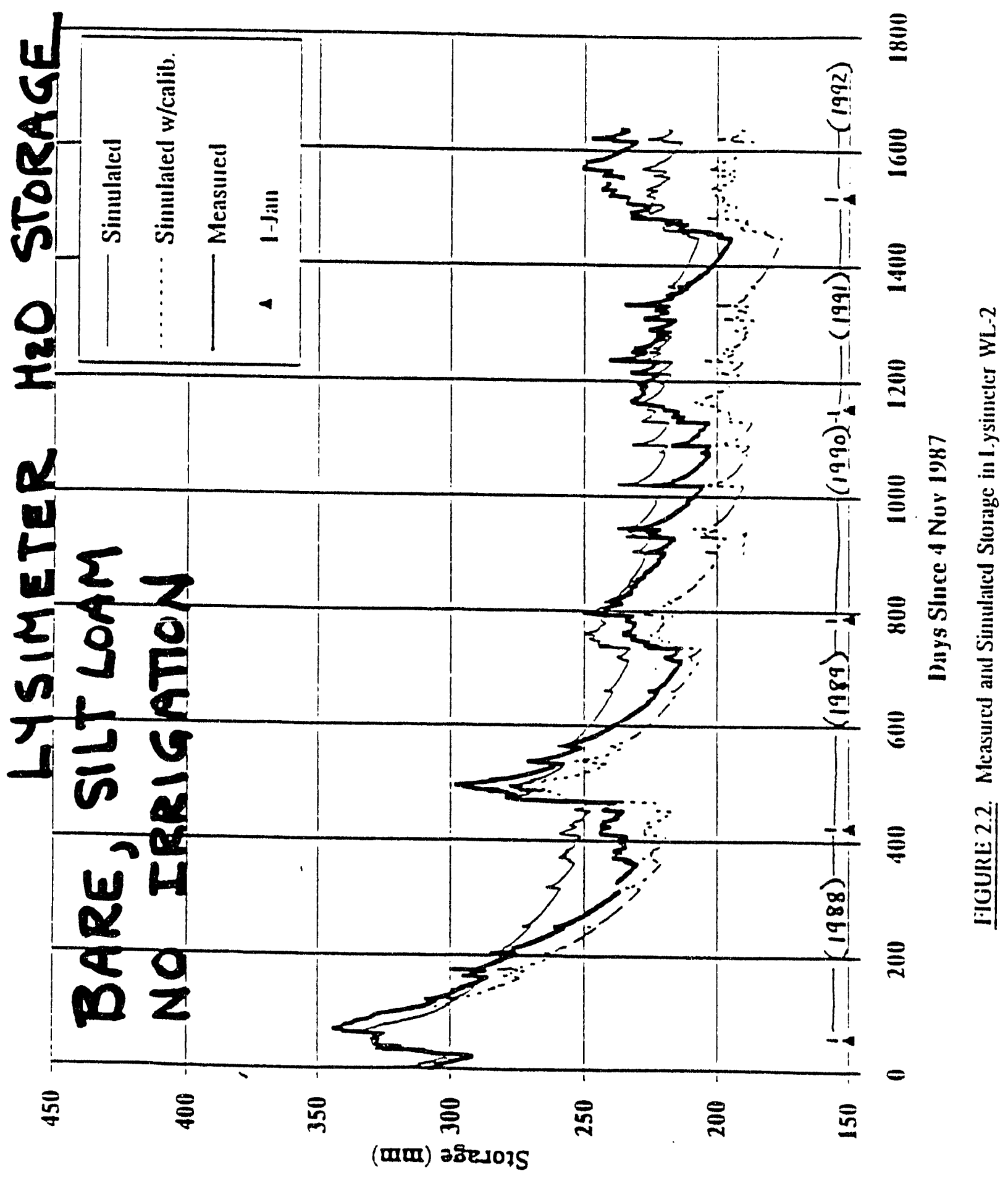




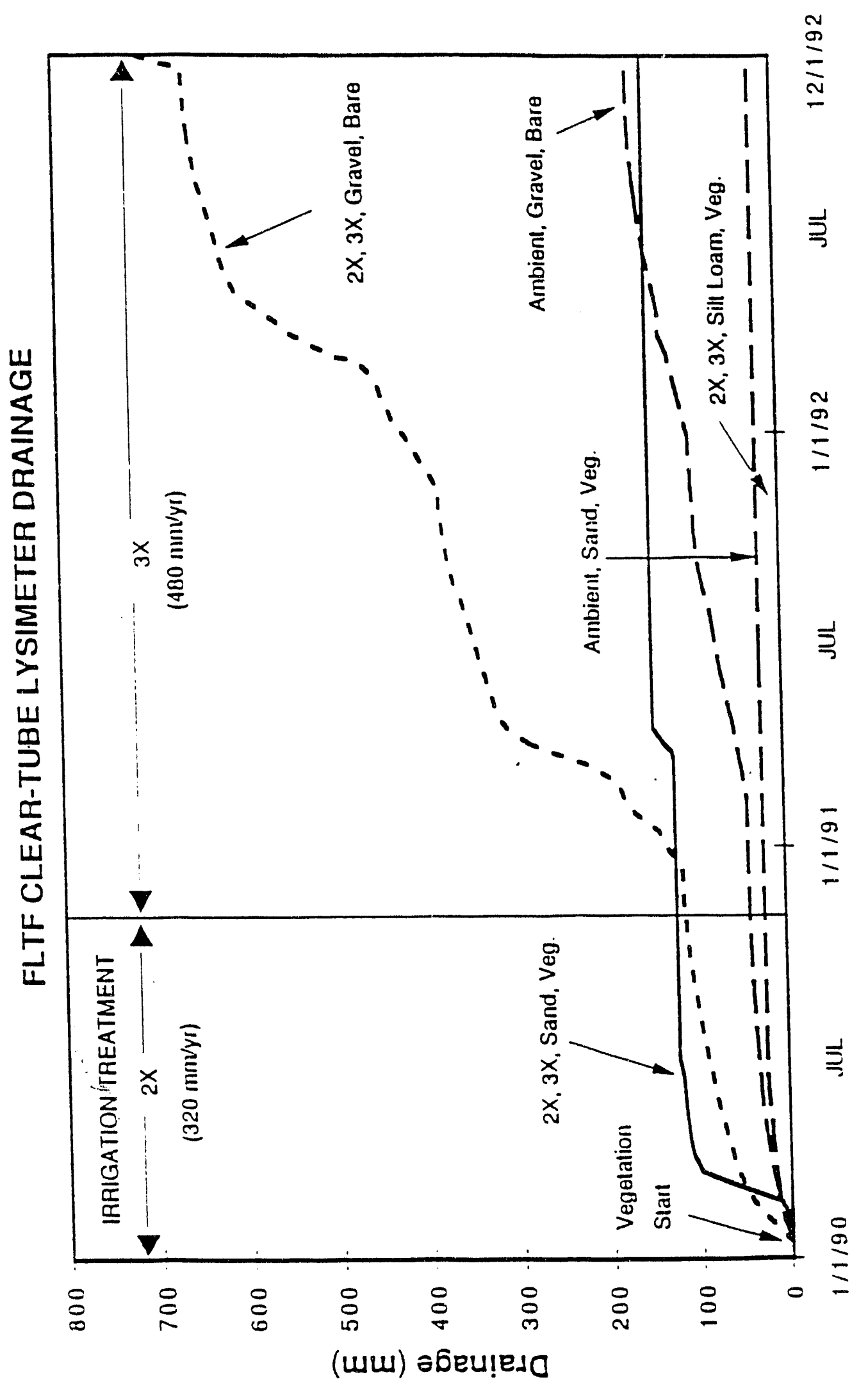


WHC-MR-0420

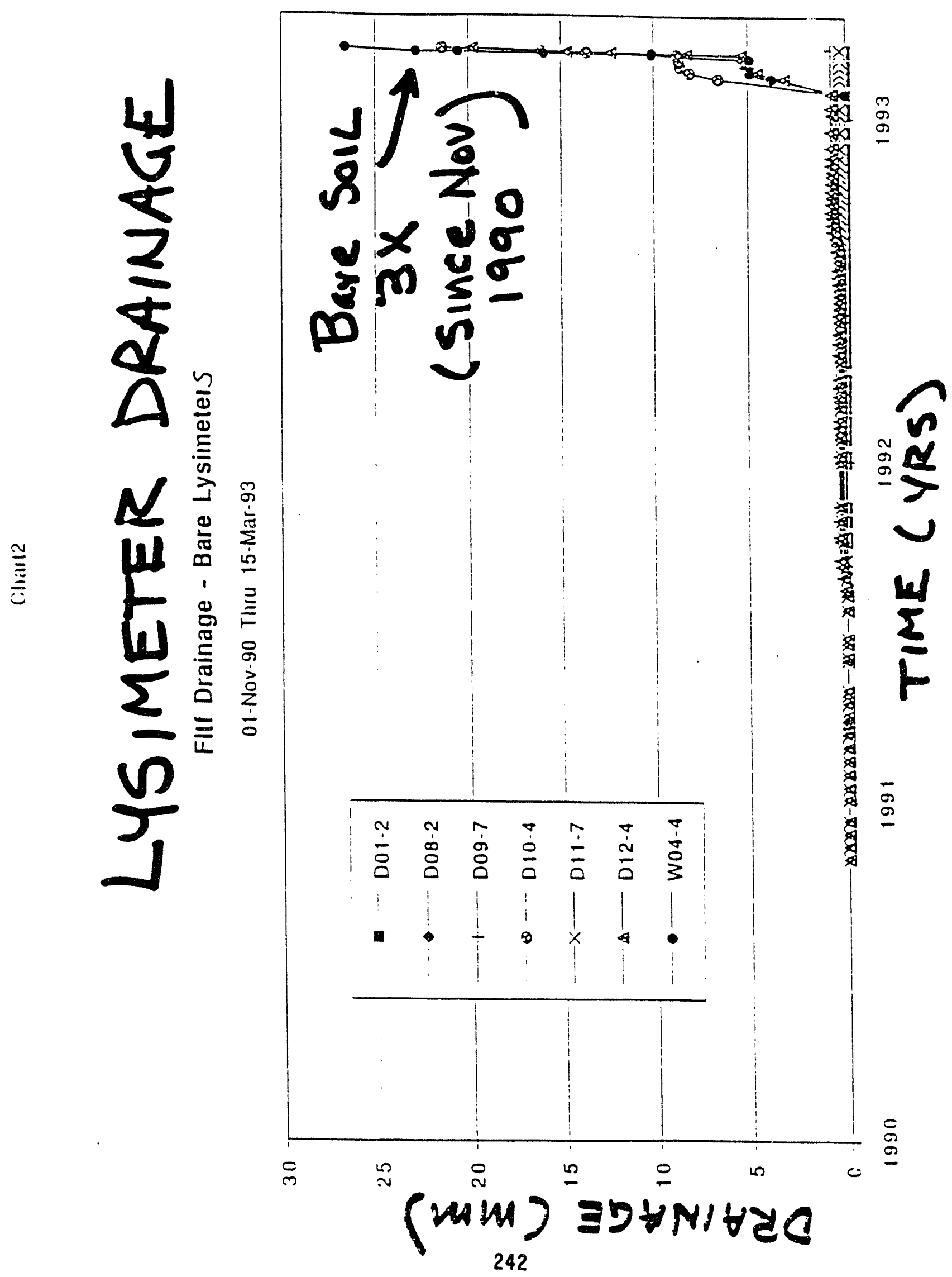




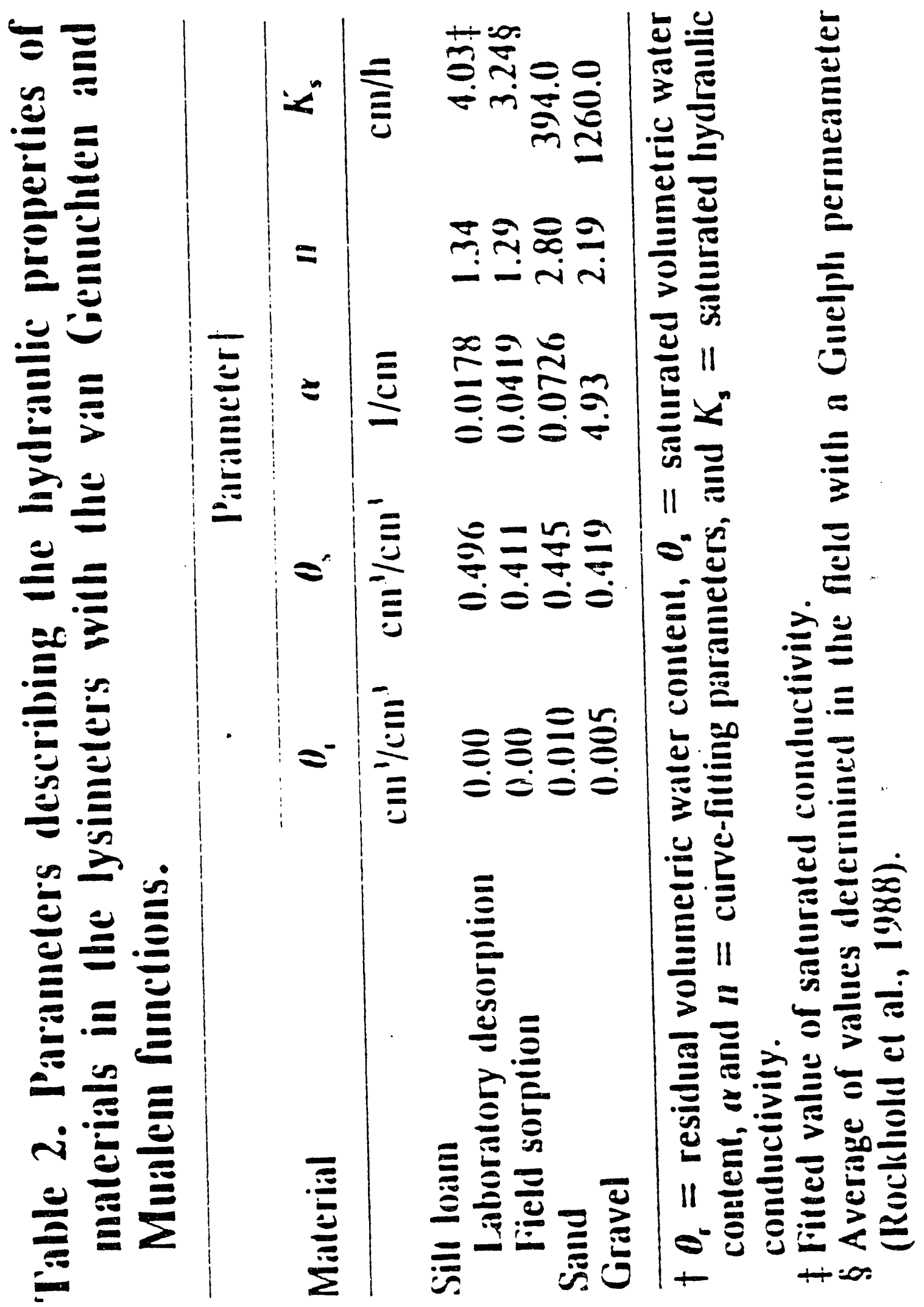


WHC-MR-0420

HYDRAULIC PROPERTIES FOR COVER MATERIALS
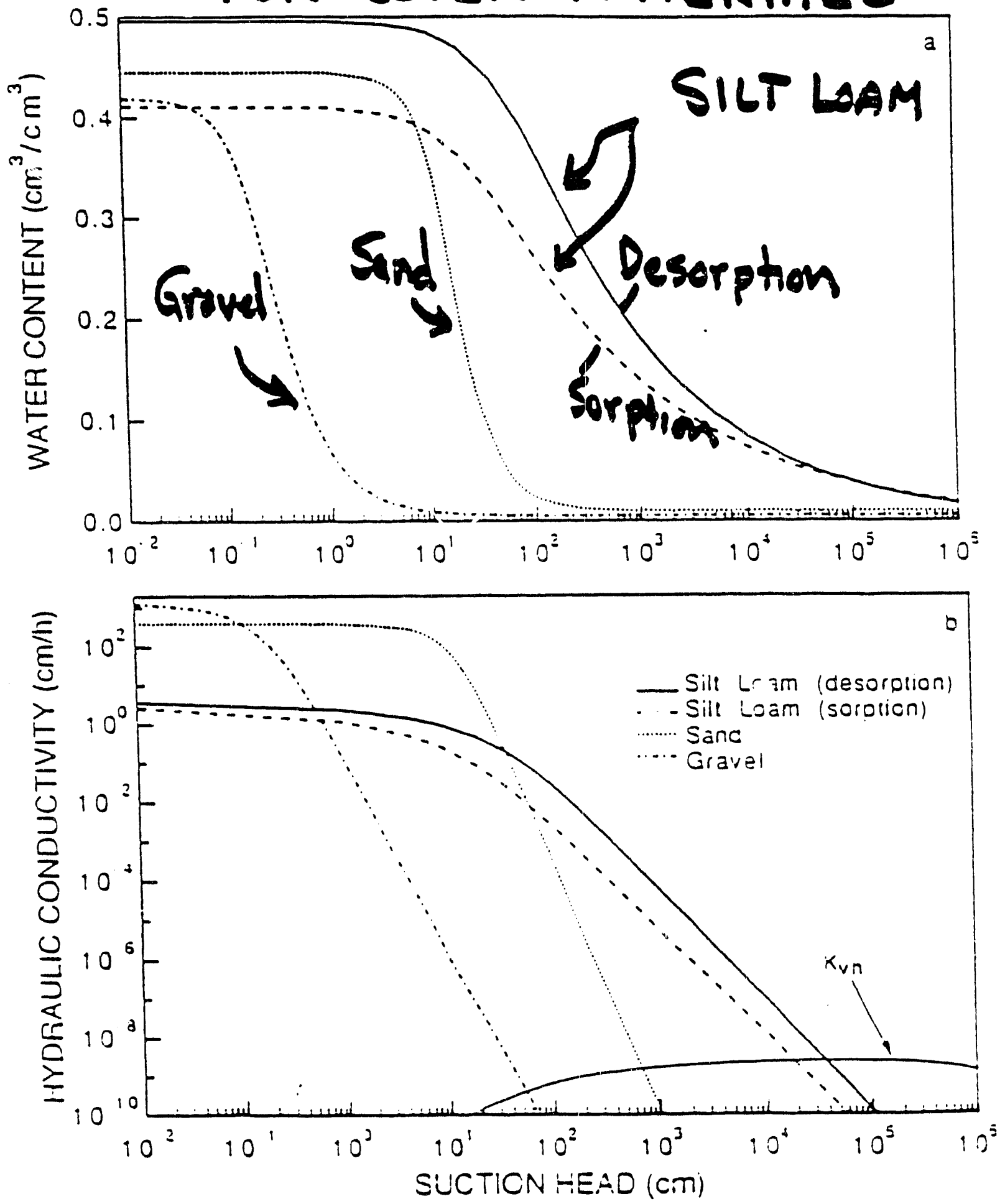

Fig. 2. (a) Water retention and (b) hydraulic conductivity of the materials in the lysimeters. The values of vapor conductivity relative to a suction-head gradient for the silt loam at $15.3^{\circ} \mathrm{C}$ are included for comparison.

244 
WHC-MR-0420

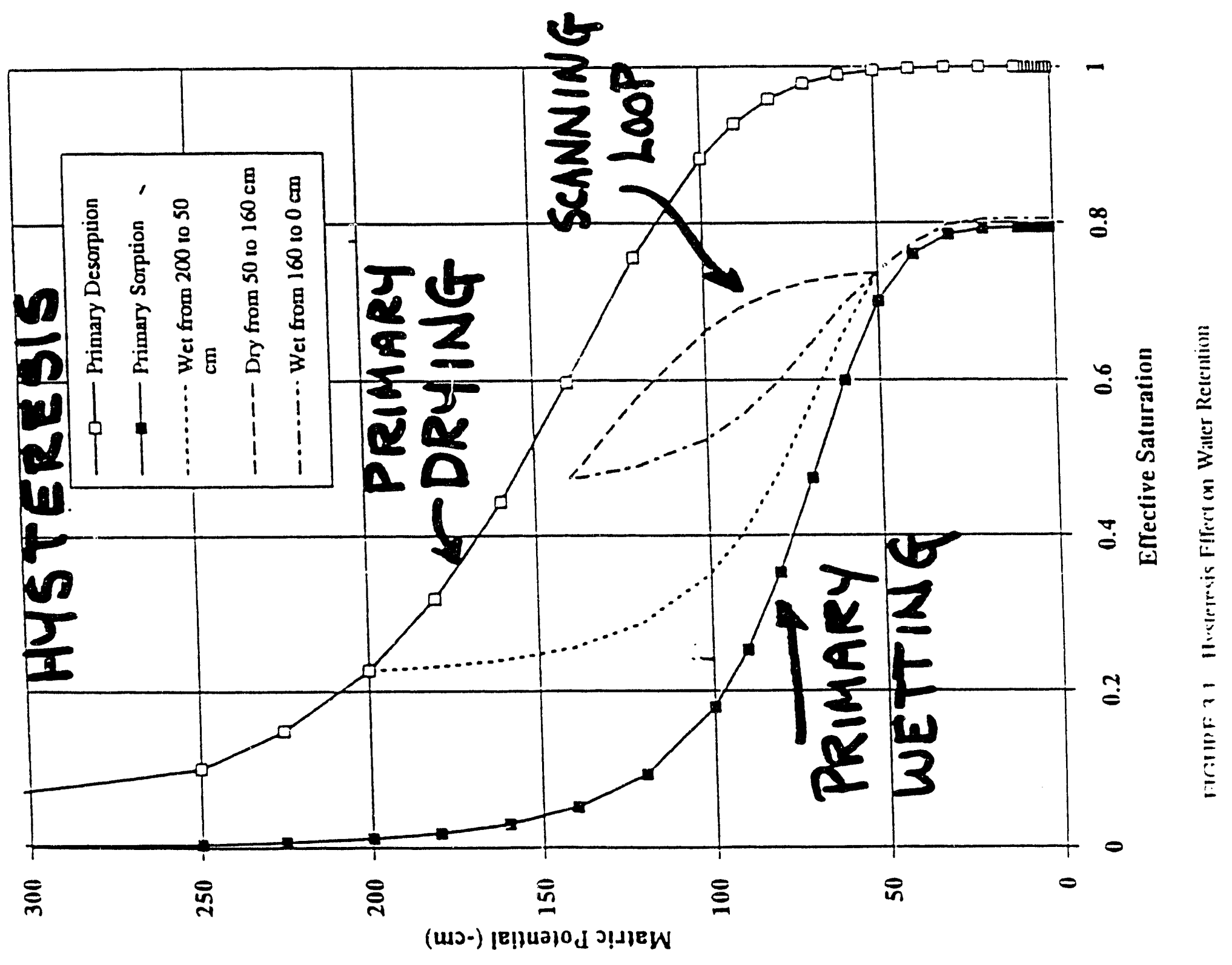




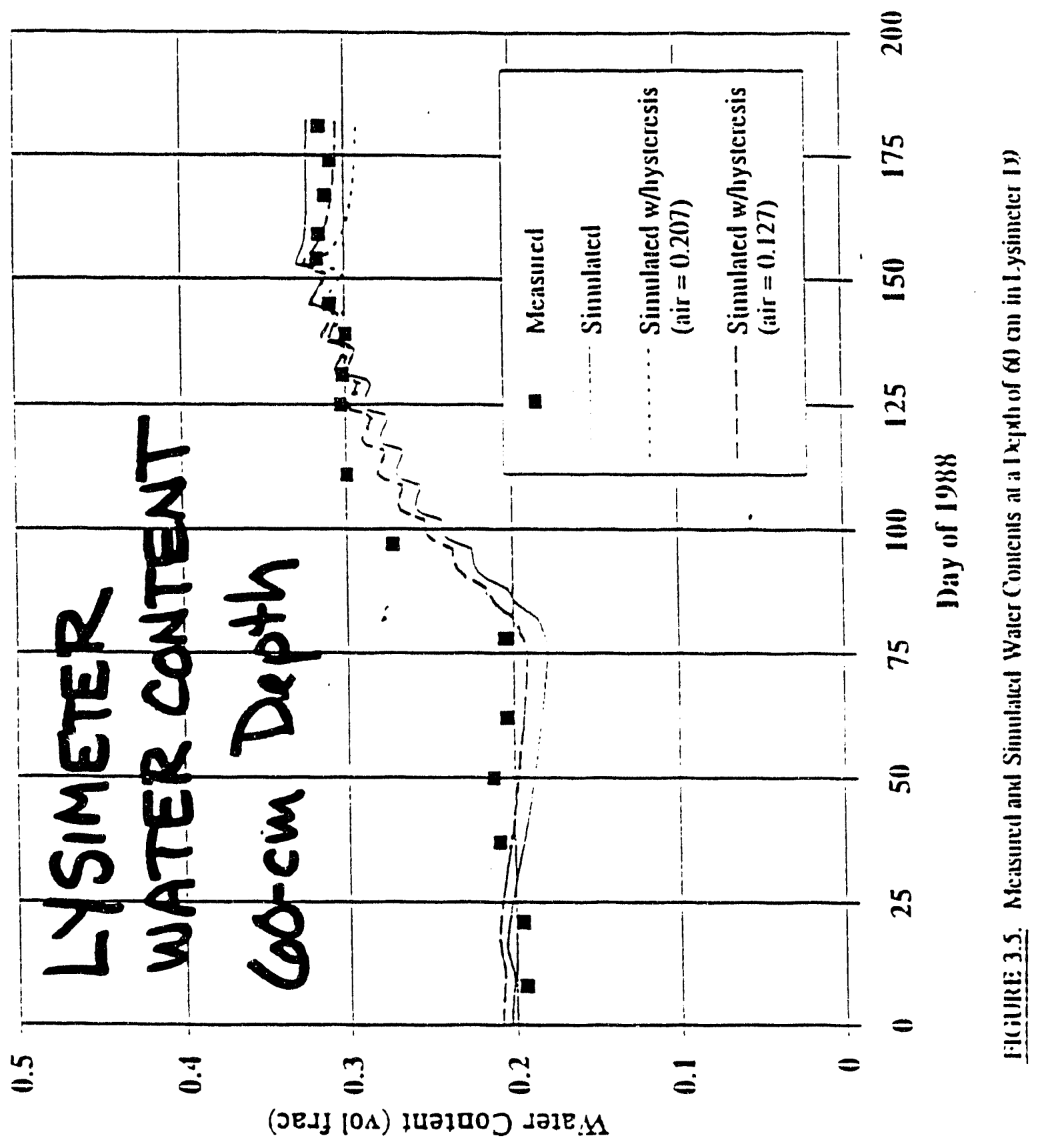




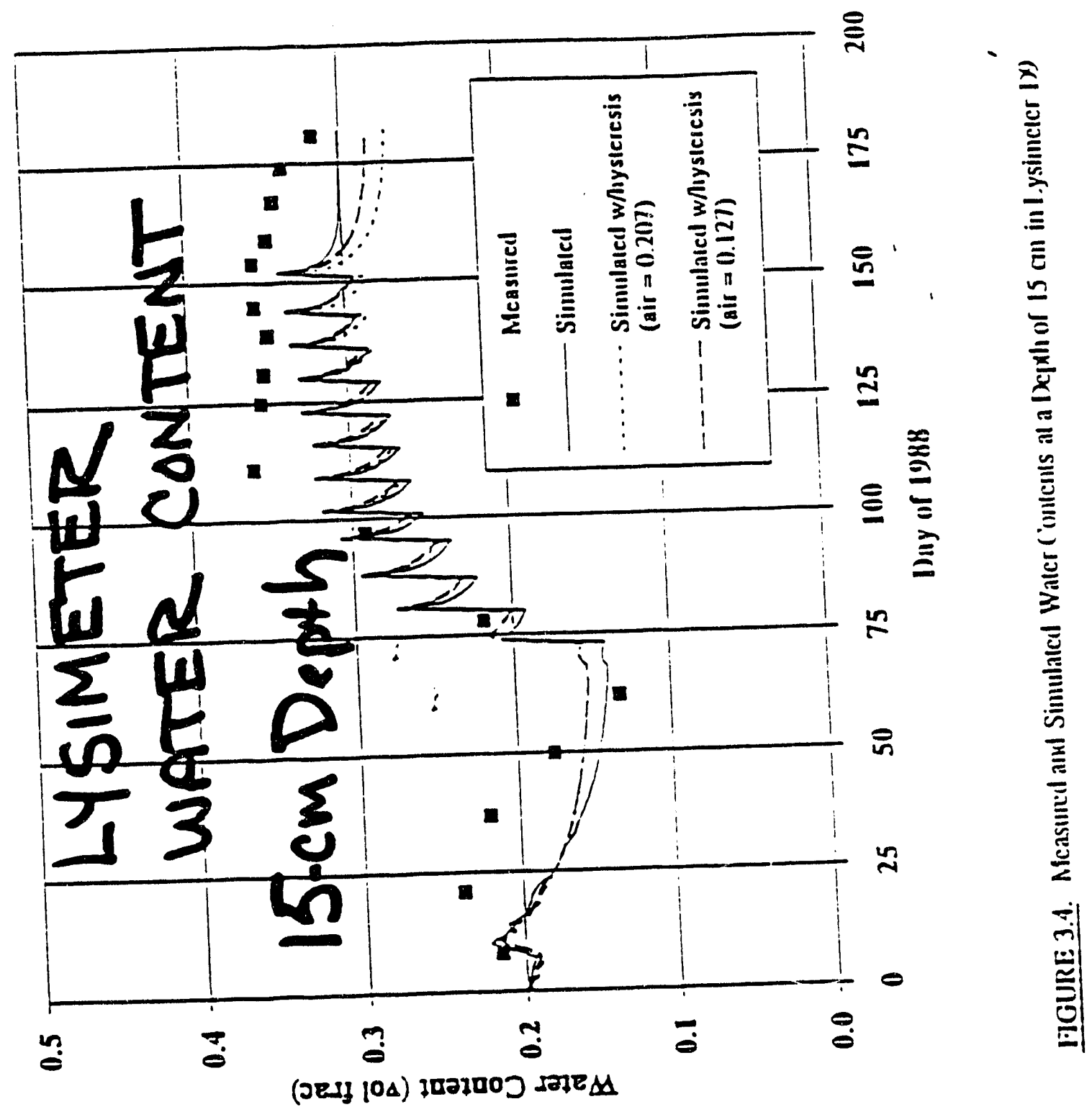


WHC-MR-0420

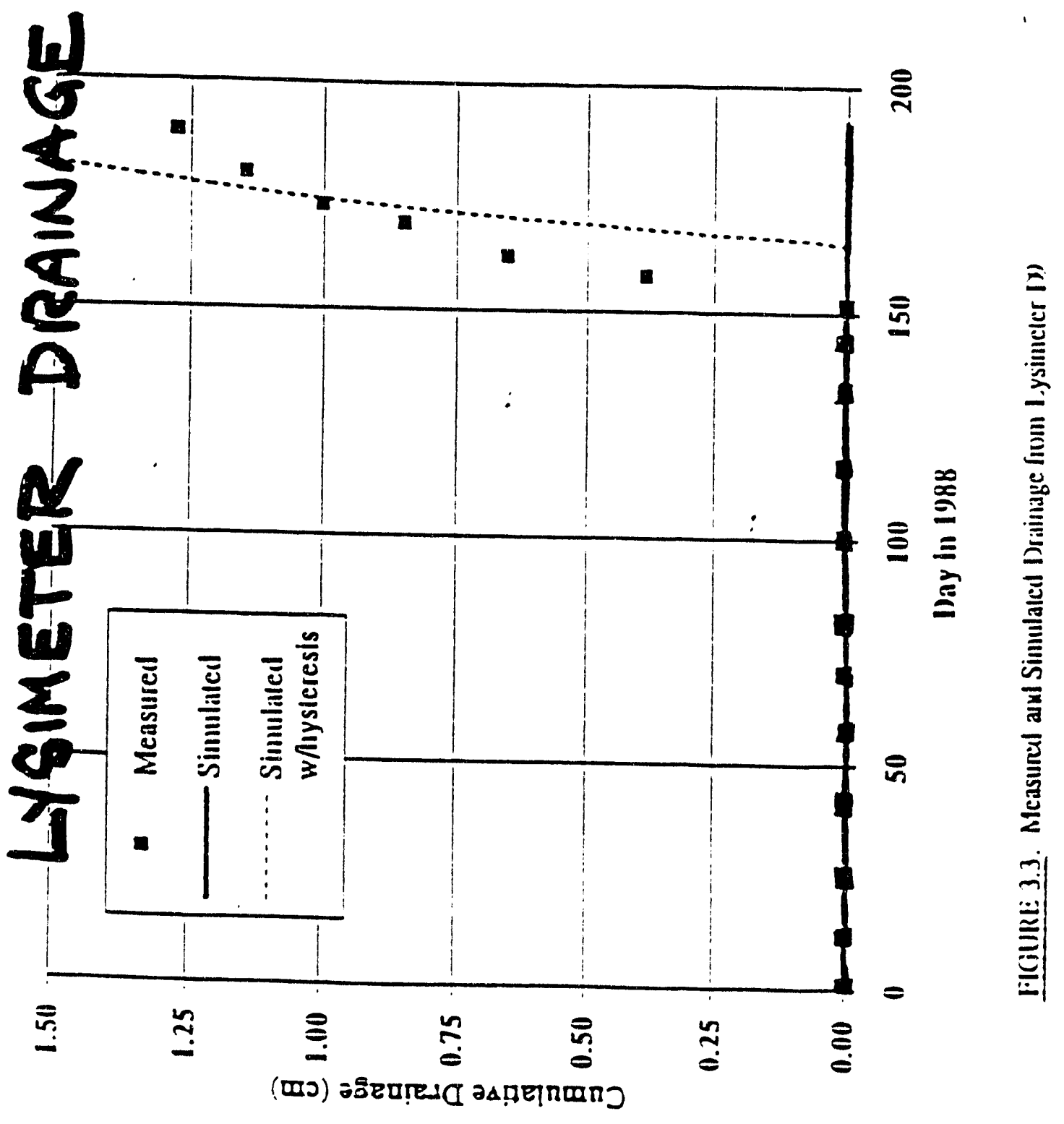




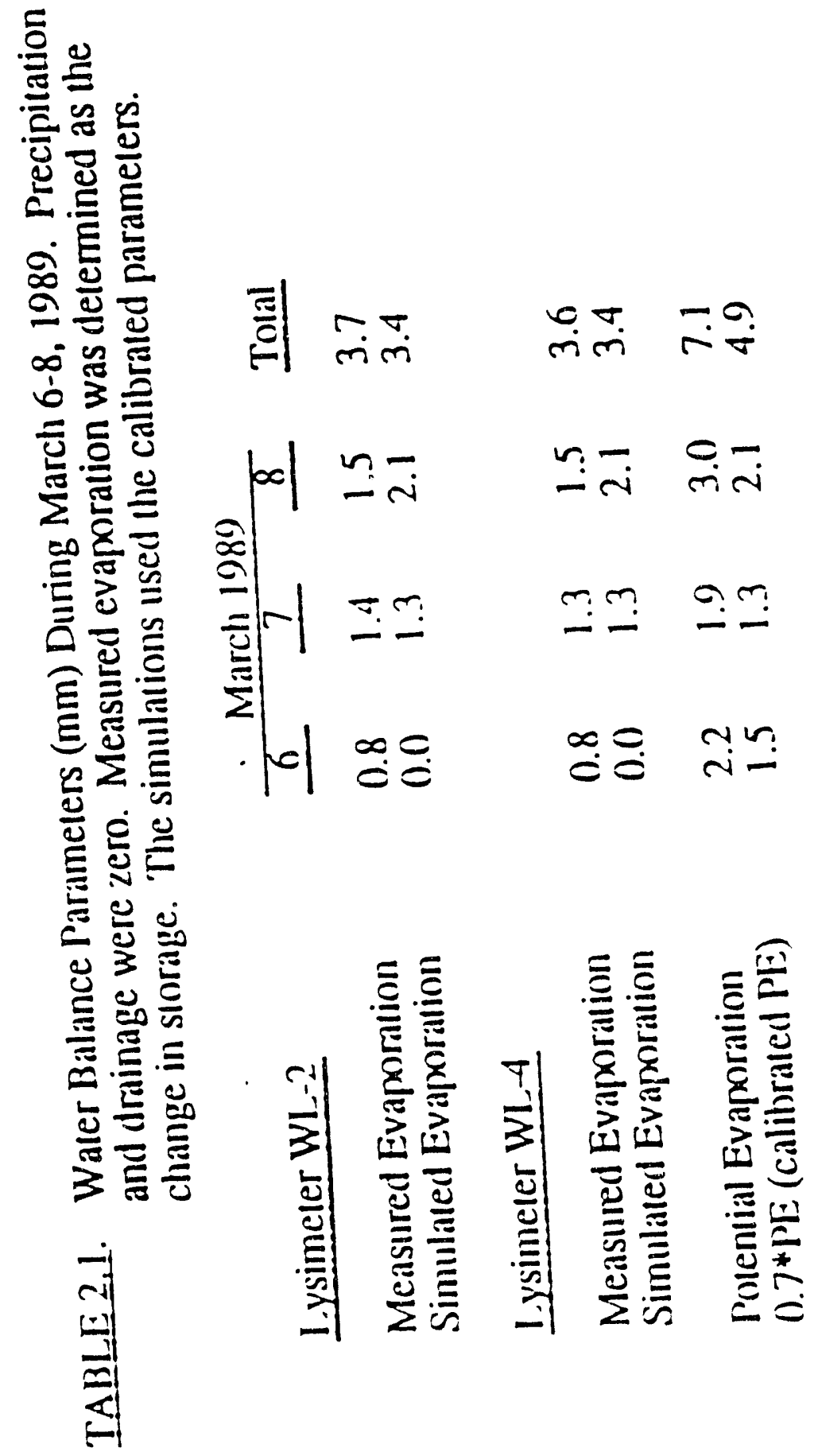




\section{WATER BALANCE (LYSIMETERS)}

\section{SUMMARY}

1. Lysimeters - only way: to Directly Measure Drainage.

2. Arid Site Predictions of Drainage Require Lysimeters (Computer Models of ET and Drainage Require Calibration Data).

3. Waste Site Recharge - Inierresi by Lysimeïer Drainage Data.

- Drainage Aïiected by Variation in Annual Precipitation (P) - Lags Noted

- Gravel - Covered Soil - Piecharge > 7.0\% P

- Bare, Coarse Sands - Perferge > 50\% P

- Grass - Covered Sands - Recriarge $>30 \%$ F

- Summer Annual (Tumbleweed) - Rechnarge $<30 \% \mathrm{P}$

- Fine Soil (Vegetated or Bare) - Recharge $\rightarrow 0$

4. Climate, Vegetation, Soii Varizbles Keg to Water Balance.

5. Drainage Data Zase Available for Model: Calibration (Additionial 5 to $\bar{I}$ yrs Continuous Fiecora"

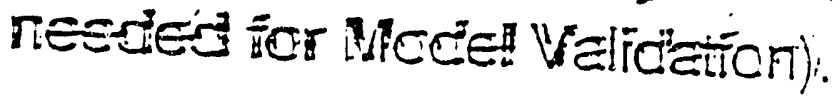


WHC-MR-0420

\section{WORKSHOP MINUTES}


WHC-MR-0420

This page left intentionally blank. 


\section{VADOSE ZONE MODELING WORKSHOP MINUTES}

March 29, 1993
$=>$ workshop attendee

* workshop presenter.

* John Rawlins - Opening Remarks

introduction

Lynn Gelhar - MIT

Peter Wierenga - University of Arizona

Rien van Genuchten - U.S. Salinity Lab., Riverside

covered workshop agenda

* Raz Khaleel - Statement of Workshop Goals and Objectives

Overview of previous workshop

history

- had similar workshop $31 / 2$ years ago

previous workshop recommendations:

1) site characterization data base

2) code calibration/validation

a) document existing database

b) some Hanford Sites aren't fully utilized

process identification:

1) validation of Van Genuchten-Mualem relationships

2) saturation-dependent anisotropy for vadose zone

Why focus on modeling issues?

1) predictive reliability

2) regulatory requirements

3) compromise between data requirements and loss of predictive reliability

\section{Objectives}

A) Objective 1

Following codes used on site:

1.) PORFLO

2.) VAM2D-CG (HydroGeologic)

3.) UNSATH (PNL)

Develop a consensus position by all "stakeho?ders"

B) Objective 2

Obtain a commitinent from all stakeholders to support the results, findings, and/or recommendations.

C) Objective 3

Identify action items

$\Rightarrow$ Ward Staubitz

There is great concern in the 200 Area Waste sites. We can not spend big dollars on all Hanford sites. We need to keep a perspective on the modeling strategy and apply model on a site specific basis. 
* Raz Khaleel - Statement of Workshop Goals and Objectives (continued)

$\Rightarrow$ Peter Wierenga

Questioned previous workshop

$\Rightarrow$ Rien van Genuchten

Need to make reports available to panel members for review.

* Raz Khaleel - Types of Wastes and Waste Sites

Showed overall Hanford site map
1) 200 plateau
2) $100 \& 300$ Area along river
3) 400 Area-FFTF

Hanford Waste Sites

1) Over 1400 waste sites have been identified, including both active TSD facilities and inactive sites.

2) Four aggregate areas are subdivided into 21 waste areas based on facility and type of operation.

3) Looked at 78 sites.

Waste Site Distribution a Hanford pie chart

Liquid Wastes Discharged \& Solid Waste Buried \& Hanford (1944-1989)

1) 5.6 million curies of radioactivity

$\Rightarrow$ Rien van Genuchten

Clarification of pie chart - $20 \%$ of total radioactive waste.

Viewgraphs of tank farms, cribs, \& trenches

Hydrogeology of Hanford Site

- fence diagram of 200 West Area

- groundwater is at 150-250 ft. depth

- Mike Connelly's viewgraphs

How much data collection will be enough for a 560 square mile site? This will hopefully be resolved from panel members.

\section{Rien Van Genuchten}

What type of data is collected?

Bulk density, etc.

* Len Collard - Performance/Risk Assessment at a CERCLA site at Hanford

200-BP-1 Cribs

1) overall RI/FS (Remedial Investigation/Feasibility Study) process

2) description of operations.

3) site sampling

4) lab testing

5) selection of sampies 
6) modeling approach

* Len Collard - Performance/Risk Assessment at a CERCLA site at Hanford 200-BP-1 Cribs (continued)

7) modeling domain

8) results

1) $\mathrm{RI} / \mathrm{FS}$

- conduct baseline risk assessment

- provide additional site characterization

2) operations

- 200-BP-1 site consists of 4 acres and contains 8 cribs

- 2 types of discharges

1) supernatant

2) evaporation

$$
\text { - cribs B43-B49 }
$$

- cribs 850 and $B 57$

- operating time $3 / 65$ - 1974

- waste quantities discharged to cribs in 200-BP-1

crib construction viewgraph

$$
\text { earth backfill }
$$

sisalkraft paper

washed gravel

$\Rightarrow$ Rien van Genuchten

Concerned about crib operation and contaminants trickling out of washed gravel.

$\Rightarrow$ Peter Wierenga

What is your philosophy of discharge?

* Len Collard

To hold contaminants in the soil column and if it did reach the groundwater, the concentration would be small.

$\Rightarrow$ Peter Wierenga

How do you keep track of what was discharged - the only to correctly determine the discharge is to compare what was pumped in vs. What was pumped out.

$\Rightarrow$ Doug Sherwood

Gave input on 200-BP-1 crib operations.

$\Rightarrow$ Allen Lu

Concentrated on radionuclides not chemical solvents.

$\Rightarrow$ Mark I. Wood

200-BP-1 input.

$\Rightarrow$ Scott Kindred

Questioned 200-BP-1 values. 
200-BP-1 radionuclides inventory viewgraph

* Len Collard - Performance/Risk Assessiment at a CERCLA site at Hanford (cont inued)

$\Rightarrow$ Lynn Gelhar

Questioned radionuclides values? Where did they come from?

liquid discharge to $200-B P-1$ cribs viewgraph

Srg0 flux for 200-BP-1 cribs viewgraph

3) site sampling activities

total of 28 boreholes
a) groundwater well - depth $>200 \mathrm{ft}$.
b) most wells had a depth of $50 \mathrm{ft}$.
c) surface sampling

$\Rightarrow$ Peter Wierenga

When did sampling take place?

* Len Collard

Sampling took place in recent years.

$\Rightarrow$ Peter Wierenga

Who is doing the drilling/testing?

* Len Collard

$\mathrm{KEH}$ is responsible for the well drilling and testing is done both on and off site.

$\Rightarrow$ Lynn Gelhar

Is there a site characterization report?

$\Rightarrow$ Ward Staubitz

There will be a site characterization report this Thursday.

$\Rightarrow$ Peter Wierenga

Questioned both the physical and chemical sampling.

$\Rightarrow$ Rien van Genuchten

Please give panel members a list of the physical and chemical properties and 1 ab resuits.

$\Rightarrow$ Ward Staubitz

Chemical sample and physical property sample techniques:

- only scanned part of the sample so they could keep moisture in

- have gravimetric moisture content results

3) site sampling activities (continued)

borehole schematic of material type

$\Rightarrow$ Rien van Genuchten

Question concerning silt layer over 200-BP-1 site. 
$\Rightarrow$ Doug Sherwood

There are about 11 deep bore wells for the 200-BP-1 site.

* Len Collard - Performance/Risk Assessment at a CERCLA site at Hanford (continued)

$\Rightarrow$ Rien van Genuchten

What was the physical and chemical data collected? A database of this information is needed.

* Len Collard

I have a list of what physical property tests were ran, but I do not have data values.

$\Rightarrow$ Rien van Genuchten

Were you able to keep gravels compact for moisture retention test?

Was the unsaturated hydraulic conductivity overestimated?

* Len Collard

The dryer regions are correct.

$\Rightarrow$ John Relyea

Throwing our larger rocks creates more voids that are filled, thus the unsaturated hydraulic conductivity will be overestimated. The unsaturated hydraulic conductivity value will be higher in model than in the field.

$\Rightarrow$ Peter Wierenga

The 200-BP-1 data looks very conservative - low conductivities.

$\Rightarrow$ Ward Staubitz

The unsaturated hydraulic conductivity is non-conservative on a flow and transport contaminant modeling philosophy.

$\Rightarrow$ Lynn Gelhar

Speculating on curves - can you do larger field testing by using pipes, thus have a larger test sample.

$\Rightarrow$ John Reiyea

I will address this tomorrow in my presentation - we currently use 20 points per curve.

$\Rightarrow$ Rien van Genuchten

What kind of " $n$ " values did you get?

$\Rightarrow$ Ward Staubitz

We need to make copies of the RI report and distribute them to the panel members. Did you need a model for the risk assessment of this site?

$\Rightarrow$ Rien van Genuchten

How did you handle absorption? Where did you get Kd values?

* Len Collard

Kd values were obtained by Hanford studies. 
* Len Collard - Performance/Risk Assessment at a CERCLA site at Hanford (continued)

$\Rightarrow$ Rien van Genuchten

How did results come out for the unsaturated zones? Where were measurements taken?

* Len Collard

Borehole.

$\Rightarrow$ Rien van Genuchten

Can you reconstruct the measurements taken vs. sample depth?

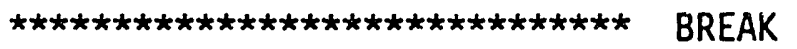

* Len Collard - Performance/Risk Assessment at a CERCLf site at Hanford (continued)

Fluid boundary conditions - geometric layout of grid viewgraph.

$\Rightarrow$ Rien van Genuchten

Do you have reasonable estimates of source?

* Len Collard

Yes.

samples were only taken at discreet points

RLS Spectral Gamma-Ray Borehole survey viewgraphs

$\Rightarrow$ Rien van Genuchten

Have you looked at geochemical controls?

$\Rightarrow$ Ward Staubitz

Straight absorption was assumed.

$\Rightarrow$ Lynn GeThar

$\therefore$ re you going to be showing Kd data?

$\Rightarrow$ Rien Van Genuchten

What was the maximum Cesium concentration in 200-BP-1 layers?

* Len Collard

$600 \mathrm{~K} \mathrm{pCi/gram}$

$\Rightarrow$ John Relyea

Do you have anything on the history of cribs and why did they cease operations?

* Len Collard

No. 
Radionuclides levels for 349A Lab Measurements viewgraph

Sampling scheme for deep boreholes B43-B49 viewgraph

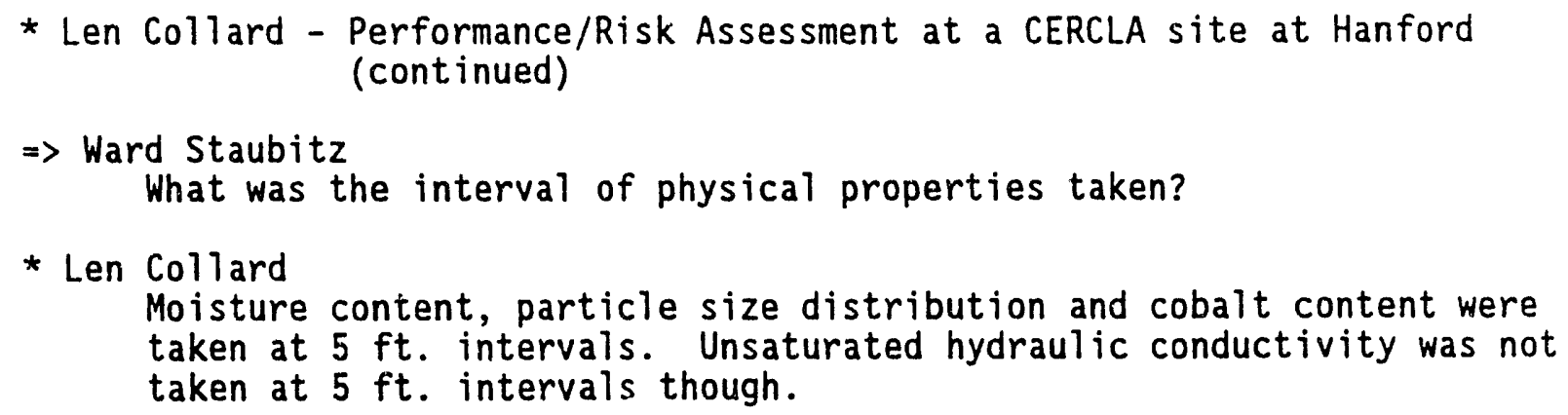

5) selection of samples for testing

1) provide data for major layers

2) problems

a) individual sample integrity and representativeness

b) use of multiple liners

c) lack of data in hot zone

d) possible overdriving of sampler

- bulk density $>2 \mathrm{~g} / \mathrm{cc}$

e) true behavior of gravels

f) sensitivity of $1 a b$ technique

g) bulk density to use with moisture retention data

- field bulk density

$\Rightarrow$ John Relyea

Two liners are currently being used.

6) modeling approach

1) conceptual model

2) computer program selection

3) contaminants of concern

a) inventory

b) screening process

4) scenarios are conservative, but not unrealistic

a) start with reported inventory

b) low Kd from range

c) vertical movement to groundwater - isotropic

d) later migration

1) anisotropic

2) world model for interactions

e) recharge rates

1) T106 Study

- average $13 \mathrm{~cm} / \mathrm{yr}$

2) $1 \mathrm{~cm} / \mathrm{yr}$

- precipitation rate $16 \mathrm{~cm} / \mathrm{yr}$

3) $10 \mathrm{~cm} / \mathrm{yr}$ (preliminary biota barrier)

$\Rightarrow$ Rien van Genuchten

No vegetation. 
mesh for single crib with horizontal symmetry viewgraph

* Len Collard - Performance/Risk Assessment at a CERCLA site at Hanford (continued)

$\Rightarrow$ Ward Staubitz

Where did you get saturated hydraulic conductivity values?

* Len Coll ard

Top gravel layer had a number of different samples in which the highest saturated hydraulic conductivity was picked. The silt layer had only one sample taken thus that saturated hydraulic conductivity was used.

$\Rightarrow$ Rien van Genuchten

Did you correct initial moisture content for gravel?

* Len Collard

No.

$\Rightarrow$ Rien van Genuchten

Then you are not being consistent.

$\Rightarrow$ Peter Wierenga

It does not matter what the initial saturated hydraulic conductivity values were for a small time scale.

$\Rightarrow$ Rien van Genuchten

I am concerned with your mechanics. I agree with your steady state calculations, however, if you overestimate your moisture content and fix boundary conditions at the top, then the velocity will be underestimated (pore velocity is smaller), thus what Len did was not the worst case scenario, but best case scenario.

$\Rightarrow$ Mark Rockhold

Questioned recharge rates.

$\Rightarrow$ Niall Kline

Below crib velocity.

$\Rightarrow$ Rien van Genuchten

Did you fix the amount of liquid or did you let the soil determine?

Should determine total amount - the flux conditions throughout the crib operation and then after operation, apply just rainfall.

Chose ambient conditions of $\mathrm{Kd}$ value.

Sr-90 concentration plots

$\Rightarrow$ Lynn Gelhar

$\mathrm{Kd}=20$ for strontium.

$\Rightarrow$ Rick Healy 


\section{WHC-MR-0420}

Do you have results for a conservative $K d$ ?

$\Rightarrow$ Ward Staubitz

Why does biota barrier accelerate?

* Len Collard - Performance/Risk Assessment at a CERCLA site at Hanford

* Len Collard (continued)

I did a world model with all cribs - 30 model.

$\Rightarrow$ Rick Healy

What were the dispersivity values and " $x$ " and " $y$ " increments?

$\Rightarrow$ Mark Rockhold

Did you calculate Peclet numbers?

world model viewgraph

surface boundary conditions independent of moisture content

$\Rightarrow$ Scott Kindred

How did the 1992 results compare with field measurements?

* Len Collard

They were not compared.

$\Rightarrow$ Lynn Gelhar

What is the horizontal and vertical distance in world map 1974 to 1992 ?

What is the volume of major infiltration?

* Len Collard

B50 crib dominates.

$\Rightarrow$ Doug Sherwood

There was 40 million liters released to the 850 crib.

$\Rightarrow$ Mark Wood

What happens when you lower the Kd value?

* Len Collard

The concentration in water increases.

$\Rightarrow$ Peter Wierenga

Did you look at nitrates and tritium?

* Len Coll lard

There is no information on the duration of operation.

* Raz Khaleel - What Are The Issues?

A) Calibration/Validation

1) flow and transport codes calibrated/validated

2) database

B) Evaluate estimates of unsaturated hydraulic conductivity (viewgraph) 
C) Dispersivity estimates

D) Uncertainty and spatial variability

E) Evaluate applicability of PSD data in estimating moisture retention (Arya-Paris model)

F) Kd/Rd approach

G) Evaluate moisture-dependent anisotropy

* Raz Khaleel - What Are The Issues? (continued)

Stochastic Theory - macroscopic anisotropy

Injection Test Site

- 1980 \& 1981 time frame

- water was injected at $15 \mathrm{ft}$. depth

saturated hydraulic conductivity histogram results

$\Rightarrow$ Lynn Gelhar

Do they have an open borehole above groundwater?

$\Rightarrow$ Rien van Genuchten

Are you concerned about the two methods? Your method gives you 1000

times the results.

$\Rightarrow$ Mark Rockhold

Question defining slug test.

$\Rightarrow$ Peter Wierenga

Three orders of magnitude is severe.

experimental and fitted variograms viewgraph

Injection Test Site data flowchart viewgraph

Kr vs Psi viewgraph

$\Rightarrow$ Rien van Genuchten

Questioned data on $\mathrm{Kr}$ vs. Psi viewgraph.

RETC viewgraph

$\Rightarrow$ Mark Rockhold

What is your procedure for combining a centrifuge sample to that of a lab sample? There are porosity problems and inconsistencies.

$\Rightarrow$ Lynn GeThar

I suggest using the data directly instead of fitting it first.

$\mathrm{Kr} / \mathrm{Kz}$ anisotropy vs. mean pressure head viewgraph

Injection Test Site borehole layout viewgraph

$\mathrm{Kr} / \mathrm{Kz}$ anisotropy based on turn ratios

$\Rightarrow$ Lynn Gelhar

Did not understand how above values were calculated.

$\Rightarrow$ Rien van Genuchten 
What gradient did you assume in Darcy's Law?

$\Rightarrow$ Rick Heaiy

Did you have lab data? Did you use vertical hydraulic conductivity or horizontal hydraulic conductivity?

* Raz Khaleel - What Are The Issues? (continued)

$\Rightarrow$ Mark Rockhold

What retention curves did you choose?

$\Rightarrow$ Peter Wierenga

Do you have water content distributions?

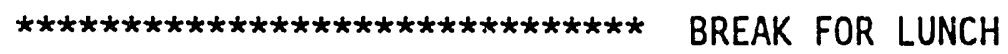

* Peter Wierenga - Las Cruces Trench Experiment

Objectives

1) test deterministic and stochastic models for water flow and solute transport

2) test site characterization and monitoring techniques

must have good control over boundary conditions

borehole parameter test

- see how long it takes a known amount of water to seep into ground

groundwater $=300 \mathrm{ft}$.

Sampling

9 soil layers

50 cores - saturated hydraulic conductivity per layer

$0.50 \mathrm{~m}$ horizontal spacing

$0.65 \mathrm{~m}$ vertical spacing average

Hydraulic properties

1) in-situ saturated hydraulic conductivity

2) core soil water retention data

3) van Genuchten parameters.

20 model to predict infiltration/evaporation

$0.50 \mathrm{~cm} /$ day

how to determine hydraulic properties?

wet end of water retention curve

one step outflow method

- problems with this method in determining unsaturated hydraulic conductivity values.

Conclusions

1) transient experiments provide good estimates of the soil 

hydraulic property relationships
a) transient tension measurements measured flux boundary conditions
2) gamma is an important parameter

$\Rightarrow$ Raz Khaleel

It looks like data is close to saturation.

* Peter Wierenga - Las Cruces Trench Experiment (continued)

* Peter Wierenga

We had data to $500 \mathrm{~cm}$. In the prediction mode, it is important to know the dry end. One must also take into account vapor flow. I believe the best method is in the "in between range", less confidence in dry end and saturation end.

$\Rightarrow$ Scott Kindred

Importance of vapor flow in unsaturated zone, i.e. Philip and Reese.

$\Rightarrow$ Glendon Gee

There needs to be a concurrence with the importance of vapor flow.

* Allen Lu - Injection Test Site at Hanford

- Injection Test Site (RHO-ST-46; Sisson + Lu, 1984)

- graphical presentation of the field data

- modeling scenarios

- model evaluation criteria

- samples of model results and comparisons

Field Experiment

1) 200 East area

2) approximately 1000 gallons of water were delivered for ten consecutive weeks to each well

3) a $20 \mathrm{~mL}$ solution of the radiological tracers ${ }^{85} \mathrm{Sr}$ and ${ }^{134} \mathrm{Cs}$ were mixed and injected

4) fiald data was obtained by lowering sensors to the desired depths in the observation wells

5) soil samples were taken from two monitoring wells

Injection Test Site viewgraphs

$\Rightarrow$ Peter Wierenga

Do you have an initial moisture content contour?

6 different model simulation scenarios

1) uniform isotropic soil

2) uniform but anisotropic soit

3) non-uniform (10 layers) but isotropic soil

4) same as scenario 3 , but hysteresis is accounted for

5) nonuniform but anisotropic soil

6) same as scenario 5 , but only saturated hydraulic conductivity were allowed to be anisotropic soil 


\section{WHC-MR-0420}

Model Evaluation Criteria

contour plots and time history plots

Injection Test Site viewgraphs

$\Rightarrow$ Rien van Genuchten

What was the anisotropy ratio?

* Allen Lu - Injection Test Site at Hanford (continued)

* Allen Lu

It was varied.

$\Rightarrow$ Lynn Gelhar

Where is the water distributed?

showed zero moment and first moment viewgraphs

$\Rightarrow$ Lynn GeThar

Why not do a complete circle?

$\Rightarrow$ Mark Rockhold

Do you have to interpolate water content values that relate with time?

$\Rightarrow$ Peter Wierenga

Did you get good mass balance? Need to apply this over the full model make a complete circle.

* AlTen Lu

Averaging over the range - get close to full mass balance.

$\Rightarrow$ John Relyea

How long did it take to get all the probe measurements?

$\Rightarrow$ Peter Wierenga

How many probes did you have?

* Allen Lu

There is a total of 32 wells.

* John Smoot - 241-T-106 Tank Leak

- occurred 4 years ago

- took place in the north central part of the 200 West area

- volume of liquid $=2000 \mathrm{~m}^{3}$

- single shell tank constructed in 1943 leaked

- tank diameter $=75 \mathrm{ft}$.

- bottom of tank = $11 \mathrm{~m}$ belaw surface

- top of tank $=2.1 \mathrm{~m}$ below surface

- tank contains wide variety of radioactive components

- leak occurred in 1973

- leak duration $=2$ months

- 1978 deepened some of the boreholes 
- 5 soil layers

$\Rightarrow$ Ward Staubitz

Did you have particle size distribution data?

30 mesh - PORFLOW 3 simulation results

$\Rightarrow$ Rien van Genuchten

Are those relative saturations?

* John Smoot - 241-T-106 Tank Leak (continued)

* John Smoot

Yes.

$\Rightarrow$ Scott Kindred

Estimate on numerical dispersion?

* John Smoot

Small retardation factor.

Conclusions

1) PORFLOW 3 results looked good

2) good match for $1973 \mathrm{Ru}-106$ plume

3) discrepancies for 1978 Ru-106 plume

4) good match for $\mathrm{Cs}-137$ plume

5) more site specific data is needed

$\Rightarrow$ John Relyea

High ionic strength?

* John Smoot

Did not take into affect the bulk density and Kd from tank contaminants.

$\Rightarrow$ Frank Schmittroth

Are you sensitive to the inventories?

$\Rightarrow$ Peter Wierenga

After the leak stopped, what was the driving force? Rainfall?

$\Rightarrow$ Scott Kindred

Was $5 \mathrm{~cm} / \mathrm{yr}$ the infiltration rate used?

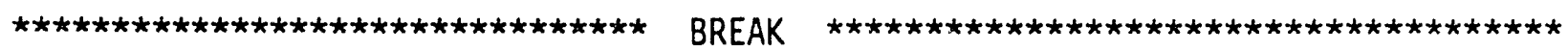

* Charley Kincaid - A Calibration/Validation Proposal

put together activity data sheet (ADS)

Purpose

1) gaining consensus from all players

$\Rightarrow$ Rien van Genuchten

What and who defines a validated model? 
$\Rightarrow$ Peter Wierenga

Who developed all the nonsense of constraints? It looks as if time and efforts are wasted on doing documentation, instead of real life work.

* Charley Kincaid

This is needed for Quality Assurance and for traceability.

$\Rightarrow$ Rien van Genuchten Question concerning validating computer code.

* Charley Kincaid - A Calibration/Validation Proposal (continued)

$\Rightarrow$ Fred Mann

Personally feels that constraint documentation is driven by Microsoft, Borland, etc., and not by the nuclear field.

$\Rightarrow$ Scott Kindred

Finds a disconnect between the RI/FS process and modeling aspect, i.e. 200-BP-1.

$\Rightarrow$ Peter Wierenga

What is the consensus?

* Charley Kincaid

Must have enough confidence in model to go to his superiors/public with it. DOE and DOE contractors can not make that decision. Feel that a consensus needs to be made with the scientific community.

$\Rightarrow$ Chuck Cline

Tried to standardize four models, i.e. several models of PORFLO on site.

This is a problem on site.

$\Rightarrow$ Ward Staubitz

What are these models going to be used for? What is too much? Need to collect less data and rely more on models.

$\Rightarrow$ Lynn Gelhar

Did not feel that 200-BP-1 was a good model.

$\Rightarrow$ Ward Staubitz

Agrees with Lynn Gelhar - also has samples stored that can be resampled and new curves collected. Do you feet expense is justified?

$\Rightarrow$ Doug Sherwood

Lynn Gelhar has not been given detailed information, i.e. \# of samples, etc., concerning 200-BP-1. Felt that Lynn Gelhar did not get a full enough picture to make a decision.

$\Rightarrow$ Rien van Genuchten We were not given information. We should go back to the earlier question concerning validation/calibration of 200-BP-1.

$\Rightarrow$ Scott Kindred Concerning risk assessment, there was plenty of data on 200-BP-1, but 
did not have enough information for validation though.

$\Rightarrow$ Ward Staubitz

Would you be comfortable in applying 200-BP-1 data to another site as a data set?

$\Rightarrow$ Scott Kindred

Depends on site itself. For sites where contaminants reach groundwater, we would want better characterization. We do however, want to use 200-BP-1 data.

* Charley Kincaid - A Calibration/Validation Proposal (continued)

$\Rightarrow$ Doug Sherwood 200-BP-1 is similar, as far as operation and length, to other sites in 200 East area.

$\Rightarrow$ Charley Kincaid

The 200 East fence is an arbitrary line - sites vary in the same areas.

$\Rightarrow$ Doug Sherwood

Money can not be spent at other sites as it was spent at 200-BP-1.

$\Rightarrow$ Rien van Genuchten

Given these complexities - do you feel that there will be a validated code for the Hanford site? You are putting a number of criteria that is desirable, but may not obtainable.

$\Rightarrow$ Ward Staubitz

This does bring up the fundamental question - can we validate a model? One way or another, a decision will have to be made using input from this panel.

$\Rightarrow$ Chuck $C 1$ ine

T106 is as close to validation as we have. It is closing in to

$\$ 2$ million for 1 borehole to $180 \mathrm{ft}$. .

$\Rightarrow$ Lynn Gelhar

Aren't you talking about $\$ 50$ billion in cost to clean the Hanford site?

* Lynn Gelhar - What Constitutes a Validation Experiment?

Does the model really predict? < = This is the key question.

Do not see calibration/validation as same process?

Possible Modeling Approaches

A. Deterministic Numerical Model

i. Classical

- ignore spatial variable

- no error measure

- model parameters not directly measurable

ii. Detailed with "actual" heterogeneity

- measurements impractical 


\section{B. Stochastic}

$$
\text { - computationally infeasible }
$$

\section{Represented by spatial random fields}

characterized with a small number of parameters

- mean

- variance

Add geologic information to data sets

Solve for average and variations about mean

Important to capture variation in $K$ vs. Sat curves

Large scale model with variable parameters

* Lynn Gelhar - What Constitutes a Validation Experiment? (continued)

Degree of spread with 20 of mean solution

New Mexico Technical lab experiment (Stephens 1988)

$\Rightarrow$ Rien van Genuchten

Has this been tried in determination sense?

* Lynn Gelhar

Yes - Mualem took ratio of harmonic mean to arithmetic mean.

* Rien Van Genuchten - Issues of Model Testing

Mathematical verification

-accuracy of numerical solution (algorithm)

Experimental validation

- simplified representation of system or process

- validation of process - not a code

- validation applies only to specific set of conditions

- space, time

- initial/boundary conditions

- models are tools

Calibration

- history matching

- parameter estimation (inverse problem)

- parameter uniqueness problems (in case of good fit)

- conceptual problems

- parameters are model/real specific

Predictions/Extrapolation

- space

- other boundary conditions

- other initial conditions

- times

$\Rightarrow$ Fred Schmittroth

How far into future extrapolate?

* Rien van Genuchten 
Length of data collection depends on site specific problems. Changing conditions - no way to appreciate long term changes based on short time of data collection.

$\Rightarrow S \operatorname{cott}$ Kindred

Comparing physical properties with recharge - what parameters are important?

$\Rightarrow$ Glendon Gee

How do you incorporate man-made effects?

* Rien Van Genuchten - Issues of Model Testing (continued)

$\Rightarrow$ Peter Wierenga

Implying a need to stabilize - do you assume non-changing of condition?

$\Rightarrow$ Scott Kindred

Baseline RI - things remain same.

$\Rightarrow$ Doug Sherwood

Regulations have not reviewed/read report - we do not endure conclusions we want help to judge modeling.

$\Rightarrow$ Charley Kincaid

Like turn. away from validation to history matching. How to build program/confidence?

* Rien van Genuchten

Use models only as management tool?

$\Rightarrow$ Charley Kincaid

Models used as tools gain acceptance. First step to gain consensus of model use, ask - What is achievable? What is needed?. All this is part of consensus building. Proposal is that, not funded now or next year.

$\Rightarrow$ Ward Staubitz

No sitewide support for modeling everything on site specific scale.

* Rien van Genuchten

Why not?

$\Rightarrow$ Ward Staubitz

Tri-Party Agreement - DOE's ability to get things done. No binding agreement/no funding.

$\Rightarrow$ Doug Sherwood

Technical building development tied to sites.

$\Rightarrow$ Ward Staubitz

Proposal put umbrella over modeling coordination/funding. Strategy of models/unofficial sharing. 
$\Rightarrow$ Peter Wierenga

Is there approach to models/unofficial sharing?

$\Rightarrow$ Ward Staubitz

No sitewide effort/coordination.

* Rien van Genuchten

A global problem is that outside agencies must go and ask.

$\Rightarrow$ Peter Wierenga

Ideally, have a small group in charge.

* Rien Van Genuchten - Issues of Model Testing (continued)

$\Rightarrow$ Charley Kincaid

Group got together 4 years ago to address question. DOE ought to see this as a sitewide problem, but DOE decided it was too much trouble. Nothing happened.

$\Rightarrow$ Al LaW Some work along this line - Groundwater Management Protection Plan. DOE-RL will implement soon. This is oriented toward characterization and modeling is mentioned.

* Rien van Genuchten Advantages of this is huge, need much more information and find weaknesses.

$\Rightarrow$ Al LaW

ERMC oriented toward site specific.

$\Rightarrow$ Ward Staubitz

Energy modeler on own, depends on individual effort to contact

colleagues. Ought to be meeting to tie efforts together.

Meeting Adjourned 


\section{VADOSE ZONE MODELING WORKSHOP MINUTES}

March 30, 1993

attendee

* John Rawlins - Introduction

* John Reiyea - Laboratory Measurement of Hydrologic Properties

Physical property test on site:

1) particle size distribution

-nevar know what we are going to get

- Hanford history includes volcanoes, wind, flood, glaciers

2) porosity \& bulk density

3) specific gravity \& grain density

4) moisture content

-liners do not hold moisture in soil when temper itures

exceed 140 degrees Fahrenheit

5) saturated hydraulic conductivity methods

1) falling head

2) constant head

3) falling head-rising tail

$\Rightarrow$ Rien van Genuchten

How do you control pressure to control channeling?

* John Relyea

Do not over pressurize.

6) moisture retention curve

CX-2 measures relative humidity

$\Rightarrow$ Rien van Genuchten

Do you deair the water to get complete saturation?

Do you use the first drying curve or the main drying curve?

* John Relyea

We measure first drying curve for saturation.

$\Rightarrow$ Raz Khaleel

What is the main drying curve?

$\Rightarrow$ Rien Var Genuchten

The drying curve that is done after completing we ting curve.

7) unsaturated hydraulic conductivity

$\Rightarrow$ Lynn Gelhar

Do you have good hydraulic contac: ?

* John Relyea

Yes 
hydraulic conductivity values

* Jim Conca - Unsaturated Hydraulic Conductivity Measurement (continued)

$\Rightarrow$ Rien van Genuchten

Do you have equal confidence in wet range as in dry range?

* Jim Conca

We are concentrating on the unsaturated hydraulic conductivity part of the curve, but traditional methods allow for a good calculation of saturated hydrautic conductivity.

$\Rightarrow$ Rien van Genuchten

Could you go up to saturaition if you wanted?

* Jim Conca

Yes

there is not a great effect on the hydraulic conductivity from the packing method used. Example: W7-9 \#0-072

$\Rightarrow$ Lynn Gelhar

Do you have a report that discusses your findings?

* Jim Conca

Yes

$\Rightarrow$ Peter Wierenga

Does the flow come from one point?

* Jim Conca

The flow hits the frorit and then gets dispersed.

$\Rightarrow$ Ward Staubitz

This is an important piece of information into guiding us on site characterization.

we are currently running samples for Z-Plant

* Raz Khaleel - Use of van Genuchten-Mualem Relationships to Predict Unsaturated Hydraulic Conductivity

covered van Genuchten-Mualem equation

Objective

-validate van Genuchten-Mualem relationship to estimate

unsaturated hydraulic conductivit; for Hanford soils, especially at low moisture contents

Current approach

- Tibrary of moisture retention curves of the Hanford site is very limited

-moisture retention curve + van Genuchten-Mualem relations + 


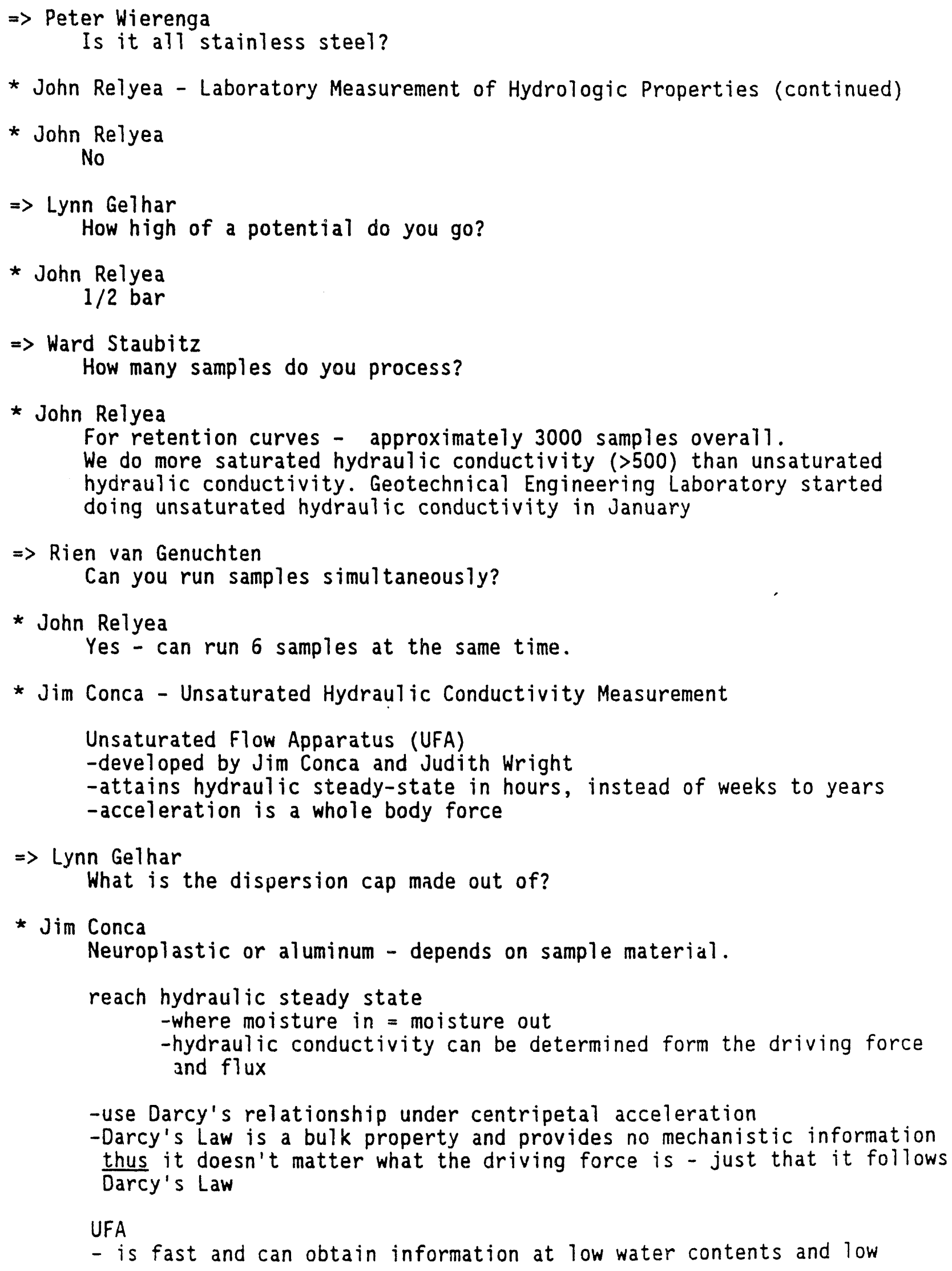


saturated hydraulic conductivity = unsaturated hydraulic conductivity curves

* Raz Khaleel - Use of van Genuchten-Mualem Relationships to Predict Unsaturated Hydraulic Conductivity (continued)

acquire data from 3 labs

1) Ksat measurement data

2) Jim Conca's Lab

3) John Relyea's Lab

parameter estimation

1) predicted

2) simultaneous - 5 parameter $\quad K s a t=$ fixed

3) simultaneous - 6 parameter all float

Example:

compare moisture retention curves generated by above methods simultaneous - 6 parameter method proves to be best fit

$\Rightarrow$ Rien van Genuchten

Most of your data points are in the dry end not the wet range. I would be nervous about the fitting.

$\Rightarrow$ John Relyea

We realize we are not getting enough points in the wet range, but that has changed because we are getting more points now.

$\Rightarrow$ Rien van Genuchten

Definitely need more points in wet range.

$\Rightarrow$ Raz Khaleel

How many data points would you consider enough?

$\Rightarrow$ Rien van Genuchten

Whatever it takes to define the curve.

$\Rightarrow$ Allen Lu

Importance here is that the simultaneous - 6 parameter is best.

$\Rightarrow$ Jim Conca

It is hard to get the wet end in Hanford soils.

$\Rightarrow$ Ward Staubitz

We are mostly concerned in dry end.

$\Rightarrow$ John Relyea

We see moisture contents down to $4 \%$ - it depends on soil texture and pressure. The $10 \%$ moisture content that is stated is an old value. Hanford sand is less than $10 \%$.

$\Rightarrow$ Ward Staubitz

Mainly looking at drainage curve at the sites we are interested. 
$\Rightarrow$ Len Collard

Volumetric moisture content for the 200BP1 site is $4 \%-7 \%$.

* Raz Khaleel - Use of van Genuchten-Mualem Relationships to Predict Unsaturated Hydraulic Conductivity (continued)

$\Rightarrow$ Rick Healy

What is the matric potential that corresponds with that?

$\Rightarrow$ Len Collard

It is low - I'm not exactly sure of the specific value.

$\Rightarrow$ Rien van Genuchten

If you are interested in $K(\theta)$ at low moisture content, why are you using the saturated hydraulic conductivity to measure or predict the curve.

$\Rightarrow$ John Relyea

We can not measure the unsaturated hydraulic conductivity at $10 \%$.

$\Rightarrow$ Peter Wierenga

Match hydraulic conductivity at 50 millibar would be more realistic and safer.

$\Rightarrow$ John Relyea

Referenced last page of his presentation handout.

$\Rightarrow$ Ward Staubitz

Since the lower end of curve is what we are interested in, it would be more realistic to not fit to the saturated hydraulic conductivity.

$\Rightarrow$ Peter Wierenga

I feel much better fitting to the unsaturated hydraulic conductivity.

$\Rightarrow$ John Relyea

The problem with that is that it is time consuming.

viewgraph - fitted hydraulic conductivity vs. measured hydraulic conductivity with regression line

$\Rightarrow$ Lynn Gelhar

The current approach of fitting to saturated hydraulic conductivity in reference to the predictive method shows a lot of errors.

viewgraph - Injection Test Site plots

This is on going - more data needs to be analyzed before a conclusion can be made.

Simultaneous fitting considerable improves fitting.

$\Rightarrow$ Rien van Genuchten

Remember $1=0.5$ is an average, it can range between -10 and 10 . Do not trust fitted 1 . 
* Ben Volk - Use of Arya-Paris Model to Estimate Moisture Retention Data

passed out presentation handout

Only the drying curve was used for this model, but the wetting could also be done.

$\Rightarrow$ Lynn Gelhar

Why is your contact angle $=0$ ?

* Ben Volk

Not sure what it is.

$\Rightarrow$ Lynn Gelhar

To set it to 0 is not justifiable then.

fractal dimension

$\Rightarrow$ Rien van Genuchten

Did you adjust for entrapped air?

* Ben Volk

Fully saturated porosity was used.

$\Rightarrow$ Rien van Genuchten

Using $90 \%$ of saturated water content might improve data considerable.

$\Rightarrow$ Rien van Genuchten

What is the calculated value?

* Ben Volk

Ran calculations on lab samples

What is the predicted value?

* Ben Volk

Plug in alpha and generate curve.

Hydrometer would give more points in smaller sieve ranges.

$\Rightarrow$ Rick Healy

I'm concerned with moisture retention curves being run on a percentage of sample, but other hydrogeologic properties are being measured on entire sample? There are inconsistencies.

$\Rightarrow$ John Relyea

There are papers on both side of the fence. Concerned with $\%>2 \mathrm{~mm}$ of the moisture retention curves we generate, even though they are not corrected by our $7 a b$ - they should be corrected. Sieve data is included as part of lab package. Moisture contents are done on ali of sample in liners. 
* Ben Volk - Use of Arya-Paris Model to Estimate Moisture Retention Data (continued)

$\Rightarrow$ John Relyea

Samples need a radiological release before transportation can be done, thus they do sit in liners. The best would be to have the liners come in after lab sample is taken.

$\Rightarrow$ Peter Wierenga

Neutron probe measurement in borehole in the field would be useful.

$\Rightarrow$ Ward Staubitz

Problems are encountered with neutron probe due to borehole diameters which are large, have different casings, etc.. We do have problems with field moisture content and water retention curves.

$\Rightarrow$ Peter Wierenga

You also have problem with field measured tension. Have you tried filter paper?

$\Rightarrow$ Ward Staubitz

We did try filter paper for awhile.

$\Rightarrow$ John Relyea

We are trying to consolidate the entire lab group to one location.

$\Rightarrow$ Peter Wierenga

Is there a protocol to a lab sample?

$\Rightarrow$ Ward Staubitz

The procedure can be found in the EIIs and should be followed. The EIIs can be changed if need to be.

$\Rightarrow$ Rien van Genuchten

Experience with fractal scaling - it is a useful tool, but not sure how it is going to help. What about relating tension to $\%$ silt, $\%$ clay, etc.?

* Ben Volk

We have a large enough database, thus we might be able to utilize this.

$\Rightarrow$ Rick Healy

You said your results are sensitive to bulk density, did you account for larger fraction in regards to particle size distribution $>2 \mathrm{~mm}$ ?

* Ben Volk

Yes - should adjust moisture retention curve. 
* Lynn Gelhar - Dispersivity Estimates

dispersivity

-a measure of contaminant spreading \& dilution

-an unpredictable fudge factor

-depends on scale

-determined by small scale heterogeneity?

Stochastic Methodology

- In $K$ variability represented by $3 D . .$. .

-macrodispersivity

Cape Cod Experiments

-depth of aquifer $=100 \mathrm{ft}$

-mass sampling -15 samples per borehole

-truly illustrates dispersion

-dilution - not smooth as in a mode?

- concentration values aren't as high as before

-a negative correlation between retardation and conductivities

-it is not right to use the same dispersivity for reactive and nonreactive cases

-need different dispersivities for retarded and non-retarded cases -a lot of spreading and dilution occur with higher longitudinal dispersivity values

-this is all saturated data

$\Rightarrow$ Scott Kindred

The problem with upper end at Hanford is due to large formation changes.

dispersivity also cepends on size of plume

unsaturated dispersion

-macro anisotropy

-macro hysteresis

-macro dispersivity

people are just beginning to see the light - give them 5 years

saturation dependance on dispersivity is a question - there is no confirmation on relationship

viewgraph - scales of variability vs. overall scale

-increase in correlation scale as overall scale increases

$\Rightarrow$ Raz Khaleel

Do not have a lot of spatial data - there is i relation to correlate the spatial and temporal variances?

* Lynn Gelhar

Skeptical - more unsaturated hydraulic conductivity work is done here at Hanford than any other places I have seen. 
* Lynn GeThar - Dispersivity Estimates (continued)

$\Rightarrow$ Rien van Genuchten

Does it depend on a steady state system?

* Lynn Gelhar

Transverse dispersion.... have not gotten that far yet to answer.

$\Rightarrow$ John Relyea

Small dispersion vertically - could it really be a 20 problem?

* Lynn Gelhar

No - even though it is small it is important to have.

$\Rightarrow$ Scott Kindred

In saturated zone, we are trying to make predictions in regard to plumes

15 miles up gradient from Columbia River. We get bad results in regards

to dispersivity.

* Lynn Gelhar

Select dispersivity in regards to 15 miles not 1 mile.

Naff result is more...

just superimpose it...

there are theories that deal w/dispersivity over a long range

$\Rightarrow$ Len Collard

Should we be looking at different dispersivities for each break or part of model?

* Lynn GeThar

Look at overall model and choose a dispersivity over a range that increases Tinearly.

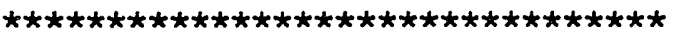

LUNCH BREAK

* Glendon Gee - Hanford Recharge

viewgraph - Hanford Site Water Balance

$\Rightarrow$ Rien van Genuchten

Why is it allowed to go on like this?

* Glendon Gee

DOE

for the past 4 years they have been looking at interim covers

$\Rightarrow$ Doug Sherwood

What are you talking about as far as area?

* Glendon Gee

West side of river. 
* Glendon Gee - Hanford Recharge (continued)

viewgraph - Groundwater Recharge

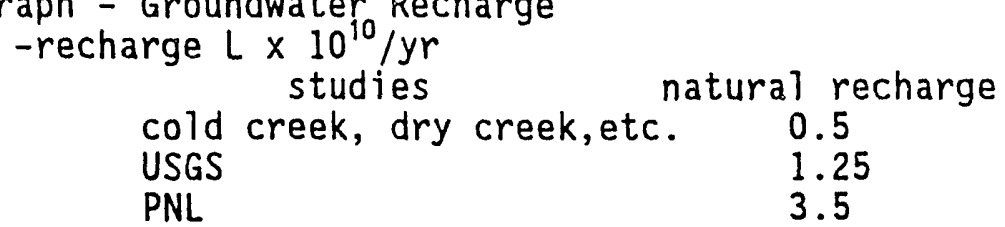

recharge is significant on Hanford site

-liquid effluents are decreasing

Past Hanford Site Recharge Studies were done in 1970s and 1980s.

Can we model recharge into the future?

\section{Conclusions}

1) Lysimeters-only way to directly measure drainage

2) arid site predictions of drainage require lysimeters

3) waste site recharge

4) $\ldots \ldots \ldots \ldots$

5) $\ldots \ldots \ldots \ldots$

$\Rightarrow$ Len Collard

What do you feel are appropriate recharge rates to use? Do you have any documentation for defense?

* Glendon Gee

It changes all over site and climate.

$\Rightarrow$ Ward Staubitz

Look at current soil cover for modeling site.

* Glendon Gee

Mode 1 is deterininistic - we shouldn't average a recharge rate. If you know precipitation rates then use that. Have to take a look at events and get a response from the system.

$\Rightarrow$ Peter Wierenga Not only depends on moisture falling, but also how it falls, i.e. if soil is dry or wet, etc..

* Glendon Gee

It is a fairly confident model - it is as good as we got.

$\Rightarrow$ Rien van Genuchten

Does the soil freeze?

* Glendon Gee

Yes

Is there a preferential flow?

* Glendon Gee

Hard to answer that. 
* Glendon Gee - Hanford Recharge (continued)

$\Rightarrow$ Peter Wierenga

May be should calibrate it over a period of both wet and dry events.

How is your vegetation by lysimeters?

* Glendon Gee

We had roots $10 \mathrm{ft}$. $-15 \mathrm{ft}$. when we dug trenches.

$\Rightarrow$ Scott Kindred

Talking of 2 things

1) natural vegetation

2) engineered design

Sagebrush roots in one lysimeter went $3 \mathrm{ft}$. .

$\Rightarrow$ Scott Kindred

Maybe we want to look at $15 \mathrm{ft}$. of sandy loam.

$\Rightarrow$ Mel Piepho

If it drains over 100 years, it is not significant.

I agree if it only happened every 100 yrs, but I am not convinced. We had the same amount of precipitation this last winter as 5 winters ago.

The difference is there was more snow this past winter.

$\Rightarrow$ Rien van Genuchten

Getting back to calibrating - Question, if you need to continue to recalibrate?

Use the model as a tool and continue.

Tracer studies are good for a natural site condition, but problems exist in terms of waste management.

$\Rightarrow$ Rien van Genuchten

Why not spray chloride on it and sample periodically?

* John Rawlins - Should summarize about workshop.

$\Rightarrow$ Raz Khaleel

Started with 3 objectives, only 1 of which was technical.

-provide audience update of some of the issues

-get feedback

Hopefully in executive session we can speak about future and speak later of funding aspects.

$\Rightarrow$ Ward Staubitz

-get an update of site characterization in vadose zone

(state of the art)

-get independent comment

-gain some confidence in that we are taking a reasonable approach and

that there is not a quick and easy way to do the work

-met most of my expectations 
$\Rightarrow$ Lynn Gelhar

-borehole injection experiment with transport simulations are great

-see more of the real database, have not seen enough of the guts

- unsaturated property characterization is important and progress is good. Need to see more screening type measurements.

-always interested in surrogate type measurements to tell you about detail

$\Rightarrow$ Raz Khaleel

Do you see an opportunity in using CERCLA type work in model val idation/cal ibration?

$\Rightarrow$ Lynn Gelhar

Like to see more on the parameters before being convinced on

validation. Problems are the base of data - calculations are relatively simple.

$\Rightarrow$ Ward Staubitz

Is model validation do-able? Is detail testing of a model necessary to increase confidence in that model? In next investigation - can you use less data?

$\Rightarrow$ Lynn Gelhar

It has to be done.

$\Rightarrow$ Doug Sherwood

-agree with Lynn Geihar

-we can not do it for unit to unit

-we can not characterize each site and get a risk for the whole site

- -need to learn to get better

$\Rightarrow$ Chuck Cline

It is critical that we know at a minimum what data is needed for a performance data.

$\Rightarrow$ John Smoot

Agree and feel that this is definitely critical.

$\Rightarrow$ Lynn Gelhar

Useful to speak about specific problems. Is tank leak a problem and what can we do to solve it?

$\Rightarrow$ Chuck Cline

Even to use a tank leak as a tracer - how much is enough data to make it sufficien'?

$\Rightarrow$ Lynn Gelhar

Right now fudging a factor into T106 study - anisotropy.

$\Rightarrow$ Rick Healy

How is this model to be used to help us solve the system? 
$\Rightarrow$ Peter Wierenga

A model is nothing more than a formalization of thoughts. Need model to make predictions or generalizations.

$\Rightarrow$ Glendon Gee

All of the waste is stored relatively close to surface and is not at the groundwater. It probably will not surface until our grandkids are dead. Personal feel that drilling wells is useless spending.

$\Rightarrow$ Ward Staubitz

Not an issue of well drilling vs. modeling.

Does modeling really tell us much?

$\Rightarrow$ Lynn Gelhar

If you can put it in to context the nature of the problems, how the sites are similar, etc., then see if you can put an answer to it.

$\Rightarrow$ Ward Staubitz

We do it by operable unit - should we model site wide? Right now it is hap hazard and there is no continuity?

$\Rightarrow$ Scott Kindred

We have actually only done vadose zone modeling on one site.

$\Rightarrow$ Peter Wierenga

Use steady state model, average recharge, and less emphasis on saturated hydraulic conductivity.

$\Rightarrow$ Peter Wierenga

If I had to choose between unsaturated hydraulic conductivity and recharge - it would be "recharge".

$\Rightarrow$ Doug Sherwood

The panel only heard about $K d$, not solubility processes. Not all contaminants at 200BPl were not Kd controlled. Did not here enough about chemistry?

$\Rightarrow$ Peter Wierenga

Should look at nitrate data.

$\Rightarrow$ Doug Sherwood

Good suggestion - we should look at it. We have 30 year old boreholes

for the objective of studying contaminants.

$\Rightarrow$ Len Collard

Problem - arrival time of data vs. when to embark on modeling aspect.

Problem - try to use a rechange that was realistic.

Problem - taking gravel into account. 
$\Rightarrow$ Rien van Genuchten

Good work is going on.

Extremely useful to sit down and have this workshop.

A lot of sitewide coordination is needed, i.e. database.

This database should be a centralized effort.

Look into other methods, i.e. Arya-Paris.

Coordination will pay off.

Recharge efforts should be supported.

Model calibration/validation - still somewhat confused, work needs to be done.

Do not only work with $\mathrm{Kd}$

$\Rightarrow$ Len Collard

Do you feel in a risk assessment environment, what degree of calibration should be done and what is defensible?

$\Rightarrow$ Rien van Genuchten

Try to calibrate as best as I could. Whatever model is chosen, calibrate using Richards calculations and use Jim Conca's data. Round off what ever gives you a higher velocity and use that as risk assessment. Have to play with model and it takes time. Look into chemistry too. Look at programs that will couple with current codes. Need a team to do it.

$\Rightarrow$ Allen Lu

Calibration/validation - feel that Injection Test Site data is still the best data.

$\Rightarrow$ Lynn Gelhar

Have not seen enough of Injection Test Site to judge it or make comments on the results. How do you actually use field data?

$\Rightarrow$ Chuck Cline

What do you tell the borehole people?

$\Rightarrow$ Lynn Gelhar

Have not used correlation between $\alpha$ and in Ks for Injection Test Site.

It is difficult for me to comment because I have not seen the data.

$\Rightarrow$ Rien van Genuchten

Get as much information as you can without drilling r.ew borehole. Then there is a modeling equation. Try to improve things as time goes on.

$\Rightarrow$ Doug Sherwood Come to Len Collard's defense. Orilling was 7 months behind schedule.

$\Rightarrow$ Ward Staubitz Len Collard was alone for funding of 200BP1. How does database address this problem?

$\Rightarrow$ Rien van Genuchten

Spend a million for these issues of database. Instead of drilling new borehole, put funds toward developing database - this is the key. 
$\Rightarrow$ Ward Staubitz

Good recommendation.

$\Rightarrow$ Peter Wierenga

-should be more effort in coordinating

-look at nitrate data

-drill borehole downstream of tank

-do not make modeling so complex

-do not need all unsaturated hydraulic conductivity models

-go about it in a simplified approach

-continue with seminars : suggest vadose zone seminars

-speak to politiciaris

$\Rightarrow$ Rick Healy

Agree with stressing simplified modeling approach.

$\Rightarrow$ Peter Wierenga

In terms of model simulation/validation, I would be inclined to set up a new experiment. Bring group together and do a detailed design - hard to do mass balance on existing crib.

$\Rightarrow$ Scott Kindred

Do simple calculations and keep model simple. I feel that spending money on a detail field experiment is a good idea.

$\Rightarrow$ Rien Van Genuchten

Research is very important.

$\Rightarrow$ Scott Kindred

Do we have enough information to make these kind of decisions.

$\Rightarrow$ Ward Staubitz

There is no support for research right now.

$\Rightarrow$ John Rawlins

Concentrate on CERCLA issues.

$\Rightarrow$ Chuck Cline

Defend what we have to do to DOE to get funding.

$\Rightarrow$ John Rawlins

We have a couple months to get together to develop a proposal for funding.

$\Rightarrow$ Chuck Cline

Keep in mind that funding is for 2 years away.

$\Rightarrow$ Doug Sherwood

We have not looked into other sites - grout, etc..

DOE keeps those out of CERCLA realm for some reason.

$\Rightarrow$ Raz Khaleel

We need to get copies made from all the presentations and a report will be done. 
$\Rightarrow$ Ward Staubitz Where do we go from here? Are semi-monthly meetings agreeable?

$\Rightarrow$ Raz Khaleel We need to talk about that. A lot of work is being done by several individuals in different groups. We need to see if we can get everyone involved and get upper management support.

$\Rightarrow$ Ward Staubitz

Bob Stewart is the new technica! branch chief - DOE contact.

Meeting closed 
WHC-MR-0420

This page left intentionally blank. 
WHC-MR-0420

\section{LIST OF WORKSHOP ATTENDEES}


WHC-MR-0420

This page left intentionally blank. 


\section{VADOSE ZONE MODELING WORKSHOP \\ Richland, WA}

\section{LIST OF ATTENDEES}

$\begin{array}{ll}\text { Chuck Cline } & \text { Washington Dept. of ECOLOGY } \\ \text { Len Collard } & \text { WHC } \\ \text { James Conca } & \text { Washington State University, Tri-Cities } \\ \text { Jerry Davis } & \text { WHC } \\ \text { Truc De Lorenzo } & \text { WHC } \\ \text { David Duranceau } & \text { WHC } \\ \text { Eugene Freeman } & \text { WHC } \\ \text { Glendon Gee } & \text { PNL } \\ \text { Lynn Gelhar } & \text { MIT } \\ \text { Rien van Genuchten } & \text { USDA, ARS } \\ \text { Dori Harris } & \text { WHC } \\ \text { Rick Healy } & \text { USGS-Denver } \\ \text { Paula Heller } & \text { WHC } \\ \text { Raz Khaleel } & \text { WHC } \\ \text { Charley Kincaid } & \text { PNL } \\ \text { Scott Kindred } & \text { GOIder Associates } \\ \text { Niall Kline } & \text { WHC } \\ \text { Al Law } & \text { WHC } \\ \text { Allen Lu } & \text { WHC } \\ \text { Fred Mann } & \text { WHC } \\ \text { William McMahon } & \text { WHC } \\ \text { Clarence Oster } & \text { BCSR } \\ \text { Mel Piepho } & \text { WHC } \\ \text { John Rawlins } & \text { WHC } \\ \text { John Relyea } & \text { WHC } \\ \text { Mark Rockhold } & \text { PNL } \\ \text { Frank Schmittroth } & \text { WHC } \\ \text { Doug Sherwood } & \text { EPA } \\ \text { John Smoot } & \text { PNL } \\ \text { Ward Staubitz } & \text { USGS-Tacoma } \\ \text { Howard Sutherland } & \text { WHC } \\ \text { Peter Wierenga } & \text { Univ. of Arizona } \\ \text { Marc Wood } & \text { WHC } \\ \end{array}$


WHC-MR-0420

This page left intentionally blank. 


\section{DISTRIBUTION}

Number of copies

\section{OFFSITE}

1

Massachusetts Institute of Technology Ralph M. Parsons Laboratory

Department of Civil Engineering

Cambridge, Massachusetts 02139

Dr. L. W. Gelhar

1

University of Arizona

Department of Soil and Water Sciences

Tucson, frizona 85721

Dr. P. J. Wierenga

2

U.S. Geological Survey 1201 Pacific Avenue, Suite 600

Tacoma, washington 98402

B. W. Drost

W. W. Staubitz

1

U.S. Geological Survey

District Office

Building 53, Denver Federal Center

MS 415, Box 25046

Lakewood, Colorado 80225

Dr. R. W. Healy

1

U.S. Salinity Laboratory

4500 Glenwood Drive

Riverside, Cal ifornia 92501

Dr. M. van Genuchten

1

Washington State Department of Ecology P.0. Box 47600

01ympia, Washington 98504-7600

C. Cline 
DISTRIBUTION (cont.)

Number of copies

ONSITE

4

U.S. Department of Energy, Richland Operations office

E. D. Goller

A5-19

A. C. Harris

A5-19

R. K. Stewart

A5- 19

RL Public Reading Room

Al-65

1

U.S. Army Corps of Engineers

M. P. Johansen

A5-19

2

U.S. Environmental Protection Agency

P. R. Beaver

B5-01

D. R. Sherwood

B5-01

5

Pacific Northwest Laboratory

M. J. Fayer

K6-77

G. W. Gee

$\mathrm{K} 6-77$

C. T. Kincaid

$\mathrm{K} 6-77$

M. L. Rockhold

$\mathrm{K} 6-77$

J. L. Smoot

K6-77

1

Washington State University, Tri-Cities

J. Conca

H2-52

46

Westinghouse Hanford Company

H. L. Benny

L7-09

D. J. Brown

B4-63

J. W. Cammann

$\mathrm{H} 4-14$

S. W. Clark

H6-01

L. B. Collard

M. P. Connelly

J. D. Davis

$\mathrm{H} 6-01$

H6-06

T. H. Delorenzo

H6-01

D. A. Duranceau

HO-36

C. H. Eccleston

H4-14

S. H. Finfrock

H6-26

R. D. Fox

HO-38

E. J. Freeman

H6-07

E. W. Giese

HO-36

P. R. Heller

B5-25

R. T. Hirano

L4-69

B5-25

Distr-2 


\section{DISTRIBUTION (cont.)}

\section{Number of copies}

R. L. Jackson

R. Khaleel

N. W. Kline

A. J. Knepp

D. W. Langford

M. J. Lauterbach

A. G. Law

T. Legore

A. H. Lu

F. M. Mann

W. J. McMahon

C. A. Oster

S. J. Phillips

M. G. Piepho

R. F. Raidl

J. A. Rawl ins

J. F. Relyea

K. D. Reynolds

P. D. Rittmann

F. A. Schmittroth

M. R. Schwab

J. C. Sonnichsen

W. H. Sutherland

H. Toffer

D. E. Wood

M. I. Wood

Central Files

Information Release

Administration

Document Processing

and Distribution (2)
H6-06

HO-36

HO-36

H6-06

B5-25

H6-01

H6-06

B4-63

HO-36

HO-36

H6-06

B5-25

H4-14

HO-36

H6-06

HO-36

L4-69

H6-06

HO-36

HO- 35

H6-07

H4-14

L5-06

HO-38

HO-33

N3-13

L8-04

A3-36

L8-15 

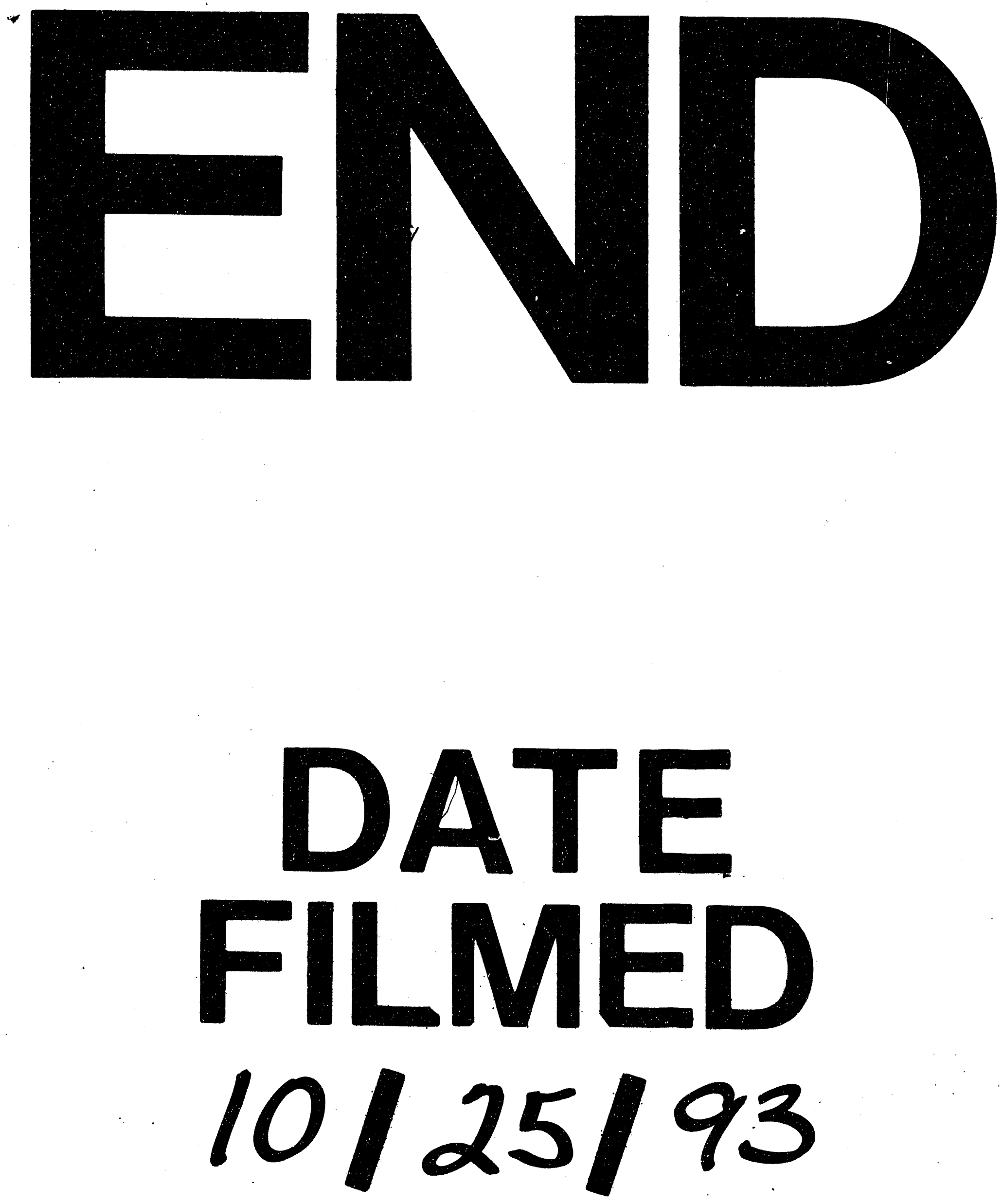

1 
